SERI/STR-253-2859

DE86010703

\title{
Wind Load Reduction for Heliostats
}

\section{A Subcontract Report}

\author{
J. A. Peterka \\ N. Hosoya \\ B. Bienkiewicz \\ J. E. Cermak \\ Colorado State University
}

Prepared under Subcontract No. XX-4-04029-1

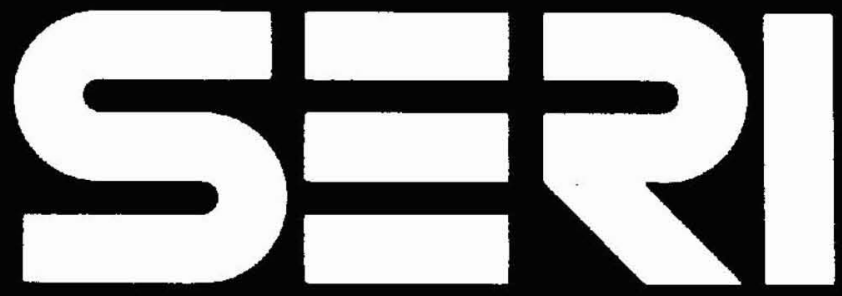

Solar Energy Research Institute

A Division of Midwest Research Institute

1617 Cole Boulevard

Golden, Colorado 80401-3393

Operated for the

U.S. Department of Energy

under Contract No. DE-AC02-83CH10093 


\section{NOTICE}

This report was prepared as an account of work sponsored by the United States Government. Neither the United States nor the United States Department of Energy, nor any of their employees, nor any of their contractors, subcontractors, or their employees, makes any warranty, expressed or implied, or assumes any legal liability or responsibility for the accuracy, completeness or usefulness of any information, apparatus. product or process disclosed, or represents that its use would not infringe privately owned rights.

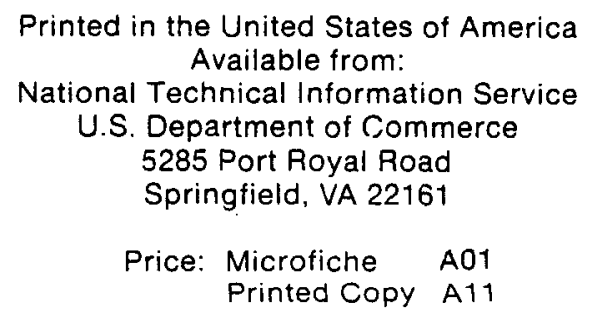

Codes are used for pricing all publications. The code is determined by the number of pages in the publication. Information pertaining to the pricing codes can be found in the current issue of the following publications, which are generally available in most libraries: Energy Research Abstracts, (ERA); Government Reports Announcements and Index (GRA and 1); Scientific and Technical Abstract Reports (STAR); and publication. NTIS-PR-360 available from NTIS at the above address. 
SERI/STR-253-2859

UC Category: 62a

DE86010703

\title{
Wind Load Reduction for Heliostats
}

\section{A Subcontract Report}

\author{
J. A. Peterka \\ N. Hosoya \\ B. Bienkiewicz \\ J. E. Cermak \\ Colorado State University
}

May 1986

SERI Technical Monitor:

L. M. Murphy

Prepared under Subcontract No. XX-4-04029-1

Solar Energy Research Institute

A Division of Midwest Research Institute

1617 Cole Boulevard

Golden, Colorado 80401-3393

Prepared for the

U.S. Department of Energy

Contract No. DE-AC02-83CH-10093 


\section{FOREWORD}

The research and development described in this document was conducted within the U.S. Department of Energy's Solar Thermal Technology Program. The goal of this program is to advance the engineering and scientific understanding of solar thermal technology and to establish the technology base from which private industry can develop solar thermal power production options for introduction into the competitive energy market.

Solar thermal technology concentrates the solar flux using tracking mirrors or lenses onto a receiver where the solar energy is absorbed as heat and converted into electricity or incorporated into products as process heat. The two primary solar thermal technologies, central receivers and distributed receivers, employ various point and 1 ine-focus optics to concentrate sunlight. Current central receiver systems use fields of heliostats (two-axis tracking mirrors) to focus the sun's radiant energy onto a single, towermounted receiver. Point focus concentrators up to 17 meters in diameter track the sun in two axes and use parabolic dish mirrors or Fresnel lenses to focus radiant energy onto a receiver. Troughs and bowls are line-focus tracking reflectors that concentrate sunlight onto receiver tubes along their focal lines. Concentrating collector modules can be used alone or in a multimodule system. The concentrated radiant energy absorbed by the solar thermal receiver is transported to the conversion process by a circulating working fluid. Receiver temperatures range from $100^{\circ} \mathrm{C}$ in low-temperature troughs to over $1500^{\circ} \mathrm{C}$ in dish and central receiver systems.

The Solar Thermal Technology Program is directing efforts to advance and improve each system concept through solar thermal materials, components, and subsystems research and development and by testing and evaluation. These efforts are carried out with the technical direction of DOE and its network of field laboratories that works with private industry. Together they have established a comprehensive, goal-directed program to improve performance and provide technically proven options for eventual incorporation into the Nation's energy supply.

To successfully contribute to an adequate energy supply at reasonable cost, solar thermal energy must be economically competitive with a variety of other energy sources. The Solar Thermal Program has developed components and system-level performance targets as quantitative program goals. These targets are used in planning research and development activities, measuring progress, assessing alternative technology options, and developing optimal components. These targets will be pursued vigorously to ensure a successful program.

This report presents the results of wind-tunnel tests supported through the Solar Energy Research Institute (SERI) by the Office of Solar Thermal Technology of the U.S. Department of Energy as part of the SERI research effort on innovative concentrators. As gravity loads on drive mechanisms are reduced through stretched-membrane technology, the wind-load contribution of the required drive capacity increases in percentage. Reduction of wind loads can provide economy in support structure and heliostat drive. Wind-tunnel tests have been directed at finding methods to reduce wind loads on heliostats. The tests investigated primarily the mean forces, the moments, 
and the possibility of measuring fluctuating forces in anticipation of reducing those forces. A significant increase in ability to predict heliostat wind loads and their reduction within a heliostat field was achieved.

The work reported here was monitored by L. M. Murphy of SERI.

Approved for

SOLAR ENERGY RESEARCH INSTITUTE

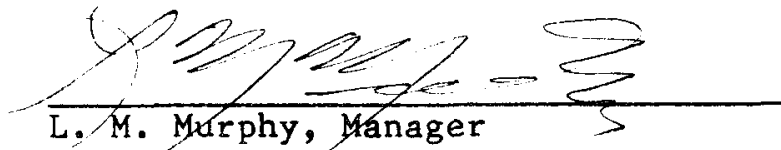

Thermal Systems Research Branch

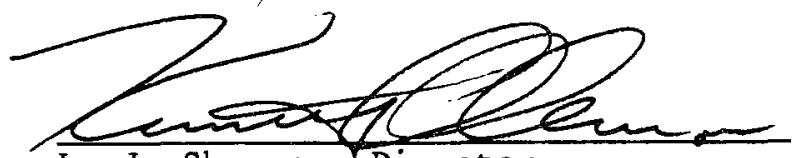

L. J. Shannon, Director

Solar Heat Research Division 
SUMMARY

The purpose of this study was to develop a more complete understanding of wind loading on solar collectors with a major emphasis on investigating methods of reducing wind loads on heliostats in typical array fields. The reason for decreasing wind loads is to improve the economy of heliostat support structure and drive mechanisms, both of which will become more sensitive to wind loads as gravity loads decrease through stretched membrane technology. Concepts investigated included perimeter fences or berms, fences or other wind-blockage elements within the field, field density, and drag-modifying devices attached to the heliostat. The primary method of investigation was wind-tunnel tests in a boundary-layer wind tunnel designed to model atmospheric surface layer winds.

Wind loads on specific heliostat field geometries had been obtained in earlier wind-tunnel studies, for mean (time averaged) loads. Those tests were not sufficient to provide designers with methods to optimize heliostat field performance and cost by including wind loading as a variable under design control. Wind-tunnel tests in this study were performed on isolated and within-field heliostats mounted on a six-component force and moment balance. The influence of perimeter fences, in-field fences, and field density on heliostat wind loads were determined for mean heliostat loads. Some fluctuating load measurements on heliostats were obtained on a balance for the first time. Initial indications of peak wind loads on a heliostat were obtained to replace gust factor approaches used in the past to determine peak loads from mean loads.

Results of the investigation for reduction of mean heliostat wind loads are summarized in Figure S-1. In this figure $C_{F}$ represents wind force, $\mathrm{C}_{\mathrm{M}}$ represents elevation torque and $\mathrm{C}_{\mathrm{M}}$ represents azimuthal torque about the support post. The ordinates in S-1 are the forces or moments in the field divided by the isolated heliostat loads and show the reduction in load as a function of upwind blockage which is the abscissa. The generalized blockage area is the surface area of upwind obstacles such as heliostats, fences, or berms projected onto a plane perpendicular to the wind direction per unit ground area. The data collapse quite well onto or below a single curve. This finding provides a powerful tool for optimization of field layout by designers.

Fluctuating load measurements were limited in scope and complicated by wind-tunnel scaling requirements. The measurements showed that the fluctuating part of the wind load decreases within a field environment in comparison to that on an isolated heliostat. The decreased fluctuating load component combined with a decreased mean load resulted in a net decrease in peak load.

Major conclusions from the study are:

- Mean wind loads on heliostats can be reduced to below 30 percent of isolated heliostats by appropriate design of the field and external fences or berms. 
- A simple design-oriented prediction method for mean heliostat wind loads in a field has been developed.

- Peak dynamic loads are significantly lower within a field than at the edge for heliostats in operational positions.

- Limited analysis of dynamic loads has not identified a loading mechanism indicating that on-heliostat spoilers would be beneficial in decreasing mean and dynamic wind loads.

- The strength of a collector should be based on the peak load rather than a mean load multiplied by an assumed gust factor.

- Full-scale wind loads are not available for comparison with windtunnel data.

- Design forces perpendicular to the mirror plane for an isolated heliostat are controlled by operational winds $(50 \mathrm{mph})$ while design drive moments are controlled by survival winds ( $90 \mathrm{mph})$.

Recommendations for future study include further work on fluctuating loads, local and integral loads on typical isolated stretched membrane heliostats, and additional synthesis of wind-load data into a design-oriented methodology for control of wind loads. Specific tasks include

- Application of the generalized blockage area concept to fluctuating loads

- Better resolution of stow position loads

- Development of a design guideline

- Comparison of wind-tunnel loads with full-scale loads. 

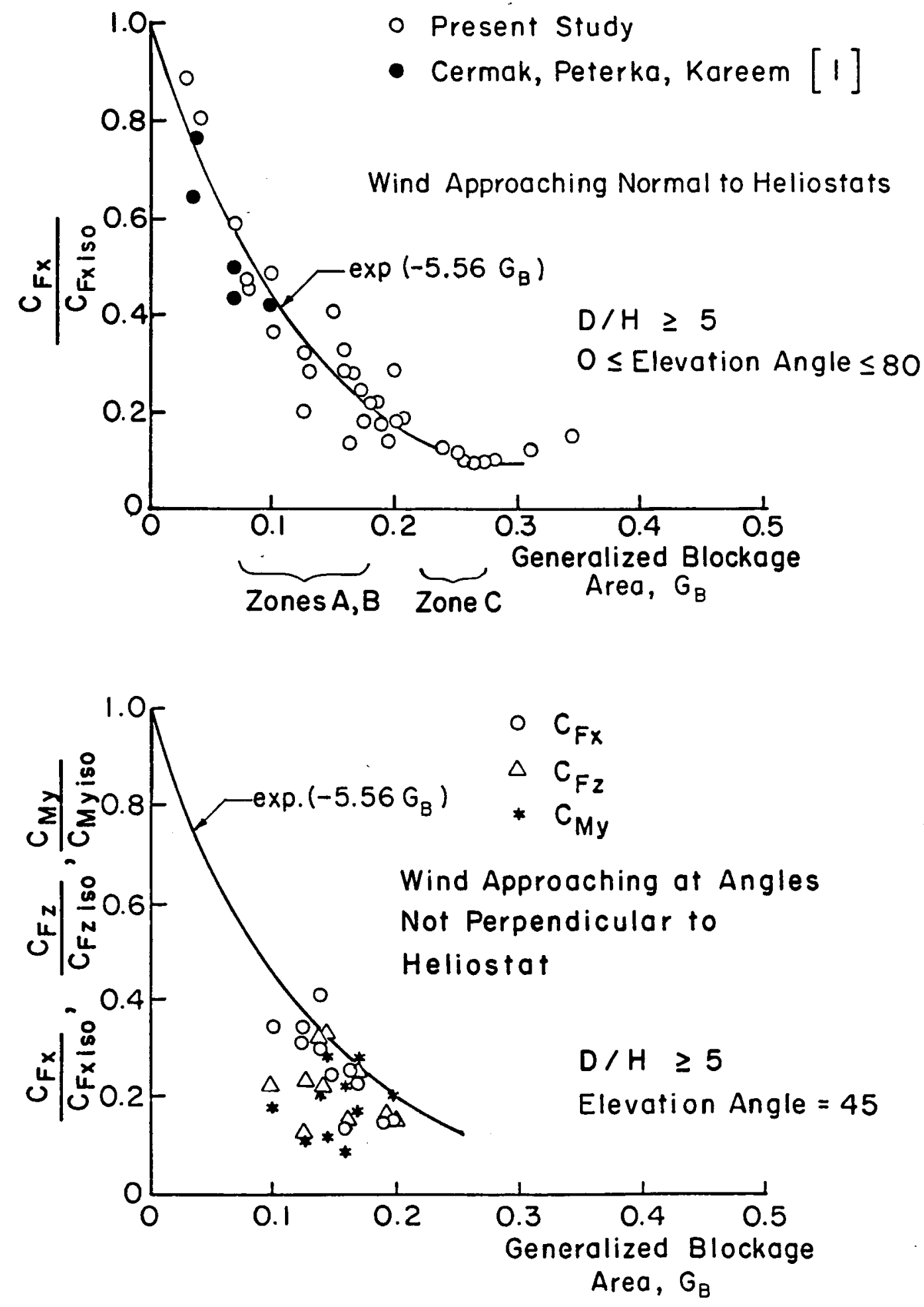

Figure S-1. Mean Load Reduction as a Function of Generalized Blockage 


\section{TABIE OF CONTENTS}

\section{Page}

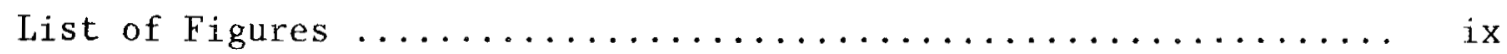

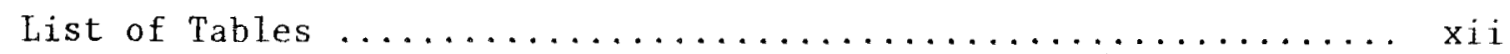

Nomenclature $\ldots \ldots \ldots \ldots \ldots \ldots \ldots \ldots \ldots \ldots \ldots \ldots \ldots \ldots \ldots \ldots \ldots \ldots \ldots \ldots \ldots$

$1.0 \quad$ Introduction $\ldots \ldots \ldots \ldots \ldots \ldots \ldots \ldots \ldots \ldots \ldots \ldots \ldots \ldots \ldots$

1.1 Previous Work $\ldots \ldots \ldots \ldots \ldots \ldots \ldots \ldots \ldots \ldots \ldots \ldots \ldots \ldots$

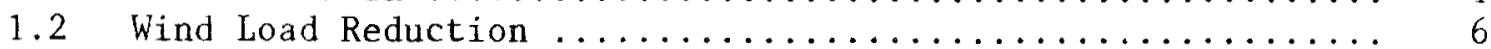

1.3 Modeling of Atmospheric Wind Loads ................. 9

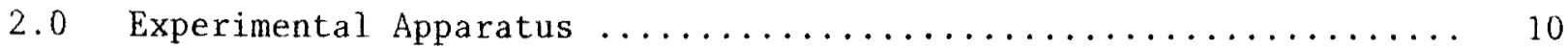

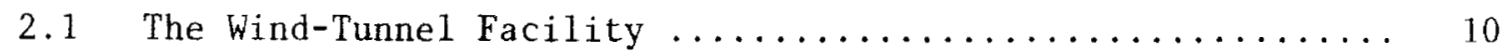

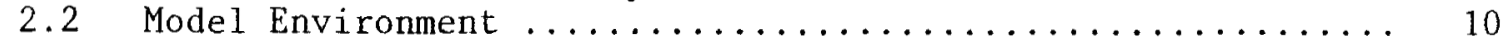

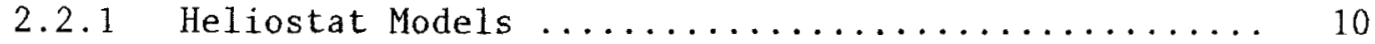

2.2.2 Wind Protective Fences .................. 17

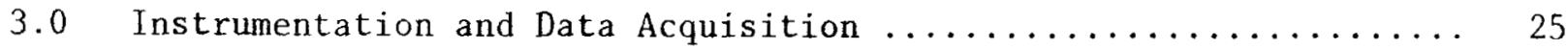

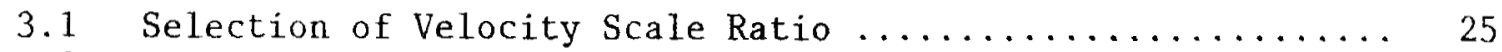

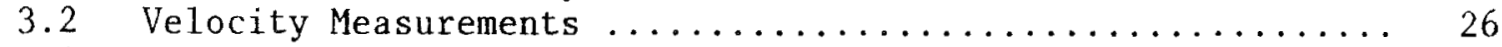

3.3 Force and Moment Measurements ................... 28

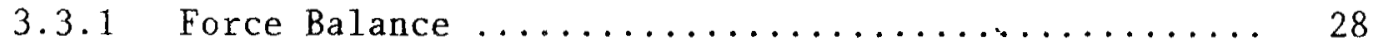

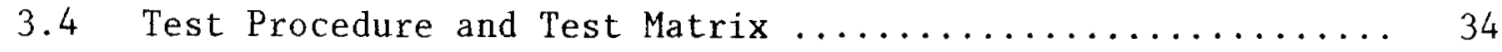

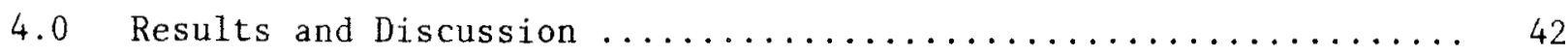

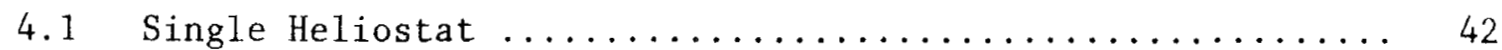

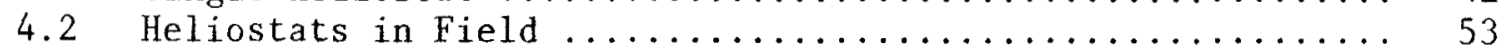

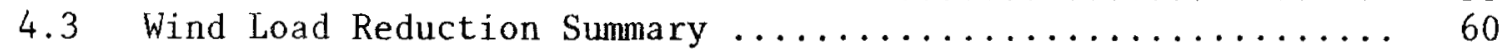

4.4 Comparison of Model Data with Full Scale .............. 65

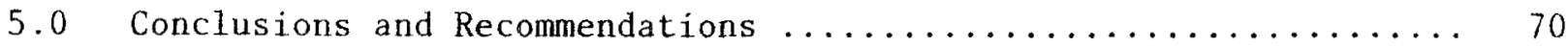

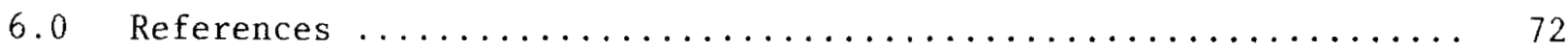

Appendix A - Validation of Wind-Tunnel Testing in Civil Engineering

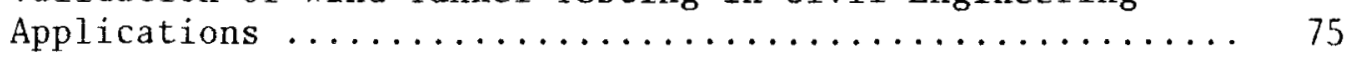

Appendix B - Wind-Tunnel Data for Isolated Heliostat ............ 90

Appendix C - Wind-Tunnel Data for Field Heliostats ............. 114 
LIST OF FIGURES

Figure

$\underline{\text { Page }}$

1-1 Velocity Reductions within a Field of Heliostats ......... 2

1-2 Influence of Fence Height on Front Collector Drag Force ..... 3

1-3 Heliostat Field for Tests of Reference [2] $\ldots \ldots \ldots \ldots \ldots \ldots \ldots$

1-4 Moment Coefficients for a High Density Heliostat

Field Array - Zone A ........................ 5

1-5 Moment Coefficients for a Low Density Heliostat

Field Array - Zone B ....................... 5

1-6 Concepts for Interior Field Wind Protection $\ldots \ldots \ldots \ldots \ldots \ldots$

1-7 Fence Configuration for Lowering Velocities Interior

to Field ............................... 7

1-8 Concepts for Reducing Fluctuating Loads ............. 8

2-1 Meteorological wind Tunnel $\ldots \ldots \ldots \ldots \ldots \ldots \ldots \ldots \ldots \ldots \ldots \ldots$

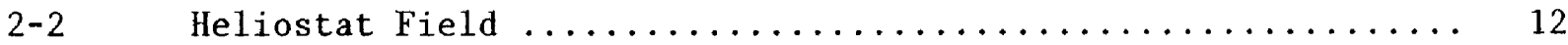

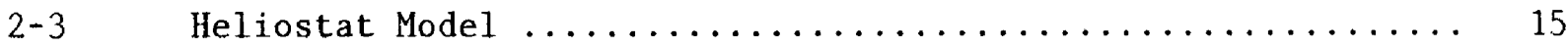

2-4 Metric Heliostat Mounted on a Six-Component Balance ....... 16

2-5 Fence Arrangement for Heliostat $1 \ldots \ldots \ldots \ldots \ldots \ldots \ldots$

2-6 Fence Arrangement for Heliostat $5 \ldots \ldots \ldots \ldots \ldots \ldots \ldots$

2-7 Arrangement of Field Density around Heliostat $3 \ldots \ldots \ldots$

2-8 Fence Arrangement for Heliostat 3 with Reduced Field

2-9 Fence Arrangement for Heliostat 3 with Reduced Field

2-10 Model Installed in the Wind Tunnel $\ldots \ldots \ldots \ldots \ldots \ldots \ldots \ldots$

2-11 Porous Fence Segments Used with the Model ............. 24

3-1 Approach Wind Profiles $\ldots \ldots \ldots \ldots \ldots \ldots \ldots \ldots \ldots \ldots \ldots \ldots \ldots$

3-2 Turbulence Power Spectrum of Approach wind . . . . . . . . . 29

3-3 Force and Moment Coordinate System ............... 30 
3-4 Force Balance Frequency Response $\ldots \ldots \ldots \ldots \ldots \ldots \ldots \ldots$

4-1 Mean Force and Moment Coefficients for an Isolated

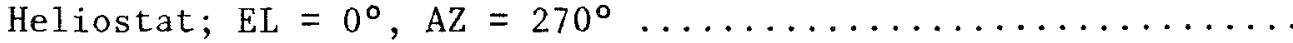

4-2 Mean Force and Moment Coefficients for an Isolated

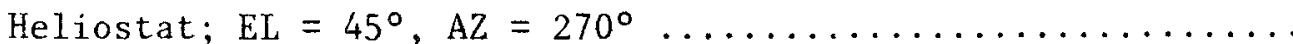

4-3 Mean Force and Moment Coefficients for an Isolated

Heliostat; $\mathrm{EL}=80^{\circ}, \mathrm{AZ}=270^{\circ} \ldots \ldots \ldots \ldots \ldots \ldots \ldots \ldots \ldots \ldots \ldots \ldots \ldots$

4-4 Mean Force and Moment Coefficients for an Isolated

Heliostat; $\mathrm{EL}=84^{\circ}, \mathrm{AZ}=270^{\circ} \ldots \ldots \ldots \ldots \ldots \ldots \ldots \ldots \ldots \ldots \ldots \ldots \ldots \ldots$

4-5 Mean Force and Moment Coefficients for an Isolated

Heliostat; $\mathrm{EL}=87^{\circ}, \mathrm{AZ}=270^{\circ} \ldots \ldots \ldots \ldots \ldots \ldots \ldots \ldots \ldots \ldots \ldots$

4-6 Mean Force and Moment Coefficients for an Isolated

Heliostat; $\mathrm{EL}=90^{\circ}, \mathrm{AZ}=270^{\circ} \ldots \ldots \ldots \ldots \ldots \ldots \ldots \ldots \ldots \ldots \ldots \ldots \ldots$

4-7 Mean Force and Moment Coefficients for an Isolated

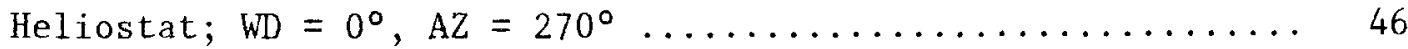

4-8 Mean Force and Moment Coefficients for an Isolated

Heliostat; $\mathrm{WD}=45^{\circ}, \mathrm{AZ}=270^{\circ} \ldots \ldots \ldots \ldots \ldots \ldots \ldots \ldots \ldots \ldots \ldots \ldots \ldots$

4-9 Comparison of Measured $C_{F}$ to Previous Measurements ...... 47

4-10 Maximum, Mean and Minimum Force and Moment Coefficients;

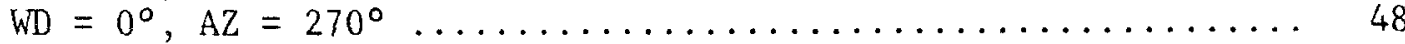

4-11 Maximum, Mean and Minimum Force and Moment Coefficients;

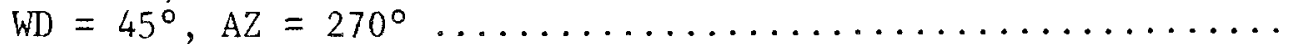

4-12 Power Spectrum of Force Coefficients; WD $=0^{\circ}$,

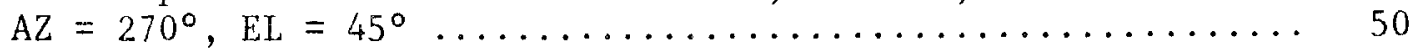

4-13 Power Spectrum of Force Coefficient for an Isolated

Heliostat; $\mathrm{WD}=45^{\circ}, \mathrm{AZ}=270^{\circ}, \mathrm{EL}=45^{\circ} \ldots \ldots \ldots \ldots \ldots$

4-14 Heliostat 1 - Representative Mean Force and Moment

Coefficients, Summer AM ..................... 54

4-15 Heliostat 1 - Representative Mean Force and Moment

Coefficients, Summer Noon .................... 55

4-16 Heliostat 5 - Representative Mean Force and Moment

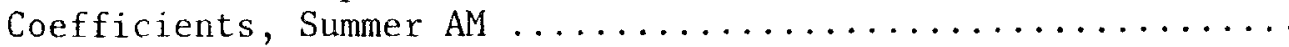

4-17 Heliostat 5 - Representative Mean Force and Moment

Coefficients, Summer Noon ..................... 58 
4-18 Mean, Max and RMS CFX for Heliostat 3--with Varying

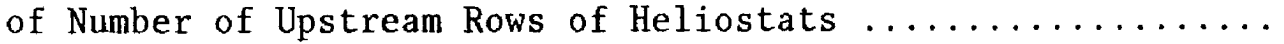

4-19 Mean, Max and RMS CFX for Heliostat 3--with Various Field Densities .......................... 61

4-20 Definition of Generalized Blockage Area ............. 61

4-21 Effects of Fences and Number of Upstream Rows of Heliostats on Mean CFX, Heliostat 3 within Low Density Field ......... 62

4-22 Effects of Fences on Mean CFX, Heliostat 3 within Low Density Field with Two Upstream Rows of Heliostats ........ 63

4-23 Effects of Fences on Mean CFX, Heliostat 3 within Low Density Field with Two Upstream Rows of Heliostats .........

4-24 Wind Loads on Heliostat 4 for a Summer Noon Case for Wind Direction 265 Degrees ............................ 64

4-25 Mean Load Reduction as a Function of Generalized Blockage ... 66

4-26 Full-Scale Wind Data $[\operatorname{Ref} .31] \ldots \ldots \ldots \ldots \ldots \ldots \ldots \ldots \ldots \ldots \ldots \ldots$

4-27 Model and Full-Scale wind Speed Comparison ............ 69 


\section{LIST OF TABLES}

Table $\quad \underline{\text { Page }}$

3-1 Test Matrix for Single Heliostat $\ldots \ldots \ldots \ldots \ldots \ldots \ldots \ldots \ldots$

3-2 Test Matrix for In-Field Heliostats $\ldots \ldots \ldots \ldots \ldots \ldots \ldots \ldots$

3-3 Description of Fences $\ldots \ldots \ldots \ldots \ldots \ldots \ldots \ldots \ldots \ldots \ldots \ldots \ldots \ldots \ldots \ldots \ldots \ldots$ 


\section{NOMENCLATURE}

Symbo1

\section{Definition}

A

$\mathrm{AZ}$

$\mathrm{A}_{\mathrm{B}}$

${ }^{\mathrm{A}} \mathrm{F}$

${ }^{A}$ ref

B

C

$\mathrm{C}_{\mathrm{x}, \mathrm{y}, \mathrm{z}}$

$\mathrm{C}_{\mathrm{x}, \mathrm{y}, \mathrm{z}}$

D

E

EL

$F_{x, y, z}$

f

$\tilde{\mathrm{f}}$

$g(t)$

G

$\mathrm{G}_{\mathrm{B}}$

$G(f)$
Constant

Azimuth angle of heliostat, $270^{\circ}$ faces south, $180^{\circ}$ faces west

Blockage area projected onto a plane perpendicular to approach wind direction

Field area containing blocking elements used for $A_{B}$

Reference area 19.6 in $^{2}$ model, $489.9 \mathrm{ft}^{2}$ full scale

Constant

Constant

Force coefficient, $\frac{F_{x, y, z}}{\left(\frac{\mathrm{U}_{\text {ref }}^{2}}{2}\right)\left(A_{\text {ref }}\right)}$

Moment coefficient, $\frac{M_{x, y, z}}{\left(\frac{\rho U_{\text {ref }}^{2}}{2}\right)\left(A_{\text {ref }}\right)\left(L_{\text {ref }}\right)}$

Distance from heliostat under consideration to the external fence

Hot-wire output voltage

Vertical angle of heliostat, zero degree for vertical

Measured force along axis, positive force in positive axis direction

Frequency, $\mathrm{Hz}$

Nondimensional frequency, $\frac{\mathrm{fL}}{\mathrm{U}}$

Fluctuating part of a time varying signal

Gust factor for load or velocity, $\frac{\text { peak }}{\text { mean }}$

Generalized blockage, $\frac{A_{B}}{A_{F}}$

Power spectral density 


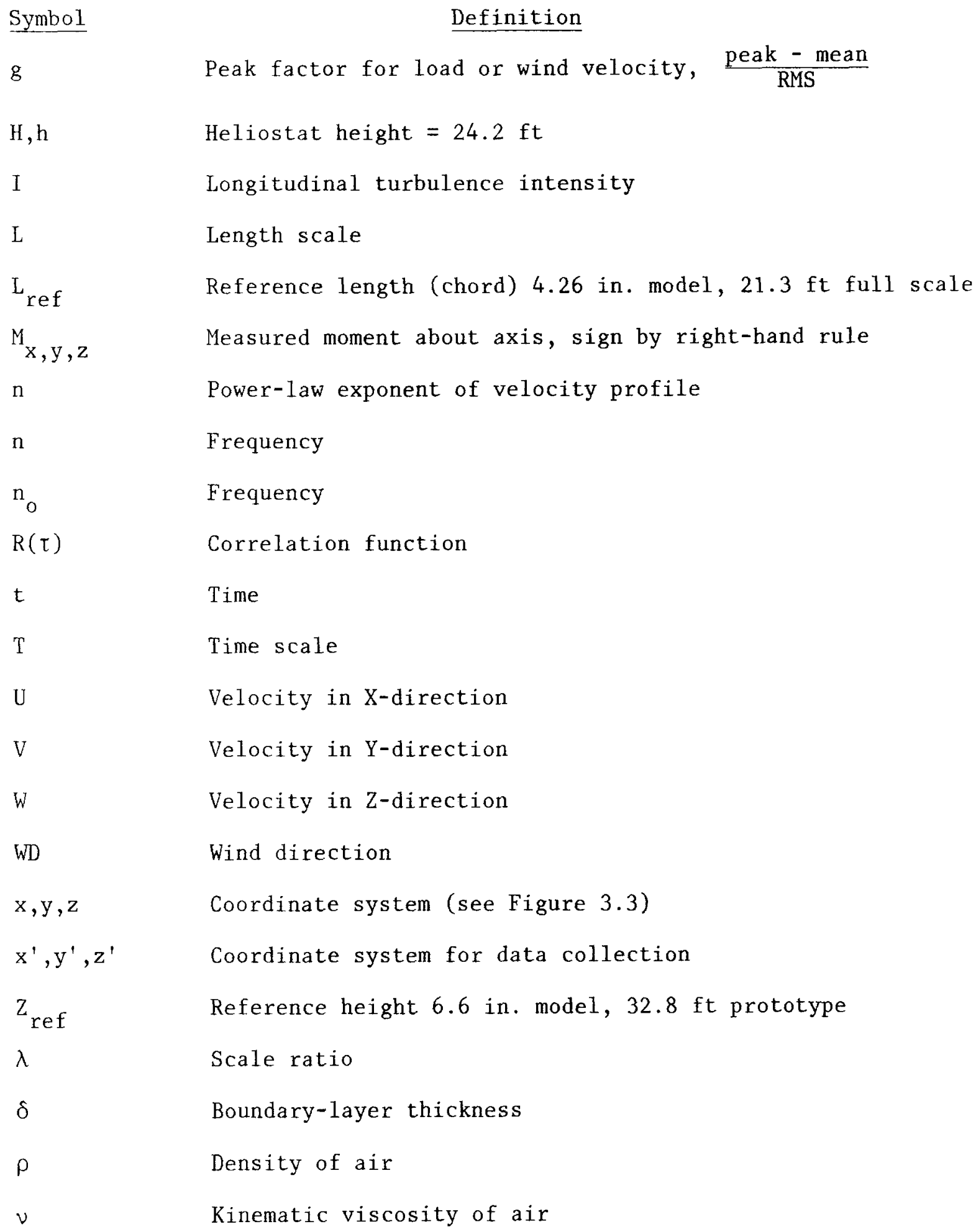


Symbol

$\sigma_{u, v, w}$
$\beta$
$\zeta$
$w$

Symbol

Subscript

$\begin{array}{ll}\text { rms } & \text { Root-mean-square } \\ \mathrm{X}, \mathrm{Y}, \mathrm{Z} & \text { Directional indicator } \\ \mathrm{L} & \text { Length } \\ \mathrm{T} & \text { Time } \\ \mathrm{f} & \text { Frequency } \\ \text { ISO } & \text { Isolated } \\ \text { mean } & \text { Mean value } \\ \text { ref } & \text { Reference } \\ \mathrm{U} & \text { Velocity } \\ \mathrm{p} & \text { Prototype } \\ \mathrm{m} & \text { Model }\end{array}$

Wind angle to the deck

Damping ratio

$2 \pi f$

Definition

RMS velocity in $\mathrm{x}, \mathrm{y}, \mathrm{z}$ directions

\section{Definition}


SECTION 1.0

INTRODUCTION

The need to understand and quantify wind loading effects on individual and fields of tracking solar collectors has emerged as an important design and development issue. This understanding is important in the cost effective design of both conventional concentrators and innovative low-cost concepts which can be considerably less robust than their conventional counterparts. Moreover, to aid in the development of that understanding, methods for the adequate modeling and simulation of wind loading on individual concentrators within the actual field environment are needed.

The quantification and understanding of wind loading effects are needed in support of the low-cost development effort from two perspectives. First, realistic design requirements must be set to allow efficient, nonconservative, and thereby low-cost, structural designs which still result in good concentrator performance. The structural design must consider the design of the drive (for tracking) support structure, and foundation in addition to the reflector structure. Second, this knowledge base is needed to ultimately allow the design of collector fields which avoid or minimize the effect of wind loading both during solar operation and when the collector field is being protected in survival loading conditions.

This study was commissioned mainly to find methods for reducing wind loads within a heliostat field to values well below those acting on an isolated unit. The methods for reduction, based on earlier work discussed below, were anticipated to rely on perimeter wind fences, in-field wind fences, field density, and possibly spoilers attached to the heliostats. Primary efforts were directed to mean wind loads; however, initial efforts were expanded to determine the magnitude of fluctuating loads. The results of this study provide a basis for reduction of mean heliostat wind loads and show that fluctuating loads decrease with distance into a field.

\subsection{PREVIOUS WORK}

A number of studies have addressed the wind loads on ground-based solar collectors and means of reduction of those wind loads [1-14]. These studies measured wind loads on heliostats $[1,2]$ photovoltaic collectors $[3,5-7,10-14]$, and parabolic trough collectors $[4,8-9]$. Other studies have addressed roofbased collectors [15-18], and older studies of dish antennas [19-21]. A review of wind load studies is given in reference [22].

Design wind loads for photovoltaic panels and parabolic collectors in large fields have been reduced substantially below those which would be required by typical wind codes by appropriate application of wind engineering analysis. Wind-tunnel tests of array fields modeled at small scale in boundary-layer wind tunnels have revealed that the dense packing of the photovoltaic and parabolic array fields provided a natural reduction in wind load below that of an isolated collector module by blocking wind from penetrating into the 
central area of the field at the height of the collectors. Collector units at the edges of the field were not protected by the field but their wind loads could be reduced to levels comparable to those in the field interior by a properly designed porous wind fence around the field periphery.

In studies of heliostats [1,2], wind-tunnel tests revealed that some reduction in wind load did occur in the interior of the heliostat field. However, the relatively loose packing of the heliostats in the field, in comparison to the photovoltaic or parabolic collector fields, prevented the large reduction in wind loads that were observed in the denser fields. Wind fences at the edge of the field did provide significant reduction in wind loads for heliostats at the edge of the field, but wind loads on units in the center of the field remained unaffected by the periphery wind fence.

The interaction of winds with a heliostat field is shown schematically in Figure 1-1. In Figure 1-1a, turbulent boundary layer winds are shown approaching a field on the left and within the field on the right. The presence of heliostats causes a decrease within the field of mean wind speed over the height of the heliostats as a consequence of wind impingement on upwind heliostats. The reduction in mean wind is accompanied by an increase in turbulent kinetic energy (gustiness) of the wind. In comparison to the heliostat at the edge of the field, units interior to the field experience lower mean wind loads and often decreased fluctuations in wind load about the mean. The reduction in mean wind loads within solar collector fields has been measured in wind-tunnel tests cited above; dynamic loads have only been measured in a limited way for photovoltaic collectors $[13,14]$. The mean load reduction within the field depends greatly on field density and heliostat pitch and azimuthal angle. Insufficient data were available to generalize the loads in a predictive formula.
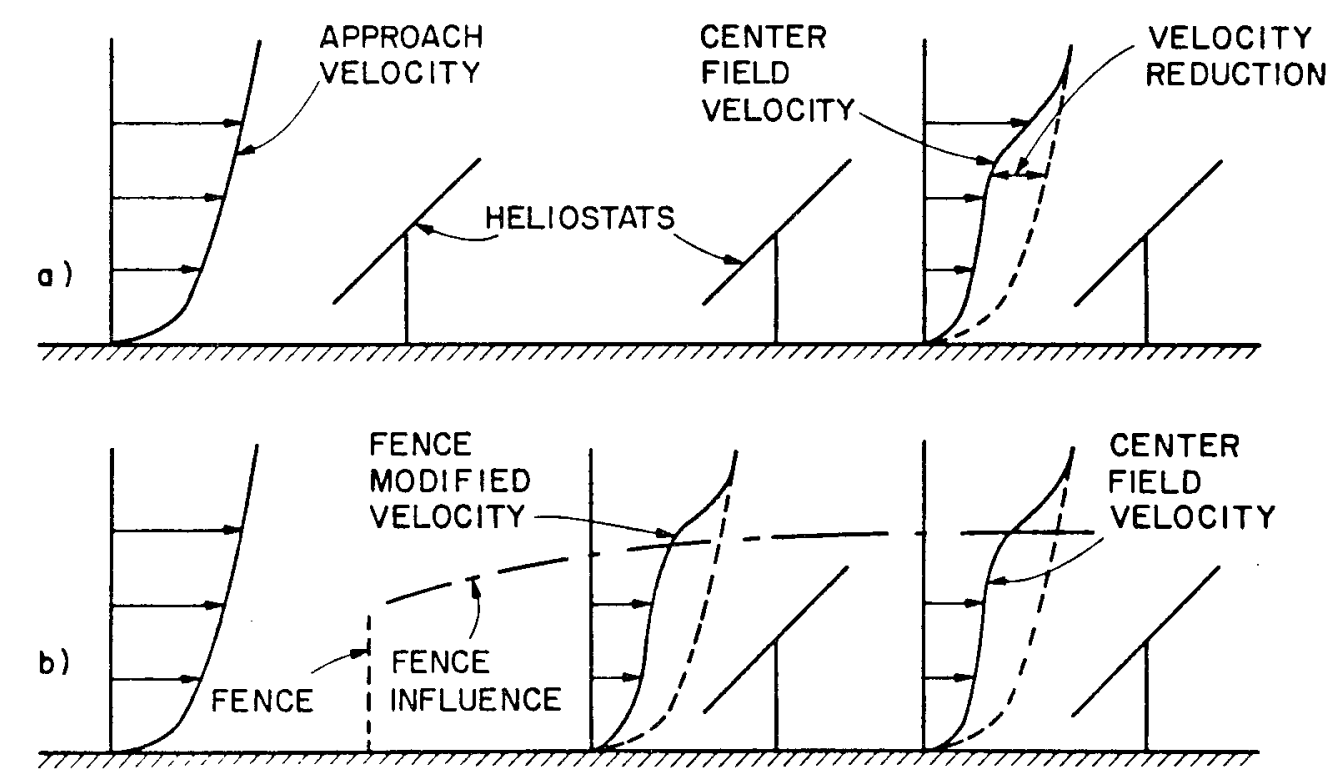

Figure 1-1. Velocity Reductions within a Field of Heliostats 
Mean wind loads on collectors near the edge of the field can be substantially reduced with an appropriately designed perimeter fence of 30 to 50 percent porosity, Figure 1-lb. Reduction of wind load on the first collector with increasing fence height is shown in Figure 1-2. The effectiveness of the fence decreases with decreasing angle between wind vector and fence line and with decreasing fence height. In addition, corners in fences, if not properly designed with spoilers, can cause increases in wind loads above those with no fence [2]. Fence costs may be realistic if heliostat costs can be reduced.

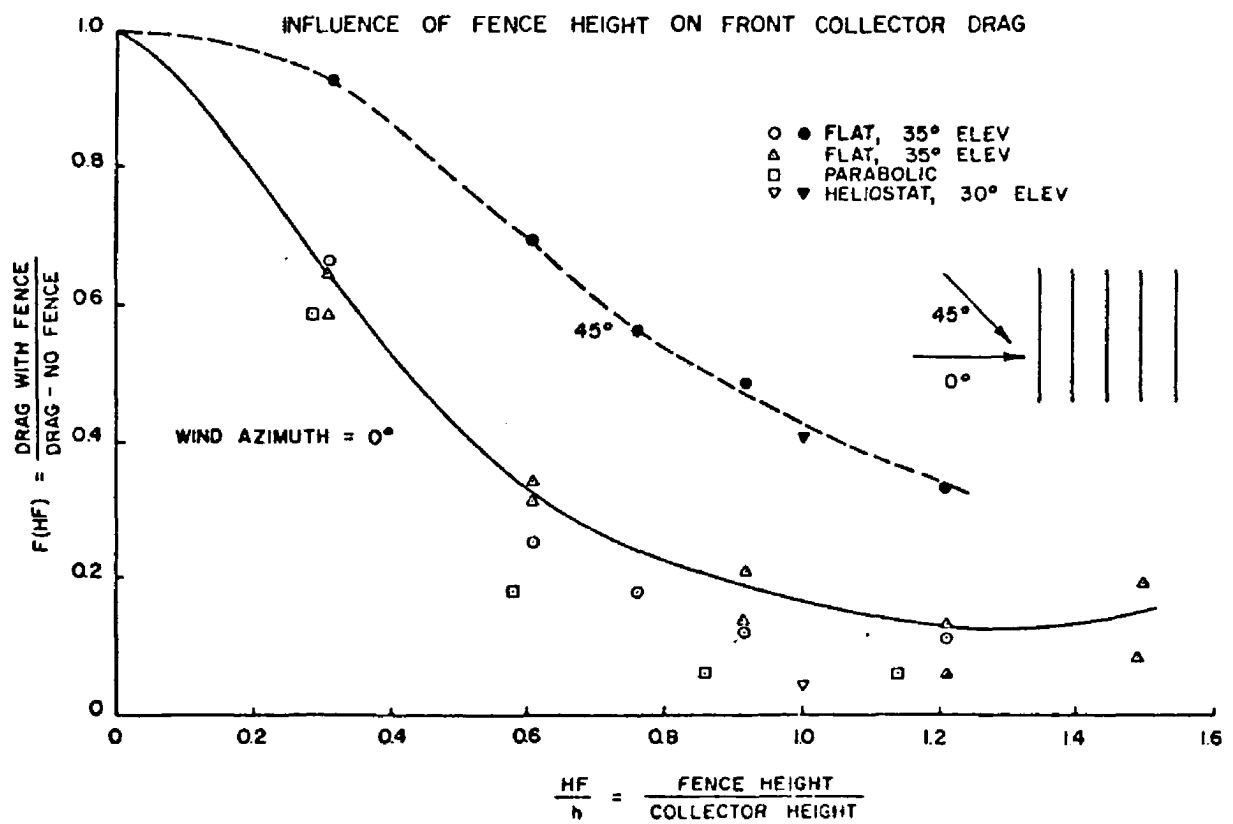

Figure 1-2. Influence of Fence Height on Front Collector Drag Force (data from references $1,4,7,10$ )

One part of the current study is to evaluate the studies cited above to determine possible methods for wind load reduction. Previous tests provided a measure of the need for reducing interior loads below those resulting naturally from protection by surrounding heliostats. In reference [2], mean wind loads were measured for several heliostats with varying distance from the edge of the field, with, and without protective perimeter fences. Two portions, A and $B$, of a possible field arrangement, Figure 1-3, were studied. Key findings of the study are illustrated in Figures 1-4 and 1-5. Both show base moment coefficients (nondimensional base moments) as a function of distance into the field for various perimeter fences and no perimeter fence. Several conclusions can be drawn from the figures and other data in references $[1,2]$ :

- Edge heliostats have larger mean loads without a perimeter fence than do heliostats within the field.

- The addition of a perimeter fence can cause large reductions in edge heliostat mean loads.

- Heliostat loads in the interior of the field in the dense portion of the field (field A in Figure 4) are substantially smaller, Figure 1-4, than those in the interior of the less dense portion of the field (area B), Figure 1-5. 


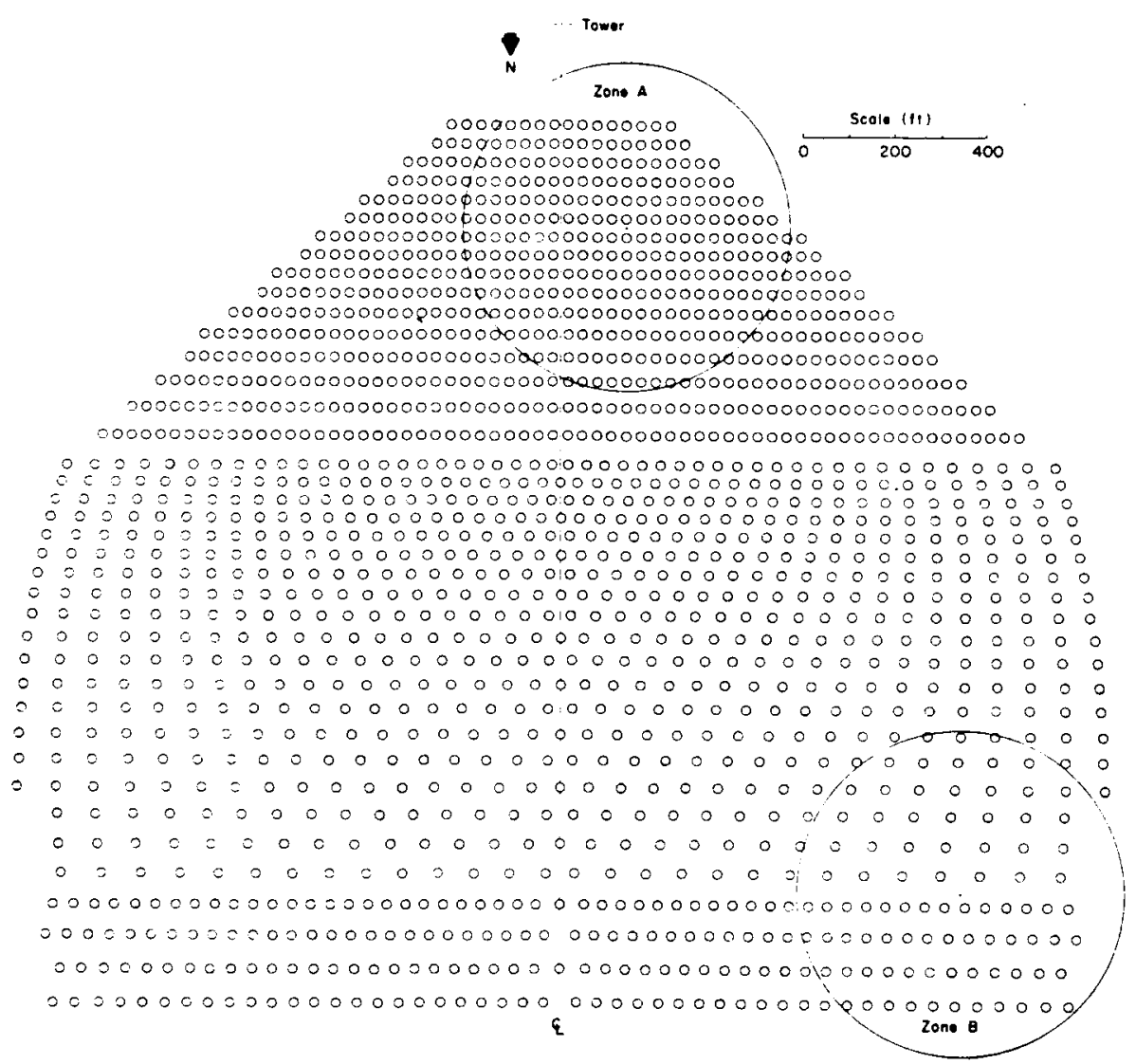

Figure 1-3. Heliostat Field for Tests of Reference [2] 

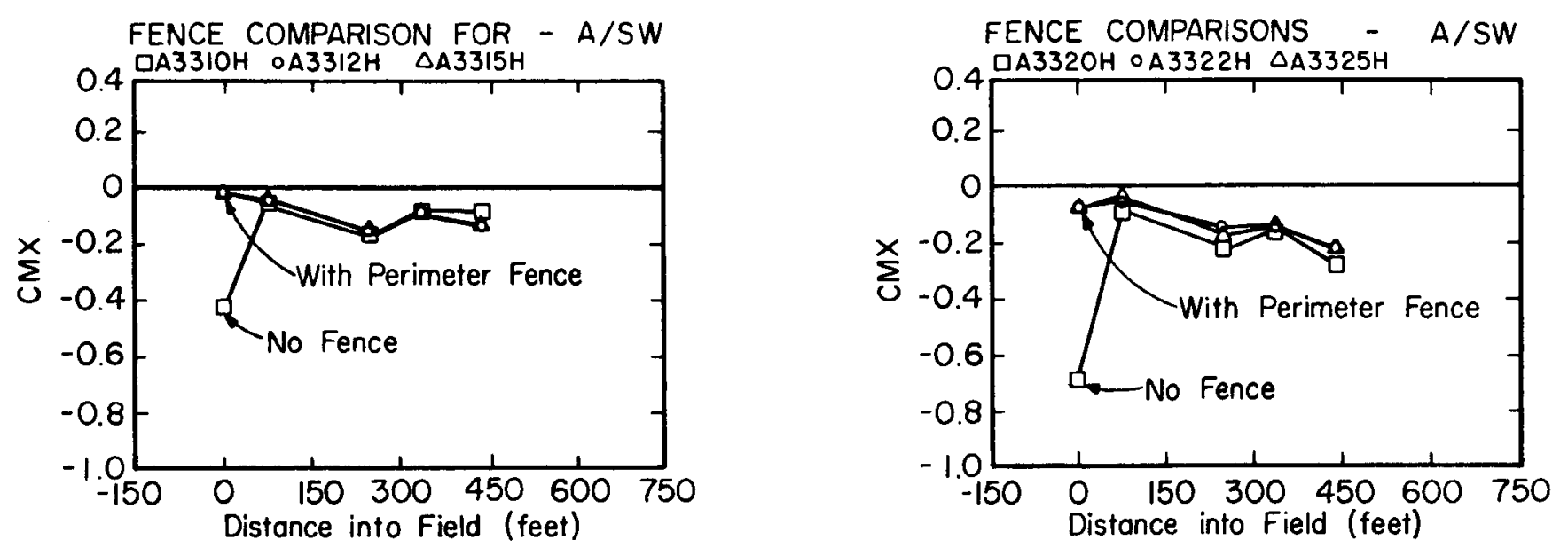

Figure 1-4. Moment Coefficients for a High Density Heliostat Field Array - Zone A
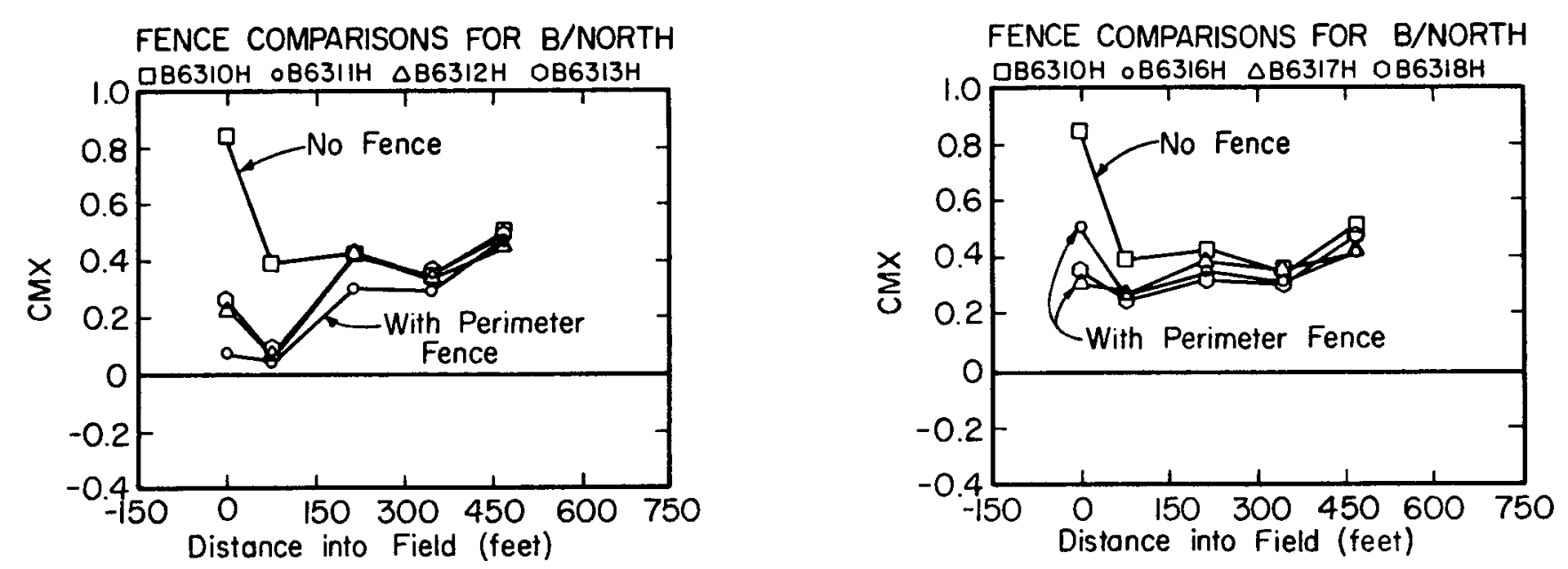

Figure 1-5. Moment Coefficients for a Low Density Heliostat Scale Model Test Field Array - Zone B (Fig. 1-3) 
- Interior heliostat loads in field A were comparable to the edge heliostat loads after reduction by perimeter fence.

- Heliostat loads in the interior of field B were lower than unprotected edge heliostats but were not affected by the perimeter fence.

The use of fences to decrease wind loads has been in use for many years. Reference [23] provides an early summary of porous fence effects directed at agricultural uses, but has more universal application. More recent references [23-27] provide data and access to additional literature describing the mean wind and turbulence structure downwind of porous fences. While a large number of references exist describing flow behind porous fences, there still remains a need for additional research for wind not perpendicular to a fence, short fence lengths, corners in fences, rows of fences and crossing fences. Use of published data on fences has been used in this and previous studies. However, this information is of limited use when predicting the load on an in-field heliostat whose wind load is determined by upwind heliostats, perimeter fence and in-field fences.

\subsection{WIND LOAD REDUCTION}

Based on review of previous work, the potential for mean load reduction on heliostats is in the edge units and in the low density field interior units. Edge units can be protected by perimeter fences as previously illustrated $[1,2]$. It is evident that mean load reduction within the field depends on velocity reduction within the field. This in turn requires additional blockage area in the field interior. Several generic concepts are shown in Figure 1-6 which could represent solid or porous fences. The fences could surround a single, several or no heliostats, could meander through the field in a straight or curved line, or could be a series of fence segments. The fences could vary with height along their length to prevent shadows on the mirrors. One possible scheme is shown in Figure 1-7 in which porous fences are included within the field to increase blockage area. These in-field fences were designed to provide minimal interference with reflected light. Modifications to this concept involve inclusion of fences in only one direction to permit easier access to the field for maintenance. In the horizontal stow mode, selected lines of strengthened heliostats could be left upright to provide fence action in place of actual fences.

Dynamic loads result from two phenomena: 1) from buffeting due to turbulence in wind approaching the field and from disturbances from upwind heliostats, and 2) from 'wake turbulence' generated by separated wind flow as it passes over the heliostat under consideration. No previous dynamic load measurements are available for this specific geometry. However, based on dynamic loading on other shapes, it is expected that reduction of mean loads will have the effect of increasing buffeting through increased turbulence in the approach wind and decreased wake excitation through decreased mean velocity. It is not certain that a decreased mean load will result in a net decrease in dynamic load; however, measurements on other geometries indicate this possibility. Efforts were made during wind-tunnel testing to measure some fluctuating loads so that the total design load, including both mean and dynamic loads, could be determined. 


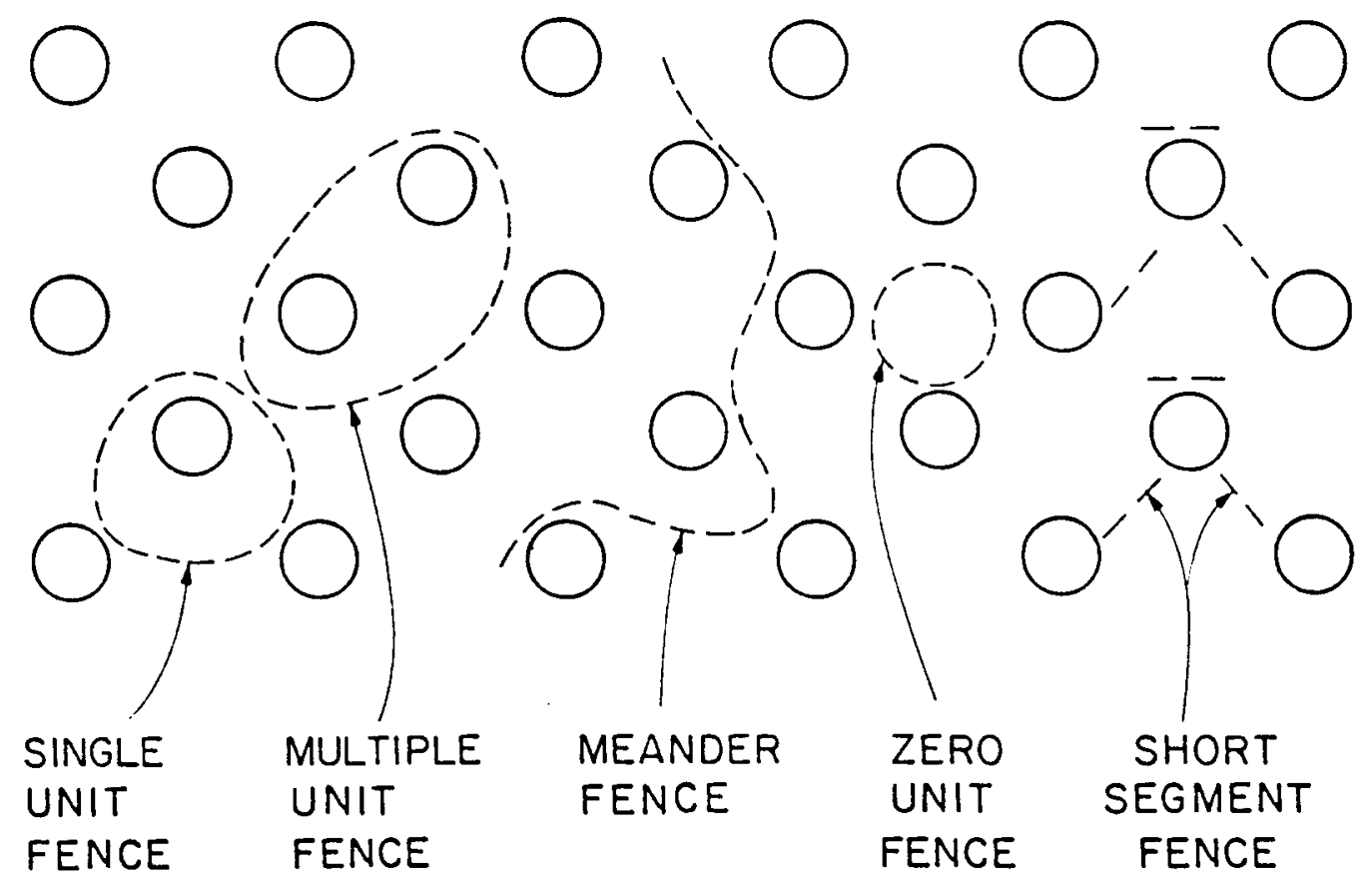

POROUS OR SOLID FENCES

FENCE HEIGHTS MAY VARY ALONG FENCE LENGTH

Figure 1-6. Concepts for Interior Field Wind Protection

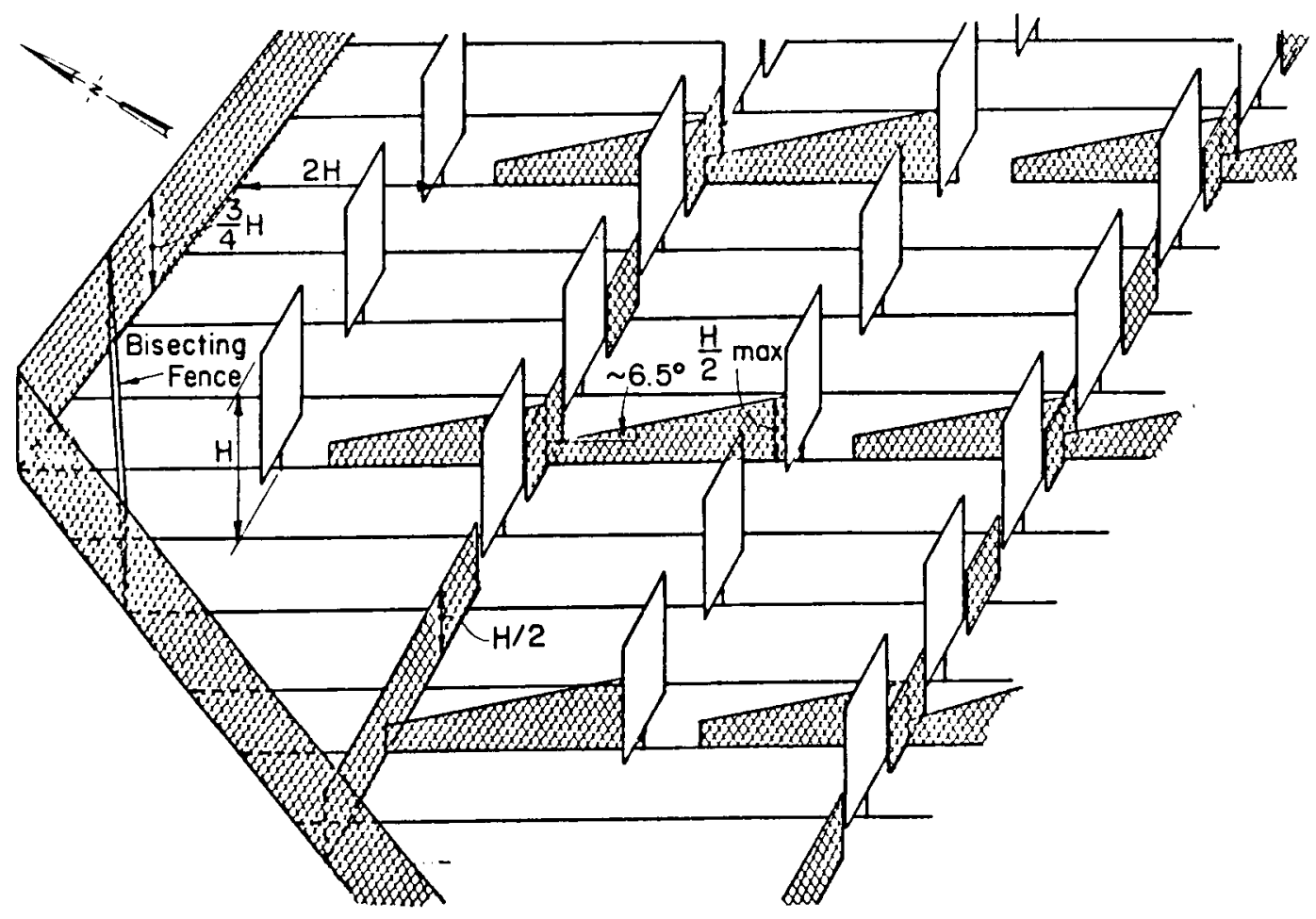

Figure 1-7. Fence Configuration for Lowering Velocities Interior to Field 
Reduction of dynamic loads depends critically on the specific mechanism responsible for the dynamic loading. In some cases, wind flow separation from the edge of the heliostat can result in increased dynamic loading. For these cases, attachments to the heliostat such as those suggested in Figure 1-8 might be beneficial in disrupting the shear layer and reducing dynamic loading. These devices are likely, however, to increase mean loading.

UPPER

SURFACE GUARDS
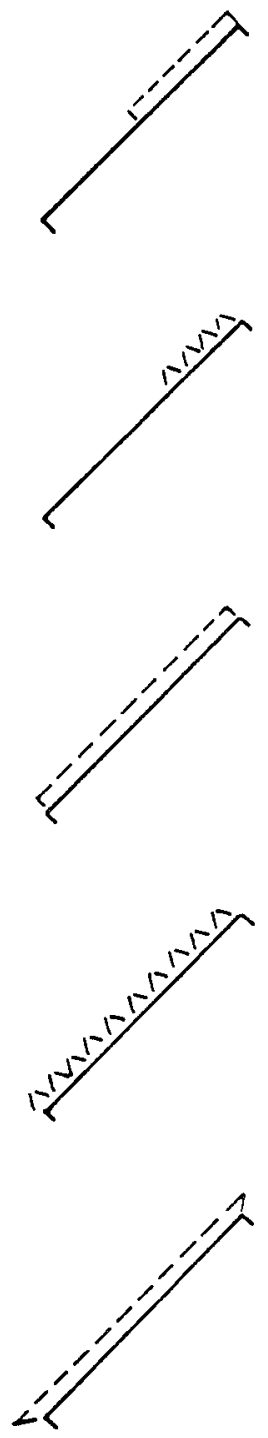

LOWER

SURFACE GUARDS
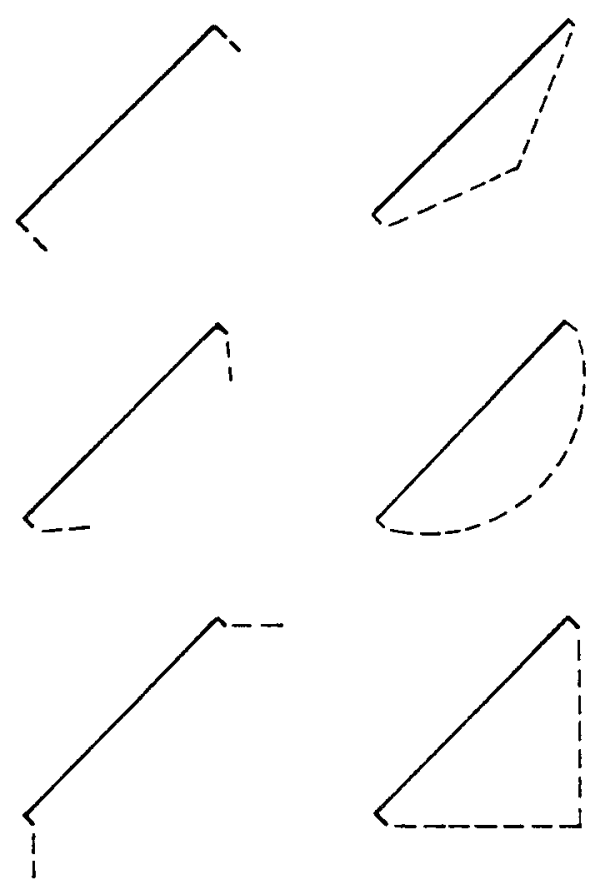

LOWER ENCLOSURES
EDGE

\section{FAIRINGS}




\subsection{MODELING OF ATMOSPHERIC WIND LOADS}

Measurements of wind loads on ground-based solar collectors outlined previously have all been obtained in wind-tunnel tests. Wind tunnels specifically designed to model atmospheric boundary-layer winds and wind loads on structures in the 1000-2000 ft above the earth's surface have been developed over the past 30 years. Numerous references are available, [28-30] for example, which describe the modeling criteria required for boundary-layer wind tunnels to model atmospheric boundary-layer winds and resulting wind loads. In general, the requirements are that the model and prototype be geometrically similar, that the approach mean velocity at the building site have a vertical profile shape similar to the full-scale flow, that the turbulence characteristics of the flows be similar, and that the Reynolds number for the model and prototype be equal.

These criteria are satisfied by constructing a scale model of the structure and its surroundings and performing the wind tests in a wind tunnel specifically designed to model atmospheric boundary-layer flows. Reynolds number similarity requires that the quantity $U L / \nu$ be similar for model and prototype. Since $v$, the kinematic viscosity of air, is identical for both, Reynolds numbers cannot be made precisely equal with reasonable wind velocities. To accomplish this the air velocity in the wind tunnel would have to be as large as the model scale factor times the prototype wind velocity, a velocity which would introduce unacceptable compressibility effects. However, for sufficiently high Reynolds numbers $\left(>2 \times 10^{4}\right)$ the pressure coefficient at any location on the structure will be essentially constant for a large range of Reynolds numbers. Typical values encountered are $10^{5}-10^{6}$ for the fullscale and $10^{4}-10^{5}$ for the wind-tunnel model. In this range acceptable flow similarity is achieved without precise Reynolds number equality.

The wind tunnel used for this study is described in Section 2.0. Appendix A presents comparisons between model and full-scale studies which illustrate the ability of wind-tunnel models to predict full-scale wind speeds and wind loads. This extensive data showing excellent comparison and the great economy of wind-tunnel tests in comparison with full-scale tests has driven a steadily increasing use of boundary-layer wind-tunnel testing. 
SECTION 2.0

\section{EXPERIMENTAL APPARATUS}

\subsection{THE WIND-TUNNEL FACILITY}

The present study was conducted in the Meteorological Wind Tunnel (MWT) of the Fluid Dynamics and Diffusion Laboratory (FDDL) at Colorado State University. Plan and elevation views of this tunnel are shown in Figure 2-1.

The MWT was designed specifically to model atmospheric boundary-layer flow. The tunnel is a closed circuit facility with a 9-to-1 contraction ratio driven by a $250 \mathrm{HP}$ variable-pitch, variable-speed propeller. The test section is $96 \mathrm{ft}$ in length and nominally $6 \mathrm{ft}$ square. The test section walls diverge approximately $1 \mathrm{in.} / 10 \mathrm{ft}$, and the roof is adjustable to maintain a zero pressure gradient along the test section. The blockage created by the model was less than 2 percent of the tunnel cross section. Hence, it was not necessary to adjust the roof to compensate for the blockage effect. Though the tunnel is capable of simulating thermally stratified planetary boundary layers, all the experiments included in this report were performed with a neutral boundary-layer stratification.

The turbulent boundary layer was tripped at the entrance section of the MWT with a $1.5 \mathrm{in}$. high sawtooth vortex generator and allowed to develop over the long test section with certain roughness (smooth Masonite with 0.25 in. holes and 0.25 in. diameter $x \quad 0.5$ in. long dowels placed in a 2 in. $x \quad 8$ in. pattern). In addition, four evenly spaced $6 \mathrm{ft}$ tall spires were installed at the tunnel entrance to create the desired atmospheric boundary layer within the test section. The turbulent boundary layer developed in this way has been shown to model the atmosphere boundary layer for model scales smaller than about 1:100 (28-30). At a 1:60 scale the larger turbulence scales are not completely represented, as discussed below, but should not affect mean wind load measurements.

\subsection{MODEL ENVIRONMENT}

\subsubsection{Heliostat Models}

As discussed in Section 1.0, a major reason for examining wind loads on heliostats is the emergence of stretched membrane heliostats which have a circular shape. It might logically follow that this study would use circular heliostat models. However, more than 200 rectangular models of glass-mirror heliostats at a $1: 60$ scale were available from a previous study [2]. In addition, typical heliostat field layouts were available from reference [2] and from the existing Barstow demonstration site and no specific field layouts have been published for the circular shape. For these reasons and because results from one flat-plate shape (for example for rectangular to circular) to the next are expected to be quite similar, the rectangular shape was selected for this study. The Barstow site layout, Figure 2-2a, was selected over that from reference [2] since some data on the reference [2] layout had been obtained, since the Barstow site may be more typical of future field geometries, and since a possibility existed for obtaining some field data for 


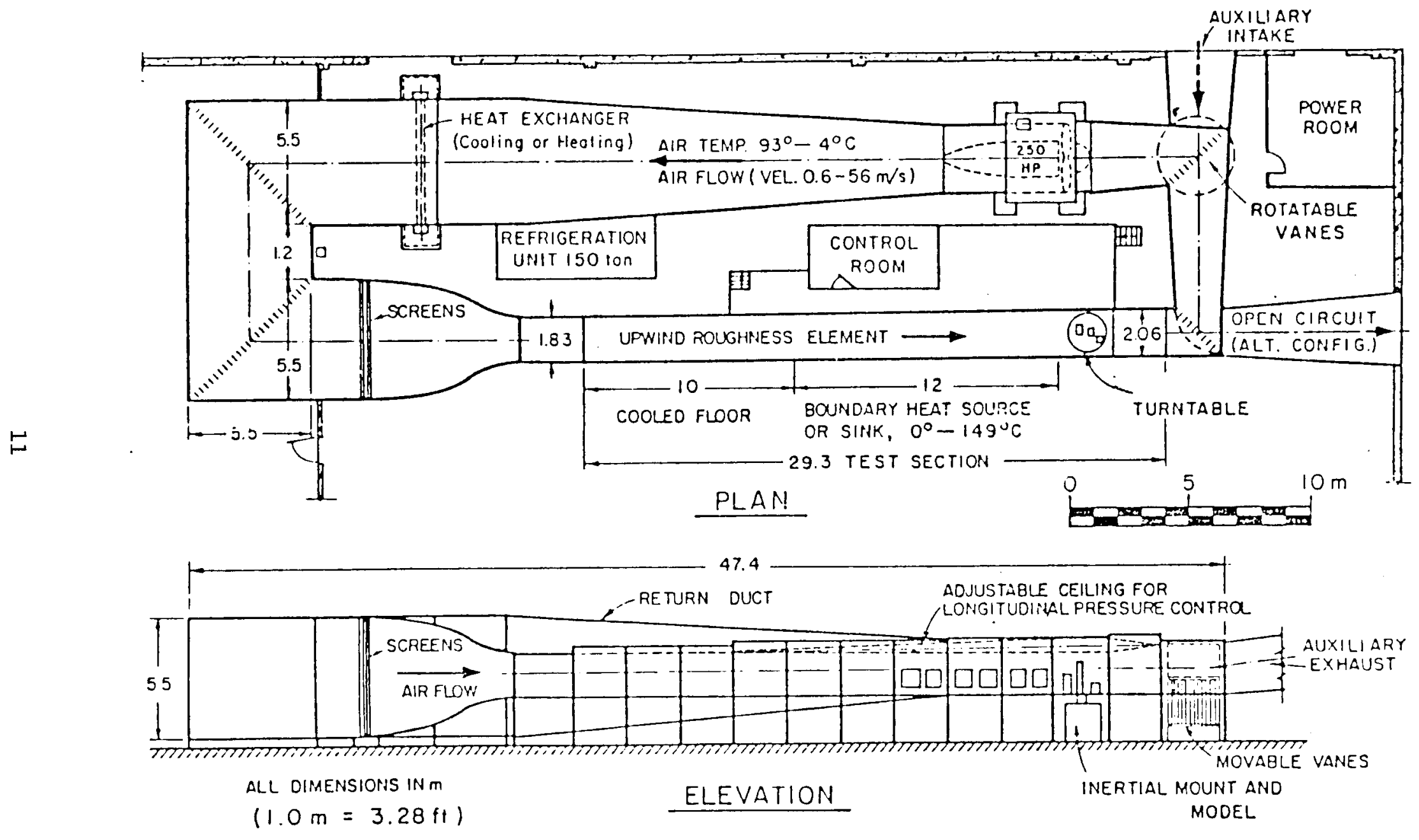

Figure 2-1. Meteorological Wind Tunnel 


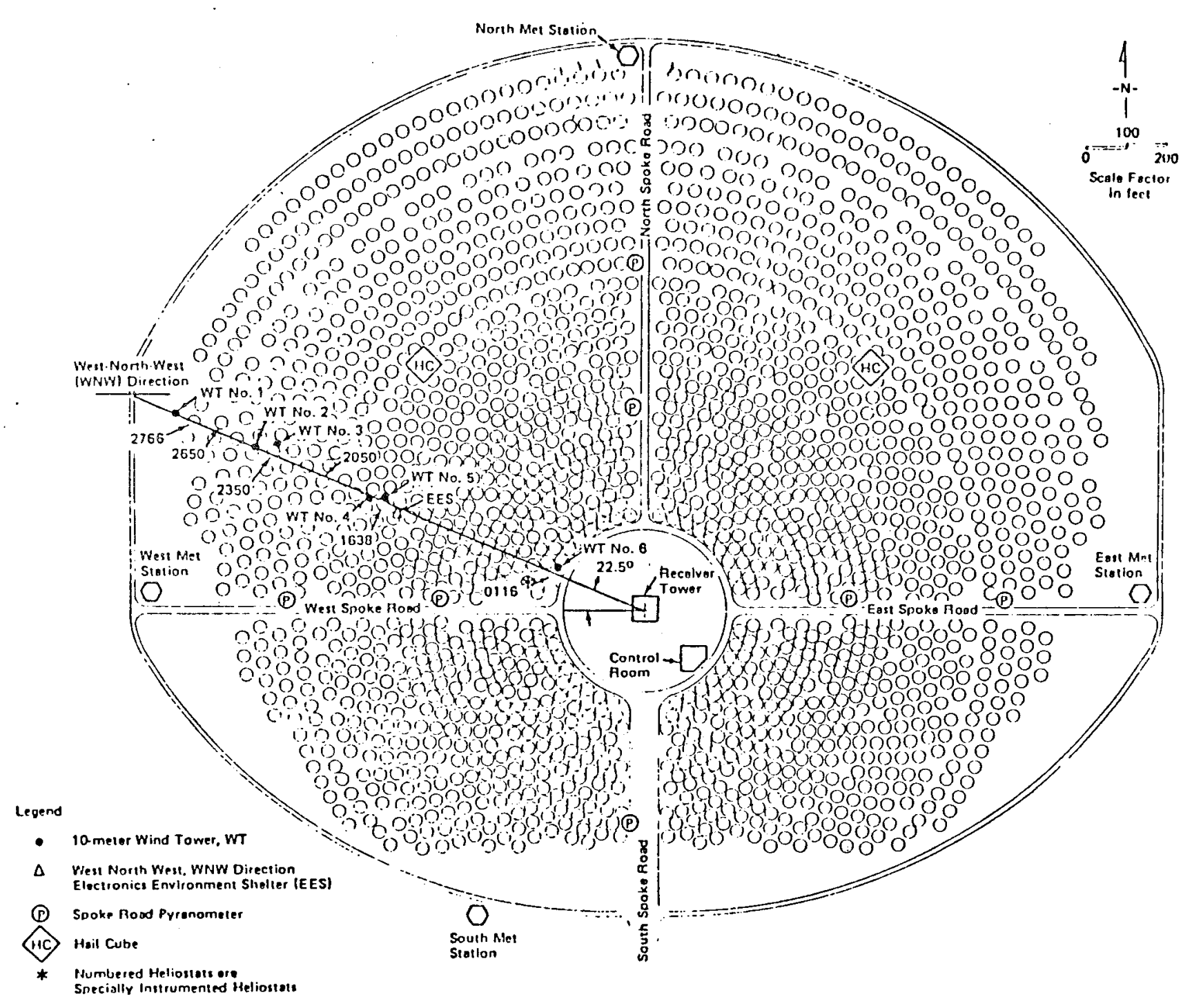

Figure 2-2a. Heliostat Field 
comparison with wind-tunnel data for specific heliostats which have been instrumented at the Barstow site.

Five heliostats in the Barstow field were selected for modeling in this test program, see Figure 2-2b. These heliostats are representative of five different environments in the field representing three different densities $(A, B, C)$ :

1) edge unit exposed to prevailing WNW winds

2) intermediate density $B$ region in the field

3) high density region $C$ in the field

4) inner edge of the high density region

5) lowest density portion $A$ of the field

Three of the five heliostats $(1,3,4)$ are ones which have been instrumented in the Barstow field for wind loads. Heliostats 1-4 lie close to a line of anemometer towers in the full-scale field, Figure 2-2a, which have been measuring full-scale wind data. Thus, comparisons between model and fullscale wind measurements are possible.

The circles surrounding each heliostat in Figure 2-2b represent the $6 \mathrm{ft}$ diameter turntable (and wind-tunnel width) for the model study. For some experiments, only those heliostats on the turntable area were used. For others, heliostats were added upstream of the turntable for specific wind directions to obtain an upwind fetch of heliostats which extended to the edge of the field. Specific field layouts are identified in the section on the test matrix in Section 3.4 .

A photo of the 1:60 heliostat models used in the study is shown in Figure 2-3a while drawings of the models are shown in Figure 2-3b. The field layout was placed on a sheet of plywood and holes drilled for each heliostat support post. The field could then be established and changed quickly by inserting heliostats into their respective holes. Heliostats could be set to a specific day and hour by setting azimuthal and elevation angles. Angled blocks were prefabricated for desired angles and quickly set next to heliostats for setting angles. Horizontal and vertical angles were held in place by friction in the bearings.

The heliostat at the center of the turntable (1-5 in Figure 2-2b) was mounted on a six-component strain-gaged force balance, Figure 2-4. The balance was designed and constructed by the Fluid Mechanics and Wind Engineering Program at Colorado State University for general purpose use in measuring mean wind loads. By constructing the instrumented heliostat of balsa wood with a brass post, it was possible to obtain a model/balance natural frequency of $28 \mathrm{~Hz}$. By low-pass filtering the output of the balance, the resonant response of the model heliostat could be virtually eliminated from the output signal permitting the fluctuating loads on the model to be measured up to a frequency of about $18 \mathrm{~Hz}$. Setting wind-tunnel speed to take advantage of velocity scaling laws, see Section 3.1 , permitted a reasonable approximation to the true fluctuating forces on the model to be obtained. Additional details of the balance operation are contained in Section 3.1. 

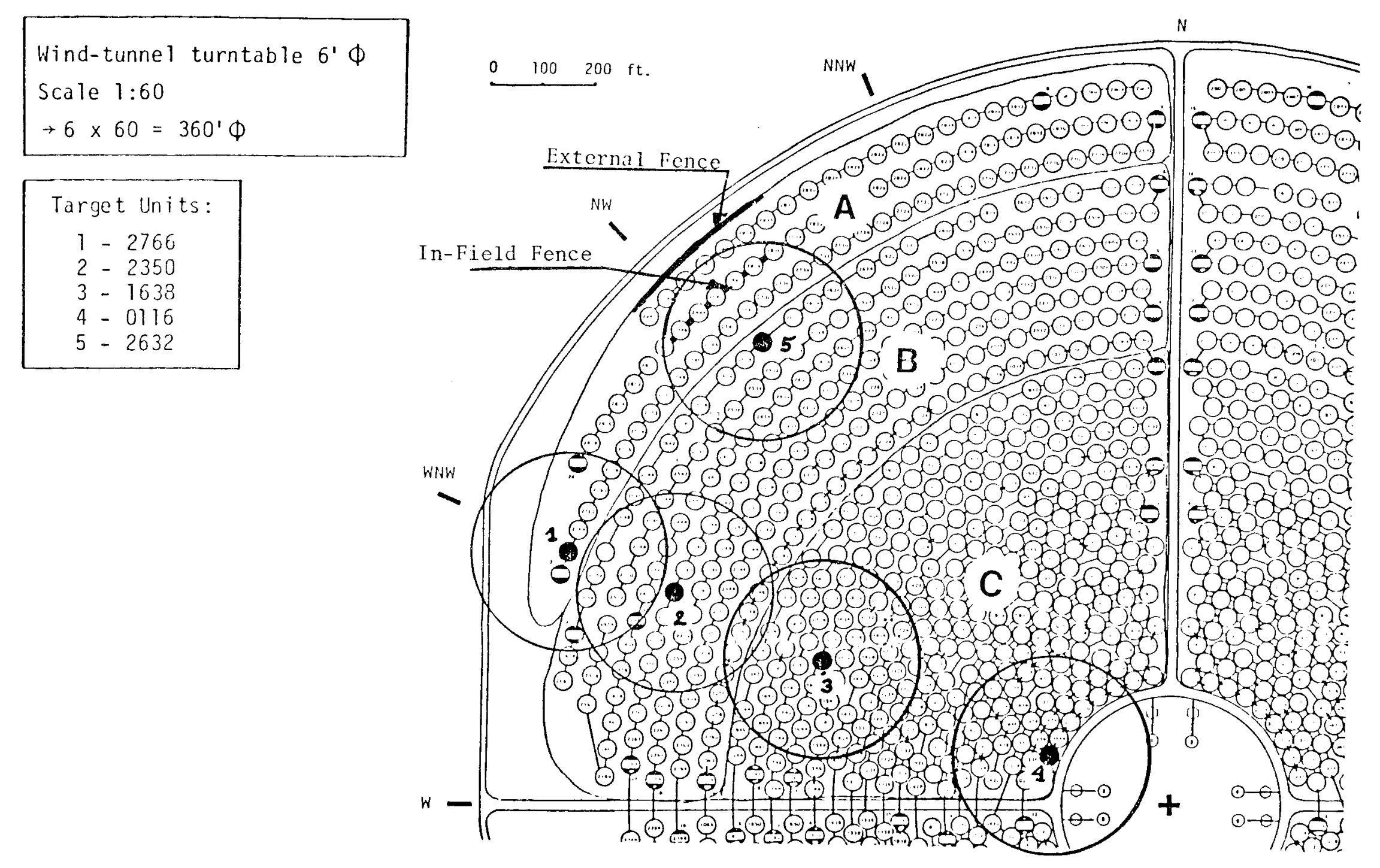

Figure 2-2b. Heliostat Field 
(a)

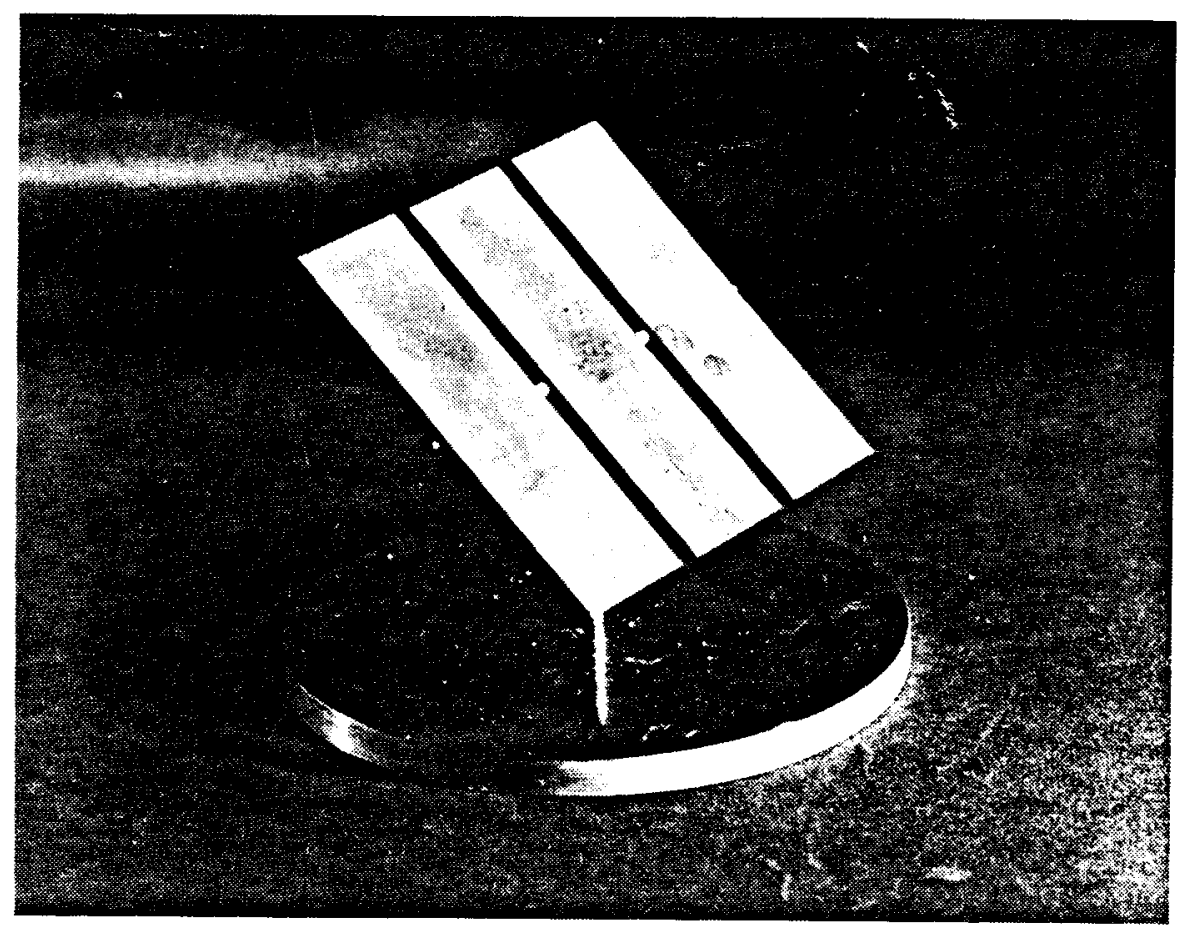

(b)

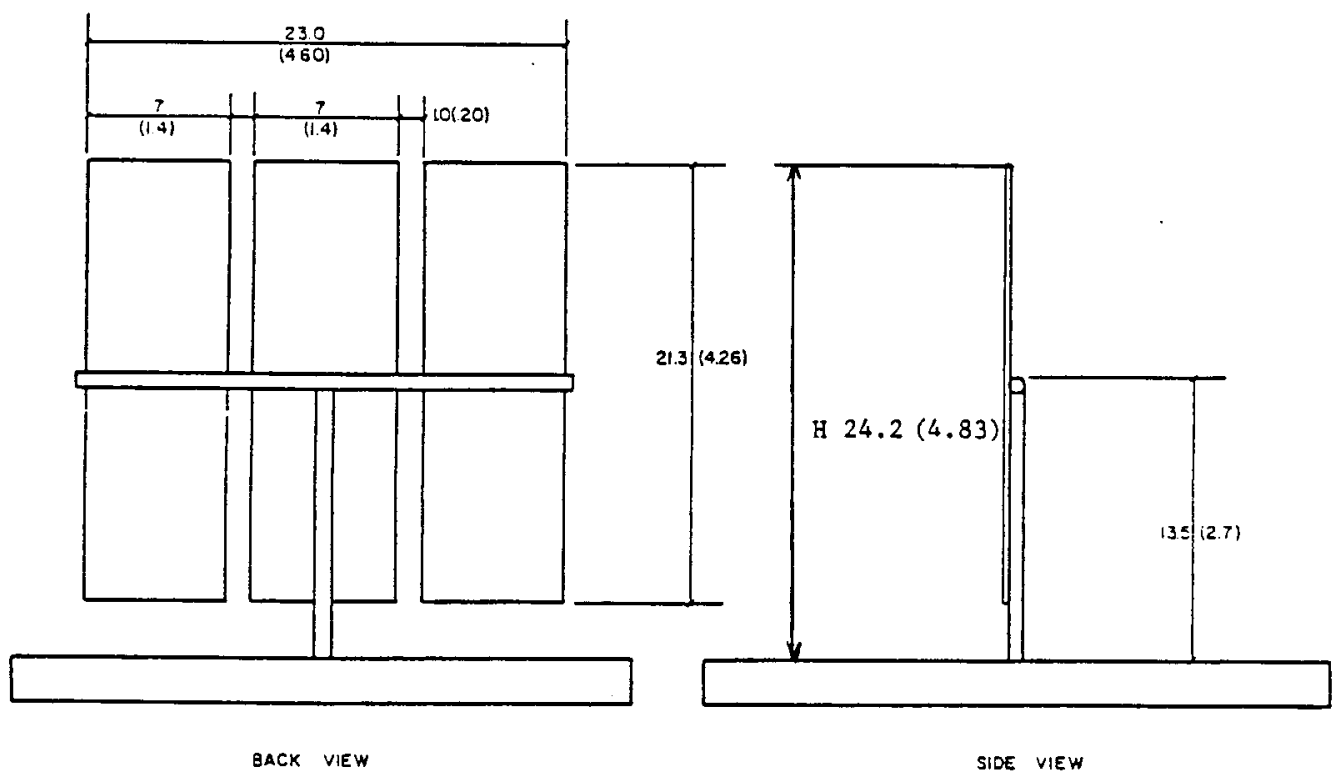

Figure 2-3. Heliostat Model 


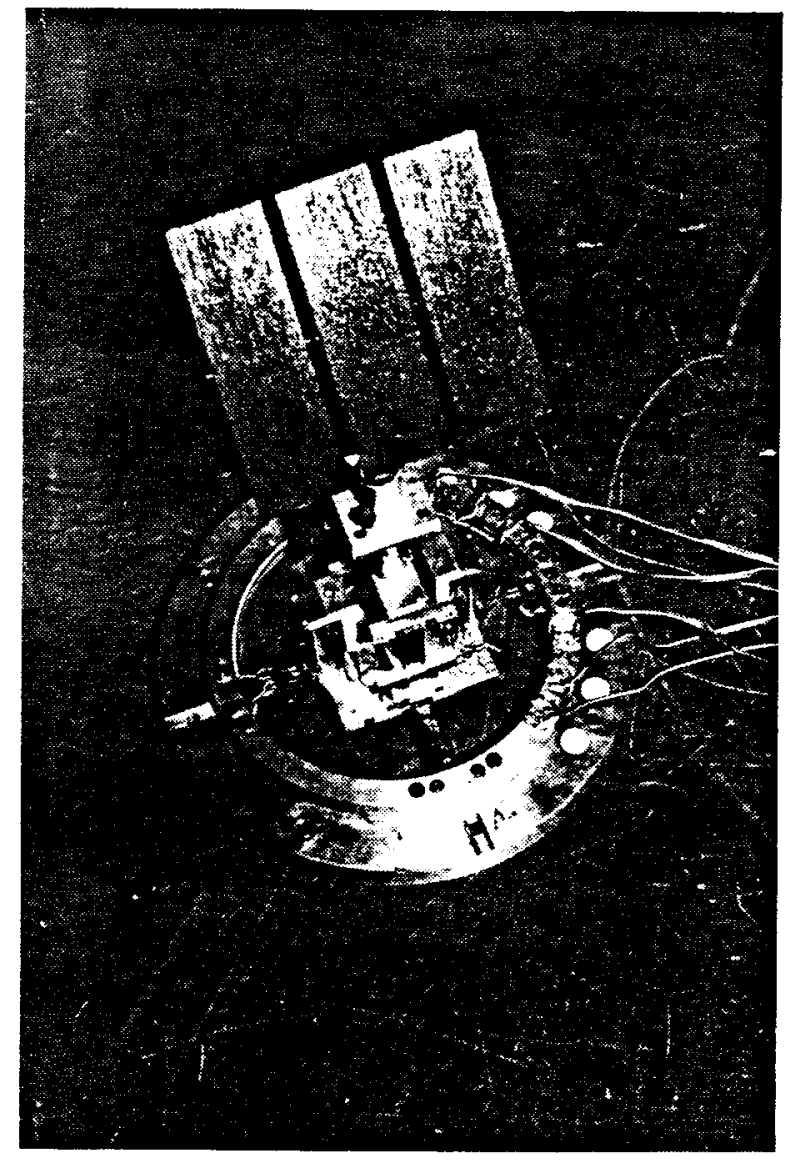

Figure 2-4. Metric Heliostat Mounted on a Six-Component Balance 


\subsubsection{Wind Protective Fences}

Four types of wind fences were used in the test program to reduce wind loads on the heliostats. They vere fences of 40 percent, 50 percent and 60 percent porosity and a solid berm with a side sloping 45 degrees to the horizontal. Heights of the fences used were 18.2 and $12.1 \mathrm{ft}$ full scale. One berm height of $18.2 \mathrm{ft}$ was used. Holes in the fence forming the porosity were sufficiently small that jets due to wind flow through a single hole in the fence could not affect heliostat loads. Some fences were placed as external fences outside the field perimeter at a distance of $2 \mathrm{H}$ from the edge heliostat posts ( $H$ is the heliostat nominal height of $24.2 \mathrm{ft}$ ). Figures $2-5$ through $2-9$ show external fence locations for use with heliostats 1,3 and 5 .

Fences were placed within the heliostat field for some data runs. In line with concepts discussed in Section 1.0, fences were aligned with rows of neliostats and placed every other row as shown in Figures 2-6 through 2-9. These figures also show variable densities of field used in the study. These are discussed more fully in Section 4.0. Data obtained during test runs showed that the additional fences perpendicular to those of Figure 2-6 were not necessary to obtain major load reductions in a field except at low field density (see Figure 2-9). The particular fences used for each data run are identified in Section 4.0, Appendix $C$, and Tables $3-2$ and $3-3$ where the run matrix and data tabulations are given.

The model installed in the wind tunnel is shown in Figure 2-10. Porous fences used with the model are shown in Figure 2-11. 


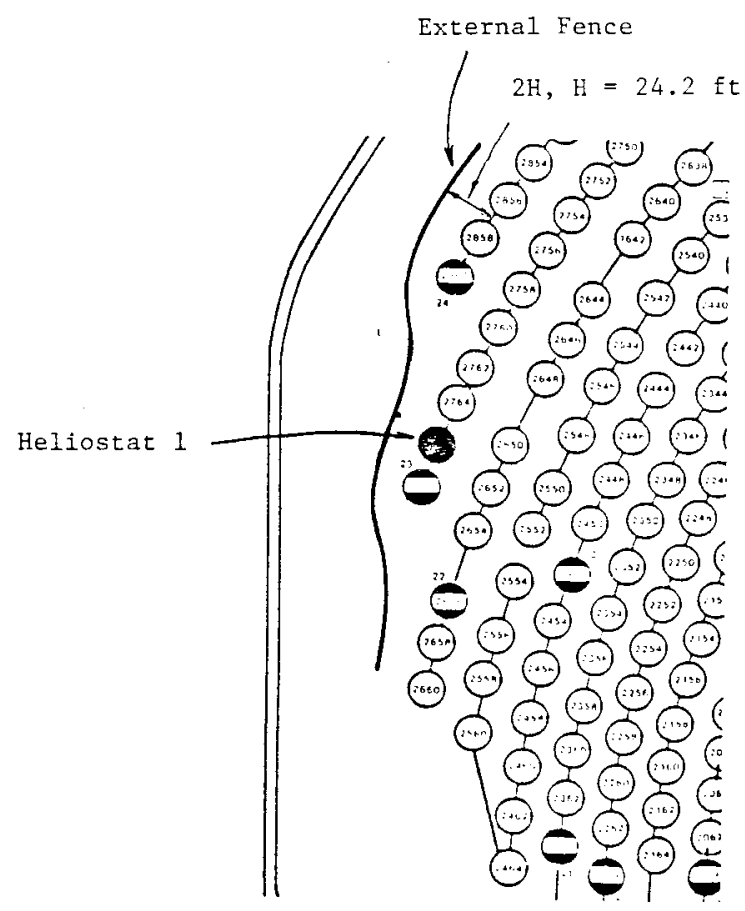

Figure 2-5. Fence Arrangement for Heliostat 1

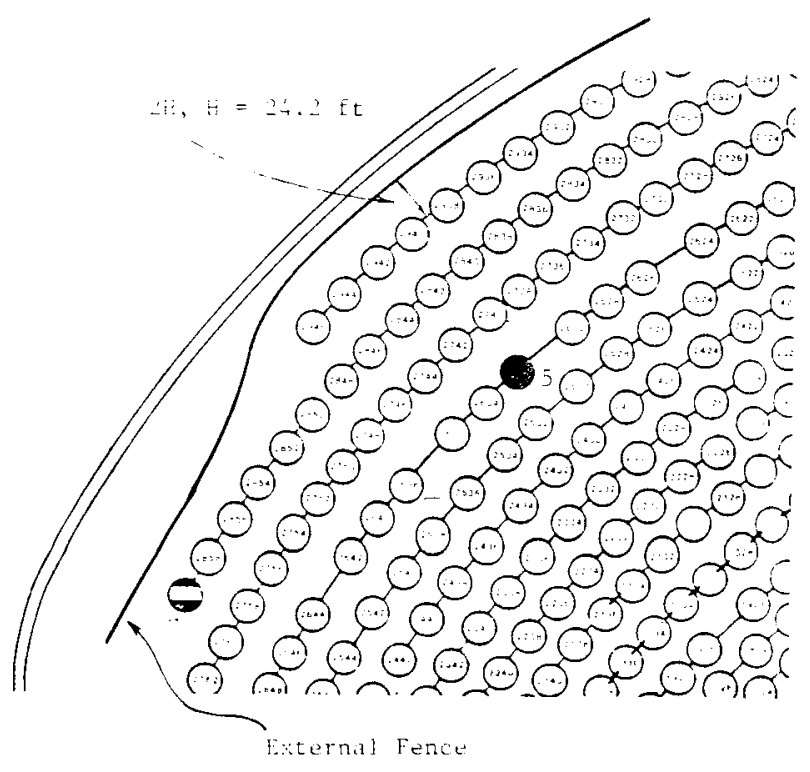

(a)

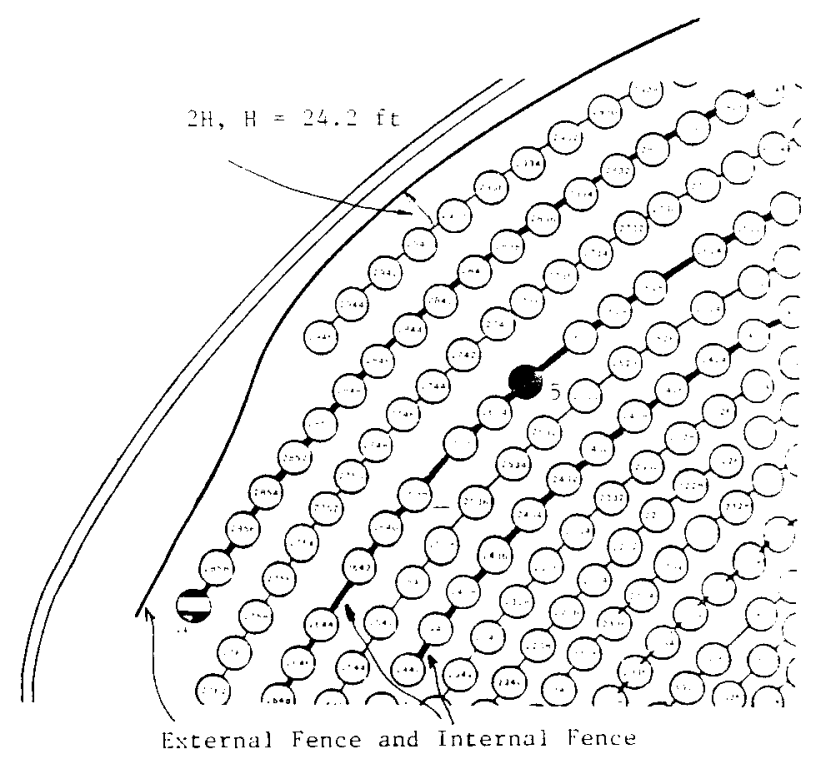

(b)

Figure 2-6. Fence Arrangement for Heliostat 5 

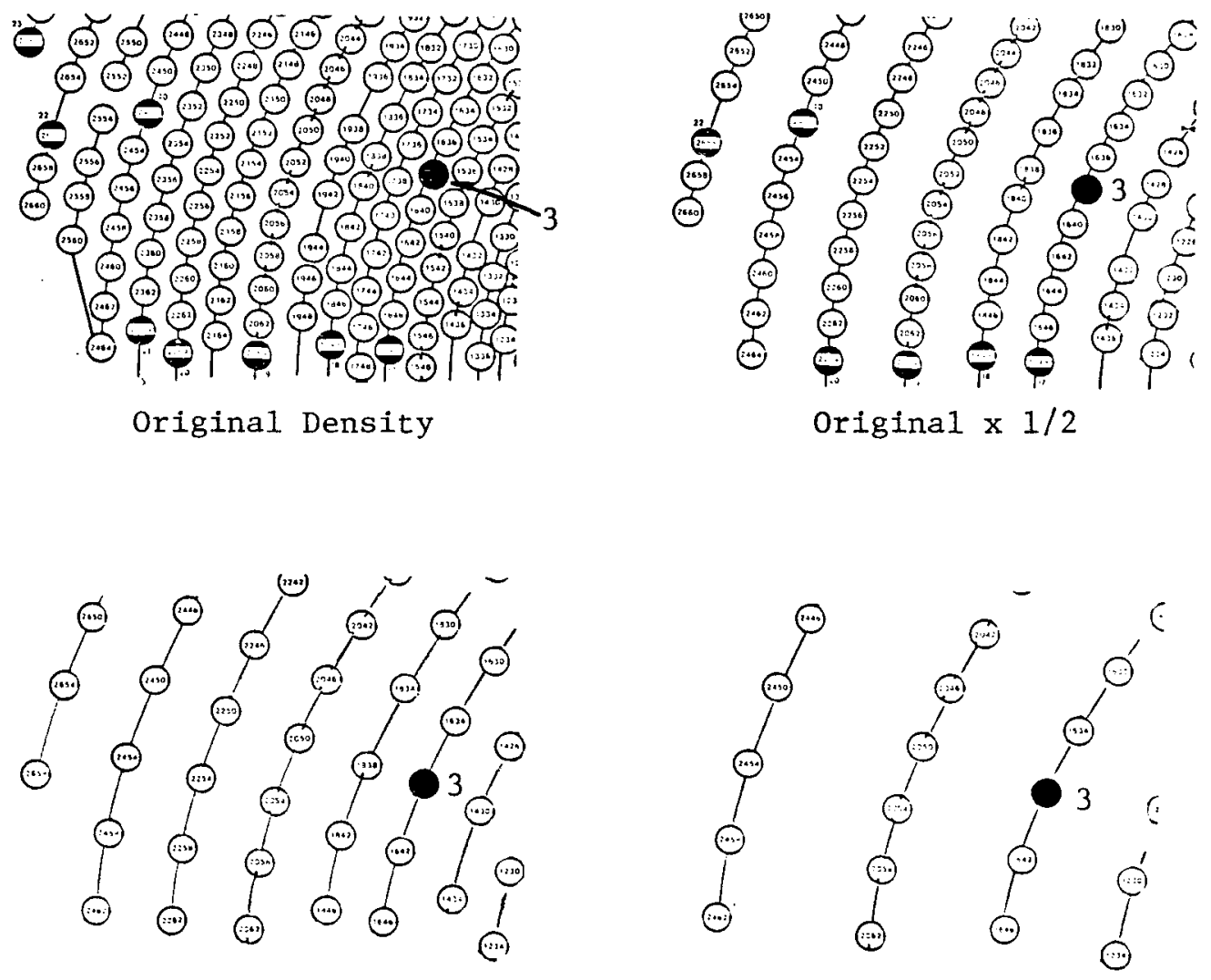

Original $\times 1 / 4$

Original $\times 1 / 8$

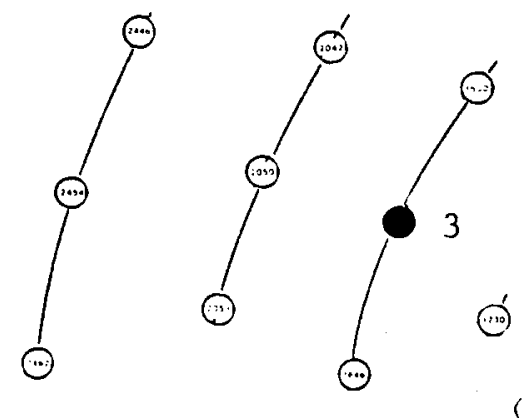

Original $\times 1 / 16$

Figure 2-7. Arrangement of Field Density around Heliostat 3 

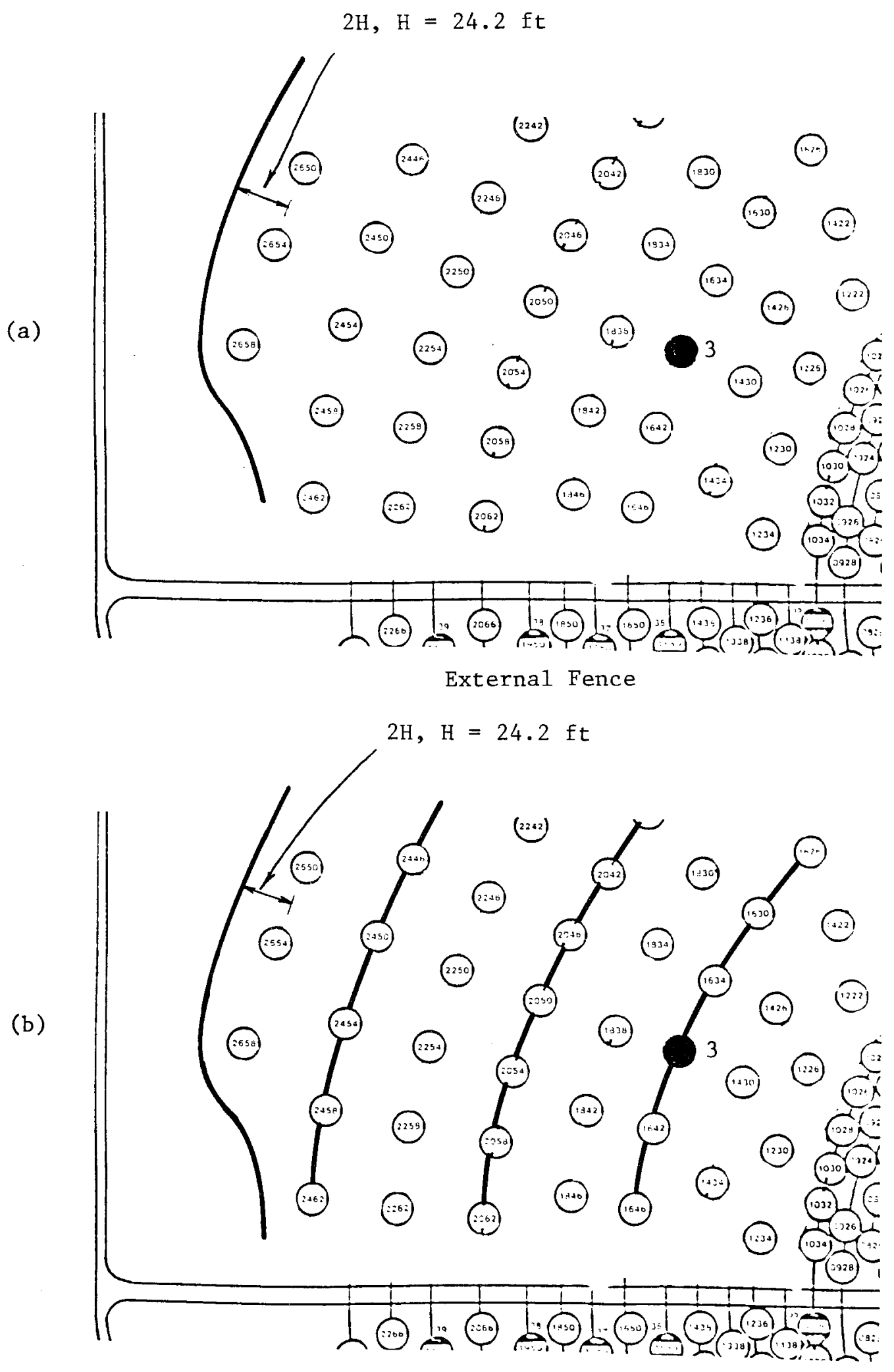

External Fence and Internal Fence

Figure 2-8. Fence Arrangement for Heliostat 3 with Reduced Field Density (original x 1/4) 


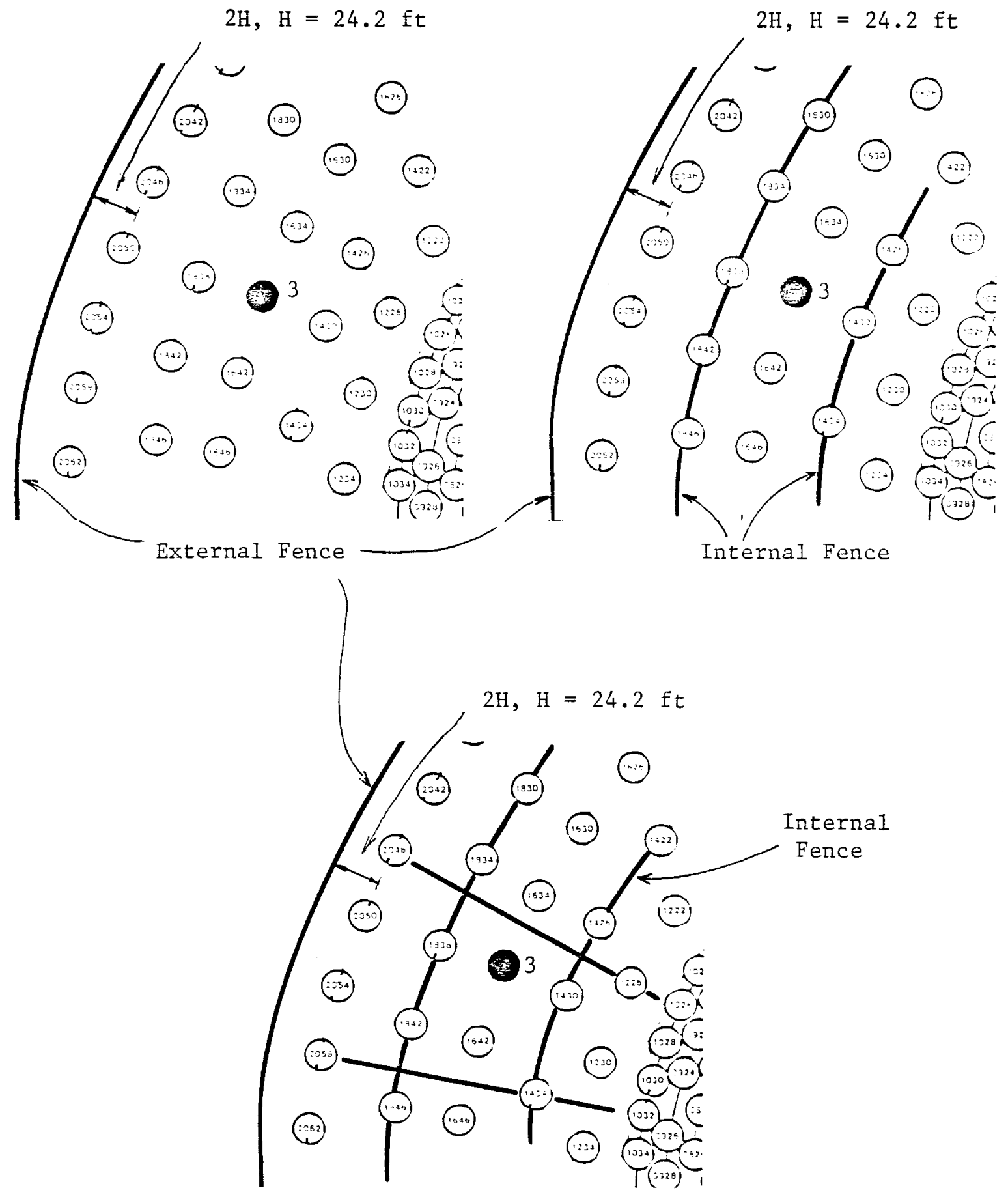

Figure 2-9. Fence Arrangement for Heliostat 3 with Reduced Field Density (original x $1 / 4$ ) 
(a)

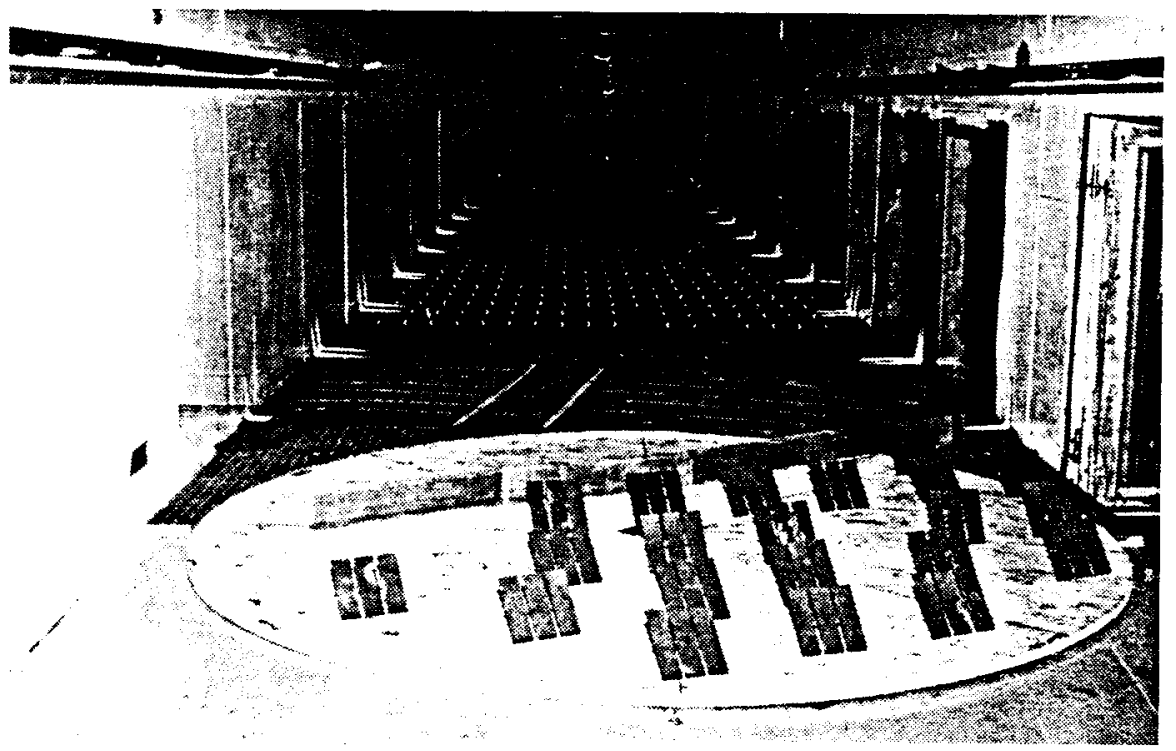

(b)

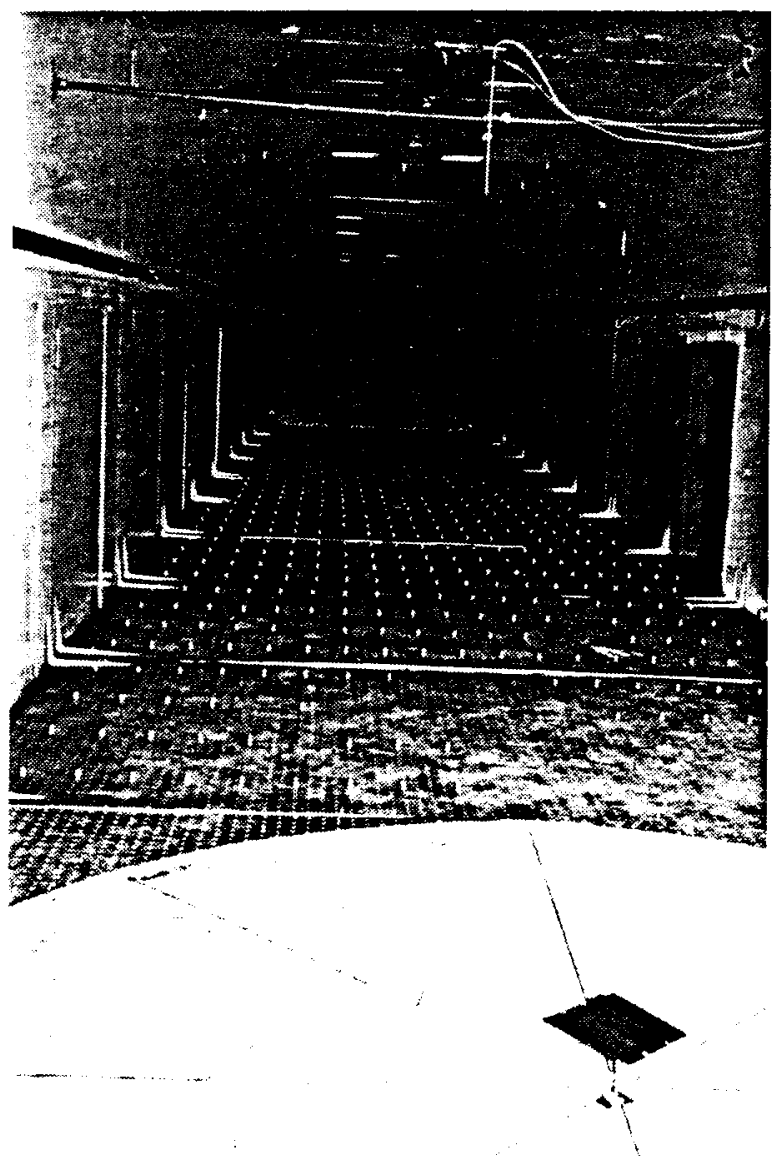

Figure 2-10. Model Installed in the Wind Tunnel 


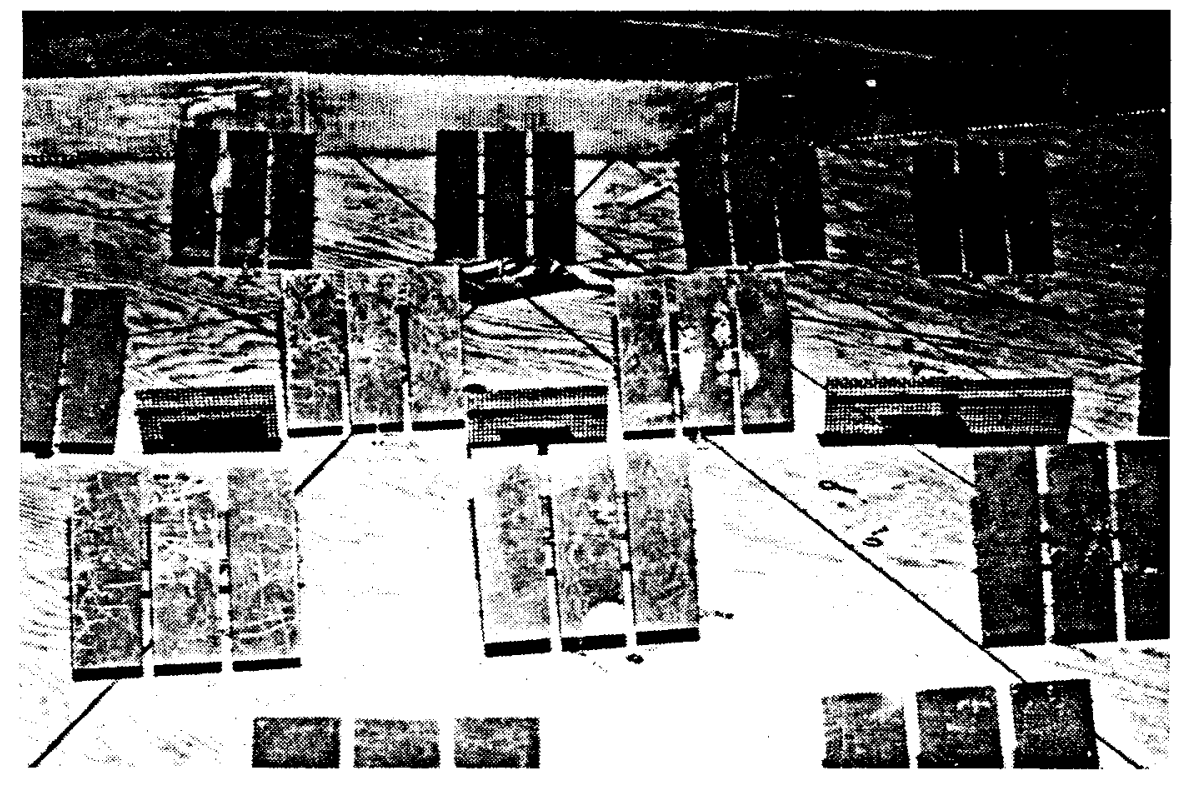

(c)

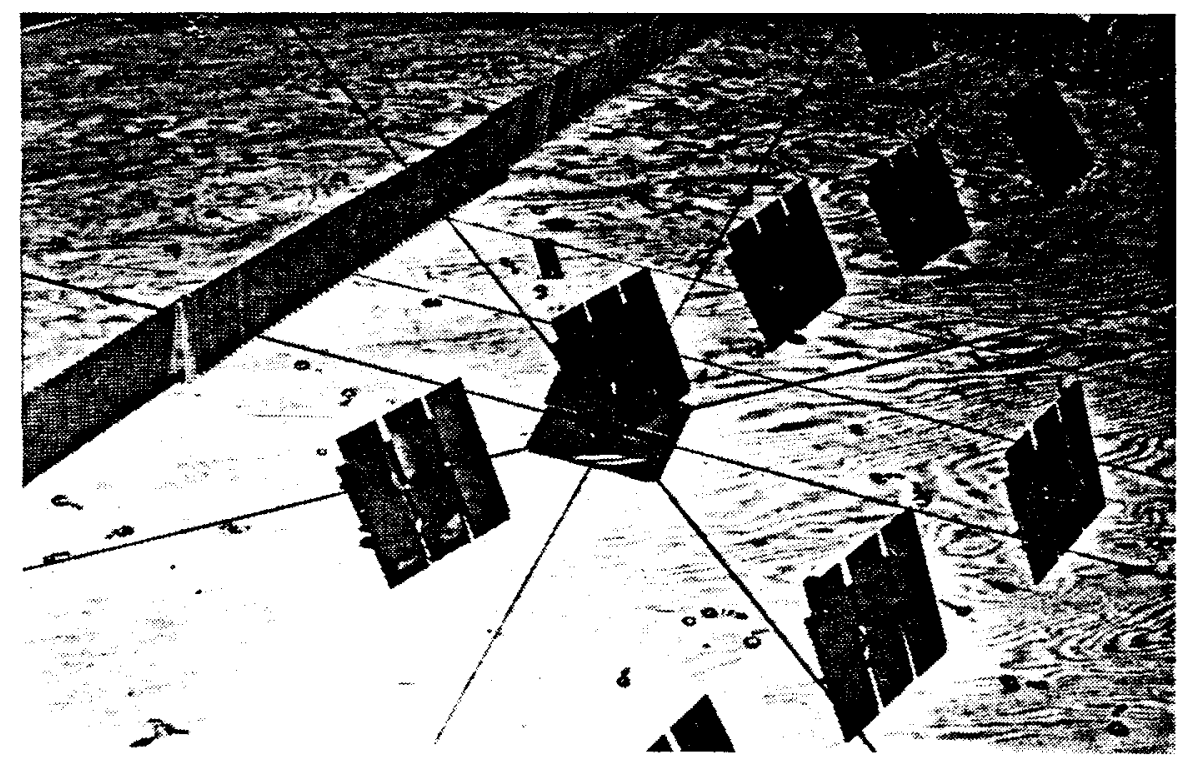

(d)

Figure 2-10. Model Installed in the Wind Tunnel 


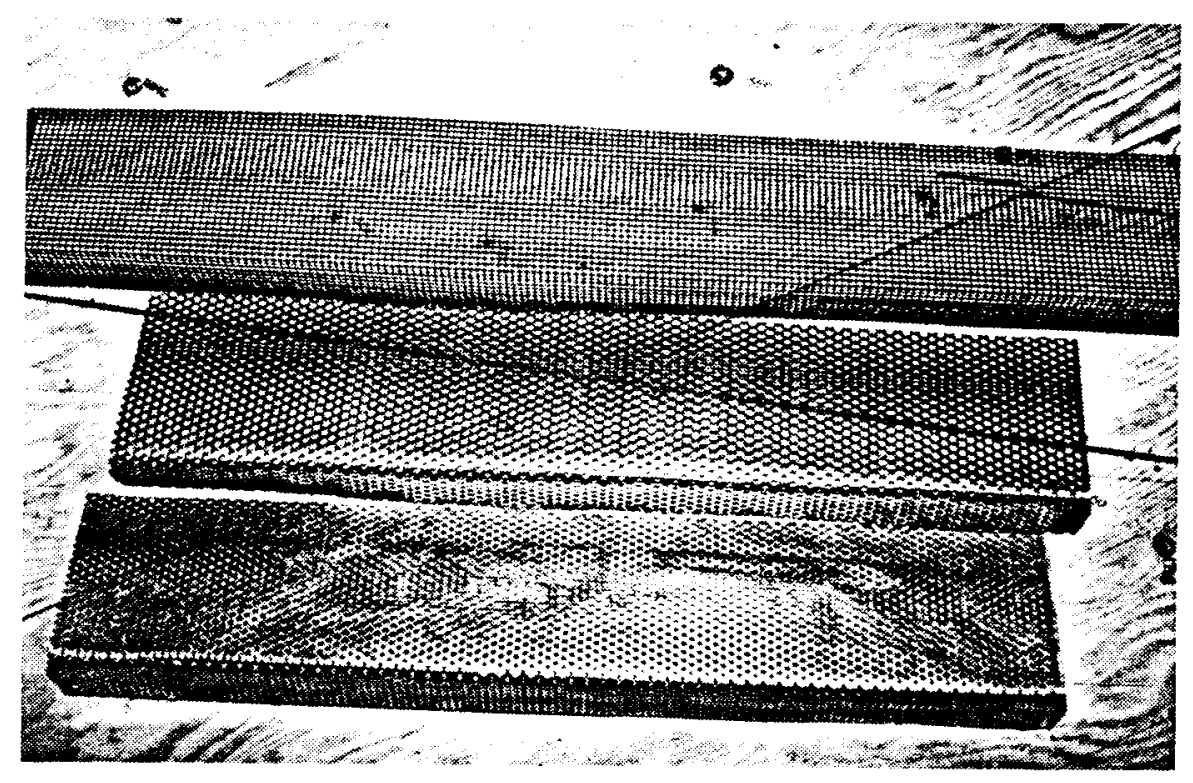

Figure 2-11. Porous Fence Segments Used with the Model 
SECTION 3.0

INSTRUMENTATION AND DATA ACQUISITION

\subsection{SELECTION OF VELOCITY SCALE RATIO}

In addition to the boundary-layer simulation, similitude of velocity between the model and prototype is considered in order to interpret the wind-tunnel results. The velocity scale ratio may be obtained from dimensionless analysis

$$
\lambda_{\mathrm{U}}=\frac{\mathrm{U}_{\mathrm{m}}}{\mathrm{U}_{\mathrm{p}}}=\frac{\mathrm{L}_{\mathrm{m}}}{\mathrm{L}_{\mathrm{p}}} \frac{\mathrm{T}_{\mathrm{p}}}{\mathrm{T}_{\mathrm{m}}}=\lambda_{\mathrm{L}} \lambda_{\mathrm{T}}^{-1},
$$

where $\mathrm{L}, \mathrm{U}$ and $\mathrm{T}$ represent length, velocity and time, and subscripts $\mathrm{m}$ and $\mathrm{p}$ represent the model and prototype parameters. $\lambda_{\mathrm{U}}, \lambda_{\mathrm{L}}$ and $\lambda_{\mathrm{T}}$ are the scale ratios of velocity, length and time between the model and prototype.

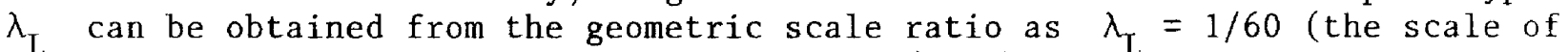
the heliostat model as noted in Section 2.2). $\lambda_{\mathrm{T}}$ is related to the scale ratio of frequency, $\lambda_{\mathrm{T}}=\lambda_{\mathrm{f}}^{-1}$. The velocity ratio between model and prototype is then

$$
\lambda_{\mathrm{U}}=\lambda_{\mathrm{L}} \lambda_{\mathrm{f}}
$$

The frequency ratio between model and prototype is established by considering the nondimensional frequency $\widetilde{f}=\mathrm{fL} / \mathrm{U}$. Similarity requirements [28-30] for wind-tunnel modeling require that $\widetilde{f}$ be the same in model and full scale, $\widetilde{\mathrm{f}}_{\mathrm{m}}=\widetilde{\mathrm{f}}_{\mathrm{p}}$ :

$$
\lambda_{\mathrm{f}}=\lambda_{\mathrm{U}} \lambda_{\mathrm{L}}^{-1}
$$

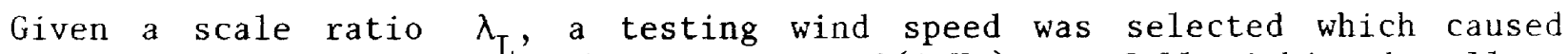
frequencies of interest in the prototype, $0(1 \mathrm{~Hz})$, to fall within the allowable frequency range of the model/balance combination, $0-18 \mathrm{~Hz}$ (see Section 3.3). By appropriate selection of wind-tunnel speed, a single measurement of the loading spectrum (frequency decomposition of the time varying loading) permits the spectral loading for a range of full-scale velocities to be determined. Thus for $\lambda_{\mathrm{L}}=1 / 60, \mathrm{f}_{\mathrm{p}} \cong 1 \mathrm{~Hz}$ and $\mathrm{U}_{\mathrm{m}}=$ $20 \mathrm{fps}$, the range of full-scale velocities represented in a spectrum is $45 \mathrm{mph}$ and up. A larger frequency range in the model would permit lower full-scale velocities to be simulated. Such a capability is now in final stages of completion.

The wind-tunnel Reynolds number is approximately $3-7 \times 10^{4}$ at the testing wind speeds used for this study which are sufficiently large to achieve Reynolds number independence of the aerodynamic coefficient. Hence, the wind load data measured in the wind tunnel are directly applicable to the design of the ful1-scale heliostat structure.

The largest limitations of the dynamic model tests were the relatively low model/balance natural frequency ( $18 \mathrm{~Hz}$ limit on frequency--a value of $100 \mathrm{~Hz}$ or higher would provide a wider range of frequency in the spectral loading) 
and a modest mismatch between wind-tunnel boundary-layer turbulence scale and model scale. The latter limitation causes a decrease in low frequency quasi-static gust amplitudes for collectors near the edge of the collector field. Further discussion of these issues and methods for their resolution are contained in Sections 3.2 and 4.0 .

\subsection{VELOCITY MEASUREMENTS}

Mean velocity and turbulence intensity profiles were measured without the presence of the model to determine that an approach boundary-layer flow appropriate to the site had been established. Tests were made at one wind velocity in the tunnel. This velocity was well above that required to produce Reynolds number similarity between the model and the prototype as discussed in Section 1.0 .

Measurements were made with a single hot-wire anemometer mounted with its axis vertical. The instrumentation used was a Thermo Systems constant temperature anemometer (Model 1050) with a 0.001 in. diameter platinum film sensing element $0.020 \mathrm{in.}$ long. Output was directed to the on-line data acquisition system for analysis.

Calibration of the hot-wire anemometer was performed by comparing output with the pitot-static tube in the wind tunnel. The calibration data were fit to a variable exponent King's Law relationship of the form

$$
\mathrm{E}^{2}=\mathrm{A}+\mathrm{BU} \mathrm{C}^{\mathrm{C}}
$$

where $E$ is the hot-wire output voltage, $U$ the velocity and $A, B$, and $C$ are coefficients selected to fit the data. The above relationship was used to determine the mean velocity at measurement points using the measured mean voltage. The fluctuating velocity in the form $U_{r m s}$ (root-mean-square velocity) was obtained from

$$
U_{r m s}=\frac{2 E E_{r m s}}{B \subset U^{c-1}}
$$

where $E_{r m s}$ is the root-mean-square voltage output from the anemometer.

Velocity and turbulence profiles are shown in Figure 3-1. The boundary-layer thickness in the wind tunnel, $\delta$, is shown in Figure $3-1$ as $220 \mathrm{ft}$ full scale. This depth is not the full-scale boundary-layer height but represents a partial-depth modeling of the atmosphere boundary layer. The mean velocity profile approaching the modeled area has the form

$$
\frac{\mathrm{U}_{\text {mean }}}{\mathrm{U}_{\text {ref }}}=\left(\frac{\mathrm{Z}}{\mathrm{Z}_{\text {ref }}}\right)^{\mathrm{n}},
$$

where $U$ indicates the local mean velocity and $U_{\text {ref }}$ is the mean wind speed at the reference height, $Z_{\text {ref }}$. The value of velocity at $Z_{\text {ref }}=6.56$ in. 

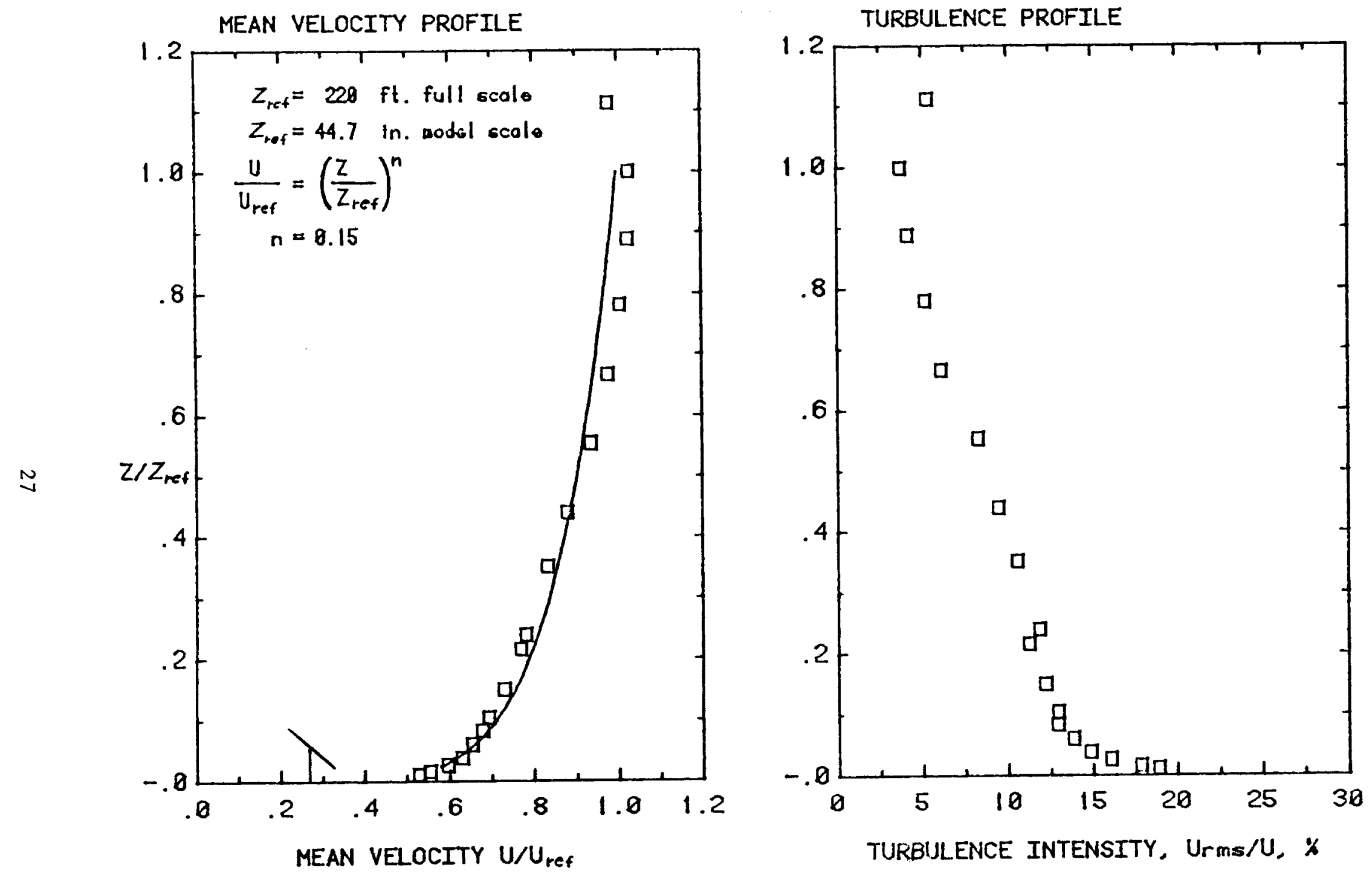

Figure 3-1. Approach Wind Profiles 
in the model, which corresponds to $10 \mathrm{~m}$ in prototype, was used to calculate the force and moment coefficients. The exponent $n$ for the approach flow established for this study is $1 / 7$ which is representative of an open-field environment. Turbulence intensity in the velocity profile measurement is defined as:

$$
\mathrm{I}=\frac{\mathrm{U}_{\mathrm{rms}}}{\mathrm{U}_{\text {mean }}} \times 100 .
$$

Uncertainties in velocity were within $1-3$ percent of the maximum within the boundary layer.

Longitudinal turbulence spectra were measured at the $10 \mathrm{~m}$ height used for reference velocity. Longitudinal refers to velocity fluctuations in the direction of the wind. The turbulence spectrum is compared to the atmospheric turbulence spectrum in Figure 3-2. A discussion of spectra and the presentation format is discussed in Section 4.1. The integral length scale was four times larger than the characteristic length of the heliostat model. The wind-tunnel does not simulate the lower frequency gustiness due to the limitation of the tunnel cross section size for a 1:60 model scale. This can result in underestimation of peak fluctuating wind load on the heliostat from the lack of the low-frequency spectral content. However, inside the heliostat field, the turbulence characteristics are dominated by the small eddies generated by upstream heliostats which are no greater than the size of the heliostat. Thus, while the peak fluctuating loads on edge-field heliostats may be slightly underestimated, loads on in-field heliostats should be well represented. The quantitative evaluation of the effect of the missing low-frequency turbulence can be accounted for with additional research.

\subsection{FORCE AND MOMENT MEASUREMENTS}

\subsubsection{Force Balance}

The force balance used in this project is shown in Figure 2-4. It is a strain-sensing apparatus consisting of four main parts: a reaction or inertial ring, a steel sprung plate supported by steel cross-beams, two nested portal gages and a stem of aluminum tubing. The reaction ring is bolted to the wind-tunnel turntable below the floor level. The entire balance rotates along with the model on the turntable, and thus defines a body-centered coordinate system. A right-handed coordinate system (Figure 3-3) is oriented with the z-axis coinciding with the model and force balance vertical axis, and the $x$ and $y$ axes in the horizontal plane at the pivot point of the heliostat $13.5 \mathrm{ft}$ above ground level. Moments about the $x$ and $y$ axes were actually sensed about $x^{\prime}$ and $y^{\prime}$ axes parallel to the $x, y$ axes but at the height of the reaction ring placed below floor level. Moments were transferred from $x^{\prime}, y^{\prime}$ axes to $x, y$ axes at each data sample point in time by classical methods of statics.

The model was designed to be as light as possible to obtain a high natural frequency of the model/balance permitting measurement of dynamic loading without excessive resonant amplification. Figure 3-4 shows the response of the balance to fluctuating load inputs for the six components. The response of all six channels can be represented by two curves: the shape of Figure $3-4 a$ occurs from a flat response attenuated at higher frequencies by an 


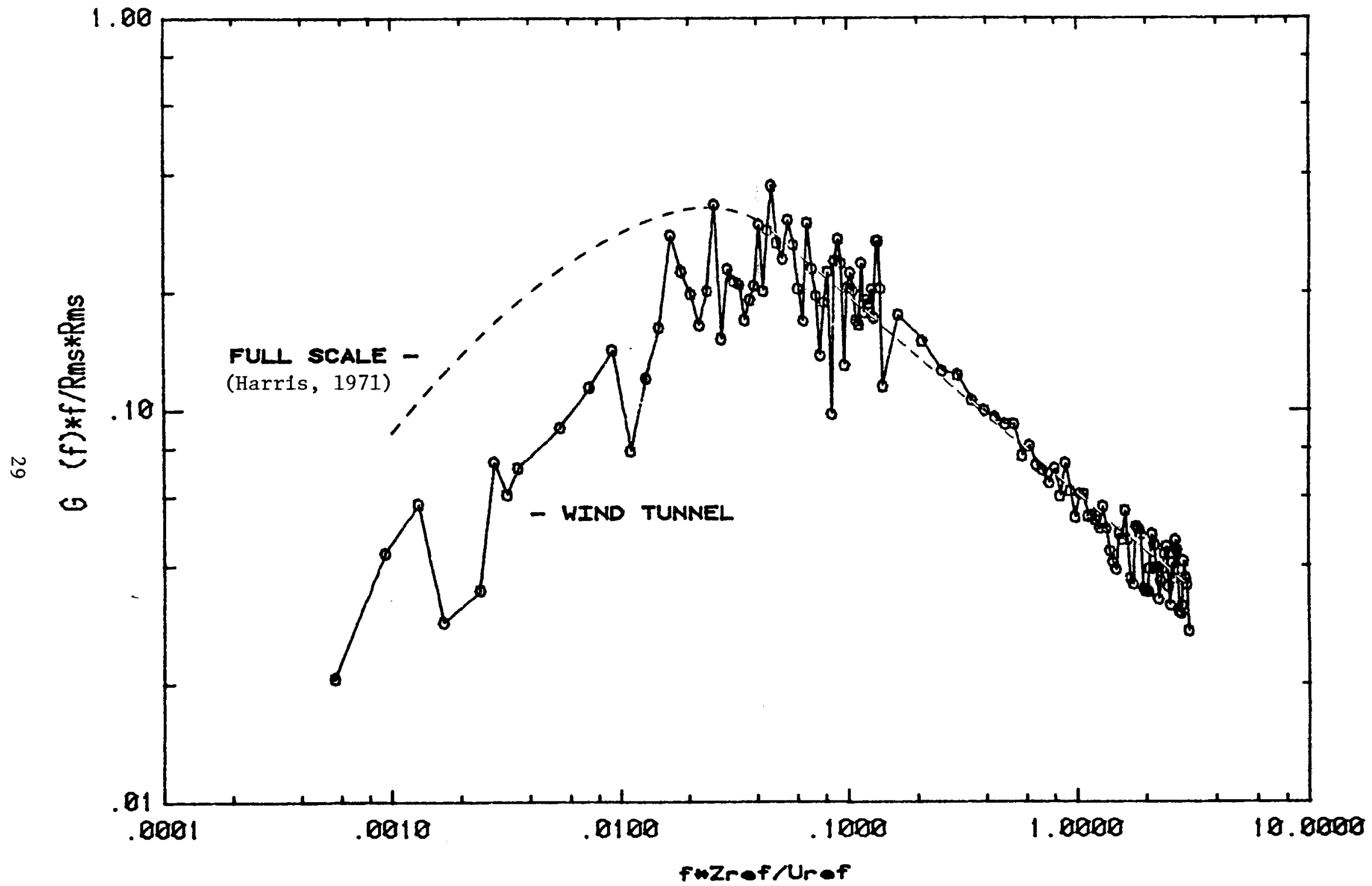

Figure 3-2. Turbulence Power Spectrum of Approach Wind 


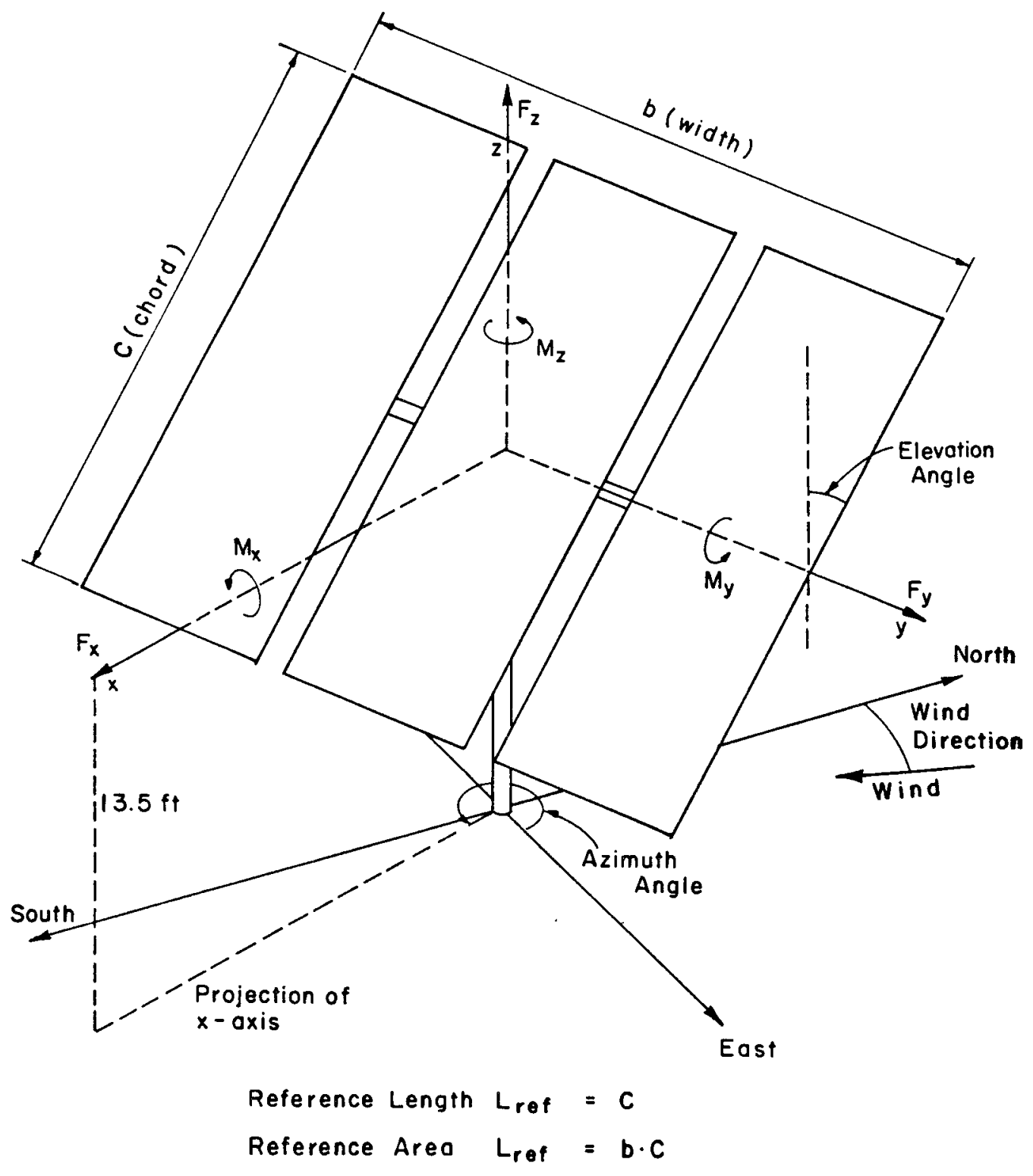

Figure 3-3. Force and Moment Coordinate System 


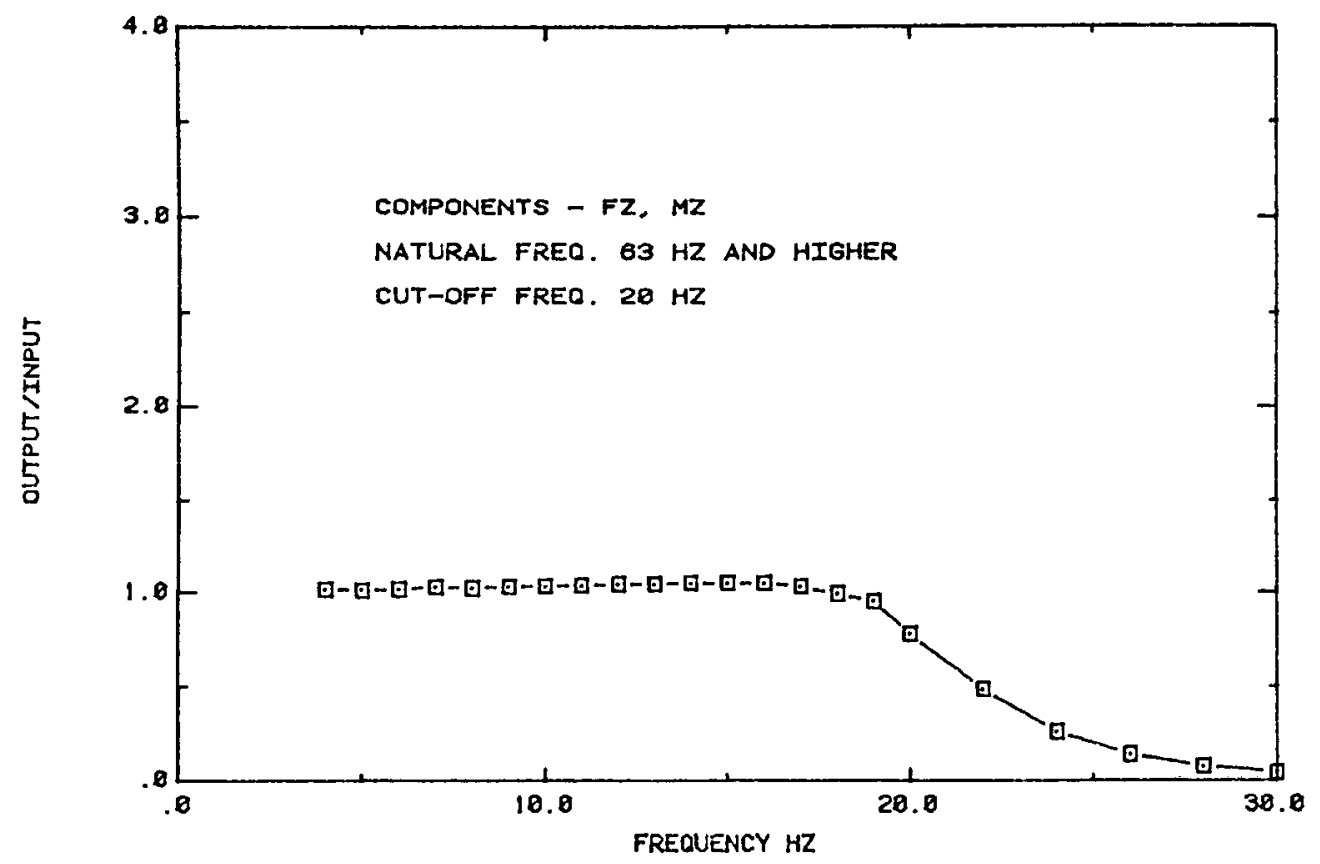

FREOUENCY RESPONSE OF FDRCE BALANCE WITH HELIIOSTAT MODEL

(a)

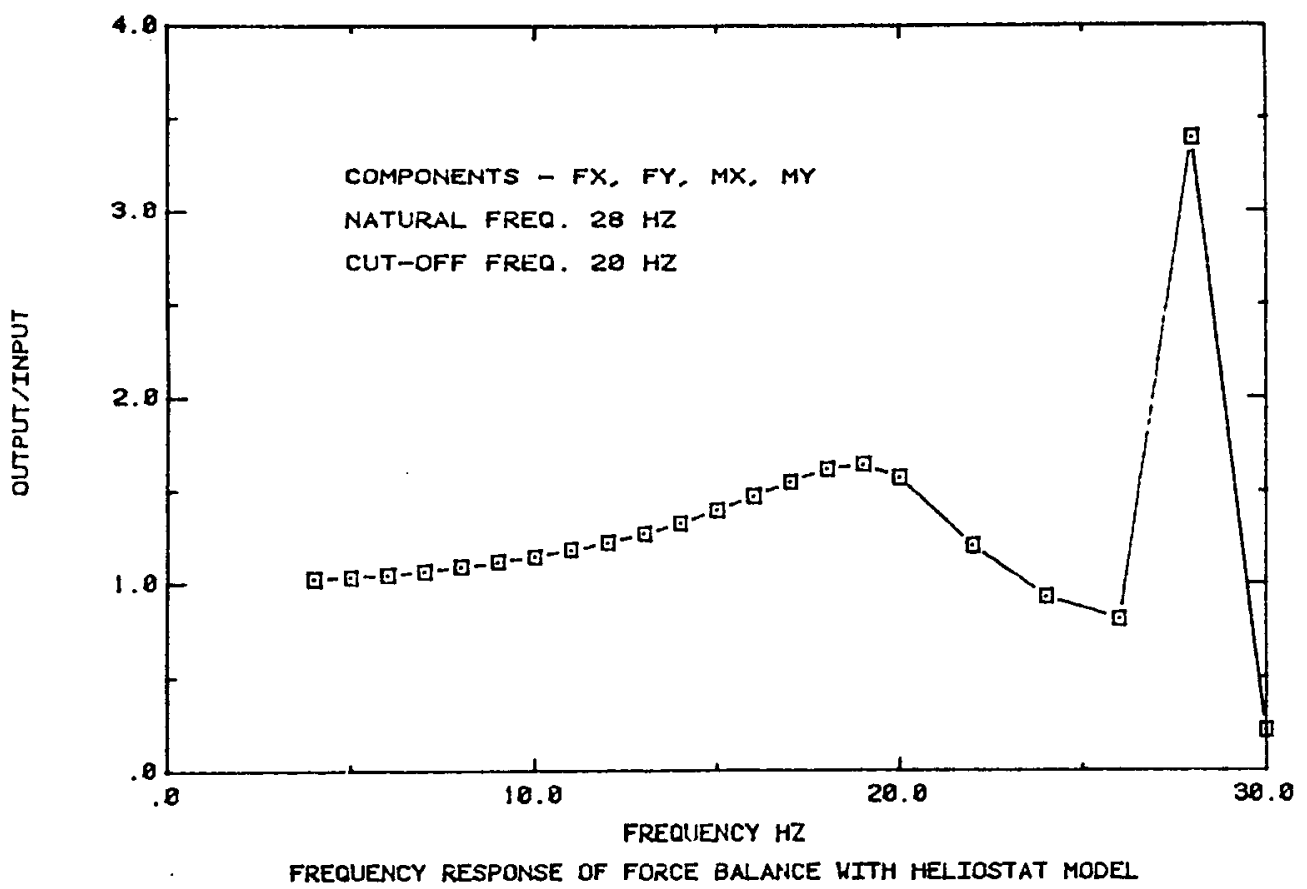

(b)

Figure 3-4. Force Balance Frequency Response 
anti-aliasing roll-off filter; the shape in Figure $3-4 b$ occurs as a combination of a $28 \mathrm{~Hz}$ model natural frequency for the four components represented with a roll-off filter. The response of the $z$ force and moment about $\mathrm{Z}$ was flat to $18 \mathrm{~Hz}$ where a low-pass filter cut the signal. The response for the other four components was not as satisfactory: the $28 \mathrm{~Hz}$ natural frequency caused distortion in the response curve, even with the low-pass filter, which would not be acceptable for determining design quality information. However, the approximate magnitudes of dynamic loading and trends of loading with various variables can be determined. The difficulties with the response shown in Figure 3-4b can now be eliminated with the completion of a new balance where response in all six components should be flat to $100 \mathrm{~Hz}$ or more.

Calibration of the entire force balance system was performed in the wind tunnel using the same electronics and data-acquisition system used during testing. Weights and a fish-line were used to pull on the stem at a certain position and the output was monitored at the same time. The resulting calibration curves were very linear over the measurement range. Interactions between the six channels were always less than 2-3 percent and linear. Interactions were removed using standard balance measurement techniques.

Accuracy of measurement was about 5 to 10 percent or better of the maximum value recorded in that channel. Thus the only data subject to concern because of accuracy are the stow position loads. Most of the effort in this measurement program was directed at operational conditions.

The forces and moments measured on the heliostat model are expressed, respectively, in terms of the nondimensional coefficients $C_{F_{x}}, C_{F_{y}}, C_{F_{z}}, C_{M_{x}}$, $\mathrm{C}_{\mathrm{M}}, \mathrm{C}_{\mathrm{M}_{\mathrm{z}}}$. They are defined as follows:

force coefficient along the $x$-axis

$$
C_{F_{x}}=\frac{F_{x}}{\left(\frac{\rho U_{\text {ref }}^{2}}{2}\right)\left(A_{\text {ref }}\right)},
$$

force coefficient along the y-axis

$$
C_{\mathrm{F}}=\frac{F_{y}}{\left(\frac{\rho U_{\text {ref }}^{2}}{2}\right)\left(A_{\text {ref }}\right)},
$$

force coefficient along the $z$-axis

$$
\mathrm{C}_{\mathrm{z}}=\frac{\mathrm{F}_{\mathrm{z}}}{\left(\frac{\rho \mathrm{U}_{\text {ref }}^{2}}{2}\right)\left(\mathrm{A}_{\text {ref }}\right)},
$$

moment coefficient about the $x$-axis

$$
C_{M_{x}}=\frac{M_{x}}{\left(\frac{\rho U_{\text {ref }}^{2}}{2}\right)\left(A_{\text {ref }}\right)\left(L_{\text {ref }}\right)},
$$


moment coefficient about the $y$-axis

$$
C_{M}=\frac{M}{\left(\frac{\rho U_{\text {ref }}^{2}}{2}\right)\left(A_{\text {ref }}\right)\left(L_{\text {ref }}\right)},
$$

moment coefficient about the $z$-axis

$$
C_{M_{z}}=\frac{M_{z}}{\left(\frac{\rho U_{\text {ref }}^{2}}{2}\right)\left(A_{\text {ref }}\right)\left(L_{\text {ref }}\right)} \text {, }
$$

where

$$
\begin{aligned}
& \mathrm{U}_{\text {ref }} \quad \text { reference mean velocity at } 10 \mathrm{~m}(6.6 \mathrm{in} \text {. model }) \\
& \rho \\
& \quad=\text { density of air, } \\
& A_{\text {ref }}=\text { reference area } 19.6 \mathrm{in}^{2} \text { model, } 489.9 \mathrm{ft}^{2} \mathrm{full} \text { scale } \\
& \mathrm{L}_{\text {ref }}=\text { reference length } 4.26 \mathrm{in} .^{2} \text { model, } 21.3 \mathrm{ft} \text { full scale } \\
& \mathrm{F}_{\mathrm{x}}, \mathrm{F}_{\mathrm{y}}, \mathrm{F}_{\mathrm{z}}=\text { measured force along axis, positive force in positive } \\
& \mathrm{M}_{\mathrm{z}}, \mathrm{M}_{\mathrm{x}}, \mathrm{M}_{\mathrm{y}}=\text { measured moment about axis, sign by right-hand rule }
\end{aligned}
$$

For each coefficient component, five values were computed:

- mean - time average

- rms - root-mean-square of the fluctuating value about the mean

- peaks - the largest and smallest values recorded during a time of roughly 10 to 30 minutes full scale (32 seconds model scale)

- gust factor - G, peak divided by mean

- peak factor - g, $\frac{(\text { peak - mean) }}{\text { rms }}$

The significance of the gust factor is that current wind code formulations use a gust factor approach to obtain peak values from mean coefficients. This approach has significant limitations in that fluctuating loads are not always proportional to the mean load. The peak factor is the number of standard deviations of the peak from the mean. This calculation approach permits dynamic loads to be analyzed separately from the mean load and then added to the mean. This approach is associated with random vibration theory, an approach to analyzing dynamic loading which has the greatest promise for systematically defining peak loads. 


\subsection{TEST PROCEDURE AND TEST MATRIX}

Prior to the data acquisition phase, a tentative test plan was established whose intent was to guide the data acquisition. The test plan revolved about the recognized need to measure data cross sections across several variables:

- heliostat setting angles (day of year, time of day)

- position of heliostat in field

- approach wind direction

- presence of external fence

- presence of internal fence along lines of heliostats

- presence of internal fences perpendicular to lines of heliostats

- height of fences

- porosity of fences (including solid berms)

- density of field (number of heliostats per unit area of ground surface)

In order to adequately cover the ranges of variables, it was found necessary to change the test plan somewhat during the testing in response to findings earlier in the test program. For example, the protection afforded to heliostats in the denser portions of the Barstow field by upwind heliostats was sufficiently high that low sensitivity to in-field fences was noted. For this reason, additional field density experiments were added to show how in-field fences provided protection. The result of the modified test plan was a set of curves which effectively collapsed mean load data from most of the variables listed above onto very few curves.

The test plan can be broken into two basic parts--wind loads on isolated heliostats and wind loads on heliostats in a field of units. Both mean and dynamic loads were measured for the two situations.

The test matrix for the isolated heliostat is shown in Table 3-1. This data was needed to obtain the baseline loads against which the loads in the heliostat field can be measured. Mean, rms and peak loads were measured for each case. Spectra were obtained for only a limited set of conditions for the purpose of evaluating the dynamic measurements. Data values corresponding to the runs listed in Table 3-1 are listed in Appendix B.

The test matrix for the in-field heliostats is shown in Table 3-2. Because of the large number of individual runs, Table 3-2 provides a summary of test conditions but omits the details of individual values for heliostat angle settings and wind directions. These values are listed in an expanded test matrix form for each configuration in Appendix $C$. Mean, rms and peak loads were measured for each case. Spectra were measured for selected cases.

The interpretation of the data of Appendices $B$ and $C$ is presented in Section 4.0 . 
Table 3-1. Test Matrix for Single Heliostat

\begin{tabular}{c|rrrrrrrrrr}
\hline \multirow{2}{*}{ Elevation Angle } & \multicolumn{7}{|c}{ Wind Direction } \\
\cline { 2 - 9 } 0 & 0 & 22.5 & 45 & 67.5 & 90 & 112.5 & 135 & 157.5 & 180 \\
\hline 15 & 115 & 113 & 111 & 109 & 107 & 105 & 103 & 101 & 99 \\
30 & 246 & - & 244 & - & 242 & - & 240 & - & 238 \\
45 & 228 & - & 230 & - & 232 & - & 234 & - & 236 \\
60 & 136 & 138 & 140 & 142 & 144 & 146 & 148 & 150 & 152 \\
75 & 226 & - & 224 & - & 222 & - & 220 & - & 218 \\
80 & 208 & - & 210 & - & 212 & - & 214 & - & 216 \\
84 & 81 & 83 & 85 & 87 & 89 & 91 & 93 & 95 & 97 \\
87 & 170 & 168 & 166 & 164 & 162 & 160 & 158 & 156 & 154 \\
90 & 172 & 174 & 176 & 178 & 180 & 182 & 184 & 186 & 188 \\
& 206 & 204 & 202 & 200 & 198 & 196 & 194 & 192 & 190 \\
\hline
\end{tabular}

Note: The numbers in the table indicate the test run number.

Azimuth angle was set at $270^{\circ}$ for all runs--see Figure 3-3.

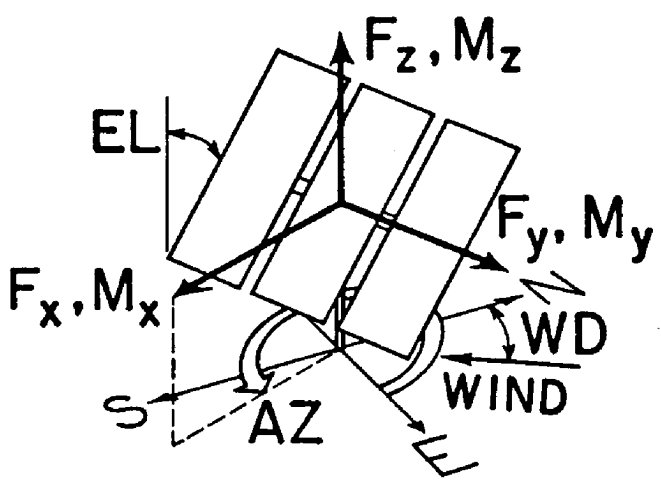


Table 3-2. Test Matrix for In-Field Heliostats

\begin{tabular}{|c|c|c|c|c|c|c|c|}
\hline Heliostat & Conf. & Case & $\begin{array}{l}\text { \# of Wind } \\
\text { Directions }\end{array}$ & Fence*t & Note & $\begin{array}{l}\text { Data } \\
\text { File }\end{array}$ & Page \\
\hline \multirow[t]{7}{*}{1} & \multirow[t]{7}{*}{$1-\mathrm{A}$} & Smr AM & 7 & \multirow[t]{7}{*}{ None } & \multirow[t]{7}{*}{$\begin{array}{l}\text { See Figure } 2-2 \\
\text { without fences }\end{array}$} & \multirow[t]{7}{*}{$\mathrm{H} 1100$} & 115 \\
\hline & & Smr Noon & 7 & & & & 115 \\
\hline & & Whtr AM & 7 & & & & 115 \\
\hline & & Wntr Noon & 7 & & & & 116 \\
\hline & & Wntr PM & 7 & & & & 116 \\
\hline & & Ver. Stow & 1 & & & & 116 \\
\hline & & Hor. Stow & 1 & & & & 116 \\
\hline \multirow[t]{7}{*}{1} & \multirow[t]{7}{*}{$1-B$} & Smr AM & 7 & \multirow[t]{7}{*}{1} & \multirow{7}{*}{$\begin{array}{l}\text { See Figure } 2-5 \\
\text { with perimeter } \\
\text { fence }\end{array}$} & \multirow[t]{7}{*}{ H1101 } & 127 \\
\hline & & Smr Noon & 7 & & & & 127 \\
\hline & & Wntr AM & 7 & & & & 127 \\
\hline & & Whtr Noon & 7 & & & & 128 \\
\hline & & Whtr PM & 7 & & & & 128 \\
\hline & & Ver. Stow & 1 & & & & 128 \\
\hline & & Hor. Stow & 1 & & & & 128 \\
\hline \multirow[t]{5}{*}{5} & \multirow[t]{5}{*}{$5-A$} & Whtr AM & 6 & \multirow[t]{5}{*}{ None } & \multirow{5}{*}{$\begin{array}{l}\text { Simulation of } \\
\text { heliostat field } \\
\text { within circle } \\
\text { on Figure } 2-2 \\
\text { without fences }\end{array}$} & \multirow[t]{5}{*}{ H500O } & 139 \\
\hline & & Whtr Noon & 6 & & & & 139 \\
\hline & & Wntr PM & 6 & & & & 139 \\
\hline & & Ver. Stow & 1 & & & & 139 \\
\hline & & Hor. Stow & 1 & & & & 139 \\
\hline
\end{tabular}

$\therefore$ See Table $3-3$ 
Table 3-2 (continued)

\begin{tabular}{|c|c|c|c|c|c|c|c|}
\hline Heliostat & Conf. & Case & $\begin{array}{l}\text { \# of Wind } \\
\text { Directions }\end{array}$ & Fence* & Note & $\begin{array}{l}\text { Data } \\
\text { File }\end{array}$ & Page \\
\hline \multirow[t]{7}{*}{5} & \multirow[t]{7}{*}{$5-B$} & Smr AM & 6 & \multirow[t]{7}{*}{1} & \multirow{7}{*}{$\begin{array}{l}\text { Simulation of } \\
\text { heliostat field } \\
\text { within circle } \\
\text { on Figure } 2-2 \\
\text { with perimeter } \\
\text { fence }\end{array}$} & \multirow[t]{7}{*}{$\mathrm{H} 5001$} & 145 \\
\hline & & Smr Noon & 6 & & & & 145 \\
\hline & & Wntr AM & 6 & & & & 145 \\
\hline & & Wntr Noon & 6 & & & & 145 \\
\hline & & Wntr PM & 6 & & & & 146 \\
\hline & & Ver. Stow & 1 & & & & 146 \\
\hline & & Hor. Stow & 1 & & & & 146 \\
\hline \multirow[t]{7}{*}{5} & $5-C$ & Smr AM & 6 & \multirow[t]{7}{*}{ None } & \multirow{7}{*}{$\begin{array}{l}\text { Simulation to } \\
\text { edge of field } \\
\text { without fences, } \\
\text { see Figure 2-2 }\end{array}$} & \multirow[t]{7}{*}{$\mathrm{H} 5100$} & 155 \\
\hline & & Smr Noon & 6 & & & & 155 \\
\hline & & Wntr AM & 6 & & & & 155 \\
\hline & & Wntr Noon & 6 & & & & 155 \\
\hline & & Whtr PM & 6 & & & & 156 \\
\hline & & Ver. Stow & 1 & & & & 156 \\
\hline & & Hor. Stow & 1 & & & & 156 \\
\hline \multirow[t]{7}{*}{5} & $5-D$ & Smr AM & 6 & \multirow[t]{7}{*}{1} & \multirow{7}{*}{$\begin{array}{l}\text { Simulation to } \\
\text { edge of field } \\
\text { with perimeter } \\
\text { fence, see } \\
\text { Figures } 2-2,2-6\end{array}$} & \multirow[t]{7}{*}{ H5101 } & 165 \\
\hline & & Smr Noon & 6 & & & & 165 \\
\hline & & Whtr AM & 6 & & & & 165 \\
\hline & & Wntr Noon & 6 & & & & 165 \\
\hline & & Wntr PM & 6 & & & & 166 \\
\hline & & Ver. Stow & 1 & & & & 166 \\
\hline & & Hor. Stow & 1 & & & & 166 \\
\hline
\end{tabular}

*See Table 3-3 
Table 3-2 (continued)

\begin{tabular}{|c|c|c|c|c|c|c|c|}
\hline Heliostat & Conf. & Case & $\begin{array}{l}\text { 非 of Wind } \\
\text { Directions }\end{array}$ & Fence $*$ & Note & $\begin{array}{l}\text { Data } \\
\text { File }\end{array}$ & Page \\
\hline \multirow[t]{7}{*}{5} & $5-E$ & Smr AM & 6 & 2 & $\begin{array}{l}\text { Simulation to } \\
\text { edge of field }\end{array}$ & H5 102 & 175 \\
\hline & & Smr Noon & 6 & & $\begin{array}{l}\text { with perimeter } \\
\text { and internal }\end{array}$ & & 175 \\
\hline & & Wntr AM & 6 & & fences, see & & 175 \\
\hline & & Whtr Noon & 6 & & 1 Igutes $<2,<0$ & & 175 \\
\hline & & Whtr PM & 6 & & & & 176 \\
\hline & & Ver. Stow & 1 & & & & 176 \\
\hline & & Hor. Stow & 1 & & & & 176 \\
\hline 3 & $3-A$ & Ver. Stow & 1 & None & $\begin{array}{l}\text { See Figures } 2-2 \text {, } \\
2-7 \text {, simulation } \\
\text { to edge of field, } \\
\text { no fences }\end{array}$ & H3100 & 185 \\
\hline 3 & $3-B$ & Ver. Stow & 1 & None & $\begin{array}{l}\text { Effect of number } \\
\text { of rows upstream, } \\
\text { see Figure } 2-7\end{array}$ & $\mathrm{H} 3200$ & 187 \\
\hline 3 & $3-c$ & Ver. Stow & 1 & None & $\begin{array}{l}\text { Effect of field } \\
\text { density, } \\
\text { see Figure 2-7 }\end{array}$ & $\mathrm{H} 3300$ & 191 \\
\hline 3 & $3-D$ & Ver. Stow & 1 & None & $\begin{array}{l}\text { Effect of number } \\
\text { of rows upstream } \\
\text { in field of } \\
\text { reduced density } \\
\text { (original x } 1 / 4 \text { ), } \\
\text { see Figure } 2-7\end{array}$ & $\mathrm{H} 3400$ & 194 \\
\hline 3 & $3-E$ & Ver. Stow & 1 & 1 & $\begin{array}{l}\text { Effect of number } \\
\text { of rows upstream } \\
\text { in field of } \\
\text { reduced density } \\
\text { (original x } 1 / 4 \text { ), } \\
\text { see Figure } 2-8 \text {, } \\
\text { w/perimeter fence }\end{array}$ & H3401 & 196 \\
\hline
\end{tabular}


Table 3-2 (continued)

\begin{tabular}{|c|c|c|c|c|c|c|c|c|}
\hline Heliostat & Conf. & Cas & & $\begin{array}{l}\text { 非 of Wind } \\
\text { Directions }\end{array}$ & Fence* & Note & $\begin{array}{l}\text { Data } \\
\text { File }\end{array}$ & Page \\
\hline 3 & $3-F$ & Ver. & Stow & 1 & 2 & $\begin{array}{l}\text { Effect of number } \\
\text { of rows upstream, } \\
\text { in field of } \\
\text { reduced density } \\
\text { (original } 1 \text { x } 1 / 4) \text {, } \\
\text { see Figure } 2-8 \\
\text { with perimeter and } \\
\text { internal fence }\end{array}$ & H3402 & 198 \\
\hline 3 & $3-G$ & Ver. & Stow & 1 & 5 & $\begin{array}{l}\text { Effect of number } \\
\text { of rows upstream } \\
\text { in field of } \\
\text { reduced density } \\
\text { (original } x 1 / 4 \text { ), } \\
\text { see Figure } 2-8 \text {, } \\
\text { same as } 3-\mathrm{F} \text { with } \\
50 \% \text { fence porosity }\end{array}$ & H3405 & 200 \\
\hline 3 & $3-\mathrm{H}$ & Ver. & Stow & 1 & 6 & $\begin{array}{l}\text { Effect of number } \\
\text { of rows upstream } \\
\text { in field of } \\
\text { reduced density } \\
\text { (original } \times 1 / 4 \text { ), } \\
\text { see Figure } 2-8 \text {, } \\
\text { same as } 3-F \text { with } \\
40 \% \text { fence porosity }\end{array}$ & H3406 & 202 \\
\hline 3 & $3-I$ & Ver. & Stow & 7 & None & $\begin{array}{l}\text { Effect of reduced } \\
\text { density } \\
\text { (origina1 } 11 / 4 \text { ), } \\
2 \text { rows upstream, } \\
\text { see Figure } 2-9 \text {, } \\
\text { without fences }\end{array}$ & H3500 & 204 \\
\hline 3 & $3-J$ & Ver. & Stow & 7 & 1 & $\begin{array}{l}\text { Field of reduced } \\
\text { density } \\
\text { (original } 1 \text { } 1 / 4 \text { ), } \\
2 \text { rows upstream, } \\
\text { see Figure } 2-9, \\
\text { with external } \\
\text { fence }\end{array}$ & H3501 & 207 \\
\hline
\end{tabular}

$\because$ See Table 3-3 
Table 3-2 (continued)

\begin{tabular}{|c|c|c|c|c|c|c|c|}
\hline Heliostat & Conf. & Case & $\begin{array}{l}\text { \#k of Wind } \\
\text { Directions }\end{array}$ & Fence* & Note & $\begin{array}{l}\text { Data } \\
\text { File }\end{array}$ & Page \\
\hline 3 & $3-K$ & Ver. Stow & 7 & 2 & $\begin{array}{l}\text { Field of reduced } \\
\text { density } \\
\text { (original x } 1 / 4 \text { ), } \\
2 \text { rows upstream, } \\
\text { see Figure } 2-9, \\
\text { with external and } \\
\text { internal fences } \\
\text { parallel to rows }\end{array}$ & $\mathrm{H} 3502$ & 210 \\
\hline 3 & $3-\mathrm{L}$ & Ver. Stow & 7 & 3 & $\begin{array}{l}\text { Field of reduced } \\
\text { density } \\
\text { (original } x 1 / 4 \text { ), } \\
2 \text { rows upstream, } \\
\text { see Figure } 2-9, \\
\text { with external, } \\
\text { internal and } \\
\text { crossing fences }\end{array}$ & H3503 & 213 \\
\hline 3 & $3-M$ & Ver. Stow & 7 & 4 & $\begin{array}{l}\text { Field of reduced } \\
\text { density } \\
\text { (original x } 1 / 4 \text { ), } \\
2 \text { rows upstream, } \\
\text { see Figure } 2-9, \\
\text { with perimeter } \\
\text { berm, without } \\
\text { internal fences }\end{array}$ & H3504 & 216 \\
\hline 3 & $3-N$ & Ver. Stow & 7 & 5 & $\begin{array}{l}\text { Field of reduced } \\
\text { density } \\
\text { (original x } 1 / 4 \text { ), } \\
2 \text { rows upstream, } \\
\text { see Figure } 2-9, \\
\text { same as } 3-\mathrm{K} \text { with } \\
50 \% \text { fence porosity }\end{array}$ & H3505 & 219 \\
\hline \multirow[t]{7}{*}{4} & \multirow[t]{7}{*}{$4-A$} & Smr AM & 1 & \multirow[t]{7}{*}{ None } & \multirow{7}{*}{$\begin{array}{l}11 \text { rows upstream } \\
\text { were present, } \\
\text { see Figure } 2-2, \\
11 \text { rows modeled } \\
\text { upstream, } \\
265^{\circ} \text { wind }\end{array}$} & \multirow[t]{7}{*}{ H4100 } & \multirow[t]{7}{*}{222} \\
\hline & & Smr Noon & 1 & & & & \\
\hline & & Whtr AM & 1 & & & & \\
\hline & & Wntr Noon & 1 & & & & \\
\hline & & Whtr PM & 1 & & & & \\
\hline & & Ver. Stow & 1 & & & & \\
\hline & & Hor. Stow & 1 & & & & \\
\hline
\end{tabular}

*See Table 3-3 
Table 3-3. Description of Fences

\begin{tabular}{|c|c|c|c|c|c|}
\hline Configuration & Elements & Porosity & Height & Distance & Figures \\
\hline 1 & External Fence & $40 \%$ & $3 / 4 \mathrm{H}$ & $2 \mathrm{H}$ & $\begin{array}{l}2-5,2-6 \\
2-8,2-9\end{array}$ \\
\hline \multirow[t]{2}{*}{2} & External Fence & $40 \%$ & $3 / 4 \mathrm{H}$ & $2 \mathrm{H}$ & $\begin{array}{l}2-6,2-8 \\
2-9\end{array}$ \\
\hline & Internal Arc Fence & $60 \%$ & $1 / 2 \mathrm{H}$ & - & \\
\hline \multirow[t]{3}{*}{3} & External Fence & $40 \%$ & $3 / 4 \mathrm{H}$ & $2 \mathrm{H}$ & $2-9$ \\
\hline & Internal Arc Fence & $60 \%$ & $1 / 2 \mathrm{H}$ & - & \\
\hline & Internal Cross Fence & $60 \%$ & $1 / 2 \mathrm{H}$ & - & \\
\hline 4 & External Solid Berm & $0 \%$ & $3 / 4 \mathrm{H}$ & - & $2-9$ \\
\hline \multirow[t]{2}{*}{5} & External Fence & $40 \%$ & $3 / 4 \mathrm{H}$ & $2 \mathrm{H}$ & $2-9$ \\
\hline & Internal Arc Fence & $50 \%$ & $3 / 4 \mathrm{H}$ & - & \\
\hline \multirow[t]{2}{*}{6} & External Fence & $40 \%$ & $3 / 4 \mathrm{H}$ & $2 \mathrm{H}$ & $2-9$ \\
\hline & Internal Arc Fence & $40 \%$ & $3 / 4 \mathrm{H}$ & - & \\
\hline
\end{tabular}

Note: $\quad H=24.2 \mathrm{ft}$ 


\section{SECTION 4.0}

\section{RESULTS AND DISCUSSION}

\subsection{SINGLE HELIOSTAT}

Wind loads in coefficient form for an isolated heliostat are shown graphically in Figures 4-1 through 4-13. The heliostat was always pointing directly south $\left(A Z=270^{\circ}\right)$. The elevation angle and wind direction were systematically varied. Force and moment coefficients as a function of approach wind direction are shown in Figures 4-1 through 4-6 for elevation angles of $0^{\circ}$ (heliostat vertical), $45^{\circ}, 80^{\circ}, 84^{\circ}, 87^{\circ}$ and $90^{\circ}$ (heliostat horizontal). Figures 4-7 and 4-8 show forces and moments as a function of elevation angle for wind directions of $0^{\circ}$ and $45^{\circ}$. Wind directions are specified as wind azimuths with the wind approaching from the quoted direction with the angle measured clockwise from true north (see Figure 3-3).

$\mathrm{C}_{\mathrm{F}}$, the force coefficient in the $\mathrm{x}$ direction, is maximum for a vertical heliostat with wind approaching perpendicular to its broad face and decreases uniformly as heliostat tends to the horizontal or as wind direction approaches tangent to the heliostat surface at $90^{\circ} . \mathrm{C}_{\mathrm{F}}$, the force coefficient in the $y$ direction, is always small because of a small projected area in that direction. $C_{F_{z}}$ reaches a maximum at $60^{\circ}$ elevation angle, or $30^{\circ}$ to the horizontal in a manner characteristic of an airfoil. Moment coefficients reflect the movement of the center of wind pressure away from the pivot center which is also the center of heliostat area. The movement is due to two primary effects: the higher wind speeds at higher elevations which can increase wind loads near the top of the heliostat and more importantly the aerodynamic lift caused by the flow separation near the upstream edge of the heliostat when placed at an angle to the flow (roughly the same mechanism causing lift on a wing).

Conclusions can be obtained from the isolated heliostat data shown in Figures 4-1 through 4-8 about whether stow position loads will drive the design of heliostats. We can calculate the ratio of velocity in stow position to velocity in operational position which, for maximum loading orientation, will cause particular mean or peak forces or moments to reach the design strength. These ratios are somewhat tentative because the stow loads in this study were not far above the resolution level of the balance. These ratios are:

$\begin{array}{cccccc}\text { Mean Force or Moment } & \mathrm{F}_{\mathrm{x}} & \mathrm{F}_{\mathrm{z}} & \mathrm{M}_{\mathrm{x}} & \mathrm{M}_{\mathrm{y}} & \mathrm{M}_{\mathrm{z}} \\ \begin{array}{c}\text { Stow Position Velocity } \\ \text { Operational Position Velocity }\end{array} & 4.2 & 4.0 & 2.1 & 1.5 & 1.6 \\ \text { Peak Force or Moment } & \mathrm{F}_{\mathrm{x}} & \mathrm{F}_{\mathrm{z}} & \mathrm{M}_{\mathrm{x}} & \mathrm{M}_{\mathrm{y}} & \mathrm{M}_{\mathrm{z}} \\ \begin{array}{c}\text { Stow Position Velocity } \\ \text { Operational Position Velocity }\end{array} & 4.4 & 2.1 & 1.3 & 1.5 & 1.5\end{array}$



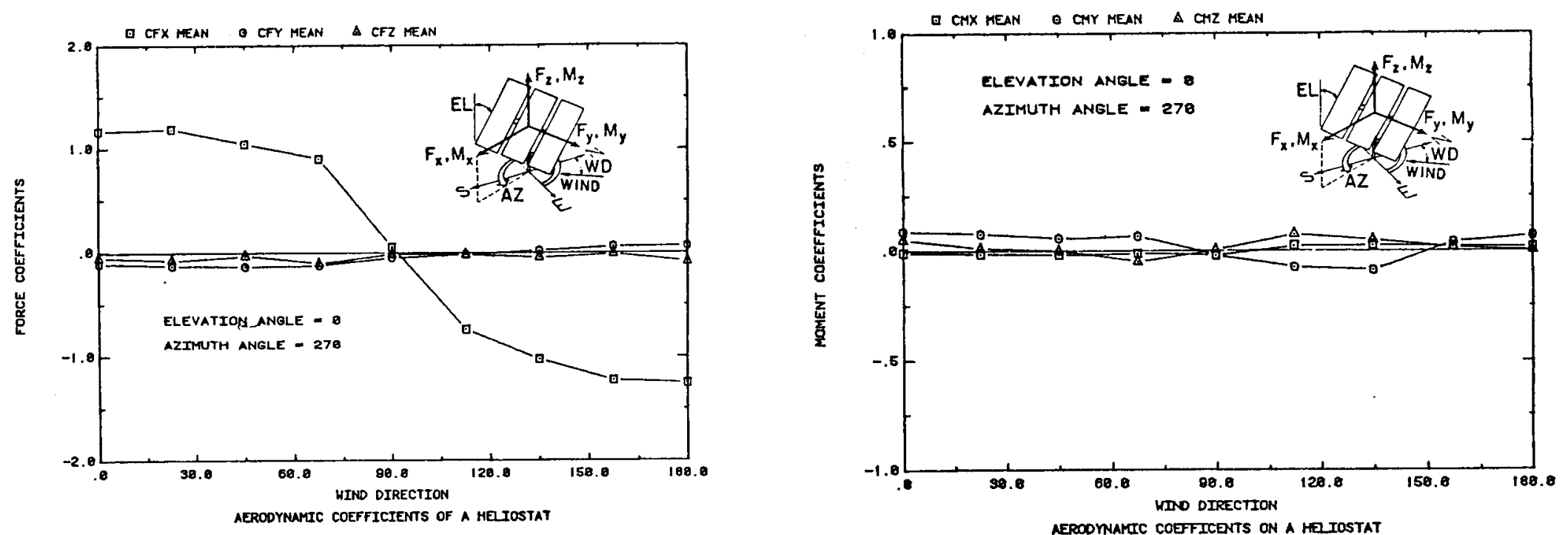

Figure 4-1a,b. Mean Force and Moment Coefficients for an Isolated Heliostat; $\mathrm{EL}=0^{\circ}, \mathrm{AZ}=270^{\circ}$
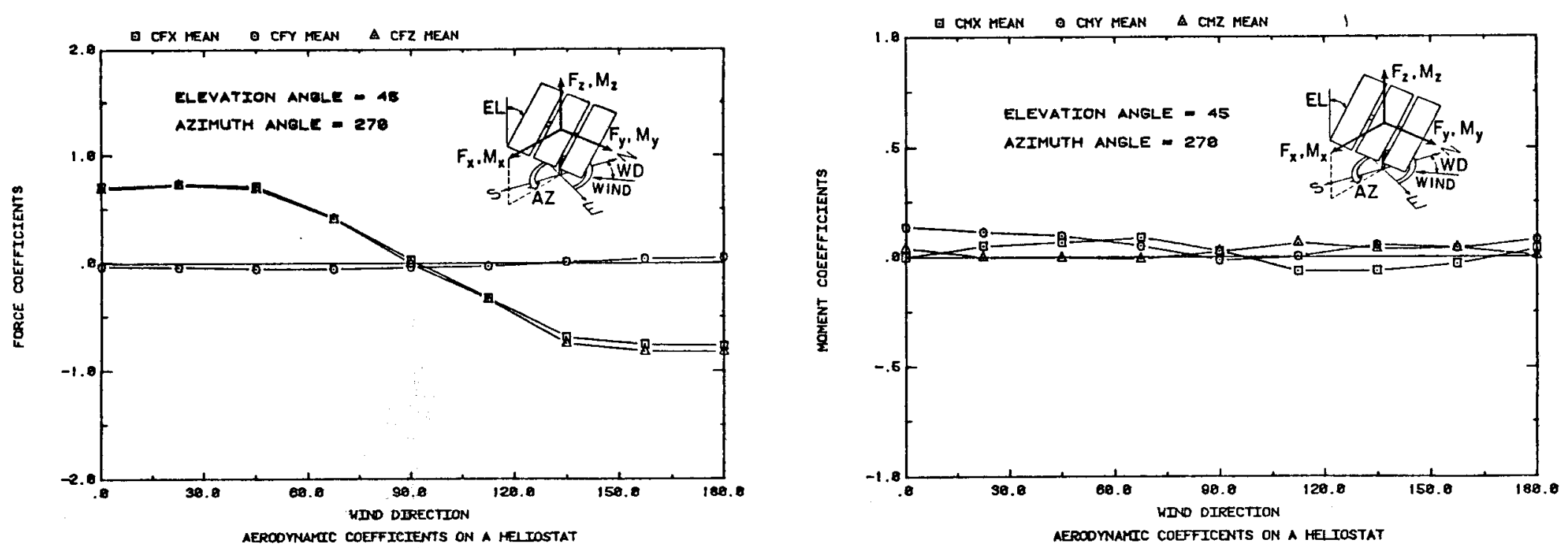

Figure 4-2a,b. Mean Force and Moment Coefficients for an Isolated Heliostat; $\mathrm{EL}=45^{\circ}, \mathrm{AZ}=270^{\circ}$ NOTE: Data values are listed in the Appendix. 

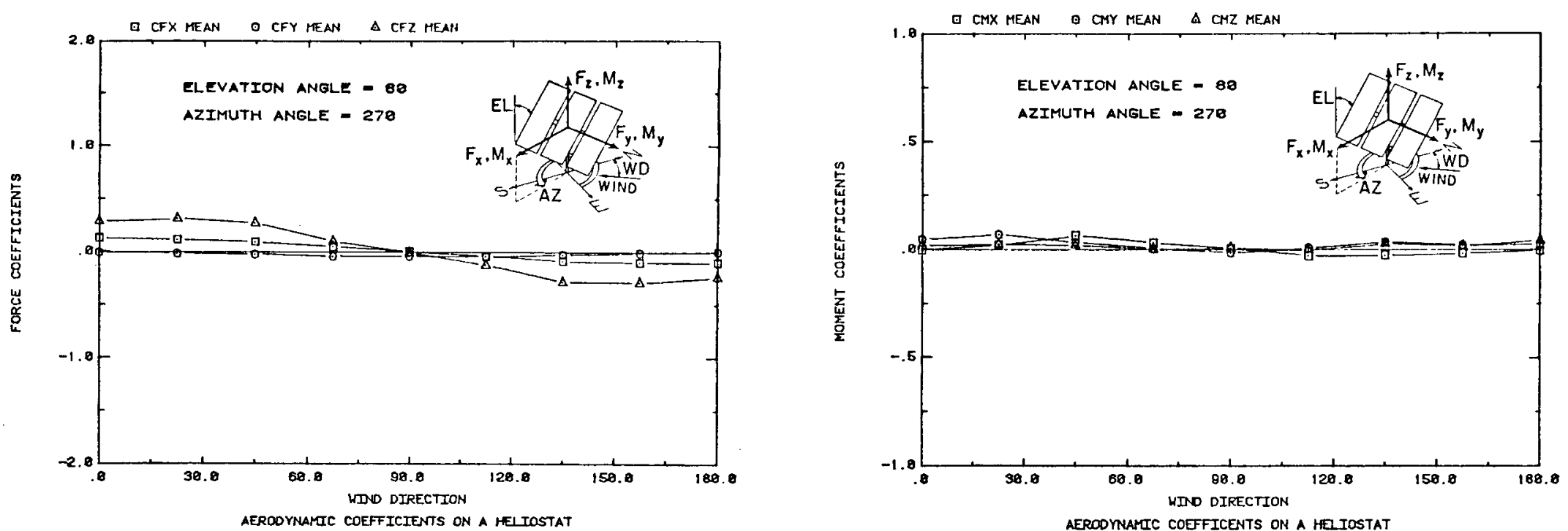

Figure 4-3a,b. Mean Force and Moment Coefficients for an Isolated Heliostat; $\mathrm{EL}=80^{\circ}, \mathrm{AZ}=270^{\circ}$
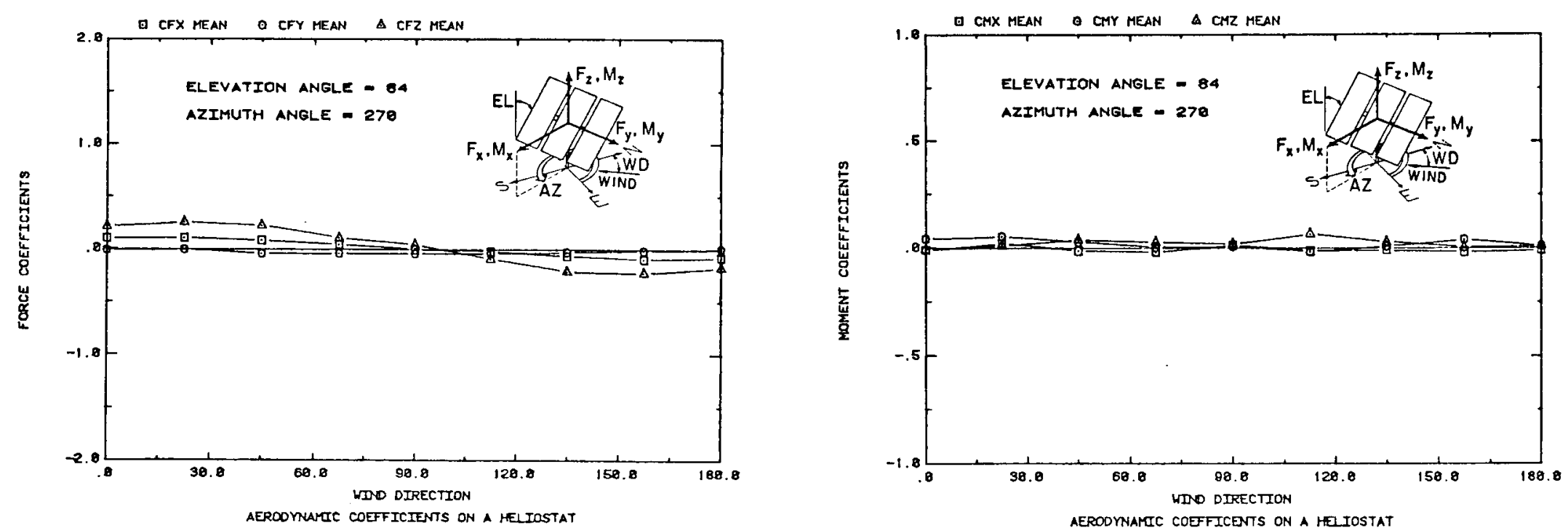

Figure 4-4a,b. Mean Force and Moment Coefficients for an Isolated Heliostat; EL $=84^{\circ}, A Z=270^{\circ}$ 

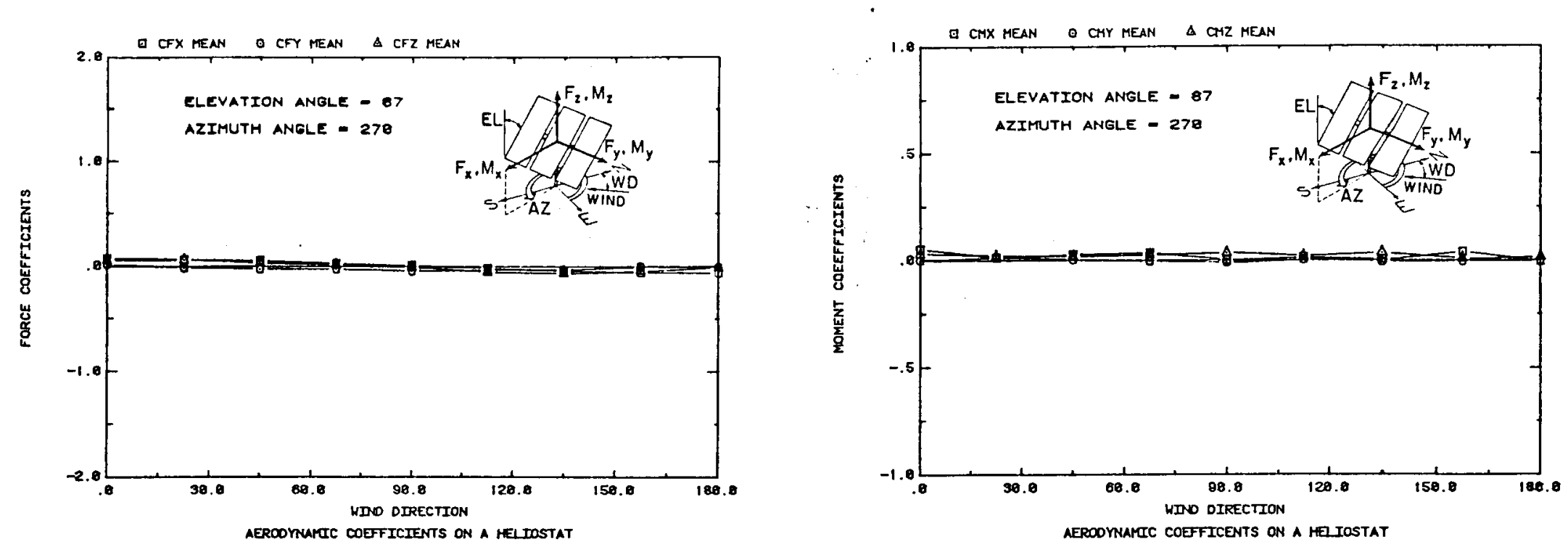

Figure 4-5a,b. Mean Force and Moment Coefficients for an Isolated Heliostat; EL $=87^{\circ}, A Z=270^{\circ}$
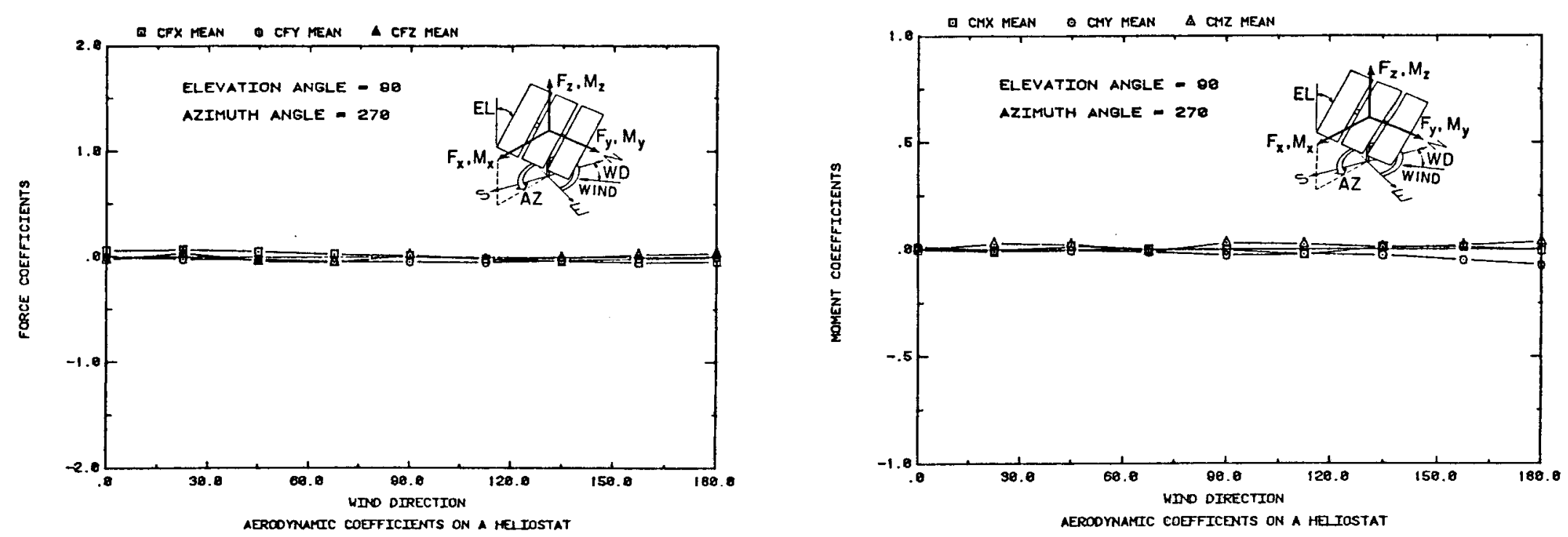

Figure 4-6a,b. Mean Force and Moment Coefficients for an Isolated Heliostat; EL $=90^{\circ}, A Z=270^{\circ}$ 

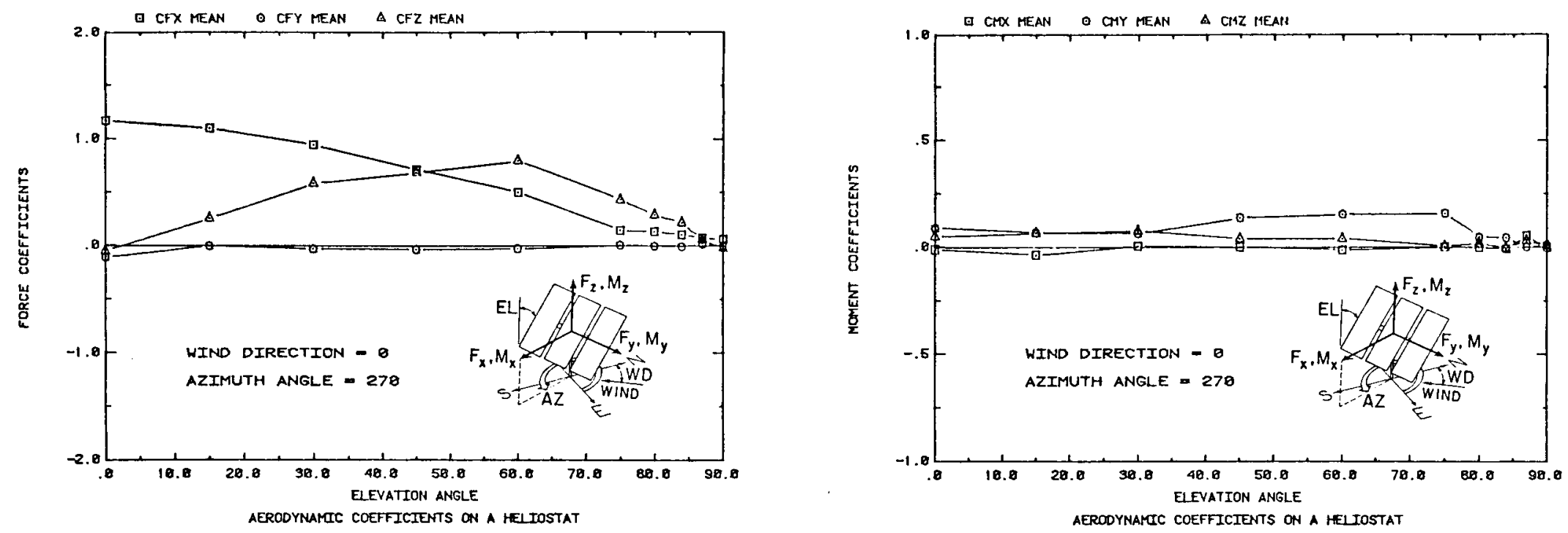

Figure 4-7a,b. Mean Force and Moment Coefficients for an Isolated Heliostat; $W D=0^{\circ}, A Z=270^{\circ}$
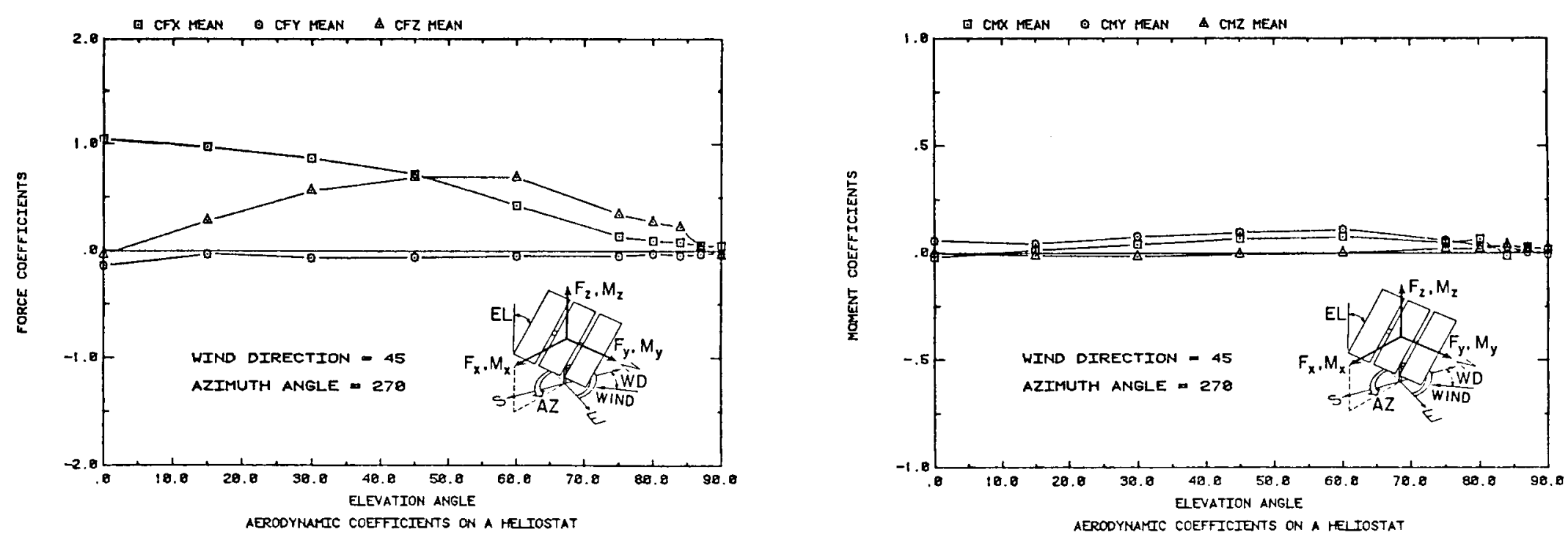

Figure 4-8a,b. Mean Force and Moment Coefficients for an Isolated Heliostat; $W D=45^{\circ}, A Z=270^{\circ}$ 

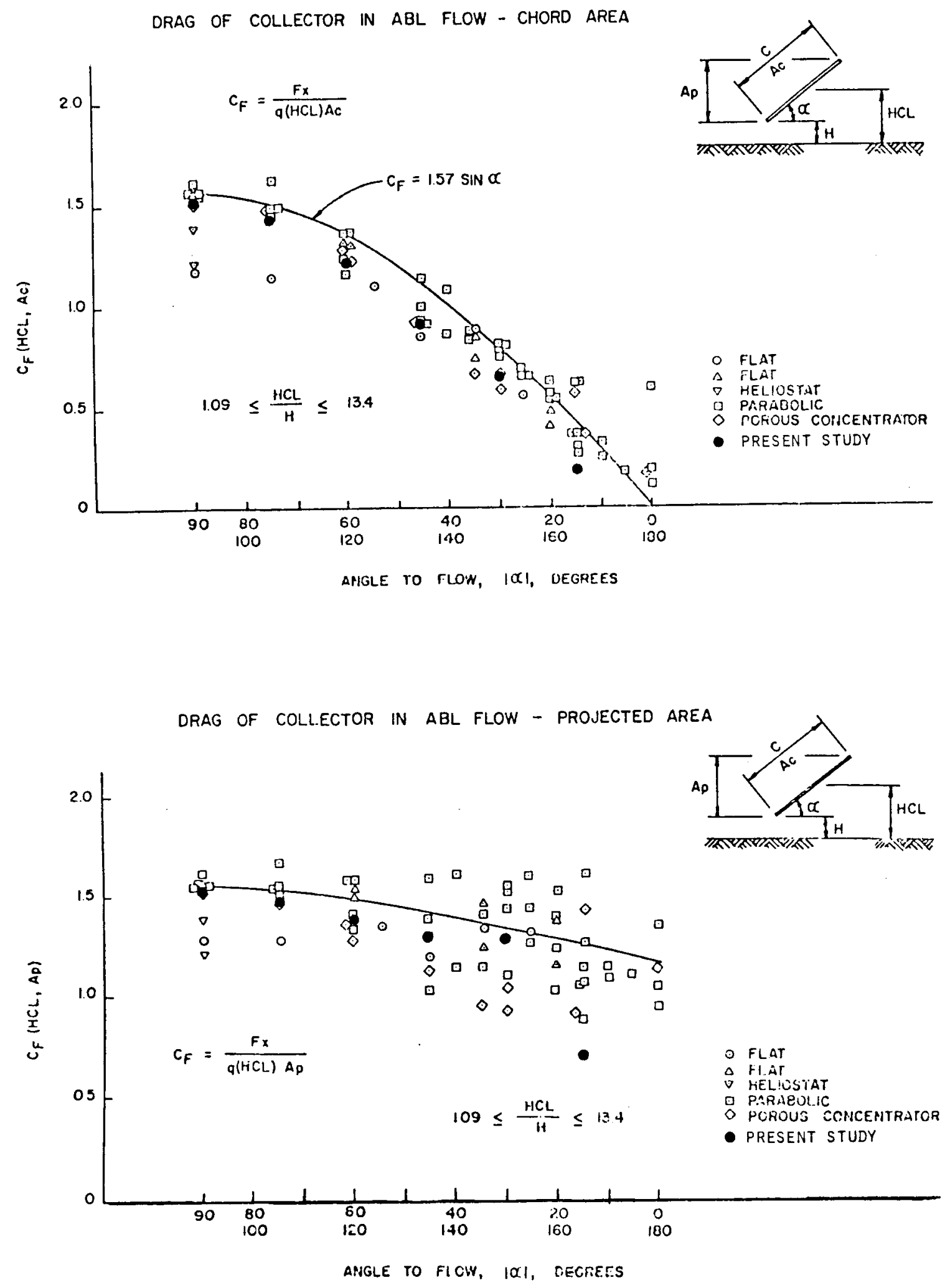

Figure 4-9. $\begin{aligned} & \text { Comparison of Measured } C_{F} \\ & \text { references } 1,3,4,7,10)\end{aligned}$ to Previous Measurements (data from 

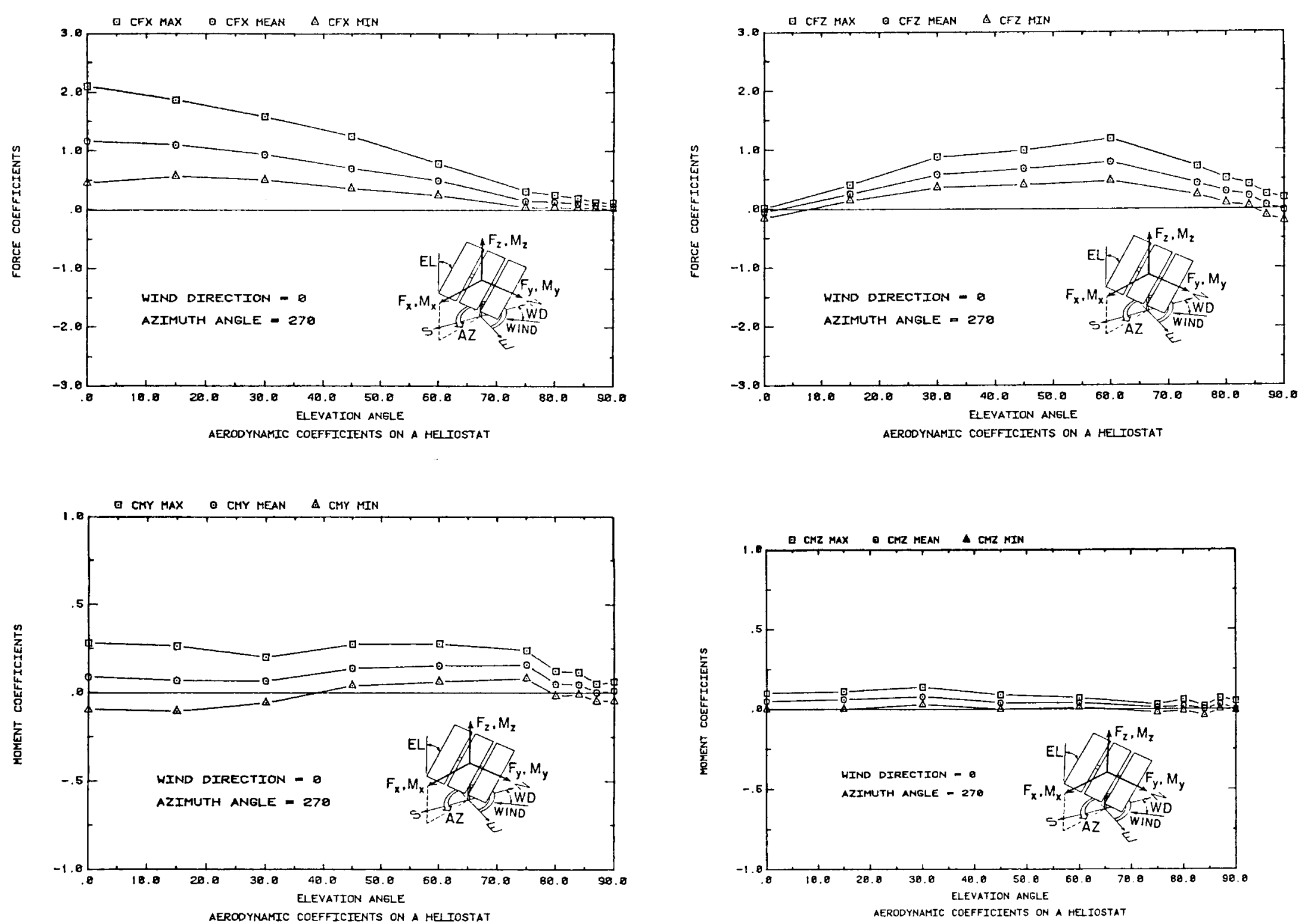

Figure 4-10a,b,c,d. Maximum, Mean and Minimum Force and Moment Coefficients; $W D=0^{\circ}, A Z=270^{\circ}$ 

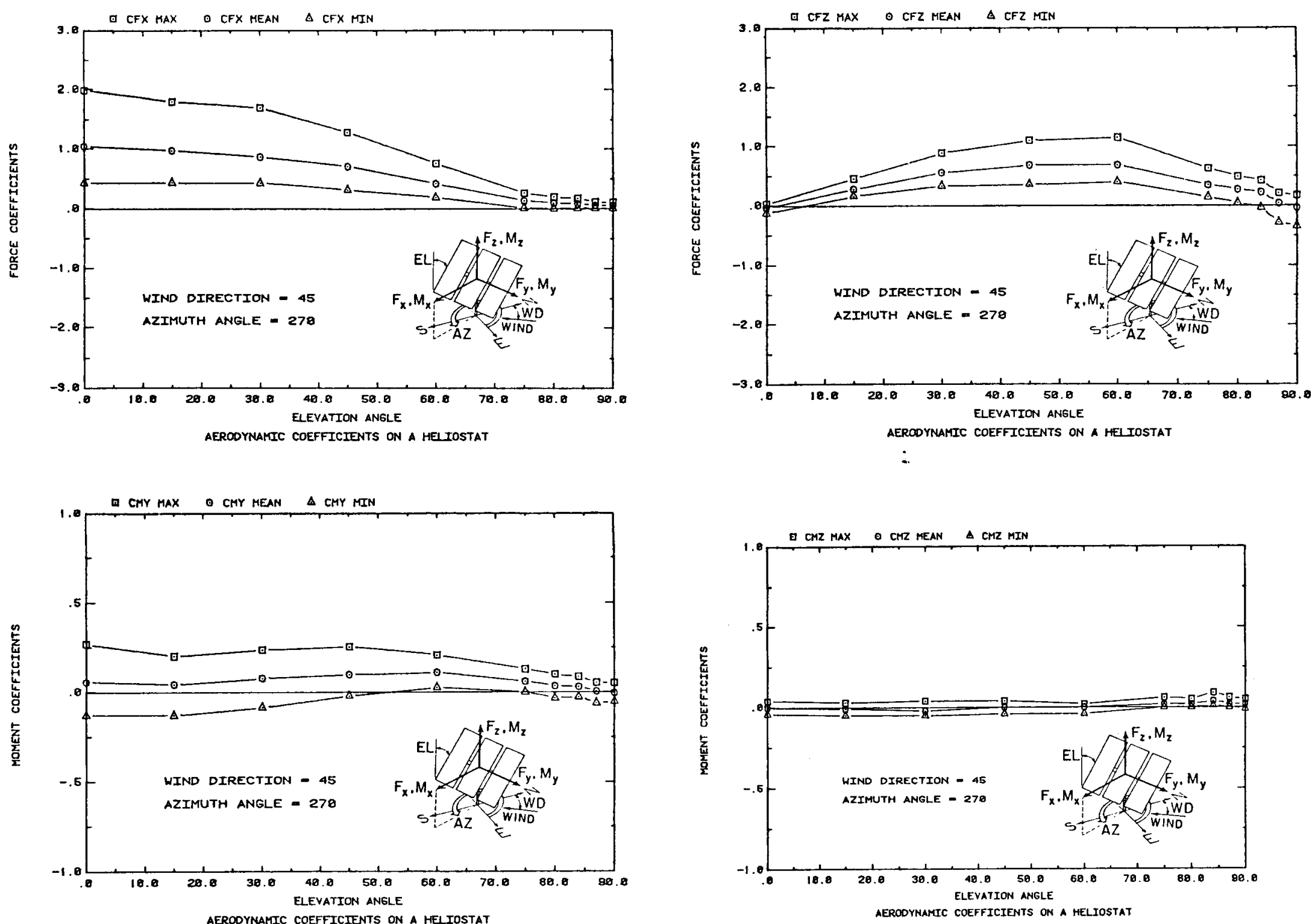

Figure 4-11a,b,c,d. Maximum, Mean and Minimum Force and Moment Coefficients; $W D=45^{\circ}, A Z=270^{\circ}$ 

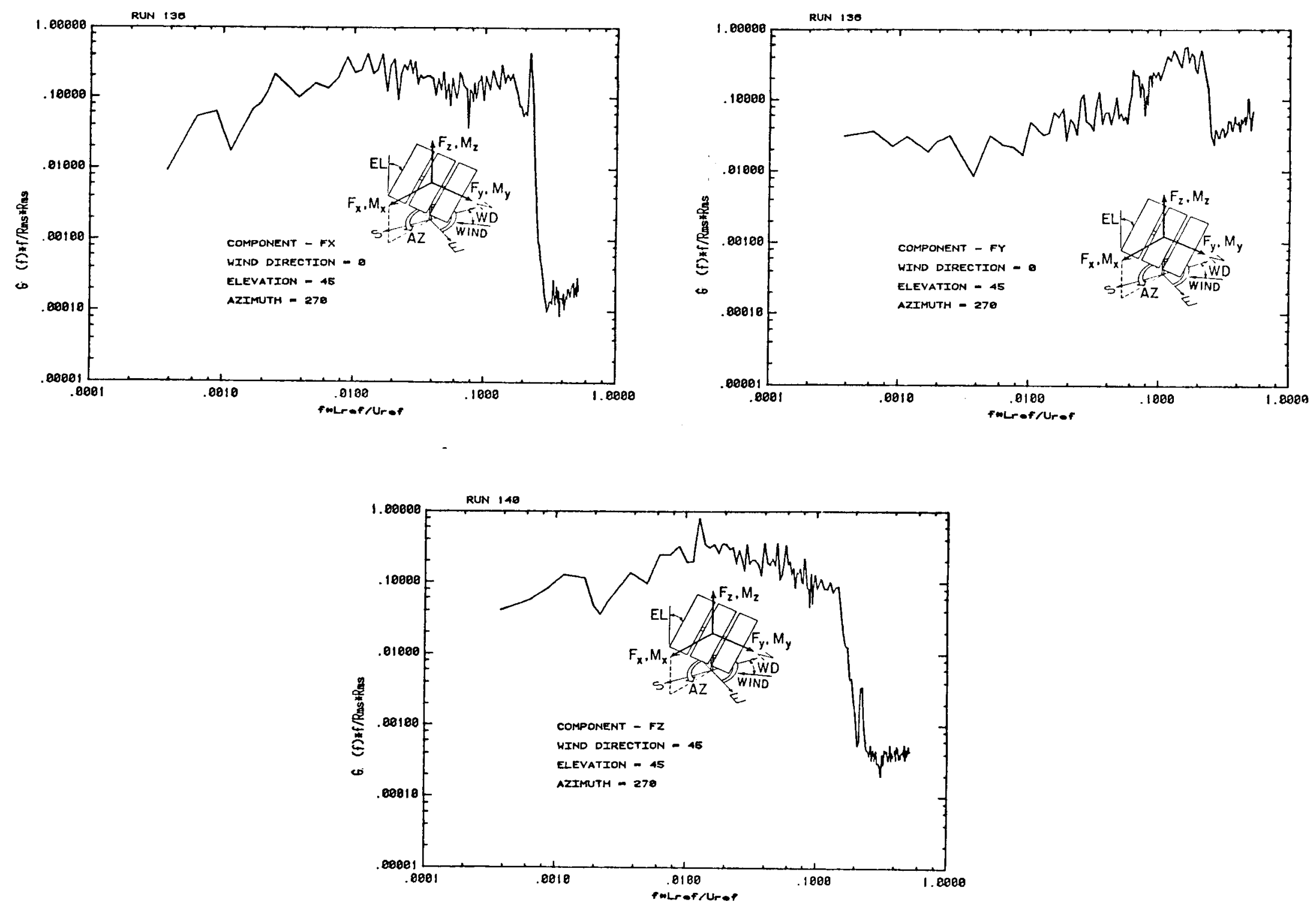

Figure 4-12a,b,c. Power Spectrum of Force Coefficients; $W D=0^{\circ}, A Z=270^{\circ}, E L=45^{\circ}$ 

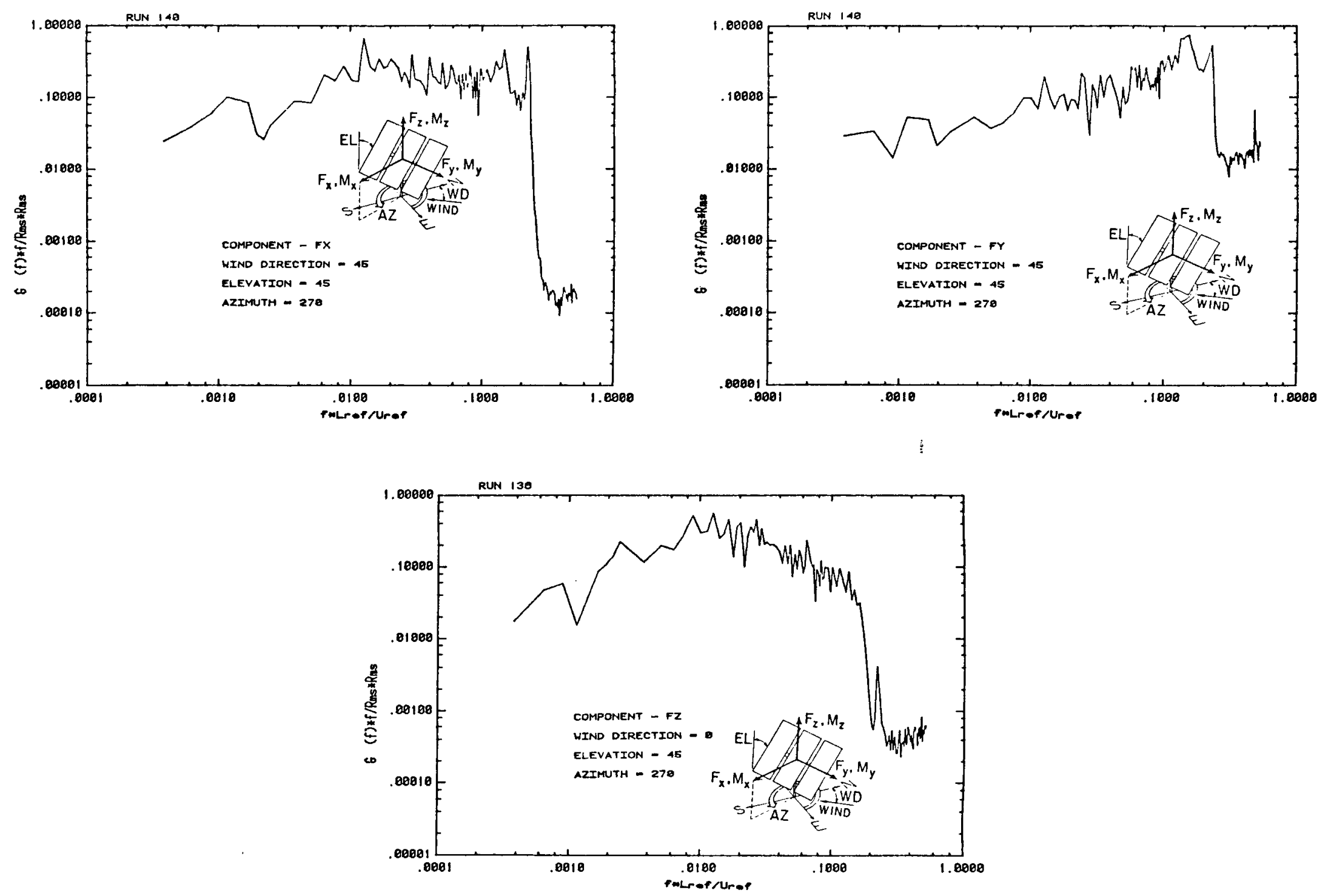

Figure 4-13a,b,c. Power Spectrum of Force Coefficient for an Isolated Heliostat; $\mathrm{WD}=45^{\circ}, \mathrm{AZ}=270^{\circ}, \mathrm{EL}=45^{\circ}$ 
These data indicate, for example, that the velocity which would cause the design horizontal peak force with the heliostat in horizontal stow position would be 4.4 times the velocity which would cause the design horizontal peak force in the most sensitive heliostat operational position. For a maximum operational wind of $50 \mathrm{mph}$, the stow wind speed to cause the same horizontal force would have to be at least $220 \mathrm{mph}$.

- Thus for an isolated (or field-edge) heliostat, operational winds tend to control design forces. The opposite is true for moments: moments are controlled by $90 \mathrm{mph}$ stow position loads.

Similar comparisons can be made within the heliostat field. However, insufficient data are available in this study to perform this calculation.

More detailed dynamic measurements on a larger scale model need to be made in order to determine whether or not the current practice of establishing stow moments by using a wind at 6 degrees to the horizontal is too conservative. Tentative measurements in this study showed a possibility that current practice is conservative.

Figure 4-9 shows the value of $C_{F}$ in this study in comparison to $C_{F_{X}}$ values measured on other isolated ground-based solar collectors in previous studies. The dynamic pressure here is referred to velocity at mid-height of the projected area to be consistent with the other data. The comparison is good. The present data is slightly lower in drag than some other collectors whose surfaces were not as smooth. The data is presented two ways in Figure 4-9: once showing the sinusoidal variation in $C_{F}$ with elevation angle and a second showing the approximately constant upper bound of $\mathrm{C}_{\mathrm{F}_{\mathrm{x}}}$ if
it is based on projected area instead of actual surface area. Figures 4-10 and 4-11 show mean, peak maximum, and peak minimum wind loads as a function of elevation angle for wind directions of $0^{\circ}$ and $45^{\circ}$ respectively. In Figure 4-10a, the peak $\mathrm{C}_{\mathrm{F}}$ at an elevation angle of $0^{\circ}$ is 2.11 compared to a mean of 1.17. This implies a gust factor in wind speed of 1.34. This value is lower than the value one would expect from typical gust factors quoted in the literature and is probably due to incomplete modeling of the larger eddy sizes. Thus, the peak measurement is possibly low for the isolated heliostat. Additional measurements combined with appropriate analysis, anticipated for study during the next year, can determine the magnitude of the underestimate. All data of Figures 4-9 and 4-10 indicate that fluctuating loads are due to wind gusting and do not indicate strong wake-dominated or vortex loading.

The power spectrum of a force coefficient represents the frequency decomposition of the fluctuating part of the time varying force coefficient. The power spectrum is defined as:

$$
G(\omega)=\frac{1}{2 \pi} \int_{-\infty}^{\infty} e^{-i \tau \omega} R(\tau) d \tau
$$




$$
\begin{aligned}
& \text { where } \quad R(\tau)=\frac{\int_{-T}^{T} g(t) g(t-\tau) d t}{\int_{-T}^{T} g^{2}(t) d t} \\
& w=2 \pi f \\
& g(t)=\text { fluctuating time sequence of force coefficient }
\end{aligned}
$$

Figures 4-12 and 4-13 present power spectra of each force coefficient for wind directions $0^{\circ}$ and $45^{\circ}$ at a constant elevation angle of $45^{\circ}$. The abscissa is reduced frequency--frequency in $\mathrm{Hz}$ made nondimensional with characteristic length scales. The ordinate is the spectrum multiplied by frequency in $\mathrm{Hz}$ and divided by the square of the root-mean-square of the fluctuating signal (the variance). The ordinate is dimensionless. The spectrum made dimensionless in this way applies to the full-scale heliostat as well as to the model and provides a convenient way to scale dynamic loads from model to full scale. The decrease in spectral amplitude near a reduced frequency of 0.2 is due to the sharp cutoff antialiasing low-pass filter used to delete the model natural frequency and represents the upper limit of useful frequency. The absence of large peaks at specific frequencies indicates a broad-band type of wind loading and the absence of an organized vortex shedding phenomena. This is the type of loading expected.

Improvements in the spectra which should be made before full utilization can be made are: an increase in upper frequency limit (higher natural frequency of the model) and decrease in normalized standard error (random variations in ordinate making graph look 'noisy'). The first can be accomplished by testing on a stiffer balance--a device currently in final development stages--and the second by increasing sampling time in the wind tunnel and adjusting segment and frequency averaging parameters in data analysis. Both improvements are planned for the next year testing. Improvements in dynamic load measurement capability will result in definition of peak loads on heliostats. It is the peak loads which provide the largest stresses in the support structure and hence control the design.

- The strength of a collector should be based on the peak load rather than a mean load multiplied by an assumed gust factor.

\subsection{HELIOSTATS IN FIELD}

Data in this section is presented in roughly the order of increasing complexity of upwind blockage. The intent of the various figures is to illustrate the influence of various types of upwind blockage, heliostats, fences, etc., on wind loads. The data in this section is later condensed into two graphs.

Figures 4-14 and 4-15 show the influence of a perimeter fence (see Figure 2-5) on wind loads on heliostat 1 at the edge of the field for summer AM and summer noon conditions. As found in earlier studies cited in the introduction, perimeter fences do provide significant reductions in wind load. 

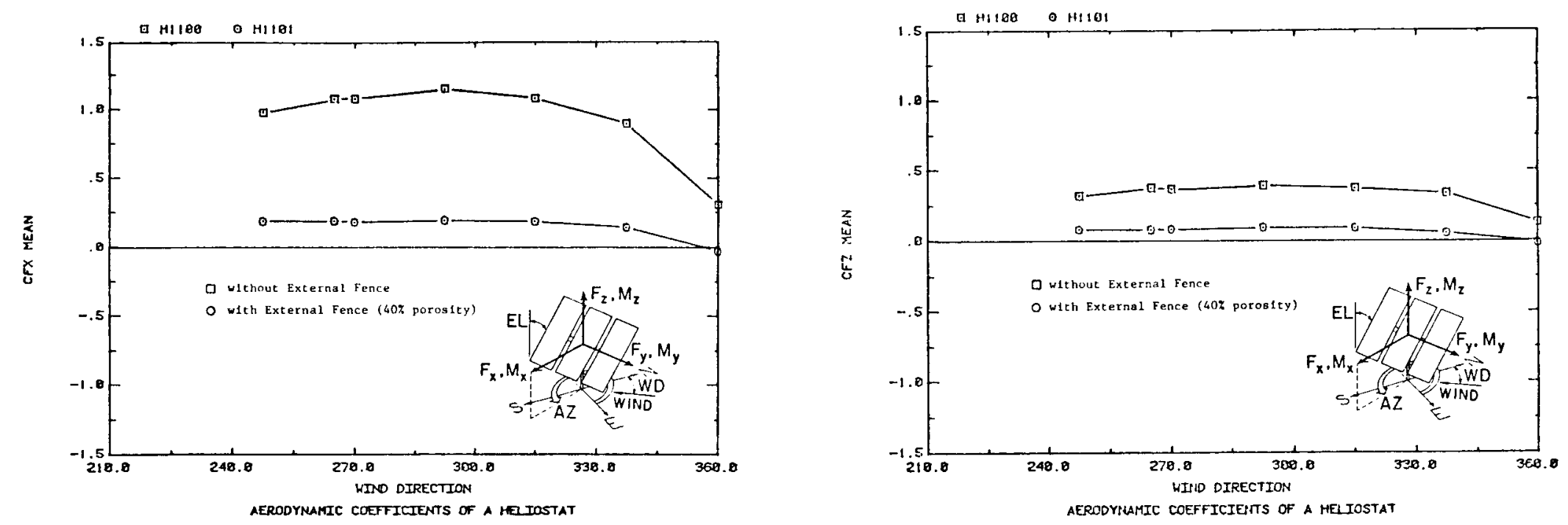

ज
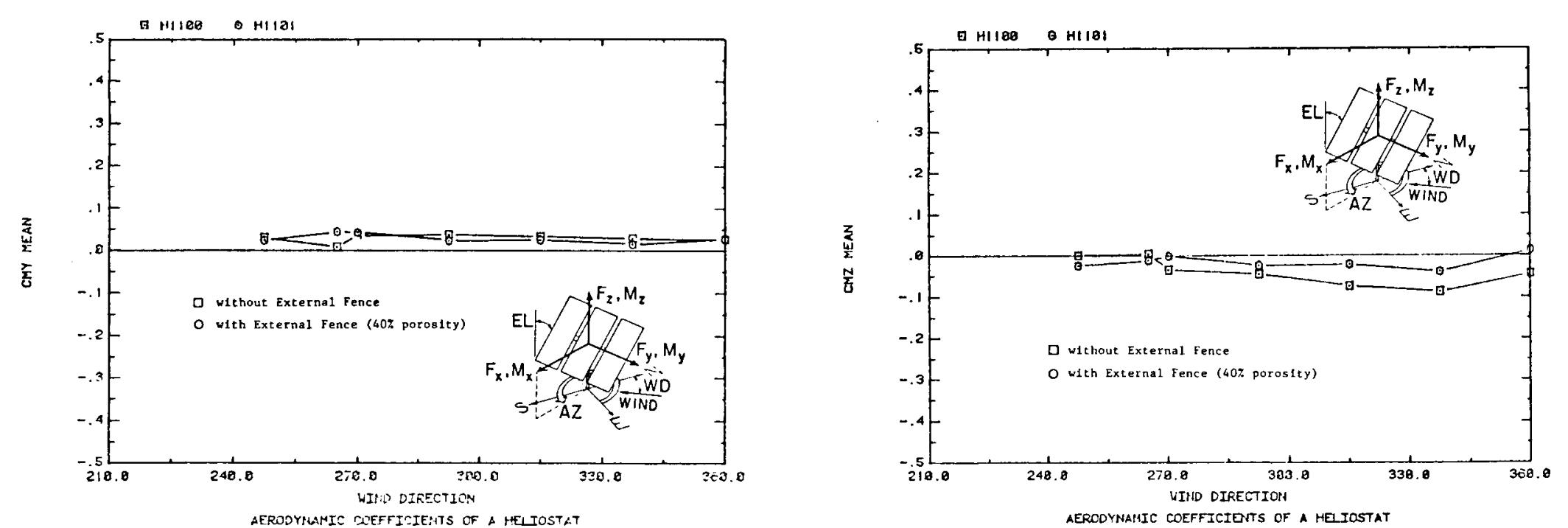

Figure 4-14a,b,c,d. Heliostat 1 - Representative Mean Force and Moment Coefficients, Summer AM 

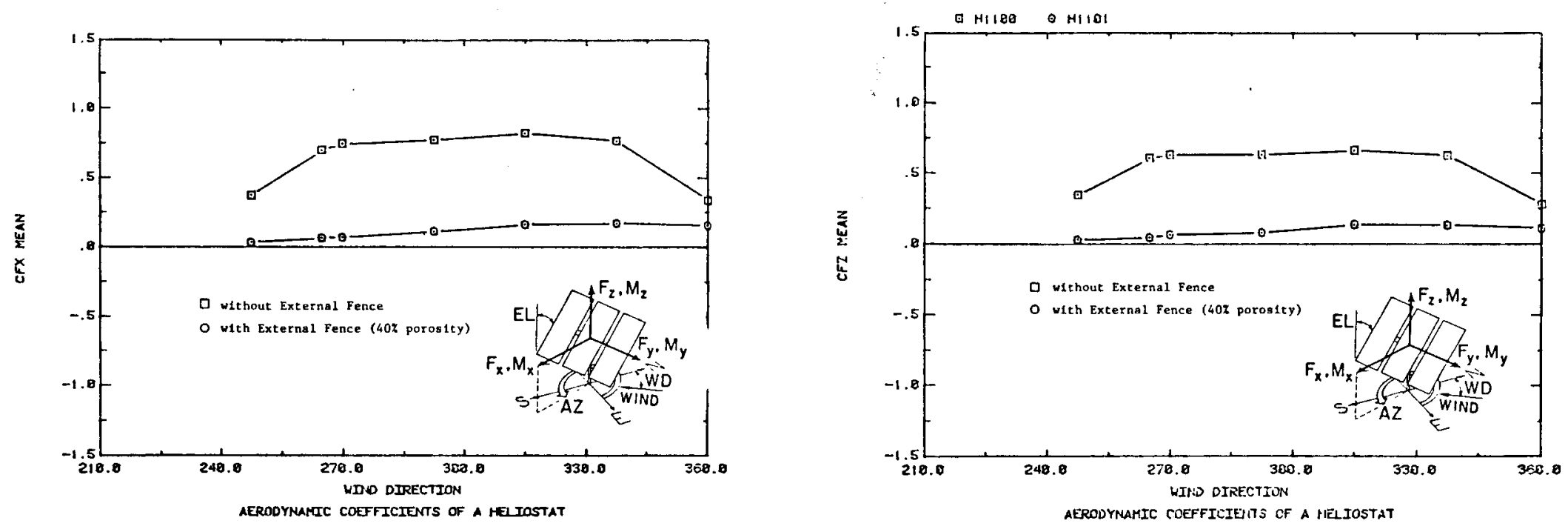

ज
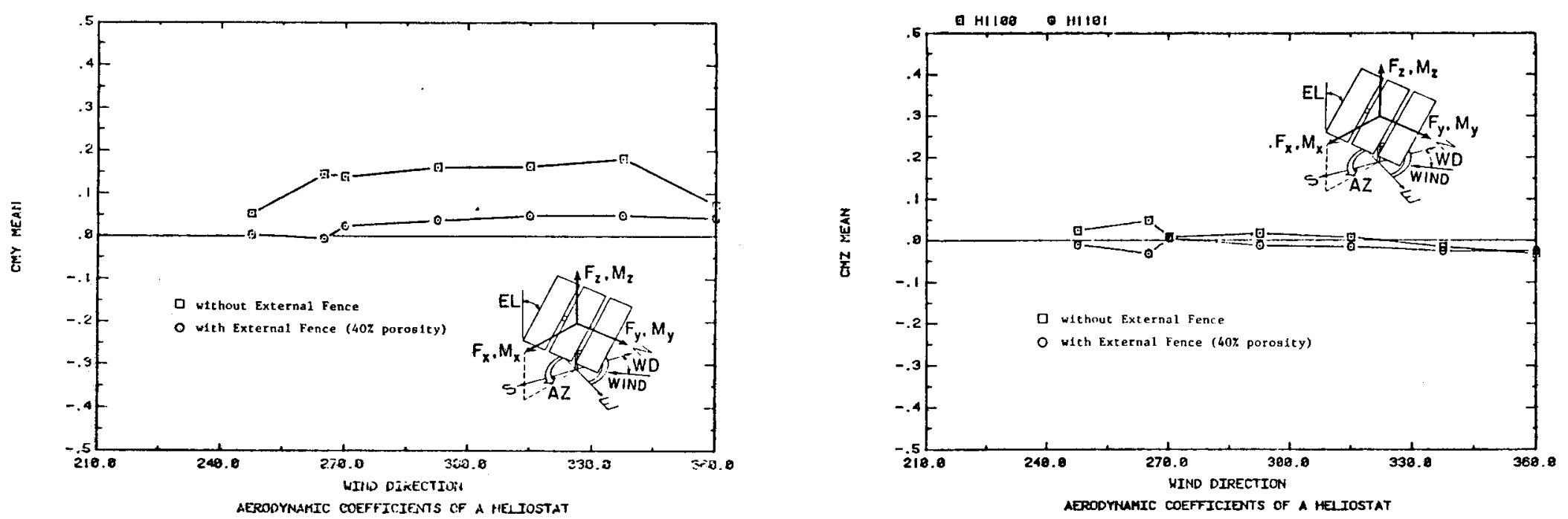

Figure 4-15a,b,c,d. Heliostat 1 - Representative Mean Force and Moment Coefficients, Summer Noon 
Heliostat 5 represents an in-field unit in the least dense area $A$ of the Barstow field (see Figure 2-6). Figures 4-16 and 4-17 show the wind loads on this heliostat for the summer AM and summer noon configuration for various fence arrangements. The highest loads shown in each figure are for an isolated heliostat for comparison purposes. The other cases in order of decreasing load are heliostat 5 in its field location without any fences in place, with external fence only and with both external and internal fence. The largest reduction in wind load from the isolated case occurred as a result. of basic field density. Both the external and internal fence provided additional load reductions. The field density about heliostat 5 is higher than the low density portion of the field used in reference [2]. In reference [2], load reductions of 20 to 30 percent in the low density field were typical without fences.

- In the Barstow low density field, mean load reductions of 50 to 70 percent were typical with additional decreases due to the addition of fences.

In order to satisfy the desired goal of providing a designer with sound guidelines on what upstream blockage is necessary to produce a particular load reduction, additional tests were run on a typical heliostat varying the properties of the field about the heliostat. Heliostat 3 was selected for this purpose. Figure 4-18 shows the effect of varying the number of upstream rows without fences on both mean and dynamic loads on heliostat 3 . The mean load decreases rapidly with number of upstream rows to a value about 20 to 25 percent of the edge case. The peak load decreases rapidly also to a value of about $1 / 3$ of the edge case. The variability in peak load with number of rows upwind from 2 to 10 rows is not due to a change in dynamic loading (the rms is remarkably constant) but is due to use of a single realization from a probability distribution with a fairly high dispersion. In random vibration peak load determination, the peak is often calculated as peak = mean $+\mathrm{g} * \mathrm{rms}$ where $g$ is a peak factor (of magnitude 3-4 for a broad band loading process) determined semi-empirically, in order to avoid the random variability in single measurement realizations. Because the dynamic loads on interior heliostats are dominated by wake turbulence generated by upstream units, the peak loads measured on these units are more likely to be closer to the correct value than those on edge units (which are believed too low--see Section 4.1 above). Thus, load reduction within the field is probably larger than shown in Figure 4-18.

- An important point shown by Figure 4-18 is that the peak wind loads acting on heliostats within the Barstow field are substantially lower than the peak loads acting on heliostats at the edge of the field.

This finding is in contrast to assumptions made about peak loads by field personnel (see Section 4.4).

- Specific dynamic load mechanisms which can be treated with spoilers on heliostats were not evident. Thus, they were not tried. Additional study in the next year should address this issue with tests. 


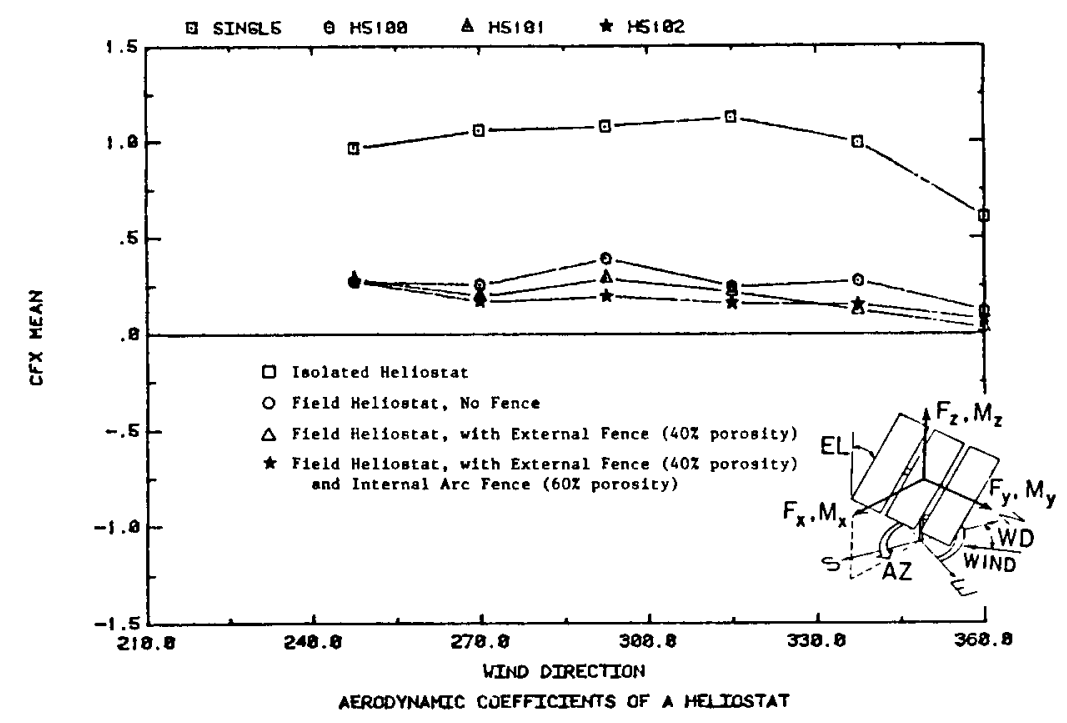

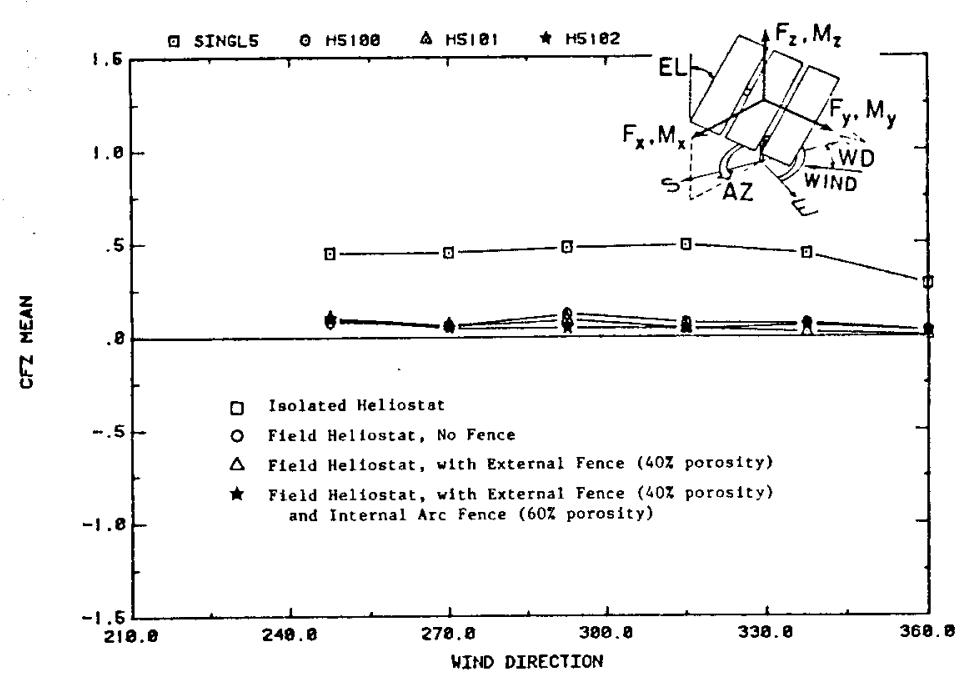

AERODYMAMIC COEFFICIENTS OF A MELTOSTAT

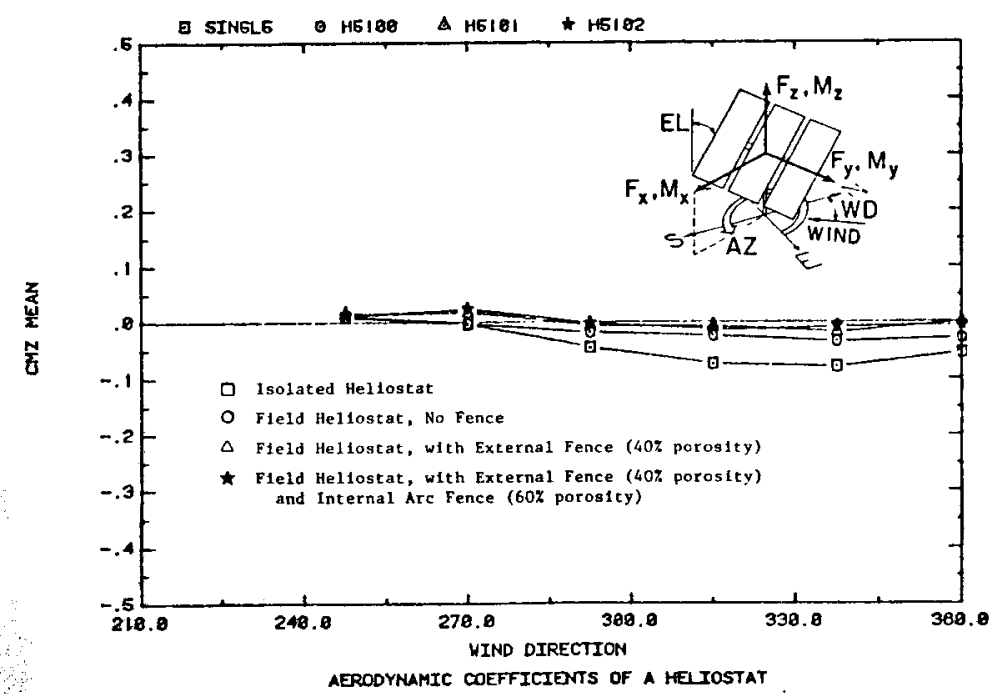

Figure 4-16a,b,c,d. Heliostat 5 - Representative Mean Force and Moment Coefficients, Summer AM 

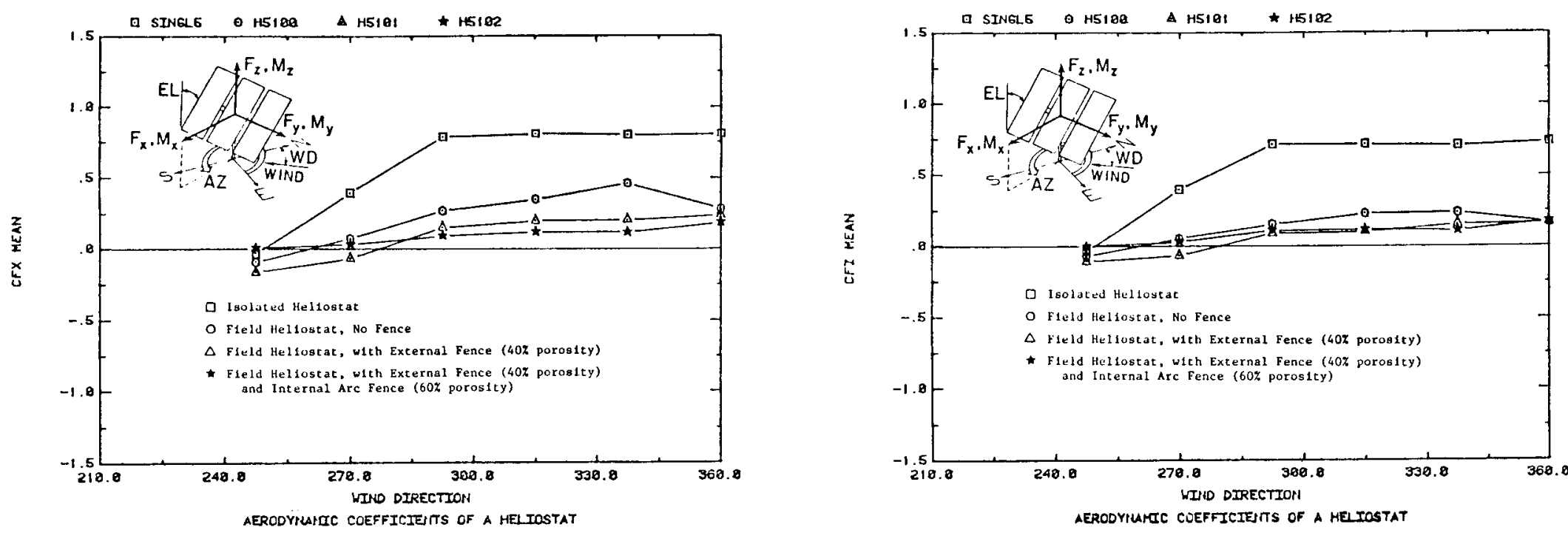

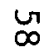
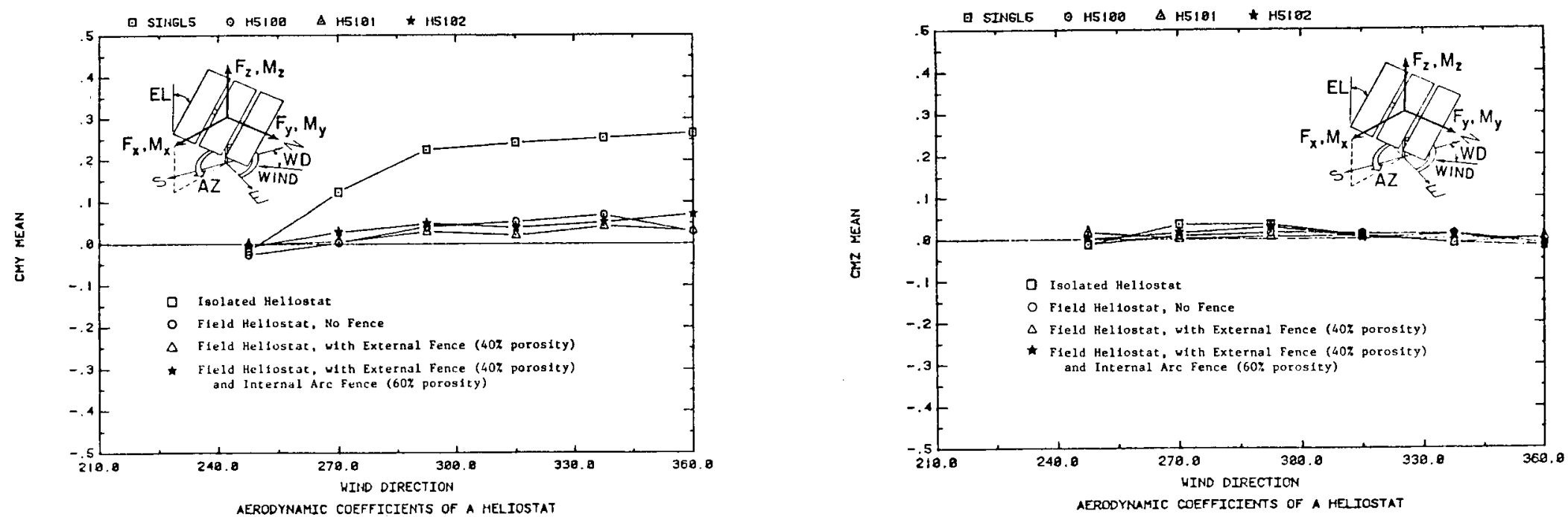

Figure 4-17a,b,c,d. Heliostat 5 - Representative Mean Force and Moment Coefficients, Summer Noon 


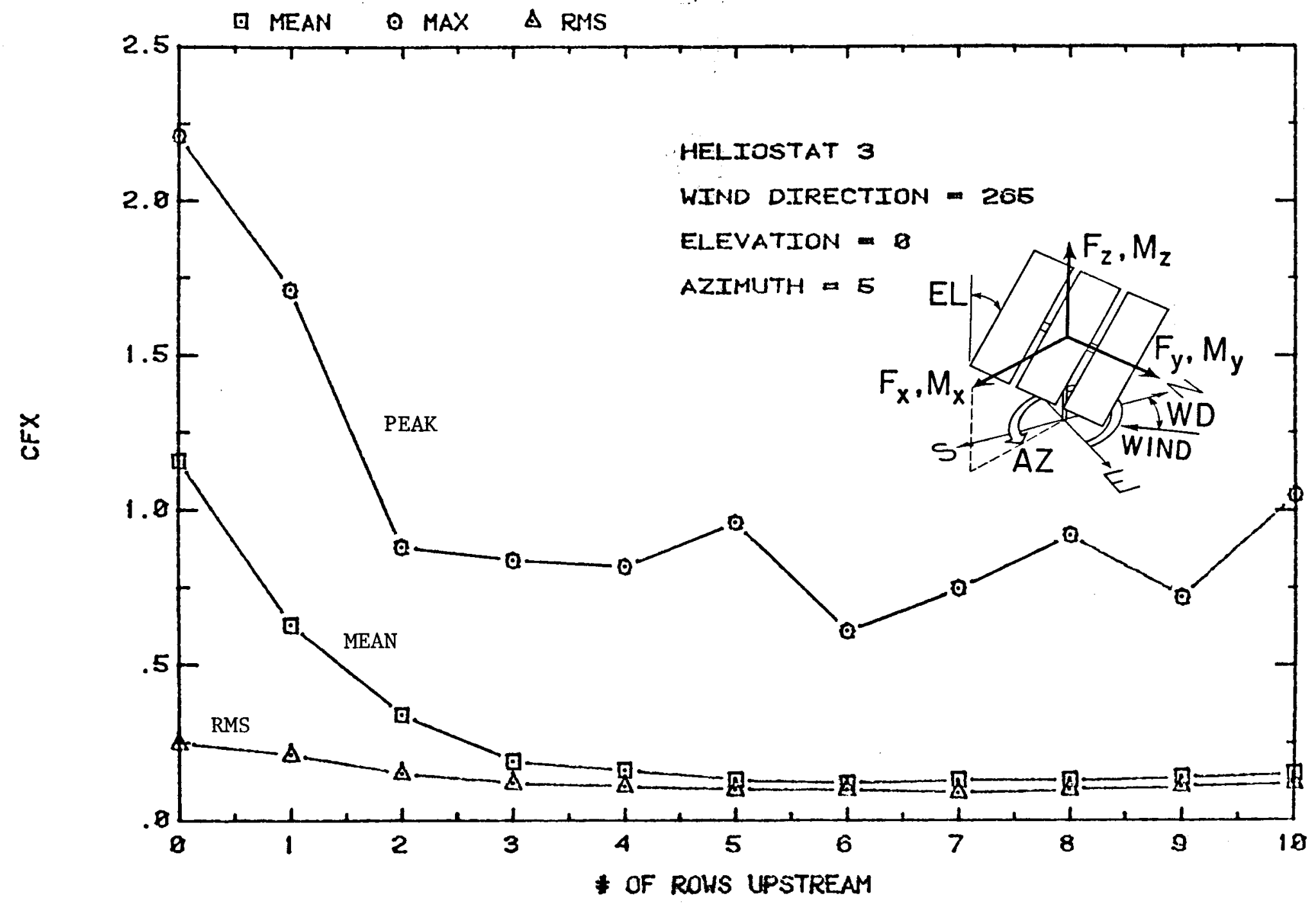

AERODYNAMIC COEFFICIENTS OF A HELIOSTAT

Figure 4-18. Mean, Max and RMS CFX for Heliostat 3--with Varying of Number of Upstream Rows of Heliostats 
- Spoilers are not likely to be effective in reducing mean loads because of increased collector area and increased turbulence in the separated shear layer which can increase loading coefficients.

The influence of field density on heliostat loads is shown in Figure 4-19. This figure shows how wind loads transition from isolated to dense field loadings. The generalized blockage area shown in the graph is defined in Figure 4-20 as

$$
G_{B}=A_{B} / A_{F}
$$

where $A_{B}=$ solid blockage area of upwind heliostats and fences projected in the approach wind direction

$A_{F}=$ ground area occupied by the upwind blockage elements included in calculation of $A_{B}$

The original density for heliostat 3 in generalized blockage area is 0.24 . Wind loads increase steadily as generalized blockage area is decreased with a rapid rise below 0.1 .

The effects of fences are more easily resolved for fields with lower density. A number of tests were performed with internal fences with various field densities and number of upstream rows. Figures 4-21 through 4-23 represent results from this series of tests for heliostat 3 . The reduced density of the field for these results is $1 / 4$ of the original Barstow field density. The results show a steady decrease in wind load with upwind blockage area. It shows that porosity in the in-field fence is important (i.e., that increase in solid area upwind decreases wind loads).

Heliostat 4 is at the inner edge of the heliostat field where velocity speedup might increase wind loads above interior units. This heliostat also is one instrumented in the field. Because of the time-consuming task of setting upwind heliostats, only one wind direction (azimuth 265 degrees) was measured--the direction where full-scale wind loads have been measured. Figure 4-24 shows the results of heliostat 4 wind loads for a summer noon case. Comparison is made to heliostats 1 and 5. Heliostat 4 shows loads significantly lower than the upwind edge heliostat 1 and slightly higher than heliostat 5. For a summer AM case (not shown in a figure), heliostat 4 had loads nearly the same as heliostat 5. The data for heliostat 4 shows that an edge heliostat on the downwind edge of the field may have loads above interior units, but well below upwind edge units.

Development of data during the testing phase indicated that the added data obtained on heliostat 3 would be more beneficial than tests on heliostat 2 . Thus no data were obtained for heliostat 2 .

\subsection{WIND LOAD REDUCTION SUMMARY}

The primary purpose of this study was to find ways to reduce mean wind loads on heliostats. One alterative is to specify specific fences required to 


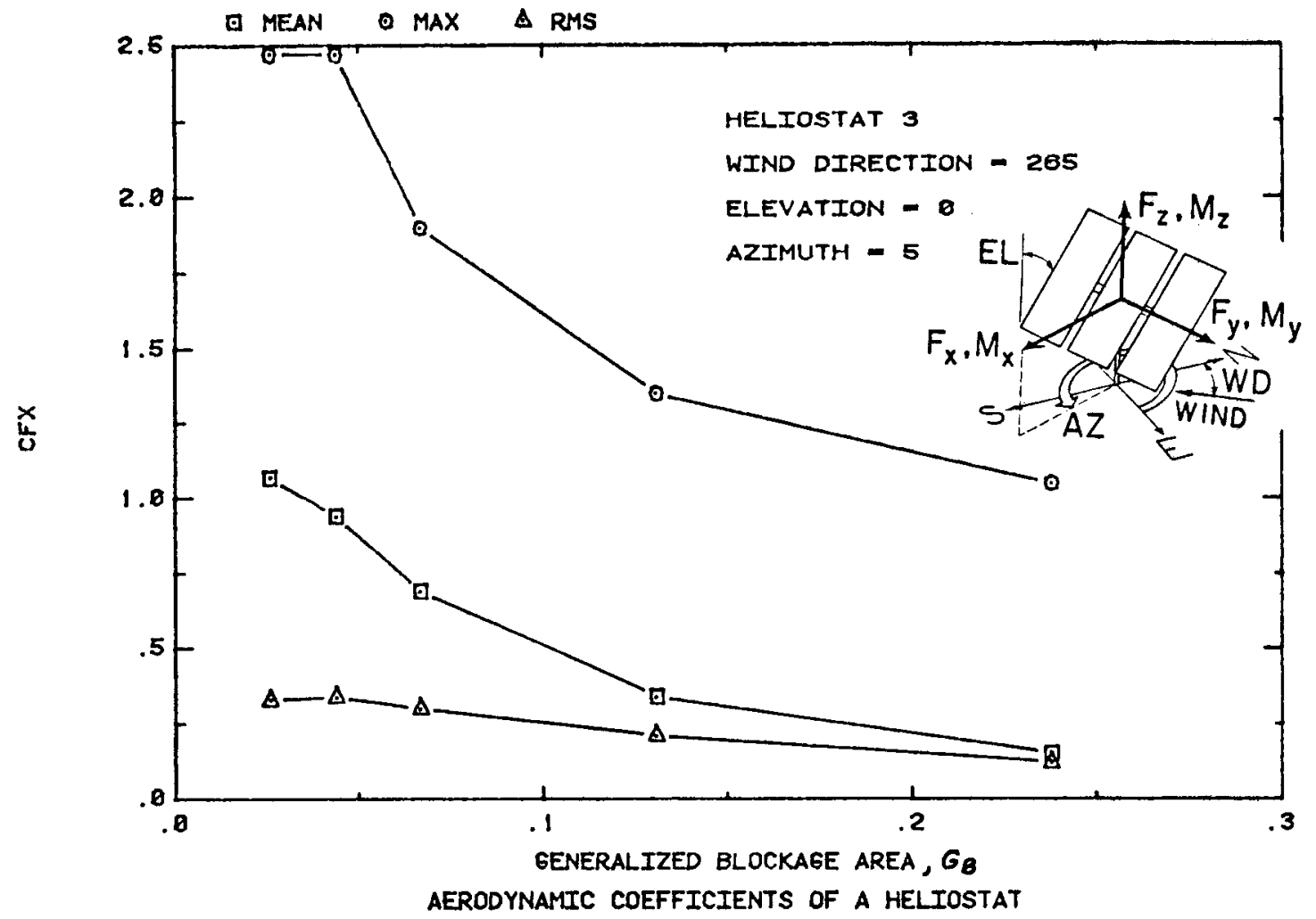

Field 4-19. Mean, Max and RMS CFX for Heliostat 3--with Various Field Densities

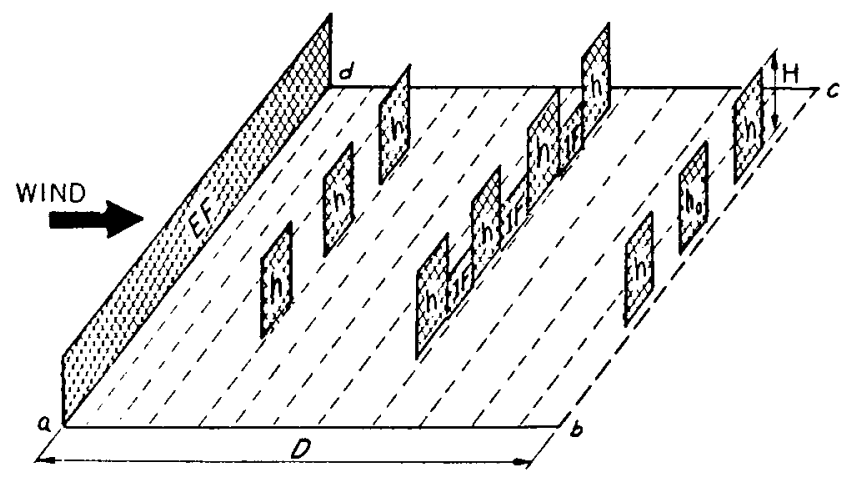

$n=$ Heliostot

$h_{0}=$ Heliostat Under Consideration

$E F=$ External Fence

$I F=$ Internal Fence

$\theta$ = Blockoge Areo for ho

$\left[3=\right.$ Field Areo (=abcd) for $h_{0}$

$A_{B}=$ Blockoge Area Projected onto a Plone Perpendicular to Approach Wind Direction

$A_{F}=$ Field Area Containing Blocking Elements Used for $A_{B}$

$D=$ Distance from $h_{0}$ to $E F$

$H=$ Height of Heliostat at Vertical Stow Position

Generalized Blockage $G_{B}=\frac{\text { Blockge Areo } A_{B}}{\text { Field Areo } A_{F}}$

Figure 4-20. Definition of Generalized Blockage Area 


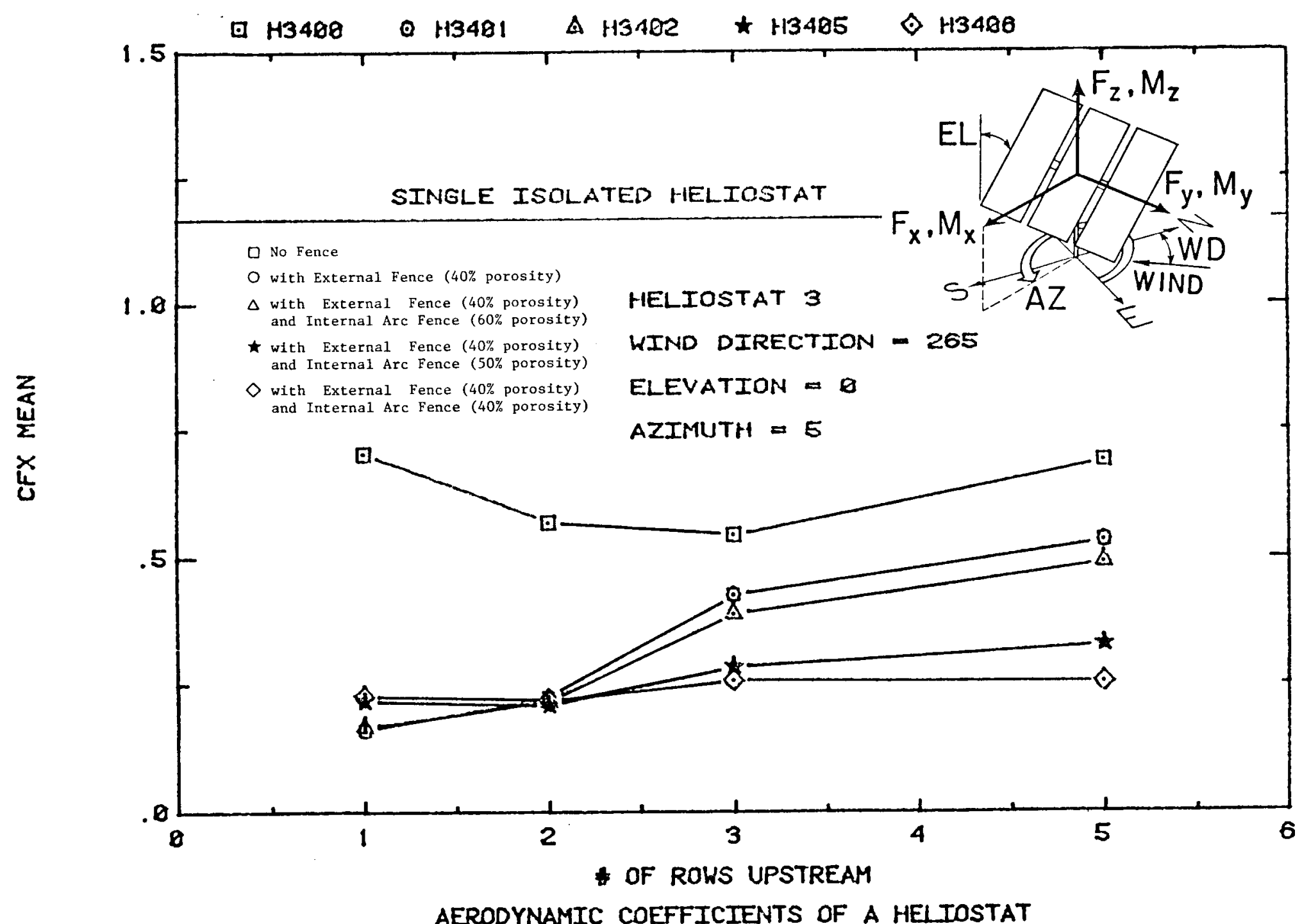

Figure 4-21. Effects of Fences and Number of Upstream Rows of Heliostats on Mean CFX, Heliostat 3 within Low Density Field 


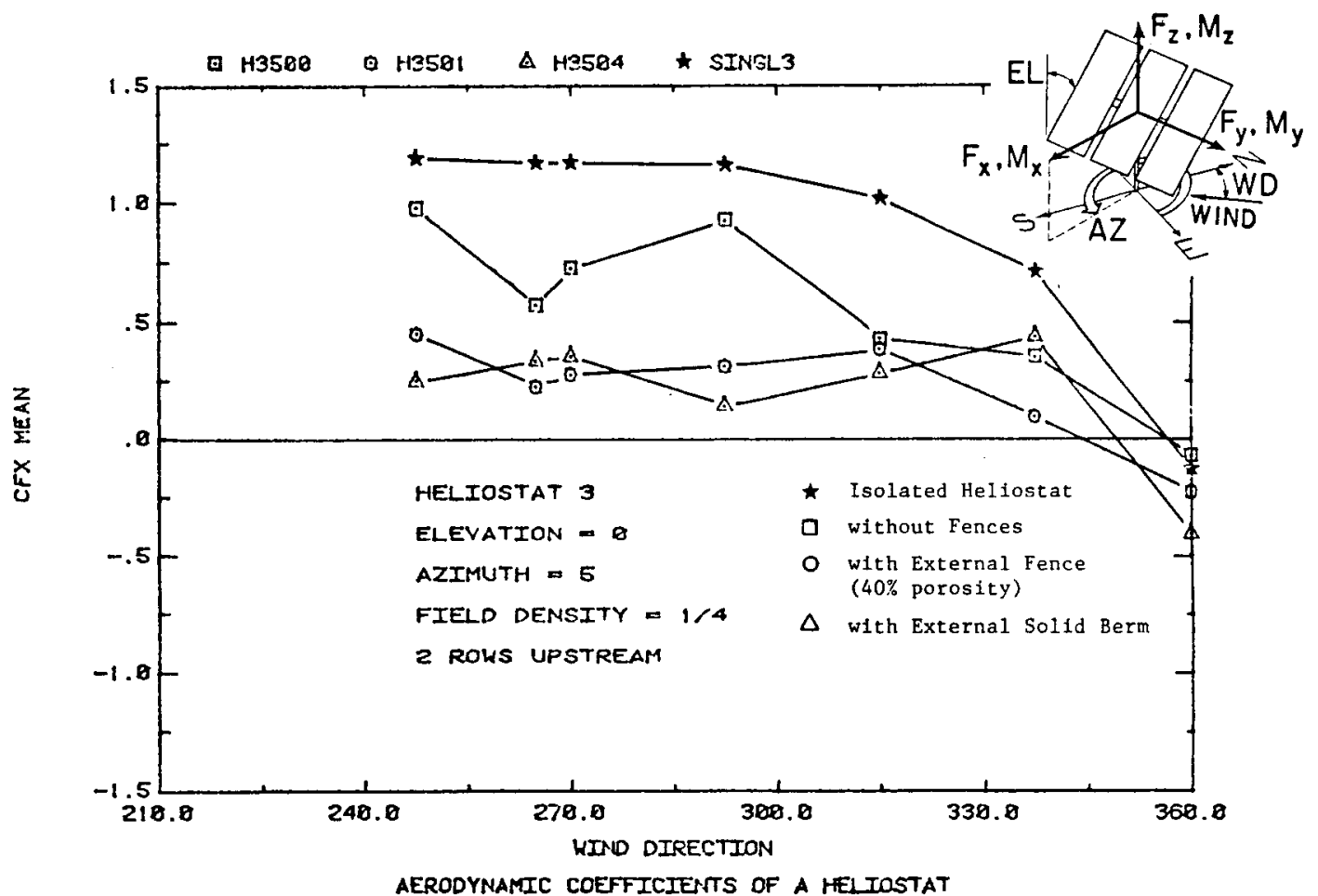

Figure 4-22. Effects of Fences on Mean CFX, Heliostat 3 within Low Density Field with Two Upstream Rows of Heliostats

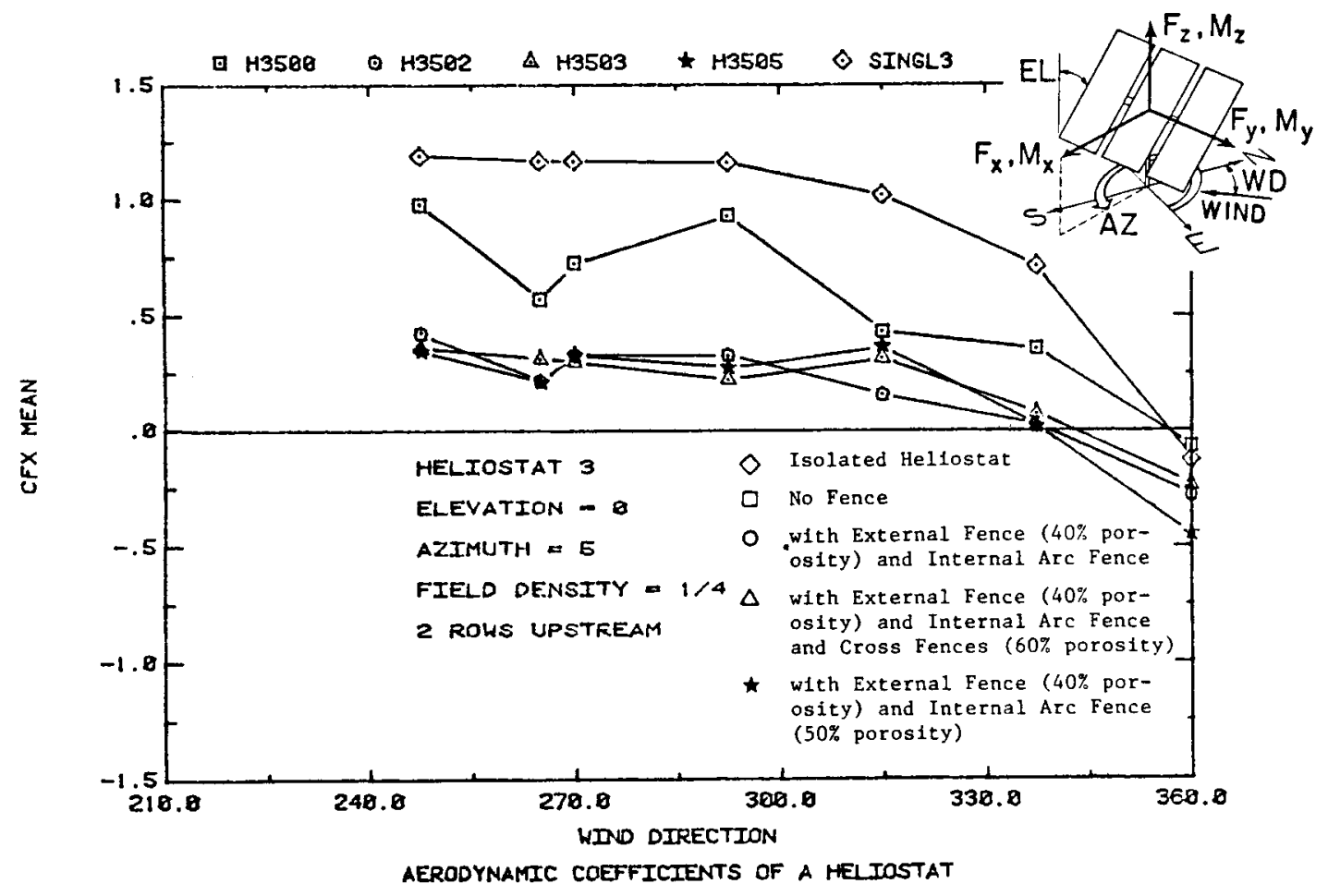

Figure 4-23. Effects of Fences on Mean CFX, Heliostat 3 within Low Density Field with Two Upstream Rows of Heliostats 

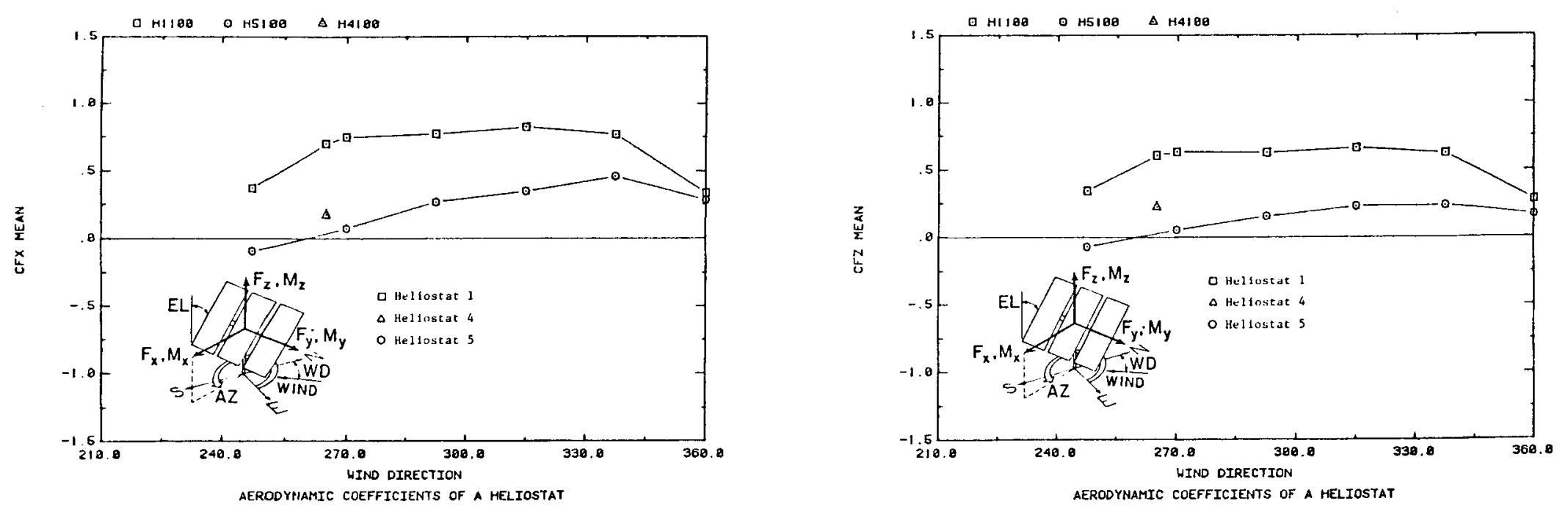

a
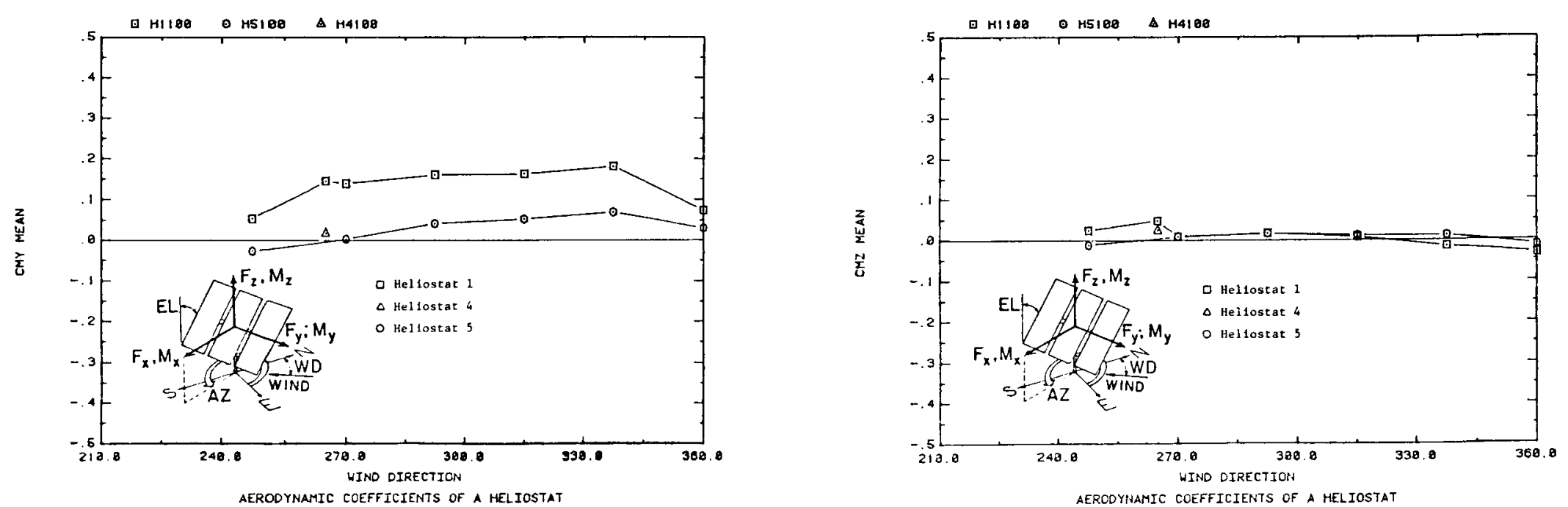

Figure 4-24a,b,c,d. Wind Loads on Heliostat 4 for a Summer Noon Case for Wind Direction 265 Degrees 
achieve specific reductions for a particular field layout. A second and more attractive alternative is to describe wind load reduction in terms of the characteristics--described in a general but quantifiable way--of the upwind devices interfering with the wind.

- During analysis of the data, it was found that a generalized quantifiable description of the upwind blockage could be found--the generalized blockage shown in Figure 4-20.

Figure 4-25 shows how data have been collapsed onto or below a single curve of load reduction using the generalized blockage area concept. The solid curve is an exponential decay with exponent $-5.56 G_{B}$ and was fit to the data in the

upper curve by regression. Only data for $\mathrm{D} / \mathrm{h} \leq 5$ did not collapse onto the curves shown and were omitted from the graphs. For $\mathrm{D} / \mathrm{h} \leq 5$, the heliostat under consideration is within the first two rows where wind can pass between upwind heliostats for some directions without significant decrease. For these cases, the perimeter fence is needed to provide adequate protection. Figure 4-25 provides a design guide which describes the quantity of upwind solid blockage required to achieve any desired level of load reduction. The blockage may be assembled from heliostats, fences, berms or other elements whose specific shapes and locations can be determined by the economics of the installation. The format used for describing load reduction leaves the field designer the maximum of latitude in selection of field geometry.

- An obvious conclusion is that an efficient load reduction mechanism is high field density (with a perimeter fence) where the upstream blockage elements are also energy producing modules. It may be desirable to trade shading losses for decreased wind loading.

\subsection{COMPARISON OF MODEL DATA WITH FULL SCALE}

One reason for selecting the Barstow site for the wind-tunnel test was that in the full scale field wind speed measurements had been made, and three heliostats had been fully instrumented with load cells for force measurements (another three had been partially instrumented). It was thus anticipated that a comparison between model and full-scale data would be made. To date, no full-scale load data has become available. Some wind speed data (mainly peak wind speeds) has been made available from anemometers installed on wind towers within the heliostat field [31]; see Figure 2-2 for the locations of the wind towers. At each of the wind towers, the anemometers were located at $10 \mathrm{ft}$, $20 \mathrm{ft}$ and $32.8 \mathrm{ft}$ above the ground. The anemometer of the west meteorological station was at a height of $32.8 \mathrm{ft}$. Wind speed data obtained on day 329 (25 Nov) of 1983 were chosen for a model and full-scale comparison of wind speeds for the heliostat under an operational mode.

In the Barstow field, instantaneous wind speeds were measured at three-minute intervals for two hours. Figure 4-26 from [31] shows variation of the wind speeds recorded at the west meteorological station on day 329 from 11 AM to 1 PM. The curve in the figure was obtained by a cubic curve fitting to the data points. The mean of the wind speeds was $23.5 \mathrm{mph}$ and the wind direction was 265 degrees during the period. 

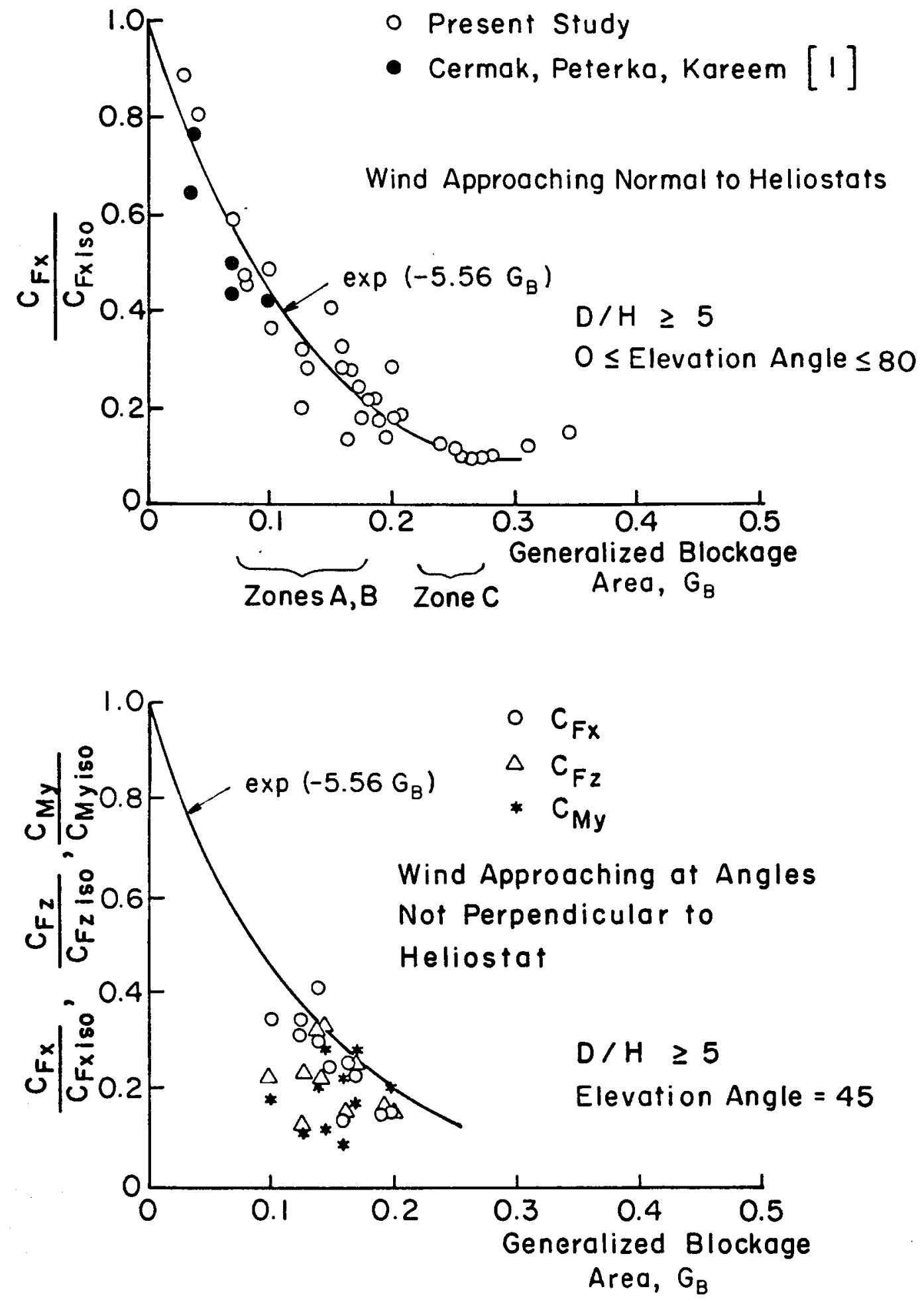

Figure 4-25. Mean Load Reduction as Function of Generalized Blockage 


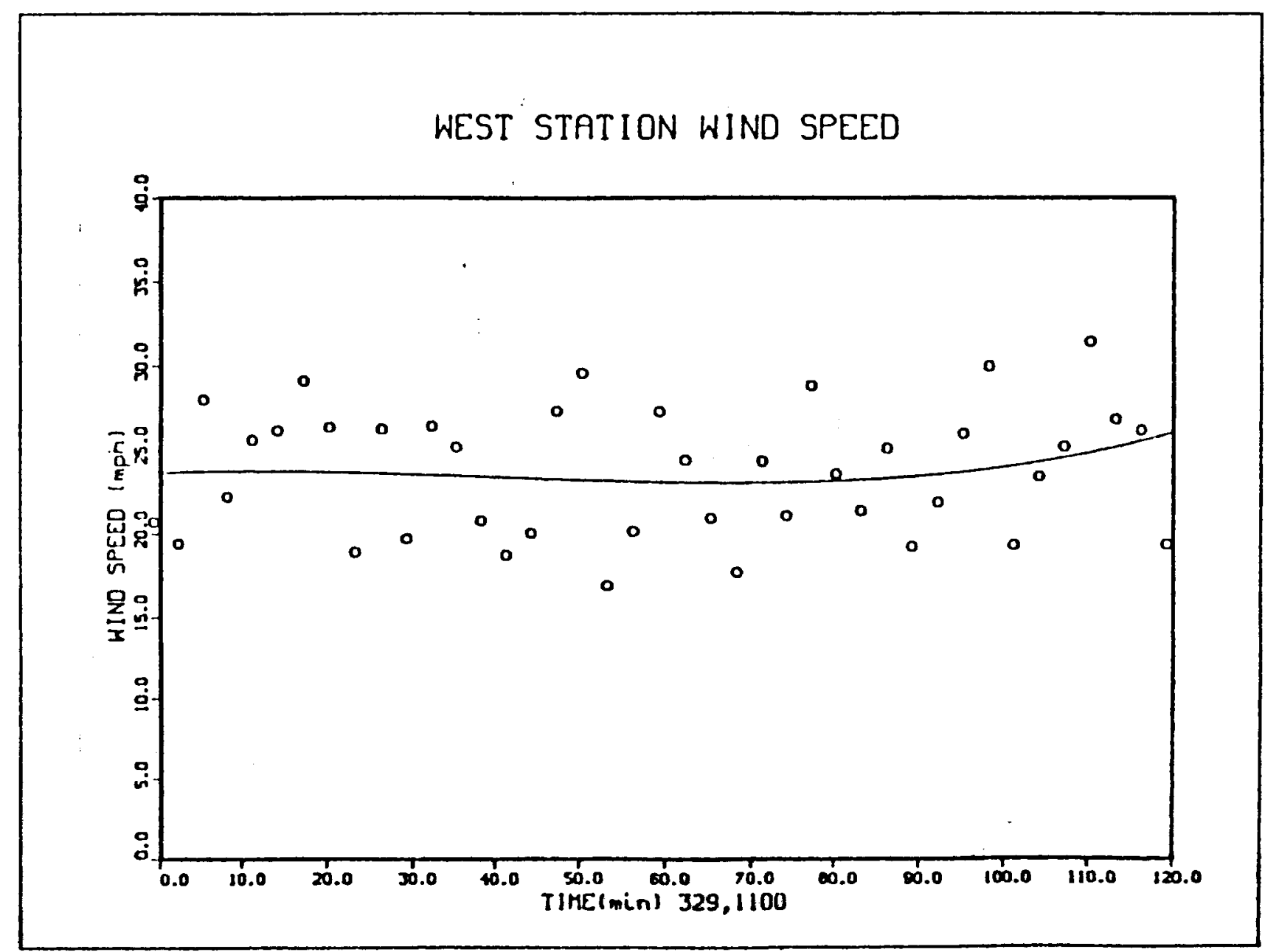

Figure 4-26. Full-Scale Wind Data [Ref. 31] 
In the wind tunnel, the heliostat field around and upstream of the wind towers 1,2 and 4 were fully simulated, and the wind speeds were measured at the corresponding heights of $10 \mathrm{ft}, 20 \mathrm{ft}$ and $32.8 \mathrm{ft}$. Measurements of wind speeds for the wind tower 6 were obtained by partially simulating the field with 11 upwind rows of heliostats (all that were available). The wind speeds were measured by a hot-film anemometer at a rate of 256 samples per second for 32 seconds for each wind tower and height. The maximum, mean and minimum wind speeds were then determined. The measurements were repeated 10 times for each configuration to obtain an ensemble average of the maximum (peak) wind speeds.

Figure 4-27 shows the full-scale peak wind speed and model peak, mean and minimum wind speeds at heights of $10 \mathrm{ft}, 20 \mathrm{ft}$ and $32.8 \mathrm{ft}$, respectively. All the wind speeds were normalized for comparisons using the mean wind speed at the west meteorological station. The full-scale and model peaks are in as good agreement as expected since the full-scale data represents a single realization of a probability distribution with a significant standard deviation. From the wind speed measurements in the wind tunnel, it is evident that the mean wind speeds decreased within the field in comparison to the west edge at $10-$ and $20-\mathrm{ft}$ levels and to a lesser extent for $32.8 \mathrm{ft}$. At the location of the wind tower 6 , however, the wind speed increased nearly to the level at the wind tower 1 . Peak wind speeds within the field did not show a tendency to decrease for either model or full scale. The lack of a decrease in peak wind speed within the full-scale field might lead incorrectly to the assumption that peak wind loads also remained constant across the field. High velocities in the field of small spatial extent cannot fully load a heliostat. Model data presented in Section 4.3 showed peak and mean loads on heliostats decreased.

- Thus, it can be concluded that peak and mean loads in the full-scale field will decrease from those at the field edge for operational positions. In other words, measurement of local wind gust peaks within the full-scale field cannot always be used to deduce peak wind loads on the heliostats. 

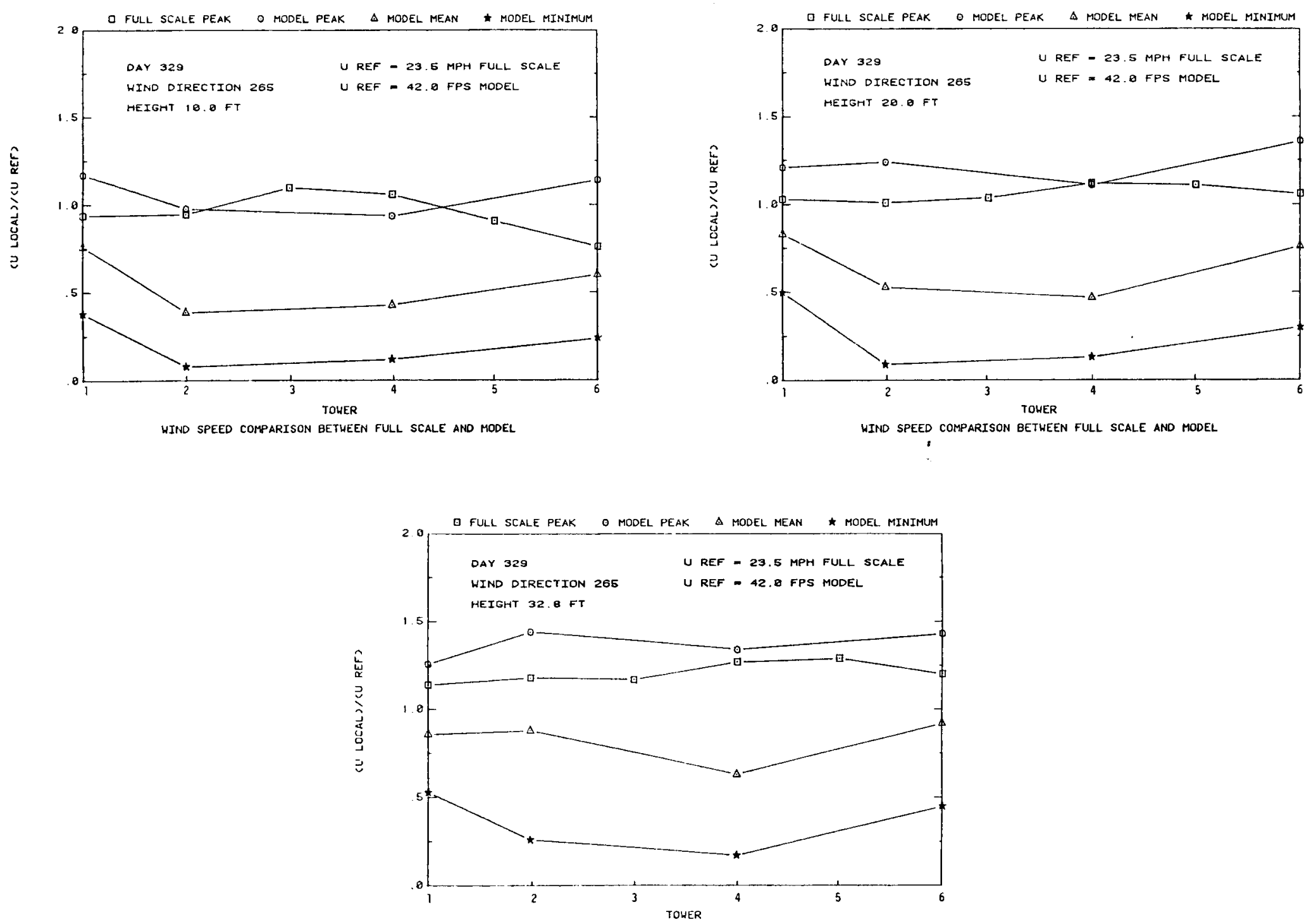

HIND SPEED COMPARISON BETHEEN FULL SCALE AND MODEL

Figure 4-27a,b,c. Model and Full-Scale Wind Speed Comparison 
SECTION 5.0

CONCLUSIONS AND RECOMMENDATIONS

A 1:60 scale simulation of wind flow over several fields of heliostats including the Barstow site was performed in a boundary-layer wind tunnel designed to model atmospheric winds. Wind loads were measured on heliostats using a six-component strain-gage balance. Wind load reductions below those of an isolated heliostat were measured as a function of heliostat setting (day of year and hour), position within the field, field density, wind-protective fence, and approach wind direction. On the basis of the data presented, the following conclusions can be made:

- Mean wind loads decrease with:

- increased distance into the field,

- increased field density,

- addition of solid or porous fences upwind.

- Mean wind load reduction data for a given load component can be collapsed onto a common curve which describes load reduction as a function of a generalized blockage calculated from upwind blockage elements.

- Mean wind loads on heliostats within the Barstow fields are substantially lower than those at the edge of the field--in many cases less than 30 percent of edge units.

- Properly designed wind fences and berms surrounding a field of heliostats can reduce edge heliostat loads to 30 percent or less of loads without the fences or berms.

- Limited investigations of fluctuating wind loads did not reveal dynamic loading mechanisms which would indicate that on-heliostat spoilers would be beneficial for mean or dynamic loads. This conclusion should be considered tentative for dynamic loads pending further testing.

- Full-scale wind loads are not available for comparison with windtunnel data.

- Design forces perpendicular to the mirror plane for an isolated heliostat are controlled by operational winds (50 $\mathrm{mph}$ ) while design drive moments are controlled by survival winds (90 mph).

- Fluctuating loads on heliostats can be measured at model scale but additional research needs to be done to decrease certain uncertainties in preliminary measurements.

- Peak wind loads are substantially lower within the heliostat field than at the edge of the field based on wind-tunnel tests in operational positions, including the Barstow field geometry. 
- The strength of a collector should be based on the peak load rather than a mean load multiplied by an assumed gust factor.

Additional research needs to be performed to fully exploit the current results:

- additional development of wind load as a function of generalized blockage area is required to develop the data into a codifiable form suitable for use by a designer not familiar with aerodynamic data.

- definition of the limits of applicability of the concept of generalized blockage area.

- complete development of techniques for dynamic force measurements including determination of the influence of model scale.

- investigate the influence of heliostat-mounted spoilers on dynamic loading.

- compare wind-tunnel loads with full-scale loads. 
SECTION 6.0

\section{REFERENCES}

1. Cermak, J. E., J. A. Peterka and A. Kareem, "Heliostat Field-Array Wind Tunnel Test," Technical Report for McDonnel1 Douglas Astronautics Company, Huntington Beach, California, Report No. CER78-79JEC-JAP-AK2, July 1978, 55 pages.

2. Ewald, R. L., J. A. Peterka and J. E. Cermak, "Heliostat-Array Wind Tunnel Study," Technical Report for Martin Marietta Aerospace, Report No. CER78-79RLE-JAP-JEC31, January 1979.

3. Peterka, J. A., J. J. Lou and J. E. Cermak, "Wind-Tunnel Test of a Photovoltaic Concentrator Array," Technical Report for Martin Marietta, Denver, Colorado, Report No. CER78-79JAP-JJL-JEC62, June 1979, 34 pages.

4. Peterka, J. A., J. M. Sinou and J. E. Cermak, "Mean Wind Forces on Parabolic-Trough Solar Collectors," Technical Report for Sandia Laboratories, Albuquerque, New Mexico, Report No. CER79-80JAP-JMS-JEC4, July 1979, 109 pages.

5. Franklin, H. A., "Wind Design of Flat-Panel Photovoltaic Array Structures - Final Report," Report No. SAND 79-7057, 83 pages.

6. Miller, R. and D. Zimmerman, "Wind Loads on Flat Plate Photovoltaic Array Fields, Phase II: Final Report," Report No. DOE/JPL 954833-79/2, September 1979,111 pages.

7. Poreh, M., J. A. Peterka and J. E. Cermak, "Wind-Tunnel Study of Wind Loads on Photovoltaic Structures," Technical Report for Bechtel National, San Francisco, California, Report No. CER79-80MP-JAP-JEC11, September 1979,83 pages.

8. Peterka, J. A., J. M. Sinou and J. E. Cermak, "Mean Wind Forces on Parabolic-Trough Solar Collectors," Report No. SAND 80-7023, May 1980, 109 pages.

9. Randall, D. E., D. D. McBride and R. E. Tate, "Steady-State Wind Loading on Parabolic-Trough Solar Collectors," Report No. SAND 79-2134, August 1980,21 pages.

10. Hosoya, N., J. A. Peterka, M. Poreh and J. E. Cermak, "Wind Pressures and Forces on Flat-Plate Photovoltaic Solar Arrays," Technical Report for Boeing Eng., Seattle, Washington, Report No. CER80-81NH-JAP-MP-JEC13, September 1980,81 pages.

11. Hosoya, N., J. A. Peterka, M. Poreh and J. E. Cermak, "Wind Pressures and Forces on Flat-Plate Photovoltaic Solar Arrays Data Supplement: Appendix" Technical Report for Boeing Eng., Seattle, Washington, Report No. CER80$81 \mathrm{NH}-J A P-M P-J E C 13 a$, September 1980, 182 pages. 
12. Miller, R. D. and D. K. Zimmerman, "Wind Loads on Flat Plate Photovoltaic Array Fields, Phase III, Final Report," Report No. DOE/JPL 954833-81/3, April 1981, 250 pages.

13. Hosoya, N., J. A. Peterka and J. E. Cermak, "Wind Pressures and Forces on Flat-Plate Photovoltaic Solar Arrays - Cross-Spectral Analysis," Technical Report for Boeing Eng., Seattle, Washington, Report No. CER80-81NHJAP-JEC57, June 1981,47 pages.

14. Miller, R. D. and D. K. Zimmerman, "Wind Loads on Flat Plate Photovoltaic Array Fields (Nonsteady Winds)," Report No. DOE/JPL 954833-81/4, August 1981,100 pages.

15. Tieleman, H. W., P. R. Sparks and R. E. Akins, "Wind Loads on Flat Plate Solar Collectors," Preprint No. 3632, ASCE Convention and Exposition, Atlanta, Georgia, 23-25 October 1979, 21 pages.

16. Tieleman, H. W., R. E. Akins and P. R. Sparks, "An Investigation of Wind Loads on Solar Collectors," Report No. VPI-E-80-1, January 1980, 155 pages.

17. Tieleman, H. W., R. E. Akins and P. R. Sparks, "An Investigation of Wind Loads on Solar Collectors, Appendix I - Data Listing for Top and Bottom of Collector," Report No. VPI-E-80-1, January 1980, 307 pages.

18. Tieleman, H. W., R. E. Akins and P. R. Sparks, "An Investigation of Wind Loads on Solar Collectors, Appendix II - Net Pressure Coefficients," Report No. VPI-E-80-1, January 1980, 165 pages.

19. Mar, J. W. and H. Liebowitz (eds.), Structures Technology for Large Radio and Radar Telescope Systems, The MIT Press, 1969.

20. Cohen, E. (ed.), Large Steerable Radio Antennas - Climatological and Aerodynamic Considerations, Annals of the New York Academy of Science, Vol. 116, October 1, 1964, pp. 1-355.

21. Jet Propulsion Laboratory, "Wind Loads on Dish Antenna," Technical Report CP-6 (unpublished).

22. Murphy, L. M., "An Assessment of Existing Studies of Wind Loading on Solar Collectors," Report No. SERI/TR-632-812, February 1981, 43 pages.

23. Eimern, J. V., R. Karshon, L. A. Razamova and G. W. Robertson, "Windbreaks and Shelterbelts," World Meteorological Organization, T.N. 59, 1964 .

24. Counihan, J., J.C.R. Hunt and P. S. Jackson, "Wakes Behind TwoDimensional Surface Obstacles," J. Fluid Mech., Vo1. 64, 1974, pp. 529563.

25. Raine, J. K. and D. C. Stevenson, "Wind Protection by Model Fences in a Simulated Atmospheric Boundary Layer," J. Indust. Aero., Vol. 2, 1977, pp. $159-180$. 
26. Perara, M.D.A.E.S., "Shelter Behind Two-Dimensional Solid and Porous Fences," J. Wind Eng. and Indust. Aero., Vol. 8, 1981, pp. 93-104.

27. Bradley, E. F. and P. J. Mulhearn, "Development of Velocity and Shear Stress Distributions in the Wake of a Porous Shelter Fence," J. Wind Eng. and Indust. Aero., Vol. 15, 1983, pp. 145-156.

28. Cermak, J. E., "Laboratory Simulation of the Atmospheric Boundary Layer," AIAA J., Vol. 9, 1971.

29. Cermak, J. E., "Applications of Fluid Mechanics to Wind Engineering," A Freeman Scholar Lecture, ASME J. of Fluids Eng., Vol. 97, No. 1, 1975.

30. Cermak, J. E. "Aerodynamics of Buildings," Annual Review of Fluid Mech., Vol. 8, 1976, pp. 75-106.

31. Green, H. J., letter with data addressed to Clay Mavis, Sandia National Laboratories, Livermore, California, 23 April 1985. 
APPENDIX A

VALIDATION OF WIND-TUNNEL TESTING

IN CIVIL ENGINEERING APPLICATIONS 
VALIDATION OF WIND-TUNNEL TESTING

IN CIVIL ENGINEERING APPLICATIONS

Boundary-layer wind tunnels have become an important tool used in physical modeling of various flow phenomena. Over the past 20 years considerable model data has been collected on wind flow over terrain and wind loads on structures. Validity of the wind-tunnel data has been evaluated by comparison with the results of full-scale measurements. Such comparisons have been recently discussed during a workshop on wind-tunnel modeling for wind engineering applications [A1] and during the Sixth International Conference on Wind Engineering [A2]. The presented data, related to wind loading and to flow characteristics, are summarized below.

Dalgliesh [A3-A5] discussed data for a tall building. He compared wind pressure on cladding and overall loading [A3,A4] as well as the building response [A5]. A typical comparison for the pressure data is shown in Figure A-1. It can be seen that the agreement between the model and fullscale data is good both for the windward and leeward locations of a pressure tap. A similar agreement was obtained for overall loading: the base shear and the overturning moment, as is depicted in Figure A-2. The degree of agreement between the model and full-scale building response was different for different response modes. The best agreement, shown in Figure A-3, was obtained for translational modes. The agreement for the other modes was not as good, especially for higher frequencies. Based on the analyzed data, Dalgleish [A3] concluded that prediction of full-scale behavior of a tall. building is possible to within 10 to 15 percent. A better agreement should not be expected due to many uncontrolled full-scale variables. Similar conclusions were reached by Lee [A6].

Holmes [A7] discussed model and full-scale tests of Aylesbury House. The full-scale study was conducted in England, while the model studies were undertaken in various wind tunnels located in Australia, Canada, U.S.A., U.K., and France. Holmes [A7] used comparative data presented by Tieleman et al. [A8] to discuss observed trends for pressure measurements. Figure A-4 shows "local" pressure coefficients based on the upwind mean wind speed at the height of the pressure tapping for center wall tapping. The mean pressure coefficients, Figure A-4a, are within a relatively narrow range. The agreement with the full-scale data is encouraging, considering the variations in model scaling ratio and boundary-layer simulation procedures used. In the case of the rms pressures, Figure A-4b, the agreement between the wind-tunnel results and full-scale data is again quite good for most of the wind directions tested.

Comparison of model prediction based on wind-tunnel tests and full-scale response of two long-span suspension bridges was presented by Davenport [A9]. The results for the Golden Gate Bridge and Bronx Whitestone Bridge are shown in Figures A-5 and A-6, respectively. It can be seen that full-scale responses fall within the response boundaries established during wind-tunnel studies of the bridge models. A similar conclusion can be drawn for the data presented by Melbourne [A10], depicted in Figure A-7.

Model/full-scale comparisons for other flow situations and flow-structure interactions were also reported in the literature. They included studies of 
(a)
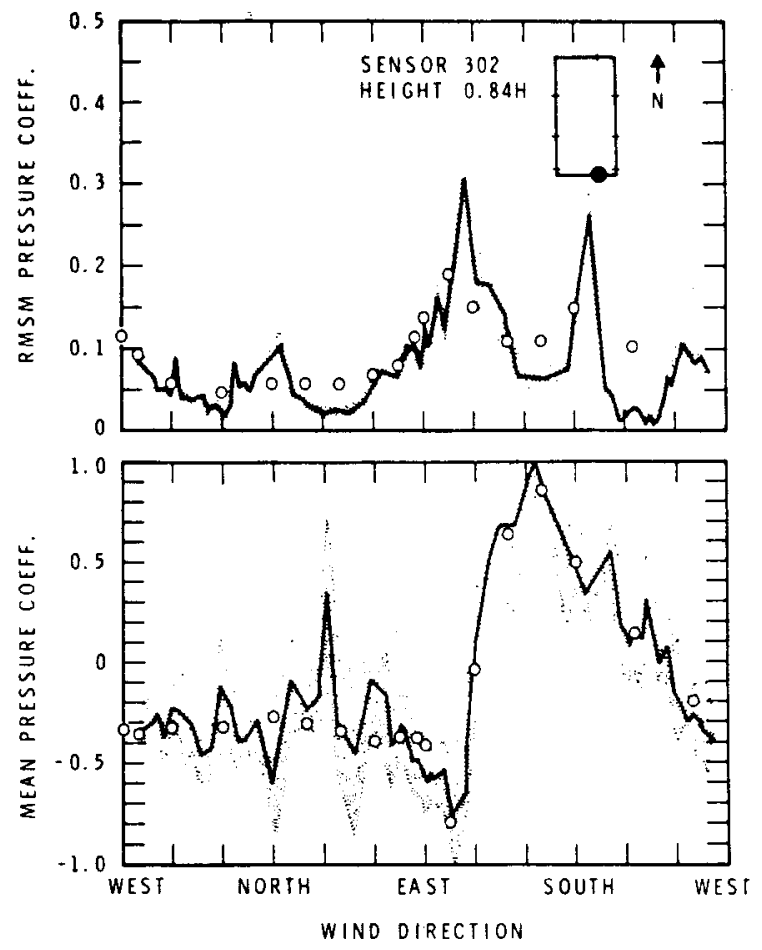

South wall tap $10.7 \mathrm{~m}$ from S.E. corner, 50 th floor.

(b)
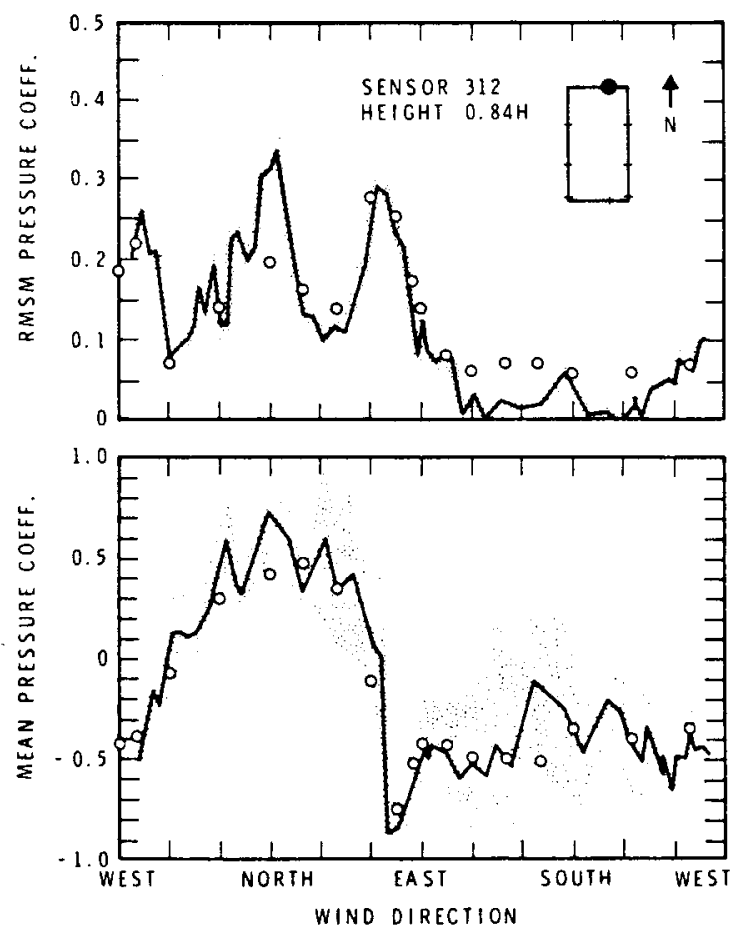

North wall tap $10.7 \mathrm{~m}$ from N.E. corner, 50th floor.

Figure A-1. Model and Full-Scale Pressure Coefficients, Ref. [A4] 
(a)
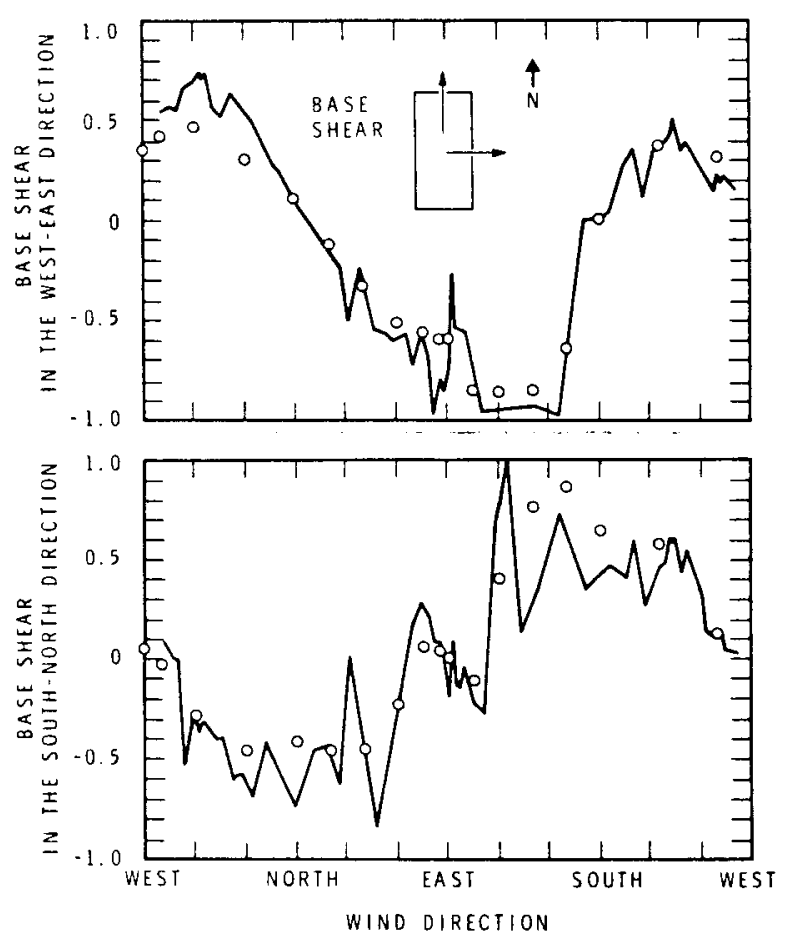

(b)
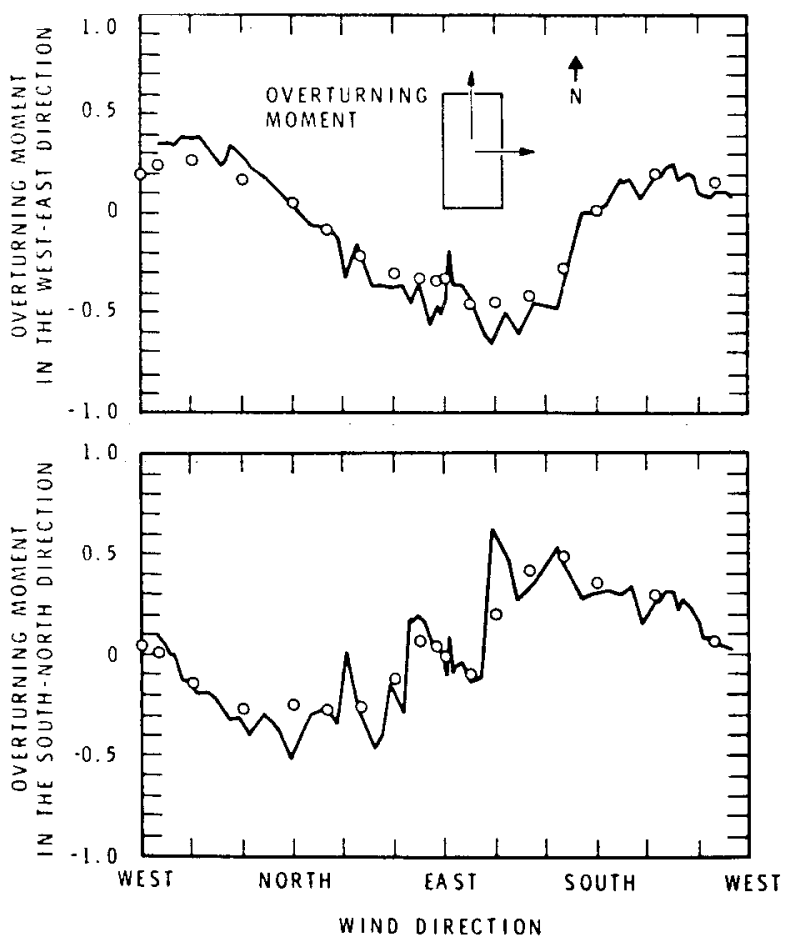

Figure A-2. Model and Full-Scale Overall Mean Loads, Ref. [A4] 


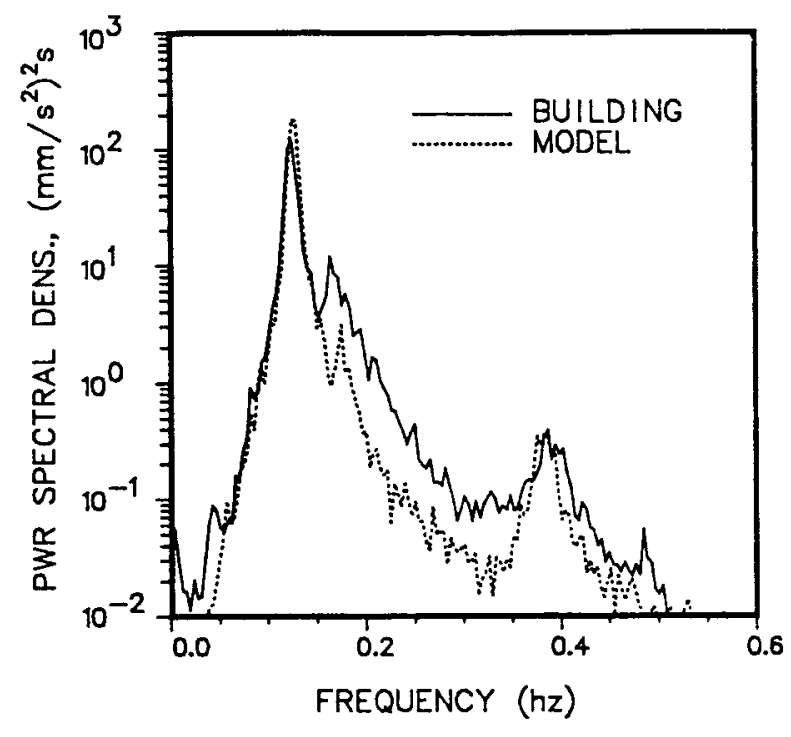

Figure A-3. Comparison of Model and Full-Scale East-West Acceleration Power Spectra - Translational

Modes, Ref. [A5] 
(a)

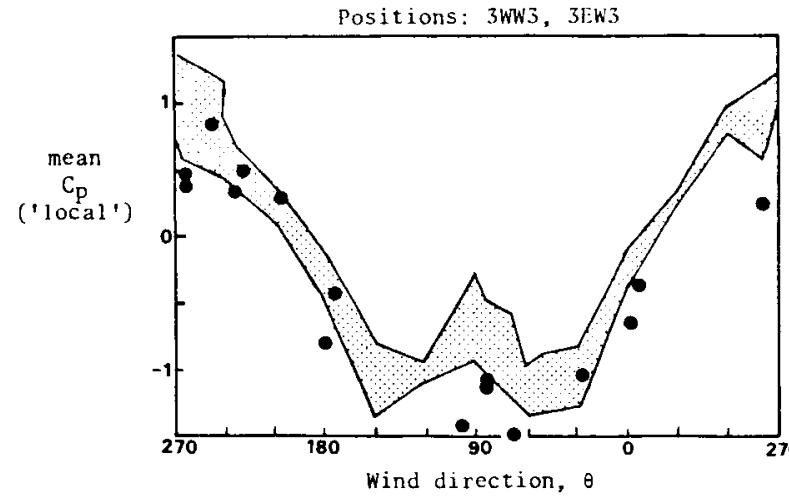

Range of wind tunnel results

- Full scale

(b)

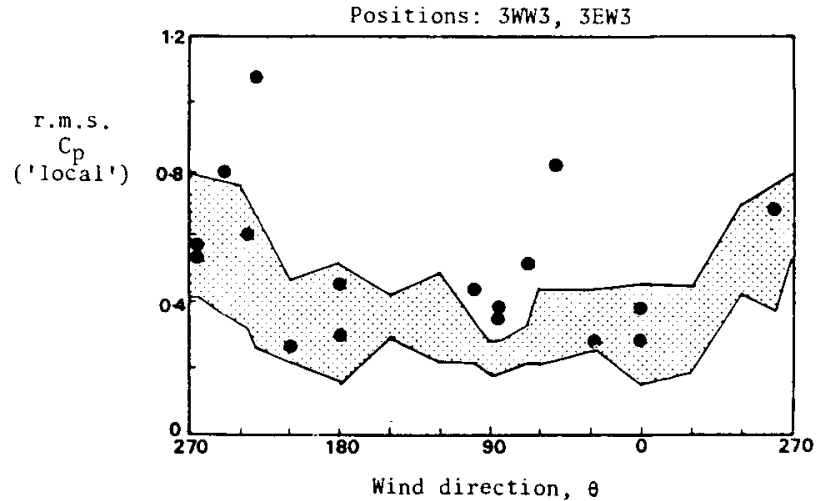

Figure A-4. Comparison of 'Local' Mean and RMS Pressure Coefficients, Refs. [A7] and [A8] 
(a)
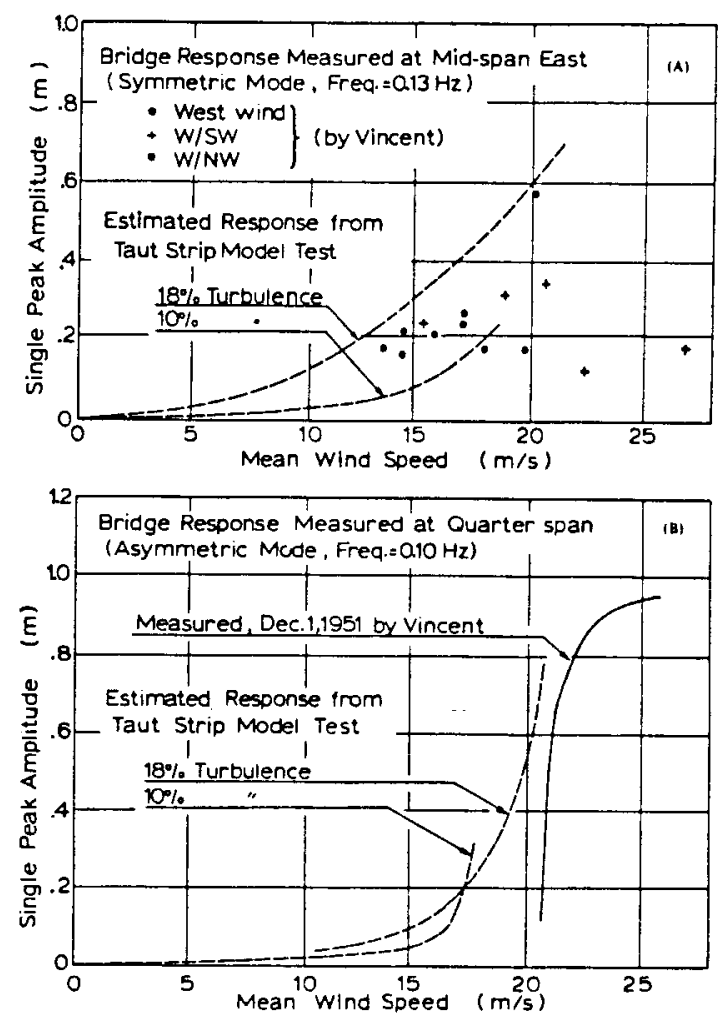

Figure A-5. Comparison of Response Amplitudes of Symmetric and Asymmetric Modes for Golden Gate Bridge, Ref. [A9] 


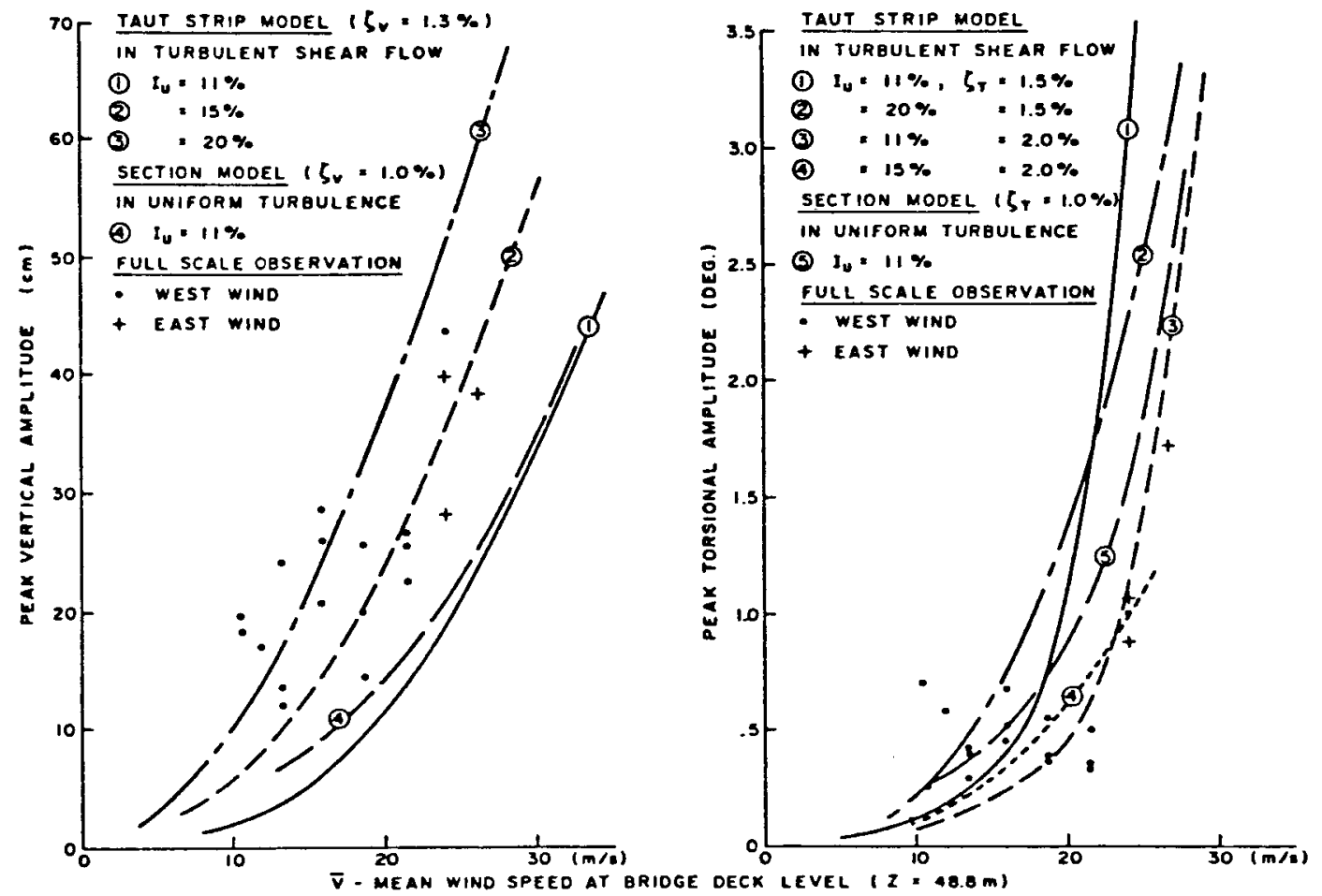

Figure A-6. Summary of Wind Tunnel and Full-Scale Bronx Whitestone Bridge Response, Wind Normal to Bridge Centerline, Ref. [A9] 


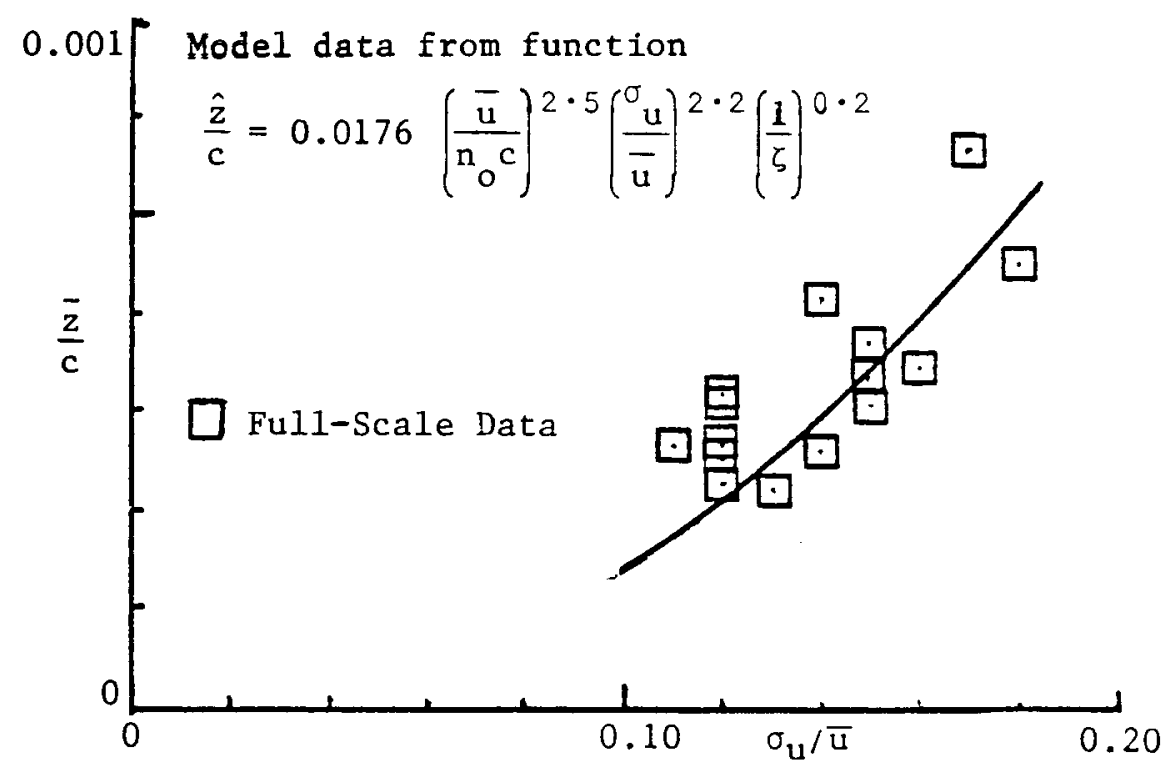

Figure A-7. Full-Scale Average Hourly Maximum Center Deck Vertical Displacement as a Function of the Turbulence Intensity of the Incident Wind Flow, at $\bar{u} / \mathrm{n}_{\mathrm{o}} \mathrm{c}=0.1, \zeta=0.01$, $\beta=0^{\circ}$, Ref. [A10] 
behavior in wind of towers and chimneys [A11], natural ventilation studies [A12], investigations of flow over various topographies [A13,A14], and others. Interesting flow data was presented by Flay and Teunissen [A13]. The authors compared simulated (in a wind tunnel) and full-scale wind structure over a suburban airport. The agreement between the compared data, as shown in Figures A-8 and A-9 was good. A similar agreement for a flow over an isolated low hill, see Figure A-10, was reported by Teunissen [A14].

As follows from the preceding discussion of a few of the many published comparisons, agreement between the model and full-scale measurements is generally good. Due to uncertainties associated with full-scale conditions and certain wind-tunnel modeling limitations, addressed by Sparks [A15], the agreement cannot be expected to be perfect. Determination of the error margins require knowledge of the full-scale data, which at the present time are available for only a very limited number of cases. More full-scale studies of flow situations and wind effects on various structures (including heliostats and stretched membrane modules) would be valuable to verify improvements in modeling techniques and to provide better documentation on validity of wind-tunnel testing. 


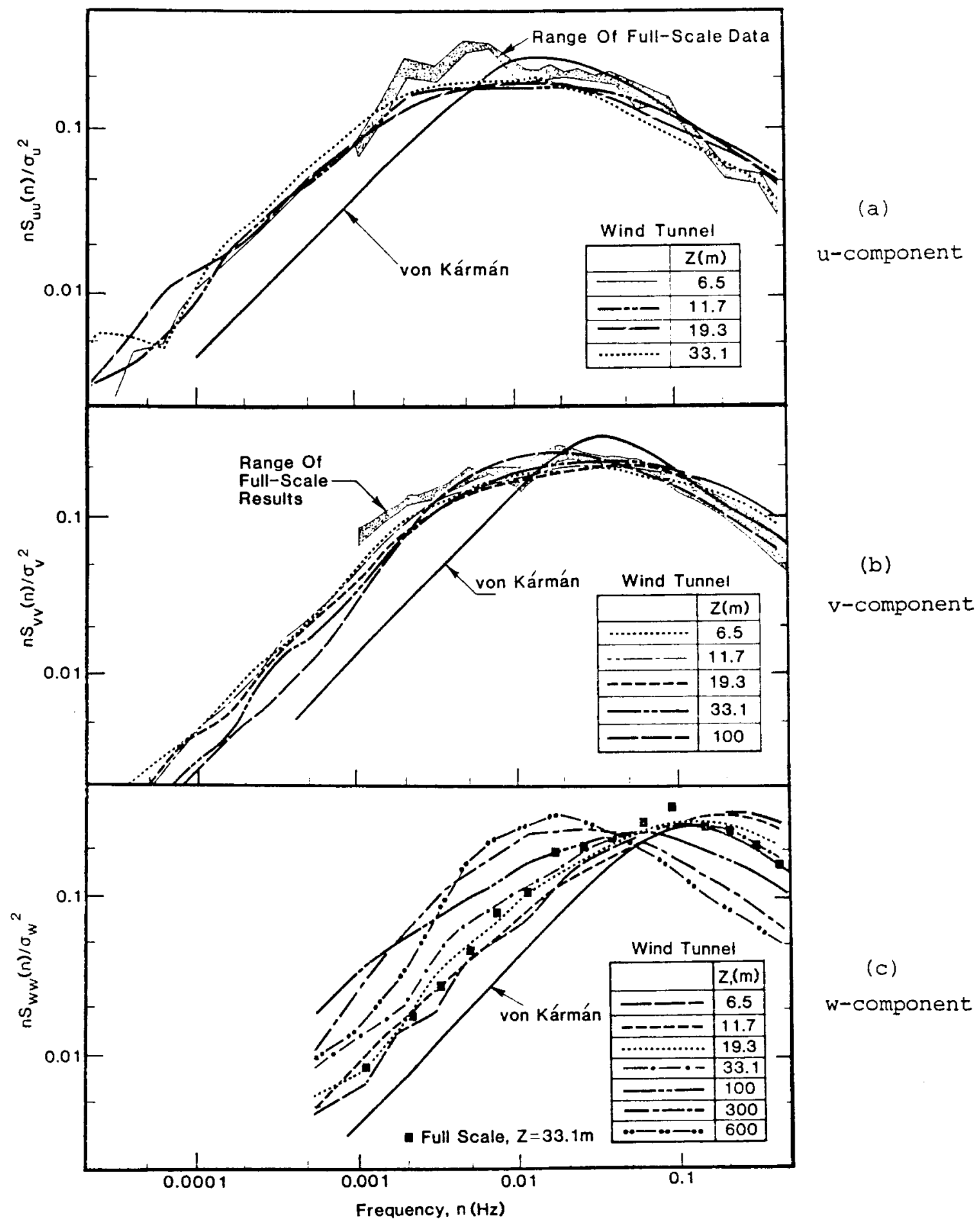

Figure A-8. Wind-Tunnel and Full-Scale Power Spectra at NAE Tower Location, Ref. [A13] 


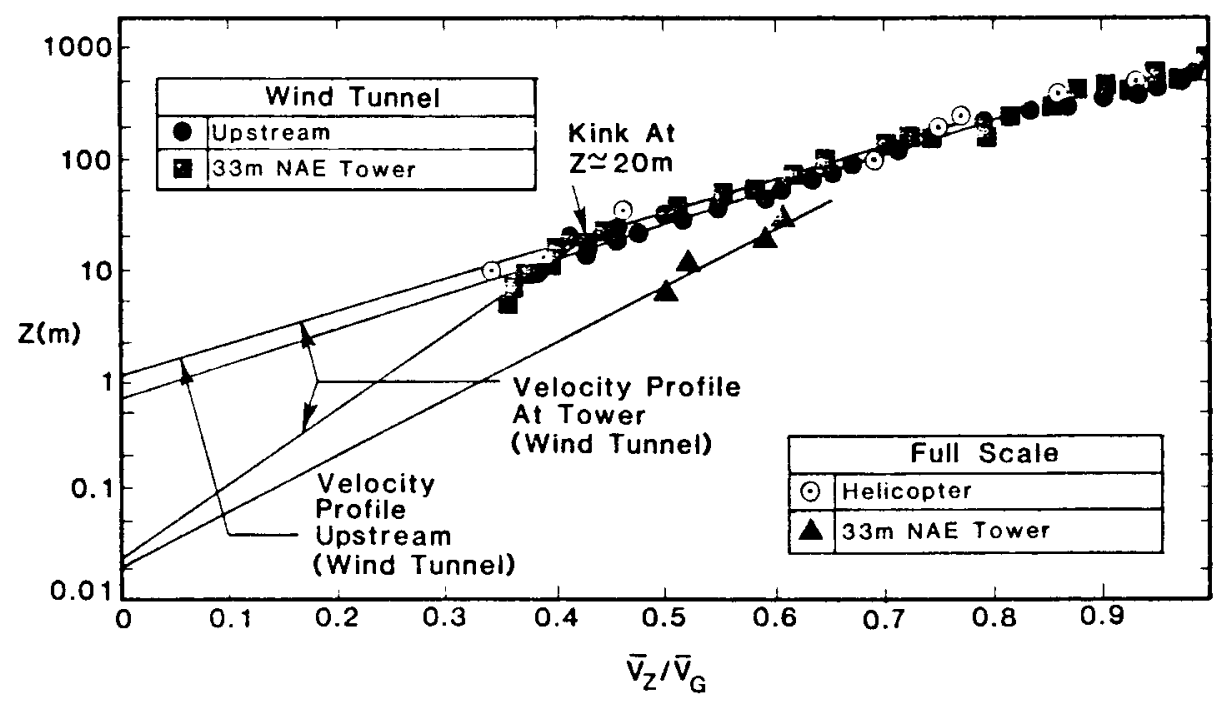

Figure A-9. Wind-Tunnel/Full-Scale Velocity Profile Comparison Above Airport, Ref. [A13] 


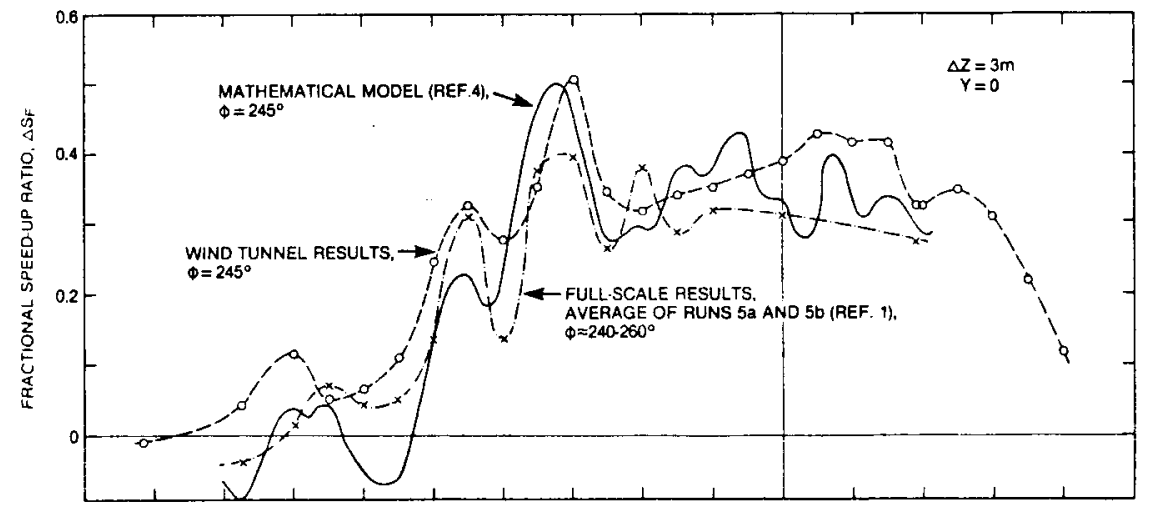

Figure A-10. Comparison of Wind-Tunnel, Full-Scale and Mathematical-Model Values of Fractional Speed-up Ratio at $\Delta Z=3 \mathrm{~m}$ Above Main EastWest Tower Line, Ref. [A14] 


\section{REFERENCES}

A1. Reinhold, T. A. (Editor), "Wind Tunnel Modeling for Civil Engineering Applications," Proceedings of the International Workshop on Wind Tunnel Modeling Criteria and Techniques in Civil Engineering Applications, Gaithersburg, Maryland, USA, Cambridge University Press, Cambridge, April 1982 .

A2. Proceedings of the Sixth International Conference on Wind Engineering, Gold Coast, Australia and Auckland, New Zealand, 21-25 March and 6-7 April 1983, printed in Journal of Wind Engineering and Industrial Aerodynamics, Vols. 13, 14, 15, December 1983.

A3. Dalgliesh, W., "Comparison of Model and Full-Scale Tests of the Commerce Court Building in Toronto," Proceedings of the International Workshop on Wind Tunnel Modeling Criteria and Techniques in Civil Engineering Applications, Gaithersburg, Maryland, USA, Cambridge University Press, Cambridge, April 1982, pp. 575-589.

A4. Dalgleish, W. A., "Comparison of Model/Full-Scale Wind Pressures on a High-Rise Building," Journal of Industrial Aerodynamics, Vol. 1, 1975, pp. 55-66.

A5. Dalgleish, W. A., K. R. Cooper and J. T. Templon, "Comparison of Model and Full-Scale Accelerations of a High-Rise Building," Proceedings of the Sixth International Conference on Wind Engineering, Gold Coast, Australia, and Auckland, New Zealand, 21-25 March and 6-7 April 1983, printed in Journal of Wind Engineering and Industrial Aerodynamics, Vols. 13, 14, 15, December 1983.

A6. Lee, B. E., "Model and Full-Scale Tests of the Arts Tower at Sheffield University," Proceedings of the International Workshop on Wind Tunnel Modeling Criteria and Techniques in Civil Engineering Applications, Gaithersburg, Maryland, USA, Cambridge University Press, Cambridge, April 1982 , pp. 590-604.

A7. Holmes, J. D., "Comparison of Model and Ful1-Scale Tests of the Aylesberry House," Proceedings of the International Workshop on Wind Tumel Modeling Criteria and Techniques in Civil Engineering Applications, Gaithersburg, Maryland, USA, Cambridge University Press, Cambridge, April 1982 , pp. 605-618.

A8. Tieleman, H. W., R. E. Akins and P. R. Sparks, "Model/Model and Ful1Scale/Model Comparison of Wind Pressures on Low-Rise Structures," Proc. Colloque, "Construive avec le Vent," Nantes, 1981, pp. IV-5-1 - IV- $\overline{5-21 .}$

A9. Davenport, A. G., "Comparison of Model and Full-Scale Tests on Bridges," Proceedings of the International Workshop on Wind Tunnel Modeling Criteria and Techniques in Civil Engineering Applications, Gaithersburg, Maryland, USA, Cambridge University Press, Cambridge, April 1982, pp. $619-636$. 
A10. Melbourne, W. H., "Comparison of Model and Full-Scale Tests of a Bridge and a Chimney Stack," Proceedings of the International Workshop on Wind Tunnel Modeling Criteria and Techniques in Civil Engineering Applications, Gaithersburg, Maryland, USA, Cambridge University Press, Cambridge, April 1982, pp. 637-653.

A11. Basu, R. I, and B. J. Vickery, "A Comparison of Model and Full-Scale Behavior in wind of Towers and Chimneys," Proceedings of the International Workshop on Wind Tunnel Modeling Criteria and Techniques in Civil Engineering Applications, Gaithersburg, Maryland, USA, Cambridge University Press, Cambridge, April 1982, pp. 654-668.

A12. Chandra, S., A. A. Kerestecioglu, P. W. Fairey, III and W. Cromer, "Comparison of Model and Full-Scale Natural Ventilation Studies," Proceedings of the International Workshop on Wind Tunnel Modeling Criteria and Techniques in Civil Engineering Applications, Gaithersburg, Maryland, USA, Cambridge University Press, Cambridge, April 1982, pp. $669-684$.

A13. Flay, R.G.J. and H. W. Teunissen, "Comparison of Simulated and Full-Scale Wind Structure over a Small Suburban Airport," Proceedings of the Sixth International Conference on Wind Engineering, Gold Coast, Australia and Auckland, New Zealand, 21-25 March and 6-7 April 1983, printed in Journal of Wind Engineering and Industrial Aerodynamics, Vols. 13, 14, 15, December 1983.

A14. Teunissen, H. W., "Wind-Tunnel and Full-Scale Comparison of Mean Wind Flow over an Isolated Low Hill," Proceedings of the Sixth International Conference on Wind Engineering, GoId Coast, Australia and Auckland, New Zealand, 21-25 March and 6-7 April 1983, printed in Journal of Wind Engineering and Industrial Aerodynamics, Vols. 13, 14, 15, December 1983.

A15. Sparks, P. R., "A Prepared Critique of Papers on the Validation of Wind Tunnel Testing," Proceedings of the International Workshop on Wind Tunnel Modeling Criteria and Techniques in Civil Engineering Applications, Gaithersburg, Maryland, USA, Cambridge University Press, Cambridge, April 1982 , pp. 685-688. 
APPENDIX B

WIND-TUNNEL DATA FOR ISOLATED HELIOSTAT 
Table B-1.

\begin{tabular}{|c|c|c|c|}
\hline WI) & EL & $\mathrm{AZ}$ & Run \\
\hline 0 & 80 & 270 & 81 \\
\hline 22.5 & 80 & 270 & 83 \\
\hline 45 & 80 & 270 & 85 \\
\hline 67.5 & 80 & 270 & 87 \\
\hline 90 & 80 & 270 & 89 \\
\hline 112.5 & 80 & 270 & 91 \\
\hline ] 35 & 80 & 270 & 93 \\
\hline 157.5 & 80 & 270 & 95 \\
\hline 180 & 80 & 270 & 97 \\
\hline 0 & 0 & 270 & 115 \\
\hline 22.5 & 0 & 270 & 113 \\
\hline 45 & 0 & 270 & 111 \\
\hline 67.5 & 0 & 270 & 109 \\
\hline 90 & 0 & 270 & 107 \\
\hline 112.5 & 0 & 270 & 105 \\
\hline 135 & 0 & 270 & 103 \\
\hline 157.5 & 0 & 270 & 101 \\
\hline 180 & 0 & 270 & 99 \\
\hline
\end{tabular}


Table B-1. continued

\begin{tabular}{|c|c|c|c|}
\hline WD & $\mathrm{EL}$ & $\mathrm{AZ}$ & Run \\
\hline 0 & 45 & 270 & 136 \\
\hline 22.5 & 45 & 270 & 138 \\
\hline 45 & 45 & 270 & 140 \\
\hline 67.5 & 45 & 270 & 142 \\
\hline 90 & 45 & 270 & 144 \\
\hline 112.5 & 45 & 270 & 146 \\
\hline 135 & 45 & 270 & 148 \\
\hline 157.5 & 45 & 270 & 150 \\
\hline 180 & 45 & 270 & 152 \\
\hline 0 & 84 & 270 & 170 \\
\hline 22.5 & 84 & 270 & 168 \\
\hline 45 & 84 & 270 & 166 \\
\hline 67.5 & 84 & 270 & 164 \\
\hline 90 & 84 & 270 & 162 \\
\hline 112.5 & 84 & 270 & 160 \\
\hline 135 & 84 & 270 & 158 \\
\hline 157.5 & 84 & 270 & 156 \\
\hline 180 & 84 & 270 & 155 \\
\hline
\end{tabular}


Table B-1. continued

\begin{tabular}{|c|c|c|c|}
\hline WD & EL & $A Z$ & Run \\
\hline 0 & 87 & 270 & 172 \\
\hline 22.5 & 87 & 270 & 174 \\
\hline 45 & 87 & 270 & 176 \\
\hline 67.5 & 87 & 270 & 178 \\
\hline 90 & 87 & 270 & 180 \\
\hline 112.5 & 87 & 270 & 182 \\
\hline 135 & 87 & 270 & 184 \\
\hline 157.5 & 87 & 270 & 186 \\
\hline 180 & 87 & 270 & 188 \\
\hline () & 90 & 270 & 206 \\
\hline 22.5 & 90 & 270 & 204 \\
\hline 45 & 90 & 270 & 202 \\
\hline 67.5 & 90 & 270 & 200 \\
\hline 90 & 90 & 270 & 198 \\
\hline 112.5 & 90 & 270 & 196 \\
\hline 135 & 90 & 270 & 194 \\
\hline 157.5 & 90 & 270 & 192 \\
\hline 180 & 90 & 270 & 190 \\
\hline
\end{tabular}


Table B-1. continued

\begin{tabular}{|c|c|c|c|}
\hline$W D$ & $\mathrm{EL}$ & $\mathrm{AZ}$ & Run \\
\hline 0 & 15 & 270 & 246 \\
\hline 45 & 15 & 270 & 244 \\
\hline 90 & 15 & 270 & 242 \\
\hline 135 & 15 & 270 & 240 \\
\hline 180 & 15 & 270 & 238 \\
\hline 0 & 30 & 270 & 282 \\
\hline 45 & 30 & 270 & 230 \\
\hline 90 & 30 & 270 & 232 \\
\hline 135 & 30 & 270 & 234 \\
\hline 180 & 30 & 270 & 236 \\
\hline 0 & 60 & 270 & 226 \\
\hline 45 & 60 & 270 & 224 \\
\hline 90 & 60 & 270 & 222 \\
\hline 135 & 60 & 270 & 220 \\
\hline 180 & 60 & 270 & 218 \\
\hline 0 & 75 & 270 & 208 \\
\hline 45 & 75 & 270 & 210 \\
\hline 90 & 75 & 270 & 212 \\
\hline 135 & 75 & 270 & 214 \\
\hline 180 & 75 & 270 & 216 \\
\hline
\end{tabular}




\begin{tabular}{|c|c|c|c|c|c|c|}
\hline $5: 4 y_{1}^{\prime}$ & $\lim$ & $=0 \frac{51}{6}$ & $-20 \div \overline{0}$ & $\begin{array}{l}\because E L \\
2 \because 50\end{array}$ & & \\
\hline $\begin{array}{l}\text { onss } \\
\text { MEAH }\end{array}$ & $\begin{array}{c}c 15 \\
13\end{array}$ & $\begin{array}{l}\cos \\
.00\end{array}$ & 05 & cho & 84 & $\begin{array}{c}c r i= \\
6\end{array}$ \\
\hline 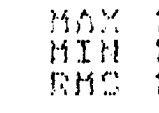 & $\begin{array}{r}95 \\
.05 \\
05\end{array}$ & .05 & .5 & $.0 \begin{array}{l}0 \\
0 \\
0\end{array}$ & 8 & 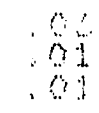 \\
\hline $\begin{array}{l}\text { PACT } \\
\text { PACT }\end{array}$ & 1.92 & 12.25 & 7.76 & 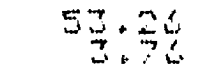 & $2 \cdot 5$ & $3: 28$ \\
\hline
\end{tabular}

MATA FOF FILE: STMOL.

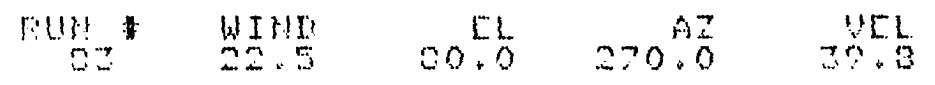

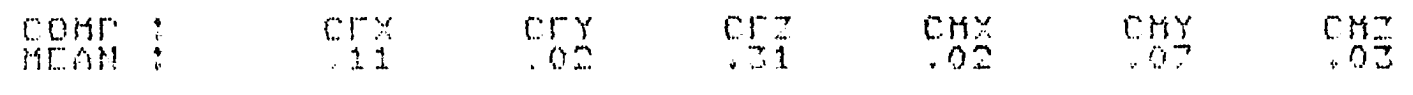

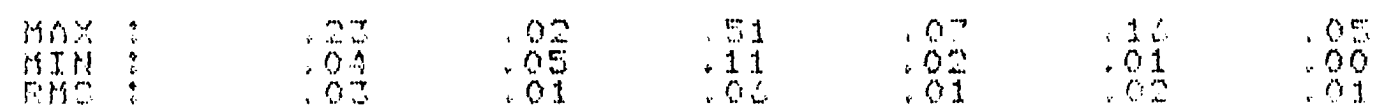

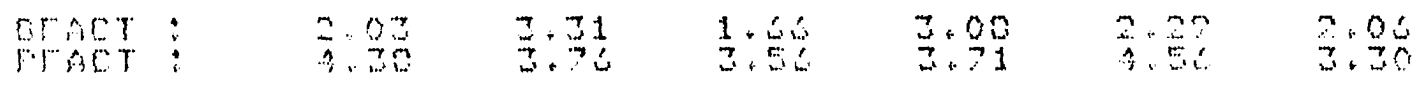

MATA FQF: FILE: : SIMGL.

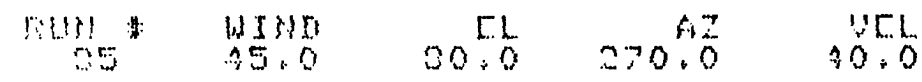

\begin{tabular}{|c|c|c|c|c|c|c|}
\hline coms & 50 & $\operatorname{cr}_{0 .}$ & $\int_{2}=$ & $\begin{array}{c}\mathrm{cis} \\
.07\end{array}$ & 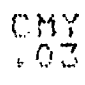 & $\cos _{0.02}$ \\
\hline $\begin{array}{l}\text { may } \\
\text { miry }\end{array}$ & .12 & $\begin{array}{l}.02 \\
.05 \\
01\end{array}$ & .03 &. $\begin{array}{l}12 \\
.01 \\
.01\end{array}$ & $\begin{array}{r}10 \\
08 \\
02\end{array}$ & $\begin{array}{r}05 \\
.00 \\
.01\end{array}$ \\
\hline
\end{tabular}

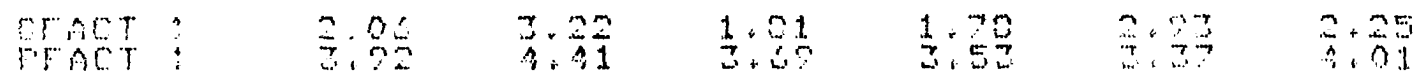

MATA FOF FILE : EISHLL

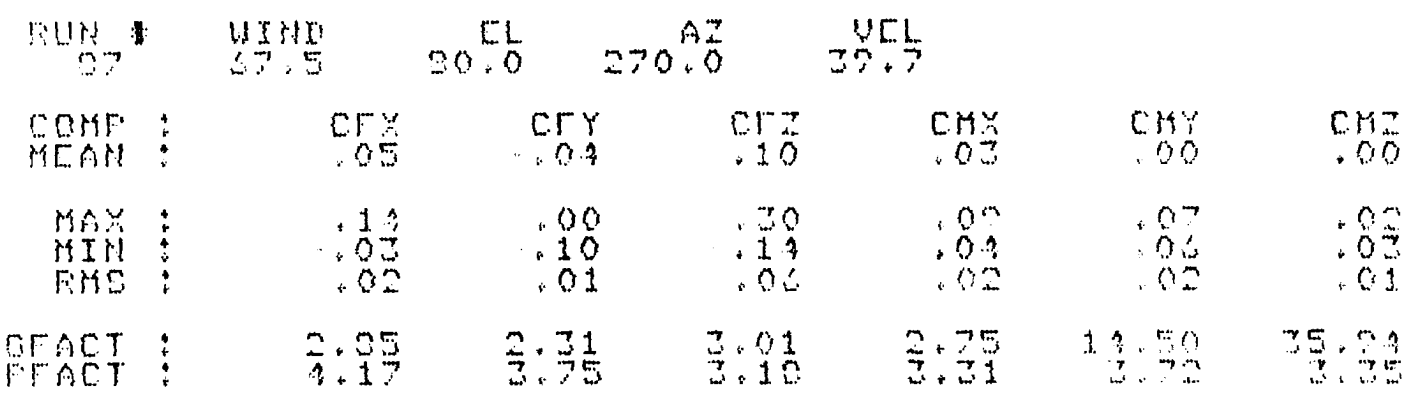




\begin{tabular}{|c|c|c|c|c|c|c|}
\hline rium & 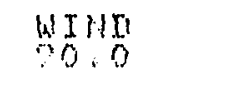 & 50.0 & $=90^{A Z}$ & 10.1 & & \\
\hline COHF : & $.0 \%$ & $\begin{array}{c}C 5 Y \\
: 09\end{array}$ & $\operatorname{cra}$ & $\begin{array}{c}\text { C.Y. } \\
.01\end{array}$ & $\begin{array}{c}c .9 Y \\
.01\end{array}$ & $\operatorname{cm}$ \\
\hline $\begin{array}{l}\text { MAY : } \\
\text { MIII } \\
\text { Fis: }\end{array}$ & $\begin{array}{l}.97 \\
.07 \\
.02\end{array}$ & $\begin{array}{l}.02 \\
10 \\
.01\end{array}$ &.$\frac{15}{2}$ & .09 & .82 & .82 \\
\hline $\begin{array}{l}\text { QFACT } \\
\text { FFACT }\end{array}$ & $=\frac{1}{3} \cdot 70$ & $\frac{9}{4} \cdot 58$ & $=1+00$ & $1 \cdot \cdot 3=$ & $\frac{7}{2} \cdot 50$ & $5 \cdot \frac{1}{3}$ \\
\hline IATA FC & SF: FILE : & SIHGL & & & & \\
\hline rut! & $11=5$ & 00.0 & $=70.0$ & $\begin{array}{l}\text { UEL } \\
30.0\end{array}$ & & \\
\hline CQME: & $\operatorname{cro}_{4}$ & $\begin{array}{r}\operatorname{cry} \\
-\quad O A\end{array}$ & $\frac{c r z}{15}$ & $\begin{array}{l}6 m y \\
63\end{array}$ & $\begin{array}{l}\text { CHY } \\
.01\end{array}$ & $\begin{array}{c}\cos \\
.00\end{array}$ \\
\hline $\begin{array}{l}\text { May } \\
\text { MISy } \\
\text { Fus : }\end{array}$ & $\begin{array}{l}.02 \\
.15 \\
.02\end{array}$ & $\begin{array}{l}.01 \\
.09 \\
.01\end{array}$ & $\begin{array}{r}.05 \\
.55 \\
.00\end{array}$ & $\begin{array}{r}.05 \\
.125 \\
.05\end{array}$ & .85 & $\begin{array}{l}.01 \\
.01 \\
.01\end{array}$ \\
\hline $\begin{array}{l}\text { QFACT } \\
\text { FFACT }\end{array}$ & $\frac{7}{4}: 35$ & $\frac{2}{3} \cdot \frac{1}{5}$ & $\begin{array}{l}7.34 \\
5.62\end{array}$ & $\begin{array}{l}3.07 \\
3.01\end{array}$ & $\Rightarrow+51$ & 15,9 \\
\hline MATA FI & OF FILE : & SINGL & & & & \\
\hline rust & $\begin{array}{r}45 n \\
135.0\end{array}$ & So: & $=70^{A 2}$ & $\begin{array}{l}V E L \\
10.5\end{array}$ & & \\
\hline COMF : & cos & $\cos$ & $\operatorname{c5}_{25}^{2}$ & $c r y$ & $.0 \%$ & $\operatorname{crs}$ \\
\hline $\begin{array}{l}\text { MAY } \\
\text { MIPE } \\
\text { Fin? }\end{array}$ & ". & $\begin{array}{r}.01 \\
-07 \\
.01\end{array}$ & $\begin{array}{l}.92 \\
.75 \\
.10\end{array}$ & $\begin{array}{l}.04 \\
.10 \\
.05\end{array}$ & .13 & .00 \\
\hline $\begin{array}{l}\text { GFACT } \\
\text { FFACT }\end{array}$ & $\frac{2}{4} \cdot 7$ & $=.38$ & $\frac{2}{4} \cdot 52$ & $9 \cdot 12$ & $\frac{3}{3} \cdot 53$ & $\frac{1}{2} \cdot 9$ \\
\hline
\end{tabular}

IATA FOF: FILE: SINGL

RUH *

COMF : CFX CFY CYZ

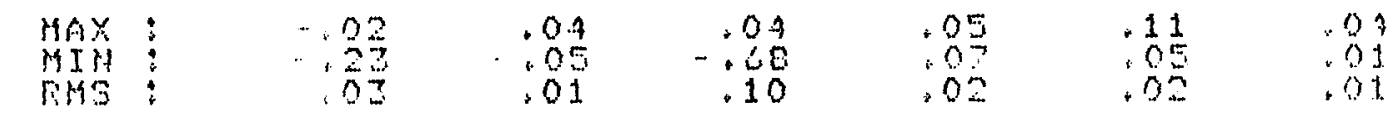

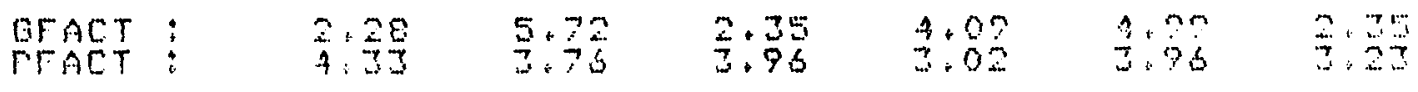




\begin{tabular}{|c|c|c|c|c|c|c|}
\hline Fun & $\begin{array}{r}\text { WINI } \\
180.0\end{array}$ & $\begin{array}{c}E L \\
30+0\end{array}$ & $\begin{array}{r}A Z \\
270.0\end{array}$ & $\begin{array}{l}\text { YEL } \\
10.5\end{array}$ & & \\
\hline $\begin{array}{l}\text { COHAF: } \\
\text { MEAN : }\end{array}$ & $\begin{array}{r}05 \\
-10\end{array}$ & $\begin{array}{l}\text { CFY } \\
.00\end{array}$ & $\frac{c^{2}}{2-3}$ & $\begin{array}{l}c 45 \\
.00\end{array}$ & $\begin{array}{l}\text { cisy } \\
02\end{array}$ & $\begin{array}{c}\cos \\
+04\end{array}$ \\
\hline $\begin{array}{l}\text { MAY } \\
\text { MIH } \\
\text { RHE }\end{array}$ & $\begin{array}{r}-02 \\
-\frac{2}{6} \\
-02\end{array}$ & $\begin{array}{r}.0 \hat{3} \\
.01 \\
.01\end{array}$ & $\begin{array}{l}.02 \\
.02 \\
107\end{array}$ & $\begin{array}{l}.05 \\
.06 \\
.02\end{array}$ & $\begin{array}{l}15 \\
05 \\
05\end{array}$ & .02 \\
\hline $\begin{array}{l}\text { QFACT } \\
\text { FACT }\end{array}$ & $\begin{array}{l}2 \\
5 \\
5\end{array}$ & $\frac{2}{3} \cdot 30$ & 3.02 & $\begin{array}{r}18.44 \\
2.55\end{array}$ & $\begin{array}{l}5.37 \\
5.39\end{array}$ & $\begin{array}{l}1.46 \\
3.0\end{array}$ \\
\hline IOTA F & DF: FILE : & SINGL & & & & \\
\hline "us? & $\begin{array}{r}4+4 \pi \\
180+6\end{array}$ & 0.5 & $270 . A^{A 2}$ & $\begin{array}{r}\text { YLL } \\
30.0\end{array}$ & & \\
\hline $\begin{array}{l}\text { COMF : } \\
\text { SIEAN : }\end{array}$ & $\begin{array}{r}c 5 x \\
-1.25\end{array}$ & $\begin{array}{l}\text { CFY } \\
.0 \%\end{array}$ & $\begin{array}{c}\operatorname{crz} \\
.09\end{array}$ & $\begin{array}{l}\text { crs } \\
.02\end{array}$ & $\begin{array}{l}\text { CHYY } \\
0 \%\end{array}$ & $.0 \%$ \\
\hline $\begin{array}{l}\text { MAX } \\
\text { MTH! } \\
\text { FMS }\end{array}$ & 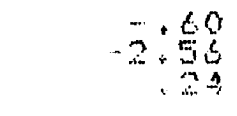 & $\begin{array}{l}15 \\
.01 \\
.02\end{array}$ & $\begin{aligned} &- 04 \\
& .10 \\
& .01\end{aligned}$ & $\begin{array}{l}06 \\
05 \\
0.5\end{array}$ & 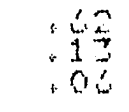 & .04 \\
\hline $\begin{array}{l}\text { OFACT } \\
\text { PFACT }\end{array}$ & $\frac{3}{5}: 0.5$ & 5.21 & $\frac{1}{5}+03$ & $\begin{array}{l}3.78 \\
3.49\end{array}$ & $\begin{array}{l}3.88 \\
8.98\end{array}$ & $\begin{array}{r}34 \cdot 91 \\
7.91\end{array}$ \\
\hline IATA F & OFi FILE : & SINGL & & & & \\
\hline Furt & $\begin{array}{r}4 I M ! M \\
157.5\end{array}$ & 0.0 & 270.0 & 70.1 & & \\
\hline $\begin{array}{l}\text { COHF } \\
\text { BEAT }\end{array}$ & $\begin{array}{r}\operatorname{cf} \\
-1,23\end{array}$ & $\begin{array}{l}\text { Cry } \\
\text { OOS }\end{array}$ & $\mathrm{CF}_{01}$ & $\begin{array}{l}\cos x \\
02\end{array}$ & $\begin{array}{l}\text { CHY } \\
\text { OSA }\end{array}$ & $\begin{array}{l}\cos 2 \\
.02\end{array}$ \\
\hline $\begin{array}{l}\text { MAX: } \\
\text { FIY } \\
\text { ris: }\end{array}$ & 2.93 & .12 & $\begin{array}{r}.02 \\
-.00 \\
.01\end{array}$ & $\begin{array}{r}.04 \\
-.05 \\
.01\end{array}$ & $\begin{aligned} .24 \\
: 05 \\
: 05\end{aligned}$ & $\begin{array}{l}.06 \\
.02 \\
.02\end{array}$ \\
\hline $\begin{array}{l}\text { GFACT: } \\
\text { PFACT }\end{array}$ & $\frac{1}{3} \cdot 5$ & $\frac{1}{3} \cdot 57$ & $\frac{6}{5}+\frac{17}{18}$ & $\frac{2}{2}+93$ & $\begin{array}{l}5.23 \\
4.00\end{array}$ & 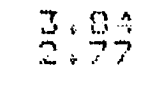 \\
\hline MATA F & OF: FILE: & SIHOL & & & & \\
\hline $\begin{array}{l}\text { run } \\
10 z\end{array}$ & $\begin{array}{r}W I Y I I \\
135+0\end{array}$ & EL & $270^{92}$ & $\begin{array}{l}\text { VEL } \\
0.2\end{array}$ & & \\
\hline $\begin{array}{c}\text { COSF } \\
\text { MEAS! }\end{array}$ & $\begin{array}{r}C F \% \\
-1.08\end{array}$ & CFY & $\begin{array}{c}\text { CFE } \\
.05\end{array}$ & $\begin{array}{l}\cos \alpha \\
.0 \%\end{array}$ & $\begin{array}{l}\text { Cry } \\
0 \%\end{array}$ & $\begin{array}{l}\operatorname{crg} z \\
.05\end{array}$ \\
\hline 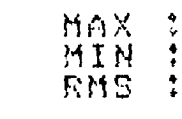 & -1.42 & $\begin{array}{r}.07 \\
.07 \\
.01\end{array}$ & $\begin{array}{l}.00 \\
.10 \\
.02\end{array}$ & .04 & $\begin{array}{l}.03 \\
.03 \\
.03 \\
.04\end{array}$ & $\begin{array}{l}.09 \\
.09 \\
.01\end{array}$ \\
\hline $\begin{array}{l}\text { OFACT } \\
\text { TFACT }\end{array}$ & $\frac{1}{4} \cdot \frac{92}{56}$ & $\begin{array}{l}3.64 \\
7 \cdot \frac{5}{5}\end{array}$ & $\begin{array}{l}2.11 \\
\approx \\
2\end{array}$ & $\frac{2}{3}: 90$ & $5.0 \%$ & $\frac{1}{3} \cdot 9 \cdot 9$ \\
\hline
\end{tabular}




\begin{tabular}{|c|c|c|c|c|c|c|}
\hline PUN & $112+5$ & 0.0 & $=70.0$ & $\begin{array}{r}\text { YCL. } \\
+0.1\end{array}$ & & \\
\hline $\begin{array}{l}\text { COMF : } \\
\text { MEAN : }\end{array}$ & CFy & $\begin{array}{l}\text { CFY } \\
.01\end{array}$ & $\operatorname{cr} \frac{2}{2}$ & $\begin{array}{l}\text { cry } \\
.02\end{array}$ & $\begin{array}{l}\text { cry } \\
.07\end{array}$ & $\begin{array}{l}\text { C.r.z } \\
.03\end{array}$ \\
\hline $\begin{array}{l}\text { MAE } \\
\text { HIN } \\
\text { RMS }\end{array}$ & $\begin{aligned}-1 & +17 \\
& +48 \\
& \cdot 20\end{aligned}$ & $\begin{array}{r}.04 \\
-.06 \\
.01\end{array}$ & $\begin{array}{r}.07 \\
-.07 \\
.01\end{array}$ & .07 &. $\begin{array}{l}10 \\
2 \\
: 03 \\
.03\end{array}$ &.$\frac{1}{0.03}$ \\
\hline $\begin{array}{l}\text { QFACT } \\
\text { FFACT }\end{array}$ & $\frac{1}{2} \cdot 96$ & $\begin{array}{l}4.44 \\
3.64\end{array}$ & $3+74$ & $\frac{2}{3} \cdot 73$ & $3 \cdot \frac{1}{3} \cdot 5$ & 1. \\
\hline IIATA F & OF: FILE: & SINGL & & & & \\
\hline${ }_{107}$ & $\begin{array}{l}\text { WIItIn } \\
90.0\end{array}$ & EL & $\because 0.0$ & צ. & & \\
\hline $\begin{array}{l}\text { CQNFF: } \\
\text { BEAN }\end{array}$ & .05 & $\begin{array}{l}\text { CFY } \\
: 05\end{array}$ & $\begin{array}{c}C 52 \\
.01\end{array}$ & chos & $\begin{array}{l}\text { CHy } \\
.02\end{array}$ & $\begin{array}{c}\mathrm{CH} \\
.0 \mathrm{O}\end{array}$ \\
\hline $\begin{array}{l}\text { MAX } \\
\text { HIN } \\
\text { FMS }\end{array}$ & . $\begin{array}{r}51 \\
: 38 \\
\div 12\end{array}$ & 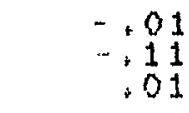 & $\begin{array}{l}.02 \\
.08 \\
.01\end{array}$ & $\begin{array}{l}0.4 \\
: 07 \\
.01\end{array}$ & $\begin{array}{l}11 \\
: 18 \\
.0 \%\end{array}$ & .06 \\
\hline $\begin{array}{l}\text { GFACT } \\
\text { PFACT }\end{array}$ & $5: 33$ & $\frac{2}{4} \cdot \frac{1}{5}$ & $\begin{array}{l}4.7 \% \\
3.89\end{array}$ & $3 \cdot 9 \frac{1}{3}$ & $\begin{array}{l}8.12 \\
4.13\end{array}$ & $15 \cdot 4 \hat{3}$ \\
\hline
\end{tabular}

\section{IIATA FOR FILE : SIMGL}

\begin{tabular}{|c|c|c|c|c|c|c|}
\hline FUN & WIMI & $\begin{array}{l}E L \\
0.0\end{array}$ & $\begin{array}{r}A Z \\
270^{A} \cdot 0\end{array}$ & $\begin{array}{r}\text { YEL } \\
33 \div 9\end{array}$ & & \\
\hline $\begin{array}{l}\text { COHF } \\
\text { MEAH! }\end{array}$ & CFY & $\begin{array}{r}C F Y \\
-112\end{array}$ & $\begin{array}{l}\text { CF } \\
+10\end{array}$ & $\begin{array}{r}\text { CMY } \\
-01\end{array}$ & $\begin{array}{l}\text { CMY } \\
0 \%\end{array}$ & cha \\
\hline $\begin{array}{l}\text { MAX } \\
\text { MIN } \\
\text { Fins: }\end{array}$ & 1. 59 & $\begin{array}{r}-.05 \\
-.25 \\
.03\end{array}$ & $\begin{array}{r}.04 \\
-.18 \\
.02\end{array}$ & $\begin{array}{r}.03 \\
.02 \\
.01\end{array}$ & $\begin{array}{l}.25 \\
.195 \\
.05\end{array}$ & .01 \\
\hline GFACT & $\frac{1}{3}: 18$ & $\begin{array}{l}1.98 \\
3 \cdot 46\end{array}$ & $\frac{1}{3} \cdot 31$ & 5.79 & $\begin{array}{l}7.49 \\
\ddot{3} \cdot 48\end{array}$ & $\frac{1}{2}: 90$ \\
\hline
\end{tabular}

IIATA FOF FTLE : SIHGL

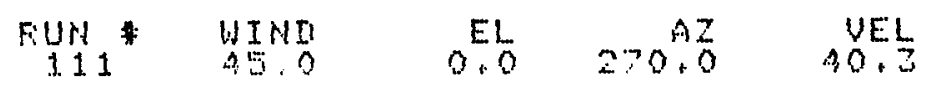

\begin{tabular}{|c|c|c|c|c|c|c|}
\hline $\begin{array}{l}\text { COMF } \\
\text { HEAH }\end{array}$ & $\begin{array}{c}c 5 x \\
1.05\end{array}$ & $\begin{array}{l}\text { CFY } \\
\cdots .14\end{array}$ & $\begin{array}{l}C F Z \\
.03\end{array}$ & .02 & $\begin{array}{l}\text { CMY } \\
.06\end{array}$ & $\begin{array}{r}0.19 z \\
.00\end{array}$ \\
\hline $\begin{array}{l}\text { MAX } \\
\text { MIUI } \\
\text { FMS }\end{array}$ & $\begin{array}{r}1.92 \\
.92 \\
122\end{array}$ & -.03 & $\begin{array}{r}.02 \\
.12 \\
.02\end{array}$ & $\begin{array}{l}07 \\
.97 \\
01 \\
01\end{array}$ & $\begin{array}{l}+27 \\
+12 \\
.05\end{array}$ & $\begin{array}{r}.94 \\
.01 \\
.01\end{array}$ \\
\hline $\begin{array}{l}\text { QFACT } \\
\text { FFACT }\end{array}$ & $\begin{array}{l}1.90 \\
4.21\end{array}$ & $\frac{1}{4} \cdot 26$ & 3.82 & $3 \cdot 9$ & $\begin{array}{l}3.35 \\
4+24\end{array}$ & $\begin{array}{l}42 \\
4: 5\end{array}$ \\
\hline
\end{tabular}




\begin{tabular}{|c|c|c|c|c|c|c|}
\hline $\operatorname{lils}_{113}$ & WIMI & 0.5 & $270.0^{A Z}$ & $\begin{array}{l}\text { UᄃL } \\
30.0\end{array}$ & & \\
\hline $\begin{array}{l}\text { CONF } \\
\text { BEAN }\end{array}$ & $1.1 \%$ & $\begin{array}{r}C F Y \\
-.1 Z\end{array}$ & $\begin{array}{r}652 \\
.08\end{array}$ & $\begin{array}{r}\text { crs } \\
-01\end{array}$ & .05 & $\begin{array}{l}\text { Crsz } \\
.01\end{array}$ \\
\hline $\begin{array}{l}\text { MAX } \\
\text { MIY } \\
\text { FHS: }\end{array}$ & $\begin{array}{r}2.24 \\
.20 \\
.24\end{array}$ & $\begin{array}{r}+03 \\
+.25 \\
+05\end{array}$ & $\begin{array}{r}.00 \\
.18 \\
.03\end{array}$ & $\begin{array}{l}.94 \\
.02 \\
.02\end{array}$ & $\begin{array}{l}32 \\
.12 \\
.06\end{array}$ & $\begin{array}{l}.09 \\
.04 \\
0.02\end{array}$ \\
\hline $\begin{array}{l}\text { GFACT } \\
\text { FACT }\end{array}$ & $\frac{1}{4} \cdot 3 \frac{6}{2}$ & $\frac{1}{3}: 94$ & $\frac{2}{3}+85$ & 4.09 & $\frac{1}{3}+93$ & $\begin{array}{l}7.74 \\
4: 4.4\end{array}$ \\
\hline
\end{tabular}

IIATA FOK FILE: SINGL

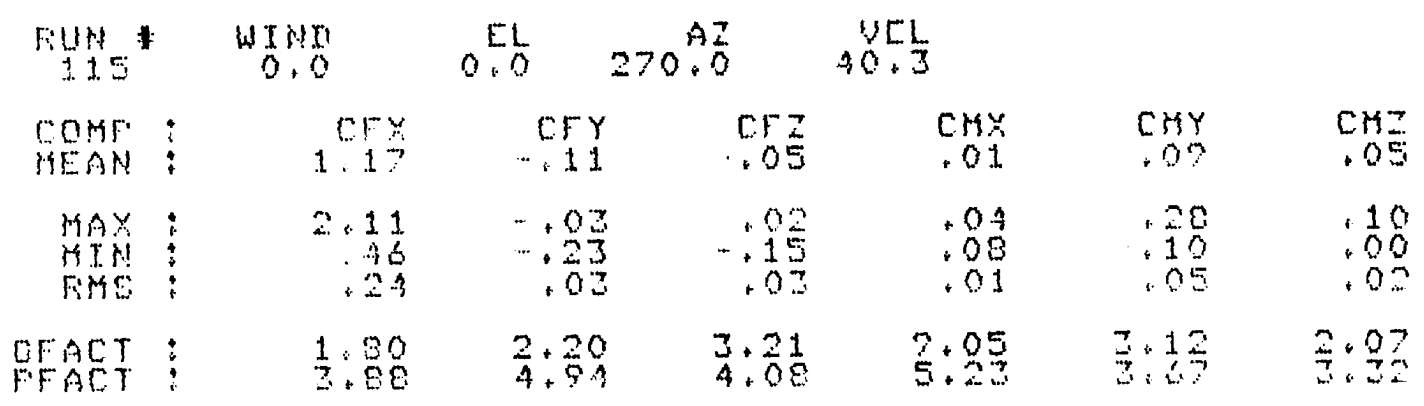

IATA FOF FILE: SINGL

\begin{tabular}{|c|c|c|c|c|c|c|}
\hline ruy & $\begin{array}{l}4 \pi \mathrm{I} \\
0.0\end{array}$ & 45.0 & & YEL & & \\
\hline CQMF : & $C F$ & $\operatorname{cFy}_{.03}$ & $\begin{array}{c}0.7 \\
.58\end{array}$ & Crs & $\begin{array}{l}C H Y \\
113\end{array}$ & ${ }_{0 \rightarrow}$ \\
\hline 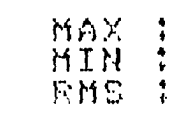 & $\begin{array}{r}1+35 \\
436 \\
15\end{array}$ & $\begin{array}{r}.00 \\
+.08 \\
+01\end{array}$ & $\begin{array}{l}.99 \\
.11 \\
.10\end{array}$ & $\begin{array}{l}.06 \\
.05 \\
.01\end{array}$ & .27 & $\begin{array}{r}.0 \% \\
.00 \\
.01\end{array}$ \\
\hline $\begin{array}{l}\text { OFACT } \\
\text { FFACT }\end{array}$ & $\frac{1}{4}: \frac{7}{2}$ & $\frac{2}{4}: 52$ & $\frac{1}{3} \cdot 0.6$ & 32.67 & $\frac{1}{5}: 99$ & $\begin{array}{l}2.20 \\
\therefore .11\end{array}$ \\
\hline
\end{tabular}

MATA FOF FILE : SINGL

\begin{tabular}{|c|c|c|c|c|c|c|}
\hline Fum & $\begin{array}{l}W \\
2\end{array}$ & $45+\frac{1}{0}$ & & & & \\
\hline $\begin{array}{l}\text { COMF : } \\
\text { MEAN : }\end{array}$ & 65 & $\begin{array}{r}\operatorname{CFY} \\
-04\end{array}$ & 85 & $\begin{array}{l}c r y \\
0.5\end{array}$ & $\begin{array}{l}C M Y \\
.11\end{array}$ & $\begin{array}{l}C M z \\
.00\end{array}$ \\
\hline $\begin{array}{l}\text { MAX } \\
\text { MINA } \\
\text { FMS }\end{array}$ & $\begin{array}{r}1+23 \\
\cdot 37 \\
12\end{array}$ & $\begin{array}{r}-.00 \\
. .09 \\
.01\end{array}$ & $\begin{array}{r}1.87 \\
+45 \\
.11\end{array}$ & $\begin{array}{l}12 \\
.00 \\
02\end{array}$ & .25 & .09 \\
\hline $\begin{array}{l}\text { ACT } \\
\text { ACT }\end{array}$ & $\frac{1}{3} \cdot \frac{6}{9}$ & $\frac{3}{3} \cdot 11$ & $\frac{1}{3} \cdot 5 \frac{5}{51}$ & $2 \cdot 35$ & $\frac{2}{4}+\frac{1}{4}$ & $21 \cdot 57$ \\
\hline
\end{tabular}


IMTA FOF: FILE: SINGL

\begin{tabular}{|c|c|c|c|c|c|c|}
\hline $\sin _{140}$ & $\begin{array}{l}41 \times n \\
45.0\end{array}$ & $45.0 \frac{1}{0}$ & & $\begin{array}{l}\text { VEL.. } \\
40.4\end{array}$ & & \\
\hline CQMF: & $C F x$ & $\begin{array}{r}\text { CFy } \\
-.06\end{array}$ & $\begin{array}{c}c .52 \\
.38\end{array}$ & $0.0 \%$ & $\begin{array}{c}\text { Cry } \\
.10\end{array}$ & $\begin{array}{c}0.07 \\
.00\end{array}$ \\
\hline $\begin{array}{l}\text { MAX } \\
\text { MIN } \\
\text { FMS }\end{array}$ & $\begin{array}{r}1.28 \\
: 13 \\
: 14\end{array}$ & $\begin{array}{r}-.01 \\
-.12 \\
.02\end{array}$ & $\begin{array}{r}1 . \frac{1}{35} \\
: 12\end{array}$ & $: \frac{1}{0}: \frac{m}{1}$ & $: \begin{array}{l}25 \\
: 02 \\
02\end{array}$ & $.0 \%$ \\
\hline $\begin{array}{l}\text { QFACT } \\
\text { FFACT }\end{array}$ & $\frac{1}{4} \cdot 3 \frac{1}{2}$ & $\frac{2}{3}: \frac{10}{7}$ & $\frac{1}{3}: 81$ & $\frac{1}{2} \cdot 83$ & $\frac{5}{4} \cdot 5 ?$ & 7.09 \\
\hline
\end{tabular}

IATA FOF FILE : SINGL

\begin{tabular}{|c|c|c|c|c|c|c|}
\hline Fus & $47 x \frac{\pi}{5}$ & 45.0 & & $\begin{array}{l}V E \frac{1}{5} \\
40.5\end{array}$ & & \\
\hline $\begin{array}{l}\text { COMF : } \\
\text { MEATH : }\end{array}$ & $C F$ & $\begin{array}{r}\text { CFY } \\
-: 06\end{array}$ & $C F Z$ & $\begin{array}{l}c 45 \\
.07\end{array}$ & $\begin{array}{l}\text { CMY } \\
.05\end{array}$ & $\begin{array}{l}\text { CHA } \\
.01\end{array}$ \\
\hline $\begin{array}{l}\text { MAYX } \\
\text { FISH } \\
\text { FHS }\end{array}$ & $.2 \frac{1}{1}$ & $\begin{array}{r}-.00 \\
-.13 \\
.02\end{array}$ & $: 7 \frac{7}{10}$ & $\begin{array}{r}15 \\
.03 \\
.02\end{array}$ & .13 & .02 \\
\hline $\begin{array}{l}\text { GFACT } \\
\text { FACT }\end{array}$ & $2 \cdot 20$ & $\frac{2}{4} \cdot 34$ & $\frac{1}{3} \cdot 85$ & $\frac{1}{2} \cdot \frac{6}{5}$ & 3.47 & $\begin{array}{l}92 \\
3.1\end{array}$ \\
\hline
\end{tabular}

IATA FOR FILE : SINGL

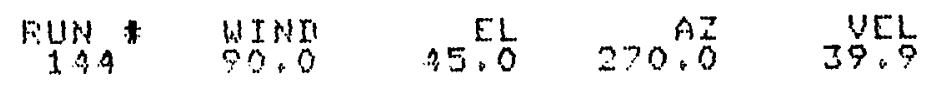

$\begin{array}{lllllll}\text { COMF: CFY CFY } & \text { CFE } & \text { CHE } & \text { CHY } & \text { CHI } \\ \text { HEAN : } & .03 & -.04 & -.01 & .0 \% & .02 & .03\end{array}$

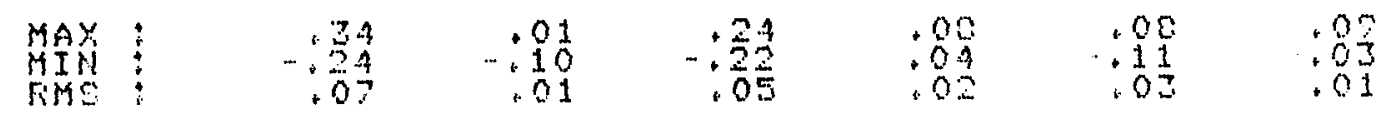

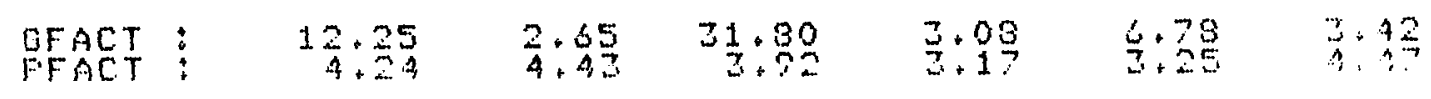

IIATA FOR FILE: SINGL

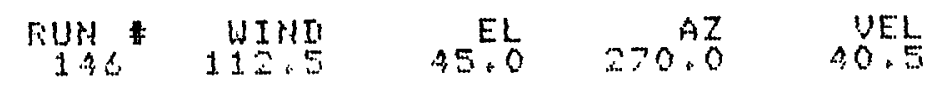

\begin{tabular}{|c|c|c|c|c|c|c|}
\hline $\begin{array}{l}\text { COMF } \\
\text { MEASt }\end{array}$ & $\begin{array}{c}C F X \\
+3 Z\end{array}$ & $\begin{array}{r}\text { CFY } \\
-.0 Z\end{array}$ & $\begin{array}{c}C F Z \\
-\quad Z 1\end{array}$ & crys & $\begin{array}{l}\text { CHY } \\
.00\end{array}$ & $c r a$ \\
\hline $\begin{array}{l}\text { MAX } \\
\text { MISS } \\
\text { RMS }\end{array}$ & $\begin{array}{r}-.02 \\
-75 \\
: 11\end{array}$ & $\begin{array}{r}.02 \\
.00 \\
.01\end{array}$ & $\begin{array}{r}-11 \\
-\quad 70 \\
.10\end{array}$ & $\begin{array}{r}.01 \\
.15 \\
.05\end{array}$ & $\begin{array}{l}15 \\
.13 \\
.03\end{array}$ &.$\frac{11}{0}$ \\
\hline FACT & $\frac{2}{3}: 28$ & $\frac{2}{3} \cdot \frac{82}{3}$ & $\frac{2}{3} .8 \hat{9}$ & $\frac{2}{5}: 38$ & 30.19 & $1: 5$ \\
\hline
\end{tabular}




\begin{tabular}{|c|c|c|c|c|c|c|}
\hline $\operatorname{lins}_{130}$ & $\begin{array}{r}\text { WINI } \\
135.0\end{array}$ & 45.0 & 270.0 & $\begin{array}{l}\text { VEL } \\
40.2\end{array}$ & & \\
\hline COMF : & $\begin{array}{l}\operatorname{cF} \\
\cdots\end{array}$ & $\begin{array}{l}\text { CFY } \\
.01\end{array}$ & $\begin{array}{l}C F Z \\
\cdots .74\end{array}$ & $\begin{array}{l}\mathrm{CH} \\
.06\end{array}$ & $\begin{array}{l}\text { CMY } \\
.05\end{array}$ & $\begin{array}{l}C H z \\
.04\end{array}$ \\
\hline $\begin{array}{l}\text { MAX } \\
\text { MITE } \\
\text { FMS }\end{array}$ & $\begin{aligned}-1 & : \frac{2}{1} \\
& : 16\end{aligned}$ & $\begin{array}{r}.08 \\
-.05 \\
.02\end{array}$ & $\begin{array}{r}+40 \\
-1.39 \\
.15\end{array}$ & $\begin{array}{l}+01 \\
+1.4 \\
.02\end{array}$ & $\begin{array}{r}+2.2 \\
+02 \\
.04\end{array}$ & $\begin{array}{r}.00 \\
.01 \\
.01\end{array}$ \\
\hline $\begin{array}{l}\text { GFACT } \\
\text { FACT }\end{array}$ & $\frac{2.05}{4.58}$ & $\begin{array}{l}5.74 \\
3.88\end{array}$ & 1.87 & $\frac{2}{3} \cdot \frac{26}{65}$ & $\begin{array}{l}4.39 \\
3.55\end{array}$ & 2.45 \\
\hline
\end{tabular}

IATA FOF FILE : SINGL

\begin{tabular}{|c|c|c|c|c|c|c|}
\hline Fun & 157.5 & ${ }_{45.0}^{E L}$ & $\begin{array}{l}A Z \\
0.0\end{array}$ & $\begin{array}{r}\text { VCL } \\
39.9\end{array}$ & & \\
\hline COMF & $\begin{array}{c}0 y \\
79\end{array}$ & $\begin{array}{l}\text { CFY } \\
.04\end{array}$ & $\begin{array}{r}\operatorname{cr} \frac{7}{2} \\
-+8 \frac{1}{2}\end{array}$ & $\begin{array}{r}c m y \\
-.03\end{array}$ & $\begin{array}{l}\text { CMY } \\
+O 4\end{array}$ & $\begin{array}{cc}C M z \\
.04\end{array}$ \\
\hline $\begin{array}{l}\text { MAX } \\
\text { FIA } \\
\text { FMS }\end{array}$ & $\begin{array}{r}-2: 29 \\
-1: 34 \\
: 12\end{array}$ & $\begin{array}{r}.02 \\
.02 \\
.01\end{array}$ & $\begin{array}{r}-12 \\
-1.36 \\
.14\end{array}$ & $\begin{array}{r}.05 \\
-11 \\
.02\end{array}$ & $\begin{array}{l}-92 \\
.15 \\
+04\end{array}$ & $\begin{array}{l}.90 \\
.01 \\
.02\end{array}$ \\
\hline GFACT & $\frac{1}{3}: 7$ & $\frac{2}{3} .07$ & $\frac{1.66}{3}$ & $\begin{array}{l}3.65 \\
3.80\end{array}$ & $5 \cdot 26$ & 2.08 \\
\hline
\end{tabular}

IMTA FOF FILE : SIHGL

\begin{tabular}{|c|c|c|c|c|c|c|}
\hline $\operatorname{Fum}_{102}$ & $\begin{array}{l}\text { WINII } \\
130.0\end{array}$ & 45.0 & $A Z$ & $\begin{array}{l}\text { VEL } \\
39.9\end{array}$ & & \\
\hline COMF: & 65 & $\begin{array}{l}\text { C.FY } \\
.05\end{array}$ & $\begin{array}{r}0.5 \\
-.82\end{array}$ & $\begin{array}{l}\text { Crys } \\
.04\end{array}$ & $\begin{array}{l}\text { Crsy } \\
.03\end{array}$ & $\begin{array}{l}\text { chy } \\
.00\end{array}$ \\
\hline $\begin{array}{l}\text { MAY } \\
\text { MIIS } \\
\text { FMS }\end{array}$ & $\begin{array}{r}\cdots+35 \\
-1.49 \\
+16\end{array}$ & $: \begin{array}{l}11 \\
0 \\
0 \\
01\end{array}$ & $\begin{array}{r}-43 \\
-1: 47 \\
+15\end{array}$ & $\begin{array}{l}10 \\
.03 \\
02\end{array}$ & $\begin{array}{l}.25 \\
.05 \\
.04\end{array}$ & $\begin{array}{l}.05 \\
.05 \\
.01\end{array}$ \\
\hline $\begin{array}{l}\text { QFACT } \\
\text { FFACT }\end{array}$ & $\frac{1}{4}+7 \frac{1}{6}$ & $\begin{array}{l}2.17 \\
4.33\end{array}$ & $\frac{1}{4.78}$ & $\frac{2}{3}+50$ & 3.17 & $1 \frac{1}{3} \cdot 15$ \\
\hline
\end{tabular}

INTA FOF FILE: SIMGL

\begin{tabular}{|c|c|c|c|c|c|c|}
\hline Pus & $\begin{array}{r}\text { WI } \\
180.0\end{array}$ & 04.6 & & $\begin{array}{r}\text { VEL } \\
40.5\end{array}$ & & \\
\hline $\begin{array}{l}\text { Cons } \\
\text { MEAH }\end{array}$ & $\begin{array}{l}C F \% \\
\cdots .08\end{array}$ & $\begin{array}{l}\text { CFY } \\
.00\end{array}$ & $\begin{array}{cr}C F \\
: 17\end{array}$ & $\begin{array}{l}\text { cry } \\
.01\end{array}$ & $\begin{array}{l}C M Y \\
.01\end{array}$ & $\begin{array}{l}\text { C.M. } \\
.01\end{array}$ \\
\hline $\begin{array}{l}\text { max } \\
\text { sisus } \\
\text { rist }\end{array}$ & $\begin{array}{r}-.02 \\
.102 \\
.02\end{array}$ & $\begin{array}{r}.04 \\
-.07 \\
.01\end{array}$ & $\begin{array}{r}.02 \\
-.55 \\
.05\end{array}$ & $\begin{array}{l}.03 \\
.05 \\
.01\end{array}$ & $\begin{array}{r}.11 \\
-.62 \\
.02\end{array}$ & $\begin{array}{r}.05 \\
.03 \\
.01\end{array}$ \\
\hline $\begin{array}{l}\text { BFACT } \\
\text { SFACT }\end{array}$ & $\frac{2}{3}+35$ & $17: 99$ & $5: 1 \%$ & 5.91 & $10 \cdot \frac{65}{5}$ & 5.79 \\
\hline
\end{tabular}


IATA FOF: FILE: SINGL

\begin{tabular}{|c|c|c|c|c|c|c|}
\hline $\ln _{156}$ & 157.5 & Q4. & & VEL & & \\
\hline COMF & $\begin{array}{r}c .5 x \\
-.09\end{array}$ & $\begin{array}{l}C F Y \\
.01\end{array}$ & $\begin{array}{c}C F \\
-.23\end{array}$ & chy & $\begin{array}{l}\text { CMY } \\
.03\end{array}$ & $\begin{array}{l}.0 \% \\
.00\end{array}$ \\
\hline $\begin{array}{l}\text { MAX } \\
\text { MIYS } \\
\text { FMS }\end{array}$ & $\begin{array}{r}\cdots \\
\cdots \\
\because \\
.02\end{array}$ & $\begin{array}{r}.05 \\
-.05 \\
.01\end{array}$ & $\begin{array}{r}\cdots \\
-.56 \\
.09\end{array}$ & $\begin{array}{r}+92 \\
+.08 \\
+02\end{array}$ & $\begin{array}{r}.11 \\
.02 \\
.02\end{array}$ & $\begin{array}{l}05 \\
.04 \\
.04\end{array}$ \\
\hline $\begin{array}{l}\text { QFACT } \\
\text { FFACY }\end{array}$ & $\frac{2}{4.08}$ & $\begin{array}{l}4.85 \\
3.58\end{array}$ & $\begin{array}{l}2.49 \\
3.56\end{array}$ & $\begin{array}{l}4.48 \\
3.46\end{array}$ & $\frac{2}{7}+37$ & 15.97 \\
\hline
\end{tabular}

IIATA FOF FILE: SINGL

\begin{tabular}{|c|c|c|c|c|c|c|}
\hline Fun & $\begin{array}{l}\text { WIMI } \\
135.0\end{array}$ & $84: 0$ & & $\begin{array}{r}\text { VEL } \\
40.0\end{array}$ & & \\
\hline $\begin{array}{l}\text { CQMF: } \\
\text { SEEAN }\end{array}$ & $\begin{array}{l}\mathrm{CF} \\
-.07\end{array}$ & $\begin{array}{r}\text { CFY } \\
-0.03\end{array}$ & $\begin{array}{l}\operatorname{cFz} \\
.21\end{array}$ & $\begin{array}{c}\operatorname{crs} \\
.01\end{array}$ & $\begin{array}{l}\text { CMY } \\
.01\end{array}$ & $\begin{array}{c}C H \\
.03\end{array}$ \\
\hline $\begin{array}{l}\text { MAX } \\
\text { MIMU } \\
\text { FMS }\end{array}$ & $\begin{array}{r}\because .00 \\
-18 \\
.02\end{array}$ & $\begin{array}{r}.02 \\
-.08 \\
.01\end{array}$ & $\begin{array}{r}.02 \\
-.58 \\
.00\end{array}$ & $\begin{array}{r}.06 \\
.09 \\
.02\end{array}$ & $\begin{array}{r}.08 \\
-.06 \\
.02\end{array}$ & .81 \\
\hline $\begin{array}{l}\text { GFACT } \\
\text { FFACT }\end{array}$ & $\begin{array}{l}2.45 \\
4.40\end{array}$ & $\frac{2}{3} .96$ & $\frac{2.71}{4.37}$ & $\begin{array}{l}8 \cdot 25 \\
.15\end{array}$ & $\begin{array}{l}3 \cdot 23 \\
\Xi\end{array}$ & $\begin{array}{l}2 \\
3.25\end{array}$ \\
\hline
\end{tabular}

IATA FOR FILE: SINGL

\begin{tabular}{|c|c|c|c|c|c|c|}
\hline Fun & 112.5 & 34.0 & & YEL & & \\
\hline COMF & CF & $\begin{array}{r}C F Y \\
-.04\end{array}$ & $\begin{array}{r}\text { CFz } \\
-.09\end{array}$ & $\begin{array}{r}\text { CHy } \\
-01\end{array}$ & $\begin{array}{l}\text { CMY } \\
.02\end{array}$ & $\begin{array}{c}\mathrm{CH} Z \\
.0 ?\end{array}$ \\
\hline $\begin{array}{l}\text { MAY } \\
\text { MIYL } \\
\text { FHS }\end{array}$ & $\begin{array}{r}.04 \\
.09 \\
+02\end{array}$ & $\begin{array}{r}.01 \\
.08 \\
.01\end{array}$ & $\begin{array}{r}.12 \\
-.34 \\
.07\end{array}$ & $\begin{array}{r}.05 \\
.05 \\
.05\end{array}$ & $\begin{array}{r}.02 \\
.09 \\
.02\end{array}$ & $\begin{array}{r}11 \\
.03 \\
.01\end{array}$ \\
\hline $\begin{array}{l}\text { GFACT } \\
\text { FFACT }\end{array}$ & $\begin{array}{l}4.33 \\
4.16\end{array}$ & $\frac{2}{3} \cdot \frac{1}{3} 5$ & $\begin{array}{l}3.66 \\
3.61\end{array}$ & $\begin{array}{l}5 \cdot 59 \\
3.12\end{array}$ & $\begin{array}{l}3.30 \\
4.18\end{array}$ & 1.73 \\
\hline
\end{tabular}

IIATA FOF: FILE: SINGL

$\begin{array}{lllll}\text { FUN } & \text { WIHI } & \text { EL } & \text { AT } & \text { UEL } \\ 132 & 90.0 & 34.0 & 270.0 & A 0.5\end{array}$

\begin{tabular}{|c|c|c|c|c|c|c|}
\hline COMF & $\begin{array}{r}\operatorname{CF} \\
-.00\end{array}$ & $\begin{array}{l}\text { CFY } \\
\cdots .04\end{array}$ & $\begin{array}{c}\text { CF } \\
.04\end{array}$ & $\begin{array}{l}C H \% \\
.01\end{array}$ & $\begin{array}{l}\text { CHY } \\
.01\end{array}$ & $C \stackrel{C}{0}$ \\
\hline $\begin{array}{l}\text { MAX } \\
\text { MINS } \\
\text { SMS }\end{array}$ & $\begin{array}{r}+05 \\
+03 \\
.01\end{array}$ & $\begin{array}{r}.00 \\
-.10 \\
.01\end{array}$ & $\begin{array}{r}20 \\
.24 \\
.05\end{array}$ & $\begin{array}{r}.00 \\
-.07 \\
.02\end{array}$ & $\begin{array}{r}.05 \\
.03 \\
.01\end{array}$ & .07 \\
\hline $\begin{array}{l}\text { GFACT } \\
\text { FFACT }\end{array}$ & $41 \cdot 56$ & $\frac{2}{4} \cdot \frac{42}{5}$ & 4.81 & 5.83 & 5.82 & 3.39 \\
\hline
\end{tabular}


IIATA FOF FILE : SINGL

\begin{tabular}{|c|c|c|c|c|c|c|}
\hline Fust & $\begin{array}{l}W I+15 \\
87.5\end{array}$ & 84.0 & & $\begin{array}{r}\text { VEL } \\
+0.2\end{array}$ & & \\
\hline $\begin{array}{l}\text { COMF" } \\
\text { MEAN : }\end{array}$ & $\begin{array}{l}C 5 x \\
.04\end{array}$ & $\begin{array}{r}\text { CFY } \\
. .04\end{array}$ & $\begin{array}{c}C F \\
.11\end{array}$ & $\begin{array}{l}\text { cho } \\
.02\end{array}$ & $\begin{array}{l}\text { CHY } \\
.00\end{array}$ & $\begin{array}{l}c r 3 \\
03\end{array}$ \\
\hline $\begin{array}{l}\text { MAX } \\
\text { MIE } \\
\text { FMS }\end{array}$ & $\begin{array}{r}.12 \\
-03 \\
.02\end{array}$ & $\begin{array}{r}.00 \\
.09 \\
.01\end{array}$ & $\begin{array}{r}.34 \\
-.15 \\
.05\end{array}$ & $\begin{array}{l}.02 \\
.08 \\
.02\end{array}$ & $\begin{array}{l}.05 \\
.05 \\
.01\end{array}$ & $\begin{array}{l}.00 \\
.00 \\
.01\end{array}$ \\
\hline $\begin{array}{l}\text { QFACT } \\
\text { FFACT }\end{array}$ & $\frac{2}{4.78}$ & $\frac{2}{3} \cdot \frac{24}{52}$ & $\begin{array}{l}3.19 \\
4.04\end{array}$ & $\begin{array}{l}9.98 \\
4.05\end{array}$ & $19 \cdot 56$ & $\begin{array}{l}2.81 \\
4+13\end{array}$ \\
\hline
\end{tabular}

WATA FOF FILE : SINOL

\begin{tabular}{|c|c|c|c|c|c|c|}
\hline Rust & $\begin{array}{l}\text { WIHI } \\
35: 0\end{array}$ & $B A \div 0$ & & 40.0 & & \\
\hline COMF & $.0 \%$ & $\begin{array}{r}\text { CFY } \\
-: 04\end{array}$ & $\begin{array}{l}\text { CF } \\
.22\end{array}$ & $\begin{array}{r}\text { EHX } \\
+01\end{array}$ & $\begin{array}{l}\text { CMY } \\
.0 Z\end{array}$ & $\begin{array}{l}\text { CHI } \\
.02\end{array}$ \\
\hline $\begin{array}{l}\text { MAX } \\
\text { WHA } \\
\text { WMS }\end{array}$ & .17 & $\begin{array}{r}.00 \\
.09 \\
.01\end{array}$ & $\begin{array}{r}+22 \\
-.04 \\
+06\end{array}$ & $\begin{array}{l}.02 \\
.02 \\
.02\end{array}$ & .00 & $\begin{array}{l}.09 \\
.01 \\
.01\end{array}$ \\
\hline $\begin{array}{l}\text { QFACT } \\
\text { FFACT }\end{array}$ & $\frac{2.09}{4.09}$ & $\frac{2}{4}+\frac{1}{25}$ & $\frac{1}{3} \cdot 30$ & $\begin{array}{l}5.80 \\
2.64\end{array}$ & 2.75 & $\frac{2}{3} \cdot 58$ \\
\hline
\end{tabular}

INATA FOF FILE: SINGL

\begin{tabular}{|c|c|c|c|c|c|c|}
\hline $\begin{array}{c}\text { Fum } \\
168\end{array}$ & $\begin{array}{l}\text { WI } \\
22.5\end{array}$ & $84: 0$ & & $\begin{array}{r}\text { VEL } \\
40.2\end{array}$ & & \\
\hline $\begin{array}{l}\text { COMF } \\
\text { MEAN }\end{array}$ & Cro & $\begin{array}{l}\text { CFY } \\
\cdots .00\end{array}$ & $\begin{array}{l}\text { C. } \\
.25\end{array}$ & $\begin{array}{l}\text { CHE } \\
.02\end{array}$ & $\begin{array}{l}\text { C.MY } \\
.05\end{array}$ & .01 \\
\hline $\begin{array}{l}\text { MAY } \\
\text { KIM } \\
\text { FMS }\end{array}$ & .21 & $\begin{array}{r}+92 \\
.04 \\
.01\end{array}$ & $\begin{array}{r}+95 \\
.04 \\
.05\end{array}$ & $\begin{array}{r}.05 \\
. .02 \\
.01\end{array}$ & .12 & .85 \\
\hline $\begin{array}{l}\text { OFACT } \\
\text { FFACT }\end{array}$ & $\begin{array}{l}1.35 \\
4.34\end{array}$ & 12.75 & $\begin{array}{l}1.80 \\
3.70\end{array}$ & $\frac{2.81}{3}+15$ & 2.27 & 5.29 \\
\hline
\end{tabular}

IATA FOF FILE: SINGL

\begin{tabular}{|c|c|c|c|c|c|c|}
\hline Eut? & WI & $84.0 L$ & & & & \\
\hline $\begin{array}{l}\text { COMF: } \\
\text { MEAN }\end{array}$ & $\begin{array}{l}\text { CFX } \\
\times 10\end{array}$ & $\begin{array}{l}\text { CFY } \\
\cdots+01\end{array}$ & $\begin{array}{l}C F z \\
21\end{array}$ & $\begin{array}{l}\text { CMY } \\
.01\end{array}$ & $\begin{array}{l}\text { CHY } \\
.04\end{array}$ & $\begin{array}{l}\cos \\
.00\end{array}$ \\
\hline $\begin{array}{l}\text { MAX } \\
\text { MAHS } \\
\text { FMS }\end{array}$ & $\begin{array}{l}15 \\
.04 \\
.02\end{array}$ & $\begin{array}{r}+02 \\
\therefore 03 \\
.01\end{array}$ & $\begin{array}{l}.41 \\
.03 \\
.05\end{array}$ & .04 & $\begin{array}{r}11 \\
.01 \\
.02\end{array}$ & $\begin{array}{l}.02 \\
.0 .4 \\
.01\end{array}$ \\
\hline QFACT: & $\begin{array}{l}1.37 \\
3.17\end{array}$ & $\begin{array}{c}4.39 \\
3.68\end{array}$ & $\begin{array}{l}1.91 \\
9.02\end{array}$ & 3.60 & $\frac{2}{4}+5$ & $\begin{array}{l}7.87 \\
4.09\end{array}$ \\
\hline
\end{tabular}


IATA FOF: FILE : SINGL

\begin{tabular}{|c|c|c|c|c|c|c|}
\hline $\operatorname{RuM}_{172}$ & UINII & 87.5 & & $\begin{array}{l}\text { VEL } \\
29.9\end{array}$ & & \\
\hline $\begin{array}{l}\text { COMF: } \\
\text { MEAN }\end{array}$ & $\begin{array}{l}C F Y \\
.08\end{array}$ & $\begin{array}{l}\text { CFY } \\
.02\end{array}$ & $\begin{array}{l}\operatorname{cFz} \\
.06\end{array}$ & $\begin{array}{l}c 4 x \\
.05\end{array}$ & $\begin{array}{l}\text { cur } \\
.00\end{array}$ & $\begin{array}{l}\mathrm{CH} \\
.05\end{array}$ \\
\hline $\begin{array}{l}\text { MAX: } \\
\text { MIHE } \\
\text { FMS: }\end{array}$ & .12 & $\begin{array}{r}.04 \\
.01 \\
.01\end{array}$ & $\begin{array}{r}.2 .3 \\
-.15 \\
.0 .\end{array}$ & .02 & $\begin{array}{l}.05 \\
.05 \\
.01\end{array}$ & .00 \\
\hline $\begin{array}{l}\text { QFACT } \\
\text { FFACT }\end{array}$ & $\frac{1}{3} \cdot \frac{6}{3}$ & $\frac{2}{3} \cdot \frac{23}{58}$ & $4: 24$ & $\frac{1}{3}: \frac{1}{2}=$ & 94.25 & $2 \cdot 41$ \\
\hline
\end{tabular}

IIATA FOF: FILE: SINGL

\begin{tabular}{|c|c|c|c|c|c|c|}
\hline $\mathrm{FUHH}_{174}$ & $\begin{array}{l}W I M I \\
22.5\end{array}$ & 87.0 & & $\begin{array}{l}\text { VEL } \\
40.0\end{array}$ & & \\
\hline $\begin{array}{l}\text { COMF : } \\
\text { MEAN }\end{array}$ & $.0 \%$ & $\begin{array}{r}\text { CFY } \\
-.01\end{array}$ & $\begin{array}{cl}\operatorname{cr} \\
.05\end{array}$ & $\begin{array}{l}\text { CMY } \\
.01\end{array}$ & $\begin{array}{l}\text { CHY } \\
.01\end{array}$ & $\begin{array}{l}\text { CHA } \\
.02\end{array}$ \\
\hline $\begin{array}{l}\text { MAX: } \\
\text { MIN } \\
\text { RHS: }\end{array}$ & $\begin{array}{r}13 \\
.00 \\
.02\end{array}$ & $\begin{array}{r}.02 \\
-.05 \\
.01\end{array}$ & $\begin{array}{r}.26 \\
-.23 \\
.05\end{array}$ & $\begin{array}{r}.05 \\
.05 \\
.01\end{array}$ & $\begin{array}{r}.04 \\
.09 \\
.02\end{array}$ & $\begin{array}{l}.06 \\
.00 \\
.02\end{array}$ \\
\hline $\begin{array}{l}\text { SFACT } \\
\text { FFACT }\end{array}$ & $\frac{2}{3} \cdot \frac{25}{9}$ & 3.36 & 4.04 & $\begin{array}{l}4.01 \\
2.93\end{array}$ & $\frac{3.52}{2} \cdot 11$ & $=50$ \\
\hline
\end{tabular}

IATA FOR: FILE ; SINGL.

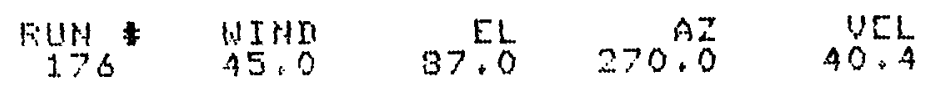

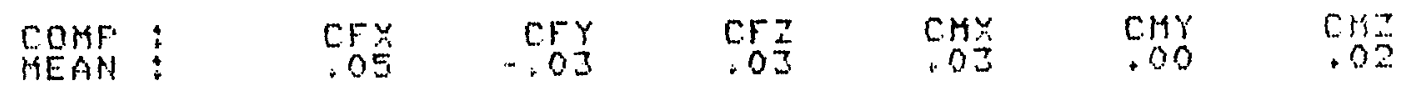

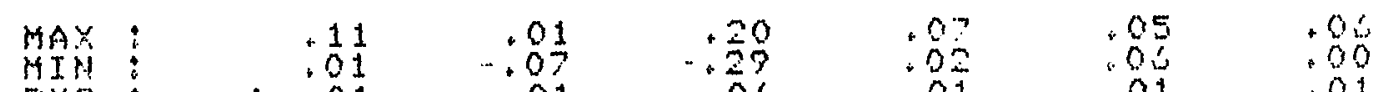

FIMS: $: .01 \quad .01 \quad .06 \quad .01 .01 \quad .01$

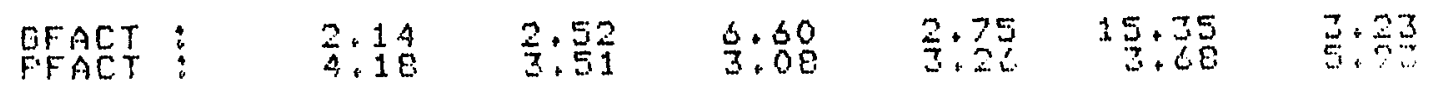

IIATA FOR FILE: SINGL

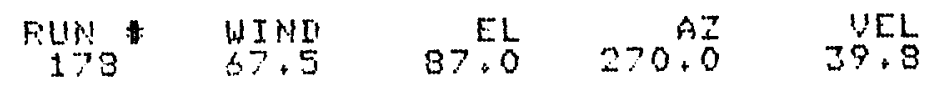

$\begin{array}{ccccccc}\text { COMF : CFY CFY } & \text { CFI } & \text { CH. } & \text { CHY } & \text { CMI } \\ \text { PGEAH : } & .03 & -.03 & .02 & .0 \% & .00 & .02\end{array}$

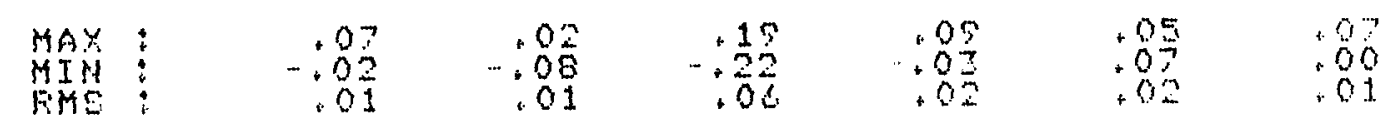

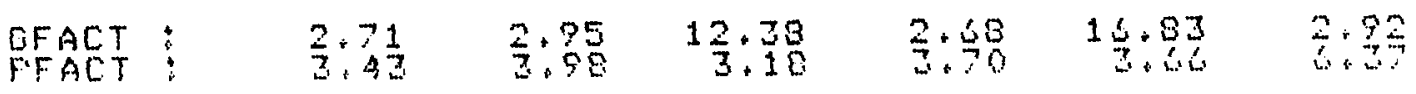




\begin{tabular}{|c|c|c|c|c|c|c|}
\hline $\operatorname{lns}_{100}=$ & $\begin{array}{l}\text { WINI } \\
90.0\end{array}$ & 37.0 & 270.0 & $\begin{array}{r}\text { YEL } \\
40.2\end{array}$ & & \\
\hline $\begin{array}{l}\text { COAF } \\
\text { : }\end{array}$ & $\begin{array}{ll}C F \\
: D 1\end{array}$ & $\begin{array}{r}C 5 Y \\
. . O S\end{array}$ & $\begin{array}{r}\text { CFZ } \\
-.01\end{array}$ & CHy & $\begin{array}{l}\text { Cry } \\
\text { :OI }\end{array}$ & $\begin{array}{l}\operatorname{crs} z \\
.04\end{array}$ \\
\hline $\begin{array}{l}\text { MAX } \\
\text { MINS } \\
\text { FMS }\end{array}$ & $\begin{array}{r}.02 \\
.02 \\
.01\end{array}$ & $\begin{array}{r}.00 \\
-.10 \\
.01\end{array}$ & $\begin{array}{l}15 \\
.20 \\
.05\end{array}$ & $\begin{array}{l}.05 \\
.08 \\
.02\end{array}$ & .02 & $\begin{array}{r}.06 \\
.01 \\
.01\end{array}$ \\
\hline $\begin{array}{l}\text { GFACT } \\
\text { FFACT }\end{array}$ & $\frac{5}{4} \cdot \frac{2}{01}$ & $\frac{2}{4}+35$ & $\begin{array}{r}34.37 \\
4.14\end{array}$ & $\begin{array}{r}880.49 \\
4: 89\end{array}$ & $3: 38$ & $\frac{1}{7} \cdot 3 \frac{1}{n}$ \\
\hline
\end{tabular}

DATA FOF: FILE: BINGL

\begin{tabular}{|c|c|c|c|c|c|c|}
\hline 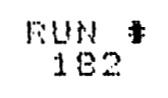 & $\begin{array}{r}\text { WISn } \\
112.5\end{array}$ & 37.5 & & $\begin{aligned} & \text { VEL } \\
& 35.7\end{aligned}$ & & \\
\hline $\begin{array}{l}\text { COMF } \\
\text { MEAN }\end{array}$ & $\begin{array}{c}C F x \\
.02\end{array}$ & $\begin{array}{r}\cos y \\
-.03\end{array}$ & $\begin{array}{cc}\mathrm{Cr} \\
.05\end{array}$ & $\begin{array}{l}\mathrm{CH} \\
.01\end{array}$ & $\begin{array}{l}\text { CHY } \\
.00\end{array}$ & $\begin{array}{l}C H z \\
.02\end{array}$ \\
\hline $\begin{array}{l}\text { MAX } \\
\text { MIN } \\
\text { FHS }\end{array}$ & $\begin{array}{r}.01 \\
.06 \\
.01\end{array}$ & $\begin{array}{r}.01 \\
.09 \\
.01\end{array}$ & $\begin{array}{r}.15 \\
-.31 \\
+06\end{array}$ & $\begin{array}{l}.07 \\
.05 \\
.02\end{array}$ & $\begin{array}{l}.06 \\
.04 \\
+02\end{array}$ & .05 \\
\hline $\begin{array}{l}\text { QFACT } \\
\text { FFACT }\end{array}$ & $\frac{2}{3} \cdot 42$ & $\frac{2.75}{4.31}$ & $\begin{array}{l}5.33 \\
7.27\end{array}$ & $\begin{array}{l}5.38 \\
3.58\end{array}$ & $14 \cdot 52$ & 2.85 \\
\hline
\end{tabular}

IATA FOF FILE : SIMGL

\begin{tabular}{|c|c|c|c|c|c|c|}
\hline rus & $\begin{array}{l}\text { WINS } \\
135.0\end{array}$ & 87.5 & & 0.1 & & \\
\hline $\begin{array}{l}\text { COMF : } \\
\text { SiEAR : }\end{array}$ & $\begin{array}{l}C F X \\
O S A\end{array}$ & $\begin{array}{r}C F Y \\
-.0 Z\end{array}$ & CFZ & $\operatorname{cmx}_{00}$ & $\begin{array}{c}\mathrm{CHY} \\
.01\end{array}$ & $\begin{array}{c}\text { Cry } \\
.03\end{array}$ \\
\hline $\begin{array}{l}\text { MAX } \\
\text { MIMU } \\
\text { rims }\end{array}$ & $\begin{array}{r}-.01 \\
-.07 \\
.01\end{array}$ & $\begin{array}{r}.01 \\
. .08 \\
.01\end{array}$ & .13 & $\begin{array}{l}.07 \\
.07 \\
.02\end{array}$ & $\begin{array}{l}.03 \\
.05 \\
.02\end{array}$ & $\begin{array}{l}.00 \\
.00 \\
.02\end{array}$ \\
\hline $\begin{array}{l}\text { EFACT } \\
\text { FACT }\end{array}$ & $\frac{2}{3} \cdot 53$ & $\frac{2}{4} \cdot 80$ & 5.80 & $20 \cdot \frac{2}{3}$ & $19 \cdot 04$ & $\begin{array}{c}-43 \\
2\end{array}$ \\
\hline
\end{tabular}

MATA FOF FILE: SIMGL

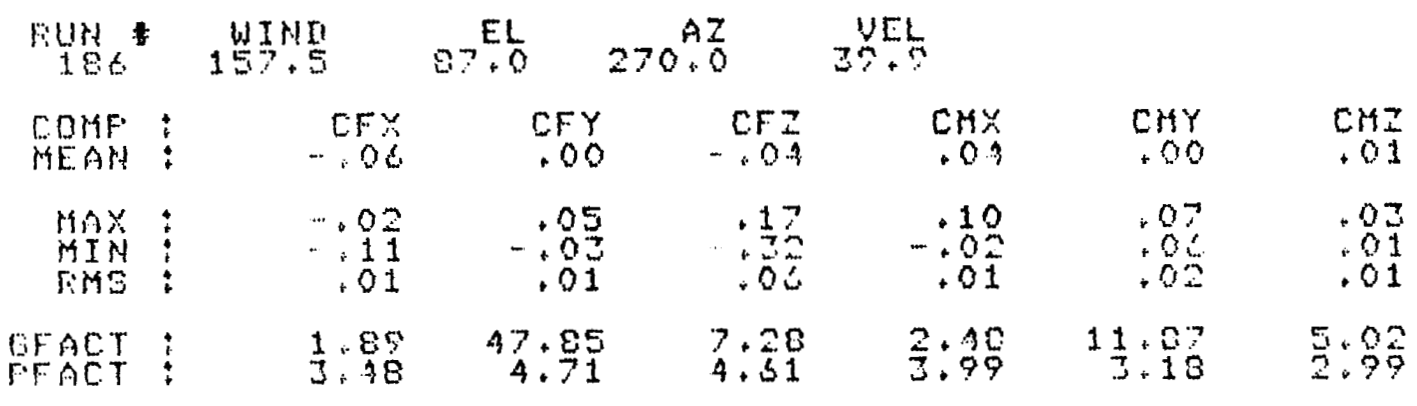




\begin{tabular}{|c|c|c|c|c|c|c|}
\hline $\begin{array}{l}\text { Find } \\
198\end{array}$ & $\begin{array}{r}W I M I \\
130.0\end{array}$ & 87.0 & $270.0^{A Z}$ & YELL & & \\
\hline $\begin{array}{l}\text { COMF } \\
\text { MEAM }\end{array}$ & $\begin{array}{r}C F Y \\
-.06\end{array}$ & $\begin{array}{c}\text { C.FY } \\
.01\end{array}$ & .01 & CMY & $\begin{array}{l}\operatorname{crs} \\
\cdots, 00\end{array}$ & $\begin{array}{c}C H z \\
.02\end{array}$ \\
\hline $\begin{array}{l}\text { MAX } \\
\text { MIN } \\
\text { RMS }\end{array}$ & $\begin{array}{r}-.02 \\
-.12 \\
.01\end{array}$ & $\begin{array}{r}.02 \\
-.01 \\
.01\end{array}$ & $\begin{array}{r}.29 \\
-.05 \\
.05\end{array}$ & $\begin{array}{r}.09 \\
-.04 \\
.01\end{array}$ & $\begin{array}{r}.06 \\
.00 \\
.01\end{array}$ & $\begin{array}{l}.01 \\
.01 \\
.01\end{array}$ \\
\hline $\begin{array}{l}\text { GFACT } \\
\text { FFACT }\end{array}$ & $\frac{1}{3}: \frac{8}{5}$ & 5.89 & $\begin{array}{r}30.32 \\
8.57\end{array}$ & $3: 50$ & 27.87 & $\begin{array}{l}5 \\
5 \\
3\end{array}$ \\
\hline
\end{tabular}

IATA FDF FILE : SIHGL

\begin{tabular}{|c|c|c|c|c|c|c|}
\hline FUN & $\begin{array}{r}W I N \Pi \\
100.0\end{array}$ & $50+\%$ & 270.0 & $\begin{array}{l}\text { VEL } \\
40.1\end{array}$ & & \\
\hline $\begin{array}{l}\text { CQMF } \vdots \\
\text { MEAM }\end{array}$ & $\begin{array}{r}C F \times \\
-.05\end{array}$ & $\begin{array}{r}\text { CFY } \\
-.0 O\end{array}$ & $\begin{array}{l}C F Z \\
+03\end{array}$ & CMY & $\begin{array}{l}\text { CHY } \\
.07\end{array}$ & $\begin{array}{l}C H z \\
.0 Z\end{array}$ \\
\hline $\begin{array}{l}\text { MAX } \\
\text { MIH } \\
\text { MHS }\end{array}$ & $\begin{array}{r}.00 \\
.01 \\
.01\end{array}$ & $\begin{array}{l}.03 \\
.03 \\
.01\end{array}$ & $+\frac{25}{25}$ & $.0 \hat{8}$ & $\begin{array}{l}01 \\
.12 \\
02\end{array}$ & $\begin{array}{l}.05 \\
.01 \\
.01\end{array}$ \\
\hline $\begin{array}{l}\text { GFACT } \\
F F A C T:\end{array}$ & $\begin{array}{l}2.48 \\
3+45\end{array}$ & $\frac{8}{2}+\frac{1}{7}$ & $\begin{array}{c}4.79 \\
3.39\end{array}$ & 10.40 & $\frac{1}{3} \cdot 97$ & $2+\frac{6}{56}$ \\
\hline NATA $F C$ & IF FILE : & SIHGL & & & & \\
\hline $\begin{array}{l}\text { FUN } \\
192\end{array}$ & $\begin{array}{r}41+40 \\
157+5\end{array}$ & $\begin{array}{c}E L \\
90.0\end{array}$ & 270.0 & $\begin{array}{r}\text { UEL } \\
37.7\end{array}$ & & \\
\hline $\begin{array}{l}\text { COMF } \\
\text { KEAN }\end{array}$ & $\begin{array}{r}\text { CFX } \\
-.05\end{array}$ & $\begin{array}{r}C F Y \\
-.01\end{array}$ & $\begin{array}{l}\text { CF } \\
.02\end{array}$ & $\begin{array}{l}c m x \\
.01\end{array}$ & $\begin{array}{l}\text { CMY } \\
\therefore 05\end{array}$ & $\begin{array}{l}C H z \\
0.02\end{array}$ \\
\hline $\begin{array}{l}\text { MAX } \\
\text { MIIH } \\
\text { RHS }\end{array}$ & $\begin{array}{l}\because 00 \\
\because 10 \\
\div 02\end{array}$ & $\begin{array}{l}.02 \\
.05 \\
.01\end{array}$ & $\therefore \frac{25}{25}$ & $\begin{array}{l}.06 \\
.05 \\
.01\end{array}$ & $\begin{array}{l}.01 \\
.10 \\
.02\end{array}$ & .05 \\
\hline $\begin{array}{l}\text { GFACT } \\
\text { FFACT }\end{array}$ & 3.01 & $\begin{array}{l}3.84 \\
4.02\end{array}$ & 10.78 & $\frac{5}{3} \cdot 15$ & $\frac{2}{3} \cdot 00$ & $2+12$ \\
\hline IATA FC & OR FILE : & SINGL & & & & \\
\hline $\begin{array}{l}\text { FiLM } \\
194\end{array}$ & $\begin{array}{r}\text { WIMI } \\
135.0\end{array}$ & $\begin{array}{r}E L \\
30.0\end{array}$ & 270.0 & $\begin{array}{r}\text { UEL } \\
39: 7\end{array}$ & & \\
\hline COMF & $\begin{array}{r}C .5 \\
-04\end{array}$ & $\begin{array}{l}\text { CFY } \\
.03\end{array}$ & $\begin{array}{c}\operatorname{crz} \\
.01\end{array}$ & $\begin{array}{l}\text { CMY } \\
.01\end{array}$ & $\begin{array}{l}\mathrm{cry} \\
.0 \mathrm{Z}\end{array}$ & .01 \\
\hline $\begin{array}{l}\text { MAX } \\
\text { MINS } \\
\text { FMS }\end{array}$ & $\begin{array}{r}.01 \\
-.09 \\
.01\end{array}$ & $\begin{array}{r}.02 \\
-.09 \\
.01\end{array}$ & $\begin{array}{r}.25 \\
-.27\end{array}$ & $\begin{array}{l}.07 \\
.05 \\
.02\end{array}$ & $\begin{array}{r}.04 \\
.10 \\
.02\end{array}$ & $\begin{array}{r}.95 \\
.05 \\
.01\end{array}$ \\
\hline $\begin{array}{l}\text { OFACT } \\
\text { FFACT }\end{array}$ & $\frac{2}{3} \cdot \frac{20}{34}$ & $\frac{2.83}{4.39}$ & $47 \cdot 18$ & $\begin{array}{l}5.93 \\
3.28\end{array}$ & $3+34$ & $\begin{array}{l}2.38 \\
2.3 .1\end{array}$ \\
\hline
\end{tabular}




\begin{tabular}{|c|c|c|c|c|c|c|}
\hline $\begin{array}{c}\text { fus } \\
196\end{array}$ & $\begin{array}{r}45 \| \pi \\
112.5\end{array}$ & 70.0 & $\begin{array}{r}\text { Az } \\
270.0\end{array}$ & $\begin{array}{r}\text { VEL } \\
39.9\end{array}$ & & \\
\hline COHF : & -65 & $\begin{array}{r}\text { CFY } \\
-.05\end{array}$ & ${ }_{02}^{C F}$ & $\begin{array}{l}\text { chx } \\
+02\end{array}$ & $\begin{array}{l}\text { cry } \\
.02\end{array}$ & $\begin{array}{l}\mathrm{CHz} \\
.0 \mathrm{I}\end{array}$ \\
\hline $\begin{array}{l}\text { MAXX: } \\
\text { NAIH } \\
\text { FMS }\end{array}$ & $\begin{array}{l}.01 \\
.05 \\
.01\end{array}$ & $\begin{aligned}- & +01 \\
\cdots & +10 \\
+ & 01\end{aligned}$ & .17 & $: 02$ & .82 & $\begin{array}{l}.06 \\
.00 \\
.01\end{array}$ \\
\hline $\begin{array}{l}\text { GFACT : } \\
\text { PFACT }\end{array}$ & $\begin{array}{l}3.04 \\
3.63\end{array}$ & $\frac{2}{3} .05$ & 13.20 & $\begin{array}{l}\overrightarrow{3} \cdot 74 \\
3.50\end{array}$ & 3.27 & $\frac{273}{3}+17$ \\
\hline XATA FO & as FILE : & SINGL & & & & \\
\hline Fug & $\begin{array}{l}\text { WIM } \\
90.0\end{array}$ & 90.5 & 270.0 & $\begin{array}{r}\text { VEL } \\
40.0\end{array}$ & & \\
\hline $\begin{array}{l}\text { COMF : } \\
\text { PEAH : }\end{array}$ & $.0 \%$ & $\begin{array}{r}\text { CFY } \\
-.04\end{array}$ & $\begin{array}{l}\operatorname{cr} 2 \\
+02\end{array}$ & $\begin{array}{l}\text { C.\$Y } \\
.00\end{array}$ & $\begin{array}{l}\text { CHY } \\
.02\end{array}$ & $\begin{array}{l}\operatorname{cm} \\
.03\end{array}$ \\
\hline $\begin{array}{l}\text { MA } \\
\text { Hiti } \\
\text { Sims }\end{array}$ & $\begin{array}{r}.05 \\
.01 \\
.01\end{array}$ & $\begin{array}{r}.00 \\
.00 \\
.01\end{array}$ & $\begin{array}{r}1.18 \\
.20 \\
.05\end{array}$ & $\begin{array}{l}.05 \\
.05 \\
.02\end{array}$ & .01 & $\begin{array}{l}.86 \\
.01\end{array}$ \\
\hline $\begin{array}{l}\text { GFACT: } \\
\text { FFACT }\end{array}$ & $3 \cdot 68$ & $\frac{2}{3} \cdot \frac{1}{3} \cdot 7$ & $2+30$ & $15 \cdot \frac{2}{3}$ & $\frac{2}{9} \cdot 25$ & $\frac{1}{3} \cdot 50$ \\
\hline
\end{tabular}

IATA FOF FILE: SIHOL

\begin{tabular}{|c|c|c|c|c|c|c|}
\hline MUN & WINI & 20.0 & $2 P 0^{A} . O^{A}$ & $\begin{array}{r}\text { VEL } \\
10.4\end{array}$ & & \\
\hline $\begin{array}{l}\text { CORF : } \\
\text { MEAH : }\end{array}$ & $\begin{array}{l}\text { CFX } \\
.03\end{array}$ & $\begin{array}{r}\text { CFY } \\
-+04\end{array}$ & CFz & $\begin{array}{l}\text { CMY } \\
.00\end{array}$ & $\begin{array}{r}\text { C.MY } \\
-.01\end{array}$ & $\begin{array}{c}\mathrm{CHz}_{2} \\
.01\end{array}$ \\
\hline $\begin{array}{l:}\text { HAXX } \\
\text { MIYH } \\
\text { SAMS }\end{array}$ & $\begin{array}{r}.07 \\
-.01 \\
.01\end{array}$ & $\begin{array}{r}-.00 \\
-.00 \\
.01\end{array}$ & $\begin{array}{r}.13 \\
-.25 \\
.05\end{array}$ & $\begin{array}{r}.05 \\
-.00 \\
.02\end{array}$ & $\begin{array}{l}.0 .9 \\
.04 \\
.01\end{array}$ & $\begin{array}{l}.01 \\
.02 \\
.01\end{array}$ \\
\hline $\begin{array}{l}\text { OFACT: } \\
\text { FFAT }\end{array}$ & $\frac{2}{3} \cdot 4 \frac{1}{3}$ & $\frac{2}{3} \cdot \frac{15}{3}$ & $7: 9 \frac{1}{3}$ & 07.07 & 3.90 & $\frac{3}{3} \cdot \frac{1}{5}-2$ \\
\hline
\end{tabular}

WATA FOF FILE: SIHGL

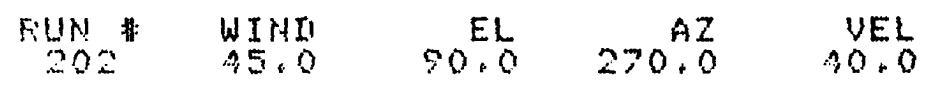

\begin{tabular}{|c|c|c|c|c|c|c|}
\hline $\begin{array}{l}\text { EOMF : } \\
\text { MEAHY }\end{array}$ & $\begin{array}{l}C F \times \\
.05\end{array}$ & $\begin{array}{l}\text { CFY } \\
- \text { to: }\end{array}$ & CFZ & $\begin{array}{l}\text { cryse } \\
+01\end{array}$ & ery & CHz \\
\hline $\begin{array}{l}\text { MAX } \\
\text { MINS } \\
\text { HMS }\end{array}$ & $\begin{array}{l}10 \\
.00 \\
+01\end{array}$ & $\begin{array}{r}.01 \\
-.02 \\
.01\end{array}$ & $\begin{array}{l}17 \\
.85 \\
.07\end{array}$ & $\begin{array}{r}.07 \\
.05 \\
.02\end{array}$ & .05 & $\begin{array}{l}.05 \\
.01 \\
.01\end{array}$ \\
\hline FACT & 2.96 & $\begin{array}{l}2.48 \\
3.48\end{array}$ & $\frac{8}{4} \div 5 \frac{2}{4}$ & 3.31 & $3+75$ & $\pm: 2$ \\
\hline
\end{tabular}


IIATA FOF: FILE : SINGL

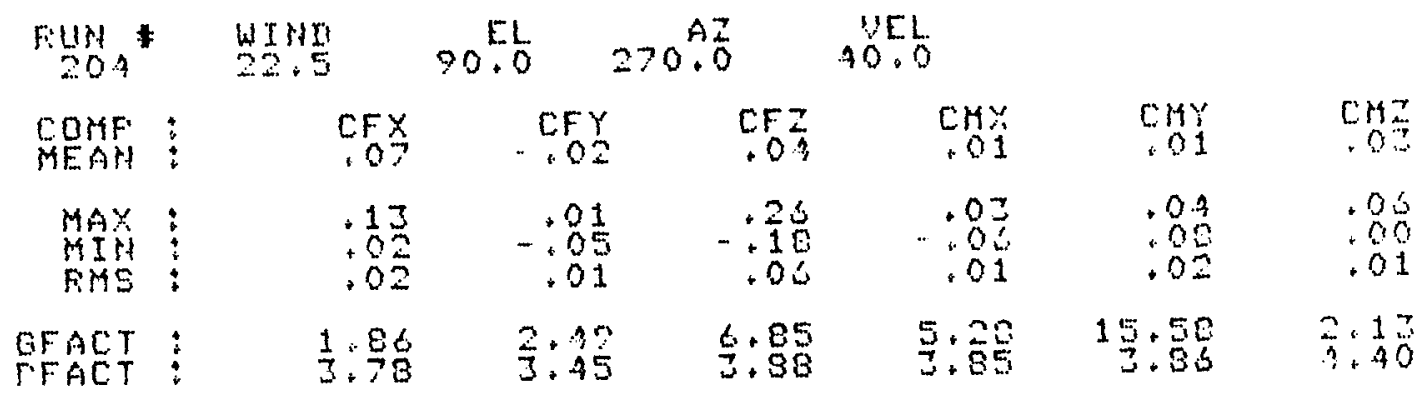

IIATA FOF: FILE: SIHOL

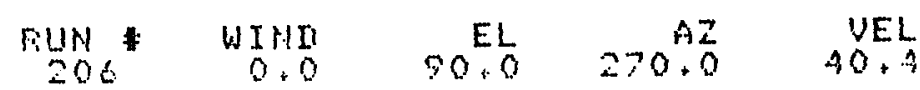

\begin{tabular}{|c|c|c|c|c|c|c|}
\hline $\begin{array}{l}\text { COMF } \\
\text { MEEAS }\end{array}$ & $\begin{array}{l}\text { CFX } \\
.0 S\end{array}$ & $\begin{array}{r}\text { CFY } \\
-.00\end{array}$ & $\begin{array}{r}C F z \\
-.03\end{array}$ & $\begin{array}{c}C K S \\
.00\end{array}$ & $\begin{array}{l}\text { C.MY } \\
.01\end{array}$ & $\begin{array}{l}C K L \\
.00\end{array}$ \\
\hline $\begin{array}{l}\text { MAY } \\
\text { MIYI } \\
\text { FisS }\end{array}$ & .12 & .03 & $\begin{array}{l}10 \\
.21 \\
.04\end{array}$ & $\begin{array}{r}.03 \\
.01\end{array}$ & $\begin{array}{l}.90 \\
.05 \\
.01\end{array}$ & \\
\hline $\begin{array}{l}\text { FACT } \\
\text { FACT }\end{array}$ & $\frac{1}{3} \cdot \frac{92}{9}$ & $\frac{21}{3} \cdot \frac{2}{54}$ & $\begin{array}{l}E .38 \\
4.29\end{array}$ & $27+8=$ & $\frac{5.42}{5.40}$ & 30 \\
\hline
\end{tabular}

IIATA FOF FILE: SIHGL

\begin{tabular}{|c|c|c|c|c|c|c|}
\hline Fun & $\begin{array}{c}\text { WIHII } \\
0.0\end{array}$ & $\begin{array}{r}E L \\
75.0\end{array}$ & $=0.9$ & $\begin{array}{l}\text { VEL } \\
10.2\end{array}$ & & \\
\hline $\begin{array}{l}\text { COMF } \\
\text { MEAH }\end{array}$ & $\begin{array}{l}\text { CFX } \\
+1\end{array}$ & $\begin{array}{l}\text { CFY } \\
.01\end{array}$ & CF & $\begin{array}{l}\text { CHy } \\
.00\end{array}$ & $\begin{array}{l}\text { chy } \\
.16\end{array}$ & $\cos$ \\
\hline $\begin{array}{l}\text { MAX : } \\
\text { MIH } \\
\text { FMS: }\end{array}$ & .31 & $\begin{aligned} .02 \\
.02 \\
.01\end{aligned}$ & $. \frac{71}{2}=\frac{1}{7}$ & $\begin{array}{r}.01 \\
-.05 \\
.01\end{array}$ & $\begin{array}{r}.24 \\
.00 \\
.02\end{array}$ & $\begin{array}{l}0 . \\
0.0 \\
.01 \\
.01\end{array}$ \\
\hline $\begin{array}{l}\text { QFACT } \\
\text { PFACT }\end{array}$ & $\frac{3}{4} \cdot 6 \frac{16}{6}$ & $\begin{array}{l}5.80 \\
4.40\end{array}$ & $\frac{1}{3} \cdot 94$ & $52 \cdot 47$ & $\frac{1}{3} \cdot \frac{51}{3}$ & $\begin{array}{l}3.04 \\
3.63\end{array}$ \\
\hline
\end{tabular}

IATA FOF: FILE: SIMGL

\begin{tabular}{|c|c|c|c|c|c|c|}
\hline Fus & $\begin{array}{l}\text { WINI } \\
45.0\end{array}$ & 75.0 & & $\begin{array}{l}\text { YEL } \\
90.5\end{array}$ & & \\
\hline COMF: & $\begin{array}{l}C F X \\
1 Z\end{array}$ & $\begin{array}{r}\text { CFY } \\
-.05\end{array}$ & $\begin{array}{l}C F Z \\
.3 .3\end{array}$ & $\begin{array}{l}\mathrm{Cm} \\
.05\end{array}$ & $\begin{array}{l}\text { CMY } \\
.06\end{array}$ & chas \\
\hline $\begin{array}{l}\text { MAX } \\
\text { HIYS } \\
\text { FMS }\end{array}$ & $\begin{array}{r}.25 \\
.01 \\
.03\end{array}$ & $\begin{array}{r}-.00 \\
-.00 \\
.01\end{array}$ & $\begin{array}{l}-62 \\
.17 \\
.07\end{array}$ & $\begin{array}{l}10 \\
.00 \\
.01\end{array}$ & .13 & $\begin{array}{l}.05 \\
.00 \\
.01\end{array}$ \\
\hline $\begin{array}{l}\text { GFACT } \\
\text { FFACT }\end{array}$ & $\frac{1}{3} \cdot 74$ & $\frac{1}{3} \cdot 7 \frac{3}{5}$ & $\begin{array}{l}1.83 \\
4.00\end{array}$ & $\frac{2}{3} \cdot \frac{15}{5}$ & $\frac{2}{5}+13$ & $2+75$ \\
\hline
\end{tabular}




\begin{tabular}{|c|c|c|c|c|c|c|}
\hline Fud & $\begin{array}{l}\text { WIMU } \\
90.0\end{array}$ & 75.0 & 270.0 & $\begin{array}{l}\text { UEL } \\
40.9\end{array}$ & & \\
\hline $\begin{array}{l}\text { COHF } \\
\text { MEAH }\end{array}$ & $.0 \%$ & $\begin{array}{r}C F Y \\
-.03\end{array}$ & $\operatorname{crz}_{-03}$ & $\begin{array}{l}\text { chy } \\
.02\end{array}$ & $\begin{array}{l}\text { CMY } \\
+01\end{array}$ & $\begin{array}{c}c+2 \\
.02\end{array}$ \\
\hline $\begin{array}{l}\text { MAX } \\
\text { MIN } \\
\text { RMS }\end{array}$ & $\begin{array}{r}.10 \\
\cdots .11 \\
.02\end{array}$ & $\begin{array}{r}.01 \\
.09 \\
.01\end{array}$ & $\begin{array}{r}-15 \\
-.25 \\
.05\end{array}$ & $\begin{array}{l}90 \\
0.3 \\
0.02\end{array}$ & $\begin{array}{r}04 \\
.02 \\
.01\end{array}$ & $\begin{array}{r}.04 \\
.01 \\
.01\end{array}$ \\
\hline $\begin{array}{l}\text { GFACT } \\
\text { FFACT }\end{array}$ & $19 \cdot 63$ & $\frac{2}{4.52}$ & $\begin{array}{l}8.45 \\
4.55\end{array}$ & $\begin{array}{l}4.58 \\
3.53\end{array}$ & $\begin{array}{r}5.03 \\
1.00\end{array}$ & $\overrightarrow{3} \cdot \frac{2}{3}$ \\
\hline
\end{tabular}

IATA FOF FILE: SINGL.

\begin{tabular}{|c|c|c|c|c|c|c|}
\hline $\begin{array}{l}\text { Fily } \\
= \pm 1\end{array}$ & 135.0 & 75.6 & & $\begin{array}{l}\text { UEL } \\
40+2\end{array}$ & & \\
\hline $\begin{array}{l}\text { COMF } \\
\text { MEAH }\end{array}$ & $\begin{array}{l}C F \\
-12\end{array}$ & $\begin{array}{r}C .5 Y \\
.04\end{array}$ & $\begin{array}{c}0.2 \\
-.36\end{array}$ & $\begin{array}{r}\text { EHS } \\
-.07\end{array}$ & $\begin{array}{l}\text { CHY } \\
.02\end{array}$ & $\begin{array}{l}\mathrm{CH}_{2} \mathrm{x} \\
.0 \mathrm{~S}\end{array}$ \\
\hline 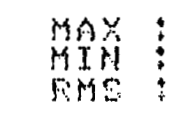 & $\begin{array}{r}-.02 \\
-.25 \\
.04\end{array}$ & $\begin{array}{r}.01 \\
-.10 \\
.01\end{array}$ & .95 & $\begin{array}{r}.00 \\
.14 \\
.02\end{array}$ & $\begin{array}{l}.12 \\
.05 \\
.02\end{array}$ & .02 \\
\hline $\begin{array}{l}\text { QFACT } \\
\text { FFACT }\end{array}$ & $\frac{2}{2}+\frac{19}{2}$ & $\frac{2}{4} .76$ & $\frac{2}{3}+\frac{19}{52}$ & $\stackrel{3}{3} .04$ & 5.25 & $\frac{1}{3} \cdot \frac{1}{00}$ \\
\hline
\end{tabular}

MATA FQF FILE: SINGL

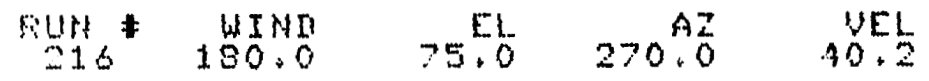

\begin{tabular}{|c|c|c|c|c|c|c|}
\hline $\begin{array}{l}\text { COMF } \\
\text { HEAM }\end{array}$ & $\begin{array}{r}\text { CF } \\
-.15\end{array}$ & $\begin{array}{l}\text { cry } \\
.01\end{array}$ & $\begin{array}{c}\operatorname{ctz} \\
.37\end{array}$ & $\begin{array}{l}C H Y \\
.02\end{array}$ & $\begin{array}{l}\text { CMY } \\
+07\end{array}$ & Craz \\
\hline $\begin{array}{l}\text { MAY } \\
\text { FTM } \\
\text { FMSE }\end{array}$ & $\begin{array}{r}-04 \\
\cdots \\
: 04 \\
.04\end{array}$ & $\begin{array}{r}.04 \\
-.04 \\
.01\end{array}$ & $\begin{array}{r}.05 \\
-75 \\
.11\end{array}$ & $\begin{array}{r}.07 \\
.04 \\
.02\end{array}$ & $\begin{array}{l}10 \\
01 \\
05\end{array}$ & $\begin{array}{l}.05 \\
.00 \\
.01\end{array}$ \\
\hline $\begin{array}{l}F A C T \\
F A C T\end{array}$ & $\frac{2}{3} \cdot \frac{2}{2}$ & $\frac{5.71}{3.82}$ & $\frac{2}{3} .90$ & $\begin{array}{l}A \cdot J^{A} \\
3 \cdot 08\end{array}$ & $\frac{2}{3} .88$ & $\frac{2}{2} \cdot 75$ \\
\hline
\end{tabular}

DIATA FOR FILE: SINGL

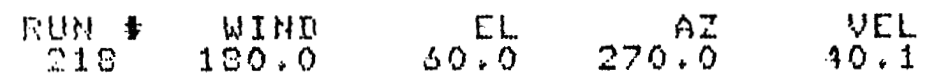

\begin{tabular}{|c|c|c|c|c|c|}
\hline $\begin{array}{l}\text { COMF } \\
\text { YIEAN }\end{array}$ & $\begin{array}{l}\operatorname{cry} \\
\cdots+99\end{array}$ & $\begin{array}{l}\text { CFY } \\
.02\end{array}$ & $\begin{array}{l}C F z \\
-80\end{array}$ & .01 & 0.12 \\
\hline $\begin{array}{l}\text { Max } \\
\text { MIN } \\
\text { riss }\end{array}$ & $\begin{array}{r}\because 17 \\
\because .05 \\
-11\end{array}$ & $\begin{array}{r}.03 \\
-.04 \\
.02\end{array}$ & $\begin{array}{r}133 \\
1+37 \\
+17\end{array}$ & $\begin{array}{r}.05 \\
-.05 \\
.02\end{array}$ & $\begin{array}{r}.20 \\
.01 \\
.04\end{array}$ \\
\hline
\end{tabular}

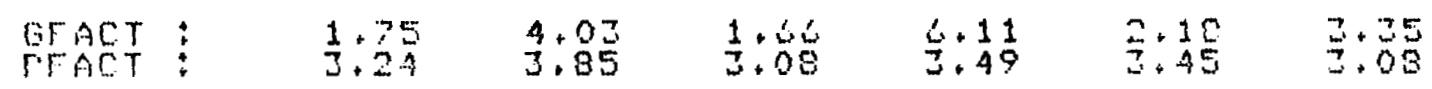




$$
\begin{aligned}
& \text { IIATA FDF FILE : SIMGL }
\end{aligned}
$$

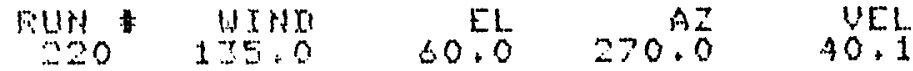

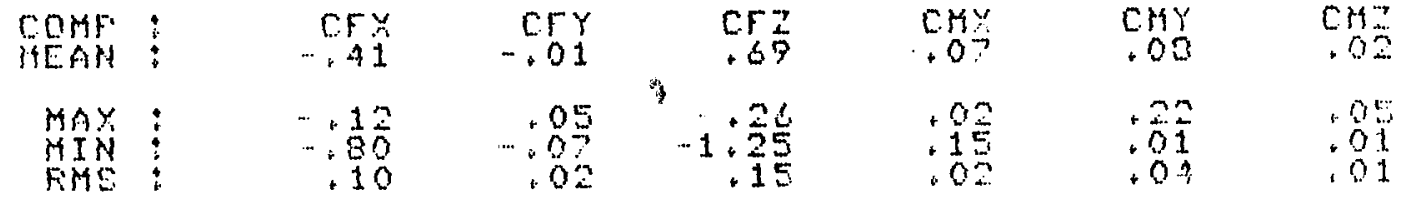

$$
\begin{aligned}
& \text { PFACT: } \quad \begin{array}{llllll}
3.96 & 3.24 & 1.82 & 2.27 & 3.57 & 2.17
\end{array}
\end{aligned}
$$$$
\text { IIATA FOF FILE: SINGL }
$$

\begin{tabular}{|c|c|c|c|c|c|c|}
\hline Fun & $\begin{array}{l}\text { WINO } \\
90.0\end{array}$ & 60.0 & 0.2 & $\begin{array}{l}\text { VEL } \\
40.1\end{array}$ & & \\
\hline COMF : & $\begin{array}{c}C F \\
.02\end{array}$ & $\begin{array}{r}\text { CFY } \\
.0 A\end{array}$ & .05 & cmy & $\begin{array}{l}\text { CHY } \\
.0 Z\end{array}$ & $\cos 2$ \\
\hline $\begin{array}{l}\text { MAX } \\
\text { MIYH } \\
\text { FMS }\end{array}$ & $\begin{array}{r}+19 \\
-.19 \\
.05\end{array}$ & $\begin{array}{r}.01 \\
.10 \\
.02\end{array}$ & $: \frac{27}{27}$ & $\begin{array}{l}.05 \\
.05 \\
.02\end{array}$ & $\begin{array}{l}.01 \\
.15 \\
.02\end{array}$ & $.0 \hat{1}$ \\
\hline $\begin{array}{l}\text { QFACT } \\
\text { FFACT }\end{array}$ & $12 \cdot \frac{87}{3}$ & $\frac{2}{4} \cdot 33$ & 11.37 & $\frac{4}{3} \cdot 31$ & $\begin{array}{l}4.49 \\
2+6\end{array}$ & $\begin{array}{l}2.96 \\
z\end{array}$ \\
\hline
\end{tabular}

IIATA FOR FILE: SIMGL

\begin{tabular}{|c|c|c|c|c|c|c|}
\hline Fust & $45+6$ & 50.0 & 270.0 & $\begin{array}{l}V E L \\
-90+3\end{array}$ & & \\
\hline COMF & CF. & $\begin{array}{l}\operatorname{cry} \\
\cdots .05\end{array}$ & $\frac{\mathrm{Cr}}{.38}$ & $\begin{array}{l}\mathrm{CH} \times \\
.03\end{array}$ & $\begin{array}{c}c m \\
.11\end{array}$ & $\begin{array}{l}\cos 2 \\
.00\end{array}$ \\
\hline $\begin{array}{l}\text { MAX } \\
\text { MIHE } \\
\text { MMS }\end{array}$ & $\begin{array}{l}75 \\
.19 \\
.08\end{array}$ & $\begin{array}{r}-.00 \\
-.10 \\
.01\end{array}$ & $\begin{array}{r}1.12 \\
: 40 \\
+12\end{array}$ & .13 & $\begin{array}{l}21 \\
.03 \\
.03\end{array}$ & .82 \\
\hline $\begin{array}{l}\text { GFACT } \\
\text { FFACT }\end{array}$ & $\frac{1}{7} \cdot \frac{30}{83}$ & $\frac{2}{3} \cdot \frac{22}{7}$ & $\frac{1}{3} \cdot \frac{6}{5}$ & $\frac{1}{3} \cdot \frac{22}{7}$ & $\frac{1}{7.87}$ & $\begin{array}{r}70 \\
3.49\end{array}$ \\
\hline
\end{tabular}

IIATA FOF: FILE : SINGL

\begin{tabular}{|c|c|c|c|c|c|c|}
\hline Kivis & WI 0.0 & 50.0 & & & & \\
\hline CoMr: & .50 & $\begin{array}{l}\text { CFY } \\
\cdots . O Z\end{array}$ & 652 & $\begin{array}{l}\operatorname{crg} x \\
.01\end{array}$ & $\begin{array}{c}\text { CHY } \\
+15\end{array}$ & $\underset{.0 \%}{C H}$ \\
\hline $\begin{array}{l}\text { MAX } \\
\text { MIH } \\
\text { FMS }\end{array}$ & .79 & $\begin{array}{r}.02 \\
-.06 \\
.01\end{array}$ & $\begin{array}{r}1.15 \\
.42 \\
.12\end{array}$ & $\begin{array}{l}.05 \\
.05 \\
.05\end{array}$ & $\begin{array}{l}28 \\
.05 \\
.03 \\
.05\end{array}$ & .09 \\
\hline $\begin{array}{l}\text { GFACT } \\
\text { FFACT }\end{array}$ & $\frac{1}{3} \cdot 59$ & $\frac{2}{3} \cdot 5 \overline{7}$ & $\frac{1}{3}: \frac{59}{32}$ & 6.58 & $\frac{1}{3.05}$ & $\frac{1}{3} \cdot 54$ \\
\hline
\end{tabular}




\begin{tabular}{|c|c|c|c|c|c|c|}
\hline furt & $\begin{array}{l}\text { WINII } \\
0.0\end{array}$ & 30.0 & & $\begin{array}{l}\text { VEL } \\
39.8\end{array}$ & & \\
\hline $\begin{array}{l}\text { COMF : } \\
\text { MEAN : }\end{array}$ & $\begin{array}{r}6 F \\
.98\end{array}$ & $\begin{array}{c}\text { CFY } \\
\ldots .03\end{array}$ & $\begin{array}{c}.52 \\
.53\end{array}$ & $\begin{array}{l}\text { CHY } \\
.01\end{array}$ & $\begin{array}{l}\text { CHY } \\
.0 ?\end{array}$ & $\begin{array}{l}\text { c.s. } \\
.03\end{array}$ \\
\hline $\begin{array}{l}\text { Max } \\
\text { HIN } \\
\text { FMS }\end{array}$ & $\begin{array}{r}1.58 \\
.51 \\
.17\end{array}$ & $\begin{array}{r}.01 \\
.07 \\
.01\end{array}$ & $\begin{array}{l}.88 \\
.95 \\
+09\end{array}$ & .07 & $\begin{array}{l}.09 \\
.02 \\
.09\end{array}$ & $.0 \%$ \\
\hline $\begin{array}{l}\text { QFACT } \\
\text { FFACT }\end{array}$ & $\frac{1}{3} \cdot 58$ & $\frac{2}{3} \cdot 79$ & $\frac{1}{3} \cdot 52$ & $7 \cdot 15$ & $\stackrel{3}{Z} \cdot 94$ & $\frac{1}{3} \cdot 7^{9}$ \\
\hline
\end{tabular}

IIATA FOR FILE: SINGL

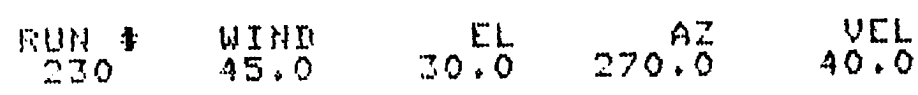

\begin{tabular}{|c|c|c|c|c|c|c|}
\hline $\begin{array}{l}\text { COMF } \\
\text { MEAN }\end{array}$ & $\begin{array}{l}\text { CFy } \\
.85\end{array}$ & $\begin{array}{c}\text { CFY } \\
-.07\end{array}$ & $\begin{array}{l}C 57 \\
.56\end{array}$ & $\begin{array}{l}\mathrm{CH} \times \\
. \mathrm{O}\end{array}$ & $\begin{array}{l}\text { CHY } \\
.00\end{array}$ & .02 \\
\hline $\begin{array}{l}\text { MAX } \\
\text { FIMSO }\end{array}$ & $\begin{array}{r}1.79 \\
.43 \\
.17\end{array}$ & $\begin{array}{r}-.01 \\
-.14 \\
.02\end{array}$ & $\begin{array}{r}.95 \\
.09\end{array}$ & $\begin{array}{l}10 \\
.01 \\
01\end{array}$ & .22 & $\begin{array}{r}.04 \\
.05\end{array}$ \\
\hline $\begin{array}{l}F A C T \\
F A C T\end{array}$ & $\begin{array}{l}1.97 \\
4.97\end{array}$ & $\begin{array}{l}2.09 \\
4.18\end{array}$ & $\begin{array}{l}1.59 \\
3.51\end{array}$ & $\frac{2}{4}+\frac{54}{4}$ & 3.05 & 焉.929 \\
\hline
\end{tabular}

IATA FOF FILE ; SINGL

\begin{tabular}{|c|c|c|c|c|c|c|}
\hline PUN $_{32}$ * & $\begin{array}{l}\text { WINn } \\
90.0\end{array}$ & 30.0 & $A Z$ & $\begin{array}{r}\text { ULL } \\
39.8\end{array}$ & & \\
\hline $\begin{array}{l}\text { COMF } \\
\text { MEAN }\end{array}$ & $\begin{array}{l}\text { CF. } \\
.05\end{array}$ & $\begin{array}{cr}C Y \\
.05\end{array}$ & $\begin{array}{c}0.2 \\
.01\end{array}$ & $\begin{array}{l}\text { CMY } \\
.01\end{array}$ & $\begin{array}{l}\text { CMY } \\
.09\end{array}$ & .03 \\
\hline $\begin{array}{l}\text { HAX } \\
\text { HIN } \\
\text { SME : }\end{array}$ & .34 & $\begin{array}{r}.00 \\
-.11 \\
.02\end{array}$ & $\begin{array}{r}.21 \\
.18 \\
.05\end{array}$ & $\begin{array}{l}.05 \\
.03 \\
.02\end{array}$ & $\begin{array}{l}.00 \\
.12 \\
.04\end{array}$ & $\begin{array}{l}.07 \\
.01 \\
.01\end{array}$ \\
\hline $\begin{array}{l}\text { BFACT } \\
\text { PFACT }\end{array}$ & 3.97 & $\frac{2}{4} \cdot 37$ & $\begin{array}{r}40.18 \\
4.07\end{array}$ & $\begin{array}{l}7.73 \\
3.20\end{array}$ & 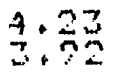 & $\begin{array}{l}2.87 \\
-4.62\end{array}$ \\
\hline
\end{tabular}

IIATA FOF FILE: SINGL

\begin{tabular}{|c|c|c|c|c|c|c|}
\hline Fus ${ }_{2 x}$ & $\begin{array}{r}\text { WINI } \\
135.0\end{array}$ & 30.0 & AL & $\begin{array}{l}\text { VELL } \\
40.2\end{array}$ & & \\
\hline $\begin{array}{l}\text { CONF } \\
\text { MEAN }\end{array}$ & $\begin{array}{r}C F Y \\
-.88\end{array}$ & $\begin{array}{l}\text { CFY } \\
.01\end{array}$ & $C F^{2}$ & $\begin{array}{l}\text { crs } \\
+0 \%\end{array}$ & $\begin{array}{l}\text { CMY } \\
01\end{array}$ & $\begin{array}{c}\text { CHI } \\
\text { OS }\end{array}$ \\
\hline $\begin{array}{l}\text { MAY } \\
\text { Ming } \\
\text { SHS }\end{array}$ & $\begin{array}{r}.36 \\
1: 37 \\
+15\end{array}$ & $\begin{array}{r}.07 \\
-.04 \\
.01\end{array}$ & $\begin{array}{r}.75 \\
-1: 05 \\
.12\end{array}$ & $\begin{array}{l}.01 \\
.13 \\
.02\end{array}$ & $\begin{array}{l}.27 \\
.05 \\
.05\end{array}$ & $\begin{array}{l}.02 \\
.02 \\
.01\end{array}$ \\
\hline $\begin{array}{l}\text { PFACT } \\
\text { FFACT }\end{array}$ & $\frac{1}{4.39}$ & $\begin{array}{l}8.23 \\
4.26\end{array}$ & $\frac{1}{3} \cdot 5 \frac{6}{5}$ & $\frac{1}{3} \cdot 30$ & $10 \cdot 50$ & $\frac{1}{3} \cdot 53$ \\
\hline
\end{tabular}




\begin{tabular}{|c|c|c|c|c|c|c|}
\hline Filust & $\begin{array}{r}\text { WIYI } \\
180.0\end{array}$ & $\begin{array}{r}E L \\
30.0\end{array}$ & 270.0 & $\begin{array}{l}\text { UEL. } \\
40.1\end{array}$ & & \\
\hline COMF: & 0.95 & $\begin{array}{l}0.5 Y \\
.04\end{array}$ & $\begin{array}{l}C F Z \\
.68\end{array}$ & CMY & $\begin{array}{l}\text { CHY } \\
.04\end{array}$ & $\begin{array}{c}c r z \\
0=\end{array}$ \\
\hline $\begin{array}{l}\text { MAX } \\
\text { MIHS } \\
\text { FMS }\end{array}$ & $\ddot{1} \div \frac{39}{15}$ & $\begin{array}{r}.08 \\
-.00 \\
.01\end{array}$ & -1.31 & $\begin{array}{l}.07 \\
: 02 \\
.02\end{array}$ & .27 & .05 \\
\hline $\begin{array}{l}\text { GFACT } \\
\text { FFACT }\end{array}$ & $\frac{1}{2} \cdot 90$ & $\begin{array}{l}2.14 \\
3.62\end{array}$ & $\begin{array}{l}1.52 \\
3.50\end{array}$ & $\begin{array}{l}5.72 \\
3.12\end{array}$ & $\begin{array}{l}4.46 \\
5: 00\end{array}$ & 3.58 \\
\hline IIATA F & DF: FILE : & SINGL & & & & \\
\hline rivis & $\begin{array}{r}45 \mathrm{IN} \\
100.0\end{array}$ & $15 \div 6$ & $=70 . A^{A}$ & $\begin{array}{r}152 L \\
40.3\end{array}$ & & \\
\hline COMF & $\begin{array}{l}C F \\
1.09\end{array}$ & $\begin{array}{l}\text { C.YY } \\
.00\end{array}$ & $\begin{array}{l}C F Z \\
-39\end{array}$ & $.0 \mathrm{~cm}$ & Coy & $\begin{array}{l}\operatorname{crs} \\
.00\end{array}$ \\
\hline $\begin{array}{l}\text { MAX } \\
\text { MIMS } \\
\text { FMS }\end{array}$ & $\begin{array}{r}5.51 \\
2.05 \\
.22\end{array}$ & $\begin{array}{l}.03 \\
.01\end{array}$ & $\begin{array}{r}.20 \\
.84 \\
.07\end{array}$ & $.0 \%$ & $\begin{array}{l}17 \\
.03 \\
.06\end{array}$ & $\begin{array}{l}.0 .9 \\
.06 \\
.01\end{array}$ \\
\hline $\begin{array}{l}\text { QFACT } \\
\text { PFACT }\end{array}$ & $\begin{array}{l}1.90 \\
4.21\end{array}$ & $\begin{array}{l}3.50 \\
4.42\end{array}$ & $\begin{array}{l}1.67 \\
3.67\end{array}$ & $\frac{2.54}{5.64}$ & $5 \cdot 57$ & 57.78 \\
\hline
\end{tabular}

IATA FOF FILE : SINGL

\begin{tabular}{|c|c|c|c|c|c|c|}
\hline Ful & $\begin{array}{r}45 n \\
135.0\end{array}$ & $15: 0$ & & $40 . \frac{1}{0}$ & & \\
\hline $\begin{array}{l}\text { COMAF : } \\
\text { MEAN : }\end{array}$ & -1.08 & $\begin{array}{r}\text { CFY } \\
. .02\end{array}$ & $\frac{C F}{24}$ & $\begin{array}{c}04 \% \\
.00\end{array}$ & $\begin{array}{l}\text { CSyY } \\
+0.4\end{array}$ & $\begin{array}{c}0.9 \\
.0 .4\end{array}$ \\
\hline $\begin{array}{l}\text { MAX : } \\
\text { MIIU: } \\
\text { VMS: }\end{array}$ & $\cdots+\frac{44}{91}$ & $\begin{array}{r}.01 \\
.05 \\
.01\end{array}$ & $\begin{array}{r}.10 \\
. \\
.08 \\
.08\end{array}$ & $\begin{array}{l}.07 \\
.04 \\
.01\end{array}$ & $\frac{24}{23}$ & $\begin{array}{l}.00 \\
.00 \\
.02\end{array}$ \\
\hline $\begin{array}{l}\text { GFACT } \\
\text { PFACT }\end{array}$ & $\frac{1}{4} \div 2 \frac{1}{7}$ & $\frac{2}{3} \cdot \frac{39}{71}$ & $\frac{1}{4}: 75$ & 19.93 & 岁: 89 & $5 \cdot 20$ \\
\hline
\end{tabular}

IIATA FOF FILE: SIMOL

\begin{tabular}{|c|c|c|c|c|c|c|}
\hline Furs & $\begin{array}{l}\text { WINII } \\
90.0\end{array}$ & 15.0 & & $\begin{array}{r}\text { YEL } \\
27.0\end{array}$ & & \\
\hline COMF : & $\begin{array}{l}\text { CFX } \\
0 \%\end{array}$ & $\begin{array}{l}C F Y \\
.04\end{array}$ & CF 2 & CMY & C.ry & $\begin{array}{c}\operatorname{crs} \\
.0 \%\end{array}$ \\
\hline $\begin{array}{l}\text { MAX } \\
\text { MIHS } \\
\text { FMS }\end{array}$ & $\begin{array}{r}.39 \\
.15 \\
.12\end{array}$ & $\begin{array}{r}-.01 \\
.10 \\
.01\end{array}$ & .12 & .03 &.$\frac{1}{1}$ & $\begin{array}{l}.00 \\
.01 \\
.01\end{array}$ \\
\hline $\begin{array}{l}\text { GFACT } \\
\text { PFACT }\end{array}$ & $21 \frac{1}{3} \cdot \frac{10}{32}$ & $\frac{2}{4} \cdot \frac{20}{72}$ & $7 \cdot 01$ & $3 \cdot 60$ & $3 \cdot 4: 2$ & $\frac{7}{3} \div 2$ \\
\hline
\end{tabular}


DATA FOF: FILE: SIHGL

\begin{tabular}{|c|c|c|c|c|c|c|}
\hline Fiup $\underbrace{}_{-44}$ & $\begin{array}{l}\text { WINI } \\
45.0\end{array}$ & 15.0 & & & & \\
\hline $\begin{array}{l}\text { CQPAF } \\
\text { TEEAH }\end{array}$ & $\begin{array}{l}\mathrm{CF}^{-} \\
.97\end{array}$ & $\begin{array}{c}\text { C.FY } \\
.03\end{array}$ & $\begin{array}{c}c 5 z \\
253\end{array}$ & $\begin{array}{l}\text { C.MY } \\
.01\end{array}$ & .04 & $\begin{array}{c}\operatorname{crg} z \\
.01\end{array}$ \\
\hline $\begin{array}{l}\text { MAX } \\
\text { MIMS } \\
\text { FHS }\end{array}$ & $\begin{array}{r}1.80 \\
.98 \\
.20\end{array}$ & $\begin{array}{r}.02 \\
.08 \\
.01\end{array}$ & $\begin{array}{l}+46 \\
104 \\
04\end{array}$ & $\begin{array}{l}.05 \\
.03 \\
.01\end{array}$ & .20 & $\begin{array}{l}.02 \\
.05 \\
.01\end{array}$ \\
\hline $\begin{array}{l}\text { GFACT } \\
\text { FFACT }\end{array}$ & $\frac{1}{4.85}$ & $\begin{array}{l}2.30 \\
4.07\end{array}$ & $1 \cdot \frac{6}{2} 0$ & $\begin{array}{l}4 \cdot 24 \\
4,37\end{array}$ & 9.81 & $\frac{3}{7}+\frac{1}{2} \frac{1}{7}$ \\
\hline
\end{tabular}

IIATA FOF FILE: SINGL

\begin{tabular}{|c|c|c|c|c|c|c|}
\hline Furt & WIIII & $15+0$ & & $\begin{array}{r}\text { vel. } \\
40.1\end{array}$ & & \\
\hline COMF: & CFy & $\begin{array}{l}\text { CFY } \\
+0 O\end{array}$ & 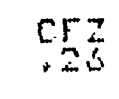 & $\begin{array}{l}\text { cry } \\
.04\end{array}$ & $\begin{array}{l}\mathrm{CHY} \\
.0 \%\end{array}$ & $\begin{array}{c}c k z \\
+05\end{array}$ \\
\hline $\begin{array}{l}\text { MAX: } \\
\text { MIHE } \\
\text { FMSE: }\end{array}$ & 1. $\frac{97}{59}$ & $\begin{array}{l}.03 \\
.03 \\
.01\end{array}$ & $\begin{array}{l}.01 \\
.15 \\
.04\end{array}$ & $\begin{array}{l}.00 \\
.00 \\
.01\end{array}$ & : &. $\begin{array}{l}11 \\
.00 \\
02\end{array}$ \\
\hline $\begin{array}{l}\text { SFACT } \\
\text { FACT }\end{array}$ & $\frac{1}{3} \cdot 29$ & 12.75 & $\frac{1}{3} .55$ & $\frac{2}{3} \cdot \frac{7}{9}$ & 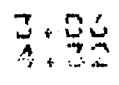 & 1.78 \\
\hline
\end{tabular}


APPENDIX $C$

WIND-TUNNEL DATA FOR ISOLATED HELIOSTAT 
Heliostat 1, Configuration 1-A

\begin{tabular}{|c|c|c|c|c|c|c|}
\hline Caste & Run 非 & WD & EL & $\mathrm{AZ}$ & Day & Time \\
\hline \multirow[t]{7}{*}{$\operatorname{Sin} r$ AM } & 348 & 0 & 25 & 350 & 172 & $8 \mathrm{AM}$ \\
\hline & 349 & 337.5 & 25 & 350 & 172 & $8 \mathrm{AH}$ \\
\hline & 350 & 315 & 25 & 350 & 172 & $8 \mathrm{AM}$ \\
\hline & 351 & 292.5 & 25 & 350 & 172 & 8 AN \\
\hline & 352 & 270 & 25 & 350 & 172 & $8 \mathrm{AN}$ \\
\hline & 353 & 265 & 25 & 350 & 172 & $8 \mathrm{AM}$ \\
\hline & 354 & 247.5 & 25 & 350 & 172 & $8 \mathrm{AN}$ \\
\hline \multirow[t]{7}{*}{ Simr Noon } & 355 & 247.5 & 45 & 305 & 172 & $0 \mathrm{PM}$ \\
\hline & 356 & 265 & 45 & 305 & 172 & $0 \mathrm{PM}$ \\
\hline & 357 & 270 & 45 & 305 & 172 & $0 \mathrm{Pl}: 1$ \\
\hline & 358 & 292.5 & 45 & 305 & 172 & $0 \mathrm{PM}$ \\
\hline & 359 & 315 & 45 & 305 & 172 & 0 PM \\
\hline & 360 & 337.5 & 45 & 305 & 172 & $0 \mathrm{PN}$ \\
\hline & 361 & 0 & 45 & 305 & 172 & $0 P M$ \\
\hline \multirow[t]{7}{*}{ Whl $\mathrm{AM}$} & 331 & 0 & 10 & 330 & 355 & $8 \mathrm{AN}$ \\
\hline & 325 & 337.5 & 10 & 330 & 355 & $8 \mathrm{AM}$ \\
\hline & 326 & 315 & 10 & 330 & 355 & $8 \mathrm{Al}$ \\
\hline & 327 & 292.5 & 10 & 330 & 355 & $8 \mathrm{ANI}$ \\
\hline & 328 & 270 & 10 & 330 & 355 & $8 \mathrm{AM}$ \\
\hline & 329 & 265 & 10 & 330 & 355 & $8 \mathrm{AM}$ \\
\hline & 330 & 247.5 & 10 & 330 & 355 & $8 \mathrm{AM}$ \\
\hline
\end{tabular}




\begin{tabular}{|c|c|c|c|c|c|c|}
\hline Case & Run 非 & WD & $\mathrm{EL}$ & $\mathrm{AZ}$ & Day & T'ime \\
\hline \multirow[t]{7}{*}{ Wintr Noon } & 345 & 0 & 20 & 305 & 355 & $0 \quad P M$ \\
\hline & 344 & 337.5 & 20 & 305 & 355 & o $\mathrm{PNH}$ \\
\hline & 343 & 315 & 20 & 305 & 355 & 0 IN \\
\hline & 342 & 292.5 & 20 & 305 & 355 & 0 Pil \\
\hline & 341 & 270 & 20 & 305 & 355 & $0 \mathrm{PN}$ \\
\hline & 340 & 265 & 20 & 305 & 355 & $0 \mathrm{PN}$ \\
\hline & 339 & 247.5 & 20 & 305 & 355 & $0 \mathrm{lil}$ \\
\hline \multirow{7}{*}{ Wutr PM } & 338 & 247.5 & 10 & 275 & 355 & $4 \mathrm{PNI}$ \\
\hline & 337 & 265 & 10 & 275 & 355 & $4 P N$ \\
\hline & 336 & 270 & 10 & 275 & 355 & $4 \mathrm{PNI}$ \\
\hline & 335 & 292.5 & 10 & 275 & 355 & $4 P N$ \\
\hline & 334 & 315 & 10 & 275 & 355 & $4 \mathrm{PN1}$ \\
\hline & 333 & 337.5 & 10 & 275 & 355 & $4 \mathrm{PN}$ \\
\hline & 332 & 0 & 10 & 275 & 355 & $4 \mathrm{PM}$ \\
\hline Ver. Stow & 346 & 337.5 & 0 & 330 & 355 & $8 A M$ \\
\hline Hor. Stow & 347 & 337.5 & 90 & 330 & 355 & $8 \mathrm{AN}$ \\
\hline
\end{tabular}




\begin{tabular}{|c|c|c|c|c|c|c|}
\hline Sư⿱ & $=\frac{U I ! I}{5}$ & $\begin{aligned} 51 \\
10\end{aligned}$ & $=20 . \overline{0}$ & 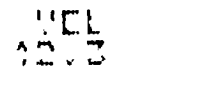 & & \\
\hline $\begin{array}{l}\text { cons: } \\
\text { ment! }\end{array}$ & cro & 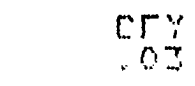 & $\mathrm{Cr}_{13}$ & çis & $\begin{array}{l}\mathrm{CH} \\
11\end{array}$ & 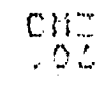 \\
\hline 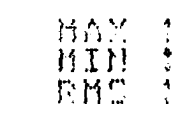 & $\begin{array}{l}30 \\
30 \\
0\end{array}$ & .01 & .78 & .50 & $\hat{j}^{4}$ & .11 \\
\hline $\begin{array}{l}\text { ACT } \\
\text { HACT }\end{array}$ & $2 \cdot 15$ & 4.00 & $\frac{1}{2} \cdot \frac{2}{2}$ & $=\frac{1}{6}$ & $\therefore 20$ & 1098 \\
\hline
\end{tabular}

MTA FOF FILE: H100

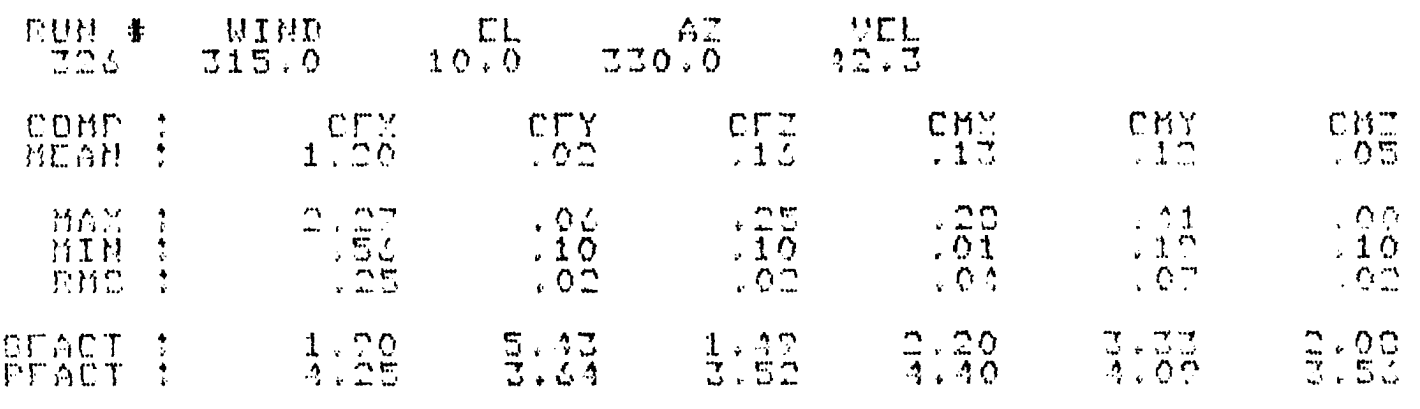

IATA TOE FILE : H1100

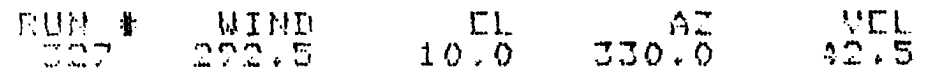

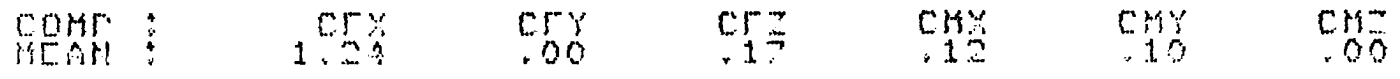

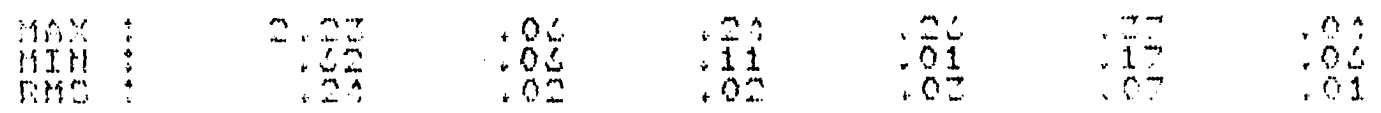

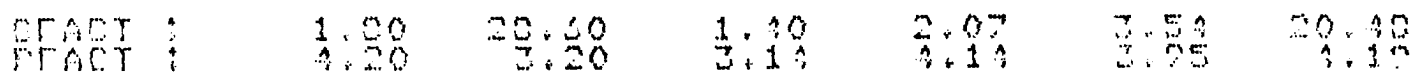

IATA FOE FILE: H1100

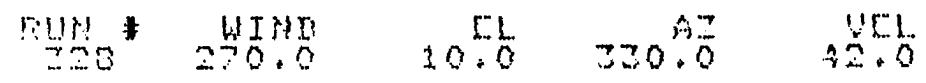

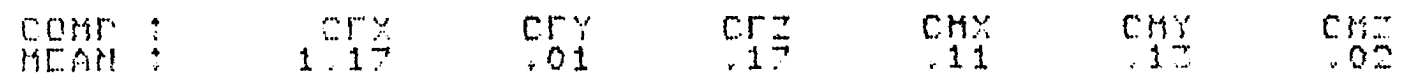

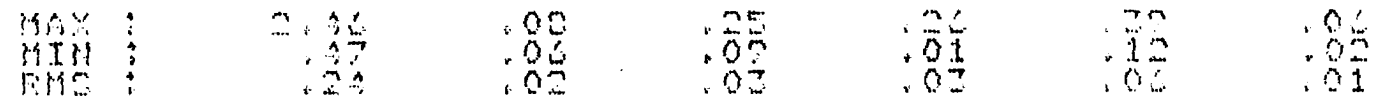

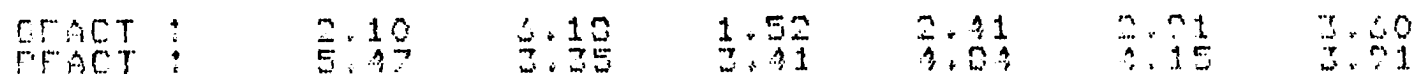




\begin{tabular}{|c|c|c|c|c|c|c|}
\hline rus! & 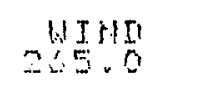 & $10 \div 0$ & $x=0: 0$ & 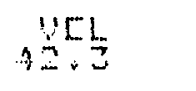 & & \\
\hline monr & cro & $\begin{array}{l}65 x \\
.02\end{array}$ & 65 & $\begin{array}{l}0 \% \% \\
0 \%\end{array}$ & $\begin{array}{l}\operatorname{csi} \\
.10\end{array}$ & 64 \\
\hline $\begin{array}{l}\text { MAY } \\
\text { MIHE } \\
\text { FMS }\end{array}$ & 1. & .09 & .20 & $\begin{array}{l}9 \\
.03 \\
03\end{array}$ & $\begin{array}{l}7 \\
13 \\
0\end{array}$ & 20 \\
\hline $\begin{array}{l}\text { GTACT } \\
\text { PFACT }\end{array}$ & $1 \cdot 71$ & $\begin{array}{l}5 \cdot 52 \\
3 \cdot 6 \\
5\end{array}$ & $\frac{1}{7} \cdot 20$ & 5.20 & $\Xi \cdot 37$ & $3: 20$ \\
\hline
\end{tabular}

IIATA FOF: FILE: H1100

\begin{tabular}{|c|c|c|c|c|c|c|}
\hline $\lim _{x \rightarrow \infty}$ & $\ln _{3}^{4} \frac{I}{9}$ & 10.5 & $3=0.0$ & 32.5 & & \\
\hline coms & $1: 01$ & $\begin{array}{l}c 5 y \\
0 y\end{array}$ & $\begin{array}{l}65 z \\
.15\end{array}$ & ${ }_{0.0}$ & $\begin{array}{l}c+4 y \\
.05\end{array}$ & 89 \\
\hline 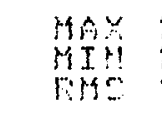 & 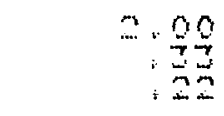 & $\begin{array}{r}10 \\
.05 \\
.05\end{array}$ & .25 & $\begin{array}{l}20 \\
07 \\
0=\end{array}$ & $=0$ & .10 \\
\hline $\begin{array}{l}\text { EACT } \\
\text { FACT }\end{array}$ & $\frac{1}{3} \cdot 5 \frac{2}{51}$ & $3.2_{-1} 1$ & $\frac{1}{4} \cdot 40$ & 2.5 & 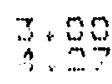 & $\therefore y$ \\
\hline
\end{tabular}

DATA FOF FILE: H1100

\begin{tabular}{|c|c|c|c|c|c|c|}
\hline$\underset{11}{ }$ & $\begin{array}{l}4 \pi ! n \\
0.0\end{array}$ & 10.5 & & $7 \div$ & & \\
\hline $\begin{array}{l}\text { CoMT } \\
\text { BEAT! }\end{array}$ & $2 \%$ & $\begin{array}{c}c 5 y \\
01\end{array}$ & .0 .3 & 04 & $\begin{array}{cc}C M Y \\
0 \%\end{array}$ & 83 \\
\hline $\begin{array}{l}\text { WAS } \\
\text { WIHE }\end{array}$ & 1. .01 & .05 & $\begin{array}{l}10 \\
.00 \\
.01\end{array}$ & .16 & $\frac{8}{80}$ & .01 \\
\hline GFACT & $\begin{array}{l}309 \\
5.32\end{array}$ & 13.78 & 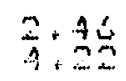 & $\begin{array}{l}3: 06 \\
3.02\end{array}$ & $19: 47$ & $3: 04$ \\
\hline
\end{tabular}

\section{IATA FDF FILE: HIIOO}

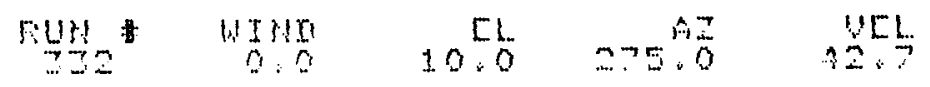

\begin{tabular}{|c|c|c|c|c|c|c|}
\hline coms & $05 \%$ & $\begin{array}{l}65 \\
609\end{array}$ & $05=$ & $\begin{array}{c}c m o \\
.00\end{array}$ & 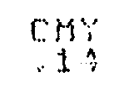 & CHA \\
\hline $\begin{array}{l}\text { MAY } \\
\text { RIHE }\end{array}$ & 1. & $\begin{array}{l}16 \\
.05 \\
.05\end{array}$ & $\begin{array}{l}10 \\
.02 \\
.02\end{array}$ & $\begin{array}{l}.05 \\
.02 \\
.03\end{array}$ & $\frac{50}{50}$ & .3 \\
\hline PFACT & $\exists \cdot \frac{1}{3}$ & $3: 3.1$ & 3.17 & $\begin{array}{r}A 1 \\
3\end{array}$ & 3.5 & $\begin{array}{l}7 \\
9\end{array}$ \\
\hline
\end{tabular}




\begin{tabular}{|c|c|c|c|c|c|c|}
\hline 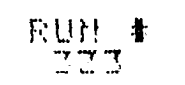 & 35 & 10.0 & 275.0 & $\begin{array}{r}\text { VEL } \\
12.0\end{array}$ & & \\
\hline $\begin{array}{l}\text { Coms } \\
\text { mEnt }\end{array}$ & $\begin{array}{r}05 \% \\
1.10\end{array}$ & .05 & .05 & $\begin{array}{l}\text { cus } \\
.0 \pm\end{array}$ & 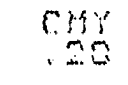 & $\cos =$ \\
\hline 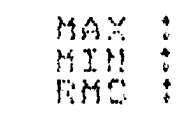 & $\begin{array}{r}1 \cdot 06 \\
\cdot 25\end{array}$ & $.0 \frac{17}{02}$ & $.0 \%$ & $\begin{array}{r}.05 \\
.09 \\
.02\end{array}$ & $\begin{aligned} 07 \\
05 \\
01\end{aligned}$ & $\begin{array}{l}02 \\
.02 \\
.01\end{array}$ \\
\hline GFACT & $\frac{1}{2}: 2$ & $\frac{1}{2} \cdot 87$ & $\frac{1}{2} \cdot \frac{59}{3}$ & 5.00 & $\frac{1}{3} \cdot 56$ & $\begin{array}{l}3.37 \\
3.00\end{array}$ \\
\hline MATA F & IF FILE: & $H 1100$ & & & & \\
\hline ritus: & $\begin{array}{l}\text { WINT } \\
315.0\end{array}$ & ${ }_{10.0}^{5 L}$ & $3 \Xi 0 . \hat{A}$ & $42 \frac{V}{2}$ & & \\
\hline COMF : & 05 & $05 \%$ & .05 & crs & CHY & 6 \\
\hline $\begin{array}{l}\text { MAS : } \\
\text { HIS! } \\
\text { PHOS: }\end{array}$ & 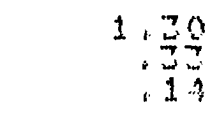 & $1: \frac{7}{5}$ &.$\frac{15}{02} .05$ & $.0 \%$ & .02 &. $\begin{array}{l}11 \\
.01 \\
.01\end{array}$ \\
\hline GFACT & $\frac{1}{4} \cdot 72$ & 4.03 & 1.52 & $\frac{1}{1}: \frac{7}{10}$ & 1.92 & $\frac{1}{3} \cdot 23$ \\
\hline
\end{tabular}

IATA FOF FILE: H1100

\begin{tabular}{|c|c|c|c|c|c|c|}
\hline Fun & $\begin{array}{r}\text { WINI } \\
292.5\end{array}$ & 10.0 & & YEL & & \\
\hline $\begin{array}{l}\text { COMO } \\
\text { MEAN }\end{array}$ & 95 & $\begin{array}{l}\text { CFY } \\
.11\end{array}$ & $\begin{array}{l}\mathrm{cr} \\
.11\end{array}$ & $\begin{array}{l}\cos y \\
.00\end{array}$ & CHY & $\begin{array}{l}\operatorname{cis} \\
.00\end{array}$ \\
\hline $\begin{array}{l}\text { MAX } \\
\text { MAIIE }\end{array}$ & $\begin{array}{r}1 \cdot 50 \\
\cdot 20 \\
-20\end{array}$ & $\begin{array}{l}20 \\
020 \\
05\end{array}$ & $\begin{array}{l}10 \\
0.02 \\
02\end{array}$ & .05 & .02 & .14 \\
\hline SACT & $\frac{1}{3} \cdot 7=$ & $\frac{1}{3} .87$ & $\frac{1}{5.29}$ & $38.5 ?$ & $\frac{1}{7}+2 \%$ & 1.8 \\
\hline
\end{tabular}

\section{IATA FOF FILE: H1100}

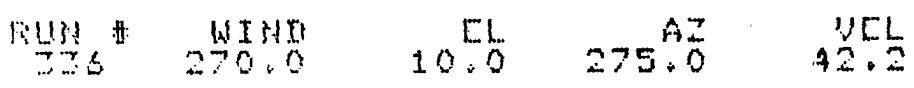

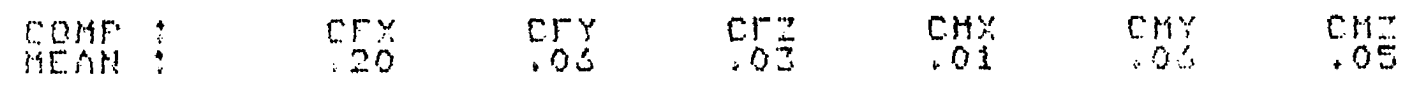

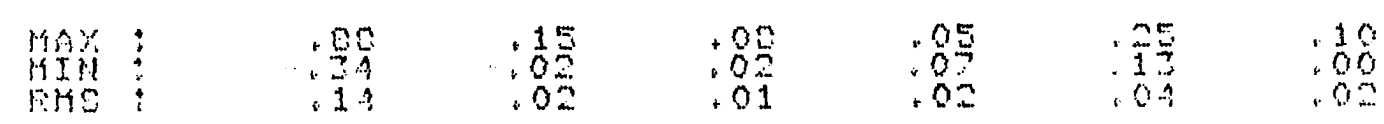

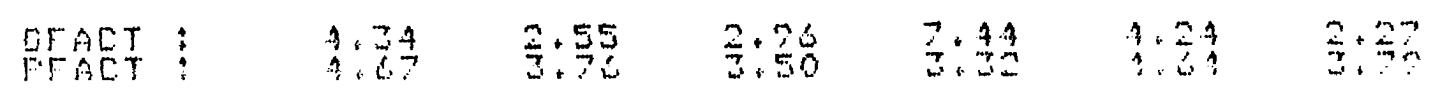




\begin{tabular}{|c|c|c|c|c|c|c|}
\hline 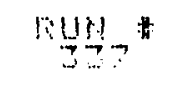 & 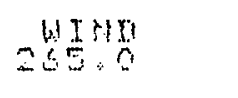 & $10 E$ & 25.0 & $4=\frac{y}{3}$ & & \\
\hline CORE & C. & $\begin{array}{c}\operatorname{cr} \\
.04\end{array}$ & $\begin{array}{cc}C 2 \\
.01\end{array}$ & $\begin{array}{c}c m \\
.01\end{array}$ & $\begin{array}{l}c r y \\
0.2\end{array}$ & $03 x$ \\
\hline $\begin{array}{l}\text { MAX: } \\
\text { Firi: } \\
\text { FMS }\end{array}$ & .62 & $\begin{array}{l}12 \\
.04 \\
.02\end{array}$ & $\begin{array}{r}.05 \\
.07 \\
.02\end{array}$ & .95 & $=\begin{array}{l}17 \\
16 \\
0.4\end{array}$ & $\begin{array}{r}98 \\
.39\end{array}$ \\
\hline GFACT & $13 \cdot 7 \cdot 3$ & $\frac{2}{3} \cdot 37$ & $1 \frac{1}{2} \cdot \frac{5}{51}$ & $\frac{9}{2}+8$ & $7+59$ & $2 \cdot 43$ \\
\hline IHATA FI & DF: FILE : & $H 1100$ & & & & \\
\hline 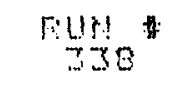 & $23=5$ & ${ }_{10.0}^{E L}$ & $=75 . A$ & $\begin{array}{l}415 \\
2.3\end{array}$ & & \\
\hline ComF : & $\begin{array}{c}C F Y \\
-54\end{array}$ & $\begin{array}{l}c .5 y \\
.01\end{array}$ & $\frac{c r}{10}$ & $\begin{array}{l}\text { cis: } \\
.01\end{array}$ & $\begin{array}{l}\text { cry } \\
.11\end{array}$ & 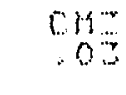 \\
\hline $\begin{array}{l}\text { MaY : } \\
\text { HMS } \\
\text { FHE: }\end{array}$ & $\begin{array}{r}: 04 \\
1: 46 \\
: 19\end{array}$ & $\begin{array}{r}+10 \\
.08 \\
.05\end{array}$ & $\cdot \frac{02}{23}$ & $+\frac{1}{61}$ & .0 .03 & $\begin{array}{r}.02 \\
.03 \\
.02\end{array}$ \\
\hline $\begin{array}{l}\text { OFACT } \\
\text { FFACT }\end{array}$ & $\frac{3}{4} \cdot 6$ & $16 \cdot 30$ & $\frac{3}{4} \cdot 25$ & $\frac{3}{2}: \frac{31}{6}$ & $=20$ & 3.01 \\
\hline INATA F & OF: FILE : & $H 3100$ & & & & \\
\hline $\operatorname{sum}_{3.9}$ & $2+7=5$ & $=0.0$ & 305.0 & $42 \cdot 5$ & & \\
\hline COMAF & $\operatorname{Cr}_{2}$ & $\begin{array}{l}\text { Cry } \\
.05\end{array}$ & $\begin{array}{cr}\mathrm{cr} z \\
.07\end{array}$ & $\begin{array}{c}c 40 \\
.03\end{array}$ & $\begin{array}{c}\operatorname{cm} Y \\
01\end{array}$ & $\begin{array}{l}\operatorname{cro} \\
.0 \%\end{array}$ \\
\hline 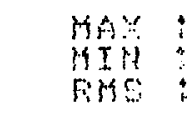 & $: \frac{1}{2}$ & $\begin{array}{l}.14 \\
.06 \\
.05\end{array}$ & $\begin{array}{l}10 \\
.02 \\
.05\end{array}$ & $\begin{array}{l}11: \\
.05 \\
+0\end{array}$ & .21 & .00 \\
\hline $\begin{array}{l}\text { GFACT } \\
\text { FFACT }\end{array}$ & $3 \cdot 5 \frac{5}{3}$ & $\dot{3} \cdot 9$ & $\frac{2}{3} \cdot 5 \frac{5}{5}$ & $3 \cdot 3$ & $1: 20$ & $\therefore .70$ \\
\hline
\end{tabular}

INATA FOF FILE: H1100

\begin{tabular}{|c|c|c|c|c|c|c|}
\hline $\operatorname{lum}_{x \rightarrow 0}$ & $=\ln 2 \ln$ & $\begin{aligned} & 51 \\
&=0.0\end{aligned}$ & 305.0 & 42.5 & & \\
\hline MONSF: & 05 & $\begin{array}{l}\text { Cry } \\
.01\end{array}$ & $\frac{c r z}{23}$ & $6 \%$ & CHS & 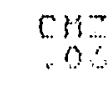 \\
\hline $\begin{array}{l}\text { MAs } \\
\text { MIHA }\end{array}$ & 1. $\frac{10}{120}$ &.$\frac{12}{10}$ & $\begin{array}{l}37 \\
.03 \\
.04\end{array}$ & .09 & $\begin{array}{r}92 \\
.85 \\
.05\end{array}$ & $\begin{array}{r}11 \\
.01 \\
.01\end{array}$ \\
\hline $\begin{array}{l}\text { FACT } \\
\text { FACT }\end{array}$ & $\frac{1}{2}: \frac{9}{9}$ & $=\frac{7}{8} \cdot 3$ & $\frac{1}{3} \cdot 65$ & 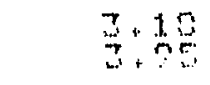 & $2 \cdot 20$ & $\begin{array}{l}1.93 \\
-1.90\end{array}$ \\
\hline
\end{tabular}




\begin{tabular}{|c|c|c|c|c|c|c|}
\hline $\operatorname{lin}_{>1}$ & $2 \rightarrow 0.0$ & $\begin{array}{r}51 . \\
20.0\end{array}$ & 305.0 & $\begin{array}{r}y[1 \\
42.3\end{array}$ & & \\
\hline $\begin{array}{l}\text { Comp : } \\
\text { MEAH : }\end{array}$ & 05 & $\begin{array}{r}c 5 y \\
-0.1\end{array}$ & $\operatorname{cr}_{2} 6$ & $\begin{array}{c}c m \\
0 \%\end{array}$ & $\begin{array}{r}\operatorname{cis} \\
10\end{array}$ & $\begin{array}{l}\mathrm{CH} \\
.05\end{array}$ \\
\hline $\begin{array}{l}\text { MAX } \\
\text { MHES }\end{array}$ & $\begin{array}{l}1.60 \\
10\end{array}$ & $\begin{array}{l}.05 \\
.12 \\
.03\end{array}$ & $\begin{array}{l}40 \\
+105 \\
.05\end{array}$ & .15 & .75 &. $\begin{array}{l}11 \\
.01\end{array}$ \\
\hline $\begin{array}{l}\text { OFACT } \\
\text { HFACT }\end{array}$ & $\begin{array}{l}1.38 \\
2.80\end{array}$ & $\frac{73}{79}$ & $\begin{array}{l}1.54 \\
7.41\end{array}$ & $\begin{array}{l}3.20 \\
3: 70\end{array}$ & $\frac{1}{3} \cdot 9$ & $\Rightarrow+10$ \\
\hline
\end{tabular}

IIATA FOF: FILE: HII0O

\begin{tabular}{|c|c|c|c|c|c|c|}
\hline${ }_{312}$ & 292.5 & $\because 5 \frac{\Gamma}{2}$ & 3.2 & पर다. & & \\
\hline $\begin{array}{l}\text { COYS } \\
\text { MEAS : }\end{array}$ & $\begin{array}{r}65 \\
1.02\end{array}$ & $\therefore \%$ & 65 & .07 & $\begin{array}{c}\text { CMY } \\
.20\end{array}$ & $\begin{array}{c}\operatorname{crs} \\
005\end{array}$ \\
\hline $\begin{array}{l}\text { MAY } \\
\text { MISH } \\
\text { FMS }\end{array}$ & $\begin{array}{r}1.90 \\
.98 \\
.21\end{array}$ & $\begin{array}{l}.05 \\
+15 \\
+05\end{array}$ & $\begin{array}{l}73 \\
10 \\
108\end{array}$ & .10 & .93 & 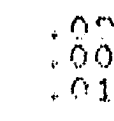 \\
\hline $\begin{array}{l}\text { OPAT } \\
\text { FिAC }\end{array}$ & $4 \cdot 95$ & $\begin{array}{l}3.49 \\
3.07\end{array}$ & $\frac{1}{2}+52$ & 5.57 & 3.16 & $2+0$ \\
\hline XATA $F$ & FE FILE: & $H 1100$ & & & & \\
\hline 048 & $\begin{array}{r}\text { bIIIS } \\
215.6\end{array}$ & $\begin{array}{r}E L \\
20.0\end{array}$ & $\begin{array}{l}A z \\
+0\end{array}$ & $420 \frac{1}{5}$ & & \\
\hline $\begin{array}{l}\text { MOMF } \\
\text { MEAM }\end{array}$ & cry & $\begin{array}{c}\operatorname{cry} \\
-.05\end{array}$ & 0.5 & $\begin{array}{l}c k \% \\
111\end{array}$ & Csy & $\mathrm{Crs}$ \\
\hline $\begin{array}{l}\text { MAY } \\
\text { MIII } \\
\text { FHE : }\end{array}$ & $\begin{array}{r}2.05 \\
.55 \\
.5\end{array}$ & .05 & $\begin{array}{r}.50 \\
.52 \\
.04\end{array}$ & $.0 \frac{1}{2}$ & .85 & .02 \\
\hline $\begin{array}{l}\text { ORACT } \\
\text { PRACT }\end{array}$ & $2: 201$ & 2.01 & 1.79 & $\frac{1}{4} \cdot 8$. & 3.90 & 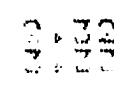 \\
\hline
\end{tabular}

WATA FOF FILE: H1100

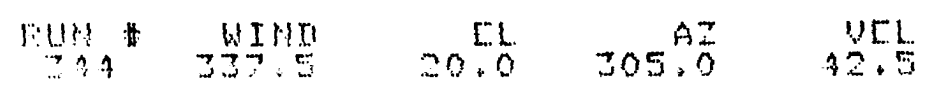

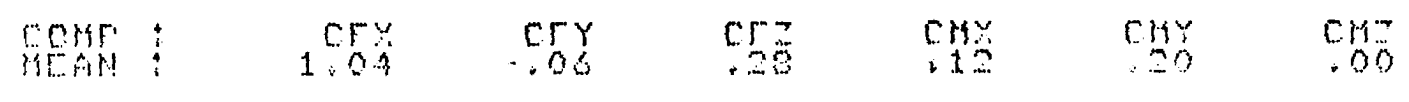

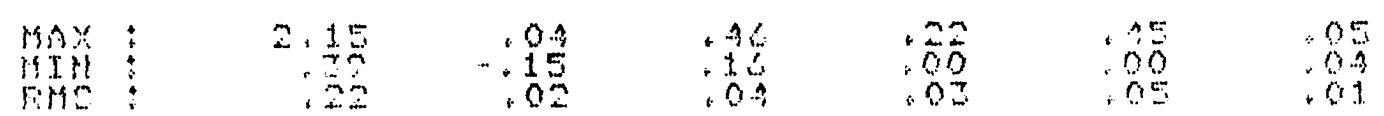

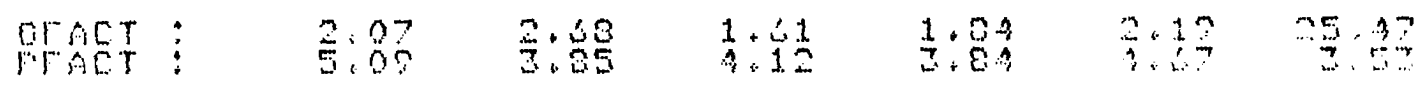




\begin{tabular}{|c|c|c|c|c|c|c|}
\hline Furt & WTin & 20.5 & 305.0 & $\begin{array}{l}251 \\
225\end{array}$ & & \\
\hline Coner : & 25 & $\cos$ & .05 & Coy & $\begin{array}{l}C M Y \\
.05\end{array}$ & $\mathrm{Cr}^{3}$ \\
\hline $\begin{array}{l}\text { MAY } \\
\text { HIHE }\end{array}$ & 1. & $\begin{array}{l}11 \\
10.4 \\
.04\end{array}$ & $\begin{array}{r}25 \\
052 \\
05\end{array}$ & $\begin{array}{r}15 \\
.05 \\
0 \%\end{array}$ & $\begin{array}{l}30 \\
81 \\
.06\end{array}$ & $\begin{array}{r}06 \\
.01\end{array}$ \\
\hline $\begin{array}{l}\text { QFACT } \\
\text { FFACT }\end{array}$ & 5.10 & 12.57 & $\overrightarrow{3} \cdot \dot{3}$ & $\frac{717}{3}: \frac{1}{2}$ & $4 \cdot 15$ & 3.00 \\
\hline
\end{tabular}

IATA FOF FILE: H1100

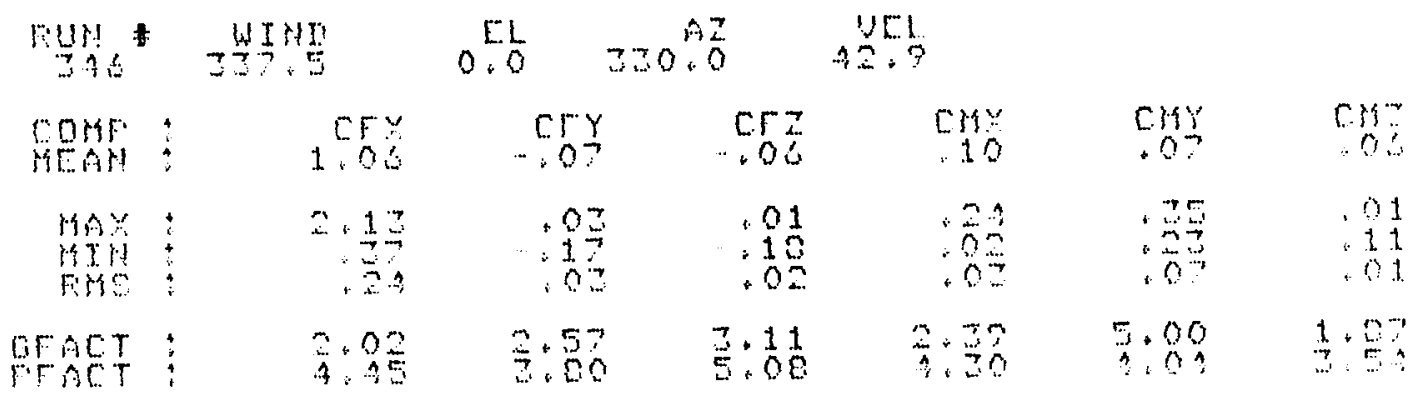

IATA FOF FILE: H1100

\begin{tabular}{|c|c|c|c|c|c|c|}
\hline $\operatorname{lum}_{y \rightarrow}$ & $-72+5$ & 90.5 & & & & \\
\hline Corsto & 856 & $\begin{array}{c}c y \\
0 s\end{array}$ & $\begin{array}{cc}\mathrm{Cr}^{2} \\
-10\end{array}$ & $\begin{array}{c}c y Y \\
.01\end{array}$ & $\begin{array}{l}C H Y \\
: O 1\end{array}$ & $8 \mathrm{CH}^{\circ}$ \\
\hline $\begin{array}{l}\text { MAY } \\
\text { MIM }\end{array}$ & .15 & .01 & $\begin{array}{l}.10 \\
.96 \\
.05\end{array}$ & .05 & $\begin{array}{r}07 \\
.05 \\
.05\end{array}$ & $\begin{array}{l}0 \% \\
01\end{array}$ \\
\hline CFACT & $=12$ & $\frac{m}{3}: \frac{m}{45}$ & 9.77 & 4.50 & 9.99 & 2.93 \\
\hline
\end{tabular}

DATA FOF FILE: H1100

\begin{tabular}{|c|c|c|c|c|c|c|}
\hline 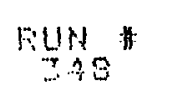 & $\begin{array}{l}4 \pi \mathrm{I} \\
0.0\end{array}$ & $\begin{array}{l}E L \\
25.0\end{array}$ & & $4 \frac{4+2}{2}$ & & \\
\hline $\begin{array}{l}\text { COMF : } \\
\text { MEAN : }\end{array}$ & CF & $\begin{array}{l}\operatorname{cry} \\
0.05\end{array}$ & $\cos$ & $\begin{array}{l}\mathrm{cm} \\
.05\end{array}$ & $\begin{array}{l}c y Y \\
0.0 ?\end{array}$ & 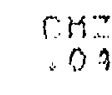 \\
\hline $\begin{array}{l}\text { MAY : } \\
\text { MIM } \\
\text { FM: }\end{array}$ & $\begin{array}{l}20 \\
10 \\
18\end{array}$ & $\begin{array}{l}.00 \\
.11 \\
.02\end{array}$ & $\begin{array}{l}29 \\
.00 \\
.05\end{array}$ & $\begin{array}{l}.12 \\
.05 \\
.05\end{array}$ & .25 & $\begin{array}{r}.01 \\
.10 \\
.00\end{array}$ \\
\hline FACT: & $\frac{7}{7} \cdot 22$ & $\frac{3}{5}+\frac{1}{3}$ & $\frac{3}{3} \cdot 5 \frac{1}{5}$ & $\frac{2}{3} \cdot \frac{1}{51}$ & $13 \cdot 70$ & $3: 3$ \\
\hline
\end{tabular}




\begin{tabular}{|c|c|c|c|c|c|c|}
\hline Fum & $\begin{array}{l}4 I N I I \\
37.5\end{array}$ & $=5.0$ & & $\begin{array}{l}\text { VEL. } \\
32.0\end{array}$ & & \\
\hline $\begin{array}{l}\text { Coms : } \\
\text { rEnts : }\end{array}$ & $\begin{array}{c}C 5 \\
.20\end{array}$ & $\begin{array}{l}\operatorname{cry} \\
405\end{array}$ & 5.5 & $\begin{array}{l}\text { CMP } \\
.06\end{array}$ & $\begin{array}{c}\text { cusy } \\
.05\end{array}$ & $.0 \%$ \\
\hline 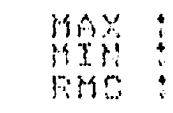 & 1.72 & .01 & $\begin{array}{l}50 \\
.17 \\
.07\end{array}$ & .15 & $\begin{array}{l}.75 \\
.25 \\
.00\end{array}$ & $\begin{array}{l}.09 \\
.03 \\
.05\end{array}$ \\
\hline $\begin{array}{l}\text { QFACT } \\
\text { FACT }\end{array}$ & $\frac{1}{2} \cdot 62$ & $\frac{2}{4.44}$ & $\frac{1}{3} \cdot 2 \cdot 6$ & $\begin{array}{l}5 \\
5\end{array}$ & $13=27$ & $\therefore 79$ \\
\hline
\end{tabular}

\section{IIATA FOF FILE: H1100}

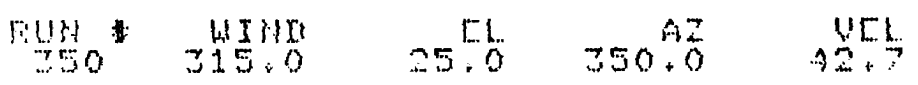

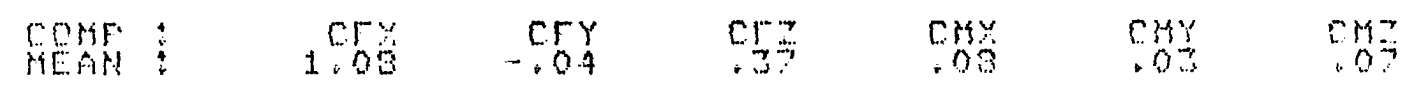

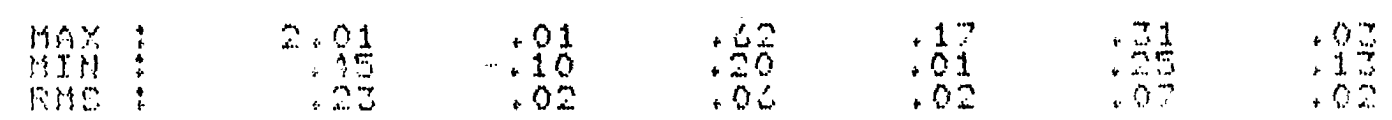

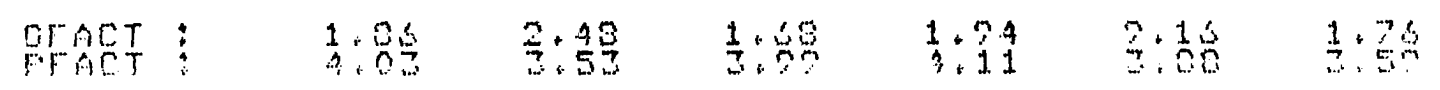

IATA FOF FILE: H1100

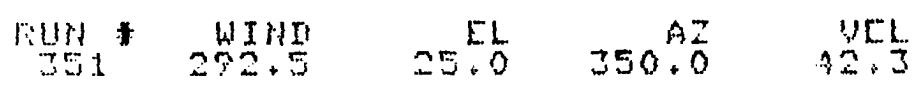

\begin{tabular}{|c|c|c|c|c|c|}
\hline Coms : & 1.5 & $\cos$ & 65 & $\begin{array}{l}c s y \\
.06\end{array}$ & $\begin{array}{c}C y Y \\
0.94\end{array}$ \\
\hline 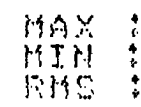 & $\begin{array}{r}2.20 \\
39 \\
25\end{array}$ & $\begin{array}{r}.01 \\
.03 \\
.01\end{array}$ & 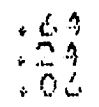 & $: \begin{array}{l}1 \\
: 01 \\
02\end{array}$ & 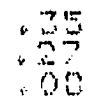 \\
\hline
\end{tabular}

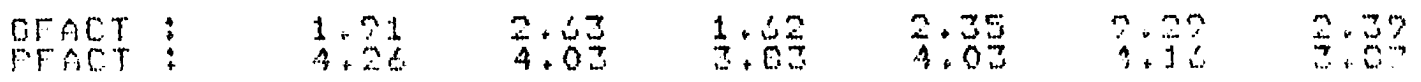

DATA FOF FILE: H1100

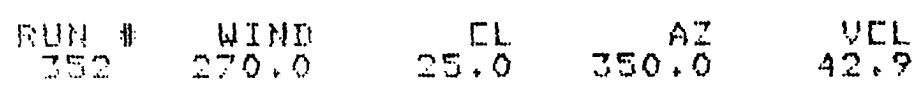

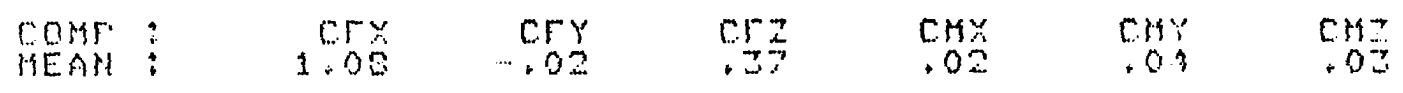

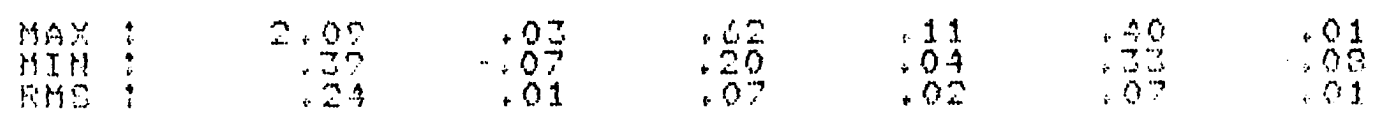

CFACT: 


\begin{tabular}{|c|c|c|c|c|c|c|}
\hline nuts & 265.15 & 25.6 & $350: 02$ & 42 도. & & \\
\hline COHF : & 1.06 & $\begin{array}{c}\cos \\
.02\end{array}$ & $\frac{05}{25}$ & $\begin{array}{l}\mathrm{CM} \\
.01\end{array}$ & cry & $\begin{array}{l}0.117 \\
.00\end{array}$ \\
\hline $\begin{array}{l}\text { MAX } \\
\text { MISH } \\
\text { RME }\end{array}$ & $\begin{array}{r}2.13 \\
.45 \\
32\end{array}$ & $\begin{array}{r}.02 \\
.03 \\
.01\end{array}$ & $\begin{array}{r}.62 \\
.20 \\
.05\end{array}$ & .05 & $\begin{array}{l}.25 \\
.28 \\
.07\end{array}$ & $\begin{array}{r}05 \\
.01 \\
01\end{array}$ \\
\hline GFACT & 1.93 & $\frac{3}{3} \cdot 20$ & $\frac{1}{4}+\frac{5}{1}$ & $\begin{array}{l}4 \\
4 \\
3\end{array}$ & 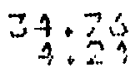 & $10+60$ \\
\hline
\end{tabular}

IMTA FOF FILE: H1100

\begin{tabular}{|c|c|c|c|c|c|c|}
\hline Fust & $\begin{aligned} 414 I I \\
237\end{aligned}$ & $=5.6$ & & UEL & & \\
\hline COMF : & .68 & $\begin{array}{l}.05 \\
.00\end{array}$ & 65 & .01 & $\begin{array}{l}C H Y \\
0.05\end{array}$ & .00 \\
\hline $\begin{array}{l}\text { WAX } \\
\text { WYS } \\
\text { SWS }\end{array}$ & $\begin{array}{l}1.97 \\
.51 \\
.51\end{array}$ & $\begin{array}{r}+85 \\
+05 \\
.01\end{array}$ & $\begin{array}{l}.54 \\
.12 \\
.05\end{array}$ & $\begin{array}{l}07 \\
60 \\
05\end{array}$ & 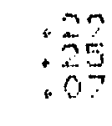 & $\begin{array}{r}61 \\
.68 \\
61\end{array}$ \\
\hline $\begin{array}{l}\text { GFACT } \\
\text { FFACT }\end{array}$ & $\frac{1.21}{4.16}$ & $37 \cdot 47$ & $\frac{1}{3} \cdot 52$ & $\begin{array}{l}5.59 \\
3.60\end{array}$ & $\frac{7}{3}+5 \%$ & 51.55 \\
\hline
\end{tabular}

IATA FOF FILE: H1100

\begin{tabular}{|c|c|c|c|c|c|c|}
\hline Ruts & $\begin{array}{r}4 I+15 \\
297.5\end{array}$ & $\begin{array}{r}E L \\
45.0\end{array}$ & & 42.5 & & \\
\hline $\begin{array}{l}\text { ComF : } \\
\text { MEAH! }\end{array}$ & $C 5$ & $\begin{array}{l}\text { CFY } \\
.08\end{array}$ & 62 & crse & $\begin{array}{c}\operatorname{crsy} \\
.05\end{array}$ & $\begin{array}{l}c+1 \\
.02\end{array}$ \\
\hline 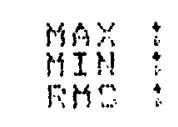 & $.0 \frac{1}{2}$ & $\begin{array}{l}12 \\
.02 \\
.05\end{array}$ & .75 & $\begin{array}{l}.07 \\
.12 \\
.03\end{array}$ & $\begin{array}{l}.15 \\
.15 \\
.02 \\
.02\end{array}$ & .00 \\
\hline GFACT & $\frac{2.35}{4}$ & 2.51 & $\frac{2}{5} \cdot 11$ & 5.97 & $\begin{array}{l}30 \\
3.76\end{array}$ & 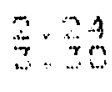 \\
\hline
\end{tabular}

IATA FOF: FILE: H1100

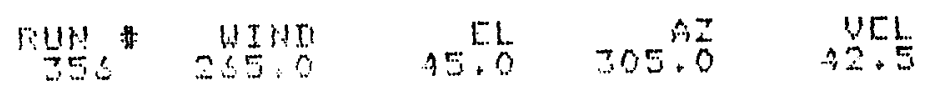

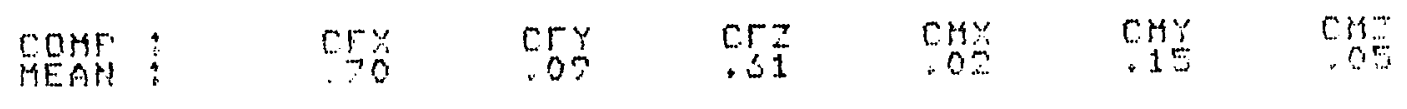

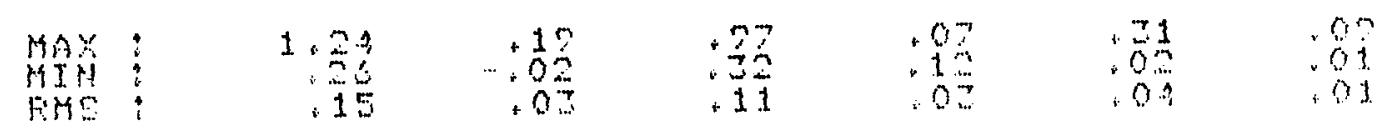

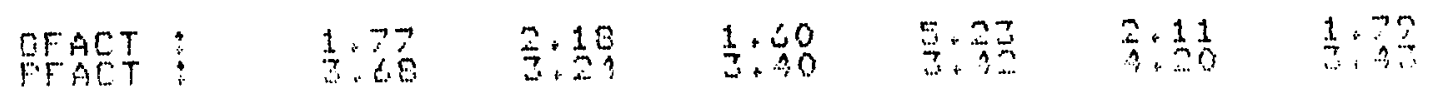




\section{MATA FOF: FILE: H1100}

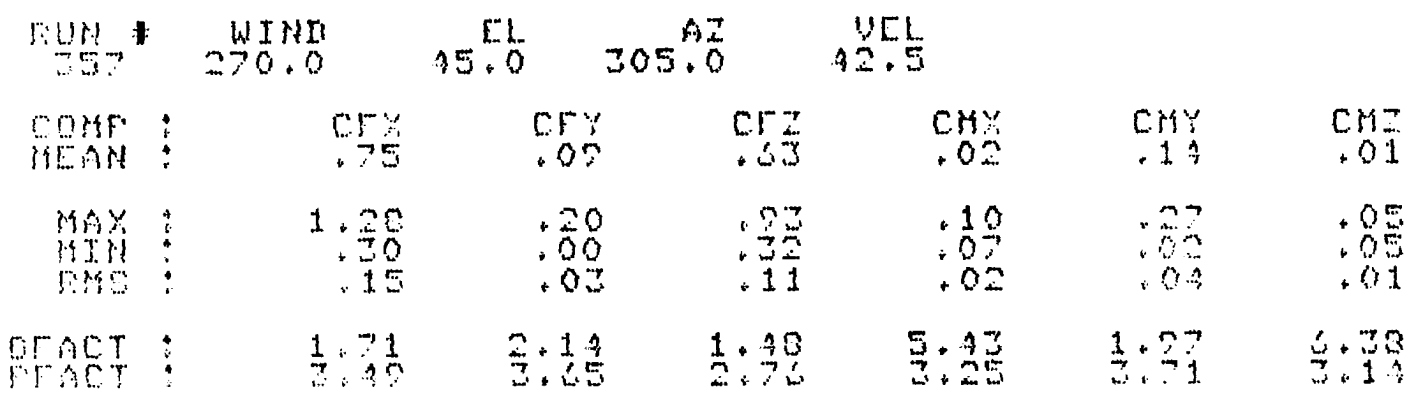

TATA FOF: FILE: H1100

\begin{tabular}{|c|c|c|c|c|c|c|}
\hline Fin! & $\frac{4510}{2}$ & $A=0$ & & $\begin{array}{r}\text { Vㄷ․ } \\
42.5\end{array}$ & & \\
\hline GOME: & 5 & $\begin{array}{l}0.5 Y \\
.07\end{array}$ & $\begin{array}{cl}c 5 \\
65\end{array}$ & $\begin{array}{l}\text { CMy } \\
+03\end{array}$ & cey & .05 \\
\hline $\begin{array}{l}\text { May } \\
\text { ming }\end{array}$ & 1. . & .012 & $\frac{94}{2}$ & $.0 \frac{12}{05}$ & $\begin{array}{r}-60 \\
.06 \\
06\end{array}$ & $\begin{array}{r}.05 \\
.02 \\
.01\end{array}$ \\
\hline $\begin{array}{l}\text { ACT } \\
\text { जिC }\end{array}$ & $\frac{1}{7} \cdot 7$ & $\frac{3}{3} \cdot 05$ & $1: 9 ?$ & 3.67 & $\begin{array}{l}1 \\
7\end{array}, \frac{7}{3}$ & 3.12 \\
\hline MAYAFC & SFILE: & $H 1100$ & & & & \\
\hline ryst & $\begin{aligned} 4515 \\
=1506\end{aligned}$ & $35: 0$ & A. & +25 & & \\
\hline oerll & 8 & $\begin{array}{l}\operatorname{cry} \\
6 \%\end{array}$ & $\begin{array}{l}\operatorname{crz} \\
+36\end{array}$ & $\begin{array}{l}\text { CHS } \\
.06\end{array}$ & crs: & $\begin{array}{c}c .45 \\
.01\end{array}$ \\
\hline 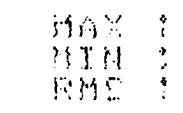 & $\begin{array}{r}1.30 \\
.38 \\
10\end{array}$ & $.0 \%$ & .93 & $\begin{array}{l}.15 \\
.05 \\
.02\end{array}$ & 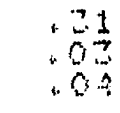 & $\begin{array}{r}.05 \\
.04 \\
.01\end{array}$ \\
\hline ACT & $\frac{1}{3} \cdot \frac{1}{6}$ & $\begin{array}{l}2 \\
3\end{array}$ & $\begin{array}{l}1 \\
2+85\end{array}$ & 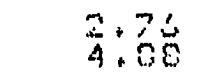 & $\begin{array}{l}1.90 \\
-61\end{array}$ & 5 \\
\hline
\end{tabular}

BATA FOF FILE: H.100

Fin WIYS

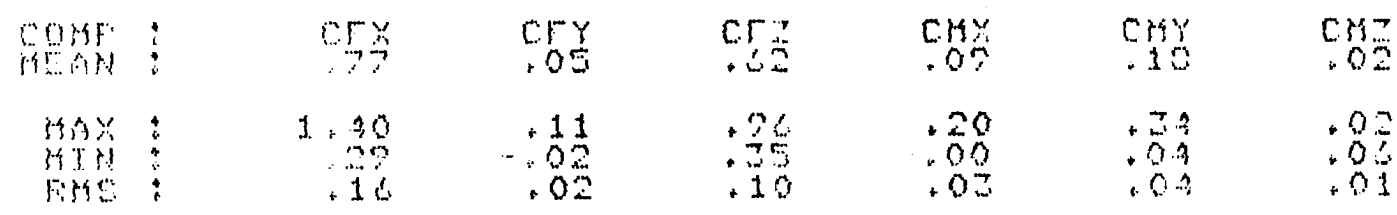

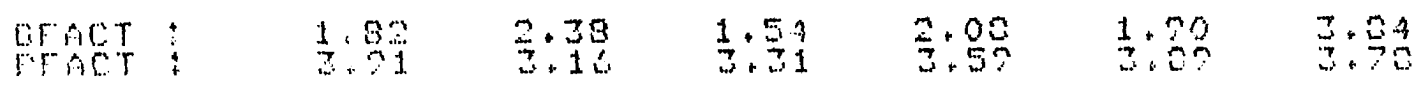




\begin{tabular}{|c|c|c|c|c|c|c|}
\hline 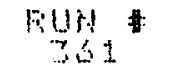 & $\begin{array}{l}\text { WTHI } \\
0.0\end{array}$ & $\begin{array}{c}E L \\
35.6\end{array}$ & $205: \frac{A}{0}$ & 42.2 & & \\
\hline $\begin{array}{l}\text { GOMF } \\
\text { MEAM }\end{array}$ & $\mathrm{CF}^{-}$ & $\begin{array}{l}C F Y \\
.02\end{array}$ & $\frac{C F}{25}$ & $\begin{array}{c}c .48 \\
.05\end{array}$ & $\begin{array}{l}\text { chy } \\
.0 \%\end{array}$ & $\begin{array}{c}C H= \\
0 \%\end{array}$ \\
\hline $\begin{array}{l}\text { MAY } \\
\text { FIIIS }\end{array}$ & $\begin{array}{r}1.05 \\
.05\end{array}$ & $\begin{array}{r}12 \\
.0 \% \\
.0 \%\end{array}$ & $\begin{array}{r}.55 \\
.07 \\
.07\end{array}$ & .29 & $\begin{array}{r}71 \\
.10 \\
.05\end{array}$ & $\begin{array}{r}.09 \\
.00 \\
.01\end{array}$ \\
\hline $\begin{array}{l}\text { GFACT } \\
\text { PFCT }\end{array}$ & $\begin{array}{l}3.09 \\
5.70\end{array}$ & $\begin{array}{l}5.73 \\
3.06\end{array}$ & $\begin{array}{l}1.99 \\
3.97\end{array}$ & $\underset{3}{3} \cdot 31$ & $\frac{3}{5}+30$ & $=32$ \\
\hline
\end{tabular}

1. 
Heliostat 1, Configuration 1-B

\begin{tabular}{|c|c|c|c|c|c|c|}
\hline Case & Run 非 & WD & $\mathrm{EL}$ & $\mathrm{AZ}$ & Day & Time \\
\hline \multirow[t]{7}{*}{ Sinr AM } & 394 & 0 & 25 & 350 & 172 & $8 \mathrm{AM}$ \\
\hline & 395 & 337.5 & 25 & 350 & 172 & 8 AN \\
\hline & 396 & 315 & 25 & 350 & 172 & $8 \mathrm{AM}$ \\
\hline & 397 & 292.5 & 25 & 350 & 172 & $8 \mathrm{AM}$ \\
\hline & 398 & 270 & 25 & 350 & 172 & $8 \mathrm{AM}$ \\
\hline & 399 & 265 & 25 & 350 & 172 & $8 \mathrm{AM}$ \\
\hline & 400 & 247.5 & 25 & 350 & 172 & $8 \mathrm{AM}$ \\
\hline \multirow[t]{7}{*}{ Smr Noon } & 385 & 247.5 & 45 & 305 & 172 & $0 \mathrm{PM}$ \\
\hline & 386 & 265 & 45 & 305 & 172 & $0 \mathrm{PH}$ \\
\hline & 387 & 270 & 45 & 305 & 172 & $0 \mathrm{PM}$ \\
\hline & 388 & 292.5 & 45 & 305 & 172 & $0 \mathrm{PM}$ \\
\hline & 389 & 315 & 45 & 305 & 172 & $0 \mathrm{PM}$ \\
\hline & 390 & 337.5 & 45 & 305 & 172 & 0 PM \\
\hline & 391 & 0 & 45 & 305 & 172 & $0 \mathrm{PH}$ \\
\hline \multirow[t]{7}{*}{ wintr AM } & 362 & 0 & 10 & 330 & 355 & $8 \mathrm{AM}$ \\
\hline & 363 & 337.5 & 10 & 330 & 355 & $8 \mathrm{AM}$ \\
\hline & 364 & 315 & 10 & 330 & 355 & $8 \mathrm{AM}$ \\
\hline & 365 & 292.5 & 10 & 330 & 355 & $8 \mathrm{AM}$ \\
\hline & 366 & 270 & 10 & 330 & 355 & $8 \mathrm{AM}$ \\
\hline & 367 & 265 & 10 & 330 & 355 & $8 \mathrm{AM}$ \\
\hline & 368 & 247.5 & 10 & 330 & 355 & $8 \mathrm{AN}$ \\
\hline
\end{tabular}




\begin{tabular}{|c|c|c|c|c|c|c|c|}
\hline Case & Run 非 & WD & EL & $\mathrm{AZ}$ & Day & & imes \\
\hline \multirow[t]{7}{*}{ Wntr Noon } & 377 & 0 & 20 & 305 & 355 & 0 & PN \\
\hline & 378 & 337.5 & 20 & 305 & 355 & 0 & PNI \\
\hline & 379 & 315 & 20 & 305 & 355 & 0 & PM \\
\hline & 381 & 292.5 & 20 & 305 & 355 & 0 & $\mathrm{PN}$ \\
\hline & 382 & 270 & 20 & 305 & 355 & 0 & $\mathrm{PN}$ \\
\hline & 383 & 265 & 20 & 305 & 355 & 0 & $\mathrm{PNI}$ \\
\hline & 384 & 247.5 & 20 & 305 & 355 & 0 & rN \\
\hline \multirow[t]{7}{*}{ Wintr PM } & 369 & 247.5 & 10 & 275 & 355 & 4 & PNI \\
\hline & 370 & 265 & 10 & 275 & 355 & 4 & PHI \\
\hline & 370 & 270 & 10 & 275 & 355 & 4 & PN \\
\hline & 372 & 292.5 & 10 & 275 & 355 & 4 & $\mathrm{PH}$ \\
\hline & 373 & 315 & 10 & 275 & 355 & 4 & PN \\
\hline & 374 & 337.5 & 10 & 275 & 355 & 4 & $\mathrm{PN}$ \\
\hline & 375 & 0 & 10 & 275 & 355 & 4 & PM \\
\hline Ver. Stow & 392 & 337.5 & 0 & 330 & 355 & 8 & AM \\
\hline Hor. Stow & 393 & 337.5 & 90 & 330 & 355 & 8 & AM \\
\hline
\end{tabular}


TATA FOR FILE: H101

\begin{tabular}{|c|c|c|c|c|c|c|}
\hline rous & $\begin{array}{r}\text { WI I HI } \\
0.0\end{array}$ & 10.0 & 0.0 & Y VEL & & \\
\hline $\begin{array}{l}\text { COMF } \\
\text { HEAH }\end{array}$ & .07 & $\begin{array}{c}\mathrm{Cr} Y \\
.02\end{array}$ & .00 & $\begin{array}{l}\text { cryox } \\
.01\end{array}$ & $\begin{array}{l}c r y \\
.0 z\end{array}$ & .01 \\
\hline $\begin{array}{l}\max \\
\text { ring } \\
\text { rins }\end{array}$ & $\begin{array}{r}50 \\
.52 \\
12\end{array}$ & $\begin{array}{l}12 \\
.1 .7 \\
.02\end{array}$ & $\begin{array}{r}.05 \\
.04 \\
.01\end{array}$ & $\begin{array}{l}+12 \\
+10 \\
+02\end{array}$ & $\begin{array}{l}+76 \\
105 \\
.05\end{array}$ & $\begin{array}{l}.02 \\
.05 \\
.01\end{array}$ \\
\hline $\begin{array}{l}\text { GECT } \\
\text { FFACT }\end{array}$ & $3+\frac{21}{15}$ & $\frac{5}{3}+94$ & $\begin{array}{r}10 \\
3\end{array}$ & 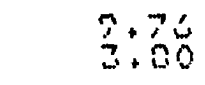 & $9+58$ & $\begin{array}{l}3.01 \\
3.45\end{array}$ \\
\hline
\end{tabular}

MATA FOF FILE: H1101

\begin{tabular}{|c|c|c|c|c|c|c|}
\hline 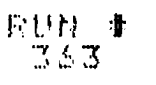 & $\begin{array}{l}W 1+\mathbb{D} \\
3\end{array}$ & $10+0$ & & 42.5 & & \\
\hline $\begin{array}{l}\text { COMF } \\
\text { MEAN }\end{array}$ & $\mathrm{Cr}_{2}$ & $\begin{array}{l}\operatorname{coy} \\
.05\end{array}$ & $.0 \frac{1}{6}$ & $\begin{array}{l}C .45 \\
.05\end{array}$ & Cry & $\begin{array}{l}C H z \\
.03\end{array}$ \\
\hline $\begin{array}{l}\text { max } \\
\text { myst } \\
\text { rise }\end{array}$ & .96 & $\begin{array}{r}+05 \\
+17 \\
.03\end{array}$ & $\begin{array}{l}.09 \\
.01 \\
.01\end{array}$ & $\begin{array}{l}.15 \\
.05 \\
.05\end{array}$ & . & $\begin{array}{l}.09 \\
.08 \\
.01\end{array}$ \\
\hline ACT & $\underline{5} \cdot 50$ & $\begin{array}{l}3 \\
3.23\end{array}$ & $\frac{3}{3}+2 \frac{2}{3}$ & 3.01 & $5+15$ & $3 \cdot 5$ \\
\hline
\end{tabular}

ISTA FOF FILE: H1101

\begin{tabular}{|c|c|c|c|c|c|c|}
\hline Fis & $\begin{array}{r}\text { WINII } \\
=15.0\end{array}$ & 10.0 & & $\begin{array}{r}45.7 \\
42.7\end{array}$ & & \\
\hline 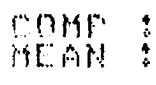 & $\begin{array}{l}0 F \\
: 26\end{array}$ & $\begin{array}{r}\text { C.5Y } \\
+.04\end{array}$ & .05 & $\begin{array}{l}\text { CPS } \\
.05\end{array}$ & $\mathrm{CMY}_{0}$ & $\begin{array}{l}c .45 \\
.00\end{array}$ \\
\hline $\begin{array}{l}\text { MAX } \\
\text { RyH } \\
\text { Fys }\end{array}$ & $.8 \frac{2}{2}$ & $\begin{array}{r}.05 \\
.15 \\
.02\end{array}$ & $\begin{array}{l}.07 \\
.00 \\
.01\end{array}$ & $\begin{array}{r}12 \\
.05 \\
.02 \\
.02\end{array}$ & 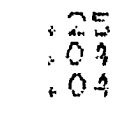 & .05 \\
\hline TACT & $\frac{3}{4.82}$ & 3.03 & $\frac{3}{3} \cdot \frac{24}{4}$ & $\frac{2}{3} \cdot 51$ & 3.79 & $\frac{7}{7}+5$ \\
\hline
\end{tabular}

IIATA FOF: FILE : H1101

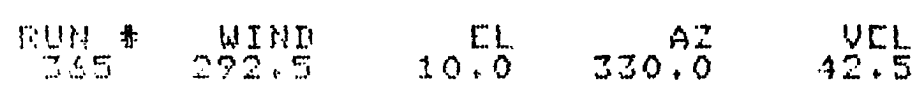

\begin{tabular}{|c|c|c|c|c|c|}
\hline $\begin{array}{l}\text { compo } \\
\text { rensy }\end{array}$ & $\operatorname{crs}$ & $\begin{array}{c}\mathrm{Cr} Y \\
-0.4\end{array}$ & $\begin{array}{l}\mathrm{Cr}_{0} \\
\mathrm{OS}\end{array}$ & $\begin{array}{l}\mathrm{CHO} \\
. \mathrm{OS}\end{array}$ & CM: \\
\hline $\begin{array}{l}\text { Max } \\
\text { mitis } \\
\text { Fiss }\end{array}$ & $\begin{array}{r}.00 \\
.01 \\
.07\end{array}$ & $\begin{array}{l}.02 \\
: 10 \\
+02\end{array}$ & $\begin{array}{l}.00 \\
.01 \\
.01\end{array}$ & .10 & .12 \\
\hline
\end{tabular}

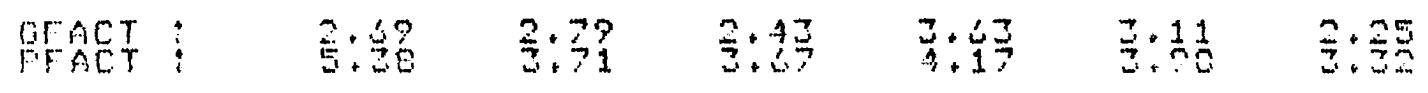




\begin{tabular}{|c|c|c|c|c|c|c|}
\hline Fust & $\begin{array}{l}45.15 \\
=6.0\end{array}$ & 10.5 & $\begin{array}{r}A 2 \\
30.0\end{array}$ & 42.3 & & \\
\hline Gors: & $\operatorname{crs}$ & $\begin{array}{cr}C r \\
.0\end{array}$ & $\begin{array}{c}\operatorname{cr} 2 \\
.04\end{array}$ & $\begin{array}{l}\csc \\
+05\end{array}$ & $\begin{array}{l}\text { cyy } \\
.04\end{array}$ & $\begin{array}{l}C H 2 \\
00\end{array}$ \\
\hline $\begin{array}{l}\text { MAO } \\
\text { FME }\end{array}$ & $\begin{array}{r}147 \\
.02 \\
+07\end{array}$ & $\begin{array}{r}+05 \\
.05 \\
.02\end{array}$ & $\begin{array}{r}.09 \\
: 01 \\
.01\end{array}$ & $\begin{array}{r}11 \\
.03 \\
.05\end{array}$ & $\begin{array}{l}17 \\
.08 \\
.03\end{array}$ & $\begin{array}{r}93 \\
.83 \\
.03\end{array}$ \\
\hline $\begin{array}{l}\text { gFACT } \\
\text { FHAT }\end{array}$ & $\frac{2}{4}+\frac{7}{2}$ & $\begin{array}{l}4.37 \\
3.13\end{array}$ & $2 \cdot 51$ & $\frac{3}{2}: \frac{1}{5}$ & 3.09 & 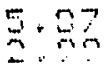 \\
\hline
\end{tabular}

IATA FOF: FILE: H1101

\begin{tabular}{|c|c|c|c|c|c|c|}
\hline $\operatorname{los}_{26}$ & $\begin{aligned} & 4 T+11 \\
= & 65.0\end{aligned}$ & $10: 0$ & & 42.5 & & \\
\hline COME : & CFY & $\begin{array}{r}\operatorname{crs} \\
-.02\end{array}$ & $\begin{array}{cr}c r z \\
.00\end{array}$ & cry & $\begin{array}{l}\text { cry } \\
\text {. }\end{array}$ & $\begin{array}{l}.43 \\
.00\end{array}$ \\
\hline $\begin{array}{l}\text { Max } \\
\text { ring } \\
\text { rus }\end{array}$ & $\begin{array}{r}.92 \\
.05 \\
.06\end{array}$ & $\begin{array}{r}.03 \\
.05 \\
.02\end{array}$ & $\begin{array}{l}.02 \\
.05 \\
.01\end{array}$ & .05 & $\begin{array}{r}17 \\
.09 \\
.07\end{array}$ & 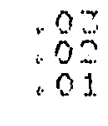 \\
\hline FFACT & 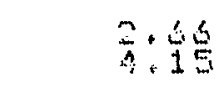 & 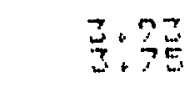 & $\frac{2}{3}: 3.1$ & $\overrightarrow{3} \cdot \overrightarrow{3}$ & 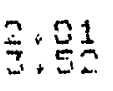 & 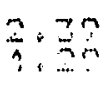 \\
\hline
\end{tabular}

MATA FOF FILE : H1101

\begin{tabular}{|c|c|c|c|c|c|c|}
\hline Fun & $287=0$ & 10.0 & & $\frac{y C L}{2}$ & & \\
\hline CoMs: & $\begin{array}{c}\text { CFy } \\
.95\end{array}$ & $\begin{array}{l}\mathrm{Cry} \\
.\end{array}$ & 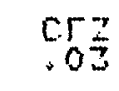 & $\begin{array}{l}c k y \\
.01\end{array}$ & $\begin{array}{l}\text { CHYY } \\
0 \%\end{array}$ & $\begin{array}{c}c+4 \\
0=\end{array}$ \\
\hline $\begin{array}{l}\text { mas } \\
\text { ming }\end{array}$ & $: \frac{3}{6}$ & $\begin{array}{r}.06 \\
.05 \\
.02\end{array}$ & .09 & .02 & .05 & .00 \\
\hline $\begin{array}{l}\text { GFACT } \\
\text { FACT }\end{array}$ & 9.86 & $\begin{array}{l}4.49 \\
3: 30\end{array}$ & $\begin{array}{l}3.00 \\
3.57\end{array}$ & $1 \frac{1}{4}, 30$ & $3: 92$ & 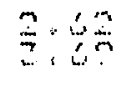 \\
\hline
\end{tabular}

IATA FOF: FILE: H1101

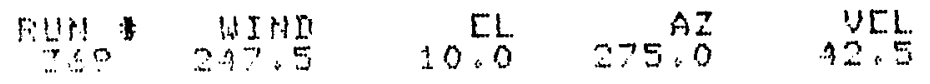

\begin{tabular}{|c|c|c|c|c|c|c|}
\hline Coms & CFY & $\begin{array}{l}\text { cry } \\
.00\end{array}$ & $\mathrm{cr}_{0}^{2}$ & $\begin{array}{l}\text { CHX } \\
.01\end{array}$ & $\begin{array}{l}\text { Cry } \\
000\end{array}$ & crsz \\
\hline $\begin{array}{l}\text { MaY } \\
\text { MHE }\end{array}$ & 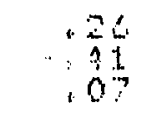 & $\begin{array}{r}.05 \\
.05 \\
+02\end{array}$ & $\begin{array}{r}.07 \\
.05 \\
.01\end{array}$ & $\begin{array}{l}.09 \\
.06 \\
05\end{array}$ & .09 & .09 \\
\hline$A C T$ & $1 \frac{2}{5} \cdot 5 \frac{3}{5}$ & 12.79 & $\frac{2}{3} \cdot 37$ & 19.75 & $12 \cdot 82$ & 111 \\
\hline
\end{tabular}




\begin{tabular}{|c|c|c|c|c|c|c|}
\hline rous & $\begin{array}{r}41+1 ! \\
265.0\end{array}$ & 10.5 & 5.0 & บㄷㄴ는 & & \\
\hline corif & .01 & $\begin{array}{l}\text { Cry } \\
.02\end{array}$ & ${ }_{0}^{c}=$ & $\begin{array}{l}\text { C.s. } \\
.04\end{array}$ & $\begin{array}{l}\operatorname{crsy} \\
+02\end{array}$ & cha \\
\hline $\begin{array}{l}\text { MEX } \\
\text { Fus }\end{array}$ & 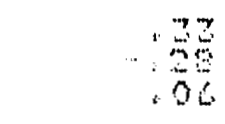 & $\begin{array}{r}.06 \\
-.05 \\
.01\end{array}$ & $\begin{array}{r}.05 \\
.05 \\
.02\end{array}$ & $\begin{array}{l}.00 \\
.00 \\
.01\end{array}$ & .12 & .02 \\
\hline GFAT & $\begin{aligned} 78 \\
5\end{aligned}$ & $2: 74$ & $4 \frac{4}{3}+\frac{2}{12}$ & $\frac{2}{3}+\frac{12}{2}$ & 5.52 & $\frac{6}{5}+13$ \\
\hline
\end{tabular}

WOTA FQF: FILE: H1101

\begin{tabular}{|c|c|c|c|c|c|c|}
\hline $\ln _{x \rightarrow 1}$ & 200.0 & 10.0 & $\begin{array}{r}A z \\
5.0\end{array}$ & $\begin{array}{l}4[5,5 \\
32.5\end{array}$ & & \\
\hline 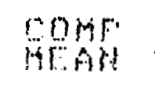 & .05 & $\begin{array}{l}c y \\
.01\end{array}$ & $\begin{array}{l}65 \\
+00\end{array}$ & $\begin{array}{l}\text { cry } \\
.00\end{array}$ & $\begin{array}{l}\text { CMY } \\
00\end{array}$ & CHI \\
\hline $\begin{array}{l}\text { Mas } \\
\text { ming } \\
\text { ans }\end{array}$ & $\frac{70}{20}$ & $\begin{array}{l}+95 \\
65 \\
05 \\
.05\end{array}$ & $\begin{array}{l}.05 \\
.05 \\
.01\end{array}$ & $\begin{array}{l}.94 \\
.04 \\
.02\end{array}$ & $\begin{array}{l}1.7 \\
.0 \% \\
0 \%\end{array}$ & .95 \\
\hline $\begin{array}{l}A C T \\
A N A T\end{array}$ & 12.77 & 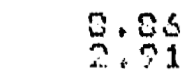 & $149 \cdot 51$ & $\begin{array}{r}12.20 \\
3.00\end{array}$ & 78,93 & 3.93 \\
\hline
\end{tabular}

MATA FOF: FILE: H1101

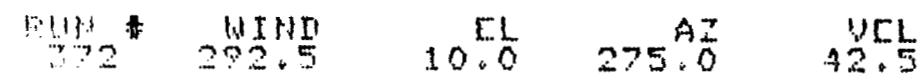

\begin{tabular}{|c|c|c|c|c|c|c|}
\hline $\begin{array}{l}\text { cons } \\
\text { manan }\end{array}$ & $\begin{array}{l}C F \\
12\end{array}$ & $\begin{array}{l}\text { CFY } \\
.02\end{array}$ & $\operatorname{crz}$ & $\begin{array}{l}\text { CMX } \\
.01\end{array}$ & $\begin{array}{l}\text { cryy } \\
.06\end{array}$ & $\cos =$ \\
\hline Mơ & $\begin{array}{r}01 \\
-12 \\
02\end{array}$ & $\begin{array}{r}.07 \\
.03 \\
.01\end{array}$ & $\begin{array}{r}.05 \\
.05 \\
.01\end{array}$ & $\begin{array}{l}.01 \\
.04 \\
01\end{array}$ & $\begin{array}{l}1 \\
03 \\
03 \\
02\end{array}$ & .82 \\
\hline $\begin{array}{l}\text { FACT } \\
\text { FACT }\end{array}$ & 3.30 & $\begin{array}{l}\vec{J} .07 \\
\square .21\end{array}$ & $\frac{7}{2} \cdot 4.4$ & 4.01 & $\frac{2}{3} \cdot \frac{3}{0}$ & $7:$ \\
\hline
\end{tabular}

IATA FOF FILE: H1101

ritss * MISI

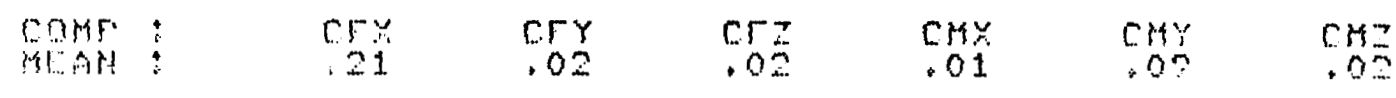

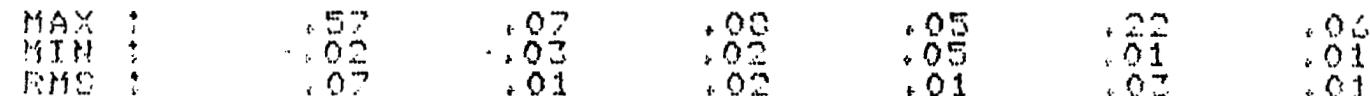

FrACT: 
IATA FOF: FILE: H1101

\begin{tabular}{|c|c|c|c|c|c|c|}
\hline Fus & $24 I+9$ & 10.0 & & 42.5 & & \\
\hline $\begin{array}{l}\text { COMF } \\
\text { MEAM }\end{array}$ & CF & $\begin{array}{l}\mathrm{Cry} \\
.09\end{array}$ & 05 & $\begin{array}{c}\mathrm{CH} \\
.01\end{array}$ & CMY & CH: \\
\hline $\begin{array}{l}\text { MAY } \\
\text { MIME } \\
\text { FMS }\end{array}$ & $\begin{array}{r}1+10 \\
\quad 119 \\
\quad 13\end{array}$ & 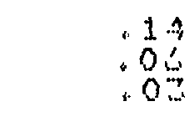 & $\begin{array}{l}10 \\
.00 \\
.01\end{array}$ & .11 & $\begin{array}{l}.32 \\
.07 \\
.05\end{array}$ & .07 \\
\hline DFACT & $5:-11$ & 莒.台台 & $\frac{2}{3} \cdot 52$ & 2.99 & $2 \cdot 03$ & 3.85 \\
\hline
\end{tabular}

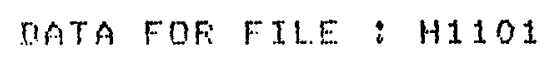




\begin{tabular}{|c|c|c|c|c|c|c|}
\hline 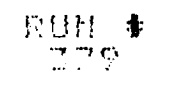 & $\begin{array}{r}45311 \\
215.0\end{array}$ & 20.0 & 305.0 & $\begin{array}{c}\cup[1 \\
3 \geq .7\end{array}$ & & \\
\hline $\begin{array}{l}\text { COMF : } \\
\text { MEAM : }\end{array}$ & 05 & $\begin{array}{l}\mathrm{cry} \\
.02\end{array}$ & $\begin{array}{l}\text { c. } \\
.15\end{array}$ & C.MY & cry & CHI \\
\hline $\begin{array}{l}\text { mox } \\
\text { ming }\end{array}$ & .85 & $\begin{array}{r}02 \\
.02 \\
.02\end{array}$ & $\begin{array}{l}17 \\
.02 \\
.02\end{array}$ & $\begin{array}{r}10 \\
.00 \\
.09\end{array}$ & $\begin{array}{r}23 \\
03 \\
0 \\
0\end{array}$ & .02 \\
\hline $\begin{array}{l}\text { OFCT } \\
\text { FACT }\end{array}$ & $\frac{25}{4}: 3$ & $\begin{array}{l}4 \cdot 47 \\
4.20\end{array}$ & $\begin{array}{l}1.32 \\
4.11\end{array}$ & $\frac{2}{2} \cdot 02$ & 4.02 & $3 \cdot \frac{1}{2}$ \\
\hline MATA F & QE: FILE: & $H 1101$ & & & & \\
\hline $\operatorname{mos}_{01}$ & $\begin{array}{l}\text { WIMD } \\
272.5\end{array}$ & $=0.0$ & $305 . A Z$ & UटL & & \\
\hline $\begin{array}{l}\text { consf } \\
\text { Mín }\end{array}$ & Cr: & $\begin{array}{l}\text { CFY } \\
.00\end{array}$ & .05 & $\begin{array}{l}\cos x \\
0.8\end{array}$ & $\begin{array}{l}\text { CisY } \\
.06\end{array}$ & $\begin{array}{l}\text { crz } \\
.00\end{array}$ \\
\hline $\begin{array}{l}\text { Max : } \\
\text { mins: } \\
\text { FHS }\end{array}$ & 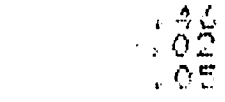 & $\begin{array}{r}.05 \\
.05 \\
.02\end{array}$ &.$\frac{1}{01}$ & .89 & .17 & .89 \\
\hline $\begin{array}{l}\text { GET } \\
\text { FFAC }\end{array}$ & 5.56 & $\begin{array}{r}14.67 \\
4.5\end{array}$ & $\frac{2}{3}: \frac{1}{6}$ & $\frac{2}{3}+20$ & $\frac{3}{3}+29$ & $2 \frac{2}{5} \cdot 7 \frac{1}{11}$ \\
\hline
\end{tabular}

IATA FOF FILE : H1101

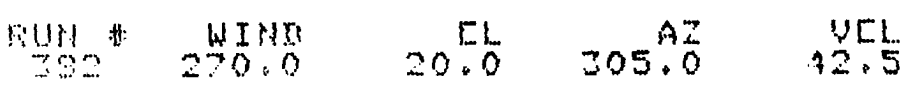

\begin{tabular}{|c|c|c|c|c|c|}
\hline Cong : & $.0 \%$ & $\begin{array}{l}\text { C.TY } \\
.01\end{array}$ & $\begin{array}{l}\cos \\
.04\end{array}$ & $\begin{array}{l}\text { CHy } \\
.04\end{array}$ & $\begin{array}{l}0.45 \\
03\end{array}$ \\
\hline $\begin{array}{l}\text { MAd } \\
\text { Fis? } \\
\text { Figs }\end{array}$ & $\begin{array}{r}76 \\
06 \\
05\end{array}$ & $\begin{array}{l}.05 \\
.05 \\
.02\end{array}$ & $\begin{array}{l}.00 \\
.01 \\
.01\end{array}$ & .00 & $.0=$ \\
\hline
\end{tabular}

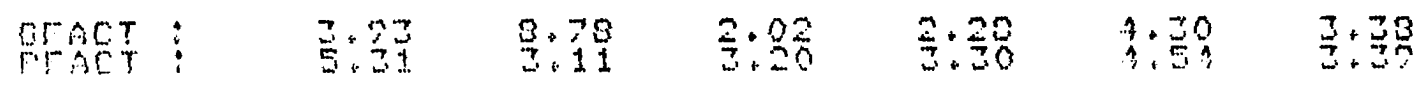

MITA FOF: FILE : H1101

\begin{tabular}{|c|c|c|c|c|c|c|}
\hline nupt & $\begin{array}{rl} & 4 I H I \\
2 & 55.0\end{array}$ & 20.0 & & $\therefore \frac{1}{5}$ & & \\
\hline $\begin{array}{l}\text { romp } \\
\text { minh }\end{array}$ & .05 & $\begin{array}{l}\text { CFY } \\
.00\end{array}$ & 0.05 & $\begin{array}{l}C M Y \\
.01\end{array}$ & $\begin{array}{l}\text { CMY } \\
.02\end{array}$ & ${ }_{0}^{\mathrm{CH}}$ \\
\hline $\begin{array}{l}\text { MaY } \\
\text { MHA }\end{array}$ & $\begin{array}{l}32 \\
10 \\
10\end{array}$ & .05 & $\begin{array}{l}10 \\
.01 \\
01\end{array}$ & $\begin{array}{l}.07 \\
.07 \\
.02\end{array}$ & $\begin{array}{l}13 \\
.02 \\
.02\end{array}$ & $\begin{array}{l}01 \\
.04 \\
.01\end{array}$ \\
\hline $\begin{array}{l}\text { GFCT } \\
\text { AnCT }\end{array}$ & $4 \cdot 2 \cdot 9 \frac{5}{7}$ & $2 \frac{1}{2} \cdot 7.7$ & $\frac{2}{3} .05$ & $\stackrel{Q}{9} \cdot 0]$ & $3 \cdot \frac{5}{2}$ & $\frac{1}{7}+\frac{1}{2}$ \\
\hline
\end{tabular}




\begin{tabular}{|c|c|c|c|c|c|c|}
\hline $9 \underset{7}{9}$ & $\begin{array}{r}41+\pi \\
=37\end{array}$ & 20.5 & 305.0 & $4=\frac{1}{3}$ & & \\
\hline $\begin{array}{l}\text { CoYSF } \\
\text { MEATS }\end{array}$ & $\operatorname{cry}$ & ${ }_{0 O O}$ & .01 & $\begin{array}{l}\operatorname{crs} \\
-01\end{array}$ & $\begin{array}{c}\text { CMY } \\
.01\end{array}$ & $\mathrm{CH}^{\circ}$ \\
\hline $\begin{array}{l}\text { MAY } \\
\text { MIU } \\
\text { FHS }\end{array}$ & 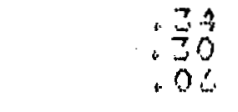 & $\begin{array}{r}.09 \\
.09 \\
.02\end{array}$ & $\begin{array}{l}.04 \\
.02 \\
.01\end{array}$ & $\begin{array}{l}.92 \\
.02 \\
.02\end{array}$ & $\begin{array}{l}1 . \hat{2} \\
.00 \\
.02\end{array}$ & $\begin{array}{r}97 \\
.05 \\
.01\end{array}$ \\
\hline $\begin{array}{l}\text { GFACT } \\
\text { FFACT }\end{array}$ & $12 \cdot \frac{2}{4} \cdot 68$ & $2 \frac{1}{5}+65$ & $\begin{array}{l}4.71 \\
3.1\end{array}$ & 12.20 & $10 \cdot 15$ & ․․요 \\
\hline MATA F & OF: FILE : & $H 1101$ & & & & \\
\hline 5up & $\begin{array}{r}45 r y \\
2475\end{array}$ & ${ }_{35}^{E L}$ & $\begin{array}{r}A z \\
305.0\end{array}$ & $\begin{array}{r}\text { VEL } \\
42.5\end{array}$ & & \\
\hline $\begin{array}{l}\text { Comf: } \\
\text { SEAH }\end{array}$ & CFY & $\begin{array}{c}c y \\
01\end{array}$ & $\operatorname{cr}_{0}$ & $\begin{array}{l}\text { cras } \\
005\end{array}$ & $\begin{array}{l}\text { CMY } \\
.00\end{array}$ & $\cos x$ \\
\hline $\begin{array}{l}\text { MAY : } \\
\text { MAIS: } \\
\text { nus: }\end{array}$ & $\begin{array}{l}.75 \\
: 17 \\
+06\end{array}$ & $\begin{array}{r}+8 z \\
-80 \\
+02\end{array}$ & $\begin{array}{l}.11 \\
.0 \frac{4}{2} \\
.02\end{array}$ & $\begin{array}{l}10 \\
.05 \\
.05\end{array}$ & .02 & 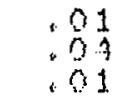 \\
\hline $\begin{array}{l}\text { GTACT } \\
\text { FFACT }\end{array}$ & $\frac{7}{4}: 13$ & $\frac{1}{2}+\frac{1}{5}=$ & 3.74 & 3.55 & $23 \cdot 25$ & $3: 31$ \\
\hline DATA F & OF: FILE: & $H 1101$ & & & & \\
\hline $\operatorname{lip}_{76} 64$ & $\begin{array}{r}41510 \\
265.0\end{array}$ & $\begin{array}{r}E L \\
45.0\end{array}$ & 305.0 & 42.3 & & \\
\hline romps & $\begin{array}{c}c r y \\
0 \%\end{array}$ & ${ }_{05}^{c 5}$ & .05 & $\begin{array}{l}\text { chy } \\
.05\end{array}$ & $\begin{array}{l}\text { CMY } \\
.01\end{array}$ & $\begin{array}{c}\cos ^{\prime}= \\
.0 \mathrm{z}\end{array}$ \\
\hline $\begin{array}{l}\text { May } \\
\text { Mind } \\
\text { rins: }\end{array}$ & .75 & $\begin{array}{l}.07 \\
.04 \\
.03\end{array}$ & .20 & $\begin{array}{l}11 \\
.01 \\
.02\end{array}$ & $.0 \%$ & $\begin{array}{r}99 \\
67 \\
61\end{array}$ \\
\hline $\begin{array}{l}\text { GFACT } \\
\text { FFACT }\end{array}$ & $5: 36$ & $2 \cdot 30$ & $\begin{array}{l}4.29 \\
7.64\end{array}$ & $\frac{2}{3} \cdot \frac{1}{5}$ & $1 \div 2 ?$ & $\therefore 1 ?$ \\
\hline WATA & OF FILE : & $H 1101$ & & & & \\
\hline Fust & $24 \pi+\pi$ & ${ }_{45.5}{ }^{1}$ & 205.0 & $\begin{array}{r}\text { VEL } \\
42+7\end{array}$ & & \\
\hline $\begin{array}{l}\text { Conts } \\
\text { MEAn! }\end{array}$ & $.0 \%$ & $\begin{array}{l}\text { Cry } \\
.00\end{array}$ & $\begin{array}{l}\text { c. } 2 \\
.07\end{array}$ & $\begin{array}{l}\text { CHx } \\
.02\end{array}$ & $\begin{array}{l}\text { CHY } \\
.0=\end{array}$ & 0.00 \\
\hline $\begin{array}{l}\text { MAY } \\
\text { PSIME: }\end{array}$ & $\begin{array}{l}25 \\
.15 \\
.05\end{array}$ & $\begin{array}{r}.85 \\
.02 \\
.02\end{array}$ & $\begin{array}{l}.10 \\
.01 \\
.02\end{array}$ & $\begin{array}{r}.00 \\
.05 \\
.05\end{array}$ & $\frac{11}{05}$ & .02 \\
\hline GFACT & $\vec{B}+1 \overrightarrow{0}$ & $25+13$ & $2.7 ?$ & $3: \frac{9}{3}$ & $\begin{array}{l}3.45 \\
3.85\end{array}$ & 7.97 \\
\hline
\end{tabular}




\begin{tabular}{|c|c|c|c|c|c|c|}
\hline Fut? & $\begin{array}{l}\text { WIM } \\
292.5\end{array}$ & $45: 0$ & 205.0 & $\begin{array}{l}U[1 \\
72.7\end{array}$ & & \\
\hline $\begin{array}{l}\text { COAF } \\
\text { MEAH }\end{array}$ & $C F$ & $\begin{array}{l}\text { C.FY } \\
.00\end{array}$ & $\begin{array}{l}\mathrm{cl}^{3} \\
.0 \mathrm{~s}\end{array}$ & $\begin{array}{l}\text { Crs. } \\
.02\end{array}$ & $\begin{array}{l}\text { Cry } \\
.0 A\end{array}$ & $\frac{\operatorname{cm}}{.01}$ \\
\hline $\begin{array}{l}\text { MAX } \\
\text { HTH } \\
\text { Fin }\end{array}$ & 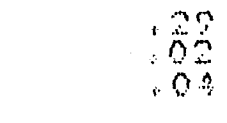 & $\begin{array}{l}.05 \\
.05 \\
.02\end{array}$ & $\begin{array}{l}15 \\
.05 \\
+02\end{array}$ & .85 & 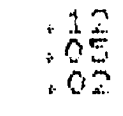 & $\begin{array}{l}.01 \\
.04 \\
.01\end{array}$ \\
\hline $\begin{array}{l}\text { GFACT } \\
\text { FFACT }\end{array}$ & $\bar{z} \cdot \frac{5}{2}$ & $150 \cdot 34$ & $\frac{1}{3} \cdot \frac{29}{7}$ & $3 \cdot 2 \frac{6}{3}$ & $3: 09$ & $\frac{38}{3} \cdot 51$ \\
\hline MATA F & OF: FILE: & $H 1101$ & & & & \\
\hline roly & $\begin{array}{r}\text { WISII } \\
315=0\end{array}$ & $\begin{array}{r}51 \\
45: 0\end{array}$ & 305.0 & 든. & & \\
\hline $\begin{array}{l}\text { Coms : } \\
\text { MEAst }\end{array}$ & $\begin{array}{l}\text { CFY } \\
.18\end{array}$ & $\begin{aligned} C F y \\
.01\end{aligned}$ & $\begin{array}{l}C 5 I \\
+14\end{array}$ & $\begin{array}{l}\text { Cry } \\
\text { DOA }\end{array}$ & $\begin{array}{c}C \mu Y \\
.05\end{array}$ & $\begin{array}{l}\text { C.Mz } \\
.02\end{array}$ \\
\hline $\begin{array}{l}\text { MAQ } \\
\text { MTHS } \\
\text { rins }\end{array}$ & $\begin{array}{l}.36 \\
.80 \\
.05\end{array}$ & $\begin{array}{r}.05 \\
. .05 \\
.03\end{array}$ & $\begin{array}{l}.29 \\
.05 \\
.05\end{array}$ & .02 & $\begin{array}{l}15 \\
602 \\
.02\end{array}$ & $\begin{array}{l}.90 \\
.05 \\
.00\end{array}$ \\
\hline $\begin{array}{l}\text { OFAT } \\
\text { FFACT }\end{array}$ & $2+\frac{2}{4}$ & 3.57 & $\frac{1}{4} \cdot 50$ & $3 \cdot 3$ & $\begin{array}{l}30 \\
31\end{array}$ & $201 \frac{1}{3}$ \\
\hline
\end{tabular}

MATA FOF FILE: H H101

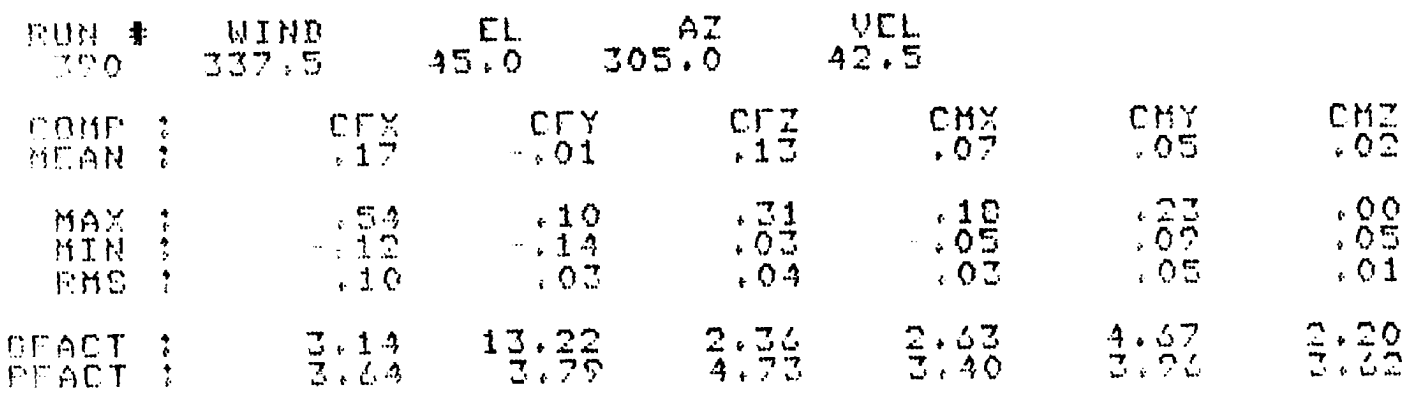

MATA FOF: FILE: H1101

\begin{tabular}{|c|c|c|c|c|c|c|}
\hline $\operatorname{lin}_{2}$ & WI $0: 0$ & $\Rightarrow E=0$ & & $42: \frac{1}{7}$ & & \\
\hline CONF : & 05 & $\begin{array}{r}\text { CFY } \\
-: 01\end{array}$ & $.05 z$ & $\begin{array}{l}\text { CMx } \\
.04\end{array}$ & $\begin{array}{l}\text { CHY } \\
.09\end{array}$ & $\begin{array}{l}\mathrm{CHz} \\
.02\end{array}$ \\
\hline $\begin{array}{l}\text { MAX } \\
\text { MIHS } \\
\text { FHS }\end{array}$ & $\begin{array}{r}72 \\
-105\end{array}$ & $\begin{array}{r}.04 \\
\div 11 \\
.02\end{array}$ & $\begin{array}{r}72 \\
0.01 \\
.04\end{array}$ & $\begin{array}{l}13 \\
.05 \\
.02\end{array}$ & $\begin{array}{l}00 \\
.00 \\
.05\end{array}$ & $\begin{array}{l}.01 \\
.02 \\
.01\end{array}$ \\
\hline $\begin{array}{l}\text { GFACT } \\
\text { FFACT }\end{array}$ & $4: \frac{6}{4}$ & $\begin{array}{l}8+59 \\
4+14\end{array}$ & $\frac{2}{5}: 92$ & 5.28 & $\begin{array}{l}4.57 \\
4.35\end{array}$ & $2 \cdot 25$ \\
\hline
\end{tabular}




\begin{tabular}{|c|c|c|c|c|c|c|}
\hline $\operatorname{lip}_{2}+$ & $\begin{array}{r}45 \pi \\
275\end{array}$ & $E, 0$ & $=\begin{array}{r}A z \\
=30.0\end{array}$ & 42.3 & & \\
\hline $\begin{array}{l}\text { COMf } \\
\text { rEAH! }\end{array}$ & 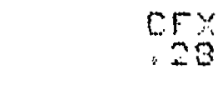 & $\begin{array}{l}\text { CFY } \\
.02\end{array}$ & $\begin{array}{c}\operatorname{cr} \\
.01\end{array}$ & cms & CMY & $\begin{array}{c}c m s \\
05\end{array}$ \\
\hline $\begin{array}{l}M G Y \\
M T H \\
\text { Fins }\end{array}$ & 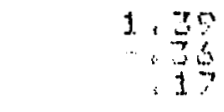 & $\cdot \frac{12}{0}$ & $\begin{array}{r}.03 \\
.12 \\
.02\end{array}$ & 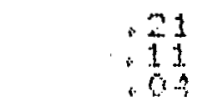 & $\frac{10}{20}$ & $\begin{array}{l}01 \\
10 \\
01\end{array}$ \\
\hline QFACT & 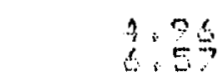 & $\frac{5}{3} \cdot 80$ & $8: 61$ & $4 \cdot 93$ & $\begin{aligned} 13 \\
3+40\end{aligned}$ & $\frac{1}{3}: 80$ \\
\hline
\end{tabular}

INTA FOF: FILE: H1101

\begin{tabular}{|c|c|c|c|c|c|c|}
\hline $\log _{72}$ & $\frac{4519}{3}$ & 90.0 & & 2.5 & & \\
\hline Conf: & CFY & $\begin{array}{c}\text { CFY } \\
.00\end{array}$ & .02 & $\begin{array}{l}\cos \\
.00\end{array}$ & $\begin{array}{c}C Y Y \\
.01\end{array}$ & $\operatorname{crs}$ \\
\hline $\begin{array}{l}\text { MAX } \\
\text { HHA } \\
\text { FHE }\end{array}$ & $\begin{array}{r}.05 \\
.05\end{array}$ & $\begin{array}{r}.04 \\
.01 \\
.01\end{array}$ & $\begin{array}{r}.25 \\
-25 \\
.05\end{array}$ & $\begin{array}{l}85 \\
05 \\
01\end{array}$ & $\begin{array}{r}05 \\
.93 \\
01\end{array}$ & .85 \\
\hline $\begin{array}{l}\text { PCT } \\
\text { PACT }\end{array}$ & 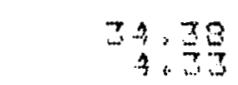 & 40,68 & 9.09 & $\begin{array}{r}74.95 \\
7.76\end{array}$ & $\begin{array}{l}5.47 \\
3+46\end{array}$ & $13 \cdot 9=$ \\
\hline
\end{tabular}

NATA FOF FILE: H1101

\begin{tabular}{|c|c|c|c|c|c|c|}
\hline $\sin$ & 4100 & $25: 0$ & & 95 & & \\
\hline COYF & $\begin{array}{c}\mathrm{Cr} \\
93\end{array}$ & $\begin{array}{c}\text { cry } \\
. .02\end{array}$ & $.05 \frac{2}{2}$ & $0 \%$ & cry & $\cos$ \\
\hline $\begin{array}{l}\text { MAY } \\
\text { HISH } \\
\text { WMS }\end{array}$ & $\begin{array}{r}45 \\
+15 \\
+15\end{array}$ & $\begin{array}{r}.05 \\
.05 \\
.01\end{array}$ & $\begin{array}{r}11 \\
: 14 \\
0 \%\end{array}$ & .05 & $\begin{array}{l}30 \\
.51 \\
.07\end{array}$ & .05 \\
\hline PFACT & $\therefore 9,93$ & 3.13 & $7 \cdot 52$ & $5 \cdot 5$ & 13.31 & $\frac{3}{7}: 3$ \\
\hline
\end{tabular}

MATA FQF: FILE: H1101

\begin{tabular}{|c|c|c|c|c|c|c|}
\hline Fuss & $\begin{array}{l}450 \\
37=5\end{array}$ & 25.0 & 350.0 & $\begin{array}{r}4[1 \\
72+7\end{array}$ & & \\
\hline $\begin{array}{l}\text { COMF } \\
\text { MEAS! }\end{array}$ & $\begin{array}{c}C H \\
: 19\end{array}$ & $\begin{array}{l}\text { Cry } \\
.00\end{array}$ & $\begin{array}{l}.52 \\
.05\end{array}$ & 64 & crsy & $6 \%$ \\
\hline $\begin{array}{l}\text { MAX } \\
\text { Mat } \\
\text { mas }\end{array}$ & $\begin{array}{r}.46 \\
-30 \\
11\end{array}$ & $\begin{array}{r}+05 \\
.03 \\
.01\end{array}$ & $\begin{array}{l}.17 \\
.02 \\
.03\end{array}$ & $\begin{array}{l}.09 \\
.92 \\
.02\end{array}$ & $\cdot \frac{20}{2}$ & $\begin{array}{l}.00 \\
.00 \\
.01\end{array}$ \\
\hline $\begin{array}{l}\text { FACT } \\
\text { FACT }\end{array}$ & $4: 51$ & $1 \frac{1}{3} \cdot \frac{3}{2}$ & $\begin{array}{l}3.15 \\
4.56\end{array}$ & 3.5 & $19 \cdot 47$ & $3: 07$ \\
\hline
\end{tabular}




\begin{tabular}{|c|c|c|c|c|c|c|}
\hline Fils. & 3300 & $25 . \frac{1}{0}$ & $\begin{array}{r}A Z \\
350.0\end{array}$ & $\begin{array}{r}V C 1 \\
42.3\end{array}$ & & \\
\hline COMF & Cro & $\begin{array}{r}\text { CFY } \\
\cdots, 00\end{array}$ & $\begin{array}{l}\operatorname{coz} 2 \\
.05\end{array}$ & cres & $\begin{array}{l}64 y \\
.02\end{array}$ & cri \\
\hline $\begin{array}{l}\text { May : } \\
\text { Mist } \\
\text { Fis: }\end{array}$ & $\begin{array}{r}50 \\
.54 \\
.07\end{array}$ & $\begin{array}{r}.03 \\
-.04 \\
.01\end{array}$ & $\begin{array}{l}15 \\
.04 \\
.02\end{array}$ & .07 & .12 & $\begin{array}{l}.01 \\
.05 \\
.01\end{array}$ \\
\hline GFACT & $\frac{5}{4}: 79$ & 2.32 & $\begin{array}{l}1.30 \\
4.20\end{array}$ & $\begin{array}{l}3.0 \% \\
3.7 \%\end{array}$ & $7 \cdot 59$ & $=\div 3$ \\
\hline BATA $F$ & FF: FILE : & $H 1101$ & & & & \\
\hline 5난 & $\begin{array}{r}41400 \\
292.5\end{array}$ & 25.0 & $350 .{ }^{A I}$ & $\begin{array}{r}U C L \\
+2.3\end{array}$ & & \\
\hline $\begin{array}{l}\text { COMF } \\
\text { MEAH : }\end{array}$ & CF. & $\begin{aligned} \text { CFy } \\
-.01\end{aligned}$ & $.0 \%$ & .01 & CMY & $\begin{array}{c}\text { CHz } \\
.02\end{array}$ \\
\hline 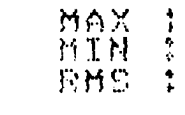 & $\begin{array}{l}.51 \\
.51 \\
.06 \\
.06\end{array}$ & $\begin{array}{r}.07 \\
.04 \\
.01\end{array}$ & $\begin{array}{l}14 \\
.02 \\
.02\end{array}$ & $\begin{array}{l}+.55 \\
.03 \\
.01\end{array}$ & .05 & $\begin{array}{l}.99 \\
.05 \\
.01\end{array}$ \\
\hline GFACT & 咅: & 5.37 & $\frac{1}{3}: 00$ & $\frac{5}{9} \cdot 77$ & 4.55 & $\frac{1}{2} \cdot 08$ \\
\hline
\end{tabular}

MATA FOF FILE: H1101

\begin{tabular}{|c|c|c|c|c|c|c|}
\hline fur! & $\begin{array}{l}\text { WIMS } \\
200.0\end{array}$ & 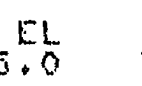 & & $42 \cdot \frac{1}{2}$ & & \\
\hline $\begin{array}{l}\text { corif: } \\
\text { rents: }\end{array}$ & $\begin{array}{ll}C F \\
: 15\end{array}$ & $\begin{array}{l}\text { Cry } \\
.00\end{array}$ & .05 & .02 & $\begin{array}{l}\text { cisy } \\
.0 .9\end{array}$ & $\begin{array}{c}c .32 \\
.00\end{array}$ \\
\hline $\begin{array}{l}\text { MAO } \\
\text { MIH } \\
\text { BHSO }\end{array}$ & $\begin{array}{r}95 \\
.51 \\
0\end{array}$ & $\begin{array}{r}.02 \\
.03 \\
.01\end{array}$ & .10 & $\begin{array}{l}.06 \\
.02 \\
.01\end{array}$ & .07 & .02 \\
\hline GFACT & $\frac{27}{5}: 17$ & 7.5 .4 & $\frac{1}{4}+13$ & $\stackrel{51}{5}: 30$ & 3.95 & $17407=$ \\
\hline
\end{tabular}

WATA FOF FILE: H1101

nur! $265.0 \quad 25.0 \quad 350.0$ AZ

\begin{tabular}{|c|c|c|c|c|c|c|}
\hline $\begin{array}{l}\text { comp : } \\
\text { Sentil }\end{array}$ & $\begin{array}{l}\mathrm{Cr} \\
.17\end{array}$ & $\begin{array}{c}.5 y \\
.01\end{array}$ & .05 & .01 & $\begin{array}{l}\text { CMY } \\
0.4\end{array}$ & .01 \\
\hline $\begin{array}{l}\text { MAY } \\
\text { MTHA } \\
\text { Fims }\end{array}$ & $\begin{array}{l}132 \\
: 02\end{array}$ & $\begin{array}{l}.04 \\
.02 \\
.01\end{array}$ & $: \frac{1}{0}: 0$ & $\begin{array}{l}.05 \\
.04 \\
.01\end{array}$ & $\begin{array}{l}17 \\
.10 \\
.02\end{array}$ & $\begin{array}{l}.01 \\
.03 \\
.01\end{array}$ \\
\hline SFACT & $\frac{3}{3} \cdot 59$ & $3 \cdot 31$ & 1.83 & $\begin{array}{l}5.01 \\
3.05\end{array}$ & $\begin{array}{l}2 \\
3\end{array}$ & $9 \cdot \frac{1}{3} \cdot \frac{1}{4}$ \\
\hline
\end{tabular}




\begin{tabular}{|c|c|c|c|c|c|c|}
\hline ruts & $39 \frac{4}{4} 5$ & 25.6 & 350.0 & +1.5 & & \\
\hline CONAF & $\frac{c 5 x}{12}$ & $\begin{array}{l}\text { c.5 } \\
.00\end{array}$ & $\begin{array}{l}\operatorname{cog} \\
.00\end{array}$ & $\begin{array}{l}\text { crs } \\
.00\end{array}$ & cisy & $0 \times$ \\
\hline $\begin{array}{l}\text { MAY } \\
\text { KIYS } \\
\text { WME }\end{array}$ & $\begin{array}{r}51 \\
-80 \\
: 07\end{array}$ & $\begin{array}{r}.94 \\
+04 \\
+01\end{array}$ & .14 & $\begin{array}{l}.0 \hat{1} \\
.05 \\
.01\end{array}$ & $: \frac{2}{2}=\frac{9}{2}$ & .01 \\
\hline $\begin{array}{l}\text { GACT } \\
\text { FACT }\end{array}$ & $\frac{2}{4}: 6$ & 12.52 & $\frac{1}{2} \cdot \frac{29}{59}$ & 12.48 & $\begin{array}{l}3.25 \\
4+61\end{array}$ & $\frac{01}{2} \cdot 0$ \\
\hline
\end{tabular}


Heliostat 5, Configuration 5-A

\begin{tabular}{|c|c|c|c|c|c|c|}
\hline Case & Run 非 & WD & $\mathrm{EL}$ & $\mathrm{AZ}$ & Day & Time \\
\hline \multirow[t]{6}{*}{ Wutr AM } & 136 & 0 & 10 & 320 & 355 & $8 \mathrm{AN}$ \\
\hline & 137 & 337.5 & 10 & 320 & 355 & 8 AM \\
\hline & 138 & 315 & 10 & 320 & 355 & $8 \mathrm{AM}$ \\
\hline & 139 & 292.5 & 10 & 320 & 355 & $8 \mathrm{AM}$ \\
\hline & 140 & 270 & 10 & 320 & 355 & $8 \mathrm{AM}$ \\
\hline & 141 & 247.5 & 10 & 320 & 355 & $8 \mathrm{AM}$ \\
\hline \multirow[t]{6}{*}{ Whtr Noon } & 148 & 0 & 20 & 290 & 355 & $0 \mathrm{PM}$ \\
\hline & 149 & 337.5 & 20 & 290 & 355 & $0 \mathrm{PM}$ \\
\hline & 150 & 315 & 20 & 290 & 355 & $0 \mathrm{PM}$ \\
\hline & 151 & 292.5 & 20 & 290 & 355 & 0 PM \\
\hline & 152 & 270 & 20 & 290 & 355 & $0 \mathrm{PM}$ \\
\hline & 153 & 247.5 & 20 & 290 & 355 & $0 \mathrm{PM}$ \\
\hline \multirow{6}{*}{ Wntr PM } & 142 & 247.5 & 10 & 265 & 355 & $4 \mathrm{PHI}$ \\
\hline & 143 & 270 & 10 & 265 & 355 & $4 \mathrm{PM}$ \\
\hline & 144 & 292.5 & 10 & 265 & 355 & $4 \mathrm{PM}$ \\
\hline & 145 & 315 & 10 & 265 & 355 & $4 \mathrm{PM}$ \\
\hline & 146 & 337.5 & 10 & 265 & 355 & $4 \mathrm{PM}$ \\
\hline & 147 & 0 & 10 & 265 & 355 & $4 \mathrm{PN}$ \\
\hline Ver. Stow & 154 & 315 & 0 & 320 & 355 & $8 \mathrm{AM}$ \\
\hline Hor. Stow & 155 & 315 & 90 & 320 & 355 & $8 \mathrm{AM}$ \\
\hline
\end{tabular}

This set to check the effectiveness of presence of the upstrean heliostat. 


\begin{tabular}{|c|c|c|c|c|c|c|}
\hline rust" & $\begin{array}{l}4 \pi+! R \\
0.0\end{array}$ & 10.0 & $\therefore=0^{\circ}: \overline{0}$ & $\begin{array}{l}32 E L \\
32.5\end{array}$ & & \\
\hline $\begin{array}{l}\text { Comr } \\
\text { MEAH }\end{array}$ & $c r y$ & Cry & cra & $\begin{array}{l}\mathrm{CMO} \\
. \mathrm{O}\end{array}$ & $\begin{array}{l}C M Y \\
.0 ?\end{array}$ & Cro: \\
\hline $\begin{array}{l}\text { MA: } \\
\text { MAIIG } \\
\text { FMS }\end{array}$ & $\begin{array}{r}1 \cdot 15 \\
\cdot 15 \\
+15\end{array}$ & $\begin{array}{l}92 \\
.02 \\
.02\end{array}$ & $\begin{array}{l}09 \\
.02 \\
.01\end{array}$ & $\begin{array}{r}13 \\
: 03 \\
0\end{array}$ & 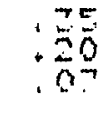 & .01 \\
\hline $\begin{array}{l}\text { PFACT } \\
\text { FACT }\end{array}$ & $\frac{2}{9} \cdot 2$ & ت. & $\begin{array}{l}2.41 \\
3.57\end{array}$ & $\frac{m}{3} \cdot \overline{\hat{O}}$ & 3.00 & 3.2 \\
\hline
\end{tabular}

INATA FOR FILE: H500O

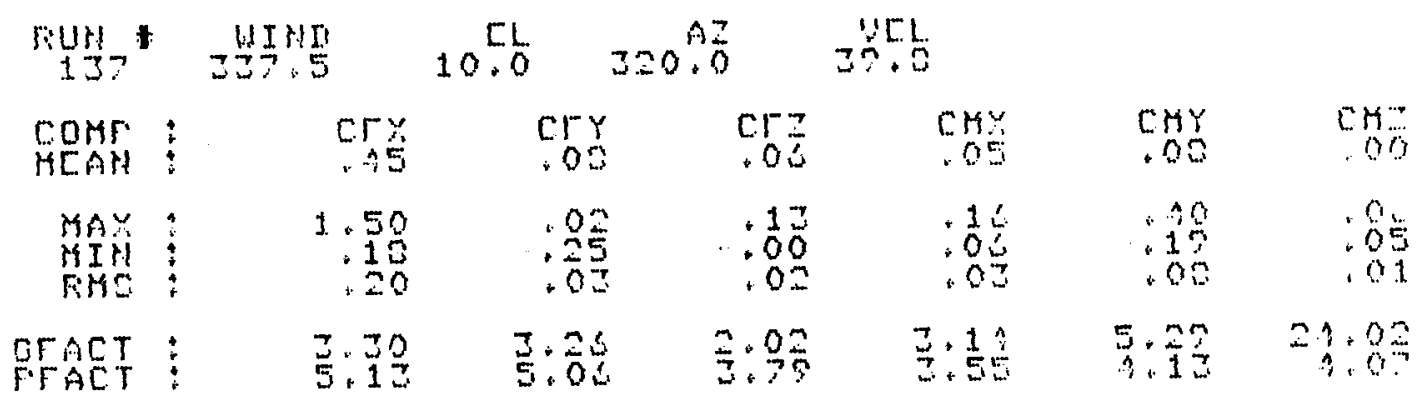

IATA FOF: FILE: H50OO

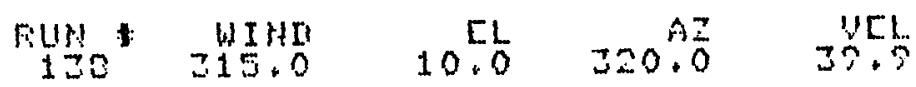

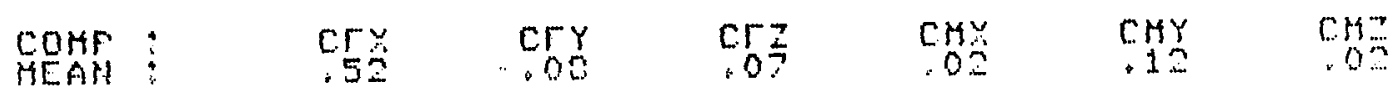

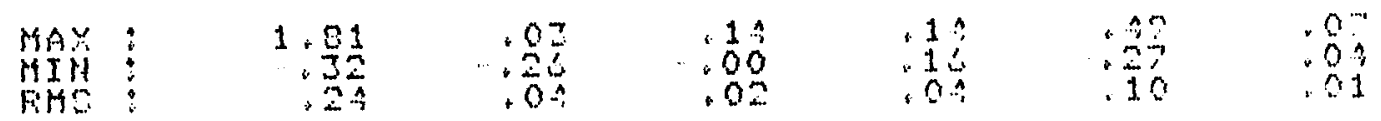

GPACT:

IATA FOF FILE: H5000

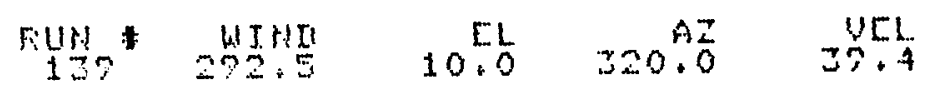

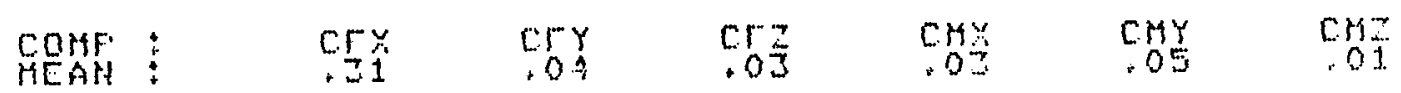

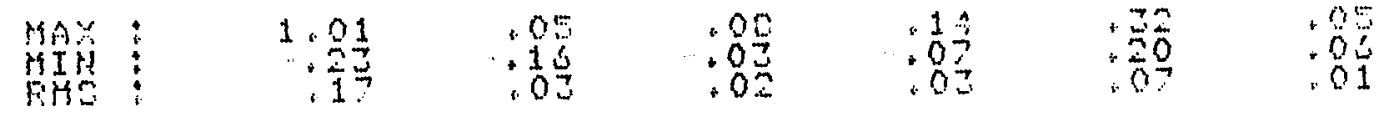

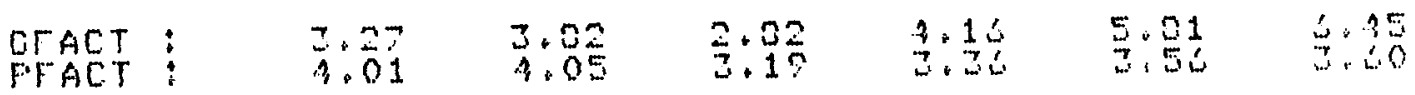




\begin{tabular}{|c|c|c|c|c|c|c|}
\hline fius & $2 \begin{array}{l}\text { WIIT } \\
270.0\end{array}$ & 10.5 & 320.0 & $\begin{array}{l}\text { YLL } \\
35.2\end{array}$ & & \\
\hline $\begin{array}{l}\text { COMF } \\
\text { BEAH }\end{array}$ & 25 & Cry & $\operatorname{cr}_{02}^{2}$ & $\begin{array}{l}\text { C.MX } \\
.01\end{array}$ & $\begin{array}{l}\text { cisy } \\
.0 \mathrm{~s}\end{array}$ & $\begin{array}{c}\operatorname{cHg} \\
.05\end{array}$ \\
\hline $\begin{array}{l}\text { MAY } \\
\text { MUIS }\end{array}$ & $\begin{array}{r}1.10 \\
: 33 \\
: 21\end{array}$ & $\begin{array}{l}.05 \\
.15 \\
.05\end{array}$ & $\begin{array}{l}.09 \\
.04 \\
.02\end{array}$ & $\begin{array}{l}17 \\
017 \\
09\end{array}$ & : & .09 \\
\hline $\begin{array}{l}\text { QFACT } \\
\text { FFACT }\end{array}$ & $\frac{4}{2} \cdot \frac{21}{75}$ & 5.95 & 3.97 & 17.40 & $\begin{array}{l}2+8 z \\
x+1\end{array}$ & $\begin{array}{l}9.08 \\
7.75\end{array}$ \\
\hline IIATA F & JF FILE : & $\mathrm{H} 5000$ & & & & \\
\hline Filus & $\begin{array}{r}W I H I \\
24\end{array}$ & 10.0 & 320.0 & บ단 & & \\
\hline $\begin{array}{l}\text { COHS : } \\
\text { MEAH : }\end{array}$ & $\begin{array}{l}c r \% \\
.10\end{array}$ & $\begin{array}{l}\text { CFy } \\
.01\end{array}$ & 65 & $\begin{array}{l}\text { CMY } \\
.00\end{array}$ & $\begin{array}{l}\mathrm{CMr} \\
.01\end{array}$ & $\begin{array}{l}\mathrm{CH}_{1} \\
.01\end{array}$ \\
\hline $\begin{array}{l}\text { MAAX } \\
\text { HIYI } \\
\text { FMS }\end{array}$ & $\begin{array}{r}30 \\
-37 \\
.12\end{array}$ & $\begin{array}{l}.07 \\
.00 \\
.02\end{array}$ & $\begin{array}{l}.07 \\
0.1 \\
+02\end{array}$ & $\begin{array}{l}10 \\
.15 \\
.05\end{array}$ & $\begin{array}{l}10 \\
0 \\
0 \\
0 \\
05\end{array}$ & .00 \\
\hline $\begin{array}{l}\text { GFACT } \\
\text { FFACT }\end{array}$ & 7.05 & $\begin{array}{l}5.80 \\
3.41\end{array}$ & $\begin{array}{l}4.41 \\
3.49\end{array}$ & $25 \cdot 74$ & 38.81 & $\frac{5}{3} \cdot 39$ \\
\hline
\end{tabular}

IATA FOF: FILE: $H 5000$

\begin{tabular}{|c|c|c|c|c|c|c|}
\hline $\operatorname{FuH}_{142}$ & $\begin{array}{r}W I H n \\
2.77 .5\end{array}$ & 10.0 & & $\begin{array}{r}\text { vㄷ. } \\
40.0\end{array}$ & & \\
\hline CORAF : & $\begin{array}{l}\operatorname{cry} \\
-16\end{array}$ & $\begin{array}{l}\text { CFY } \\
+0.7\end{array}$ & $\begin{array}{l}\operatorname{ctz} \\
.03\end{array}$ & $\begin{array}{l}c r y \\
.01\end{array}$ & Cry & $\begin{array}{c}\mathrm{CH} \\
.00\end{array}$ \\
\hline $\begin{array}{l}\text { MAX } \\
\text { MIHE } \\
\text { RMS }\end{array}$ & $\begin{array}{r}.32 \\
-185 \\
+16\end{array}$ & $\begin{array}{r}13 \\
01 \\
01\end{array}$ & $\begin{array}{r}.03 \\
+13 \\
+05\end{array}$ & .07 & .92 & $\begin{array}{l}.05 \\
.03 \\
.02\end{array}$ \\
\hline $\begin{array}{l}\text { QFACT } \\
\text { FFACT }\end{array}$ & $\frac{5}{4} \cdot 32$ & $\frac{3}{5} \cdot 25$ & $\begin{array}{l}4.53 \\
3.81\end{array}$ & $\begin{array}{l}5.89 \\
4.8 \%\end{array}$ & $\therefore 28$ & $18+21$ \\
\hline
\end{tabular}

IATA FOF: FILE: H500O

\begin{tabular}{|c|c|c|c|c|c|c|}
\hline $\operatorname{lovit}_{13}$ & $\begin{array}{r}W I+I I \\
270.0\end{array}$ & ${ }_{10.0}^{E L}$ & & $\begin{array}{r}\text { UCL. } \\
40.4\end{array}$ & & \\
\hline $\begin{array}{l}\text { COMF : } \\
\text { MEAH : }\end{array}$ & $\begin{array}{r}\text { cry } \\
-.08\end{array}$ & $\begin{array}{c}\text { c. } Y \\
.03\end{array}$ & .02 & CMy & $\begin{array}{l}\text { CHY } \\
.02\end{array}$ & chiz \\
\hline $\begin{array}{l}\text { MAX } \\
\text { MIHS } \\
\text { FYS }\end{array}$ & -.22 & $\begin{array}{l}.03 \\
.01 \\
.01\end{array}$ & $\begin{array}{l}.07 \\
.05 \\
.01\end{array}$ & $\begin{array}{l}.03 \\
.01 \\
.01\end{array}$ & $\begin{array}{l}11 \\
.15 \\
.05\end{array}$ & .02 \\
\hline $\begin{array}{l}\text { GFACT } \\
\text { FFACT }\end{array}$ & $\begin{array}{l}2.10 \\
5: 08\end{array}$ & $\frac{2}{3}+7 \frac{7}{3}$ & $\frac{3.10}{2}+10$ & $\frac{2}{4}+30$ & 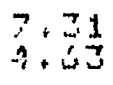 & 3.53 \\
\hline
\end{tabular}

1 


\begin{tabular}{|c|c|c|c|c|c|c|}
\hline num & $\begin{array}{r}4 I 3 \pi \\
292.5\end{array}$ & 10.0 & $=65.0$ & $\begin{array}{r}\text { YCL } \\
40.1\end{array}$ & & \\
\hline $\begin{array}{l}\text { COMF } \\
\text { MEAN }\end{array}$ & $\begin{array}{l}\text { CFX } \\
.10\end{array}$ & $\begin{array}{l}\text { CFY } \\
.02\end{array}$ & $\begin{array}{cl}C F \\
.02\end{array}$ & $\begin{array}{l}\text { Cry } \\
.00\end{array}$ & $\begin{array}{l}\text { cry } \\
.0 \mathrm{~S}\end{array}$ & $\begin{array}{l}\text { cha } \\
.00\end{array}$ \\
\hline $\begin{array}{l}\text { MAX } \\
\text { MIS } \\
\text { FHS }\end{array}$ & $\begin{array}{r}73 \\
.52 \\
.13\end{array}$ & $\begin{array}{r}.00 \\
+80 \\
.01\end{array}$ & .10 & .05 & $\begin{array}{l}32 \\
.13 \\
0\end{array}$ & .04 \\
\hline $\begin{array}{l}\text { GFACT } \\
\text { FFACT }\end{array}$ & $7.5 \frac{5}{3}$ & $\frac{3}{3}: 75$ & $\frac{2.40}{3} \cdot 67$ & $13 \cdot \frac{8}{3}$ & 8.72 & $39 \cdot \frac{6}{58}$ \\
\hline
\end{tabular}

IIATA FOF: FILE: H50OO

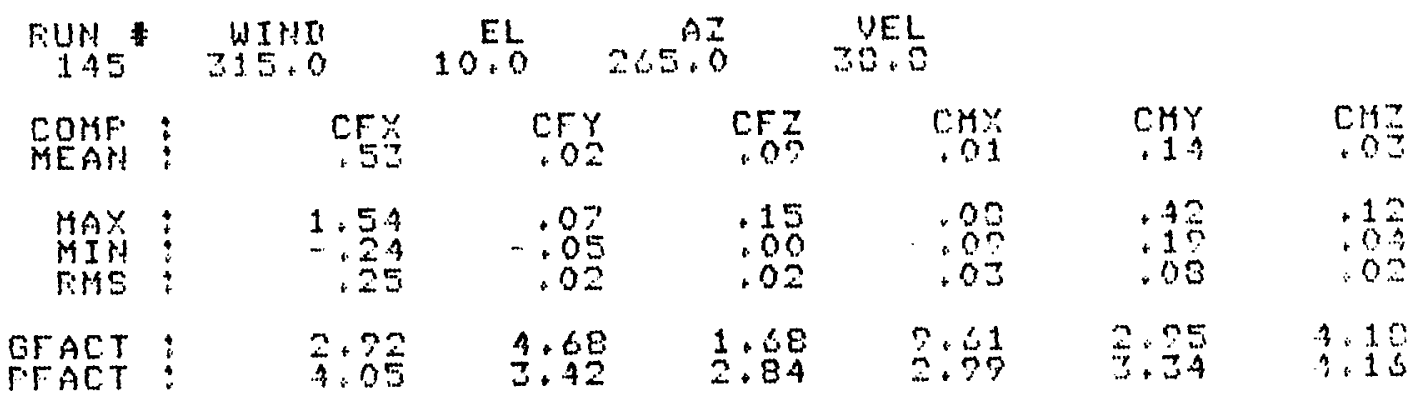

IMATA FOF FILE: H5OOO

\begin{tabular}{|c|c|c|c|c|c|c|}
\hline Furs & $\begin{array}{r}W I M I \\
2 Z 7\end{array}$ & 10.6 & & UEL & & \\
\hline $\begin{array}{l}\text { COMF: } \\
\text { MEAHI }\end{array}$ & $\begin{array}{l}\text { CFY } \\
.44\end{array}$ & $\begin{array}{l}\text { CFY } \\
.01\end{array}$ & $\begin{array}{l}\text { CFZ } \\
.06\end{array}$ & CH\% & $\begin{array}{c}\mathrm{CHY} \\
.11\end{array}$ & $\mathrm{CHz}$ \\
\hline $\begin{array}{l}\text { MAX } \\
\text { MIYH } \\
\text { rimS }\end{array}$ & $\begin{array}{r}1+28 \\
-13\end{array}$ & $\begin{array}{l}.03 \\
.05 \\
.02\end{array}$ & .13 & .00 & $\begin{array}{r}23 \\
13 \\
103 \\
0\end{array}$ & $\begin{array}{l}.02 \\
01 \\
02\end{array}$ \\
\hline MFACT & 2.90 & $\begin{array}{l}5.58 \\
3.58\end{array}$ & $\frac{2}{3}: \frac{1}{89}$ & $13 \cdot \frac{8}{3} \cdot 83$ & 3.09 & 3 \\
\hline
\end{tabular}

IIATA FOF FILE: H500O

\begin{tabular}{|c|c|c|c|c|c|c|}
\hline $\operatorname{rum}_{12}$ & WIMI & $10+0$ & & VEL & & \\
\hline COMF & CFX & $\begin{array}{r}\text { CFY } \\
-.02\end{array}$ & .01 & $\begin{array}{l}\text { cry } \\
.05\end{array}$ & $\begin{array}{l}\mathrm{CHY} \\
+0 \%\end{array}$ & $\begin{array}{r}\operatorname{crg} 2 \\
+0.3\end{array}$ \\
\hline $\begin{array}{l}\text { HAX } \\
\text { MINA } \\
\text { FMS }\end{array}$ & $\begin{array}{r}.70 \\
-.18 \\
.14\end{array}$ & $\begin{array}{r}.05 \\
.02 \\
.01\end{array}$ & .07 & $\begin{array}{r}.03 \\
.08 \\
.02\end{array}$ & .25 & .08 \\
\hline $\begin{array}{l}\text { QFACT } \\
\text { FFACT }\end{array}$ & $\begin{array}{l}2.80 \\
\frac{1}{4} .06\end{array}$ & $\frac{2}{3} .5 \%$ & $\begin{array}{l}5.06 \\
2.72\end{array}$ & 2.22 & $3+83$ & $\begin{array}{l}210 \\
\approx .92\end{array}$ \\
\hline
\end{tabular}




\begin{tabular}{|c|c|c|c|c|c|c|}
\hline rut ${ }_{1.8} *$ & WIMn & 20.0 & 290.0 & $\begin{array}{l}\text { UEL } \\
39.3\end{array}$ & & \\
\hline $\begin{array}{l}\text { COMF } \\
\text { MEAN }\end{array}$ & $\begin{array}{l}.0 \% \\
.40\end{array}$ & $\begin{array}{r}\text { CFY } \\
.04\end{array}$ & 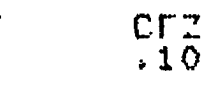 & $\begin{array}{c}\text { crss } \\
.06\end{array}$ & $\begin{array}{l}\text { C.MY } \\
.09\end{array}$ & $\begin{array}{l}\text { Criz } \\
.03\end{array}$ \\
\hline $\begin{array}{l}\text { MAX } \\
\text { FHS } \\
\text { FHS }\end{array}$ & $\begin{array}{r}1.10 \\
-05 \\
.15\end{array}$ & $\begin{array}{r}.03 \\
.13 \\
.02\end{array}$ & $\begin{array}{r}.20 \\
.00 \\
.03\end{array}$ & $\begin{array}{l}12 \\
.00 \\
.02\end{array}$ & $\begin{array}{r}29 \\
+28 \\
+10 \\
+05\end{array}$ & $\begin{array}{l}.01 \\
.03 \\
.01\end{array}$ \\
\hline $\begin{array}{l}\text { OFACT } \\
\text { FFACT }\end{array}$ & $\frac{2}{4} \cdot 76$ & $\begin{array}{l}3.73 \\
3.86\end{array}$ & $\frac{2}{3} \cdot 9 \frac{0}{3}$ & $\frac{1}{3} \cdot \frac{25}{3}$ & 3.30 & $\begin{array}{l}2.90 \\
4+31 \\
+71\end{array}$ \\
\hline IIATA F & IFi FILE : & $H 5000$ & & & & \\
\hline$\underset{198}{\text { rut }}$ & 337.5 & 20.0 & 290.0 & บㄷ․ & & \\
\hline $\begin{array}{l}\text { COMF } \\
\text { MEAN }\end{array}$ & $\begin{array}{l}C F x \\
.48\end{array}$ & $\begin{array}{r}\mathrm{CFY}^{-y} \\
. .03\end{array}$ & $\begin{array}{l}\text { CF } \\
.10\end{array}$ & $\begin{array}{l}.025 \\
.02\end{array}$ & CMY & $\begin{array}{l}\text { CHZ } \\
+01\end{array}$ \\
\hline $\begin{array}{l}\text { MAY } \\
\text { FIN } \\
\text { FMS }\end{array}$ & $\begin{array}{r}1+23 \\
: 105 \\
\cdot 18\end{array}$ & $\begin{array}{r}.07 \\
-.09 \\
.02\end{array}$ & .02 & $\begin{array}{r}.09 \\
.04 \\
.02\end{array}$ & $\begin{array}{r}74 \\
.14 \\
04\end{array}$ & .07 \\
\hline $\begin{array}{l}\text { QFACT } \\
\text { FFACT }\end{array}$ & $\begin{array}{l}2.33 \\
4.87\end{array}$ & $\begin{array}{l}3.43 \\
3.54\end{array}$ & $\frac{2.39}{5.35}$ & $\begin{array}{l}3.70 \\
3.17\end{array}$ & $\begin{array}{l}5.13 \\
1.67\end{array}$ & $\begin{array}{l}4.30 \\
4.20\end{array}$ \\
\hline
\end{tabular}

\section{IATA FOF FILE: H5000}

\begin{tabular}{|c|c|c|c|c|c|c|}
\hline Kun & WIMI & 20.0 & $\begin{array}{l}A 2 \\
.0\end{array}$ & 39.0 & & \\
\hline $\begin{array}{l}\text { COMF : } \\
\text { MEAM : }\end{array}$ & 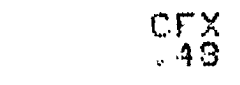 & $\begin{array}{r}\text { CFY } \\
-+02\end{array}$ & $\begin{array}{c}\text { C.F } \\
.11\end{array}$ & CHY & $\begin{array}{l}\text { crsy } \\
: 11\end{array}$ & $\begin{array}{l}\text { crsz } \\
.01\end{array}$ \\
\hline $\begin{array}{l}\text { MAX } \\
\text { RIN } \\
\text { FHS }\end{array}$ & $\begin{array}{r}1.56 \\
\cdot .23 \\
-21\end{array}$ & $\begin{array}{r}.07 \\
. .13 \\
.03\end{array}$ & $.2 \frac{1}{8}$ & $\begin{array}{l}11 \\
.08 \\
.02\end{array}$ & $\begin{array}{l}.11 \\
.15 \\
.07\end{array}$ & $\begin{array}{l}.09 \\
.03 \\
.01\end{array}$ \\
\hline $\begin{array}{l}\text { GFACT } \\
\text { FFACT }\end{array}$ & $\frac{3}{5}+\frac{27}{2}$ & $\begin{array}{l}5.21 \\
3.87\end{array}$ & $\begin{array}{l}2.01 \\
3.76\end{array}$ & $\begin{array}{l}4.04 \\
3.39\end{array}$ & $\begin{array}{l}3.34 \\
4.20\end{array}$ & $\begin{array}{r}-53 \\
7.52\end{array}$ \\
\hline
\end{tabular}

INTA FOF FILE : H500O

\begin{tabular}{|c|c|c|c|c|c|c|}
\hline Fum 151 & $\begin{array}{r}\text { WIMII } \\
292.5\end{array}$ & 20.0 & & $\begin{array}{r}\text { Y.L } \\
38.9\end{array}$ & & \\
\hline $\begin{array}{l}\text { COMF: } \\
\text { MEAN }\end{array}$ & $\begin{array}{l}65 \% \\
+16\end{array}$ & $\begin{array}{r}\text { CFY } \\
-.00\end{array}$ & .02 & $\begin{array}{l}\text { CMY } \\
.02\end{array}$ & $\begin{array}{l}\text { CHY } \\
.02\end{array}$ & ${ }_{0}^{C M}=$ \\
\hline $\begin{array}{l}\text { MAX } \\
\text { MIN } \\
\text { FISS }\end{array}$ & $\begin{array}{r}1+05 \\
+33 \\
+13\end{array}$ & $\begin{array}{r}.10 \\
. .07 \\
.01\end{array}$ & $\begin{array}{r}10 \\
.05 \\
+02\end{array}$ & $\begin{array}{l}.06 \\
.05 \\
.02\end{array}$ & $\begin{array}{l}25 \\
105 \\
105\end{array}$ & .017 \\
\hline $\begin{array}{l}\text { GFACT } \\
\text { FFACT }\end{array}$ & $\begin{array}{l}3.58 \\
6.34\end{array}$ & $\begin{array}{r}339.19 \\
4.84\end{array}$ & $\begin{array}{l}7.58 \\
6.00\end{array}$ & $\begin{array}{l}3.88 \\
3.10\end{array}$ & 10.59 & $\begin{array}{l}2.08 \\
3.06\end{array}$ \\
\hline
\end{tabular}




\begin{tabular}{|c|c|c|c|c|c|c|}
\hline $\begin{array}{l}\text { Fust } \\
152\end{array}$ & $\begin{array}{r}\text { Win } \\
270.0\end{array}$ & 20.5 & $\begin{array}{r}A z \\
290.0\end{array}$ & $\begin{array}{r}\text { VEL } \\
39.0\end{array}$ & & \\
\hline COMF & $\begin{array}{l}C F y \\
.01\end{array}$ & $\begin{array}{l}\text { CFY } \\
.01\end{array}$ & $\begin{array}{l}\operatorname{cog} \\
.03\end{array}$ & $\begin{array}{l}C H K \\
.01\end{array}$ & CHY & $\begin{array}{c}\mathrm{cm} \\
.01\end{array}$ \\
\hline $\begin{array}{l}\text { MAX } \\
\text { MIN } \\
\text { FME }\end{array}$ & $\begin{array}{r}+44 \\
-\quad 54 \\
+11\end{array}$ & $\begin{array}{r}+16 \\
-.09 \\
.02\end{array}$ & $\begin{array}{r}.08 \\
-.15 \\
.04\end{array}$ & $\begin{array}{l}.09 \\
.03 \\
.02\end{array}$ & $\begin{array}{l}10 \\
.20 \\
.04\end{array}$ & $\begin{array}{l}.03 \\
.03 \\
.01\end{array}$ \\
\hline $\begin{array}{l}\text { EFACT } \\
\text { FFACT }\end{array}$ & $\begin{array}{r}33.98 \\
4.92\end{array}$ & 13.89 & $\begin{array}{l}4.92 \\
2.97\end{array}$ & $11 \cdot 18$ & $\begin{array}{l}9.98 \\
3.00\end{array}$ & $\begin{array}{l}5.38 \\
1.11\end{array}$ \\
\hline IATA F & BF: FILE : & 45000 & & & & \\
\hline Fund & $2475 \frac{10}{5}$ & 20.5 & 290.02 & $\begin{array}{l}\text { YCL. } \\
39.3\end{array}$ & & \\
\hline $\begin{array}{l}\text { CONF: } \\
\text { MEAN }\end{array}$ & $\begin{array}{r}C F x \\
-25\end{array}$ & $\begin{array}{l}\text { CFY } \\
.04\end{array}$ & $\begin{array}{l}\operatorname{crz} \\
.08\end{array}$ & $\begin{array}{l}\operatorname{crs} \\
.03\end{array}$ & $\begin{array}{r}\text { CHY } \\
-.09\end{array}$ & .01 \\
\hline $\begin{array}{l}\text { MAX } \\
\text { MIN } \\
\text { RMS }\end{array}$ & $\begin{array}{r}.29 \\
-7 \frac{2}{3} \\
.13\end{array}$ & $\begin{array}{r}.17 \\
-: 00 \\
.03\end{array}$ & .02 & .04 & .07 & .09 \\
\hline $\begin{array}{l}\text { GFACT } \\
\text { FFACT }\end{array}$ & $\frac{2}{3} \cdot \frac{97}{51}$ & $\frac{3}{3}: \frac{85}{4}$ & $\frac{2}{3}+\frac{45}{51}$ & $3+\frac{6}{5}$ & $5 \cdot 17$ & 5.59 \\
\hline
\end{tabular}

IIATA FOF FILE: H5OOO

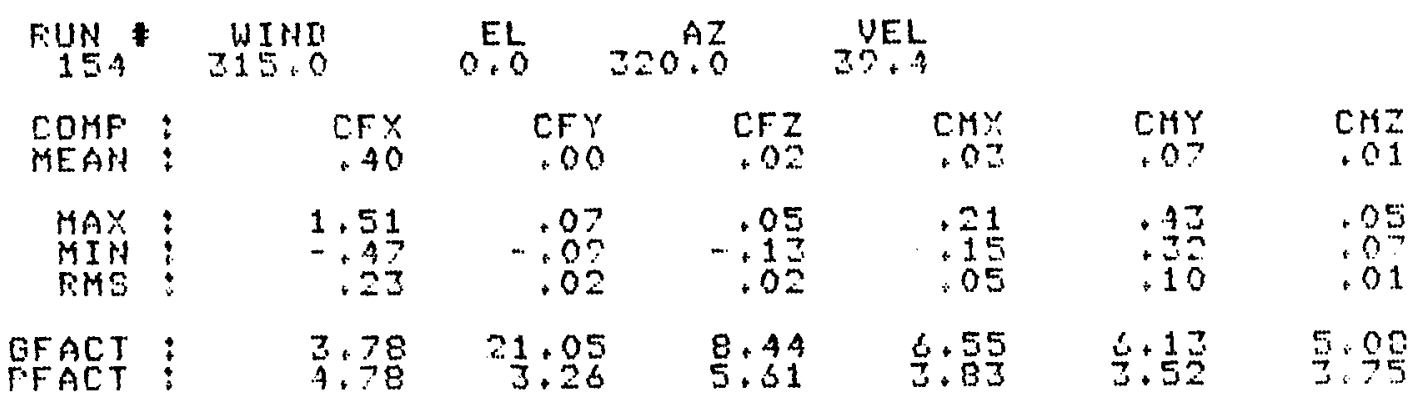

IIATA FOF FILE: H500O

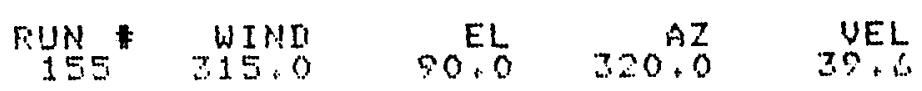

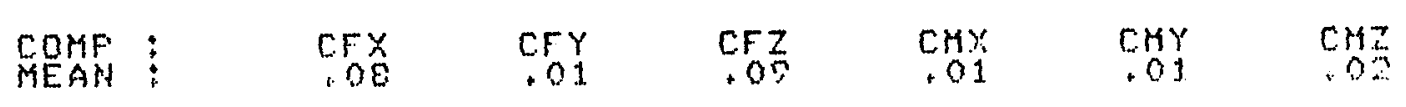

\begin{tabular}{|c|c|c|c|c|c|}
\hline $\begin{array}{l}\text { MAX } \\
\text { MIH } \\
\text { TMS }\end{array}$ & $\begin{array}{l}15 \\
.02 \\
.02\end{array}$ & $\begin{array}{r}.04 \\
-.03 \\
.01\end{array}$ & $\begin{array}{r}.25 \\
-.15 \\
.05\end{array}$ & $\begin{array}{r}.05 \\
-.05 \\
.05\end{array}$ & $\begin{array}{r}.00 \\
.02 \\
.02\end{array}$ \\
\hline ACT: & $\begin{array}{l}1 \cdot 80 \\
4: 14\end{array}$ & $3: 93$ & $\frac{2}{3} \cdot 72$ & $\hat{2} \div \frac{4}{9}$ & $5 \cdot 31$ \\
\hline
\end{tabular}

1 
Heliostat 5, Configuration 5-B

\begin{tabular}{|c|c|c|c|c|c|c|}
\hline Case & Run 非 & WD & EL & $\mathrm{AZ}$ & Day & Time \\
\hline \multirow[t]{6}{*}{ Smr AM } & 176 & 0 & 25 & 340 & 172 & $8 \mathrm{AM}$ \\
\hline & 178 & 337.5 & 25 & 340 & 172 & $8 \mathrm{AM}$ \\
\hline & 179 & 315 & 25 & 340 & 172 & $8 \mathrm{AM}$ \\
\hline & 180 & 292.5 & 25 & 340 & 172 & $8 \mathrm{AM}$ \\
\hline & 181 & 270 & 25 & 340 & 172 & $8 \mathrm{AM}$ \\
\hline & 182 & 247.5 & 25 & 340 & 172 & $8 \mathrm{AM}$ \\
\hline \multirow[t]{6}{*}{ Smr Noon } & 183 & 247.5 & 45 & 290 & 172 & $0 \mathrm{PM}$ \\
\hline & 184 & 270 & 45 & 290 & 172 & $0 \mathrm{PM}$ \\
\hline & 185 & 292.5 & 45 & 290 & 172 & $0 \mathrm{PM}$ \\
\hline & 186 & 315 & 45 & 290 & 172 & $0 \mathrm{PM}$ \\
\hline & 187 & 337.5 & 45 & 290 & 172 & $0 \mathrm{PM}$ \\
\hline & 188 & 0 & 45 & 290 & 172 & $0 \mathrm{PM}$ \\
\hline \multirow[t]{6}{*}{ Whtr AM } & 158 & 0 & 10 & 320 & 355 & $8 \mathrm{AM}$ \\
\hline & 159 & 337.5 & 10 & 320 & 355 & $8 \mathrm{AM}$ \\
\hline & 160 & 315 & 10 & 320 & 355 & $8 \mathrm{AM}$ \\
\hline & 161 & 292.5 & 10 & 320 & 355 & $8 \mathrm{AM}$ \\
\hline & 162 & 270 & 10 & 320 & 355 & $8 \mathrm{AM}$ \\
\hline & 163 & 247.5 & 10 & 320 & 355 & $8 \mathrm{AM}$ \\
\hline \multirow[t]{6}{*}{ Whitr Noon } & 170 & 0 & 20 & 290 & 355 & $0 \mathrm{PM}$ \\
\hline & 171 & 337.5 & 20 & 290 & 355 & $0 \mathrm{PM}$ \\
\hline & 172 & 315 & 20 & 290 & 355 & $0 \mathrm{PM}$ \\
\hline & 173 & 292.5 & 20 & 290 & 355 & $0 \mathrm{PM}$ \\
\hline & 174 & 270 & 20 & 290 & 355 & $0 \mathrm{PM}$ \\
\hline & 175 & 247.5 & 20 & 290 & 355 & $0 \mathrm{PM}$ \\
\hline \multicolumn{7}{|c|}{145} \\
\hline
\end{tabular}




\begin{tabular}{|c|c|c|c|c|c|c|}
\hline Case & Run 非 & WD & EL & $\mathrm{AZ}$ & Day & T'ime \\
\hline \multirow[t]{6}{*}{ Wntr PM } & 164 & 247.5 & 10 & 265 & 355 & $4 \mathrm{PN}$ \\
\hline & 165 & 270 & 10 & 265 & 355 & $4 P M$ \\
\hline & 166 & 292.5 & 10 & 265 & 355 & $4 \mathrm{PM}$ \\
\hline & 167 & 315 & 10 & 265 & 355 & $4 \mathrm{PM}$ \\
\hline & 168 & 337.5 & 10 & 265 & 355 & $4 \mathrm{PM}$ \\
\hline & 169 & 0 & 10 & 265 & 355 & $4 \mathrm{PM}$ \\
\hline Ver. Stow & 157 & 315 & 0 & 320 & 355 & 8 AM \\
\hline Hor. Stow & 156 & 315 & 90 & 320 & 355 & $8 \mathrm{AM}$ \\
\hline
\end{tabular}




\begin{tabular}{|c|c|c|c|c|c|c|}
\hline Fiurs & $\begin{array}{r}\text { WINI } \\
315.0\end{array}$ & $=0.0$ & 0.2 & 35.6 & & \\
\hline $\begin{array}{l}\text { COMF } \\
\text { MEAN }\end{array}$ & $\begin{array}{c}\cos \\
.01\end{array}$ & $\begin{array}{l}\text { CFY } \\
: 00\end{array}$ & .01 & $\begin{array}{l}\text { CMY } \\
.00\end{array}$ & $\begin{array}{c}\operatorname{cis} \\
.01\end{array}$ & .01 \\
\hline $\begin{array}{l}\text { MAX } \\
\text { MINS } \\
\text { FHE }\end{array}$ & $\begin{array}{r}.07 \\
.03 \\
.01\end{array}$ & $\begin{array}{r}.04 \\
-.04 \\
.01\end{array}$ & $\begin{array}{r}.70 \\
.45 \\
.09\end{array}$ & $\begin{array}{r}.07 \\
.07\end{array}$ & $\begin{array}{r}.04 \\
.09 \\
.02\end{array}$ & $\begin{array}{l}.07 \\
.81 \\
.01\end{array}$ \\
\hline $\begin{array}{l}\text { QFACT } \\
\text { FFACT }\end{array}$ & $5: \frac{2}{5}$ & $953+23$ & $\begin{array}{r}30.97 \\
4.91\end{array}$ & $\begin{array}{r}441.80 \\
4.94\end{array}$ & $11 \cdot 47$ & 7.83 \\
\hline
\end{tabular}

IIATA FOF FILE: H5OO1

\begin{tabular}{|c|c|c|c|c|c|c|}
\hline $\operatorname{Rum}_{35}$ & $\begin{array}{l}\text { WINII } \\
315: 0\end{array}$ & 0.5 & & & & \\
\hline $\begin{array}{l}\text { CONF : } \\
\text { MEAN : }\end{array}$ & $c_{21}$ & $\begin{array}{l}\text { CFY } \\
.01\end{array}$ & $\frac{\operatorname{cog}}{.02}$ & $\begin{array}{l}\mathrm{CH} \\
. \mathrm{OS}\end{array}$ & $\begin{array}{l}\text { Cryy } \\
.04\end{array}$ & $\begin{array}{l}\cos z \\
01\end{array}$ \\
\hline $\begin{array}{l}\text { MAX } \\
\text { HIH } \\
\text { FMS }\end{array}$ & $\begin{array}{l}1.84 \\
\cdots: \frac{4}{17}\end{array}$ & $\begin{array}{l}.08 \\
.05 \\
.02\end{array}$ & $\begin{array}{l}.03 \\
.12 \\
.02\end{array}$ & $\begin{array}{l}.20 \\
.08 \\
.02\end{array}$ & $\begin{array}{l}.35 \\
.18 \\
.06\end{array}$ & $\begin{array}{l}+02 \\
.06 \\
.01\end{array}$ \\
\hline $\begin{array}{l}\text { BFACT } \\
\text { FFACT }\end{array}$ & $\begin{array}{l}7.32 \\
6 \cdot 92\end{array}$ & $\frac{5}{3} \cdot \frac{21}{9}$ & $\begin{array}{l}5.78 \\
4.78\end{array}$ & $\begin{array}{c}0.44 \\
5.25\end{array}$ & $\begin{array}{r}10.06 \\
5.11\end{array}$ & $\begin{array}{l}3+85 \\
3+65\end{array}$ \\
\hline
\end{tabular}

IMTA FOF FILE: H5001

FuIS WIMI

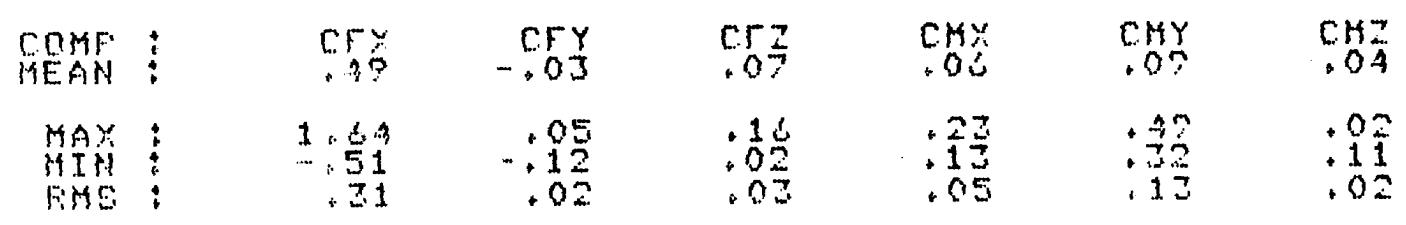

GFACT: $\begin{array}{llllll}3.31 & 3.50 & 2.27 & 4.12 & 5.17 & 5.58\end{array}$

IIATA FOF: FILE: H5001

FuS $15 \%$ WIYI $\quad 10.0 \quad 320.0$ AZ 39.3

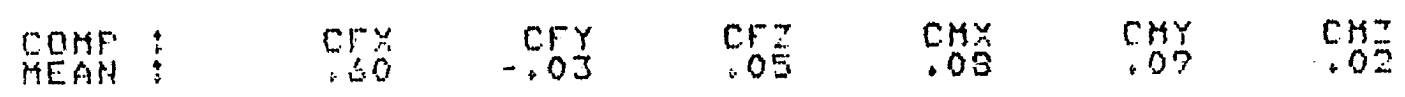

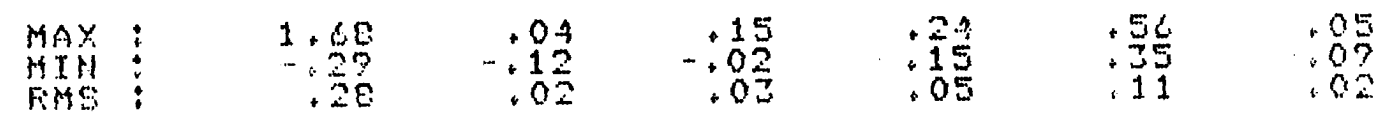

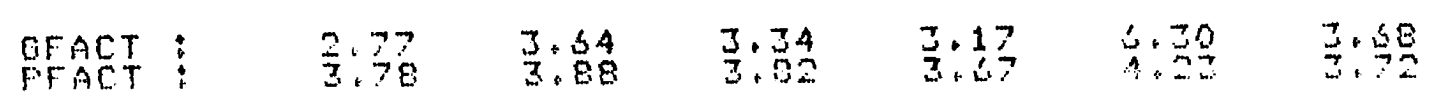




\begin{tabular}{|c|c|c|c|c|c|c|}
\hline Nun & $315: 0$ & $10+\frac{E 1}{0}$ & $=20^{A 2}$ & 39.3 & & \\
\hline $\begin{array}{l}\text { COMF } \\
\text { MEASt }\end{array}$ & $\operatorname{cF}_{22}$ & $\begin{array}{r}\text { CFY } \\
.00\end{array}$ & .05 & $\begin{array}{c}C M y \\
.02\end{array}$ & $\begin{array}{l}c+3 r \\
.02\end{array}$ & $\begin{array}{l}0.37 \\
.01\end{array}$ \\
\hline $\begin{array}{l}\text { MAX } \\
\text { MIIN } \\
\text { FMS }\end{array}$ & $\begin{array}{r}1 \cdot \frac{11}{3} \\
\cdot \frac{3}{2}\end{array}$ & $\begin{array}{r}.05 \\
-.07 \\
.02\end{array}$ & $\begin{array}{r}.08 \\
-.03 \\
.02\end{array}$ & $\begin{array}{r}.1 \% \\
.11 \\
.02\end{array}$ & $.2 \frac{20}{23}$ & .05 \\
\hline $\begin{array}{l}\text { QFACT } \\
\text { FACT }\end{array}$ & 5.05 & $\begin{array}{r}17.90 \\
3.28\end{array}$ & 3.90 & $\frac{4}{3} \div \frac{19}{8}$ & $11 \cdot 89$ & $\begin{array}{l}1.61 \\
2.50\end{array}$ \\
\hline
\end{tabular}

IIATA FOF: FILE : H50O1

\begin{tabular}{|c|c|c|c|c|c|c|}
\hline FiUn & 292.5 & 10.0 & & $\begin{array}{r}\text { UEL } \\
39.1\end{array}$ & & \\
\hline $\begin{array}{l}\text { COMF: } \\
\text { MEATt }\end{array}$ & CFY & $\begin{array}{l}\text { CFY } \\
.00\end{array}$ & .01 & $\begin{array}{l}\text { C.s. } \\
.04\end{array}$ & $\begin{array}{l}\mathrm{CHY} \\
. \mathrm{OS}\end{array}$ & $\begin{array}{l}\text { CHa } \\
.00\end{array}$ \\
\hline $\begin{array}{l}\text { MAX } \\
\text { MIYH } \\
\text { FMSE }\end{array}$ & $\begin{array}{r}1.38 \\
\cdots: \frac{29}{15}\end{array}$ & $\begin{array}{r}.07 \\
-.07 \\
.02\end{array}$ & $\begin{array}{r}.07 \\
.07 \\
.02\end{array}$ & $\begin{array}{l}10 \\
.00 \\
.05\end{array}$ & $\begin{array}{l}+36 \\
.57 \\
+07\end{array}$ & $\begin{array}{r}05 \\
.05 \\
+01\end{array}$ \\
\hline $\begin{array}{l}\text { GFACT } \\
\text { FFACT }\end{array}$ & $\frac{3.54}{6.73}$ & $\begin{array}{r}37.89 \\
2.98\end{array}$ & $\begin{array}{l}5.36 \\
2: 65\end{array}$ & 5.05 & 12.28 & $1+23$ \\
\hline
\end{tabular}

IATA FOF FILE: H5OOI

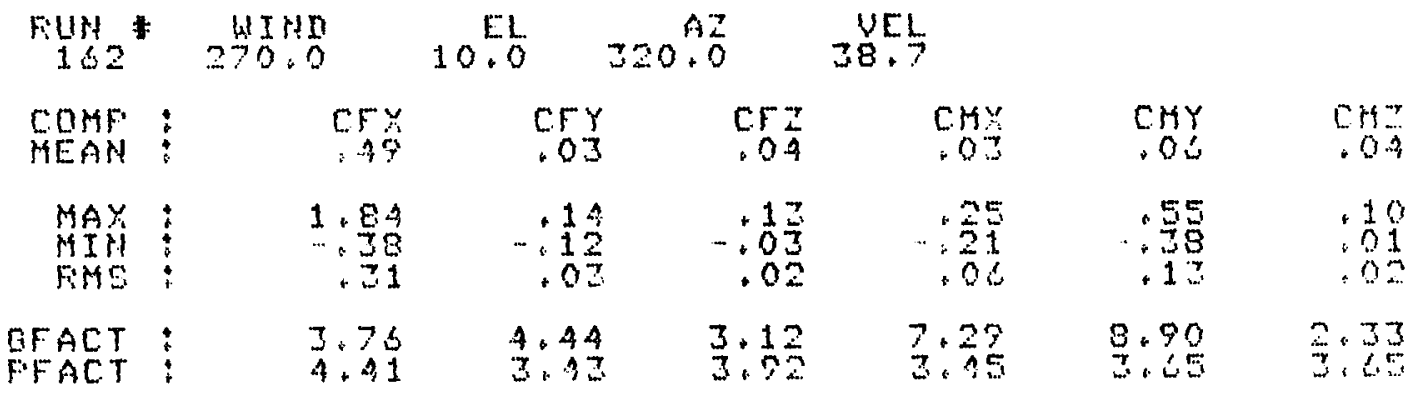

IIATA FOR FILE: H5001

\begin{tabular}{|c|c|c|c|c|c|c|}
\hline Fun & $\begin{array}{r}\text { WI } \$ 1 ! \\
247.5\end{array}$ & 10.0 & & $\begin{array}{r}\text { VEL } \\
39.3\end{array}$ & & \\
\hline COMF : & CFy & CFY & $\begin{array}{l}0.7 \\
.03\end{array}$ & $\begin{array}{l}\text { cmy } \\
.02\end{array}$ & $\begin{array}{l}\mathrm{cmy} \\
.02\end{array}$ & $\begin{array}{l}\cos 2 \\
.01\end{array}$ \\
\hline $\begin{array}{l}\text { MAX } \\
\text { MINS } \\
\text { RMS }\end{array}$ & $\begin{array}{r}.93 \\
-.93 \\
.18\end{array}$ & $\begin{array}{r}.10 \\
-.08 \\
.02\end{array}$ & $\begin{array}{r}.05 \\
. .03 \\
.02\end{array}$ & $\begin{array}{r}.15 \\
-.15 \\
.04\end{array}$ & .32 & $\begin{array}{r}.05 \\
.03 \\
.03\end{array}$ \\
\hline $\begin{array}{l}\text { GFACT } \\
\text { FFACT }\end{array}$ & 5.56 & $\begin{array}{l}4.63 \\
3.85\end{array}$ & $\frac{3}{3} \cdot 22$ & $\begin{array}{l}7+24 \\
3+18\end{array}$ & 17.19 & 4.17 \\
\hline
\end{tabular}


IATA FOF FILE ; H5OOI

\begin{tabular}{|c|c|c|c|c|c|c|}
\hline $\begin{array}{l}\text { Funt } \\
1.44\end{array}$ & $\begin{array}{r}\text { WINII } \\
247.5\end{array}$ & $\begin{array}{r}E L \\
10: 0\end{array}$ & & $\begin{array}{r}\text { VEL } \\
40.2\end{array}$ & & \\
\hline $\begin{array}{l}\text { COMF } \\
\text { MEAN }\end{array}$ & $\begin{array}{r}\text { CFy } \\
-.28\end{array}$ & $\begin{array}{l}\text { CFY } \\
+O A\end{array}$ & $\begin{array}{cc}C F \\
-.04\end{array}$ & $\begin{array}{l}\text { cry } \\
+02\end{array}$ & $\begin{array}{l}\text { CHYY } \\
: 10\end{array}$ & ${ }_{6}^{C H I}$ \\
\hline $\begin{array}{l}\text { MAX } \\
\text { MIN } \\
\text { Fins }\end{array}$ & $\begin{array}{r}: 1: 25 \\
: 10\end{array}$ & $\begin{array}{r}+23 \\
.04 \\
.02\end{array}$ & $\begin{array}{r}+99 \\
+15 \\
+04\end{array}$ & $\begin{array}{r}16 \\
.02 \\
.02\end{array}$ & $\begin{array}{r}18 \\
+38 \\
+68\end{array}$ & $\begin{array}{l}.07 \\
.10 \\
.02\end{array}$ \\
\hline $\begin{array}{l}\text { GFACT } \\
\text { FACT }\end{array}$ & 3.99 & 5.39 & $\overrightarrow{3}+91$ & 皇.71 & $\begin{array}{l}3.43 \\
3.40\end{array}$ & $4 \div 21$ \\
\hline
\end{tabular}

IIATA FOF FILE : H5001

\begin{tabular}{|c|c|c|c|c|c|c|}
\hline Furt & $\begin{array}{l}\text { WINI } \\
270.0\end{array}$ & 10.0 & 5.0 & $\begin{array}{r}\text { UEL } \\
40.0\end{array}$ & & \\
\hline COMF : & $\begin{array}{l}\mathrm{CF} \\
+01\end{array}$ & $\begin{array}{c}\text { C.TY } \\
.0 .4\end{array}$ & $.0 \%$ & $\begin{array}{c}\text { Cs. } \\
.02\end{array}$ & $\begin{array}{l}C M Y \\
01\end{array}$ & $\mathrm{CH}_{\mathrm{O}}$ \\
\hline $\begin{array}{l}\text { MAX: } \\
\text { MIM } \\
\text { FMS }\end{array}$ & $\begin{array}{r}.52 \\
. .45 \\
.10\end{array}$ & $\begin{array}{r}+10 \\
.01 \\
.01\end{array}$ & $\begin{array}{l}.07 \\
.03 \\
.02\end{array}$ & $\begin{array}{l}.00 \\
.01 \\
.01\end{array}$ & $\begin{array}{l}17 \\
: 17 \\
: 04\end{array}$ & .09 \\
\hline $\begin{array}{l}\text { QFACT } \\
F F A C T\end{array}$ & $4 \frac{2}{5} \cdot \frac{25}{5}$ & $\begin{array}{l}2.73 \\
4.55\end{array}$ & $\begin{array}{r}20.48 \\
3.63\end{array}$ & $\begin{array}{l}3.61 \\
5.52\end{array}$ & $\begin{array}{r}15.38 \\
4.10\end{array}$ & $\frac{3}{3} \cdot 53$ \\
\hline
\end{tabular}

IIATA FOF FILE : $H 5001$

\begin{tabular}{|c|c|c|c|c|c|c|}
\hline Rum & $\begin{array}{r}\text { WI } 32 \pi \\
292.5\end{array}$ & 10.0 & & $\begin{array}{r}\text { UE. } \\
39.7\end{array}$ & & \\
\hline $\begin{array}{l}\text { COMF } \\
\text { MEAN }\end{array}$ & $\begin{array}{l}C F x \\
14\end{array}$ & $\begin{array}{c}\text { CFY } \\
.03\end{array}$ & $\begin{array}{l}\mathrm{cr} z \\
.02\end{array}$ & $\begin{array}{l}\text { CHY } \\
.02\end{array}$ & $\begin{array}{l}\text { CHY } \\
.06\end{array}$ & $\begin{array}{c}\mathrm{CHz} \\
.00\end{array}$ \\
\hline $\begin{array}{l}\text { MAX } \\
\text { MIH } \\
\text { SME }\end{array}$ & $\begin{array}{r}.72 \\
-\quad 49 \\
.14\end{array}$ & $\begin{array}{r}.11 \\
-.04 \\
.02\end{array}$ & $\begin{array}{r}.08 \\
-.05 \\
.02\end{array}$ & $\begin{array}{l}.05 \\
.05 \\
.01\end{array}$ & .70 & $\begin{array}{l}.04 \\
.07 \\
.01\end{array}$ \\
\hline GFACT & $\begin{array}{l}5: 01 \\
4.10\end{array}$ & 3.99 & $\frac{5}{3} \cdot 18$ & 3.89 & 5.90 & $1 ?: 57$ \\
\hline
\end{tabular}

MATA FOR FILE : HSOO1

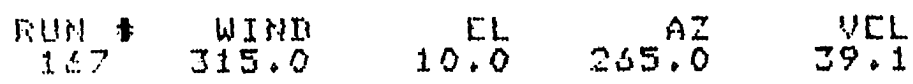

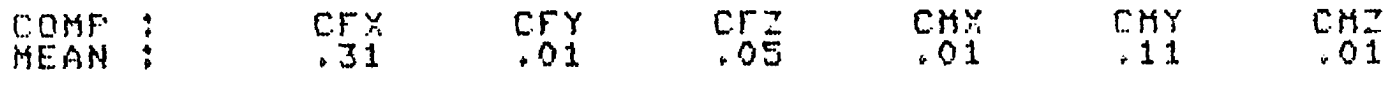

\begin{tabular}{|c|c|c|c|c|c|}
\hline $\begin{array}{l}\text { MAY } \\
\text { MIN } \\
\text { FMS }\end{array}$ & 1.26 & $\begin{array}{r}.08 \\
.05 \\
.02\end{array}$ & $\begin{array}{l}12 \\
.03 \\
.02\end{array}$ & .05 & $\begin{array}{r}30 \\
: 20 \\
: 00\end{array}$ \\
\hline
\end{tabular}

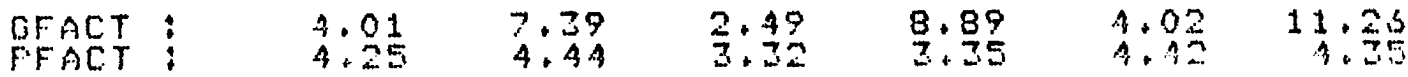




\begin{tabular}{|c|c|c|c|c|c|c|}
\hline Fut & $\begin{array}{r}\text { WIn } \\
337.5\end{array}$ & 10.0 & 255.0 & $=\frac{Y L}{8}$ & & \\
\hline COMF: & $\begin{array}{l}0.5 \\
23\end{array}$ & $\begin{array}{l}\text { CFY } \\
.02\end{array}$ & $\begin{array}{l}\operatorname{cr} \\
+03\end{array}$ & $\begin{array}{l}\operatorname{cis} x \\
.04\end{array}$ & $\begin{array}{l}\text { CHY } \\
.04\end{array}$ & $\begin{array}{l}\mathrm{CH} \\
.01\end{array}$ \\
\hline $\begin{array}{l}\text { MAX } \\
\text { MIM } \\
\text { PME }\end{array}$ & $\begin{array}{r}1.11 \\
\cdots: 19 \\
: 15\end{array}$ & $\begin{array}{r}.07 \\
.02 \\
.01\end{array}$ & $\begin{array}{l}.00 \\
.03 \\
.02\end{array}$ & $\begin{array}{r}.07 \\
.01 \\
.01\end{array}$ & $\begin{array}{r}.32 \\
.05 \\
1.05\end{array}$ & \\
\hline $\begin{array}{l}\text { QFACT } \\
\text { FFACT }\end{array}$ & $\begin{array}{l}3.81 \\
5.80\end{array}$ & $\begin{array}{l}3.89 \\
1.79\end{array}$ & $\frac{2}{2}+\frac{61}{7}$ & $\frac{1}{2} \cdot \frac{75}{2}$ & $7 \cdot 78$ & \\
\hline
\end{tabular}

\begin{tabular}{|c|c|c|c|c|c|c|}
\hline rupt & WIIII & 10.0 & & UEL & & \\
\hline $\begin{array}{l}\text { COMF } \\
\text { MEAN }\end{array}$ & $\operatorname{crs}$ & $\begin{array}{l}\text { CFY } \\
.00\end{array}$ & .07 & $\begin{array}{l}\text { C.MY } \\
.00\end{array}$ & $\begin{array}{l}\text { CHY } \\
12\end{array}$ & .01 \\
\hline $\begin{array}{l}\text { MAY } \\
\text { SIMS }\end{array}$ & $\begin{array}{r}1+58 \\
+25 \\
+24\end{array}$ & $\begin{array}{r}.07 \\
-.04 \\
.01\end{array}$ & $\begin{array}{r}+14 \\
.01 \\
.05\end{array}$ & $\begin{array}{l}.07 \\
.03 \\
.02\end{array}$ & $\begin{array}{r}1.97 \\
: 2.9 \\
.00\end{array}$ & .85 \\
\hline $\begin{array}{l}\text { GFACT } \\
\text { FFACT }\end{array}$ & 3.19 & $2 \frac{1}{4} \cdot \frac{1}{6}=$ & $\frac{2}{2} \cdot \frac{11}{7}$ & $\begin{array}{r}71 \cdot 7 \cdot 1 \\
3 \\
\therefore\end{array}$ & $\begin{array}{r}7.00 \\
11.71\end{array}$ & $\begin{array}{c}6 \\
2 \\
-3\end{array}$ \\
\hline
\end{tabular}

IIATA FOF FILE: H5001

\begin{tabular}{|c|c|c|c|c|c|c|}
\hline Fus & $\begin{array}{c}\text { WIMU } \\
0.0\end{array}$ & 20.5 & & $\begin{array}{r}\text { U.1. } \\
39.7\end{array}$ & & \\
\hline $\begin{array}{l}\text { COMF: } \\
\text { HEAM : }\end{array}$ & $\mathrm{Cr}_{4}$ & $\begin{array}{c}\mathrm{Cry} \\
\cdots .03\end{array}$ & $\begin{array}{l}\mathrm{CF} \\
.07\end{array}$ & 0.05 & $\begin{array}{l}\text { CHY } \\
.13\end{array}$ & $\cos ^{\circ}=3$ \\
\hline $\begin{array}{l}\text { MAX } \\
\text { MIN } \\
\text { FME }\end{array}$ & $\begin{array}{r}1 \cdot \frac{6}{25} \\
\cdots .25\end{array}$ & $\begin{array}{r}.07 \\
-.12 \\
.02\end{array}$ & $\begin{array}{r}.21 \\
.02 \\
.02\end{array}$ & $\begin{array}{l}15 \\
.05 \\
.02\end{array}$ & .17 & $\begin{array}{l}.02 \\
.03 \\
.05\end{array}$ \\
\hline $\begin{array}{l}\text { GFACT } \\
\text { FFACT }\end{array}$ & $\begin{array}{l}3.93 \\
5.67\end{array}$ & $4: 71$ & $\begin{array}{l}2.78 \\
4.12\end{array}$ & $\frac{2}{3}: 22$ & 3.19 & 2.73 \\
\hline
\end{tabular}

IIATA FOF FILE: HSOOI

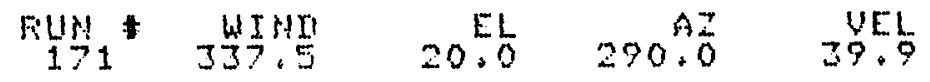

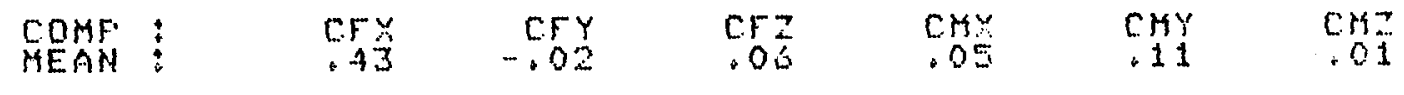

\begin{tabular}{|c|c|c|c|c|c|}
\hline $\begin{array}{l}\text { MAX : } \\
\text { MIT } \\
\text { SHSS }\end{array}$ & $\begin{array}{l}1.43 \\
-13 \\
.22\end{array}$ & $\begin{array}{r}.07 \\
-.11 \\
.03\end{array}$ & $\begin{array}{r}10 \\
.04 \\
.02\end{array}$ & $\frac{1}{2} \frac{\hat{z}}{0}$ & $+\frac{35}{12}$ \\
\hline
\end{tabular}

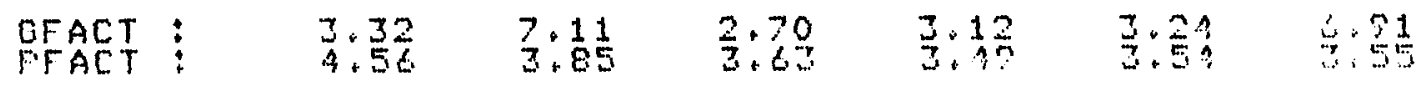


IIATA FOF FILE: HSOOI

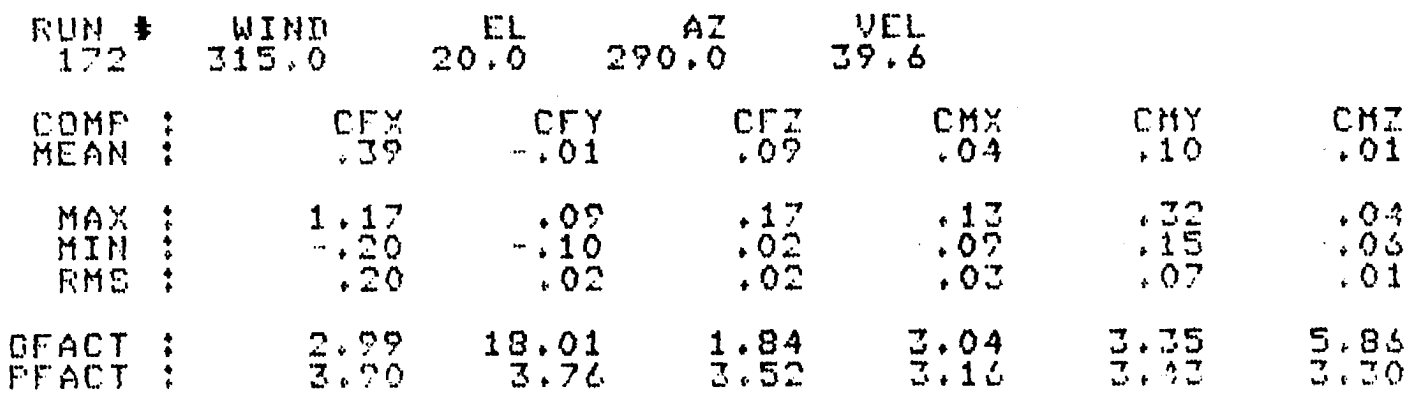

IIATA FOE FILE : H5001

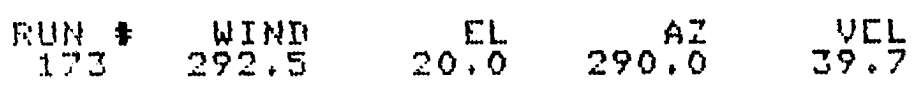

COMF: C.FX CFY CFE

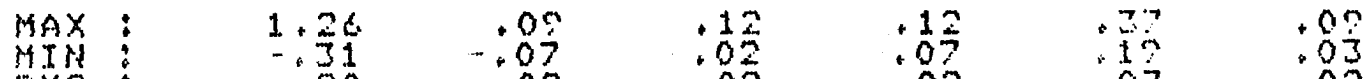

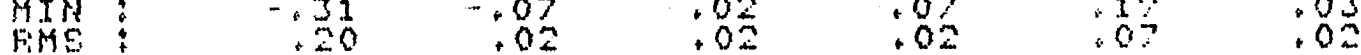

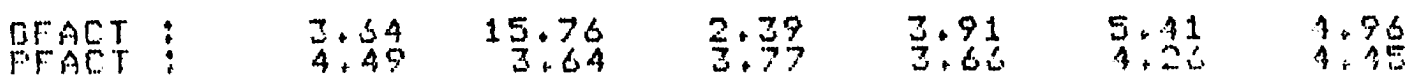

IIATA FOR FILE: HSOO1

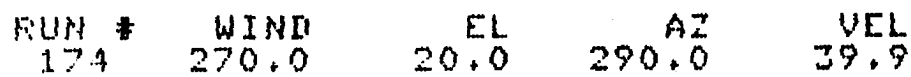

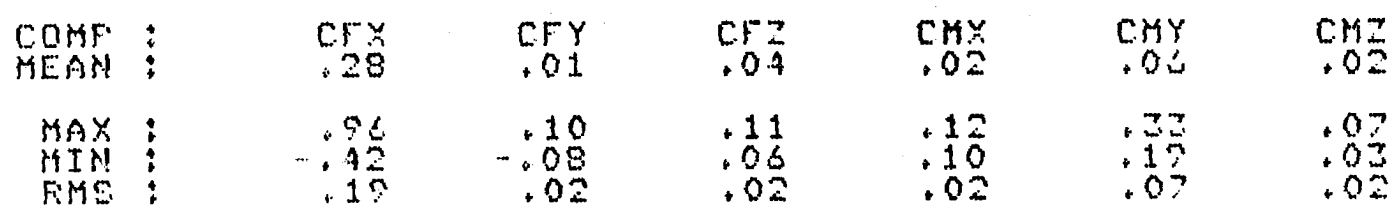

$\begin{array}{lllllll}\text { GFACT: } & 3.42 & 9.50 & 2.79 & 7.27 & 5.32 & 9.18\end{array}$

IATA FOF FILE : H5001

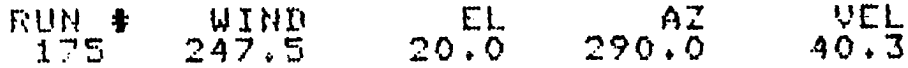

\begin{tabular}{|c|c|c|c|c|c|}
\hline $\begin{array}{l}\text { COMF } \\
\text { MEAH }\end{array}$ & $\begin{array}{r}c 5 x \\
-.05\end{array}$ & $\begin{array}{l}\text { CEY } \\
.03\end{array}$ & $\begin{array}{r}C .5 z \\
.04\end{array}$ & $\begin{array}{l}\text { C.MY } \\
.00\end{array}$ & $\begin{array}{l}C M Y \\
11\end{array}$ \\
\hline $\begin{array}{l}\text { HAX } \\
\text { RMA } \\
\text { RMS }\end{array}$ & $\begin{array}{r}.32 \\
-11\end{array}$ & $\begin{array}{r}.17 \\
.08 \\
.07\end{array}$ & $\begin{array}{r}.09 \\
-.14 \\
.03\end{array}$ & $\begin{array}{l}.09 \\
.08 \\
.02\end{array}$ & $\begin{array}{l}00 \\
: \frac{20}{6} \\
04\end{array}$ \\
\hline
\end{tabular}

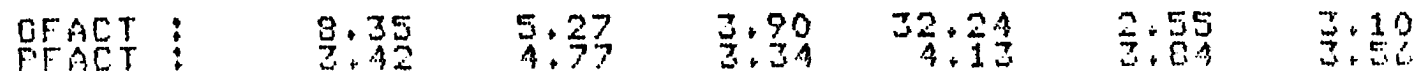




\begin{tabular}{|c|c|c|c|c|c|c|}
\hline FiUH & WIMI & 25.0 & $=40.92$ & UEL & & \\
\hline CQRF & $\begin{array}{c}\text { CFy } \\
.03\end{array}$ & $\begin{array}{rr}\text { CFY } \\
.02\end{array}$ & .04 & $\begin{array}{l}C H Y \\
.01\end{array}$ & $\begin{array}{l}\mathrm{Cmy} \\
.01\end{array}$ & $\begin{array}{c}\operatorname{crs} \\
.01\end{array}$ \\
\hline $\begin{array}{l}\text { MAX } \\
\text { MIA } \\
\text { FMS }\end{array}$ & $\begin{array}{r}1+\frac{1}{3} \\
: 21\end{array}$ & $\begin{array}{r}.04 \\
.09 \\
.02\end{array}$ & $\begin{array}{r}.27 \\
.125 \\
.05\end{array}$ & $.1 \frac{19}{15}$ & $\begin{array}{r}50 \\
.58 \\
.11\end{array}$ & .05 \\
\hline $\begin{array}{l}\text { GFACT } \\
\text { FFACT }\end{array}$ & $3 \frac{3}{5} \cdot \frac{2}{5}$ & $\frac{5}{4.27}$ & 4.53 & $\begin{array}{r}17.70 \\
3+64\end{array}$ & $\begin{array}{l}35.53 \\
4.52\end{array}$ & 8.85 \\
\hline
\end{tabular}

IIATA FOF: FILE : H5001

\begin{tabular}{|c|c|c|c|c|c|c|}
\hline Fun & 337.5 & 25.0 & & WEL & & \\
\hline $\begin{array}{l}\text { COMF: } \\
\text { SIEAN }\end{array}$ & $\begin{array}{c}C F . \\
+31\end{array}$ & $\begin{array}{r}\text { CFY } \\
. .02\end{array}$ & $\begin{array}{c}C .7 \\
.1 .9\end{array}$ & $\begin{array}{l}C M= \\
D=\end{array}$ & $\begin{array}{l}\text { CMY } \\
.0 \Xi\end{array}$ & $\mathrm{CH}_{.02}$ \\
\hline $\begin{array}{l}\text { MAX } \\
\text { MIN } \\
\text { FME }\end{array}$ & $\begin{array}{r}1 \cdot 3 \frac{1}{3} \\
\cdot: \frac{3}{2}\end{array}$ & $\begin{array}{r}.02 \\
.09 \\
.02\end{array}$ & $\begin{array}{l}.35 \\
.00 \\
.05\end{array}$ & $\begin{array}{l}.15 \\
.03 \\
.05\end{array}$ & .41 & $\begin{array}{r}+93 \\
.03 \\
.02\end{array}$ \\
\hline $\begin{array}{l}\text { GFACT } \\
\text { FFACT }\end{array}$ & 4.18 & $\begin{array}{l}4.72 \\
1.10\end{array}$ & $\frac{2}{4}: 53$ & $\begin{array}{l}5.79 \\
3.54\end{array}$ & $15 \cdot 52$ & $3 \cdot 25$ \\
\hline
\end{tabular}

IIATA FOF FILE: H5001

\begin{tabular}{|c|c|c|c|c|c|c|}
\hline $\operatorname{Fup}_{1 \rightarrow 9}$ & WISH & 25.0 & & $\begin{array}{r}\text { VEL. } \\
39.8\end{array}$ & & \\
\hline COMF : & $\begin{array}{l}C F Y \\
.48\end{array}$ & $\begin{array}{l}\text { CFY } \\
-.02\end{array}$ & $\begin{array}{l}\mathrm{CF} \\
.18\end{array}$ & Cry & $\begin{array}{l}\mathrm{CHY} \\
.01\end{array}$ & $\begin{array}{cc}\operatorname{cm} \\
007\end{array}$ \\
\hline $\begin{array}{l}\text { MAX } \\
\text { MIH } \\
\text { RHE }\end{array}$ & $\begin{array}{r}1.58 \\
\cdots .30 \\
.25\end{array}$ & $\begin{array}{r}.02 \\
.07 \\
.02\end{array}$ & $\begin{array}{l}.43 \\
.02 \\
.06\end{array}$ & .15 & $\begin{array}{l}50 \\
.13 \\
11\end{array}$ & .02 \\
\hline $\begin{array}{l}\text { GFACT } \\
\text { FFACT }\end{array}$ & $\begin{array}{l}3.28 \\
4.40\end{array}$ & $\frac{3}{3} \cdot 40$ & 2.35 & $1 \cdot 12$ & $3 \cdot 55$ & $\frac{5}{7}$ \\
\hline
\end{tabular}

IIATA FOF: FILE: H5OOI

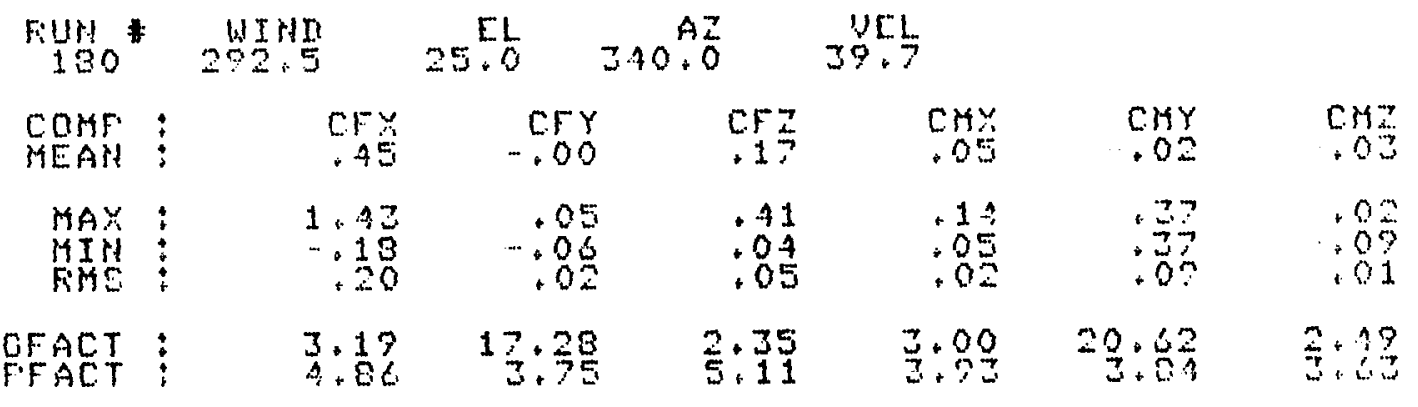


IIATA FOF FILE： H5001

\begin{tabular}{|c|c|c|c|c|c|c|}
\hline 내연 & $\begin{array}{r}\text { WI INI } \\
270.0\end{array}$ & 5.0 & & $\begin{array}{l}\text { VEL. } \\
40.0\end{array}$ & & \\
\hline $\begin{array}{l}\text { Comp : } \\
\text { MEASt }\end{array}$ & .38 & ${ }_{-01}$ & $\begin{array}{l}\text { CFZ } \\
.14\end{array}$ & $\begin{array}{l}\text { CHX } \\
.01\end{array}$ & $\begin{array}{l}\text { CHY } \\
.01\end{array}$ & $\begin{array}{l}\text { c.sz } \\
.00\end{array}$ \\
\hline $\begin{array}{l}\text { MAX: } \\
\text { MIII } \\
\text { RME: }\end{array}$ & $\begin{array}{l}1+31 \\
\cdots, 28 \\
.15\end{array}$ & $\begin{array}{r}.00 \\
-.07 \\
.02\end{array}$ & .87 & $\begin{array}{l}.00 \\
.10 \\
.02\end{array}$ & . & $\begin{array}{l}.04 \\
.0 .9 \\
.01\end{array}$ \\
\hline $\begin{array}{l}\text { QFACT } \\
\text { FFACT }\end{array}$ & $\frac{3}{5}+51$ & $13+08$ & $\frac{2}{5} \cdot 25$ & $\begin{array}{r}10.35 \\
4.17\end{array}$ & $\begin{array}{r}49.49 \\
4.99\end{array}$ & $12 \div \frac{60}{4}$ \\
\hline
\end{tabular}

IATA FOF: FILE: H5001

\begin{tabular}{|c|c|c|c|c|c|c|}
\hline $\operatorname{lum}_{12}$ & $\begin{array}{r}W I H \pi \\
247.5\end{array}$ & 25.0 & $\begin{array}{l}A= \\
+0\end{array}$ & $\begin{array}{l}\text { UCL } \\
70.1\end{array}$ & & \\
\hline $\begin{array}{l}\text { Cons: } \\
\text { MEAH: }\end{array}$ & .95 & $\begin{array}{l}\text { cry } \\
.01\end{array}$ & $\frac{C F}{12}$ & .01 & $\begin{array}{l}\text { EtgY } \\
.01\end{array}$ & $\begin{array}{l}\operatorname{crt} \\
\text { os }\end{array}$ \\
\hline 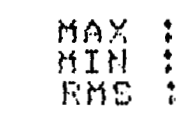 & $\begin{array}{r}1.36 \\
-28 \\
.23\end{array}$ & $\begin{array}{r}.08 \\
. .07 \\
.02\end{array}$ & 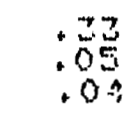 & $\begin{array}{l}11 \\
+15 \\
.02\end{array}$ & $\begin{array}{l}.99 \\
.56 \\
.12\end{array}$ & $\begin{array}{l}.05 \\
.02 \\
.01\end{array}$ \\
\hline $\begin{array}{l}\text { QFACT } \\
\text { FFACT }\end{array}$ & 3.12 & $\begin{array}{l}7 \cdot 48 \\
3 \cdot 54\end{array}$ & $\frac{1}{3}+23$ & $14+14$ & 93.95 & 2.86 \\
\hline
\end{tabular}

IIATA FOF: FILE: HSOO1

\begin{tabular}{|c|c|c|c|c|c|c|}
\hline Fust & $\begin{array}{r}454 \pi \\
247.5\end{array}$ & 45.0 & & $\begin{array}{l}\text { VIL } \\
40.8\end{array}$ & & \\
\hline $\begin{array}{l}\text { COHF } \\
\text { MEAN }\end{array}$ & $\begin{array}{r}\operatorname{cry} \\
-.13\end{array}$ & $\begin{array}{l}\text { CFY } \\
.04\end{array}$ & $\begin{array}{c}C 5 \\
+12\end{array}$ & $\begin{array}{l}\text { CMx } \\
.01\end{array}$ & $\begin{array}{l}\text { CHY } \\
.0 D\end{array}$ & $\cos _{01}$ \\
\hline $\begin{array}{l}\text { MAX } \\
\text { KIVI } \\
\text { FMS }\end{array}$ & $\begin{array}{r}+31 \\
-\quad 48 \\
112\end{array}$ & $\begin{array}{r}.20 \\
-13 \\
.04\end{array}$ & $\begin{array}{r}.05 \\
-.35 \\
.06\end{array}$ & $\therefore \frac{1}{15}$ & $\begin{array}{l}12 \\
.25 \\
.02\end{array}$ & $\begin{array}{r}.02 \\
+05 \\
+01\end{array}$ \\
\hline $\begin{array}{l}\text { GFACT } \\
\text { FFACT }\end{array}$ & $5 \cdot 25$ & $\begin{array}{l}5.54 \\
4.43\end{array}$ & $\frac{2}{3} .90$ & 21.73 & $\frac{8}{4}+\frac{15}{7}$ & $\begin{array}{l}1.14 \\
2+40\end{array}$ \\
\hline
\end{tabular}

IATA FOR FILE : HSOOI

\begin{tabular}{|c|c|c|c|c|c|c|}
\hline $\lim _{194} *$ & 270.0 & $\begin{array}{r}E L \\
45.0\end{array}$ & $0.9 Z$ & $40 \frac{1}{4}$ & & \\
\hline COMF : & $\begin{array}{c}C x^{\circ} \\
.02\end{array}$ & $\begin{array}{l}\mathrm{CFY} \\
.03\end{array}$ & $\begin{array}{l}\operatorname{crz} \\
.01\end{array}$ & $\begin{array}{l}.04 x \\
.01\end{array}$ & $\begin{array}{l}\text { CHY } \\
.00\end{array}$ & $\begin{array}{l}\operatorname{crg} z \\
.01\end{array}$ \\
\hline $\begin{array}{l}\text { MAX } \\
\text { WIHS } \\
\text { FNS }\end{array}$ & $\begin{array}{r}.53 \\
-.39 \\
.12\end{array}$ & $\begin{array}{r}.15 \\
-10 \\
.04\end{array}$ & $\begin{array}{l}28 \\
.16 \\
.06\end{array}$ & $\begin{array}{l}13 \\
.12 \\
.03\end{array}$ & $\begin{array}{r}10 \\
.20 \\
.05\end{array}$ & $\begin{array}{r}.02 \\
.09 \\
.01\end{array}$ \\
\hline $\begin{array}{l}\text { QFACT } \\
\text { FFACT }\end{array}$ & $2 \frac{2}{4}: 58$ & $\begin{array}{l}5.48 \\
3.42\end{array}$ & 26.56 & 10.53 & $3448 \cdot 22$ & $\begin{array}{l}4.08 \\
3.54\end{array}$ \\
\hline
\end{tabular}




\begin{tabular}{|c|c|c|c|c|c|c|}
\hline Fun & 292.5 & 45.0 & 290.0 & UEL & & \\
\hline COMF: & $\begin{array}{r}.05 \\
.25\end{array}$ & $\begin{array}{l}\mathrm{CFY} \\
.01\end{array}$ & $\frac{C r}{.15}$ & .05 & $\begin{array}{l}\text { CHY } \\
.04\end{array}$ & $\begin{array}{l}\mathrm{CH}^{-} \\
\mathrm{O}=\end{array}$ \\
\hline $\begin{array}{l}\text { MAY } \\
\text { MISH } \\
\text { FMS }\end{array}$ & $\begin{array}{r}+71 \\
.32 \\
.12\end{array}$ & $\begin{array}{r}+14 \\
-11 \\
.03\end{array}$ & $\begin{array}{r}50 \\
.00 \\
.07\end{array}$ & $\begin{array}{l}+\frac{1}{5} \\
.15 \\
+05\end{array}$ & $\begin{array}{r}9 \\
+25 \\
+20 \\
+06\end{array}$ & $\begin{array}{r}07 \\
.01 \\
.01\end{array}$ \\
\hline $\begin{array}{l}\text { GFACT } \\
\text { FFACT }\end{array}$ & 3.58 & $1 \frac{2}{3} \cdot 18$ & $\frac{2}{4} \cdot 58$ & $\begin{array}{l}3+94 \\
3.60\end{array}$ & 4.47 & 4.82 \\
\hline
\end{tabular}

IIATA FOF FILE: HSOOI

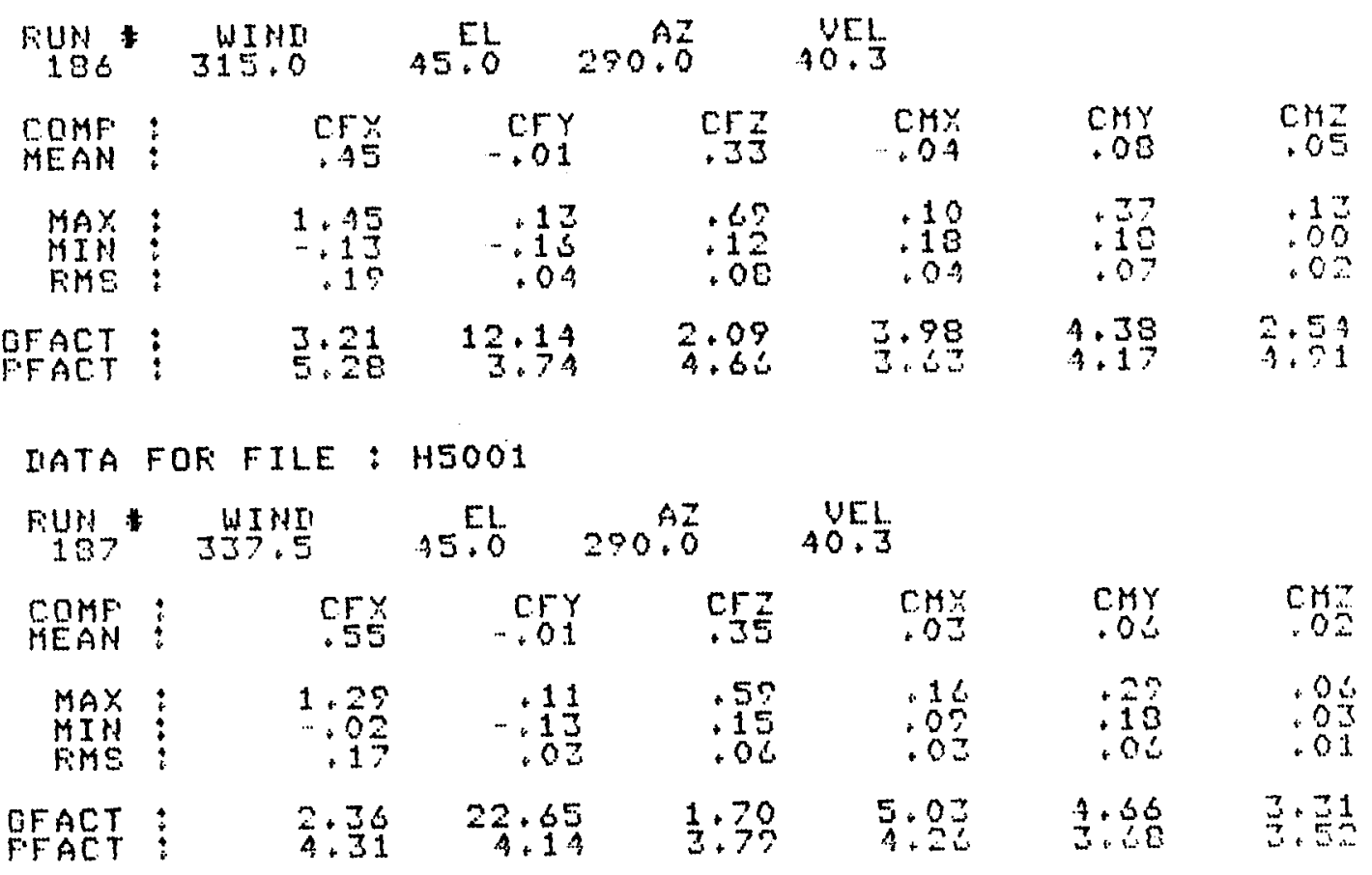

IATA FOF FILE: H5001

\begin{tabular}{|c|c|c|c|c|c|c|}
\hline $\begin{array}{c}\text { Fius } \\
188\end{array}$ & $\begin{array}{l}\text { WND } \\
0.0\end{array}$ & 45.5 & & $\begin{array}{l}\text { VEL.. } \\
-40.6\end{array}$ & & \\
\hline $\begin{array}{l}\text { COMF } \\
\text { MEAN }\end{array}$ & $\begin{array}{cc}\mathrm{CF}_{2} \\
.42\end{array}$ & $\begin{array}{c}\operatorname{cry} \\
-.03\end{array}$ & $\frac{C^{2}}{.29}$ & $\begin{array}{l}\text { CHS } \\
\text { OH }\end{array}$ & $\begin{array}{l}.04 Y \\
.05\end{array}$ & $\begin{array}{c}c k z \\
.03\end{array}$ \\
\hline $\begin{array}{l}\text { MAX } \\
\text { MIN } \\
\text { FISE }\end{array}$ & $\begin{array}{r}1.28 \\
: 13 \\
: 18\end{array}$ & $\begin{array}{r}.09 \\
.14 \\
.04\end{array}$ & .57 & $\begin{array}{l}.10 \\
.05 \\
.02\end{array}$ & .27 & .00 \\
\hline $\begin{array}{l}\text { QFACT } \\
\text { FFACT }\end{array}$ & $\begin{array}{l}3.07 \\
4.75\end{array}$ & $\begin{array}{l}4.58 \\
3.06\end{array}$ & 2.03 & $\frac{2}{3}+33$ & 5.75 & $2+\frac{2}{4}$ \\
\hline
\end{tabular}


Heliostat 5, Configuration 5-C

\begin{tabular}{|c|c|c|c|c|c|c|}
\hline Case & Run 非 & WD & $\mathrm{EL}$ & $\mathrm{AZ}$ & Day & Time \\
\hline \multirow[t]{6}{*}{$\operatorname{Sin} r \quad A M$} & 213 & 0 & 25 & 340 & 172 & $8 \mathrm{AM}$ \\
\hline & 210 & 337.5 & 25 & 340 & 172 & $8 \mathrm{AN}$ \\
\hline & 209 & 315 & 25 & 340 & 172 & $8 \mathrm{AM}$ \\
\hline & 206 & 292.5 & 25 & 340 & 172 & $8 \mathrm{AM}$ \\
\hline & 205 & 270 & 25 & 340 & 172 & $8 \mathrm{AM}$ \\
\hline & 202 & 247.5 & 25 & 340 & 172 & $8 \mathrm{AM}$ \\
\hline \multirow[t]{6}{*}{ Sinr Noon } & 201 & 247.5 & 45 & 290 & 172 & 0 PN \\
\hline & 198 & 270 & 45 & 290 & 172 & $0 \mathrm{PM}$ \\
\hline & 197 & 292.5 & 45 & 290 & 172 & $0 \mathrm{PM}$ \\
\hline & 194 & 315 & 45 & 290 & 172 & $0 \mathrm{PM}$ \\
\hline & 191 & 337.5 & 45 & 290 & 172 & $0 \mathrm{PM}$ \\
\hline & 190 & 0 & 45 & 290 & 172 & $0 \mathrm{PM}$ \\
\hline \multirow[t]{6}{*}{ Wilte AM } & 253 & 0 & 10 & 320 & 355 & $8 \mathrm{AM}$ \\
\hline & 252 & 337.5 & 10 & 320 & 355 & $8 \mathrm{ANI}$ \\
\hline & 249 & 315 & 10 & 320 & 355 & 8 AM \\
\hline & 248 & 292.5 & 10 & 320 & 355 & $8 \mathrm{AM}$ \\
\hline & 245 & 270 & 10 & 320 & 355 & $8 \mathrm{AM}$ \\
\hline & 244 & 247.5 & 10 & 320 & 355 & $8 \mathrm{AM}$ \\
\hline \multirow[t]{6}{*}{ Whtr Noon } & 230 & 0 & 20 & 290 & 355 & $0 \mathrm{PM}$ \\
\hline & 227 & 337.5 & 20 & 290 & 355 & $0 \mathrm{PMI}$ \\
\hline & 226 & 315 & 20 & 290 & 355 & $0 \mathrm{PM}$ \\
\hline & 222 & 292.5 & 20 & 290 & 355 & $0 \mathrm{PM}$ \\
\hline & 221 & 270 & 20 & 290 & 355 & $0 \mathrm{PM}$ \\
\hline & 218 & 247.5 & 20 & 290 & 355 & $0 \mathrm{PM}$ \\
\hline \multicolumn{7}{|c|}{155} \\
\hline
\end{tabular}


Heliostat 5, Configuration 5-C. continued

\begin{tabular}{|c|c|c|c|c|c|c|}
\hline Case & Run 非 & WD & $\mathrm{EL}$ & $\mathrm{AZ}$ & Day & 'T' ime \\
\hline \multirow[t]{6}{*}{ Whtr PM } & 241 & 247.5 & 10 & 265 & 355 & $4 \mathrm{INH}$ \\
\hline & 240 & 270 & 10 & 265 & 355 & $4 P N$ \\
\hline & 237 & 292.5 & 10 & 265 & 355 & 4 PN \\
\hline & 236 & 315 & 10 & 265 & 355 & $4 \mathrm{PMI}$ \\
\hline & 234 & 337.5 & 10 & 265 & 355 & $4 \mathrm{PM}$ \\
\hline & 231 & 0 & 10 & 265 & 355 & $4 P M$ \\
\hline Ver. Stow & 217 & 315 & 0 & 320 & 355 & $8 \mathrm{AM}$ \\
\hline Hor. Stow & 215 & 315 & 90 & 320 & 355 & $8 \mathrm{AM}$ \\
\hline
\end{tabular}




\begin{tabular}{|c|c|c|c|c|c|c|}
\hline rusp & WI 0.0 & 35.5 & & 는. & & \\
\hline $\begin{array}{l}\text { COMF } \\
\text { MEAN }\end{array}$ & 050 & .05 & .15 & Cry & $\cos y$ & .01 \\
\hline $\begin{array}{l}\text { MAX } \\
\text { HIME } \\
\text { FHE }\end{array}$ & .97 & $\begin{array}{r}.05 \\
.13 \\
.03\end{array}$ & +. & $\begin{array}{l}.12 \\
.05 \\
.05\end{array}$ & : & \\
\hline DFACT: & 5.45 & $\frac{5}{3} .67$ & 象. & $\begin{array}{l}5.35 \\
3.75\end{array}$ & 7.07 & \\
\hline
\end{tabular}

DATA FOF FILE: H5100

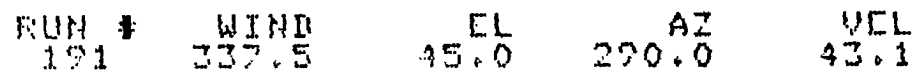

\begin{tabular}{|c|c|c|c|c|c|}
\hline COMF: & 65 & $\begin{array}{l}C r Y \\
.02\end{array}$ & $c c^{2}$ & $\begin{array}{l}\text { CHX } \\
.05\end{array}$ & CMY \\
\hline $\begin{array}{l}\text { MAY } \\
\text { KIHE } \\
\text { KMS }\end{array}$ & $\begin{array}{r}1.17 \\
.08 \\
.15\end{array}$ & $\begin{array}{l}.05 \\
.12 \\
.05\end{array}$ & $\begin{array}{l}.12 \\
.10 \\
.05\end{array}$ & $: 05$ & $. \frac{2}{1} . \frac{1}{0}$ \\
\hline FACT & $\begin{array}{r}2.19 \\
4.49\end{array}$ & $\begin{array}{l}5.54 \\
3.47\end{array}$ & $\begin{array}{l}1.77 \\
3.90\end{array}$ & $3.7-7$ & $ت .7 \overrightarrow{7}$ \\
\hline
\end{tabular}

IATA FOF FILE : H5100

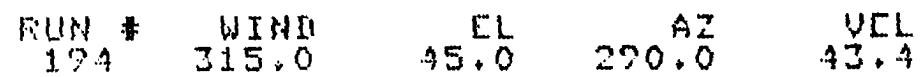

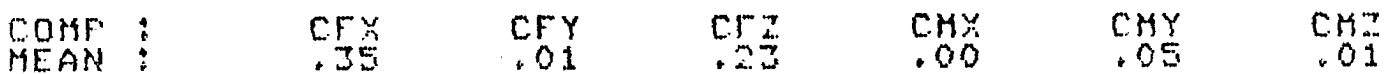

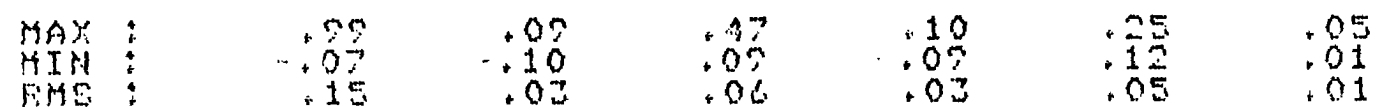

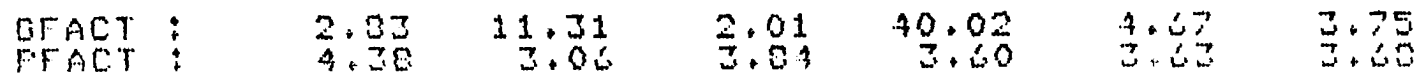

IIATA FOR FILE: HS100

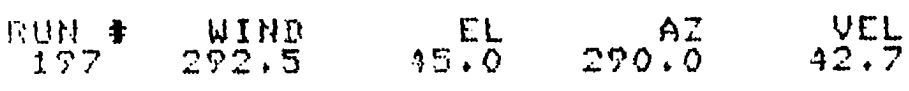

COMF: CFE

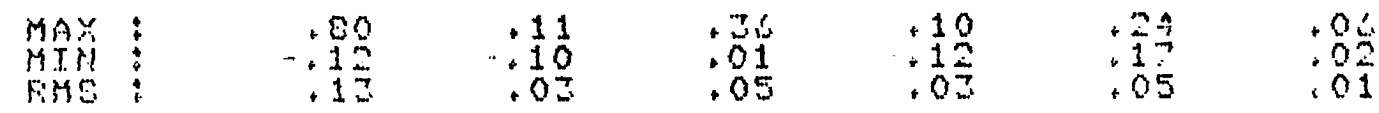

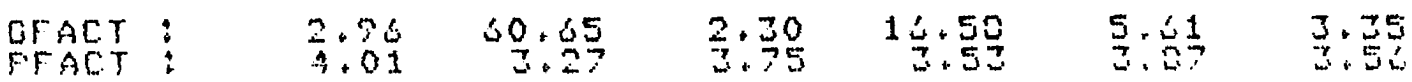




\begin{tabular}{|c|c|c|c|c|c|c|}
\hline Fiust & $\begin{aligned} \text { WIMn } & \\
2>0.0 & \end{aligned}$ & $45.0 \frac{1}{0}$ & 290.0 & $\begin{array}{r}\text { VEL } \\
43.4\end{array}$ & & \\
\hline $\begin{array}{l}\text { COMF } \\
\text { MEAM }\end{array}$ & $\begin{array}{l}\text { CFE } \\
.08\end{array}$ & $\begin{array}{l}\mathrm{CFY} \\
.01\end{array}$ & $\begin{array}{c}\operatorname{crz} \\
00\end{array}$ & .02 & $\begin{array}{l}\text { CHY } \\
.00\end{array}$ & $\begin{array}{l}\operatorname{cr} 2 \\
.01\end{array}$ \\
\hline $\begin{array}{l}\text { MAX } \\
\text { HIH } \\
\text { PHS }\end{array}$ & $\begin{array}{r}+49 \\
-13 \\
.10\end{array}$ & $\begin{array}{r}+10 \\
-\quad 08 \\
.02\end{array}$ & $\begin{array}{r}.20 \\
.14 \\
.04\end{array}$ & $\begin{array}{l}.00 \\
.11 \\
.03\end{array}$ & $\begin{array}{l}+37 \\
+13 \\
04\end{array}$ & .05 \\
\hline $\begin{array}{l}\text { QFACT } \\
F F A C T\end{array}$ & 4.37 & $\begin{array}{l}3.58 \\
3.01\end{array}$ & 3.61 & $\frac{A}{2}: \overrightarrow{3}$ & 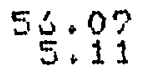 & 5.32 \\
\hline
\end{tabular}

IIATA FOF FILE:

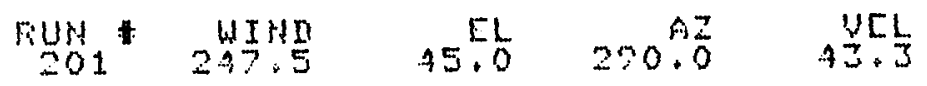

\begin{tabular}{|c|c|c|c|c|c|c|}
\hline $\begin{array}{l}\text { COMF } \\
\text { MEAN }\end{array}$ & $\begin{array}{l}\text { CFY } \\
\cdots .09\end{array}$ & $\begin{array}{l}\text { CFY } \\
.02\end{array}$ & $\begin{array}{c}\mathrm{Cr} \\
.07\end{array}$ & $\begin{array}{l}\text { C.x } \\
.00\end{array}$ & $\begin{array}{l}\text { Criy } \\
.03\end{array}$ & .01 \\
\hline $\begin{array}{l}\text { MAX } \\
\text { MIHE } \\
\text { FHS }\end{array}$ & $\begin{array}{r}+34 \\
50 \\
10 \\
10\end{array}$ & $\begin{array}{r}.10 \\
.00 \\
.03\end{array}$ & $\begin{array}{l}.95 \\
.25 \\
.04\end{array}$ & .09 &. $\begin{array}{ll}1 & 1 \\
2 & 0 \\
0 & 4\end{array}$ & $\begin{array}{l}.02 \\
.05 \\
.01\end{array}$ \\
\hline $\begin{array}{l}\text { QFACT } \\
F F A C T\end{array}$ & 5.50 & 5.00 & 3.58 & $39+22$ & 7.74 & $\begin{array}{l}3 \\
7.99 \\
9\end{array}$ \\
\hline
\end{tabular}

IATA FOF: FILE: H5100

\begin{tabular}{|c|c|c|c|c|c|c|}
\hline$\underset{202}{\text { Eurt }}$ & $\begin{array}{r}45 H \pi \\
2-75: 5\end{array}$ & 25.0 & & 11. & & \\
\hline $\begin{array}{l}\text { COMF : } \\
\text { MEAH : }\end{array}$ & $\begin{array}{c}C F \\
.27\end{array}$ & $\begin{array}{l}\text { CFY } \\
.01\end{array}$ & $\begin{array}{c}C F z \\
.05\end{array}$ & .00 & $\begin{array}{l}\text { CHY } \\
.00\end{array}$ & $\begin{array}{l}\text { CHz } \\
\text { OI }\end{array}$ \\
\hline $\begin{array}{l}\text { MAY } \\
\text { MIH } \\
\text { MMS }\end{array}$ & $\begin{array}{r}.92 \\
-.25 \\
.12\end{array}$ & $\begin{array}{r}.00 \\
.04 \\
.02\end{array}$ & $\begin{array}{l}.22 \\
.04 \\
.04\end{array}$ & $\begin{array}{l}.02 \\
.10 \\
.02\end{array}$ & $\begin{array}{l}35 \\
.33 \\
.09\end{array}$ & $\begin{array}{r}.95 \\
.85 \\
.01\end{array}$ \\
\hline FACT & $3 \cdot \frac{21}{79}$ & $12 \cdot 37$ & $\frac{2}{3} \cdot 73$ & 230.63 & $508 \cdot 18$ & $\begin{array}{c}5.11 \\
4 \\
4\end{array}$ \\
\hline
\end{tabular}

IIATA FOF: FILE: H5100

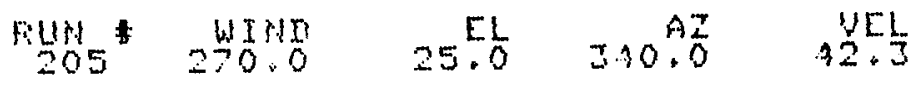

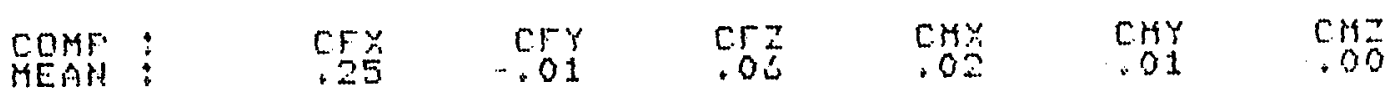

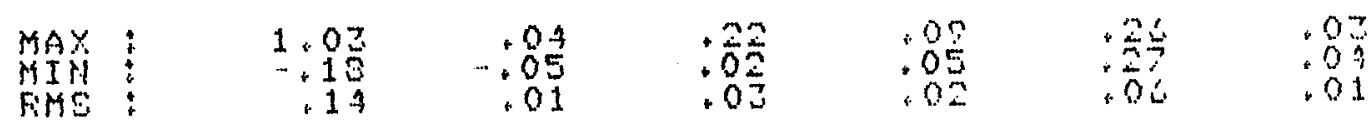

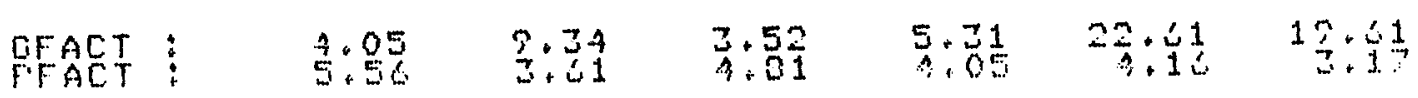




\begin{tabular}{|c|c|c|c|c|c|c|}
\hline Futs $\frac{150}{20}$ & $29 I+5$ & 25.0 & $340 .{ }^{A 2}$ & $4 \frac{V}{2}: \frac{1}{3}$ & & \\
\hline $\begin{array}{l}\text { CoMF : } \\
\text { MEAM : }\end{array}$ & $\frac{c 14}{.39}$ & $\begin{array}{l}\text { CFY } \\
.02\end{array}$ & $\begin{array}{c}C F \\
.12\end{array}$ & $\begin{array}{l}\mathrm{CM} \\
+\mathrm{OS}\end{array}$ & $\begin{array}{l}\text { CHY } \\
.01\end{array}$ & $\operatorname{crg}_{02}$ \\
\hline $\begin{array}{l}\text { MAX } \\
\text { MIH } \\
\text { RMS }\end{array}$ & $\begin{array}{r}1.08 \\
.08 \\
.18\end{array}$ & $\begin{array}{r}.03 \\
-.07 \\
.01\end{array}$ & .25 & $\begin{array}{l}.11 \\
+03 \\
02\end{array}$ & $\begin{array}{l}.54 \\
.028 \\
.07\end{array}$ & $\begin{array}{l}.02 \\
.0 \% \\
.01\end{array}$ \\
\hline $\begin{array}{l}\text { GFACT: } \\
\text { FFACT }\end{array}$ & $2+38$ & $\frac{4.41}{3} \cdot 68$ & 2.2. & $\begin{array}{l}3.04 \\
3.40\end{array}$ & $27+02$ & $\begin{array}{l}3.94 \\
2.85\end{array}$ \\
\hline IMATA FE & DF FILE : & $H 5100$ & & & & \\
\hline nut & $\begin{array}{r}\text { WINn } \\
315.0\end{array}$ & 25.0 & $\begin{array}{r}A 2 \\
340.0\end{array}$ & $\begin{array}{r}\text { VEL } \\
72.5\end{array}$ & & . \\
\hline $\begin{array}{l}\text { COMF: } \\
\text { MEAN }\end{array}$ & $\begin{array}{l}\mathrm{CF} \\
.24\end{array}$ & $\begin{array}{l}\text { CFy } \\
.01\end{array}$ & .05 & $\begin{array}{l}\text { CMs } \\
.02\end{array}$ & $\begin{array}{l}\text { CSYY } \\
.01\end{array}$ & $\mathrm{CH}_{2}$ \\
\hline $\begin{array}{l}\text { SAA. } \\
\text { HIM } \\
\text { FHE }\end{array}$ & $\begin{array}{r}.93 \\
-13 \\
+1.13\end{array}$ & $\begin{array}{r}+03 \\
.05 \\
.01\end{array}$ & $\begin{array}{l}.21 \\
.02 \\
.02\end{array}$ & $\begin{array}{l}11 \\
.05 \\
.02\end{array}$ & + $+\frac{2}{30}$ & $\begin{array}{l}.02 \\
.04 \\
.01\end{array}$ \\
\hline $\begin{array}{l}\text { DFACT } \\
\text { FFACT }\end{array}$ & $\begin{array}{l}3.36 \\
5.08\end{array}$ & $\frac{4}{3}+\frac{17}{42}$ & $\frac{2.84}{3.25}$ & $\begin{array}{l}4.56 \\
3.57\end{array}$ & $\begin{array}{r}13.74 \\
3.65\end{array}$ & 2.72 \\
\hline IIATA FO & OF FILE : & 45100 & & & & \\
\hline Fum & $3 \frac{45}{37}$ & 25.0 & $\begin{array}{r}A Z \\
340.0\end{array}$ & $\begin{array}{r}V E L \\
42.5\end{array}$ & & \\
\hline $\begin{array}{l}\text { COMF: } \\
\text { MEAH : }\end{array}$ & 25 & $\begin{array}{r}\text { CFY } \\
-.02\end{array}$ & $\begin{array}{l}\mathrm{Cr} \\
.05\end{array}$ & $\begin{array}{l}C M X \\
.03\end{array}$ & $\begin{array}{l}\text { CryY } \\
.01\end{array}$ & $\begin{array}{l}\mathrm{Cryz} \\
.0 \mathrm{z}\end{array}$ \\
\hline $\begin{array}{l}\text { MAX } \\
\text { MIYI } \\
\text { FME }\end{array}$ & $\begin{array}{r}1.11 \\
: .27 \\
.14\end{array}$ & $\begin{array}{r}.02 \\
-.03 \\
.01\end{array}$ & $\begin{array}{l}.23 \\
.02 \\
.02\end{array}$ & $\begin{array}{l}12 \\
.05 \\
.02\end{array}$ & $\begin{array}{l}39 \\
.87 \\
07\end{array}$ & $\begin{array}{l}.09 \\
.09 \\
.01\end{array}$ \\
\hline $\begin{array}{l}\text { GFACT } \\
\text { FFACT }\end{array}$ & 4.05 & $\begin{array}{l}3.84 \\
4.63\end{array}$ & $\begin{array}{l}3.44 \\
5.06\end{array}$ & $\begin{array}{l}3.88 \\
4.22\end{array}$ & 20.53 & $\begin{array}{l}2 \\
1 \\
1\end{array}$ \\
\hline IIATA FC & OF FILE : & 45100 & & & & \\
\hline Futs & $\begin{array}{l}W 1+\pi \\
0.0\end{array}$ & 25.0 & $\begin{array}{r}\mathrm{AZ} \\
340.0\end{array}$ & $\begin{array}{r}\text { VEL } \\
42.7\end{array}$ & & \\
\hline COMF : & 6.5 & $\begin{array}{r}\text { CFY } \\
-.02\end{array}$ & $\begin{array}{l}\operatorname{coz} \\
.03\end{array}$ & .02 & $\begin{array}{l}\text { CMY } \\
.02\end{array}$ & $\begin{array}{c}\mathrm{CHz} \\
. \mathrm{OS}\end{array}$ \\
\hline $\begin{array}{l}\text { MAX } \\
\text { MAIH } \\
\text { FHSS: }\end{array}$ & $\begin{array}{r}.87 \\
. \quad 54 \\
+17\end{array}$ & $\begin{array}{r}.05 \\
.09 \\
.02\end{array}$ & $\begin{array}{l}17 \\
.07 \\
.03\end{array}$ & $.0 \%$ & .10 & $\begin{array}{l}.01 \\
.03 \\
.01\end{array}$ \\
\hline $\begin{array}{l}\text { EFACT } \\
\text { FFACT }\end{array}$ & $\begin{array}{l}7.43 \\
4+48\end{array}$ & $4: 71$ & $\begin{array}{l}5.59 \\
4.61\end{array}$ & 皇. 39 & $12.5 \%$ & 2.83 \\
\hline
\end{tabular}




\begin{tabular}{|c|c|c|c|c|c|c|}
\hline FuH & $\begin{aligned} 4 I+I I \\
215.0\end{aligned}$ & $\begin{aligned} E L \\
90.0\end{aligned}$ & & $\begin{array}{l}\text { WEL } \\
\text { SI. }\end{array}$ & & \\
\hline COMF: & $\begin{array}{c}0.0 \% \\
.06\end{array}$ & $\begin{array}{l}\text { CFY } \\
.00\end{array}$ & 0.07 & $\begin{array}{l}\text { chy } \\
.00\end{array}$ & $\begin{array}{l}\text { CHY } \\
.02\end{array}$ & $\begin{array}{l}\text { CHa } \\
.00\end{array}$ \\
\hline $\begin{array}{l}\text { MAY } \\
\text { MIYL } \\
\text { FME }\end{array}$ & $\begin{array}{l}\cdot 18 \\
\cdots 02 \\
.02\end{array}$ & $\begin{array}{r}.06 \\
-.05 \\
.01\end{array}$ & $\begin{array}{r}37 \\
.20 \\
.00\end{array}$ & $\begin{array}{l}.05 \\
.05 \\
.02\end{array}$ & $\begin{array}{l}.09 \\
.06 \\
.02\end{array}$ & $\begin{array}{l}.02 \\
.01 \\
.01\end{array}$ \\
\hline $\begin{array}{l}\text { GFACT } \\
\text { FFACT }\end{array}$ & $\frac{2.42}{4.02}$ & $\begin{array}{r}24 \\
3.07\end{array}$ & $4+46$ & $1 \stackrel{4}{3} \cdot 22$ & $5 \cdot 31$ & 11.34 \\
\hline
\end{tabular}

DATA FOF FILE:

\begin{tabular}{|c|c|c|c|c|c|c|}
\hline Fury $_{217}$ & $\begin{array}{r}W I+I I \\
215.0\end{array}$ & 0.0 & & $\begin{array}{l}\text { UEL } \\
42.2\end{array}$ & & \\
\hline $\begin{array}{l}\text { COMF : } \\
\text { MEAN }\end{array}$ & CFY & $\begin{array}{l}\text { CFY } \\
.00\end{array}$ & $\begin{array}{c}c^{2} \\
.01\end{array}$ & $\begin{array}{l}\text { CHy } \\
.03\end{array}$ & CHY & $\operatorname{crg}=1$ \\
\hline $\begin{array}{l}\text { MAX } \\
\text { MINE } \\
\text { FHE }\end{array}$ & $\begin{array}{r}1.07 \\
.30 \\
.10\end{array}$ & $\begin{array}{r}.00 \\
-.09 \\
.03\end{array}$ & $\begin{array}{l}+05 \\
+0.4 \\
.01\end{array}$ & . $1 \frac{1}{1} 1 \frac{1}{1}$ & $\begin{array}{r}.30 \\
.31 \\
.08\end{array}$ & $\begin{array}{l}.02 \\
05 \\
01 \\
01\end{array}$ \\
\hline $\begin{array}{l}\text { QFACT } \\
\text { FFACT }\end{array}$ & $\frac{2.90}{3.95}$ & 20.85 & $\frac{3}{3} \cdot \frac{21}{51}$ & 5.20 & $-1 \cdot 17$ & $\dot{4} \cdot 15$ \\
\hline
\end{tabular}

IIATA FOF FILE: H5100

\begin{tabular}{|c|c|c|c|c|c|c|}
\hline Eum & $\begin{array}{r}\text { WINII } \\
247.5\end{array}$ & 20.0 & $\begin{array}{l}A Z \\
0.0\end{array}$ & $\begin{array}{r}\text { UEL } \\
43.0\end{array}$ & & \\
\hline $\begin{array}{l}\text { CaMF } \\
\text { MEAH }\end{array}$ & $\begin{array}{l}\text { CFY } \\
.05\end{array}$ & $\begin{array}{l}\text { CFY } \\
.02\end{array}$ & .01 & $\begin{array}{l}\text { C.M. } \\
.00\end{array}$ & $\begin{array}{l}.04 Y \\
.00\end{array}$ & $\begin{array}{l}\text { crit } \\
.00\end{array}$ \\
\hline $\begin{array}{l}\text { MAX } \\
\text { MIN } \\
\text { FHS }\end{array}$ & $\begin{array}{r}.50 \\
.54 \\
+14\end{array}$ & $\begin{array}{r}.15 \\
-11 \\
.04\end{array}$ & .115 & .11 &.$\frac{12}{2}$ & $\begin{array}{l}.06 \\
.05 \\
.01\end{array}$ \\
\hline $\begin{array}{l}\text { QFACT } \\
\text { FFACT }\end{array}$ & $\begin{array}{r}12.39 \\
4.08\end{array}$ & $\begin{array}{l}7.42 \\
3.45\end{array}$ & $\begin{array}{r}14.59 \\
3.85\end{array}$ & $35+\frac{42}{5}$ & 107.45 & $11 \cdot 61$ \\
\hline
\end{tabular}

IIATA FOF FILE: H5100

\begin{tabular}{|c|c|c|c|c|c|c|}
\hline Fust & 270.0 & 20.0 & $250 .{ }^{A I}$ & $\begin{array}{l}V C L \\
42.5\end{array}$ & & \\
\hline COHF : & $\begin{array}{l}C F^{-} \\
.15\end{array}$ & $\begin{array}{l}\text { CFY } \\
.02\end{array}$ & $\begin{array}{c}c t z \\
.04\end{array}$ & $\begin{array}{c}\mathrm{CH} \\
.01\end{array}$ & C.MY & Crst \\
\hline $\begin{array}{l}\text { MAX } \\
\text { MIN } \\
\text { FMS }\end{array}$ & 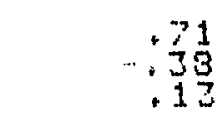 & $\begin{array}{r}12 \\
-11 \\
02\end{array}$ & .17 & $.1 \frac{1}{13}$ & $\begin{array}{l}.22 \\
.15 \\
.05\end{array}$ & $\begin{array}{l}.05 \\
.02 \\
.01\end{array}$ \\
\hline $\begin{array}{l}F A C T \\
F A C T\end{array}$ & $\frac{4}{4} \cdot 3 \frac{32}{37}$ & $\begin{array}{l}7.95 \\
3.48\end{array}$ & $3 \cdot \frac{9}{3}$ & $\begin{array}{r}10.25 \\
2.01\end{array}$ & $\begin{array}{l}3.55 \\
4+20\end{array}$ & 5.05 \\
\hline
\end{tabular}




\begin{tabular}{|c|c|c|c|c|c|c|}
\hline fium & $\begin{array}{r}413 I \\
292.5\end{array}$ & 20.0 & $270^{A 2} .0^{\circ}$ & $\begin{array}{l}\text { VEL } \\
72.6\end{array}$ & & \\
\hline $\begin{array}{l}\text { COMF } \\
\text { MEAM }\end{array}$ & $\operatorname{cr}_{2}$ & $\begin{array}{r}\mathrm{CFY} \\
-.01\end{array}$ & .10 & crs & $\begin{array}{l}\text { Cris } \\
.05\end{array}$ & $\begin{array}{l}\text { CHa } \\
.05\end{array}$ \\
\hline $\begin{array}{l}\text { MAX } \\
\text { MIH } \\
\text { FMS }\end{array}$ & $\begin{array}{r}1.04 \\
.32 \\
.17\end{array}$ & $\begin{array}{r}.12 \\
-.10 \\
.04\end{array}$ & $\begin{array}{r}+25 \\
-.02 \\
+03\end{array}$ & $\begin{array}{r}+00 \\
.21 \\
.04\end{array}$ & $\begin{array}{l}+3.4 \\
132 \\
03\end{array}$ & $\begin{array}{r}10 \\
.00 \\
.02\end{array}$ \\
\hline $\begin{array}{l}\text { QFACT } \\
\text { FACT }\end{array}$ & 3.26 & 17.15 & $\frac{2}{3}+3 \frac{1}{57}$ & 3.70 & 3.97 & $\begin{array}{l}7 \\
-95\end{array}$ \\
\hline
\end{tabular}

IIATA FOF FILE : H5100

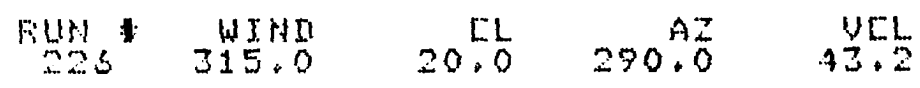

\begin{tabular}{|c|c|c|c|c|c|c|}
\hline $\begin{array}{l}\text { COMF } \\
\text { MEAN }\end{array}$ & $6 F^{2}$ & $\begin{array}{r}\text { CFY } \\
-.02\end{array}$ & $\cos 2$ & $\begin{array}{l}\text { CHY } \\
.04\end{array}$ & $\begin{array}{l}\text { crsy } \\
.00\end{array}$ & $\begin{array}{c}\text { crsz } \\
.05\end{array}$ \\
\hline $\begin{array}{l}\text { MAX } \\
\text { SIIH } \\
\text { FHE }\end{array}$ & $\begin{array}{r}1+36 \\
\cdots: 24 \\
.18\end{array}$ & $\begin{array}{r}.09 \\
.13 \\
.04\end{array}$ & $\begin{array}{l}.25 \\
.05 \\
.03\end{array}$ & $\begin{array}{r}.07 \\
.15 \\
.02\end{array}$ & 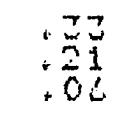 & $\begin{array}{l}10 \\
.01 \\
.01\end{array}$ \\
\hline $\begin{array}{l}\text { JFACT } \\
\text { FACT }\end{array}$ & $\begin{array}{l}3.88 \\
5.2 \%\end{array}$ & $\frac{5}{3} \cdot 4 \frac{2}{3}$ & $\begin{array}{l}2.00 \\
3.09\end{array}$ & $\frac{3}{3}+\frac{51}{37}$ & $\begin{array}{l}3.73 \\
4.12\end{array}$ & \\
\hline
\end{tabular}

IIATA FOF FILE:

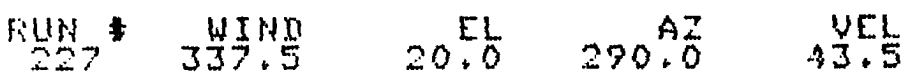

\begin{tabular}{|c|c|c|c|c|c|}
\hline $\begin{array}{l}\text { COMF } \\
\text { MEAN }\end{array}$ & CFy & $\begin{array}{r}C F Y \\
-.01\end{array}$ & $.0 \%$ & crsy & $\begin{array}{l}\text { Crsy } \\
.01\end{array}$ \\
\hline $\begin{array}{l}\text { MAX } \\
\text { MIN } \\
\text { FIMS }\end{array}$ & $\begin{array}{r}.83 \\
.02 \\
.11\end{array}$ & $\begin{array}{r}.06 \\
.09 \\
.02\end{array}$ & $\begin{array}{r}.17 \\
.00 \\
.02\end{array}$ & $\begin{array}{l}.07 \\
.00 \\
.02\end{array}$ & $\begin{array}{l}.20 \\
.09 \\
.0 .2\end{array}$ \\
\hline
\end{tabular}

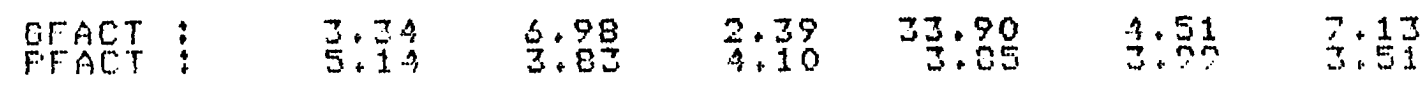

IATA FDF FILE : HS100

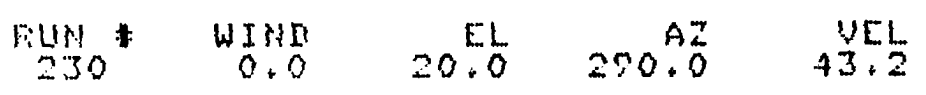

\begin{tabular}{|c|c|c|c|c|c|}
\hline $\begin{array}{l}\text { COMF } \\
\text { MEAN }\end{array}$ & $\begin{array}{l}C F x \\
.30\end{array}$ & $\begin{array}{l}\text { CFY } \\
. .02\end{array}$ & $\begin{array}{l}\text { C.T. } \\
+11\end{array}$ & $\begin{array}{l}\text { CHx } \\
.02\end{array}$ & $\begin{array}{l}\text { C.sy } \\
.00\end{array}$ \\
\hline $\begin{array}{l}\text { MAX } \\
\text { MIH } \\
\text { FMS }\end{array}$ & $\begin{array}{r}1.24 \\
-15 \\
1.14\end{array}$ & $\begin{array}{r}.09 \\
.14 \\
.02\end{array}$ & $\begin{array}{l}.27 \\
.03 \\
+03\end{array}$ & $\begin{array}{l}.13 \\
.02 \\
.02\end{array}$ & $\begin{array}{l}20 \\
.12 \\
0.04\end{array}$ \\
\hline
\end{tabular}

$\begin{array}{lllllll}\text { GFACT } & 4.11 & 6.75 & 2.54 & 4.24 & 4.45 & 3.86\end{array}$ 


\begin{tabular}{|c|c|c|c|c|c|c|}
\hline$\frac{\text { FuN }}{231}$ & WIMI & 10.0 & $2 \Delta 5.0$ & $\begin{array}{l}\text { ULL } \\
43.4\end{array}$ & & \\
\hline COMF & $\begin{array}{l}\text { CF } \\
+23\end{array}$ & $\begin{array}{r}\text { CFY } \\
-.00\end{array}$ & $\begin{array}{l}\text { CF } \\
.01\end{array}$ & $\begin{array}{l}\text { c.m. } \\
.00\end{array}$ & C.HY & $\begin{array}{l}\cos \\
.01\end{array}$ \\
\hline $\begin{array}{l}\text { MAX } \\
\text { MIIH } \\
\text { FiHS }\end{array}$ & $\begin{array}{r}1.04 \\
-.09 \\
.14\end{array}$ & $\begin{array}{r}.03 \\
-.04 \\
.01\end{array}$ & $\begin{array}{l}.05 \\
.03 \\
.01\end{array}$ & $\begin{array}{r}.05 \\
-.05 \\
.01\end{array}$ & $\begin{array}{l}20 \\
.21 \\
05\end{array}$ & $\begin{array}{l}.05 \\
.05 \\
.01\end{array}$ \\
\hline $\begin{array}{l}\text { GFACT } \\
\text { FFACT }\end{array}$ & 5.70 & $\begin{array}{r}14.34 \\
4.01\end{array}$ & $\begin{array}{l}3.73 \\
3.26\end{array}$ & $10+17$ & $\begin{array}{l}4.70 \\
4.62\end{array}$ & $\begin{array}{r}10 \cdot 21 \\
4+31\end{array}$ \\
\hline
\end{tabular}

IIATA FOF FILE: H5100

\begin{tabular}{|c|c|c|c|c|c|c|}
\hline Fun & 337.5 & 10.0 & & & & \\
\hline CQMF & .25 & $\begin{array}{l}\text { CFY } \\
.00\end{array}$ & $\begin{array}{c}c T^{2} \\
.05\end{array}$ & $\begin{array}{c}\mathrm{Cry} \\
.01\end{array}$ & .05 & $\begin{array}{l}C H z \\
.05\end{array}$ \\
\hline $\begin{array}{l}\text { MAX } \\
\text { MIMI } \\
\text { FMS }\end{array}$ & $\begin{array}{r}.98 \\
-\quad 19 \\
.12\end{array}$ & $\begin{array}{r}.04 \\
-.04 \\
.01\end{array}$ & $\begin{array}{r}.07 \\
.02 \\
.01\end{array}$ & $\begin{array}{l}.00 \\
.05 \\
.01\end{array}$ &.$\frac{32}{12}$ & $\begin{array}{r}.00 \\
.01 \\
+01\end{array}$ \\
\hline $\begin{array}{l}\text { GFACT } \\
\text { PFACT }\end{array}$ & $\begin{array}{l}3.53 \\
4.97\end{array}$ & $2 \frac{2}{3} \cdot \frac{74}{50}$ & $\frac{2}{3}+40$ & 5.53 & 3.78 & $2+72$ \\
\hline
\end{tabular}

IIATA FOF FILE:

\begin{tabular}{|c|c|c|c|c|c|c|}
\hline $\operatorname{RuN}_{236}$ & $\begin{array}{r}\text { WINI } \\
315.0\end{array}$ & 10.0 & & $\begin{array}{l}\text { YEL } \\
42.3\end{array}$ & & \\
\hline COHF : & .250 & .01 & .0 .2 & $\begin{array}{l}\mathrm{CH} \\
.01\end{array}$ & CMY & $\begin{array}{l}\operatorname{crs} z \\
.03\end{array}$ \\
\hline $\begin{array}{l}\text { MAX } \\
\text { MIHS } \\
\text { FHS }\end{array}$ & $\begin{array}{r}+96 \\
.36 \\
.16\end{array}$ & $\begin{array}{r}.05 \\
.04 \\
.01\end{array}$ & $\begin{array}{r}.04 \\
-.02 \\
.01\end{array}$ & $\begin{array}{l}.04 \\
.05 \\
.02\end{array}$ & $\begin{array}{l}.30 \\
.20 \\
.02\end{array}$ & .10 \\
\hline $\begin{array}{l}\text { GFACT } \\
\text { FFACT }\end{array}$ & $\begin{array}{l}4.79 \\
4: 64\end{array}$ & $\frac{3.85}{3.25}$ & $\frac{2.71}{3.07}$ & $\begin{array}{l}3.98 \\
3.25\end{array}$ & $\begin{array}{l}7.97 \\
8+10\end{array}$ & $\frac{2}{7} \cdot 75$ \\
\hline
\end{tabular}

IIATA FOF FILE: HS100

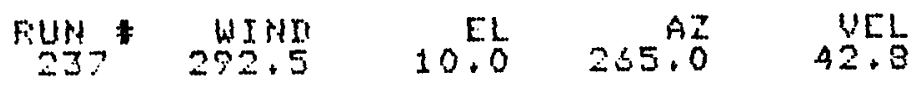

\begin{tabular}{|c|c|c|c|c|c|c|}
\hline $\begin{array}{l}\text { CQMF } \\
\text { MEAN }\end{array}$ & $\begin{array}{l}C F Y \\
+D 1\end{array}$ & $\begin{array}{l}\text { CFy } \\
.02\end{array}$ & $\begin{array}{c}\operatorname{crz} \\
\cdots .01\end{array}$ & $\begin{array}{l}\text { C.KS } \\
.00\end{array}$ & $\begin{array}{l}\text { CHY } \\
.0 .4\end{array}$ & $\begin{array}{l}\text { Crs } \\
.02\end{array}$ \\
\hline $\begin{array}{l}\text { MAX } \\
\text { KIH } \\
\text { FMS }\end{array}$ & 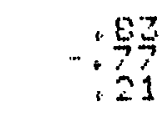 & $\begin{array}{r}.09 \\
-.05 \\
.02\end{array}$ & .04 & $\begin{array}{l}.02 \\
.00 \\
.02\end{array}$ & $\begin{array}{r}32 \\
.25 \\
00\end{array}$ & $\begin{array}{l}.09 \\
.85 \\
.02\end{array}$ \\
\hline $\begin{array}{l}A C T \\
A C T\end{array}$ & $10 \frac{1}{2} \cdot 74$ & 3.88 & $1 \frac{1}{5} \cdot 35$ & $1 ? \cdot 42$ & $\frac{0.52}{3.58}$ & \\
\hline
\end{tabular}




\begin{tabular}{|c|c|c|c|c|c|c|}
\hline Eusto & $\begin{array}{l}\text { WINII } \\
270.0\end{array}$ & $\begin{array}{r}E L \\
10.0\end{array}$ & & $\begin{array}{r}U 2 . \frac{1}{9} \\
\end{array}$ & & \\
\hline COMF & $\begin{array}{c}\cos \\
-.05\end{array}$ & $\begin{array}{l}\text { CFY } \\
.01\end{array}$ & $\begin{array}{l}C F= \\
.01\end{array}$ & $\begin{array}{c}\mathrm{CH} \\
.02\end{array}$ & $\begin{array}{l}\text { CHY } \\
.01\end{array}$ & $\begin{array}{c}\cos \\
.00\end{array}$ \\
\hline $\begin{array}{l}\text { MAX } \\
\text { MIS } \\
\text { FHS }\end{array}$ & $+2 \frac{12}{5}$ & $\begin{array}{r}.04 \\
-.04 \\
+01\end{array}$ & $\begin{array}{l}.05 \\
.07 \\
.01\end{array}$ & $\begin{array}{l}.05 \\
.05 \\
.02\end{array}$ & $\begin{array}{l}30 \\
30 \\
08 \\
0\end{array}$ & \\
\hline $\begin{array}{l}\text { OFACT } \\
\text { FFACT }\end{array}$ & $\begin{array}{r}10.57 \\
3.58\end{array}$ & $\frac{6}{3} \cdot 65$ & $4+27$ & $\begin{array}{l}4.95 \\
3.60\end{array}$ & 50.05 & 23.8 \\
\hline
\end{tabular}

IATA FOF FILE: HS100

\begin{tabular}{|c|c|c|c|c|c|c|}
\hline $\mathrm{Fin}_{2 \rightarrow 1}$ & 247.5 & 10.0 & $A Z$ & $\begin{array}{c}\text { UᄃL } \\
42+9\end{array}$ & & \\
\hline COHF: & $\begin{array}{r}c 5 s \\
-.16\end{array}$ & $\begin{array}{l}\text { CFY } \\
.03\end{array}$ & .02 & $\begin{array}{l}\operatorname{cms} \\
.03\end{array}$ & $\begin{array}{l}\mathrm{CHY} \\
.0 .3\end{array}$ & criz \\
\hline $\begin{array}{l}\text { MAX } \\
\text { HAHE } \\
\text { FASE }\end{array}$ & $-1+\frac{31}{10}$ & $\begin{array}{r}10 \\
.03 \\
.02\end{array}$ & $\begin{array}{r}03 \\
.11 \\
.02\end{array}$ & $\begin{array}{l}11 \\
.05 \\
.02\end{array}$ & . & $\begin{array}{l}.05 \\
.11 \\
.02\end{array}$ \\
\hline $\begin{array}{l}\text { QFACT } \\
\text { FFACT }\end{array}$ & $\begin{array}{c}0.45 \\
6.05\end{array}$ & $\begin{array}{l}3.73 \\
4.64\end{array}$ & 5.17 & 3.52 & 0.15 & 5.72 \\
\hline
\end{tabular}

MATA FOR FILE: HS100

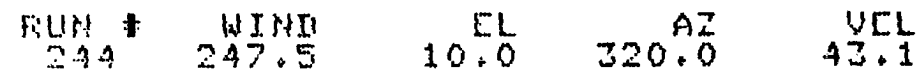

\begin{tabular}{|c|c|c|c|c|c|c|}
\hline COMHF : & $.0 F$ & $\begin{array}{l}\text { Cry } \\
.00\end{array}$ & $\begin{array}{l}\text { CFI } \\
.01\end{array}$ & $\begin{array}{l}\text { CM. } \\
.00\end{array}$ & $\begin{array}{l}C .4 Y \\
.01\end{array}$ & CHz \\
\hline $\begin{array}{l}\text { MAY } \\
\text { MIH } \\
\text { RMS }\end{array}$ & $\begin{array}{r}.95 \\
-95 \\
.14\end{array}$ & $\begin{array}{r}.09 \\
.10 \\
.03\end{array}$ & .05 & .12 & . $\frac{32}{2}$ & $\begin{array}{l}.05 \\
.0 \mathrm{D} \\
.0 \mathrm{~L}\end{array}$ \\
\hline $\begin{array}{l}\text { OFACT } \\
\text { FAACT }\end{array}$ & $\begin{array}{l}5.72 \\
5.01\end{array}$ & $\begin{array}{r}128.58 \\
3.01\end{array}$ & $\frac{8.21}{2} .20$ & $34+25$ & $23+\frac{1}{7}$ & 3.97 \\
\hline
\end{tabular}

IATA FOF FILE:

\begin{tabular}{|c|c|c|c|c|c|c|}
\hline$x_{-45}$ & $\begin{array}{l}\text { WINII } \\
2>0.0\end{array}$ & 10.0 & 320.0 & $\begin{array}{r}Y\left[\frac{1}{3}\right. \\
4\end{array}$ & & \\
\hline $\begin{array}{l}\text { COMF } \\
\text { MEAN }\end{array}$ & C. 2.9 & $\begin{array}{l}\text { CFY } \\
.01\end{array}$ & .05 & $\begin{array}{l}C M x_{2} \\
.02\end{array}$ & $\begin{array}{l}\text { CHY } \\
.05\end{array}$ & $\begin{array}{c}\cos z \\
.01\end{array}$ \\
\hline $\begin{array}{l}\text { MAX } \\
\text { MIM } \\
\text { FMS }\end{array}$ & $\begin{array}{r}1.10 \\
: 10 \\
.10\end{array}$ & $\begin{array}{r}11 \\
-11 \\
03\end{array}$ & $\begin{array}{l}.00 \\
.01 \\
.01\end{array}$ & $\begin{array}{l}17 \\
+13 \\
04\end{array}$ & . & $\begin{array}{r}10 \\
.00 \\
.01\end{array}$ \\
\hline FACT & $\frac{-1}{5} \cdot \frac{1}{0} \bar{\varepsilon}$ & $17 \div \frac{1}{3}$ & 2.53 & $\begin{array}{r}2 \\
2 \\
7\end{array}$ & 采: & $\frac{3}{4} \cdot \frac{2}{6} ?$ \\
\hline
\end{tabular}


IATA FOF FILE: HS100

\begin{tabular}{|c|c|c|c|c|c|c|}
\hline Filn & WIIII & 10.0 & & $\begin{array}{l}\text { YEL } \\
42.6\end{array}$ & & \\
\hline $\begin{array}{l}\text { COMF : } \\
\text { HEAH }\end{array}$ & .39 & $\begin{array}{l}\text { CFY } \\
.01\end{array}$ & $\operatorname{cr}_{4}$ & $\begin{array}{l}\mathrm{CH} 5 \\
.05\end{array}$ & $\begin{array}{l}\text { CHY } \\
.03\end{array}$ & $\begin{array}{l}\text { C.M. } \\
.00\end{array}$ \\
\hline $\begin{array}{l}\text { MAO } \\
\text { MIN } \\
\text { FMS }\end{array}$ & $\begin{array}{r}1+14 \\
\quad+13 \\
\quad+17\end{array}$ & $\begin{array}{l}+12 \\
+11 \\
03\end{array}$ & $\begin{array}{l}.08 \\
.00 \\
.01\end{array}$ & $.2 \frac{2}{7} .04$ & $\begin{array}{r}+71 \\
+5 \frac{2}{2} \\
+07\end{array}$ & .05 \\
\hline $\begin{array}{l}\text { OFACT } \\
\text { FFACT }\end{array}$ & $\begin{array}{l}2.39 \\
4.45\end{array}$ & $\begin{array}{r}14 \cdot 91 \\
3.57\end{array}$ & $\begin{array}{l}2.00 \\
3.28\end{array}$ & 1.07 & 5.35 & $=25+15$ \\
\hline
\end{tabular}

IMTA FDF: FILE : H5100

\begin{tabular}{|c|c|c|c|c|c|c|}
\hline $\operatorname{Fun}_{249}$ & WIMn & 10.0 & & $\begin{array}{l}\text { VEL } \\
43.1\end{array}$ & & \\
\hline CQMF: & $\begin{array}{l}\text { CF. } \\
.23\end{array}$ & $\begin{array}{l}\text { CFY } \\
\cdots .00\end{array}$ & .05 & $\begin{array}{l}\text { CMY } \\
.0 \%\end{array}$ & $\begin{array}{l}\text { CMY } \\
.01\end{array}$ & crsa \\
\hline $\begin{array}{l}\text { MAX } \\
\text { MIIE } \\
\text { FMS }\end{array}$ & $\begin{array}{r}1.05 \\
.18 \\
.18\end{array}$ & $\begin{array}{r}.09 \\
.12 \\
.02\end{array}$ & .07 & $\begin{array}{l}17 \\
.17 \\
.0 .4\end{array}$ & 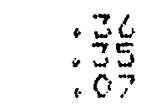 & $\begin{array}{l}.02 \\
.06 \\
.01\end{array}$ \\
\hline $\begin{array}{l}\text { QFACT } \\
\text { FFACT }\end{array}$ & 3.75 & $\begin{array}{r}23.79 \\
3.50\end{array}$ & $2 \cdot 29$ & $\begin{array}{l}1.30 \\
2.70\end{array}$ & 51.70 & $\begin{array}{l}3.05 \\
3.01\end{array}$ \\
\hline
\end{tabular}

IIATA FDE FILE: H5100

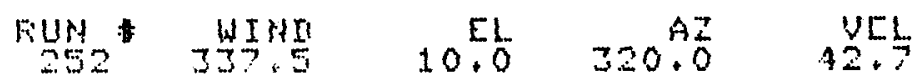

\begin{tabular}{|c|c|c|c|c|c|c|}
\hline $\begin{array}{l}\text { COMF } \\
\text { MEAH }\end{array}$ & $\begin{array}{l}C F X \\
.32\end{array}$ & $\begin{array}{r}\text { CFY } \\
-.01\end{array}$ & $\begin{array}{cr}c 5 \\
.03\end{array}$ & $\begin{array}{l}\text { CHY } \\
.03\end{array}$ & CMY & $\begin{array}{c}\text { CHz } \\
\text { OI }\end{array}$ \\
\hline $\begin{array}{l}\text { MAY } \\
\text { MIHE } \\
\text { FiMS }\end{array}$ & $\begin{array}{l}1.21 \\
* 15 \\
* 15\end{array}$ & $\begin{array}{r}.07 \\
-10 \\
.03\end{array}$ & $\begin{array}{l}.07 \\
.02 \\
.01\end{array}$ & $\begin{array}{l}15 \\
.05 \\
.04\end{array}$ &.$\frac{24}{23}$ & $\begin{array}{l}+92 \\
+02 \\
01\end{array}$ \\
\hline $\begin{array}{l}\text { QFACT } \\
\text { FFACT }\end{array}$ & $\frac{3}{5} \cdot \frac{32}{3}$ & $\begin{array}{l}6.53 \\
3.04\end{array}$ & $\begin{array}{l}2.35 \\
3.48\end{array}$ & $\begin{array}{l}4.35 \\
3.25\end{array}$ & $\frac{5}{3} \cdot 58$ & $\begin{array}{l}5.72 \\
4+25\end{array}$ \\
\hline
\end{tabular}

IATA FOF FILE: H5100

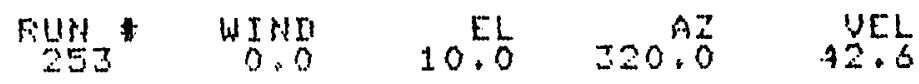

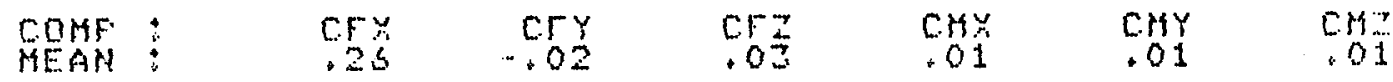

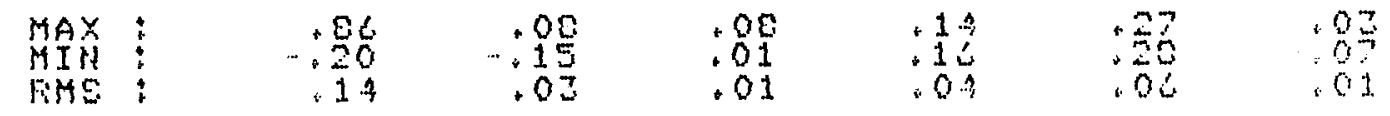

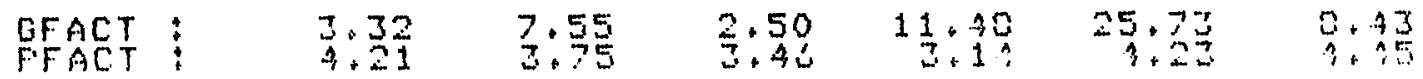


Heliostat 5, Configuration 5-D

\begin{tabular}{|c|c|c|c|c|c|c|c|}
\hline$C_{i 1 s e}$ & Run & WD & $\mathrm{EL}$ & $\mathrm{AZ}$ & Day & & ime \\
\hline \multirow[t]{6}{*}{ Sillr AM } & 212 & 0 & 25 & 340 & 172 & 8 & $\mathrm{AM}$ \\
\hline & 211 & 337.5 & 25 & 340 & 172 & 8 & $\mathrm{AN}$ \\
\hline & 208 & 315 & 25 & 340 & 172 & 8 & $\mathrm{AM}$ \\
\hline & 207 & 292.5 & 25 & 340 & 172 & 8 & $A M$ \\
\hline & 204 & 270 & 25 & 340 & 172 & 8 & $\mathrm{AM}$ \\
\hline & 203 & 247.5 & 25 & 340 & 172 & 8 & $\mathrm{AM}$ \\
\hline \multirow[t]{6}{*}{ Smr Noon } & 200 & 247.5 & 45 & 290 & 172 & 0 & $\mathrm{PM}$ \\
\hline & 199 & 270 & 45 & 290 & 172 & 0 & $\mathrm{PM}$ \\
\hline & 196 & 292.5 & 45 & 290 & 172 & 0 & $\mathrm{PM}$ \\
\hline & 193 & 315 & 45 & 290 & 172 & 0 & $P M$ \\
\hline & 195 & 337.5 & 45 & 290 & 172 & 0 & $P M$ \\
\hline & 189 & 0 & 45 & 290 & 172 & 0 & PN \\
\hline \multirow[t]{6}{*}{ Whitr AM } & 254 & 0 & 10 & 320 & 355 & 8 & $\mathrm{AN}$ \\
\hline & 251 & 337.5 & 10 & 320 & 355 & 8 & AN \\
\hline & 250 & 315 & 10 & 320 & 355 & 8 & $\mathrm{AN}$ \\
\hline & 247 & 292.5 & 10 & 320 & 355 & 8 & AM \\
\hline & 246 & 270 & 10 & 320 & 355 & 8 & AM \\
\hline & 243 & 247.5 & 10 & 320 & 355 & 8 & AM \\
\hline \multirow[t]{6}{*}{ Whtr Noon } & 229 & 0 & 20 & 290 & 355 & 0 & PM \\
\hline & 228 & 337.5 & 20 & 290 & 355 & 0 & PM \\
\hline & 225 & 315 & 20 & 290 & 355 & 0 & PM \\
\hline & 223 & 292.5 & 20 & 290 & 355 & 0 & $\mathrm{PM}$ \\
\hline & 220 & 270 & 20 & 290 & 355 & 0 & $\mathrm{PN}$ \\
\hline & 219 & 247.5 & 20 & 290 & 355 & 0 & PN \\
\hline \multicolumn{8}{|c|}{165} \\
\hline
\end{tabular}


Heliostat 5, Configuration 5-D. continued

\begin{tabular}{|c|c|c|c|c|c|c|}
\hline Case & Run 排 & WD & EL & $\mathrm{AZ}$ & Day & Time \\
\hline \multirow[t]{6}{*}{ Wntr PM } & 242 & 247.5 & 10 & 265 & 355 & $4 P N$ \\
\hline & 239 & 270 & 10 & 265 & 355 & $4 P N$ \\
\hline & 238 & 292.5 & 10 & 265 & 355 & $4 P N$ \\
\hline & 235 & 315 & 10 & 265 & 355 & $4 \mathrm{PM}$ \\
\hline & 233 & 337.5 & 10 & 265 & 355 & $4 m$ \\
\hline & 232 & 0 & 10 & 265 & 355 & $4 p$ \\
\hline Ver. Stow & 216 & 315 & 0 & 320 & 355 & $8 \mathrm{~A}$ \\
\hline Hor. Stow & 215 & 315 & 90 & 320 & 355 & $8 \mathrm{AM}$ \\
\hline
\end{tabular}




\begin{tabular}{|c|c|c|c|c|c|c|}
\hline rust & WImI & 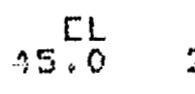 & $2=0.0$ & $\begin{array}{r}\text { VEL } \\
42.7\end{array}$ & & \\
\hline $\begin{array}{l}\text { COMF } \\
\text { HEAN }\end{array}$ & $\begin{array}{l}C F \\
2 A\end{array}$ & $\begin{array}{r}\text { CFY } \\
-.05\end{array}$ & $\mathrm{Cr}_{17}$ & $\begin{array}{l}\text { chs } \\
.02\end{array}$ & cry & $\begin{array}{l}\text { CHI } \\
.00\end{array}$ \\
\hline $\begin{array}{l}\text { MAX } \\
\text { SISUE }\end{array}$ & $\begin{array}{r}1.19 \\
: 13 \\
: 18\end{array}$ & $\begin{array}{r}.09 \\
.13 \\
.03\end{array}$ & $\begin{array}{r}.80 \\
.02 \\
.0 \%\end{array}$ & $\begin{array}{l}13 \\
.04 \\
.02\end{array}$ & .20 & $\begin{array}{r}.05 \\
.09 \\
.01\end{array}$ \\
\hline $\begin{array}{l}\text { GFACY } \\
\text { FFACY }\end{array}$ & $\frac{4}{5} \cdot \frac{31}{7}$ & $\begin{array}{l}4 \\
3 \\
3.31 \\
31\end{array}$ & $\frac{3.01}{5.05}$ & $\begin{array}{l}4.70 \\
3.77\end{array}$ & 5.17 & $13 \cdot 92$ \\
\hline
\end{tabular}

IATA FOF FILE: H5101

\begin{tabular}{|c|c|c|c|c|c|c|}
\hline rum & HIIHI & 95.0 & $=9 A^{A Z}$ & $\begin{array}{r}\text { VEL } \\
43.6\end{array}$ & & \\
\hline $\begin{array}{l}\text { COMF: } \\
\text { MEAN }\end{array}$ & $\begin{array}{l}\text { CF } \\
.20\end{array}$ & $\begin{array}{l}\mathrm{CFY} \\
.01\end{array}$ & $\begin{array}{l}.52 \\
.10\end{array}$ & .01 & $\begin{array}{l}\text { cris } \\
.02\end{array}$ & $\begin{array}{c}\operatorname{cra} z \\
.01\end{array}$ \\
\hline $\begin{array}{l}\text { MAX } \\
\text { MIUS } \\
\text { SHSS }\end{array}$ & $\begin{array}{r}.93 \\
-13 \\
.13\end{array}$ & $\begin{array}{r}.02 \\
-.08 \\
.02\end{array}$ & $\begin{array}{r}+39 \\
+.58 \\
+02\end{array}$ & $\begin{array}{l}.11 \\
.05 \\
.02\end{array}$ & $\begin{array}{l}.20 \\
.18 \\
.04\end{array}$ & .05 \\
\hline $\begin{array}{l}\text { OFACT } \\
\text { PFACT }\end{array}$ & $\begin{array}{l}4.75 \\
5.58\end{array}$ & $\begin{array}{r}17.56 \\
3.40\end{array}$ & $\begin{array}{l}3.49 \\
4.53\end{array}$ & $\frac{7}{2}: \frac{43}{3}$ & 7.81 & $3+90$ \\
\hline
\end{tabular}

IIATA FOR FILE : HS101

\begin{tabular}{|c|c|c|c|c|c|c|}
\hline $\begin{array}{l}\text { Fund } \\
195\end{array}$ & 337.5 & 45. & & $\begin{array}{r}4\left[\frac{1}{2}\right. \\
43.2\end{array}$ & & \\
\hline COMF: & $\begin{array}{l}\mathrm{CF} \times \\
20\end{array}$ & $\begin{array}{r}\text { CFy } \\
-.00\end{array}$ & $\begin{array}{l}\mathrm{Cr}^{3} \\
+16\end{array}$ & $\begin{array}{l}\mathrm{CM}_{2} \\
.02\end{array}$ & 0.04 & $\begin{array}{c}\text { Criz } \\
.01\end{array}$ \\
\hline $\begin{array}{l}\text { MAY } \\
\text { HIHA } \\
\text { FHAS }\end{array}$ & $\begin{array}{r}.85 \\
-25 \\
.12\end{array}$ & $\begin{array}{r}+00 \\
-.10 \\
+02\end{array}$ & .02 & $\begin{array}{l}11 \\
.05 \\
.05\end{array}$ & $\begin{array}{l}2 \\
.15 \\
.05\end{array}$ & $\begin{array}{l}.07 \\
.03 \\
.01\end{array}$ \\
\hline $\begin{array}{l}\text { GFACT } \\
\text { FACT }\end{array}$ & $\begin{array}{l}A \cdot 18 \\
A .70\end{array}$ & $\begin{array}{r}25.12 \\
4.06\end{array}$ & $2+72$ & $\begin{array}{l}-7.74 \\
3.40\end{array}$ & 5.52 & $\stackrel{9}{3}: \frac{10}{2}$ \\
\hline
\end{tabular}

IHATA FOR FILE: H5101

\begin{tabular}{|c|c|c|c|c|c|c|}
\hline$\underset{176}{\text { Fint }}$ & $\begin{array}{r}\text { WIHn } \\
292.5\end{array}$ & $45+0$ & & $\begin{array}{l}\text { YEL } \\
72.6\end{array}$ & & \\
\hline $\begin{array}{l}\text { COMF : } \\
\text { BEAH : }\end{array}$ & $0 F$ & cry & $.0 \%$ & $\begin{array}{l}\operatorname{crs} \\
.01\end{array}$ & $\begin{array}{c}C S Y \\
0:\end{array}$ & $\begin{array}{l}\cos 2 \\
.01\end{array}$ \\
\hline $\begin{array}{l}\text { MAS } \\
\text { MIH } \\
\text { BMS }\end{array}$ & $+\frac{85}{14}$ & $\begin{array}{r}+11 \\
\because 12 \\
04\end{array}$ & $\begin{array}{l}.34 \\
.04 \\
.04\end{array}$ & $\begin{array}{l}+11 \\
.11 \\
.04\end{array}$ & $\begin{array}{l}.25 \\
.15 \\
.05\end{array}$ & $\begin{array}{l}.05 \\
.03 \\
.01\end{array}$ \\
\hline $\begin{array}{l}\text { GFACT } \\
\text { FFACT }\end{array}$ & 5.57 & 39.12 & 专:14 & $\begin{array}{r}1 \mathrm{~J} .60 \\
2.50\end{array}$ & $\begin{array}{c}0.32 \\
3.20\end{array}$ & $7+42$ \\
\hline
\end{tabular}




\begin{tabular}{|c|c|c|c|c|c|c|}
\hline Fun & 270.0 & 45.0 & $290^{A} \cdot 0^{\circ}$ & VEL & & \\
\hline Conf & $\begin{array}{r}C F \\
-.07\end{array}$ & $\begin{array}{l}\text { CFY } \\
+O 2\end{array}$ & $.0 \%$ & $\begin{array}{l}\mathrm{CH} \\
.01\end{array}$ & $\begin{array}{l}C+4 Y \\
-01\end{array}$ & $\begin{array}{l}\operatorname{crs} \\
\div 00\end{array}$ \\
\hline $\begin{array}{l}\text { MAY } \\
\text { MIHE } \\
\text { RMS }\end{array}$ & $\begin{array}{r}.52 \\
.51 \\
.11\end{array}$ & $\begin{array}{r}.12 \\
.02 \\
.02\end{array}$ & $-4 \frac{15}{25}$ & $\begin{array}{r}19 \\
.12 \\
.04\end{array}$ & .22 & $\begin{array}{r}.95 \\
.08 \\
.01\end{array}$ \\
\hline $\begin{array}{l}\text { QFACT } \\
\text { FFACT }\end{array}$ & $7: 71$ & $\begin{array}{l}7.02 \\
2.15\end{array}$ & $\begin{array}{l}3 \\
3 \\
3\end{array}$ & $13+2 \cdot 3$ & $24+27$ & 3.70 \\
\hline
\end{tabular}

IATA FOF FILE : H5101

\begin{tabular}{|c|c|c|c|c|c|c|}
\hline nutyo & 287.5 & 95.0 & & 43.5 & & \\
\hline $\begin{array}{l}\text { COMF } \\
\text { MEAN }\end{array}$ & $\begin{array}{c}C 5 \\
0.15\end{array}$ & $\begin{array}{l}\text { CFY } \\
.0 .9\end{array}$ & CFI & $\begin{array}{l}c k y \\
0=\end{array}$ & $\begin{array}{l}\text { Cry } \\
.00\end{array}$ & $\begin{array}{l}\mathrm{CM} \\
.0\end{array}$ \\
\hline $\begin{array}{l}M A Z \\
M I H Z \\
F M S:\end{array}$ & $\begin{array}{r}.25 \\
-.58 \\
.11\end{array}$ & $\begin{array}{r}.13 \\
.07 \\
.03\end{array}$ & $\begin{array}{r}.05 \\
.25 \\
.04\end{array}$ & $.10 \%$ & $\begin{array}{l}17 \\
.12 \\
.05\end{array}$ & $\begin{array}{l}.07 \\
.02 \\
.01\end{array}$ \\
\hline $\begin{array}{l}\text { GFACT } \\
\text { FFACT }\end{array}$ & $3.5 \%$ & $\begin{array}{l}3.83 \\
3.02\end{array}$ & $\frac{2}{3} \cdot 44$ & $7+34$ & $298+2 \frac{1}{7}$ & $\vec{J}+85$ \\
\hline
\end{tabular}

IATA FOF FILE: H5101

\begin{tabular}{|c|c|c|c|c|c|c|}
\hline Fun & $\begin{array}{r}41+5 \\
297.5\end{array}$ & 25.0 & & $\begin{array}{l}\text { VEL } \\
32.5\end{array}$ & & \\
\hline COEMF : & .258 & $\begin{array}{l}c .5 Y \\
.01\end{array}$ & $\begin{array}{l}\mathrm{CF} \\
.10\end{array}$ & c.s. & .01 & $\begin{array}{c}c y^{2} \\
.02\end{array}$ \\
\hline $\begin{array}{l}\text { MAYY } \\
\text { MIN } \\
\text { KMS }\end{array}$ & $\begin{array}{l}1.19 \\
: \frac{27}{15}\end{array}$ & $\begin{array}{r}.00 \\
.02 \\
.02\end{array}$ & $\begin{array}{l}20 \\
.02 \\
.04\end{array}$ & $\begin{array}{r}10 \\
.07 \\
.03\end{array}$ & $\begin{array}{l}+A z \\
: 32 \\
: 10\end{array}$ & .05 \\
\hline $\begin{array}{l}\text { GTACT } \\
\text { FFACT }\end{array}$ & 4.23 & $\frac{3}{3} .72 \frac{2}{80}$ & 2.82 & 5.05 & $55+30$ & $3 \cdot 30$ \\
\hline
\end{tabular}

IIATA FOF FILE: HS101

\begin{tabular}{|c|c|c|c|c|c|c|}
\hline Fus: & $\begin{array}{l}\text { WINI } \\
270.0\end{array}$ & 25.0 & & $\begin{array}{l}\text { VEL } \\
-12.3\end{array}$ & & \\
\hline COMF : & .20 & $\begin{aligned} c F y \\
-.01\end{aligned}$ & $\begin{array}{c}\operatorname{cF}_{0}^{2} \\
.02\end{array}$ & $\begin{array}{l}\text { C.MY } \\
.00\end{array}$ & Cry & $\begin{array}{c}\operatorname{crs} \\
\operatorname{0o}\end{array}$ \\
\hline $\begin{array}{l}\text { MAY } \\
\text { MIH } \\
\text { FMS }\end{array}$ & $\cdots: \frac{29}{3}$ & $\begin{array}{r}.03 \\
.05 \\
+01\end{array}$ & $\begin{array}{l}.22 \\
003 \\
04\end{array}$ & .07 & $\frac{27}{20}$ & $\begin{array}{l}.06 \\
.02 \\
.01\end{array}$ \\
\hline $\begin{array}{l}\text { EFACT } \\
\text { PFACT }\end{array}$ & $\begin{array}{l}4.30 \\
5.15\end{array}$ & 7.60 & $\begin{array}{l}3.50 \\
4.51\end{array}$ & $\begin{array}{r}22.40 \\
3 \cdot 69\end{array}$ & $11 \cdot 55$ & 3.47 \\
\hline
\end{tabular}




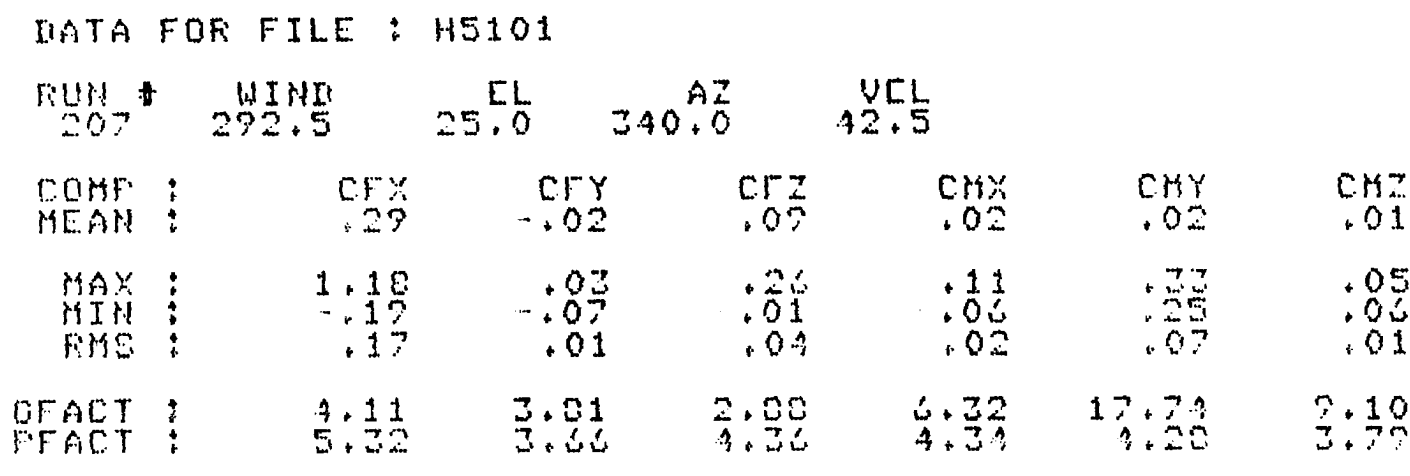

MATA FOF FILE: H5101

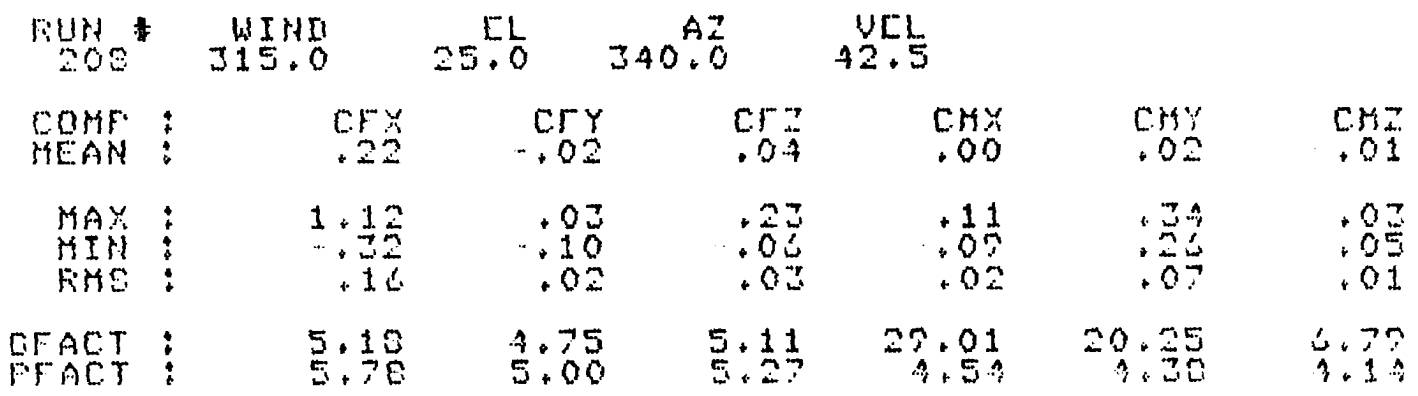

IATA FOF FILE: HSIOI

\begin{tabular}{|c|c|c|c|c|c|c|}
\hline $\begin{array}{l}\text { Furs } \\
21.1\end{array}$ & $\begin{aligned} W I+I \\
337.5\end{aligned}$ & 5.0 & & $\begin{array}{r}\text { UEL } \\
42.7\end{array}$ & & \\
\hline COMF & $\begin{array}{c}\text { C. } \\
.12\end{array}$ & $\begin{array}{r}C F Y \\
-.01\end{array}$ & .05 & $\begin{array}{l}\text { c.ry } \\
.02\end{array}$ & $\begin{array}{l}C H Y \\
.02\end{array}$ & $\begin{array}{l}c .42 \\
.02\end{array}$ \\
\hline 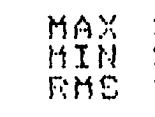 & $1+1 \frac{1}{7}$ & $\begin{array}{r}.03 \\
.08 \\
.01\end{array}$ & $\begin{array}{l}2 \\
.02 \\
.03 \\
.03\end{array}$ & $.1 \frac{1}{0} .02$ & . & $.0 \%$ \\
\hline $\begin{array}{l}\text { QFACT } \\
\text { FACT }\end{array}$ & $\frac{9}{7} \cdot \frac{99}{37}$ & $\begin{array}{l}7.77 \\
3: 49\end{array}$ & $\begin{array}{l}7+51 \\
6+01\end{array}$ & 5.30 & 活。岂总 & $\begin{array}{r}3.25 \\
90\end{array}$ \\
\hline
\end{tabular}

IIATA FOF: FILE: H5101

\begin{tabular}{|c|c|c|c|c|c|c|}
\hline Fill & WINII & $=5 \%$ & 0.2 & $\begin{array}{r}Y C L \\
32.9\end{array}$ & & \\
\hline $\begin{array}{l}\text { COMF: } \\
\text { MEAN }\end{array}$ & $.0 \%$ & $\begin{array}{l}\cos Y \\
.001\end{array}$ & .01 & $\begin{array}{l}C M E \\
.02\end{array}$ & $\begin{array}{l}\text { cry } \\
.01\end{array}$ & $\begin{array}{l}\mathrm{Cm} Z \\
.00\end{array}$ \\
\hline $\begin{array}{l}\text { MAX } \\
\text { KINS } \\
\text { KMS }\end{array}$ & . & $\begin{array}{r}.05 \\
-.07 \\
.02\end{array}$ & .10 & $\begin{array}{l}.12 \\
.05 \\
.03\end{array}$ & $\begin{array}{l}75 \\
135 \\
10\end{array}$ & $\begin{array}{l}.06 \\
.05 \\
.01\end{array}$ \\
\hline $\begin{array}{l}\text { FACT } \\
\text { FACT }\end{array}$ & $2 \frac{2}{3} \cdot 37$ & 5.72 & $2 \frac{3}{3} \cdot 20$ & 它.48 & $21+50$ & 72.77 \\
\hline
\end{tabular}




\begin{tabular}{|c|c|c|c|c|c|c|}
\hline Fivit & $\begin{array}{r}\text { HIN } \\
315.0\end{array}$ & Fo. & $320 .{ }^{A 2}$ & $\begin{array}{l}45.1 \\
12.3\end{array}$ & & \\
\hline COYAF & Cry & $\begin{array}{l}\text { CFy } \\
.00\end{array}$ & $\begin{array}{cc}\cos \\
.05\end{array}$ & $\begin{array}{l}\text { crys } \\
\text { oo }\end{array}$ & $\begin{array}{c}\text { cry } \\
.02\end{array}$ & $\begin{array}{l}\cos 2 \\
.00\end{array}$ \\
\hline $\begin{array}{l}\text { MAO } \\
\text { FIHS } \\
\text { FMS }\end{array}$ & $\begin{array}{r}.10 \\
.08 \\
.02\end{array}$ & $\begin{array}{r}.04 \\
-.06 \\
.01\end{array}$ & $\begin{array}{r}.23 \\
.30 \\
.06\end{array}$ & $\begin{array}{l}.07 \\
.03 \\
.02\end{array}$ & $\begin{array}{r}.05 \\
.05 \\
.02\end{array}$ & $\begin{array}{r}92 \\
.03 \\
01\end{array}$ \\
\hline $\begin{array}{l}\text { QFACT } \\
\text { FACT }\end{array}$ & 3.92 & $2 \frac{2}{4} \cdot \frac{2}{3} 9$ & $\frac{8}{2}+\frac{72}{7}$ & $1 \stackrel{1}{3} \cdot 1 ?$ & $5: \frac{1}{2}$ & $\begin{array}{l}3.10 \\
3.40\end{array}$ \\
\hline
\end{tabular}

IMATA FOF: FILE : HS101

\begin{tabular}{|c|c|c|c|c|c|c|}
\hline Fun & $\begin{array}{r}45 \% \\
3 \pm 5.6\end{array}$ & 0.5 & & $42+3$ & & \\
\hline $\begin{array}{l}\text { COHF: } \\
\text { HEAHE }\end{array}$ & $\frac{C F}{15}$ & $\begin{array}{r}\text { cfr } \\
-.00\end{array}$ & .01 & $\begin{array}{c}\text { csis } \\
.01\end{array}$ & $\begin{array}{l}\operatorname{cs}\{Y \\
.01\end{array}$ & $\begin{array}{l}\operatorname{crs} \\
.0 \mathrm{H}\end{array}$ \\
\hline $\begin{array}{l}\text { MAX } \\
\text { HIMU } \\
\text { FHS }\end{array}$ & $\begin{array}{r}94 \\
* \frac{2}{1}\end{array}$ & $\begin{array}{r}.00 \\
-.06 \\
.02\end{array}$ & $\begin{array}{r}.04 \\
.04 \\
.01\end{array}$ & .11 &.$\frac{25}{17}$ & $\begin{array}{l}.09 \\
.09 \\
.01\end{array}$ \\
\hline $\begin{array}{l}\text { GFACT } \\
\text { WFAT }\end{array}$ & $\begin{array}{l}5.36 \\
5.12\end{array}$ & $\begin{array}{r}57.55 \\
3.00\end{array}$ & $\frac{4.75}{4.48}$ & $19 \cdot 12$ & $=2.39$ & 2.15 \\
\hline
\end{tabular}

IATA FOF FILE: H5101

\begin{tabular}{|c|c|c|c|c|c|c|}
\hline Fur & 24505 & 20.0 & & $\begin{array}{r}\text { VEL } \\
42.8\end{array}$ & & \\
\hline $\begin{array}{l}\text { COHF : } \\
\text { MEAH : }\end{array}$ & $\begin{array}{r}C F \\
-.05\end{array}$ & $\begin{array}{l}\mathrm{CFY} \\
.03\end{array}$ & $\begin{array}{r}C F^{2} \\
.03\end{array}$ & $\begin{array}{l}\text { CMS } \\
.02\end{array}$ & ${ }_{0.02}^{\cos }$ & $c+c=$ \\
\hline $\begin{array}{l}\text { MAE } \\
\text { MIHA } \\
\text { FMS }\end{array}$ & $\begin{array}{r}49 \\
-.59 \\
: 14\end{array}$ & $\begin{array}{r}14 \\
.03 \\
.04\end{array}$ & $\begin{array}{l}.07 \\
.17 \\
.03\end{array}$ & $\begin{array}{l}13 \\
.05 \\
.09\end{array}$ & $\begin{array}{r}15 \\
.20 \\
.05\end{array}$ & $.0 \%$ \\
\hline $\begin{array}{l}\text { QFACT } \\
\text { FACT }\end{array}$ & $\begin{array}{r}10.34 \\
3.91\end{array}$ & $\begin{array}{l}5.04 \\
3.10\end{array}$ & $\begin{array}{l}5.25 \\
4.42\end{array}$ & 5.45 & 10.03 & 3.65 \\
\hline
\end{tabular}

IATA FOF FILE : HS101

\begin{tabular}{|c|c|c|c|c|c|c|}
\hline 5um & 250.0 & 20.0 & 0.0 & 42.3 & & \\
\hline $\begin{array}{l}\text { COMF : } \\
\text { MEAN : }\end{array}$ & .03 & $\begin{array}{l}\text { CFY } \\
.01\end{array}$ & .01 & $\begin{array}{l}\text { cms } \\
0 \%\end{array}$ & $\begin{array}{l}\text { Cify } \\
.01\end{array}$ & $\begin{array}{l}c .3 s \\
0=\end{array}$ \\
\hline $\begin{array}{l}\text { MAX: } \\
\text { MIYH } \\
\text { BHS }\end{array}$ & $\begin{array}{r}.45 \\
\cdots+48 \\
: 11\end{array}$ & $\begin{array}{r}+12 \\
\cdots+11 \\
+03\end{array}$ & $\begin{array}{l}12 \\
.05 \\
.05\end{array}$ & $\begin{array}{l}.02 \\
.12 \\
.05\end{array}$ & $\begin{array}{l}1.5 \\
.14 \\
.09\end{array}$ & $\begin{array}{l}.05 \\
.05\end{array}$ \\
\hline $\begin{array}{l}\text { FACT } \\
\text { FACT }\end{array}$ & $19 \cdot 39$ & $1 \frac{2}{7} \cdot \frac{1}{8} \%$ & $1 \frac{1}{4} .070$ & $\begin{array}{l}3.4 \% \\
3.10\end{array}$ & 29.96 & $\begin{array}{l}7 \\
3\end{array}$ \\
\hline
\end{tabular}




\begin{tabular}{|c|c|c|c|c|c|c|}
\hline Fust & $\begin{array}{r}\text { WIHI } \\
292.5\end{array}$ & 20.5 & 290.0 & VELL & & \\
\hline $\begin{array}{l}\text { COMF } \\
\text { MEAN }\end{array}$ & $\begin{array}{l}\text { CFY } \\
.13\end{array}$ & $\begin{array}{l}C F Y \\
. .01\end{array}$ & .04 & $\begin{array}{l}\text { CMx } \\
.05\end{array}$ & $\begin{array}{l}c .4 Y \\
.02\end{array}$ & $\begin{array}{l}\operatorname{cm} z \\
.02\end{array}$ \\
\hline $\begin{array}{l}\text { MAX } \\
\text { MIN } \\
\text { FMS }\end{array}$ & $\begin{array}{r}+87 \\
+36\end{array}$ & $\begin{array}{r}+10 \\
-14 \\
+04\end{array}$ & $\begin{array}{r}17 \\
.02 \\
.03\end{array}$ & $\begin{array}{l}.07 \\
.10 \\
.04\end{array}$ & $\begin{array}{l}27 \\
.12 \\
.05\end{array}$ & $\begin{array}{r}.05 \\
.05 \\
.01\end{array}$ \\
\hline $\begin{array}{l}\text { GFACT } \\
\text { FPACT }\end{array}$ & $\begin{array}{l}6.92 \\
4.71\end{array}$ & $\begin{array}{r}17.04 \\
2: 01\end{array}$ & 3.79 & $\begin{array}{l}3.53 \\
3.61\end{array}$ & 10.23 & $\frac{5}{4} \div 21$ \\
\hline
\end{tabular}

IMTA FOF FILE: HS101

\begin{tabular}{|c|c|c|c|c|c|c|}
\hline KบNㅗㄴ & 315.0 & 20.0 & & $\begin{array}{r}\text { VEL } \\
43.4\end{array}$ & & \\
\hline $\begin{array}{l}\text { CONF } \\
\text { HEAN }\end{array}$ & $\begin{array}{l}c .5 \\
.11\end{array}$ & $\begin{array}{l}\text { C. } \\
.01\end{array}$ & $\begin{array}{l}C 52 \\
.04\end{array}$ & $\begin{array}{l}\text { CHY } \\
.02\end{array}$ & $\begin{array}{l}\text { CHY } \\
.00\end{array}$ & .01 \\
\hline $\begin{array}{l}\text { MAX } \\
\text { HIN } \\
\text { FHE }\end{array}$ & $\begin{array}{r}.53 \\
.17 \\
.10\end{array}$ & $\begin{array}{r}.06 \\
-.05 \\
.02\end{array}$ & $\begin{array}{l}14 \\
.03 \\
.02\end{array}$ & $\begin{array}{l}.09 \\
.09 \\
.02\end{array}$ & $\begin{array}{l}15 \\
115 \\
.07\end{array}$ & $\begin{array}{l}.92 \\
.02 \\
.01\end{array}$ \\
\hline $\begin{array}{l}\text { QFACT } \\
\text { FFACT }\end{array}$ & $5 \cdot 15$ & $\begin{array}{r}10.65 \\
7.10\end{array}$ & 3.33 & $\frac{6}{3} .02$ & 3.05 & $\frac{5}{4}+\frac{1}{7} ?$ \\
\hline
\end{tabular}

IATA FOF: FILE: HS101

\begin{tabular}{|c|c|c|c|c|c|c|}
\hline rust & $\begin{array}{r}\text { WINI } \\
337.5\end{array}$ & $\begin{array}{r}E L \\
20.0\end{array}$ & $\begin{array}{l}A Z \\
.0\end{array}$ & $\begin{array}{r}U[1 \\
43 \cdot 2\end{array}$ & & \\
\hline $\begin{array}{l}\text { CQMF: } \\
\text { MEAM : }\end{array}$ & $\begin{array}{l}6 \% \\
+20\end{array}$ & $\begin{array}{r}\text { CFY } \\
. .02\end{array}$ & $\begin{array}{l}\operatorname{ctz} \\
.05\end{array}$ & $\begin{array}{l}C M Y \\
.00\end{array}$ & $\begin{array}{l}\text { C.sy } \\
.04\end{array}$ & $\begin{array}{l}\text { CHz } \\
+02\end{array}$ \\
\hline $\begin{array}{l}\text { MAX } \\
\text { MIIH } \\
\text { rMS }\end{array}$ & $\begin{array}{r}.80 \\
-113 \\
+12\end{array}$ & $\begin{array}{r}.05 \\
.11 \\
.02\end{array}$ & .13 & $\begin{array}{r}10 \\
.08 \\
.02\end{array}$ & $\begin{array}{r}22 \\
.10 \\
104\end{array}$ & $\begin{array}{r}.07 \\
.02 \\
.01\end{array}$ \\
\hline $\begin{array}{l}\text { GFACT } \\
\text { FFACT }\end{array}$ & $\begin{array}{l}3.97 \\
4.99\end{array}$ & $\begin{array}{l}7.34 \\
3.95\end{array}$ & $\frac{2}{4} \cdot \frac{27}{12}$ & $\begin{array}{r}839.58 \\
3.91\end{array}$ & $5+20$ & 3.25 \\
\hline
\end{tabular}

DATA FOF FILE: $H 5101$

\begin{tabular}{|c|c|c|c|c|c|c|}
\hline $\begin{array}{l}\text { Fus } \\
228\end{array}$ & $\begin{array}{c}\text { WI } 15 \pi \\
0.0\end{array}$ & 20.0 & .0 & VEL. & & \\
\hline COYF: & $\begin{array}{l}65 \\
.33\end{array}$ & $\begin{array}{l}C F Y \\
.02\end{array}$ & $\begin{array}{c}c 5 \\
+1\end{array}$ & $\begin{array}{l}\text { cry. } \\
.05\end{array}$ & $\begin{array}{c}\text { CHY } \\
.07\end{array}$ & $\begin{array}{l}\text { Cys } \\
.03\end{array}$ \\
\hline $\begin{array}{l}\text { MAX } \\
\text { MIYS } \\
\text { FMS }\end{array}$ & $\begin{array}{l}1.3 \\
\cdots \\
\cdots\end{array}$ & $\begin{array}{r}.10 \\
-15 \\
.04\end{array}$ & $\begin{array}{r}.30 \\
.02 \\
.04\end{array}$ & $\begin{array}{l}17 \\
.07 \\
.07\end{array}$ & .72 & $\begin{array}{l}.09 \\
.09\end{array}$ \\
\hline $\begin{array}{l}\text { GFACT } \\
F F A C T\end{array}$ & $\frac{4}{5}+\frac{22}{2}$ & $\frac{8}{2} .96$ & $2+2 \frac{1}{4}$ & $\frac{3}{3}+\frac{24}{7}$ & 5.02 & $\frac{3}{4} \cdot 48$ \\
\hline
\end{tabular}




\begin{tabular}{|c|c|c|c|c|c|c|}
\hline rust & $\begin{array}{r}\text { WIHX } \\
0.0\end{array}$ & 10.0 & $=25.0$ & $\begin{array}{l}\text { YEL } \\
\text { A3.4 }\end{array}$ & & \\
\hline COMAY & $0 F$ & $\begin{array}{ll}\mathrm{CF} \\
.02\end{array}$ & $\begin{array}{c}C 5 \\
.04\end{array}$ & $\begin{array}{l}6 \% Y \\
.05\end{array}$ & $\begin{array}{l}\text { crys } \\
.05\end{array}$ & 01 \\
\hline 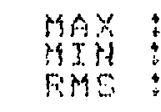 & $\begin{array}{c}1 \cdot \frac{1}{2} \\
\cdot \frac{1}{2}\end{array}$ & $\begin{array}{r}.07 \\
.03 \\
.01\end{array}$ & $\begin{array}{r}+10 \\
+02 \\
.01\end{array}$ & $\begin{array}{l}.07 \\
.05 \\
.01\end{array}$ & .25 & 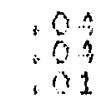 \\
\hline $\begin{array}{l}\text { QFACT } \\
\text { FACT }\end{array}$ & $3 \cdot 31$ & $\begin{array}{l}4.40 \\
4.36\end{array}$ & $\frac{2}{3} \cdot \frac{29}{7}$ & 2.72 & $5 \cdot 11$ & $7+39$ \\
\hline
\end{tabular}

IATA FOF FILE: H5101

\begin{tabular}{|c|c|c|c|c|c|c|}
\hline $\operatorname{mos}_{23}$ & 337.5 & 10.0 & $A Z$ & VEL & & \\
\hline $\begin{array}{l}\text { COSF } \\
\text { MEAS }\end{array}$ & $\begin{array}{l}\text { CF. } \\
13\end{array}$ & $\begin{array}{l}C F Y \\
.00\end{array}$ & $\begin{array}{l}C F Z \\
\therefore 0\end{array}$ & $\begin{array}{c}C H \\
.01\end{array}$ & $\begin{array}{c}C H Y \\
.03\end{array}$ & $\begin{array}{c}\cos \\
.02\end{array}$ \\
\hline $\begin{array}{l}\text { MAY } \\
\text { MISE } \\
\text { FMS }\end{array}$ & $\cdot \frac{82}{12}$ & $\begin{array}{r}.03 \\
-.03 \\
.01\end{array}$ & $\begin{array}{l}.04 \\
.04 \\
.01\end{array}$ & .09 & $: 215$ & .07 \\
\hline $\begin{array}{l}\text { QFACT } \\
\text { FFACT }\end{array}$ & $\begin{array}{l}4.47 \\
4: 78\end{array}$ & $\begin{array}{l}3.99 \\
5.9\end{array}$ & $72 \cdot 40$ & $10 \cdot 10$ & $\begin{array}{r}7.31 \\
3.65\end{array}$ & 3.50 \\
\hline
\end{tabular}

MATA FOF FILE : HS101

\begin{tabular}{|c|c|c|c|c|c|c|}
\hline 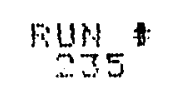 & $\begin{array}{r}11451 \\
315.0\end{array}$ & 10.5 & $A 2$ & $\begin{array}{l}\text { VEL } \\
71.3\end{array}$ & & \\
\hline COMF : & $\begin{array}{l}\mathrm{CF} \\
.10\end{array}$ & $\begin{array}{l}C F \\
.00\end{array}$ & $.0 \%$ & $\begin{array}{l}\mathrm{Cry} \\
.01\end{array}$ & $\begin{array}{l}\text { CHy } \\
.02\end{array}$ & $\begin{array}{l}\mathrm{CH}_{0}^{-\mathrm{x}} \\
.00\end{array}$ \\
\hline $\begin{array}{l}\text { MAY } \\
\text { HIM } \\
\text { FMS }\end{array}$ & .79 & $\begin{array}{r}.03 \\
.02 \\
.01\end{array}$ & $\begin{array}{l}.05 \\
.04 \\
.01\end{array}$ & .04 & $\begin{array}{l}21 \\
.15 \\
.04\end{array}$ & $\begin{array}{l}.06 \\
.04 \\
.02\end{array}$ \\
\hline GFACT & $\begin{array}{l}3.06 \\
5.28\end{array}$ & $\begin{array}{r}78: 49 \\
4.79\end{array}$ & $\begin{array}{r}10.08 \\
2.01\end{array}$ & $\frac{4}{3}: \frac{7}{6}$ & 0.50 & $1 \frac{9}{3} \cdot 32$ \\
\hline
\end{tabular}

IIATA FOF FILE: H5101

\begin{tabular}{|c|c|c|c|c|c|c|}
\hline Fus & WIma & 10.0 & & $\begin{array}{l}\text { VEL } \\
73.1\end{array}$ & & \\
\hline COMF : & $\begin{array}{c}t .5 X \\
+03\end{array}$ & $\begin{array}{l}\text { CFY } \\
.02\end{array}$ & $\begin{array}{c}C^{5} \\
-0 z\end{array}$ & $\begin{array}{l}\text { crix } \\
.01\end{array}$ & $\begin{array}{l}\text { CHY } \\
.02\end{array}$ & $\begin{array}{l}\cos z \\
.04\end{array}$ \\
\hline $\begin{array}{l}\text { MAY } \\
\text { MIYS } \\
\text { Rise }\end{array}$ & $\begin{array}{r}1.00 \\
-.75 \\
.10\end{array}$ & $\begin{array}{r}.09 \\
.09 \\
.02\end{array}$ & $\begin{array}{r}.02 \\
.11 \\
.02\end{array}$ & $\begin{array}{l}.00 \\
.05 \\
.02\end{array}$ & $\frac{29}{26}$ & $\begin{array}{l}.0 \hat{1} \\
.11 \\
02\end{array}$ \\
\hline $\begin{array}{l}\text { FACT } \\
\text { FACT }\end{array}$ & $33: 91$ & $\begin{array}{l}5.0 S \\
4.20\end{array}$ & 3.51 & 4.79 & $1 \underset{3}{14}+92$ & $=57$ \\
\hline
\end{tabular}




\begin{tabular}{|c|c|c|c|c|c|c|}
\hline 祡 & 270.0 & 10.0 & 235.0 & $42+9$ & & \\
\hline $\begin{array}{l}\text { COMS } \\
\text { MEAN }\end{array}$ & $\begin{array}{l}c 5.5 \\
-10\end{array}$ & $\begin{array}{l}\mathrm{CFY} \\
.01\end{array}$ & cry & $\begin{array}{l}\mathrm{CH} \\
.03\end{array}$ & ${ }_{02}^{\operatorname{crs} Y}$ & $\begin{array}{c}\text { Criz } \\
.01\end{array}$ \\
\hline $\begin{array}{l}\text { MAY } \\
\text { MIM }\end{array}$ & $\begin{array}{r}.48 \\
-.05 \\
.15\end{array}$ & $\begin{array}{r}.06 \\
-.05 \\
.01\end{array}$ & $\begin{array}{r}.02 \\
.02 \\
.02\end{array}$ & $\begin{array}{r}.09 \\
.09 \\
.02\end{array}$ & $=\frac{1}{2}$ & $\begin{array}{r}.05 \\
.05 \\
.05\end{array}$ \\
\hline $\begin{array}{l}\text { OFACT } \\
\text { FFACT }\end{array}$ & $3 \cdot \frac{1}{5}$ & $\frac{?}{3.53}$ & $5 \cdot \frac{30}{3}$ & $3 \cdot 41$ & $14+1 \frac{2}{4}$ & $3 \cdot 43$ \\
\hline
\end{tabular}

IATA FOF FILE: H5101

\begin{tabular}{|c|c|c|c|c|c|c|}
\hline 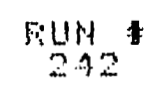 & $\begin{array}{r}W I+I I \\
247+5\end{array}$ & $\begin{array}{c}E L \\
10.0\end{array}$ & $\begin{array}{l}A z \\
.0\end{array}$ & $\begin{array}{r}\text { VEL } \\
33.0\end{array}$ & & \\
\hline $\begin{array}{l}\text { COMF : } \\
\text { MEAN : }\end{array}$ & $\begin{array}{c}c 5 x \\
-20\end{array}$ & $\begin{array}{l}\text { C.TY } \\
.02\end{array}$ & .05 & .02 & $\begin{array}{l}\text { CHY } \\
.04\end{array}$ & $\begin{array}{l}C M Z \\
.01\end{array}$ \\
\hline $\begin{array}{l}\text { MAX } \\
\text { HIM } \\
\text { Fus }\end{array}$ & $\begin{array}{r}47 \\
.79\end{array}$ & $\begin{array}{r}.00 \\
.03 \\
.01\end{array}$ & $\begin{array}{r}.0 A \\
.10 \\
.02\end{array}$ & $\begin{array}{l}.08 \\
.04 \\
.02\end{array}$ & .10 & $\begin{array}{l}.03 \\
.03 \\
.01\end{array}$ \\
\hline $\begin{array}{l}\text { GFACT } \\
\text { FFACT }\end{array}$ & $\begin{array}{l}5.97 \\
4.52\end{array}$ & $\begin{array}{r}4.00 \\
4.54\end{array}$ & $\begin{array}{l}3.56 \\
3.56\end{array}$ & 3.50 & $3+\frac{2}{6} \cdot 1$ & 3.13 \\
\hline
\end{tabular}

IATA FOF FILE : H5101

\begin{tabular}{|c|c|c|c|c|c|c|}
\hline$\underset{213}{\text { Fust }}$ & $\begin{array}{r}\text { WIHD } \\
247.5\end{array}$ & 10.0 & & $\begin{array}{l}\text { VEL } \\
+2.2\end{array}$ & & \\
\hline $\begin{array}{l}\text { COMF } \\
\text { MEAN }\end{array}$ & $.0 \%$ & .05 & $\begin{array}{c}\mathrm{Cr}^{2} \\
.02\end{array}$ & $\begin{array}{l}c .9 x \\
.04\end{array}$ & $\begin{array}{l}\text { CMY } \\
: 01\end{array}$ & $\begin{array}{l}C \mu z \\
.02\end{array}$ \\
\hline $\begin{array}{l}\text { MAx } \\
\text { Mins }\end{array}$ & $\begin{array}{r}+55 \\
-15 \\
.15\end{array}$ & $\begin{array}{r}15 \\
.05 \\
.04\end{array}$ & $\begin{array}{r}.07 \\
: 02 \\
.02\end{array}$ & $\begin{array}{l}+15 \\
+15 \\
+0 \%\end{array}$ & 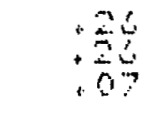 & $\begin{array}{r}.05 \\
.05 \\
.01\end{array}$ \\
\hline $\begin{array}{l}\text { FACT } \\
\text { FACT }\end{array}$ & $7+75$ & 5.57 & $\begin{array}{l}3.10 \\
2+50\end{array}$ & $\begin{array}{l}1.49 \\
3.08\end{array}$ & 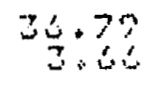 & $3: 21$ \\
\hline
\end{tabular}

IATA FOF FILE: H5101

\begin{tabular}{|c|c|c|c|c|c|c|}
\hline 닌 & $\begin{array}{r}W I N I \\
270+0\end{array}$ & 10.5 & & $4 \frac{45}{5}$ & & \\
\hline $\begin{array}{l}\text { COMF } \\
\text { MEAH : }\end{array}$ & $\frac{c 5}{.32}$ & $\begin{array}{l}\text { Cry } \\
.00\end{array}$ & Cr & $\begin{array}{l}\mathrm{CH} \\
.02\end{array}$ & $\begin{array}{l}\operatorname{cry} Y \\
.05\end{array}$ & $\begin{array}{l}\text { CHI } \\
.05\end{array}$ \\
\hline $\begin{array}{l}\text { MAX } \\
\text { MIMU } \\
\text { RMS }\end{array}$ & $\begin{array}{r}1+30 \\
-31 \\
-21\end{array}$ & $\begin{array}{r}+14 \\
.11 \\
.04\end{array}$ & $\begin{array}{l}.07 \\
.04 \\
.02\end{array}$ & $\begin{array}{l}.21 \\
.15 \\
.05\end{array}$ & $\begin{array}{r}79 \\
.53 \\
.02\end{array}$ & $\begin{array}{l}1 \\
.01 \\
.01 \\
0\end{array}$ \\
\hline $\begin{array}{l}\text { QFACT } \\
\text { FFACT }\end{array}$ & $\begin{array}{l}4.97 \\
-3.97\end{array}$ & $3 \frac{1}{3} \cdot 5 \frac{5}{50}$ & $\begin{array}{l}4.08 \\
3.32\end{array}$ & 11.49 & 7.30 & $\frac{1}{4}+7 \overrightarrow{3}$ \\
\hline
\end{tabular}




\begin{tabular}{|c|c|c|c|c|c|c|}
\hline Fum & 202.5 & 10.5 & $=0 . A Z$ & $\frac{V E L}{12.3}$ & & \\
\hline COMF: & $\frac{C 5}{2}$ & $\begin{array}{l}\mathrm{Cr} Y \\
.00\end{array}$ & .01 & $\begin{array}{l}\text { C.S. } \\
.02\end{array}$ & $\begin{array}{l}\text { cryy } \\
.02\end{array}$ & $\begin{array}{l}\operatorname{crz} \\
.02\end{array}$ \\
\hline $\begin{array}{l}\text { MAY } \\
\text { MIY } \\
\text { FMS }\end{array}$ & $\begin{array}{l}92 \\
: 14 \\
: 14\end{array}$ & $\begin{array}{r}.11 \\
-.03 \\
.02\end{array}$ & .05 & $\begin{array}{l}12 \\
.02 \\
.05\end{array}$ & $\begin{array}{l}.27 \\
.105\end{array}$ & .02 \\
\hline $\begin{array}{l}\text { GFACT } \\
\text { FFACT }\end{array}$ & 4. & 112.18 & $\begin{array}{l}4.37 \\
4.09\end{array}$ & $\frac{7}{3}+\frac{9}{3}$ & 3.98 & $3 \cdot \frac{3}{3} \cdot \frac{3}{6}$ \\
\hline IATA $F$ & OF FILE : & $H 5101$ & & & & \\
\hline $\begin{array}{l}\text { mutt } \\
250\end{array}$ & $\begin{array}{r}\text { WIIn } \\
315.0\end{array}$ & ${ }_{10.0}^{E L}$ & $\begin{array}{r}A z \\
320.0\end{array}$ & $\begin{array}{r}\text { UᄃL. } \\
42.5\end{array}$ & & \\
\hline $\begin{array}{l}\text { COMF : } \\
\text { MEAH : }\end{array}$ & $\begin{array}{c}C F \\
.13\end{array}$ & $\begin{array}{r}\operatorname{cry} \\
-.01\end{array}$ & .01 & $\begin{array}{l}\text { Crus } \\
+01\end{array}$ & c.ry & $\begin{array}{c}\cos \\
.01\end{array}$ \\
\hline $\begin{array}{l}\text { MAY } \\
\text { MIM! } \\
\text { FMS }\end{array}$ & .72 & $\begin{array}{r}.08 \\
-.08 \\
.02\end{array}$ & $\begin{array}{r}.05 \\
-.04 \\
.02\end{array}$ & $\begin{array}{l}10 \\
+10 \\
+02\end{array}$ & .21 & $\begin{array}{r}.05 \\
+03 \\
.01\end{array}$ \\
\hline FFACT & 5.26 & 군워 & $2 \cdot \frac{51}{2}$ & 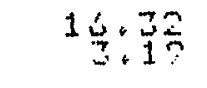 & 9.99 & \begin{tabular}{l}
4.45 \\
\hdashline .5 .5 \\
4.5
\end{tabular} \\
\hline MATA F & OF FILE : & 45101 & & & & \\
\hline Fus! & $37 \frac{4}{3}+5$ & ${ }_{10}^{E} \frac{E}{0}$ & 320.0 & $\begin{array}{r}U[L \\
42+2\end{array}$ & & \\
\hline $\begin{array}{l}\text { COMF: } \\
\text { SEAY }\end{array}$ & $\begin{array}{l}65 \% \\
20\end{array}$ & $\begin{array}{l}\text { CFY } \\
.00\end{array}$ & .01 & $\begin{array}{l}\text { CMS } \\
.04\end{array}$ & Crys & $\begin{array}{l}\operatorname{crs} \\
+02 \\
\text { on }\end{array}$ \\
\hline $\begin{array}{l}\text { MAY : } \\
\text { MIIS: }\end{array}$ & $\begin{array}{l}1.97 \\
: 16\end{array}$ & $\begin{array}{l}.05 \\
.09 \\
.03\end{array}$ & $\begin{array}{r}.07 \\
.04 \\
.02\end{array}$ & $\begin{array}{l}10 \\
.10 \\
.04\end{array}$ & $.2 \frac{2}{21}$ & $\begin{array}{l}.91 \\
.03 \\
.01\end{array}$ \\
\hline CFACT & 5.33 & 175.38 & $\begin{array}{l}7.73 \\
2: 32\end{array}$ & $\frac{A}{2} \cdot \frac{1}{5} \cdot 1$ & $\begin{array}{r}13+37 \\
3+4\end{array}$ & 3.70 \\
\hline
\end{tabular}

IATA FOF FILE : H5101

\begin{tabular}{|c|c|c|c|c|c|c|}
\hline $\operatorname{mun}_{-1}$ & $\begin{array}{l}W \| \mathrm{I} \\
0: 0\end{array}$ & 1.0 .5 & & $\begin{array}{l}\text { UCL. } \\
42.5\end{array}$ & & \\
\hline COMF : & $\begin{array}{c}C F \\
.12\end{array}$ & $\begin{array}{r}\text { cry } \\
-.02\end{array}$ & .05 & $\begin{array}{l}\operatorname{css} x \\
.00\end{array}$ & $\begin{array}{l}\text { CMY } \\
.01\end{array}$ & $\begin{array}{l}\cos \\
+00\end{array}$ \\
\hline $\begin{array}{l}\text { MAX } \\
\text { MIM } \\
\text { SMS }\end{array}$ & $\cdot \frac{92}{5}$ & $\begin{array}{r}.09 \\
.02 \\
.05 \\
.0\end{array}$ & $\begin{array}{l}.02 \\
.05 \\
.01\end{array}$ & $\begin{array}{l}15 \\
+15 \\
+04\end{array}$ & $\begin{array}{r}.20 \\
.30 \\
.06\end{array}$ & $\begin{array}{r}95 \\
.95 \\
.03\end{array}$ \\
\hline FACT & $\frac{8}{5} \cdot \frac{1}{80}$ & 6.73 & $\frac{2}{2} \cdot 53$ & $92 \cdot 29$ & $1:=20$ & $\begin{array}{r}2.53 \\
2.63\end{array}$ \\
\hline
\end{tabular}




\begin{tabular}{|c|c|c|c|c|c|c|}
\hline Cinse & Rus it & WD & $\mathrm{EL}$ & $\mathrm{AZ}$ & Day & Time \\
\hline \multirow[t]{6}{*}{ Smr AM } & 279 & 0 & 25 & 340 & 172 & $8 \mathrm{AM}$ \\
\hline & 281 & 337.5 & 25 & 340 & 172 & $8 \mathrm{AM}$ \\
\hline & 282 & 315 & 25 & 340 & 172 & $8 \mathrm{ANI}$ \\
\hline & 283 & 292.5 & 25 & 340 & 172 & $8 \mathrm{AM}$ \\
\hline & 284 & 270 & 25 & 340 & 172 & $8 \mathrm{AM}$ \\
\hline & 285 & 247.5 & 25 & 340 & 172 & $8 \mathrm{AM}$ \\
\hline \multirow[t]{6}{*}{ Simr Noon } & 286 & 274.5 & 45 & 290 & 172 & $0 \mathrm{PM}$ \\
\hline & 287 & 270 & 45 & 290 & 172 & $0 \mathrm{PM}$ \\
\hline & 288 & 292.5 & 45 & 290 & 172 & $0 \mathrm{PM}$ \\
\hline & 289 & 315 & 45 & 290 & 172 & $0 \mathrm{PM}$ \\
\hline & 290 & 337.5 & 45 & 290 & 172 & $0 \mathrm{PM}$ \\
\hline & 291 & 0 & 45 & 290 & 172 & $0 \mathrm{PM}$ \\
\hline \multirow{6}{*}{ Whtr AM } & 255 & 0 & 10 & 320 & 355 & $8 \mathrm{AM}$ \\
\hline & 256 & 337.5 & 10 & 320 & 355 & $8 \mathrm{AM}$ \\
\hline & 257 & 315 & 10 & 320 & 355 & $8 \mathrm{AM}$ \\
\hline & 258 & 292.5 & 10 & 320 & 355 & $8 \mathrm{AM}$ \\
\hline & 259 & 270 & 10 & 320 & 355 & $8 \mathrm{AN}$ \\
\hline & 260 & 247.5 & 10 & 320 & 355 & $8 \mathrm{AM}$ \\
\hline \multirow[t]{6}{*}{ Wutr Noon } & 267 & 0 & 20 & 290 & 355 & $0 \mathrm{PN}$ \\
\hline & 268 & 337.5 & 20 & 290 & 355 & o PM \\
\hline & 269 & 315 & 20 & 290 & 355 & $0 \mathrm{PM}$ \\
\hline & 270 & 292.5 & 20 & 290 & 355 & $0 \mathrm{PM}$ \\
\hline & 271 & 270 & 20 & 290 & 355 & $0 \mathrm{PlI}$ \\
\hline & 272 & 247.5 & 20 & 290 & 355 & $0 \mathrm{PH}$ \\
\hline
\end{tabular}


Heliostat 5, Configuration 5-E. continued.

\begin{tabular}{|c|c|c|c|c|c|c|}
\hline Case & Run 非 & WD & $\mathrm{EL}$ & $A Z$ & Day & Time \\
\hline \multirow[t]{6}{*}{ Wintr PM } & 261 & 247.5 & 10 & 265 & 355 & $4 \mathrm{PM}$ \\
\hline & 262 & 270 & 10 & 265 & 355 & $4 P M$ \\
\hline & 263 & 292.5 & 10 & 265 & 355 & $4 \mathrm{PM}$ \\
\hline & 264 & 315 & 10 & 265 & 355 & $4 \mathrm{PN}$ \\
\hline & 265 & 337.5 & 10 & 265 & 355 & $4 \mathrm{PM}$ \\
\hline & 266 & 0 & 10 & 265 & 355 & $4 P M$ \\
\hline Ver. Stow & 213 & 315 & 0 & 320 & 355 & 8 AI \\
\hline Hor. Stow & 274 & 315 & 90 & 320 & 355 & $8 \mathrm{AM}$ \\
\hline
\end{tabular}




\begin{tabular}{|c|c|c|c|c|c|c|}
\hline 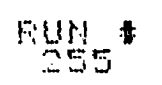 & $\begin{array}{r}W 13 \pi \\
0.0\end{array}$ & 10.0 & $=20.0$ & $\begin{array}{l}4{ }_{1} \\
.2+0\end{array}$ & & \\
\hline $\begin{array}{l}\text { COMF } \\
\text { MEAHS }\end{array}$ & Cry & $\begin{array}{r}\text { Cry } \\
-.01\end{array}$ & .01 & cro & CMY & $\begin{array}{l}\cos z \\
.01\end{array}$ \\
\hline $\begin{array}{l}\text { MAX } \\
\text { MIY } \\
\text { FMS }\end{array}$ & $\begin{array}{r}1+95 \\
.45 \\
.15\end{array}$ & $\begin{array}{r}12 \\
-14 \\
.04\end{array}$ & $\begin{array}{r}.05 \\
-.05 \\
.01\end{array}$ & $\begin{array}{l}10 \\
.15 \\
.05\end{array}$ & $.7 \frac{1}{5}$ & $\begin{array}{l}0.08 \\
.05 \\
.02\end{array}$ \\
\hline SFACT: & 8.69 & $\frac{9}{3.78}$ & 是: & $11 \frac{1}{2}: \frac{5}{7}$ & $19 \cdot 3 Z$ & $3+43$ \\
\hline
\end{tabular}

IATA FOF: FILE: HS102

\begin{tabular}{|c|c|c|c|c|c|c|}
\hline rums & 30145 & 10.0 & & $\begin{array}{r}\text { U. } \\
41.5\end{array}$ & & \\
\hline COMF: & $\begin{array}{c}\mathrm{CF}^{\circ} \\
.19\end{array}$ & $\begin{array}{l}\text { Cry } \\
.01\end{array}$ & $\begin{array}{l}.05 \\
.03\end{array}$ & CH. & $.0 \%$ & $\begin{array}{l}\text { Cryz } \\
.00\end{array}$ \\
\hline $\begin{array}{l}\text { MAX } \\
\text { HIYH }\end{array}$ & $\begin{array}{l}.97 \\
.23 \\
.15\end{array}$ & $\begin{array}{r}.87 \\
.02\end{array}$ & $\begin{array}{l}.07 \\
.01 \\
.01\end{array}$ & $\begin{array}{l}15 \\
.10 \\
.05\end{array}$ & $\cdot \frac{29}{2} \cdot \frac{2}{6}$ & $\begin{array}{l}.05 \\
.07 \\
.01\end{array}$ \\
\hline $\begin{array}{l}\text { QFACT } \\
\text { FFACT }\end{array}$ & $\begin{array}{l}7.31 \\
4+49\end{array}$ & $\begin{array}{r}10.02 \\
3.44\end{array}$ & 2.75 & $12 \cdot 52$ & $1 \frac{1}{5} \cdot \frac{1}{2}$ & $\begin{array}{r}67+85 \\
5+1\end{array}$ \\
\hline
\end{tabular}

IIATA FOF: FILE: H5102

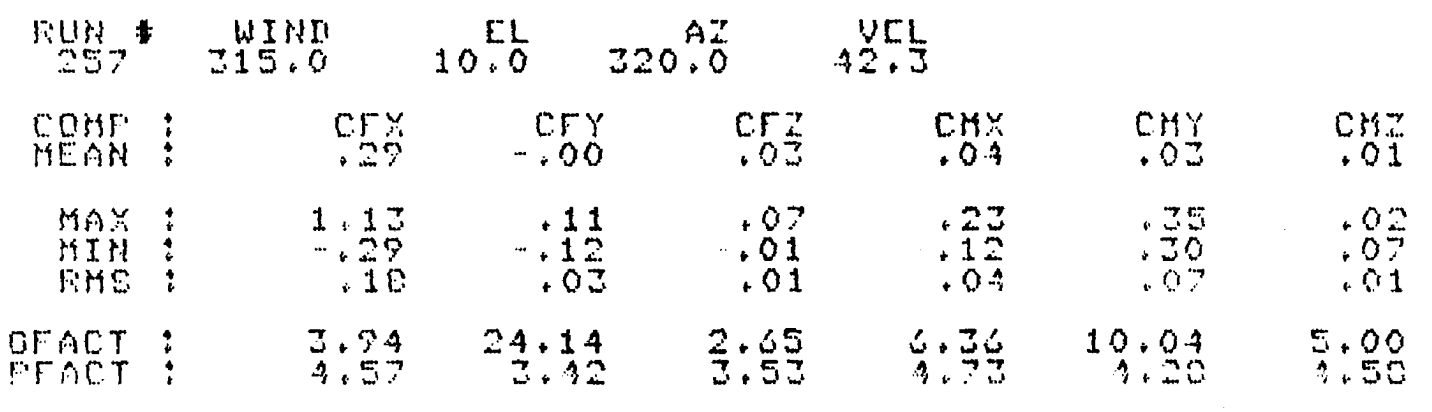

IATA FOR: FILE: $\mathrm{HS102}$

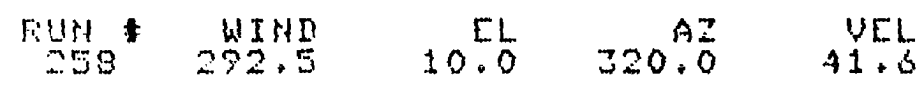

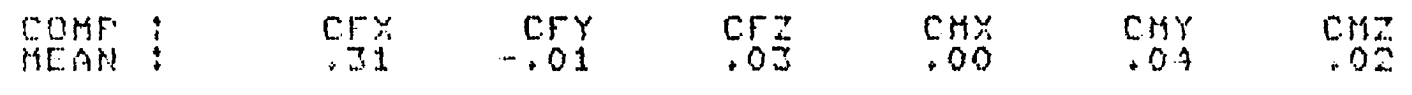

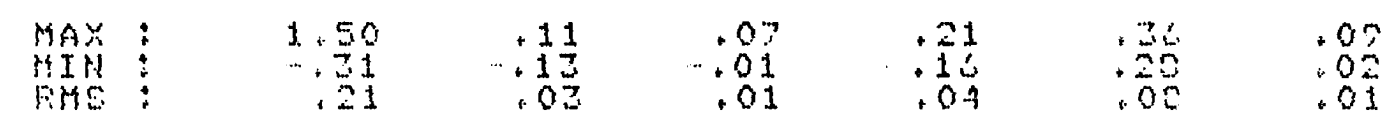

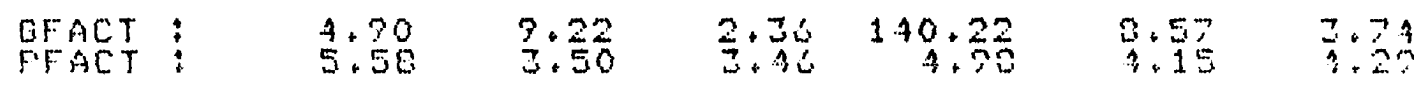




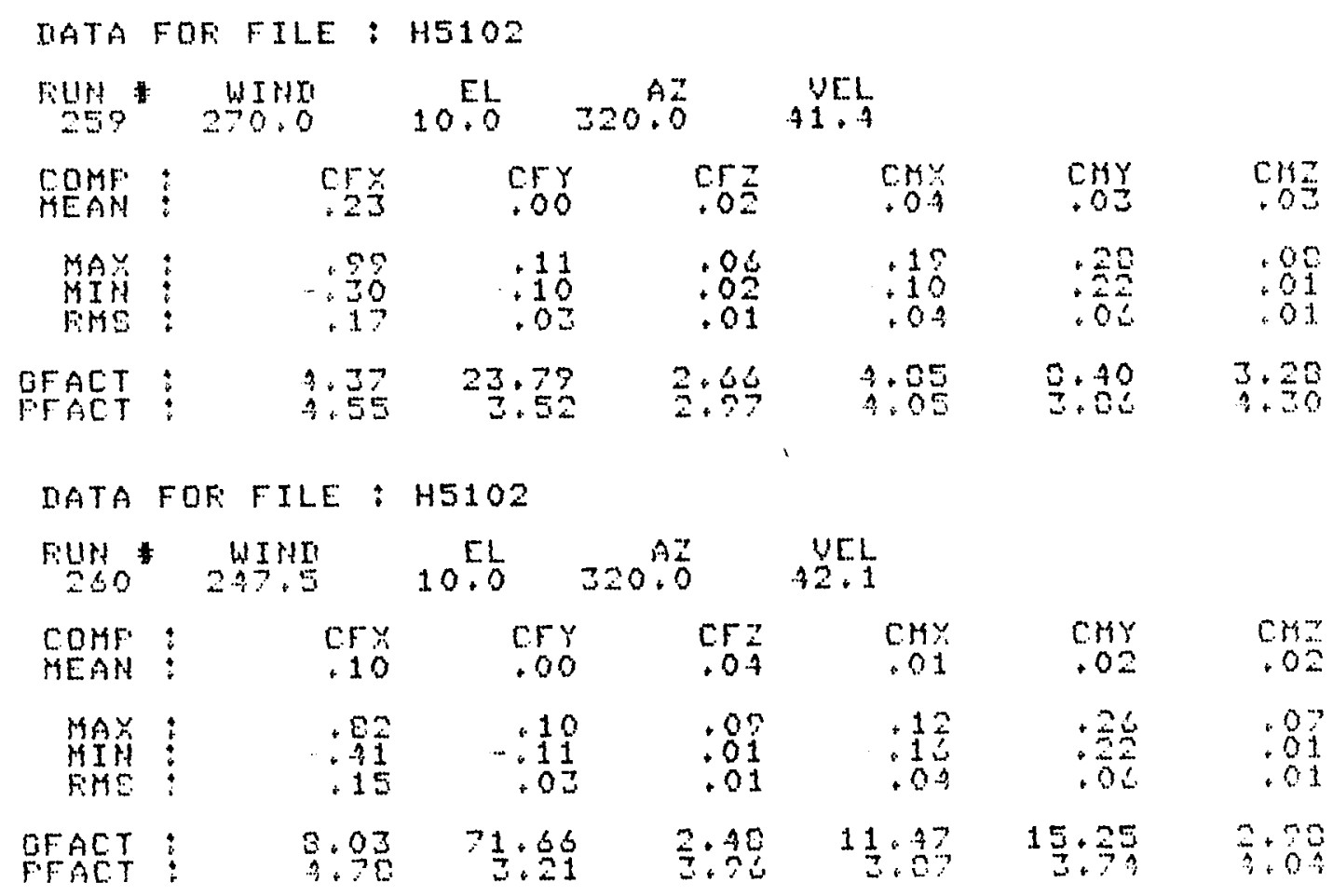

IATA FOF: FILE: HSIO2

\begin{tabular}{|c|c|c|c|c|c|c|}
\hline Rurs & 247.5 & 10.0 & & $\begin{array}{l}\text { VEL } \\
71.1\end{array}$ & & \\
\hline $\begin{array}{l}\text { COMF: } \\
\text { MEEAH }\end{array}$ & CFy & $\begin{array}{l}\text { Cry } \\
.01\end{array}$ & $\begin{array}{c}62 \\
-.02\end{array}$ & CMs & 0.05 & $\begin{array}{l}\operatorname{cox} \\
.02\end{array}$ \\
\hline $\begin{array}{l}\text { MAY : } \\
\text { FIYS }\end{array}$ & -1:告客 & $\begin{array}{r}+10 \\
-.09 \\
.02\end{array}$ & $\begin{array}{r}.05 \\
.14 \\
.02\end{array}$ & $\begin{array}{l}11 \\
.05 \\
.02\end{array}$ & .22 & .09 \\
\hline $\begin{array}{l}\text { QFACT } \\
\text { FFACT }\end{array}$ & 0.18 & $\frac{7}{3}+\frac{72}{56}$ & 0.32 & $3 \cdot 22$ & $\begin{array}{l}30 \\
\because 30\end{array}$ & $=97$ \\
\hline
\end{tabular}

IATA FOF FILE : H51O2

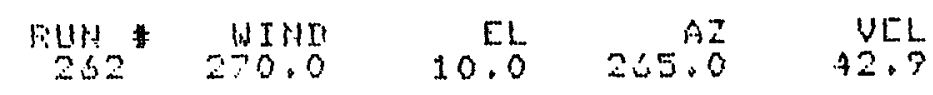

COMF:

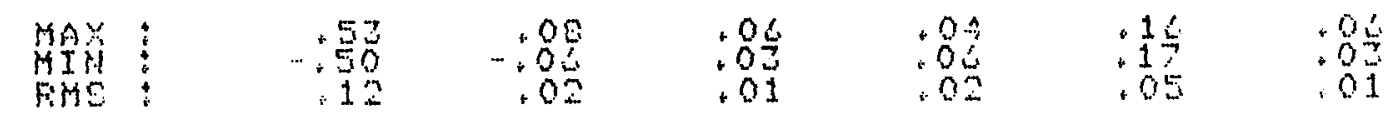

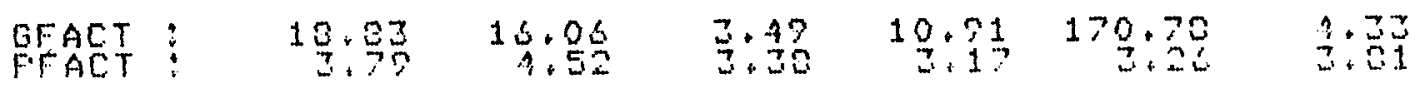

1 


\begin{tabular}{|c|c|c|c|c|c|c|}
\hline 代等3 & $\begin{array}{r}\text { WIMI } \\
292.5\end{array}$ & 10.0 & 205.0 & $\begin{array}{r}\text { UEL } \\
43.1\end{array}$ & & \\
\hline $\begin{array}{l}\text { COMF } \\
\text { MEAN }\end{array}$ & $\begin{array}{l}\text { CF } \\
.10\end{array}$ & C. & CF" & $\begin{array}{l}\text { CHK } \\
.02\end{array}$ & $\begin{array}{l}\text { Cris } \\
.02\end{array}$ & $\begin{array}{l}c .47 \\
.02\end{array}$ \\
\hline $\begin{array}{l}\text { MAY } \\
\text { MTHE }\end{array}$ & $\begin{array}{r}1.07 \\
.45 \\
.15\end{array}$ & $\begin{array}{r}.13 \\
.05 \\
.02\end{array}$ & $\begin{array}{l}.05 \\
.05 \\
.01\end{array}$ & $\begin{array}{l}.03 \\
.02 \\
.02\end{array}$ & $\frac{27}{50}$ & $\begin{array}{l}+\frac{1}{2} \\
.02 \\
.01\end{array}$ \\
\hline $\begin{array}{l}\text { OFACT } \\
\text { FFAC }\end{array}$ & $\begin{array}{r}10.57 \\
6.41\end{array}$ & 4.55 & $5 \cdot 22$ & $\frac{3}{3} .75$ & 12.59 & $\frac{1}{5} \cdot \frac{2}{6}=$ \\
\hline
\end{tabular}

\section{IIATA FOR FILE: HS102}

\begin{tabular}{|c|c|c|c|c|c|c|}
\hline Find & $\begin{array}{l}\text { WIn } \\
-15.0\end{array}$ & 10.0 & & $\begin{array}{l}\text { VEL } \\
3 Z .1\end{array}$ & & \\
\hline $\begin{array}{l}\text { CONF } \\
\text { MEAN }\end{array}$ & C. 28 & $\begin{array}{l}\text { CFY } \\
.0 Z\end{array}$ & .05 & $\begin{array}{l}\text { C.MY } \\
.01\end{array}$ & $\begin{array}{l}\text { CMY } \\
.05\end{array}$ & $.0 \%=$ \\
\hline $\begin{array}{l}\text { MAY } \\
\text { MIH } \\
\text { FHE }\end{array}$ & $\begin{array}{r}.20 \\
.20 \\
.17\end{array}$ & $\begin{array}{r}+11 \\
-02 \\
.02\end{array}$ & $\begin{array}{l}.02 \\
.02 \\
.01\end{array}$ & $\begin{array}{r}.04 \\
.04 \\
.01\end{array}$ & . 92 & $\begin{array}{l}.09 \\
.05 \\
.01\end{array}$ \\
\hline $\begin{array}{l}\text { QFACT } \\
\square A C T\end{array}$ & $\begin{array}{l}4.29 \\
4.42\end{array}$ & $\begin{array}{l}3.53 \\
3.01\end{array}$ & $\frac{2.79}{1.07}$ & 点. & $\begin{array}{l}5.32 \\
4.15\end{array}$ & J. \\
\hline
\end{tabular}

IATA FOF FILE: H5102

FUS

\begin{tabular}{|c|c|c|c|c|c|c|}
\hline $\begin{array}{l}\text { COMF } \\
\text { MEAM }\end{array}$ & .25 & $\begin{array}{cr}\text { cry } \\
.03\end{array}$ & .01 & $\begin{array}{l}\text { CMY } \\
.02\end{array}$ & $\begin{array}{l}\text { CHY } \\
\text { DOS }\end{array}$ & $\begin{array}{l}C r z \\
05\end{array}$ \\
\hline $\begin{array}{l}\text { MAX } \\
\text { MIIS } \\
\text { Fis }\end{array}$ & $\begin{array}{r}1.92 \\
+32 \\
+12\end{array}$ & $\begin{array}{r}.10 \\
.05 \\
.02\end{array}$ & $\begin{array}{l}.05 \\
.04 \\
+01\end{array}$ & $\begin{array}{l}.03 \\
.05 \\
.02\end{array}$ & : $\frac{32}{2} \frac{1}{5}$ & $\begin{array}{l}10 \\
.02 \\
.01\end{array}$ \\
\hline $\begin{array}{l}\text { CACT } \\
\text { AACT }\end{array}$ & $\frac{4}{5.25}$ & $\begin{array}{l}4.03 \\
4.20\end{array}$ & $\begin{array}{l}3.35 \\
2.58\end{array}$ & $\begin{array}{l}4.27 \\
3.04\end{array}$ & 台误 & 3.1 \\
\hline
\end{tabular}

IATA FOF: FILE: HS102

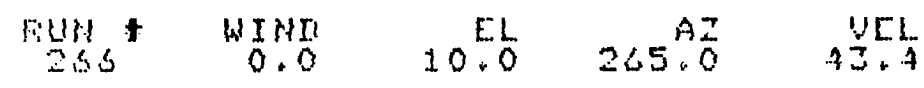

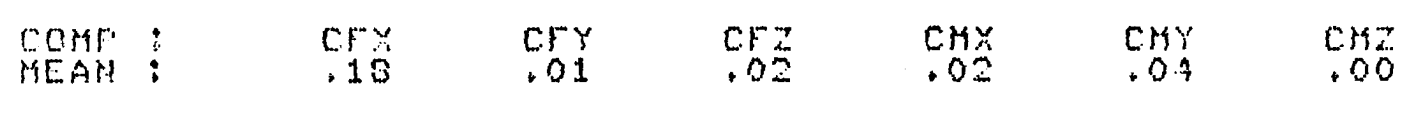

\begin{tabular}{|c|c|c|c|c|c|}
\hline $\begin{array}{l}\text { MAY } \\
\text { MIM } \\
\text { SiMS: }\end{array}$ & $\begin{array}{r}.85 \\
-.14 \\
+11\end{array}$ & $\begin{array}{r}.07 \\
.02 \\
.01\end{array}$ & $\begin{array}{l}.06 \\
.02 \\
.01\end{array}$ & .03 & $\begin{array}{l}20 \\
.10 \\
.04\end{array}$ \\
\hline
\end{tabular}

FFACT: $\quad \begin{aligned} & 4.35 \\ & \text { FPCT }\end{aligned}$ 


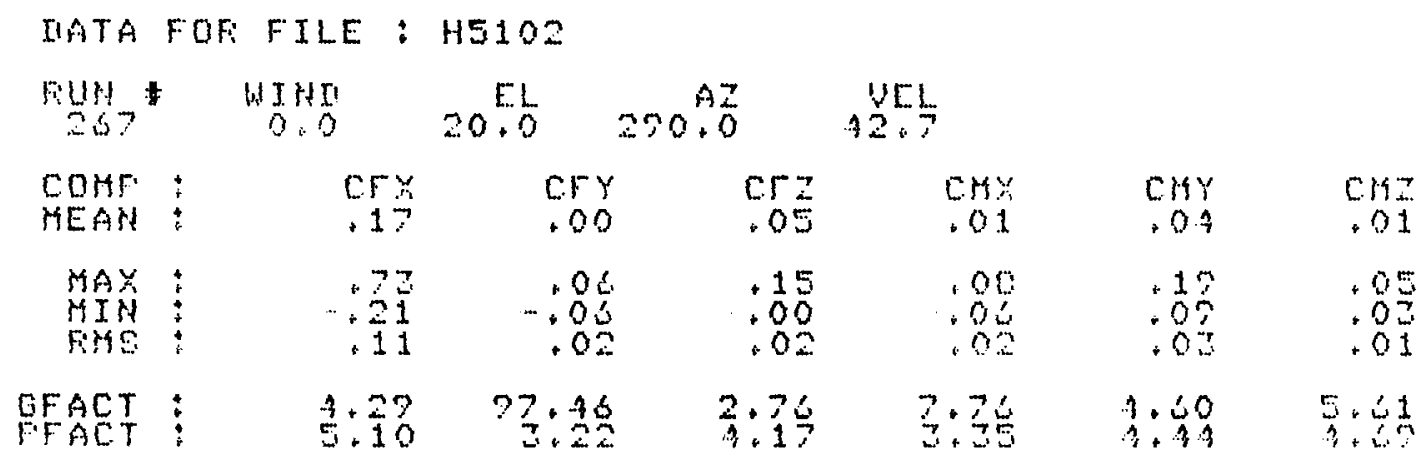

IATA FOF FILE: H5102

nuss

\begin{tabular}{|c|c|c|c|c|c|c|}
\hline COMF & $\begin{array}{l}\text { CFy } \\
.16\end{array}$ & $\begin{array}{r}\text { Cry } \\
-.01\end{array}$ & ${ }_{0}^{c} 2$ & CMy & $\begin{array}{l}\text { CMy } \\
.05\end{array}$ & $\cos 2$ \\
\hline $\begin{array}{l}\text { MAX } \\
\text { MIYH } \\
\text { BME }\end{array}$ & $\begin{array}{r}75 \\
.23 \\
.12\end{array}$ & $\begin{array}{r}.07 \\
.10 \\
.03\end{array}$ & $\begin{array}{r}11 \\
.02 \\
.02\end{array}$ & .00 & $\begin{array}{r}1 \% \\
.19 \\
.04\end{array}$ & $\begin{array}{r}.03 \\
.03\end{array}$ \\
\hline $\begin{array}{l}F A C T \\
F A C T\end{array}$ & $\begin{array}{l}4.57 \\
4.72\end{array}$ & $13 \cdot 51$ & 4.22 & $\begin{array}{c}0.09 \\
2.75\end{array}$ & 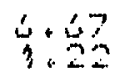 & $\stackrel{4}{y}$ \\
\hline
\end{tabular}

IATA FOF FILE: H5102

\begin{tabular}{|c|c|c|c|c|c|c|}
\hline Fus & 315.0 & $=0.0$ & & $\begin{array}{l}\text { VEL } \\
74.2\end{array}$ & & \\
\hline COMF & $\begin{array}{l}\text { CF. } \\
.13\end{array}$ & $\begin{array}{l}\text { CFy } \\
.00\end{array}$ & $\begin{array}{l}0.7 \\
.05\end{array}$ & $\begin{array}{l}\operatorname{crs} 5 \\
.00\end{array}$ & $\begin{array}{l}\text { cryy } \\
.02\end{array}$ & $\begin{array}{l}\text { cMI } \\
+01\end{array}$ \\
\hline $\begin{array}{l}\text { MAY } \\
\text { FIME }\end{array}$ & $\begin{array}{r}7 \% \\
.10 \\
.10\end{array}$ & $\begin{array}{r}.00 \\
.00 \\
.05\end{array}$ & $\begin{array}{l}17 \\
.01 \\
.02\end{array}$ & $\begin{array}{r}.05 \\
.05 \\
.02\end{array}$ & $\begin{array}{l}12 \\
: 12 \\
0 \\
0\end{array}$ & .05 \\
\hline $\begin{array}{l}\text { DFACT } \\
\text { FACT }\end{array}$ & $5+72$ & $10 \frac{1}{3} \cdot 57$ & $2+92$ & $22 \cdot 43$ & $9+27$ & 5.92 \\
\hline
\end{tabular}

IATA FOF: FILE: H5102

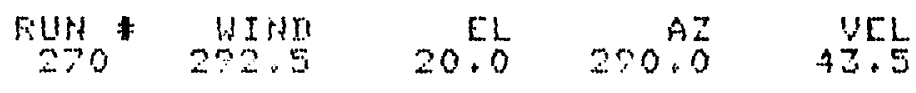

\begin{tabular}{|c|c|c|c|c|c|c|}
\hline CONF & $\begin{array}{l}64 \\
.13\end{array}$ & $\begin{array}{l}\text { CrY } \\
.01\end{array}$ & .04 & $\begin{array}{l}\text { cry } \\
.00\end{array}$ & $\begin{array}{l}\text { CHY } \\
0 \%\end{array}$ & $\begin{array}{c}\cos \\
.01\end{array}$ \\
\hline $\begin{array}{l}\text { MAX } \\
\text { MIHA } \\
\text { FMSE }\end{array}$ & $\begin{array}{r}1.91 \\
: 42 \\
: 12\end{array}$ & $\begin{array}{r}.12 \\
: 11 \\
.04\end{array}$ & $\begin{array}{r}12 \\
.04 \\
.02\end{array}$ & : 12 & .51 & .07 \\
\hline$A C T$ & 8.87 & $1 \frac{1}{9} \cdot 2 ?$ & 5.45 & 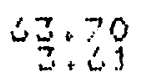 & $\overrightarrow{5}+\frac{1}{1}$ & 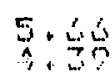 \\
\hline
\end{tabular}




\begin{tabular}{|c|c|c|c|c|c|c|}
\hline Fivst & $\begin{array}{r}W I+\pi \\
270.0\end{array}$ & 20.0 & 290.0 & VEL & & \\
\hline $\begin{array}{l}\text { COMF : } \\
\text { NiEAN : }\end{array}$ & $6 F$ & $\begin{array}{c}c .5 y \\
.02\end{array}$ & .01 & $\begin{array}{l}\text { CMY } \\
.01\end{array}$ & $\begin{array}{l}C+95 \\
02\end{array}$ & $\begin{array}{l}C M= \\
.0 O\end{array}$ \\
\hline $\begin{array}{l}\text { MAY } \\
\text { HINS } \\
\text { FWD }\end{array}$ & $\begin{array}{l}+4 \frac{1}{4} \\
\div 12 \\
12\end{array}$ & $\begin{array}{r}.14 \\
.10 \\
.04\end{array}$ & $\begin{array}{r}.10 \\
.02 \\
.02\end{array}$ & $\begin{array}{l}12 \\
: 11 \\
.04\end{array}$ & .25 & $\begin{array}{l}.05 \\
.04 \\
.01\end{array}$ \\
\hline QFACT & $\begin{array}{r}41 \\
7.37\end{array}$ & $\begin{array}{l}3 \\
3\end{array}$ & $\begin{array}{l}4 \\
3\end{array} 40$ & $19+70$ & $13 \cdot 50$ & $\begin{array}{r}7 \\
3.55 \\
5\end{array}$ \\
\hline IATA F & JF: FILE : & 45102 & & & & \\
\hline Filn & $\begin{array}{r}\text { WINU } \\
247: 5\end{array}$ & 20.0 & 290.0 & VEL & & \\
\hline $\begin{array}{l}\text { COMF: } \\
\text { MEAH : }\end{array}$ & $\begin{array}{r}\operatorname{cr} x \\
-16\end{array}$ & $\begin{array}{l}\text { Cfy } \\
.01\end{array}$ & $\begin{array}{c}\mathrm{Cr}^{2} \\
+06\end{array}$ & $\begin{array}{l}\text { CMY } \\
+02\end{array}$ & $\begin{array}{l}\text { Crfy } \\
\text {.0? }\end{array}$ & $\begin{array}{l}\text { CHz } \\
.01\end{array}$ \\
\hline $\begin{array}{l}\text { MAX } \\
\text { HIN } \\
\text { FHS }\end{array}$ & $\begin{array}{r}.92 \\
-74 \\
.75\end{array}$ & $\begin{array}{r}.21 \\
: 12 \\
: 05\end{array}$ & $\begin{array}{l}.05 \\
.15 \\
.02\end{array}$ & $\begin{array}{r}+17 \\
.24 \\
.05\end{array}$ &.$\frac{17}{0}$ & .07 \\
\hline OFACT & 4.55 & $\begin{array}{r}17.68 \\
4.05\end{array}$ & $\frac{2}{3}+\frac{45}{5}$ & 13.07 & $\begin{array}{l}\dot{4} \\
\ddot{5} .32 \\
.3\end{array}$ & $\begin{array}{l}3+42 \\
3+2\end{array}$ \\
\hline
\end{tabular}

IIATA FOR FILE ; 15102

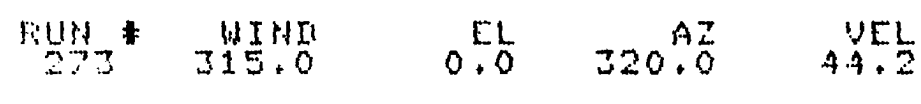

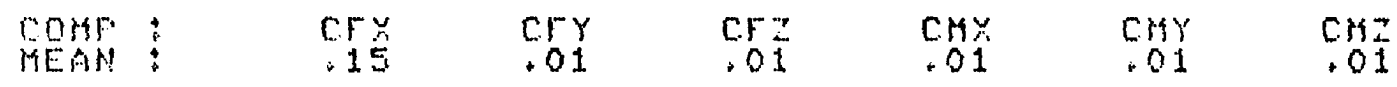

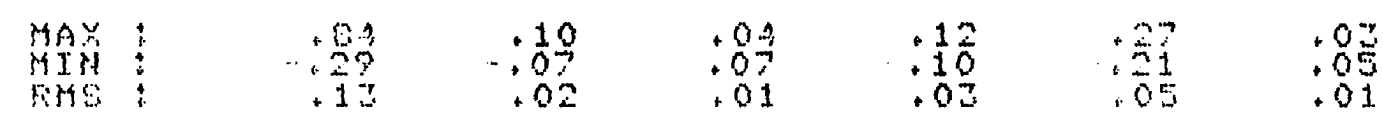

FFACT:

MATA FOF: FILE: 45102

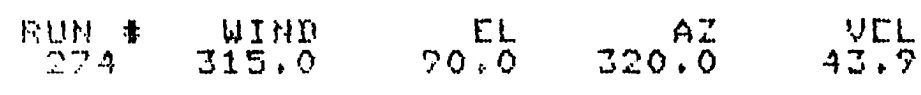

\begin{tabular}{|c|c|c|c|c|c|c|}
\hline $\begin{array}{l}\text { CoMF } \\
\text { HEAS }\end{array}$ & cry & $\begin{array}{l}\text { CFY } \\
.01\end{array}$ & .05 & .01 & $\begin{array}{l}\text { Cry } \\
.00\end{array}$ & $\begin{array}{l}\mathrm{CH} 2 \\
.00\end{array}$ \\
\hline $\begin{array}{l}\text { Mas } \\
\text { ming } \\
\text { Fins }\end{array}$ & $\begin{array}{r}.10 \\
-.02 \\
.02\end{array}$ & $\begin{array}{r}+04 \\
+04 \\
+01\end{array}$ & $\begin{array}{r}20 \\
.35 \\
.07\end{array}$ & .115 & $\begin{array}{l}.06 \\
.05 \\
.02\end{array}$ & $\begin{array}{l}.02 \\
.02 \\
.01\end{array}$ \\
\hline $\begin{array}{l}A C T \\
A C T\end{array}$ & $\begin{array}{r}10.73 \\
5.72\end{array}$ & 10.75 & 57.40 & 15.71 & 21.49 & 点: \\
\hline
\end{tabular}


IAATA FOF: FILE: 15102

\begin{tabular}{|c|c|c|c|c|c|c|}
\hline rivit & $\begin{array}{l}\text { WINT } \\
0.0\end{array}$ & $2=E \frac{E}{0}$ & & $\begin{array}{l}\text { UEL } \\
42.5\end{array}$ & & \\
\hline $\begin{array}{l}\text { COMF } \\
\text { MAEAN }\end{array}$ & $\begin{array}{l}\text { CFy } \\
.0 S\end{array}$ & $\begin{array}{r}\text { Cry } \\
-.02\end{array}$ & $\begin{array}{l}c+2 \\
.03\end{array}$ & $\begin{array}{l}\text { crss } \\
.01\end{array}$ & $\begin{array}{l}\text { chy } \\
.0 y\end{array}$ & $\begin{array}{l}\cos 2 \\
.00\end{array}$ \\
\hline $\begin{array}{l}\text { MAX } \\
\text { MIM } \\
\text { RMS: }\end{array}$ & .75 & $\begin{array}{l}.04 \\
.05 \\
.02\end{array}$ & .19 & $\begin{array}{l}.12 \\
.11 \\
.05\end{array}$ & .39 & $\begin{array}{r}.05 \\
.05 \\
.01\end{array}$ \\
\hline $\begin{array}{l}\text { QFACT } \\
\text { FFACT }\end{array}$ & 3.33 & 3.67 & 4.35 & $19+2-4$ & $1 \frac{2}{3} \cdot 05$ & $13 \cdot 20$ \\
\hline
\end{tabular}

IIATA FDF: FILE: 15102

\begin{tabular}{|c|c|c|c|c|c|c|}
\hline${ }_{301}$ & $\begin{array}{r}4 I+I I \\
237.5\end{array}$ & 25.0 & & $\begin{array}{r}Y C L \\
.2 .5\end{array}$ & & \\
\hline COHF : & $\begin{array}{l}C F \\
.15\end{array}$ & $\begin{array}{r}\operatorname{cry} \\
-.01\end{array}$ & $\begin{array}{c}\operatorname{crg} \\
.03\end{array}$ & $\begin{array}{l}\text { crus } \\
.00\end{array}$ & $\begin{array}{l}\text { CHy } \\
.02\end{array}$ & $\begin{array}{l}c+3 i= \\
.01\end{array}$ \\
\hline $\begin{array}{l}\text { MAX: } \\
\text { MIHE: }\end{array}$ & $\begin{array}{r}1.93 \\
: .50 \\
.14\end{array}$ & $\begin{array}{r}.07 \\
+.07 \\
.01\end{array}$ & .22 & $\begin{array}{l}10 \\
.10 \\
.03\end{array}$ & $.2 \frac{25}{20}$ & .06 \\
\hline $\begin{array}{l}\text { QFACT } \\
\text { FFACT }\end{array}$ & $\begin{array}{l}3.92 \\
6.22\end{array}$ & $\frac{5.42}{4.22}$ & $\begin{array}{l}3.03 \\
5.00\end{array}$ & $2 \frac{1}{7} \cdot 07$ & $\begin{array}{r}13.52 \\
4.53\end{array}$ & $5 \cdot 5$ \\
\hline
\end{tabular}

IIATA FOF FILE: H5102

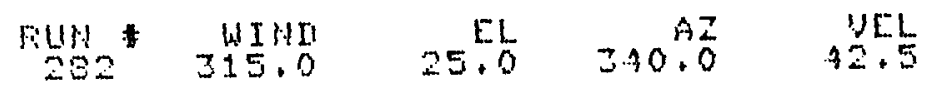

\begin{tabular}{|c|c|c|c|c|c|c|}
\hline CQMFP : & $0 F$ &.$F^{\prime}$ & $\begin{array}{c}c F \\
.04\end{array}$ & .01 & $\begin{array}{l}\text { cris } \\
\text { coz }\end{array}$ & .01 \\
\hline $\begin{array}{l}\text { MAO } \\
\text { HIMS }\end{array}$ & 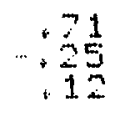 & $\begin{array}{r}.07 \\
.03 \\
.01\end{array}$ & $\begin{array}{l}17 \\
.02 \\
.02\end{array}$ & .02 & $\begin{array}{l}30 \\
+31 \\
+05\end{array}$ & $\begin{array}{l}.0 \hat{3} \\
.08 \\
.01\end{array}$ \\
\hline ACT & $\begin{array}{l}3.53 \\
3.48\end{array}$ & $9: 3 \vec{s}$ & 3.93 & 12.94 & $1 \stackrel{6}{5} \cdot 5 \frac{1}{5}$ & $\begin{array}{l}3.00 \\
3.9 .9\end{array}$ \\
\hline
\end{tabular}

IIATA FOF FILE: H5102

\begin{tabular}{|c|c|c|c|c|c|c|}
\hline $\begin{array}{l}\text { nus } \\
283\end{array}$ & $\begin{array}{rl} & 4 I M U \\
2 & 2\end{array}$ & $\begin{array}{r}E L \\
25.0\end{array}$ & & $\begin{array}{r}\text { vㄷ. } \\
42.9\end{array}$ & & \\
\hline COMF & $\begin{array}{c}c 5 x \\
+17\end{array}$ & $\begin{array}{l}\text { CFY } \\
-.00\end{array}$ & $\begin{array}{l}.52 \\
.05\end{array}$ & $\begin{array}{l}\operatorname{crs} \\
.02\end{array}$ & $\begin{array}{l}\text { cry } \\
.09\end{array}$ & $\begin{array}{l}\text { criz } \\
+06\end{array}$ \\
\hline $\begin{array}{l}\text { MAX } \\
\text { MIM } \\
\text { FIMS }\end{array}$ & $+\frac{97}{15}$ & $\begin{array}{l}.04 \\
.04 \\
.01\end{array}$ & $: 02$ & . 12 & $\begin{array}{r}31 \\
.31 \\
.06\end{array}$ & $\begin{array}{r}.05 \\
.05 \\
.03\end{array}$ \\
\hline $\begin{array}{l}\text { QFACT } \\
F A C T\end{array}$ & 5.05 & $\begin{array}{r}73.00 \\
3.00\end{array}$ & 2.75 & 5.32 & 7.70 & $\begin{array}{r}30.95 \\
9.95\end{array}$ \\
\hline
\end{tabular}


IATA FOF FILE: HSIO2

\begin{tabular}{|c|c|c|c|c|c|c|}
\hline 단 & $\begin{array}{l}\text { WINIr } \\
270.0\end{array}$ & 25.0 & 370.0 & $\begin{aligned} V \\
12\end{aligned}$ & & \\
\hline $\begin{array}{l}\text { COMF: } \\
\text { TEAAH : }\end{array}$ & $\begin{array}{l}\mathrm{CF} \\
+17\end{array}$ & $\begin{array}{l}\mathrm{CrY} \\
. .01\end{array}$ & .0 .5 & $\begin{array}{l}\text { Cr.s. } \\
+01\end{array}$ & cisy & 0.02 \\
\hline $\begin{array}{l}\text { MAX } \\
\text { WIME }\end{array}$ & $\begin{array}{r}94 \\
+70 \\
+12\end{array}$ & $\begin{array}{l}+05 \\
.05 \\
.01\end{array}$ & $\begin{array}{l}155 \\
+00 \\
.02\end{array}$ & $\begin{array}{l}.09 \\
+02 \\
.02\end{array}$ & . & $\begin{array}{l}.00 \\
.01 \\
.01\end{array}$ \\
\hline $\begin{array}{l}\text { GFACT } \\
\text { FACT }\end{array}$ & $\begin{array}{l}5.50 \\
6.28\end{array}$ & $9+3 z$ & $\begin{array}{l}3.04 \\
5.02\end{array}$ & 11.02 & 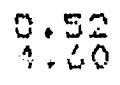 & 3.27 \\
\hline
\end{tabular}

XATA FOF FILE: 15102

\begin{tabular}{|c|c|c|c|c|c|c|}
\hline nuts & $\begin{array}{r}W I H R \\
247.5\end{array}$ & 25.0 & $\frac{A I}{0}$ & $\begin{array}{r}V E L \\
12.5\end{array}$ & & \\
\hline $\begin{array}{l}\text { COHFF : } \\
\text { HEAN }\end{array}$ & $\begin{array}{l}C F \\
.27\end{array}$ & $\begin{array}{l}\text { Cry } \\
.00\end{array}$ & $\begin{array}{l}c 5 z \\
.10\end{array}$ & $\begin{array}{l}\text { CMS } \\
.02\end{array}$ & $\begin{array}{l}\text { CHY } \\
.04\end{array}$ & $\begin{array}{l}\text { CHAz } \\
.01\end{array}$ \\
\hline $\begin{array}{l}\text { MAX } \\
\text { MIPS } \\
\text { FiMS }\end{array}$ & $\begin{array}{c}1+0 z \\
+30 \\
+13\end{array}$ & $\begin{array}{r}.05 \\
-.04 \\
.01\end{array}$ & $\begin{array}{l}22 \\
.23 \\
.02 \\
02\end{array}$ & $\begin{array}{l}11 \\
.02 \\
.03\end{array}$ & $\frac{3}{0}$ & $\begin{array}{l}02 \\
.02 \\
.01\end{array}$ \\
\hline $\begin{array}{l}\text { FACT } \\
\text { FACT }\end{array}$ & 3.78 & 38.02 & $\frac{2}{5.30}$ & 4.47 & 9.30 & 4.22 \\
\hline
\end{tabular}

IBTA FOF: FILE: HS102

\begin{tabular}{|c|c|c|c|c|c|c|}
\hline $\begin{array}{c}\text { Fun } \\
\text {-0s }\end{array}$ & $\begin{array}{r}W I+I I \\
247\end{array}$ & $\begin{array}{r}E L \\
95.0\end{array}$ & $\begin{array}{r}A 2 \\
90.0\end{array}$ & $\begin{array}{r}\text { U다 } \\
42.7\end{array}$ & & \\
\hline $\begin{array}{l}\text { COMF } \\
\text { BYEAM }\end{array}$ & $.0 \%$ & $\begin{array}{l}\text { CFY } \\
.02\end{array}$ & $\begin{array}{l}c 5 \\
.00\end{array}$ & $\begin{array}{l}\text { CMy } \\
.00\end{array}$ & $\begin{array}{l}\text { CMY } \\
.01\end{array}$ & $\begin{array}{l}\mathrm{CHz} \\
.00\end{array}$ \\
\hline $\begin{array}{l}\text { Max } \\
\text { MIM } \\
\text { Fise }\end{array}$ & $\begin{array}{r}.43 \\
-45 \\
-11\end{array}$ & $\begin{array}{r}.15 \\
.11 \\
.04\end{array}$ & $\begin{array}{l}.21 \\
.22 \\
.05\end{array}$ & $\begin{array}{l}+11 \\
: 12 \\
.03\end{array}$ & $\begin{array}{r}16 \\
.15 \\
.04\end{array}$ & $\begin{array}{l}.05 \\
.03 \\
.01\end{array}$ \\
\hline $\begin{array}{l}\text { PFACT } \\
\text { FACT }\end{array}$ & 50.05 & 3.85 & $110 \underline{0} \cdot 4$. & 27.09 & 30.3 & 20.05 \\
\hline
\end{tabular}

IATA FOF FILE: H5IO2

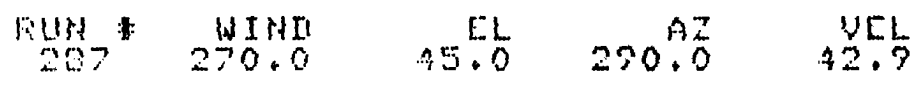

\begin{tabular}{|c|c|c|c|c|c|c|}
\hline Coms: & .03 & $\begin{array}{l}\text { efr } \\
.01\end{array}$ & .05 & $\begin{array}{l}\text { cris } \\
.00\end{array}$ & $\begin{array}{l}\text { CMy } \\
.03\end{array}$ & $\begin{array}{l}\mathrm{CMz} \\
.02\end{array}$ \\
\hline $\begin{array}{l}\text { MAX } \\
\text { MINL } \\
\text { Fims }\end{array}$ & $: \frac{1}{2}$ & $\begin{array}{l}11 \\
.07 \\
.02\end{array}$ & $\begin{array}{l}+27 \\
+15 \\
+05\end{array}$ & $\begin{array}{l}.09 \\
.05 \\
.02\end{array}$ & $\begin{array}{l}15 \\
10 \\
+05\end{array}$ & .05 \\
\hline
\end{tabular}

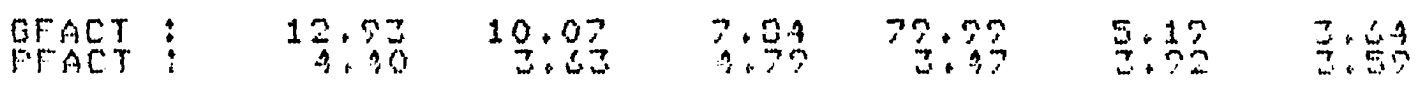




\begin{tabular}{|c|c|c|c|c|c|c|}
\hline 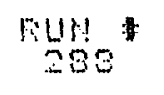 & $\begin{array}{r}4 I 4 \pi \\
272.5\end{array}$ & 45.0 & $2=0 . A^{A Z}$ & $\begin{array}{l}\text { VELL. } \\
72.7\end{array}$ & & \\
\hline $\begin{array}{l}\text { CQMPY } \\
\text { TIEAN }\end{array}$ & $\begin{array}{l}\text { CF } \\
.10\end{array}$ & $\begin{array}{l}\text { CFY } \\
.00\end{array}$ & .052 & $\begin{array}{c}\text { CHy } \\
.01\end{array}$ & $\begin{array}{l}\text { CMY } \\
.05\end{array}$ & $\begin{array}{r}0.9 \\
.02\end{array}$ \\
\hline $\begin{array}{l}\text { MAY } \\
\text { MHE }\end{array}$ & $\begin{array}{r}+40 \\
-10 \\
-10\end{array}$ & $\begin{array}{r}+50 \\
-.09 \\
.09\end{array}$ & $\begin{array}{l}.12 \\
.05 \\
+06\end{array}$ & $\begin{array}{l}.09 \\
.10 \\
.09\end{array}$ & .22 & $\begin{array}{r}.06 \\
.00 \\
.01\end{array}$ \\
\hline $\begin{array}{l}\text { GFACT } \\
\text { FACT }\end{array}$ & $4: 31$ & $23 \cdot 32$ & 4.12 & 7.52 & 9.79 & $=12$ \\
\hline
\end{tabular}

IATA FOF FILE: HS102

\begin{tabular}{|c|c|c|c|c|c|c|}
\hline 도요 & $\begin{array}{l}\text { UIHI } \\
315: 0\end{array}$ & $5: 0$ & & Uᄃㄴ. & & \\
\hline $\begin{array}{l}\text { COMT } \\
\text { MEAM }\end{array}$ & $\begin{array}{c}C F \\
.12\end{array}$ & $\begin{array}{l}\text { CFY } \\
.01\end{array}$ & $\frac{\operatorname{cr} z}{12}$ & $\begin{array}{l}\text { C.t } \\
.00\end{array}$ & $\begin{array}{l}\text { CMY } \\
.0 .\end{array}$ & CHa \\
\hline $\begin{array}{l}\text { Hay } \\
\text { RiHS } \\
\text { RHS }\end{array}$ & $\begin{array}{r}.52 \\
-12 \\
.09\end{array}$ & $\begin{array}{r}.09 \\
-.03 \\
.02\end{array}$ & $\begin{array}{l}.80 \\
.00 \\
.05\end{array}$ & $\begin{array}{r}.05 \\
.05 \\
.02\end{array}$ & $\begin{array}{l}2 \\
.02 \\
.05 \\
.05\end{array}$ & $\begin{array}{l}.02 \\
.05 \\
.01\end{array}$ \\
\hline $\begin{array}{l}\text { GFACT } \\
\text { FFACT }\end{array}$ & $4 \cdot 34$ & $14+14$ & 3.20 & $2+\frac{1}{2}+\frac{1}{6}$ & $\begin{array}{l}5.3 .2 \\
5.30\end{array}$ & $1 \frac{1}{3}, 2=$ \\
\hline
\end{tabular}

IHATA FOF: FILE: HE102

\begin{tabular}{|c|c|c|c|c|c|c|}
\hline Furt & $\begin{array}{r}M I M I I \\
327\end{array}$ & 95.0 & 0 & $\begin{array}{r}\text { VEL } \\
+2.5\end{array}$ & & \\
\hline $\begin{array}{l}\text { COMF: } \\
\text { MEAH: }\end{array}$ & $\begin{array}{c}C F \\
+12\end{array}$ & $\begin{array}{l}.5 Y \\
.00\end{array}$ & CFz & CMy & $\begin{array}{c}\operatorname{crsy} \\
.05\end{array}$ & $\cos$ \\
\hline $\begin{array}{l}\text { MAY } \\
\text { KINS } \\
\text { FIS }\end{array}$ & $\begin{array}{l}.48 \\
.09 \\
.09\end{array}$ & .95 & $.01 \frac{1}{.02}$ & $\begin{array}{r}10 \\
.02 \\
.02\end{array}$ & .25 & $\begin{array}{r}0.9 \\
.02 \\
.01\end{array}$ \\
\hline $\begin{array}{l}A C T \\
A C T\end{array}$ & $\frac{5}{5.87}$ & 20.40 & $\begin{array}{l}3.55 \\
5.48\end{array}$ & $11 \cdot 20$ & 5.72 & $3+\frac{1}{2}$ \\
\hline
\end{tabular}

IATA FOF FILE: H5102

\begin{tabular}{|c|c|c|c|c|c|c|}
\hline Fum & WIIII & 45.0 & & & & \\
\hline CQMP : & $\begin{array}{l}C F \\
.13\end{array}$ & $\begin{array}{l}C F Y \\
.00\end{array}$ & .15 & cho & $\begin{array}{l}\cos y \\
.07\end{array}$ & $\begin{array}{c}c r z \\
.03\end{array}$ \\
\hline $\begin{array}{l}\text { MAX } \\
\text { MIML } \\
\text { Fis }\end{array}$ & $\cdots \frac{6}{14}$ & $\begin{array}{l}.08 \\
+11 \\
.02\end{array}$ & $.0 \frac{1}{102}$ & $\begin{array}{l}+13 \\
.02 \\
+02\end{array}$ & $\begin{array}{l}.25 \\
.03 \\
.04\end{array}$ & $\begin{array}{r}.01 \\
.05 \\
.01\end{array}$ \\
\hline $\begin{array}{l}\text { QFACT } \\
\text { FFACT }\end{array}$ & $3 \cdot 32$ & 23.05 & $\frac{2}{4} \cdot 45$ & A.15 & $3+51$ & $\frac{3}{4} \cdot 21$ \\
\hline
\end{tabular}


Heliostat 3, Configuration 3-A

\begin{tabular}{lcccc}
\hline Case & Run & WD & EL & AZ \\
\hline Ver. Stow & 411 & 265 & 0 & 5 \\
\hline
\end{tabular}




\begin{tabular}{|c|c|c|c|c|c|c|}
\hline riul & $\begin{aligned} & W I H I \\
= & 65.0\end{aligned}$ & 0.5 & 5.20 & $\begin{array}{r}4 E L \\
3 \pm 0\end{array}$ & & \\
\hline COMA & $\begin{array}{c}0.5 \\
: 15\end{array}$ & $\begin{array}{l}\operatorname{cr} Y \\
.01\end{array}$ & ${ }_{0}^{\cos }$ & $.0 \%$ & .01 & $\begin{array}{c}c y= \\
.02\end{array}$ \\
\hline $\begin{array}{l}\text { MAY } \\
\text { MIHE } \\
\text { FME }\end{array}$ & 1. .05 & $\begin{array}{l}.05 \\
.05 \\
.02\end{array}$ & $\begin{array}{l}10 \\
.05 \\
.02\end{array}$ & $\begin{array}{l}.0 .4 \\
.04 \\
.01\end{array}$ &.$\frac{25}{6}$ & .98 \\
\hline $\begin{array}{l}\text { BFACT } \\
\text { PACT }\end{array}$ & $5: 70$ & $7 \cdot 42$ & 114.70 & $17+\frac{7-7}{7}$ & $=\frac{22}{5} \cdot 17$ & 107 \\
\hline
\end{tabular}




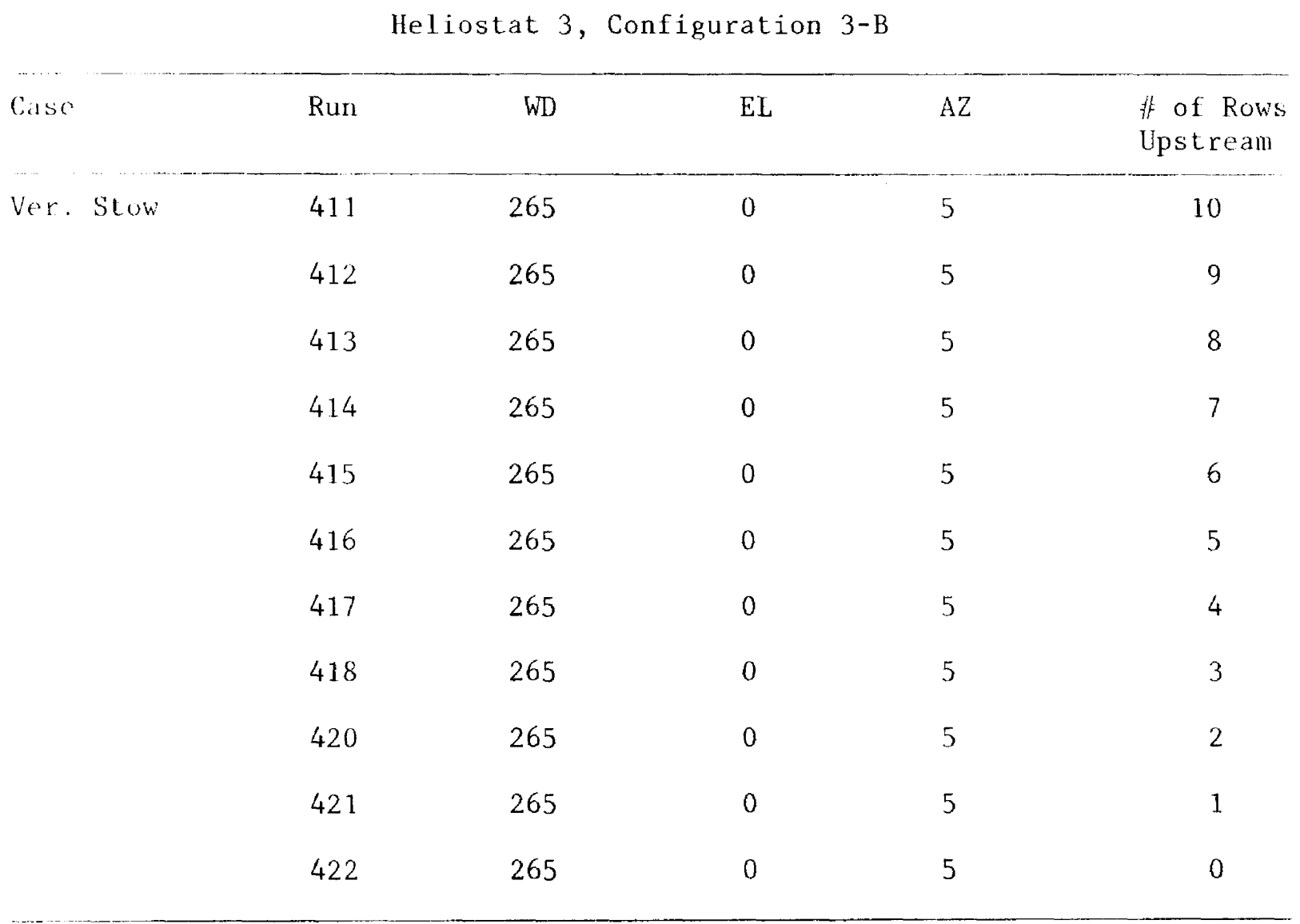




\begin{tabular}{|c|c|c|c|c|c|c|}
\hline $\operatorname{lom}_{11}$ & $=\frac{11}{5}=0$ & $5 \frac{1}{0}$ & 5.0 & 350 & & \\
\hline momar & Cr. & $\begin{array}{c}.0 Y \\
.01\end{array}$ & $\int_{00}^{6}$ & $\begin{array}{l}c 4 x \\
.00\end{array}$ & CMY & $\cos$ \\
\hline $\begin{array}{l}\text { MAY } \\
\text { MIML } \\
\text { RMS }\end{array}$ & $\begin{array}{l}1.05 \\
: \frac{1}{15}\end{array}$ & $\begin{array}{l}05 \\
.05 \\
.02\end{array}$ & $\begin{array}{l}10 \\
.05 \\
.02\end{array}$ & $\begin{array}{l}+0 A \\
+0 \% \\
+81\end{array}$ & .25 & .07 \\
\hline $\begin{array}{l}\text { GFACT } \\
\text { FFACT }\end{array}$ & 4.78 & 7.92 & $112: 70$ & $17: 3 A$ & $=2 \cdot 97$ & $5: 20$ \\
\hline
\end{tabular}

IATA FOF: FILE: Hב2OO

\begin{tabular}{|c|c|c|c|c|c|c|}
\hline Fun! & 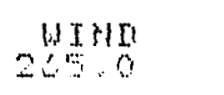 & 0.5 & 92 & 35 & & \\
\hline Gons : & $\begin{array}{cc}c r \\
13\end{array}$ & $\operatorname{cry}$ & $\operatorname{crs}$ & 0.01 & Cry & ckst \\
\hline $\begin{array}{l}\text { MAX } \\
\text { HYII } \\
\text { KHS }\end{array}$ & .72 & $\begin{array}{l}.08 \\
.00 \\
.02\end{array}$ & .11 & $\begin{array}{l}+04 \\
.05 \\
.01\end{array}$ & 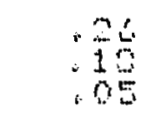 & .01 \\
\hline QFACT & $\frac{5}{5}: \frac{9}{13}$ & $12 \cdot 10$ & $2: 05$ & $4: 29$ & 15.03 & 诺 \\
\hline
\end{tabular}

IATA FOF FILE: H3200

\begin{tabular}{|c|c|c|c|c|c|c|}
\hline$\underset{y=2}{\operatorname{mos}}$ & $\begin{array}{r}4240 \\
285.0\end{array}$ & $0.5 \mathrm{~L}$ & 62 & YcL & & \\
\hline $\begin{array}{l}\text { OOHF : } \\
\text { WEAM! }\end{array}$ & $\begin{array}{l}c 5: \\
012\end{array}$ & $\begin{array}{r}6 y \\
-.00\end{array}$ & $\operatorname{cr}_{1}^{2}$ & $\begin{array}{l}c r y \\
+0=\end{array}$ & $\begin{array}{l}\text { CMY } \\
.00\end{array}$ & $\operatorname{crs}$ \\
\hline $\begin{array}{l}\text { MAY } \\
\text { MIYI } \\
\text { RHo }\end{array}$ & $-\frac{12}{10}$ & $\begin{array}{l}0.9 \\
.09\end{array}$ & $\begin{array}{l}.02 \\
.02 \\
.02\end{array}$ & .07 & .10 & .08 \\
\hline GYACT & $3: \frac{2}{31}$ & 59.38 & 4.09 & 3.20 & 71.78 & $\begin{array}{l}3.50 \\
3.95\end{array}$ \\
\hline
\end{tabular}

IATA FOF FILE: H3200

\begin{tabular}{|c|c|c|c|c|c|c|}
\hline Bum & 265.0 & $\begin{array}{cl}\mathrm{EL} \\
.0\end{array}$ & $\hat{H} 2$ & $4=\frac{1}{7}$ & & \\
\hline $\begin{array}{l}\text { Coris } \\
\text { rents }\end{array}$ & $.0 \%$ & $\operatorname{cr} \gamma$ & $\begin{array}{l}0.2 \\
.01\end{array}$ & CMO & $\begin{array}{l}\text { CMY } \\
.00\end{array}$ & 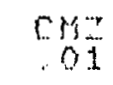 \\
\hline $\begin{array}{l}\text { MAOX } \\
\text { Matho }\end{array}$ & $4 \frac{75}{6}$ & $\begin{array}{l}.04 \\
.06 \\
.01\end{array}$ & $\begin{array}{r}10 \\
.03 \\
.02\end{array}$ & $\begin{array}{r}.05 \\
.03 \\
.01\end{array}$ &.$\frac{2}{10}$ & $\begin{array}{r}81 \\
.81 \\
.01\end{array}$ \\
\hline FACT & $\frac{50}{6}$ & $7+80$ & 0.0 .9 & $2=407$ & 3698 & 30 \\
\hline
\end{tabular}




\begin{tabular}{|c|c|c|c|c|c|c|}
\hline$\frac{118 !}{715}$ & $\begin{array}{l}410 n \\
265.0\end{array}$ & 0.5 & 5.0 & $32 \frac{1}{2}$ & & \\
\hline Cors? & $c \%$ & $\begin{array}{l}\operatorname{CFY} \\
.01\end{array}$ & $\cos _{0}$ & CM\% & cro & .02 \\
\hline $\begin{array}{l}\text { Max } \\
\text { moss }\end{array}$ & $\begin{array}{r}.61 \\
.47 \\
.16\end{array}$ & $\begin{array}{r}.04 \\
.07 \\
.01\end{array}$ & .07 & $\begin{array}{l}.04 \\
.01 \\
.01\end{array}$ & .25 & $\begin{array}{l}.02 \\
.03 \\
.01\end{array}$ \\
\hline $\begin{array}{l}\mathrm{GFACT} \\
\mathrm{rTACT}\end{array}$ & 5.02 & $12 \cdot 33$ & 3.76 & $\begin{array}{l}4.79 \\
3.17\end{array}$ & $90: 77$ & 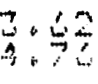 \\
\hline
\end{tabular}

IATA FOF FILE : M3200

\begin{tabular}{|c|c|c|c|c|c|c|}
\hline rums & $\begin{array}{r}\text { UISII } \\
=65.0\end{array}$ & ${ }_{0.0}^{I L}$ & $A Z$ & $4=\frac{1}{2}$ & & \\
\hline $\begin{array}{l}\text { coms } \\
\text { rement }\end{array}$ & 05 & $\begin{array}{l}\text { CFY } \\
.01\end{array}$ & $\begin{array}{c}c r \\
.02\end{array}$ & $\begin{array}{l}\text { crys } \\
.01\end{array}$ & EMY & $\cos =$ \\
\hline $\begin{array}{l}\text { MAY } \\
\text { HIME } \\
\text { RMS }\end{array}$ & . & $\begin{array}{r}.09 \\
.03 \\
.01\end{array}$ & $\begin{array}{r}12 \\
.07 \\
.02\end{array}$ & $\begin{array}{l}.04 \\
.03 \\
.01\end{array}$ & .0 & .01 \\
\hline $\begin{array}{l}\text { CACT } \\
\text { FACT }\end{array}$ & 2.21 & $15: 05$ & $\frac{6}{5}+\frac{5}{3}$ & 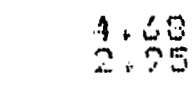 & $7+\frac{1}{5} \cdot ?$ & $\begin{array}{l}6.54 \\
6.76\end{array}$ \\
\hline
\end{tabular}

IIATA FOF FILE ? H320O

\begin{tabular}{|c|c|c|c|c|c|c|}
\hline 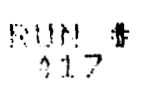 & $\begin{array}{r}4 I \pi n \\
-65.0\end{array}$ & 0.0 & Az & 41.5 & & \\
\hline $\begin{array}{l}\text { cotsp } \\
\text { mant }\end{array}$ & $\begin{array}{l}\text { CFX } \\
116\end{array}$ & Cry & .01 & $\begin{array}{l}\text { CHX } \\
.01\end{array}$ & $\begin{array}{c}\text { cisy } \\
.01\end{array}$ & .042 \\
\hline $\begin{array}{l}\text { Mas } \\
\text { Mas }\end{array}$ & $\frac{92}{12}$ & .02 & $\begin{array}{l}10 \\
.05 \\
.02\end{array}$ & $\begin{array}{l}.82 \\
.02 \\
.01\end{array}$ & $: \frac{72}{05}$ & .01 \\
\hline FACT & 3.92 & $\begin{array}{l}3 \\
y\end{array}$ & 12.45 & $3: 57$ & $2 \frac{1}{5}, \frac{3}{7}$ & $\begin{array}{l}3.49 \\
3.46\end{array}$ \\
\hline
\end{tabular}

WATA FOF: FILE: H3200

\begin{tabular}{|c|c|c|c|c|c|c|}
\hline 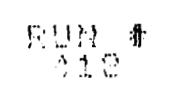 & $\begin{array}{r}45 n \\
2650\end{array}$ & 0.0 & $\begin{array}{l}A Z \\
: O\end{array}$ & 5 & & \\
\hline OOMS & 65 & $\begin{array}{r}C r y \\
.01\end{array}$ & 61 & $\begin{array}{l}\text { CMK } \\
.00\end{array}$ & $\begin{array}{l}\mathrm{CMO} \\
.0 \%\end{array}$ & $\begin{array}{l}\cos \\
.00\end{array}$ \\
\hline $\begin{array}{l}\text { May } \\
\text { ming : }\end{array}$ & . & $\begin{array}{r}.05 \\
.05 \\
.02\end{array}$ & $\begin{array}{l}.00 \\
.05 \\
.03\end{array}$ & $\begin{array}{r}.05 \\
.05 \\
.02\end{array}$ & 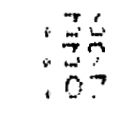 & $\begin{array}{l}0.08 \\
.01 \\
.01\end{array}$ \\
\hline $\begin{array}{l}\text { DFACT } \\
F A C T\end{array}$ & $\stackrel{5}{5} \cdot \frac{13}{2}$ & $\begin{array}{l}7.47 \\
=70\end{array}$ & $2 \cdot \frac{25}{3}$ & $\begin{array}{r}24+43 \\
7 \\
2\end{array}$ & $9 \cdot \frac{1}{7}$ & $97 \cdot 29$ \\
\hline
\end{tabular}




\begin{tabular}{|c|c|c|c|c|c|c|}
\hline Fuำ & $\begin{array}{l}15+1 \\
25.2\end{array}$ & ${ }_{0}^{E}: 0$ & 5. & $\rightarrow 2.5$ & & \\
\hline COMF & $0 \%$ & $\begin{array}{cc}\operatorname{cr} y \\
002\end{array}$ & .02 & $\begin{array}{l}C M Y \\
0.01\end{array}$ & CHy & $\cos$ \\
\hline $\begin{array}{l}\text { MAY } \\
\text { MIMS } \\
\text { RMS }\end{array}$ & $\begin{array}{r}+95 \\
:-15 \\
: \frac{5}{5}\end{array}$ & $\begin{array}{r}+05 \\
-125 \\
+05\end{array}$ & .07 & $\begin{array}{l}.06 \\
.04 \\
.01\end{array}$ & 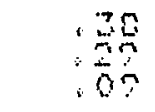 & .02 \\
\hline DEACT & $3 \cdot 62$ & $\frac{4}{5} \cdot \frac{1}{6}$ & $2.2 \frac{1}{5}$ & $\dot{2}: \overline{1}$ & 19.95 & $\begin{array}{l}3 \\
7\end{array}$ \\
\hline IATA $F$ & DF: FILE: & 43200 & & & & \\
\hline $\operatorname{lin}_{3=1}$ & $\begin{array}{l}1130 \\
250\end{array}$ & $5: 0$ & $5: 0$ & U든 & & \\
\hline $\begin{array}{l}\text { Conf } \\
\text { GEAH }\end{array}$ & $\begin{array}{l}C F \\
. S 3\end{array}$ & $\begin{array}{c}C Y Y \\
0 L\end{array}$ & .01 & $\cos$ & 0.03 & ${ }_{0.0}^{C H}=$ \\
\hline $\begin{array}{l}\text { MAX } \\
\text { Gys }\end{array}$ & 1.21 & $\begin{array}{l}.05 \\
: \frac{25}{05} \\
: 05\end{array}$ & .09 & .09 & 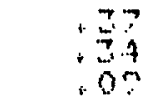 & $\begin{array}{r}01 \\
.15 \\
02\end{array}$ \\
\hline $\begin{array}{l}\text { GFACT } \\
\text { FFACT }\end{array}$ & $\frac{2}{5}: 23$ & $\begin{array}{l}3.40 \\
4.97\end{array}$ & $\frac{4}{3} \cdot \frac{9-3}{21}$ & $\frac{3}{7} .09$ & $\begin{array}{r}11 \cdot 10 \\
3 \cdot 64\end{array}$ & $=17$ \\
\hline DATA F & OF: FILE : & $H 3200$ & & & & \\
\hline nust & 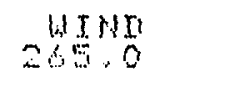 & 0.0 & $5+0$ & $\begin{array}{r}V E L \\
42.2\end{array}$ & & \\
\hline Cors: & CF & $\begin{array}{l}\text { CFY } \\
.0 \%\end{array}$ & $\begin{array}{cc}\mathrm{Cr}^{\circ} \\
\mathrm{O}=\end{array}$ & cros & $\begin{array}{l}\text { Cisy } \\
.05\end{array}$ & $\begin{array}{l}C H \\
05\end{array}$ \\
\hline $\begin{array}{l}\text { Mag : } \\
\text { MyHE } \\
\text { Muge }\end{array}$ & $\begin{array}{r}2.21 \\
: 51 \\
: 25\end{array}$ & $\begin{array}{r}.02 \\
-15 \\
+02\end{array}$ & $\begin{array}{l}12 \\
.05 \\
.02\end{array}$ & $\begin{array}{r}.05 \\
.03 \\
.01\end{array}$ & $\begin{array}{l}40 \\
.40 \\
.03 \\
.05\end{array}$ & .01 \\
\hline $\begin{array}{l}\text { FFACT } \\
\text { FFACT }\end{array}$ & $\frac{1}{4}: \frac{1}{1}$ & $\frac{2}{3} .01$ & $\begin{array}{l}4.33 \\
2.38\end{array}$ & 5.25 & $\begin{array}{l}9 \\
3 \\
3\end{array}$ & $3 \cdot 93$ \\
\hline
\end{tabular}


Heliostat 3, Configuration 3-C

\begin{tabular}{|c|c|c|c|c|c|}
\hline Case & Run & WD & $\mathrm{EL}$ & $A Z$ & $\begin{array}{c}\text { Field } \\
\text { Density } \\
\text { GA }\end{array}$ \\
\hline \multirow[t]{5}{*}{ Ver. Stow } & 411 & 265 & 0 & 5 & 0.238 \\
\hline & 423 & 265 & 0 & 5 & 0.131 \\
\hline & 424 & 265 & 0 & 5 & 0.067 \\
\hline & 425 & 265 & 0 & 5 & 0.044 \\
\hline & 426 & 265 & 0 & 5 & 0.026 \\
\hline
\end{tabular}




\begin{tabular}{|c|c|c|c|c|c|c|}
\hline Fur! & $\begin{array}{r}453 \pi \\
265.0\end{array}$ & 0.5 & 5.5 & $\begin{array}{l}Y \subset L \\
-3 \pm 0\end{array}$ & & \\
\hline Coms: & $C F$ & .01 & 0.00 & $\begin{array}{c}C M 5 \\
.00\end{array}$ & $\begin{array}{l}C M Y \\
.01\end{array}$ & $\begin{array}{l}C H= \\
0=\end{array}$ \\
\hline $\begin{array}{l}\text { MAS } \\
\text { SIMS } \\
\text { FMS }\end{array}$ & $\begin{array}{r}1.05 \\
.12\end{array}$ & $\begin{array}{r}.05 \\
.05 \\
.02\end{array}$ & $\begin{array}{l}10 \\
.05 \\
.02\end{array}$ & .901 &. $\begin{array}{l}20 \\
.15 \\
05\end{array}$ & .98 \\
\hline QFACT & $5 \cdot 23$ & $7 \times 49$ & $11 S+70$ & $17 \cdot 79$ & $2=.47$ & $\therefore \%$ \\
\hline
\end{tabular}

DATA FOF FILE: H3JOO

\begin{tabular}{|c|c|c|c|c|c|c|}
\hline 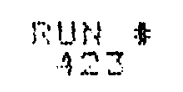 & $\begin{aligned} 4 I ! n \\
2650\end{aligned}$ & 0.5 & $A Z$ & $+\frac{V E L}{2}$ & & \\
\hline $\begin{array}{l}\text { Comf : } \\
\text { MEAH : }\end{array}$ & 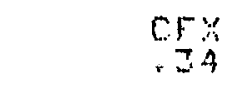 & $\begin{array}{l}C 5 y \\
.02\end{array}$ & .02 & $\begin{array}{l}\text { cro: } \\
.01\end{array}$ & CHy & $\begin{array}{c}C H 2 \\
.02\end{array}$ \\
\hline $\begin{array}{l}\text { MAY } \\
\text { MUS } \\
\text { PWS }\end{array}$ & $\begin{array}{l}1.35 \\
: 35 \\
21\end{array}$ & $\begin{array}{r}.04 \\
.12 \\
.02\end{array}$ & $\begin{array}{l}13 \\
.04 \\
0.5\end{array}$ & .05 & $\begin{array}{l}4 \\
31 \\
30 \\
05\end{array}$ & .02 \\
\hline GFACT & $4 \cdot 91$ & $4 \cdot 50$ & $5 \cdot 73$ & $\stackrel{5}{5} .5$ & 15.58 & 9.38 \\
\hline
\end{tabular}

IATA FOF FILE: 13300

laup 2050

E.L

5. AZ $\quad 42 . \mathrm{L}$

GOME:

cFy

C.2.

.00

$\cos ^{2} \quad$ criz

MAS:
Ming:

.05
-.10
.05

10
$: 0.2$
.02

.52

.02

PFACT:

275

$3 \cdot 56$

12.00

i. 30

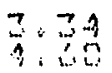

IATA FOF FILE : H3ZOO

\begin{tabular}{|c|c|c|c|c|c|c|}
\hline V:urst & $\begin{array}{l}\text { WJII } \\
2: 5.0\end{array}$ & 0.0 & 42 & 92.9 & & \\
\hline Come & $\frac{C . F}{94}$ & $\begin{array}{l}\text { CFy } \\
\cdots .0 ?\end{array}$ & ${ }_{0}^{c}=$ & .01 & $\begin{array}{l}\text { CHYY } \\
.05\end{array}$ & $\begin{array}{l}c y z \\
0.5\end{array}$ \\
\hline $\begin{array}{l}\text { MAX } \\
\text { MIHS } \\
\text { GHS }\end{array}$ & $\begin{array}{l}2.37 \\
01 \\
34\end{array}$ & 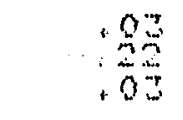 & $\begin{array}{l}.09 \\
.05 \\
.02\end{array}$ & .08 & $\begin{array}{r}50 \\
.50 \\
.14\end{array}$ & $\begin{array}{l}0.9 \\
.02 \\
.02\end{array}$ \\
\hline $\begin{array}{l}\text { GFACT } \\
\text { FPACT }\end{array}$ & $\frac{2}{4} \cdot \frac{1}{5}$ & $\begin{array}{l}3.00 \\
4.23\end{array}$ & $\begin{array}{l}7+43 \\
2 \cdot 21\end{array}$ & $\frac{5}{3} \cdot 9$ & $1 \frac{1}{7} \cdot 1 \frac{1}{4}$ & $\hat{\imath} \cdot \overrightarrow{3}=$ \\
\hline
\end{tabular}


IATA FOE FILE: H3300

\begin{tabular}{|c|c|c|c|c|c|c|}
\hline nun & $\begin{array}{l}\text { WIHO } \\
265.0\end{array}$ & 0.5 & 5.0 & $42 \frac{y}{2}-\frac{1}{7}$ & & \\
\hline $\begin{array}{c}\text { COMF } \\
\text { HEAH }\end{array}$ & CF\% & $\begin{array}{l}\text { CFY } \\
.09\end{array}$ & $\begin{aligned} C P & \\
-.02 & \end{aligned}$ & CHS & CyY & $\cos =$ \\
\hline $\begin{array}{l}\text { MAY } \\
\text { Ming } \\
\text { FHS }\end{array}$ & 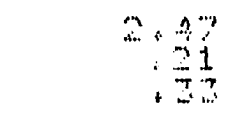 & $\begin{array}{r}.02 \\
.00 \\
.08\end{array}$ & .07 & $\begin{array}{l}+05 \\
.04 \\
+01\end{array}$ & $\begin{array}{r}.50 \\
.90 \\
.12\end{array}$ & $\begin{array}{l}.03 \\
.10 \\
.02\end{array}$ \\
\hline $\begin{array}{l}\text { FACT } \\
\text { FACT }\end{array}$ & $\frac{2}{4} \cdot 21$ & $\frac{3}{3} \cdot 3$ & $\begin{array}{l}3 \\
2 \\
2\end{array}$ & 㞻.5: & $\frac{698}{9} .99$ & 3.20 \\
\hline
\end{tabular}

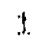


Heliostat 3, Configuration 3-D

\begin{tabular}{lccccc}
\hline Case & Run & WD & EL & AZ & $\begin{array}{l}\text { \# Rows } \\
\text { Upstream }\end{array}$ \\
\hline Ver. Stow & 424 & 265 & 0 & 5 & 5 \\
& 429 & 265 & 0 & 5 & 3 \\
& 432 & 265 & 0 & 5 & 2 \\
& 435 & 265 & 0 & 5 & 1 \\
\hline
\end{tabular}




\begin{tabular}{|c|c|c|c|c|c|c|}
\hline ling & $\begin{array}{r}\text { WIHI } \\
235.0\end{array}$ & $\begin{array}{r}5 . \\
0.0\end{array}$ & 5.0 & บㄷ․ & & \\
\hline cons : & 65 & $\begin{array}{l}\text { cry } \\
.05\end{array}$ & .01 & $\begin{array}{l}\text { CKS } \\
.00\end{array}$ & CH: & $\begin{array}{c}\cos \\
.03\end{array}$ \\
\hline 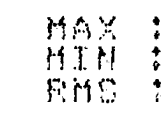 & $1: 90$ & $\begin{array}{r}.05 \\
.125 \\
.02\end{array}$ & $\begin{array}{r}10 \\
69 \\
02\end{array}$ & $\begin{array}{l}.05 \\
.04 \\
.01\end{array}$ & $\begin{array}{r}59 \\
.58 \\
.15\end{array}$ & .02 \\
\hline QFACT & 3.75 & $\begin{array}{l}3.50 \\
4.40\end{array}$ & $\begin{array}{l}12.00 \\
4.04\end{array}$ & 12.07 & 2.30 & $\begin{array}{r}3.3 \\
4.50\end{array}$ \\
\hline
\end{tabular}

\begin{tabular}{|c|c|c|c|c|c|c|}
\hline nust & $\begin{array}{r}4 I \$ D \\
265.0\end{array}$ & 0.5 & $5 . \hat{Z}$ & $\frac{461}{2}$ & & \\
\hline WEMP & $.5 \%$ & $\begin{array}{l}C .5 Y \\
.04\end{array}$ & 0.01 & .01 & $\begin{array}{l}\text { CHY } \\
.02\end{array}$ & $\frac{C H z}{.01}$ \\
\hline $\begin{array}{l}\text { SASE } \\
\text { NATH } \\
\text { Fiss }\end{array}$ & $\begin{array}{r}1.50 \\
: 55 \\
+25\end{array}$ & $\begin{array}{l}.08 \\
.02 \\
.05\end{array}$ & $\begin{array}{l}10 \\
.07 \\
.02\end{array}$ & $\begin{array}{l}.07 \\
.03 \\
.02\end{array}$ & $\begin{array}{l}50 \\
.56 \\
.12\end{array}$ & $\begin{array}{l}.05 \\
.05 \\
.02\end{array}$ \\
\hline $\begin{array}{l}\text { QFACT } \\
\text { FFCT }\end{array}$ & $\frac{2}{4} \cdot 2 \cdot 4$ & 5.23 & $15: 58$ & $\begin{array}{l}3 \\
3 \\
2 \\
75\end{array}$ & $17: 50$ & 10.12 \\
\hline
\end{tabular}

IATA FOF: FILE: HZ4OO

\begin{tabular}{|c|c|c|c|c|c|c|}
\hline xun & $\begin{array}{r}4 I H I \\
235.0\end{array}$ & 0.0 & 5.0 & $\begin{array}{l}\text { VEL. } \\
+2.5\end{array}$ & & \\
\hline $\begin{array}{l}\text { COMF } \\
\text { BEAH }\end{array}$ & $.5 \%$ & $\begin{array}{l}05 y \\
.05\end{array}$ & $\begin{array}{c}65 \\
.07\end{array}$ & .01 & $\begin{array}{l}\mathrm{Cry} \\
.03\end{array}$ & ${ }_{0}^{C M}=$ \\
\hline 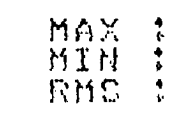 & $\begin{array}{l}1.00 \\
\cdots \\
+15\end{array}$ & $\begin{array}{r}.05 \\
.15 \\
.05\end{array}$ & $\begin{array}{r}.12 \\
-02 \\
.02\end{array}$ & $\begin{array}{l}+04 \\
.04 \\
.01\end{array}$ & +12 & .07 \\
\hline GFACY & 2.21 & $\begin{array}{l}3 \cdot 44 \\
4 \cdot 10\end{array}$ & 3.30 & 2.34 & $\begin{array}{r}17.17 \\
4+00\end{array}$ & $3+5$ \\
\hline
\end{tabular}

MATA FOF FILE:

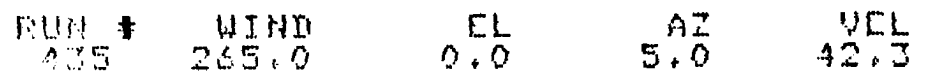

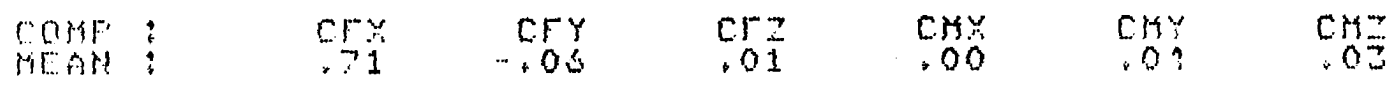

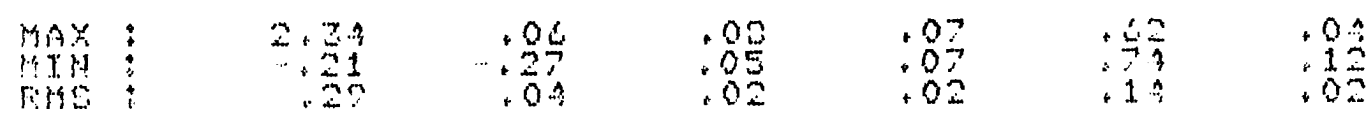

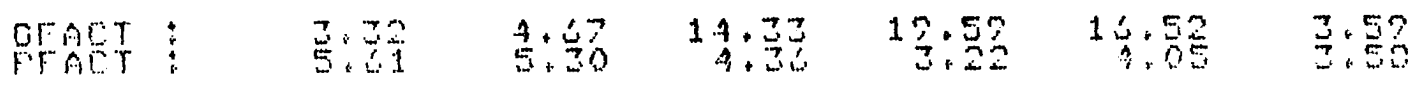


Heliostat 3, Configuration 3-E

\begin{tabular}{|c|c|c|c|c|c|}
\hline Case & Run & WD & EL & $\mathrm{A} Z$ & $\begin{array}{l}\text { If Rows } \\
\text { Upstream }\end{array}$ \\
\hline \multirow[t]{4}{*}{ Ver. Stow } & 427 & 265 & 0 & 5 & 5 \\
\hline & 430 & 265 & 0 & 5 & 3 \\
\hline & 433 & 265 & 0 & 5 & 2 \\
\hline & 436 & 265 & 0 & 5 & 1 \\
\hline
\end{tabular}


WATA FOF FILE: 11:101

\begin{tabular}{|c|c|c|c|c|c|c|c|}
\hline $\lim _{x \rightarrow 7}$ & & $\begin{array}{l}W I+15 \\
235.0\end{array}$ & $0: 0$ & $=\frac{A 2}{5.0}$ & $4: \frac{V L}{0}$ & & \\
\hline $\begin{array}{l}\text { cors } \\
\text { mints }\end{array}$ & $\vdots$ & 0.5 & $.5 y$ & $\operatorname{cr}_{0} \frac{7}{2}$ & $\begin{array}{l}\mathrm{CH} \\
.00\end{array}$ & $\begin{array}{l}C x y \\
.05\end{array}$ & $\begin{array}{r}C H z \\
0=\end{array}$ \\
\hline 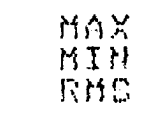 & $\vdots$ & 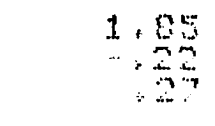 & $\begin{array}{r}.95 \\
.20 \\
.05\end{array}$ & $\begin{array}{l}.07 \\
.02 \\
.02\end{array}$ & $\begin{array}{l}.05 \\
.07 \\
.01\end{array}$ & $\begin{array}{r}59 \\
549 \\
+12 \\
+12\end{array}$ & $\begin{array}{l}+05 \\
+12 \\
.02\end{array}$ \\
\hline PACT & $\vdots$ & $\begin{array}{l}7.46 \\
4: 56\end{array}$ & $4 \div \frac{10}{4}$ & $\vec{z} \cdot \frac{21}{0}$ & $7 \underset{3}{3}+2$ & 10.02 & $\begin{array}{l}3.00 \\
.07\end{array}$ \\
\hline IATA & $\mathrm{FOF}$ & Fi FILE : & $H 3401$ & & & & \\
\hline$\because \frac{114}{30}$ & & $\begin{array}{r}\text { WIIII } \\
265.0\end{array}$ & $0: 5 \frac{1}{0}$ & $5^{A \frac{7}{O}}$ & 42.5 & & \\
\hline $\begin{array}{l}\because(1 S 3) \\
\text { mott }\end{array}$ & : & 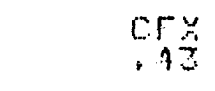 & $\begin{array}{c}\operatorname{cry} \\
.03\end{array}$ & cris & $\begin{array}{l}\text { CH: } \\
.01\end{array}$ & $\begin{array}{l}\text { EryY } \\
.01\end{array}$ & $\begin{array}{l}C M z \\
.00\end{array}$ \\
\hline $\begin{array}{l}\text { MAn } \\
\text { ring }\end{array}$ & $\vdots$ & 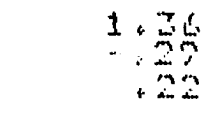 & $\begin{array}{r}04 \\
+13 \\
.02\end{array}$ & $\begin{array}{l}11 \\
.02 \\
.02\end{array}$ & $\begin{array}{l}.05 \\
.04 \\
.01\end{array}$ & $\begin{array}{l}30 \\
.11 \\
10\end{array}$ & $\begin{array}{l}.05 \\
.05 \\
+02\end{array}$ \\
\hline $\begin{array}{l}\text { GACT } \\
\text { FACT }\end{array}$ & $\vdots$ & $\begin{array}{l}3.12 \\
4.12\end{array}$ & $\begin{array}{l}7.75 \\
4.40\end{array}$ & $\frac{5}{5}+\frac{1}{5}$ & $\frac{5}{3} \cdot 20$ & $\begin{array}{r}95 \\
3.95 \\
3\end{array}$ & 103.31 \\
\hline UATA & $\mathrm{FOF}$ & FI FILE & $H 3401$ & & & & \\
\hline$x: \frac{1}{3} x+x$ & & $\begin{array}{r}W I N 5 \\
265.0\end{array}$ & 0.5 & 5.92 & $\begin{array}{r}\text { ULL } \\
72.5\end{array}$ & & \\
\hline 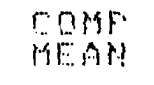 & 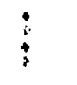 & $\begin{array}{r}C F Y \\
2.3\end{array}$ & $\begin{array}{r}\text { CFY } \\
-.02\end{array}$ & $\begin{array}{l}\cos \\
+01\end{array}$ & $\begin{array}{l}\text { CH: } \\
.00\end{array}$ & $\begin{array}{l}\text { CMY } \\
: 01\end{array}$ & c.siz \\
\hline 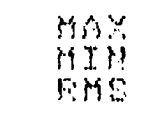 & $\vdots$ & $\begin{array}{r}.9 \\
71 \\
.14\end{array}$ & $\begin{array}{r}.05 \\
+10 \\
.02\end{array}$ & $\begin{array}{l}.10 \\
.07 \\
.02\end{array}$ & $\begin{array}{r}.04 \\
.05 \\
.01\end{array}$ & . & $\begin{array}{l}.05 \\
.05 \\
.02\end{array}$ \\
\hline $\begin{array}{l}\text { GFACT } \\
\text { FFACT }\end{array}$ & $\vdots$ & $\begin{array}{l}7 \cdot 22 \\
4 \cdot 16\end{array}$ & $\frac{6}{3} \cdot \frac{17}{8}$ & 17.44 & 173.01 & $\begin{array}{r}2.45 \\
4.00\end{array}$ & $4+51$ \\
\hline
\end{tabular}

IATA FOR FILE: H3AOI

\begin{tabular}{|c|c|c|c|c|c|c|c|}
\hline Fillst & & $\begin{array}{r}\text { WINII } \\
265.0\end{array}$ & 0.5 & $\begin{array}{l}A Z \\
.0\end{array}$ & $\frac{V E L}{2.7}$ & & \\
\hline COKF & $:$ & $\begin{array}{l}\text { CFY } \\
+16\end{array}$ & $\begin{array}{r}\text { CFY } \\
. .02\end{array}$ & $\begin{array}{c}\operatorname{cog}^{2} \\
.02\end{array}$ & $\begin{array}{l}\text { CMY } \\
: 00\end{array}$ & $\begin{array}{l}\cos 5 \\
.03\end{array}$ & $\begin{array}{l}\text { Crf } \\
.00\end{array}$ \\
\hline $\begin{array}{l}\text { MAX } \\
\text { MISE }\end{array}$ & $\vdots$ & 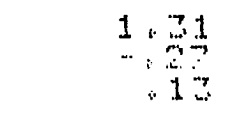 & $\begin{array}{r}.03 \\
.12 \\
.02\end{array}$ & $\begin{array}{l}+09 \\
.05 \\
.02\end{array}$ & $\begin{array}{l}.04 \\
.05 \\
.01\end{array}$ & $\begin{array}{l}45 \\
+79 \\
+3\end{array}$ & $\begin{array}{l}+0 A \\
+04 \\
+01\end{array}$ \\
\hline $\begin{array}{l}F A C T \\
F A C: T\end{array}$ & 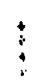 & $\begin{array}{l}5 \cdot 5 \\
0 \cdot 5 \cdot\end{array}$ & $\begin{array}{l}5.00 \\
5.69\end{array}$ & $\begin{array}{l}3.03 \\
3.05\end{array}$ & $\begin{array}{r}14.03 \\
3+50\end{array}$ & $1 \stackrel{4}{5}: 45$ & 29.53 \\
\hline
\end{tabular}


Heliostat 3 , Configuration $3-\mathrm{F}$

\begin{tabular}{lccccc}
\hline Case & Run & WD & EL & AZ & $\begin{array}{l}\text { \# Rows } \\
\text { Upstream }\end{array}$ \\
\hline Ver. Stow & 428 & 265 & 0 & 5 & 5 \\
& 431 & 265 & 0 & 5 & 3 \\
& 434 & 265 & 0 & 5 & 2 \\
& 265 & 0 & 5 & 1
\end{tabular}




\begin{tabular}{|c|c|c|c|c|c|c|}
\hline Funs & $\begin{array}{r}4 \mathrm{~J}+\mathrm{n} \\
255.0\end{array}$ & 0.0 & 5.0 & $\begin{array}{l}\text { VEL } \\
-74.0\end{array}$ & & \\
\hline Come & 65 & $\begin{array}{l}C r y \\
.04\end{array}$ & $.0 \frac{2}{2}$ & $\begin{array}{l}.035 \\
.00\end{array}$ & $\begin{array}{l}\text { CMY } \\
.05\end{array}$ & $\begin{array}{cc}\mathrm{Cm} z \\
.02\end{array}$ \\
\hline $\begin{array}{l}\text { MAX } \\
\text { MIM } \\
\text { FMs: }\end{array}$ & $\begin{array}{l}1 .-47 \\
-11 \\
0.25\end{array}$ & $\begin{array}{l}.05 \\
.15 \\
.05\end{array}$ & .05 & $\begin{array}{l}.04 \\
.07 \\
.01\end{array}$ & $: \frac{1}{3}=$ & $\begin{array}{l}.05 \\
.10 \\
.02\end{array}$ \\
\hline $\begin{array}{l}\text { GFACT } \\
\text { FFACT }\end{array}$ & $\frac{2}{4} \cdot 99$ & $3+74$ & $\begin{array}{l}3.55 \\
3.95\end{array}$ & $23 \cdot 25$ & $1 \underset{z}{4}: \frac{10}{10}$ & A. \\
\hline
\end{tabular}

IIATA FOF FILE:

\begin{tabular}{|c|c|c|c|c|c|c|}
\hline $\operatorname{lin}_{431} \neq$ & $\begin{array}{r}4 I M I \\
235.0\end{array}$ & $\begin{array}{c}E L \\
0.0\end{array}$ & $\begin{array}{l}A Z \\
0\end{array}$ & $\begin{array}{r}\text { UEL } \\
42 . ?\end{array}$ & & \\
\hline $\begin{array}{l}\text { COMF" } \\
\text { MEASt : }\end{array}$ & 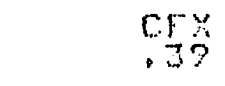 & $\begin{array}{r}\operatorname{CFY} \\
-.03\end{array}$ & $\int_{01}^{c}$ & $\begin{array}{l}\text { CHX } \\
.01\end{array}$ & cry & $\begin{array}{l}C H z \\
.02\end{array}$ \\
\hline $\begin{array}{l}\text { MAX } \\
\text { MAS } \\
\text { FHS }\end{array}$ & $\begin{array}{r}1.31 \\
-+207 \\
+21\end{array}$ & $\begin{array}{r}.05 \\
-102\end{array}$ & $\begin{array}{r}.00 \\
.00 \\
.02\end{array}$ & $\begin{array}{r}.05 \\
.04 \\
.01\end{array}$ & $\begin{array}{l}+\frac{A}{A} \\
+3.7 \\
.0 ?\end{array}$ & $\begin{array}{l}.03 \\
.03 \\
.02\end{array}$ \\
\hline $\begin{array}{l}\text { QFACT } \\
\text { FFACT }\end{array}$ & $3: 35$ & $5 \cdot 20$ & $3 \cdot 2 \frac{2}{10}$ & 4.17 & 15.80 & $5 \cdot \frac{1}{4}$ \\
\hline
\end{tabular}

IIATA FOF FILE : HZAO2

\begin{tabular}{|c|c|c|c|c|c|c|}
\hline Siut & $\begin{array}{r}\text { WIWn } \\
265.0\end{array}$ & $\begin{array}{c}E L \\
0: 0\end{array}$ & $\begin{array}{ll}\mathrm{AL}_{2} \\
10\end{array}$ & $\begin{array}{r}v[1 \\
42.5\end{array}$ & & \\
\hline $\begin{array}{l}\text { ConF } \\
\text { MEAN : }\end{array}$ & 25 & $\begin{array}{r}\text { CFY } \\
.01\end{array}$ & .01 & $\begin{array}{l}\text { CHs } \\
.02\end{array}$ & $\begin{array}{l}\text { CrgY } \\
.01\end{array}$ & $\begin{array}{l}C M I \\
.01\end{array}$ \\
\hline $\begin{array}{l}\text { MAX } \\
\text { HISI } \\
\text { Risti: }\end{array}$ & $\begin{array}{r}1+\frac{1}{2} \\
: \frac{1}{2}\end{array}$ & $\begin{array}{r}.06 \\
.10 \\
.02\end{array}$ & $\begin{array}{l}.09 \\
.04 \\
.02\end{array}$ & $\begin{array}{l}.07 \\
.04 \\
.01\end{array}$ & . & $\begin{array}{l}.05 \\
.05 \\
.01\end{array}$ \\
\hline $\begin{array}{l}\text { GFACT: } \\
\text { FFACT }\end{array}$ & 5.25 & 10.05 & $\begin{array}{l}9.40 \\
2.91\end{array}$ & 3.31 & 35.75 & $\begin{array}{c}5.02 \\
3.52\end{array}$ \\
\hline
\end{tabular}

IATA FOF FILE: $\mathrm{H3} 302$

\begin{tabular}{|c|c|c|c|c|c|c|}
\hline $\operatorname{lom}_{9 \rightarrow 3}=$ & $\begin{array}{r}\text { WIHI } \\
255.0\end{array}$ & 0.1 & 5.0 & $\begin{array}{r}\text { YEL } \\
42.2\end{array}$ & & \\
\hline $\begin{array}{l}\text { ComF : } \\
\text { MEAM : }\end{array}$ & $\begin{array}{c}c 5 x \\
.17\end{array}$ & $\begin{array}{l}\text { CFY } \\
.01\end{array}$ & $\begin{array}{l}\mathrm{CF} \\
.00\end{array}$ & $\begin{array}{l}\text { CMx } \\
.02\end{array}$ & $\begin{array}{l}\text { CHY } \\
.01\end{array}$ & $\begin{array}{c}\cos 2 \\
.01\end{array}$ \\
\hline 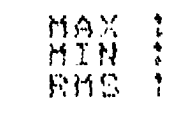 & .92 & $\begin{array}{r}.02 \\
.02 \\
.02\end{array}$ & .07 & $\begin{array}{l}.84 \\
.04 \\
.01\end{array}$ & $\begin{array}{r}-41 \\
+35 \\
.05\end{array}$ & $\begin{array}{r}.05 \\
.05 \\
.01\end{array}$ \\
\hline GFACT & $\frac{5}{5} \cdot 30$ & 10.07 & 12.71 & $\begin{array}{l}3.50 \\
3.21\end{array}$ & $=0.07$ & $7+\frac{1}{9} 7$ \\
\hline
\end{tabular}




\begin{tabular}{|c|c|c|c|c|c|}
\hline Case & Run & WD & $\mathrm{EL}$ & $\mathrm{AZ}$ & $\begin{array}{l}\text { \# Rows } \\
\text { Upstream }\end{array}$ \\
\hline \multirow[t]{4}{*}{ Ver. Stow } & 493 & 265 & 0 & 5 & 5 \\
\hline & 496 & 265 & 0 & 5 & 3 \\
\hline & 497 & 265 & 0 & 5 & 2 \\
\hline & 501 & 265 & 0 & 5 & 1 \\
\hline
\end{tabular}




\begin{tabular}{|c|c|c|c|c|c|c|}
\hline$\underset{90}{9}$ & $\begin{array}{r}\text { WISI } \\
255.0\end{array}$ & $\begin{array}{cl}E L \\
0.0\end{array}$ & $5: 0$ & $\begin{array}{l}\cup C L \\
12.7\end{array}$ & & \\
\hline $\begin{array}{l}\text { morsF : } \\
\text { mEnt: }\end{array}$ & $C F$ & $\begin{array}{l}\text { Cry } \\
.00\end{array}$ & $\begin{array}{l}\mathrm{Cr}-\mathrm{y} \\
.00\end{array}$ & .01 & ${ }_{0}^{C M Y}$ & $.0 \%$ \\
\hline $\begin{array}{l}\text { max } \\
\text { ristis } \\
\text { rist }\end{array}$ & $\begin{array}{r}1.55 \\
.95 \\
.25\end{array}$ & $\begin{array}{l}.00 \\
.03 \\
.02\end{array}$ & $\begin{array}{l}.00 \\
.03 \\
.01\end{array}$ & .07 & .92 & $\begin{array}{l}.05 \\
.05 \\
.05\end{array}$ \\
\hline GFACY & $4:-28$ & $\begin{array}{r}27 \\
4 \\
4\end{array}$ & $\begin{array}{r}352.30 \\
5.77\end{array}$ & $\stackrel{4}{4}: \frac{25}{6}$ & $\begin{array}{r}25.74 \\
7.54\end{array}$ & $2 \frac{2}{7} \cdot 07$ \\
\hline $\operatorname{IATA} F$ & IF FILE : & H34OS & & & & \\
\hline $\operatorname{lum}_{60}$ & $\begin{aligned} & \text { HIHI } \\
= & 65.0\end{aligned}$ & 0.0 & 5.92 & $\begin{array}{r}\text { YCL } \\
2.5\end{array}$ & & \\
\hline $\begin{array}{l}\text { COHF : } \\
\text { MEAN : }\end{array}$ & $6 \%$ & $\begin{array}{l}.05 \\
.00\end{array}$ & $\operatorname{cr}$ & $\begin{array}{l}\operatorname{crs} \\
.02\end{array}$ & $\begin{array}{l}\text { CMY } \\
.0 O\end{array}$ & $\cos$ \\
\hline $\begin{array}{l}\text { MAY } \\
\text { MIU: } \\
\text { FMS }\end{array}$ & $\begin{array}{r}1+39 \\
-28 \\
2.8\end{array}$ & $\begin{array}{r}.00 \\
.07 \\
.02\end{array}$ & $\begin{array}{l}.04 \\
.04 \\
.01\end{array}$ & $\begin{array}{l}.07 \\
.05 \\
.02\end{array}$ & $\begin{array}{l}+45 \\
+32 \\
+11\end{array}$ & $\begin{array}{l}.05 \\
.05 \\
.01\end{array}$ \\
\hline $\begin{array}{l}\text { gFACT } \\
\text { HACT }\end{array}$ & 4.57 & $\begin{array}{r}326 \\
3.53\end{array}$ & $\begin{array}{l}5.47 \\
3.50\end{array}$ & $\begin{array}{l}5.05 \\
3.95 \\
3.95\end{array}$ & $109 \div \frac{23}{7}$ & 崖: \\
\hline
\end{tabular}

DATA FOE FILE: H3AOS

\begin{tabular}{|c|c|c|c|c|c|c|}
\hline $\operatorname{lom}_{9}$ & $\begin{array}{l}\text { WIPI } \\
=65.0\end{array}$ & 5.0 & $\begin{array}{r}A Z \\
5.0\end{array}$ & $\begin{array}{c}4[L \\
42.5\end{array}$ & & \\
\hline $\begin{array}{l}\text { MPSF : } \\
\text { MEAM : }\end{array}$ & $\begin{array}{cc}C F \\
-2 \%\end{array}$ & $\begin{array}{l}\text { Cry } \\
.00\end{array}$ & $\begin{array}{l}\mathrm{cr} z \\
+00\end{array}$ & $\begin{array}{l}C M \% \\
.01\end{array}$ & cmy & Cris \\
\hline 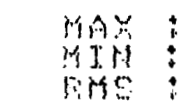 & $\begin{array}{r}1.42 \\
: 47 \\
: 21\end{array}$ & $\begin{array}{r}.00 \\
.08 \\
.02\end{array}$ & $\begin{array}{l}.97 \\
.03 \\
.01\end{array}$ & $\begin{array}{l}.04 \\
.06 \\
.02\end{array}$ & $\begin{array}{r}+1 \\
+42 \\
+11\end{array}$ & $\begin{array}{l}.02 \\
.10 \\
.02\end{array}$ \\
\hline $\begin{array}{l}\text { OFACT } \\
\text { FFACT }\end{array}$ & 6.74 & $\begin{array}{r}79.99 \\
2+41\end{array}$ & $\begin{array}{r}100 \\
5+12\end{array}$ & $\begin{array}{l}7.49 \\
2.30\end{array}$ & $\begin{array}{r}24.47 \\
3 \cdot 20\end{array}$ & $\begin{array}{l}3.53 \\
4+24\end{array}$ \\
\hline MATA FC & OF: FILE : & Hב 305 & & & & \\
\hline $\operatorname{mol}_{501}$ & $\begin{array}{l}W I H I \\
285.0\end{array}$ & 0.5 & 5.0 & $\begin{array}{l}\text { VCL } \\
.2 .5\end{array}$ & & \\
\hline $\begin{array}{l}\text { Corst: } \\
\text { MEAst }\end{array}$ & 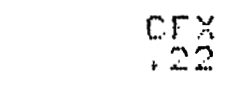 & $\begin{array}{l}\mathrm{CFY}^{2} \\
.00\end{array}$ & $\begin{array}{c}c r z \\
.02\end{array}$ & $\begin{array}{l}\text { C.M. } \\
.00\end{array}$ & $\begin{array}{l}\text { CMY } \\
.01\end{array}$ & .02 \\
\hline 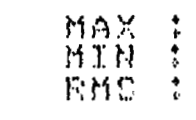 & $\begin{array}{l}1.02 \\
\cdot 10 \\
\cdot 10\end{array}$ & $\begin{array}{l}.06 \\
.03 \\
.02\end{array}$ & $\begin{array}{r}.06 \\
-.05 \\
.02\end{array}$ & $\begin{array}{l}.05 \\
.04 \\
.01\end{array}$ & $\begin{array}{l}37 \\
83 \\
: 10 \\
10\end{array}$ & $\begin{array}{l}.02 \\
.07 \\
.01\end{array}$ \\
\hline $\begin{array}{l}\text { OFACT } \\
\text { FFACT }\end{array}$ & 4.59 & 17.790 & $\frac{2}{2} \cdot 74$ & 10.27 & $\begin{array}{r}4750 \\
7.59\end{array}$ & $\frac{3}{5} \cdot \frac{1}{2}$ \\
\hline
\end{tabular}


Heliostat 3 , Configuration $3-\mathrm{H}$

\begin{tabular}{llllll}
\hline Case & Run & WD & EL & AZ & $\begin{array}{l}\text { \# Rows } \\
\text { Upstratm }\end{array}$ \\
Ver. Stow & 494 & 265 & 0 & 5 & 5 \\
& 495 & 265 & 0 & 5 & 3 \\
& 498 & 265 & 0 & 5 & 2 \\
& 499 & 265 & 0 & 5 & 1
\end{tabular}


MATA FOF FILE: HZ3OS

\begin{tabular}{|c|c|c|c|c|c|c|}
\hline Fus? & $\begin{aligned} & \text { WIHI } \\
= & 65.0\end{aligned}$ & 0.0 & 5.0 & $42 \div 5$ & & \\
\hline $\begin{array}{l}\text { COMF: } \\
\text { MEAM : }\end{array}$ & $\begin{array}{c}c 5 x \\
258\end{array}$ & $\begin{array}{l}\text { Cry } \\
.00\end{array}$ & .05 & $\begin{array}{l}\text { CMY } \\
.01\end{array}$ & $\begin{array}{l}\text { Cry } \\
.01\end{array}$ & .5 \\
\hline 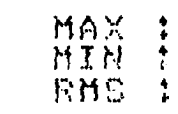 & $\begin{array}{r}1 .-35 \\
455 \\
.25\end{array}$ & $\begin{array}{l}.05 \\
.07 \\
.02\end{array}$ & $\begin{array}{l}.07 \\
.05 \\
.01\end{array}$ & $\begin{array}{l}.02 \\
.05 \\
.01\end{array}$ & : & \\
\hline FACT & 5.23 & $\begin{array}{r}27.50 \\
9.02\end{array}$ & 坮. & $\begin{array}{l}5.50 \\
4.90\end{array}$ & $A \frac{1}{5} \cdot \frac{2}{2}$ & 5 \\
\hline
\end{tabular}

IATA FOR FILE: H3406

\begin{tabular}{|c|c|c|c|c|c|c|}
\hline 944 & $\begin{array}{r}45 \% \pi \\
255.0\end{array}$ & EL & 5.0 & $\begin{array}{l}\text { VᄃL } \\
72.9\end{array}$ & & \\
\hline $\begin{array}{l}\text { comp : } \\
\text { riEnH }\end{array}$ & $\begin{array}{c}C^{2} \\
.26\end{array}$ & $\begin{array}{l}\text { cry } \\
.00\end{array}$ & .05 & $\begin{array}{c}\mathrm{CH} \\
.02\end{array}$ & $\begin{array}{l}\text { CMY } \\
.01\end{array}$ & .01 \\
\hline $\begin{array}{l}\text { MAX } \\
\text { MIH } \\
\text { FMS }\end{array}$ & $\begin{array}{r}1+30 \\
\cdots \\
+2\end{array}$ & $\begin{array}{r}.00 \\
.07 \\
+02\end{array}$ & $\begin{array}{l}.00 \\
.05 \\
.01\end{array}$ & $\begin{array}{l}.02 \\
.05 \\
.01\end{array}$ & $\begin{array}{l}17 \\
11 \\
+10\end{array}$ & \\
\hline $\begin{array}{l}A A C T \\
A A C T\end{array}$ & $\begin{array}{l}5.93 \\
4.50\end{array}$ & 105,34 & 10.71 & $3+3=3$ & -34.52 & \\
\hline
\end{tabular}

OATA FOF FILE: HZAOG

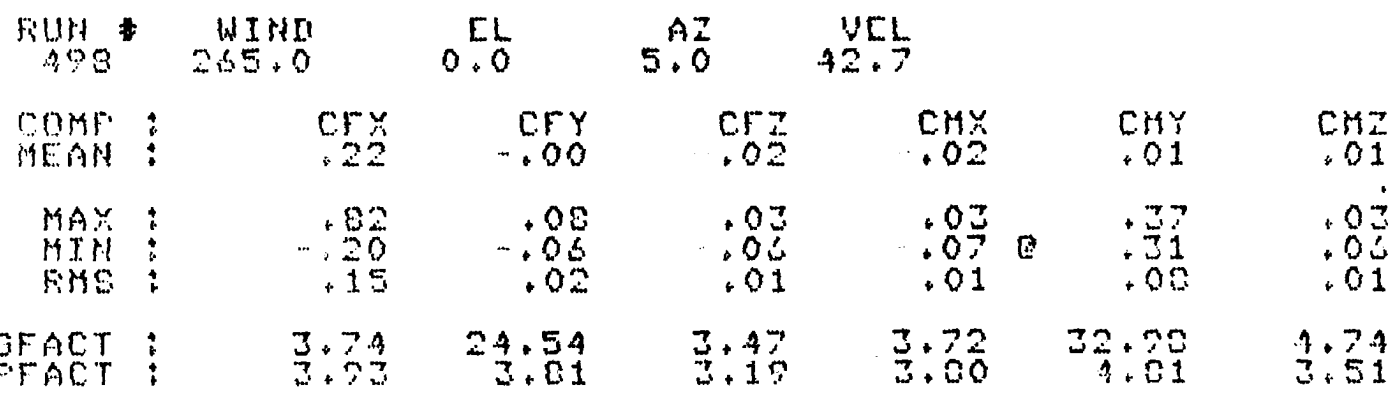

MATA FOF FILE : H3.00S

$\begin{array}{rrrr}\text { NUHHI } & E L & A Z & \text { ULL } \\ 235.0 & 0.0 & 5.0 & 42.5\end{array}$

\begin{tabular}{|c|c|c|c|c|c|}
\hline $\begin{array}{l}\text { COHF : } \\
\text { MEAS : }\end{array}$ & $\begin{array}{l}\text { CFY } \\
23\end{array}$ & $\begin{array}{l}\text { CFy } \\
. .00\end{array}$ & 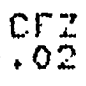 & $\begin{array}{l}\text { C.HE } \\
.02\end{array}$ & $\begin{array}{l}\text { crsy } \\
.00\end{array}$ \\
\hline $\begin{array}{l}\text { MAY : } \\
\text { MIHS } \\
\text { FMS: }\end{array}$ & 93 & $\begin{array}{r}.07 \\
.09 \\
.02\end{array}$ & $\begin{array}{r}.04 \\
.06 \\
.01\end{array}$ & $\begin{array}{l}.03 \\
.02 \\
.02\end{array}$ & $\begin{array}{r}40 \\
: 39 \\
10 \\
10\end{array}$ \\
\hline
\end{tabular}

GFACT: $\quad 4.97$ 43.85 栗 
Heliostat 3, Configuration 3-I

\begin{tabular}{lllll}
\hline Case & Run & WD & EL & AZ \\
\hline Ver. Stow & 432 & 265 & 0 & 5 \\
& 439 & 247.5 & 0 & 5 \\
& 440 & 270 & 0 & 5 \\
441 & 292.5 & 0 & 5 \\
442 & 315 & 0 & 5 \\
& 443 & 337.5 & 0 & 5 \\
& 444 & 0 & 0 & 5 \\
\hline
\end{tabular}


IRTA FOR FILE: 135500

\begin{tabular}{|c|c|c|c|c|c|c|}
\hline $\operatorname{lims}_{92}=$ & $\begin{array}{r}\text { 4INn } \\
265.0\end{array}$ & 0.5 & 5.0 & $\begin{array}{l}\text { VCL } \\
72.5\end{array}$ & & \\
\hline $\begin{array}{l}\text { COMS } \\
\text { MEAN }\end{array}$ & 65 & cry & .04 & $\begin{array}{l}\text { CHy } \\
.01\end{array}$ & $\begin{array}{l}\mathrm{CHY} \\
.03\end{array}$ & $\begin{array}{l}C M z \\
.03\end{array}$ \\
\hline $\begin{array}{l}\text { MAX } \\
\text { HMS } \\
\text { nMs }\end{array}$ & $\begin{array}{r}1.40 \\
-15 \\
.25\end{array}$ & $\begin{array}{l}.05 \\
.13 \\
.03\end{array}$ & $\begin{array}{r}17 \\
.02 \\
.02\end{array}$ & $\begin{array}{l}.04 \\
.04 \\
.01\end{array}$ & +41 & .03 \\
\hline $\begin{array}{l}\text { GFACT } \\
\text { FFACT }\end{array}$ & 2.31 & $\begin{array}{l}3: 44 \\
4: 10\end{array}$ & 3.30 & $? \cdot 3 \frac{1}{3}$ & $17: 170$ & $3: 5$ \\
\hline
\end{tabular}

IDATA FOF FILE: H3500

\begin{tabular}{|c|c|c|c|c|c|c|}
\hline fills & $24 \frac{41 \pi}{24}$ & ${ }_{0 .}^{E L}$ & 5.0 & 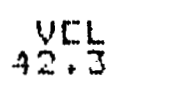 & & \\
\hline GOME : & .98 & $\begin{array}{c}c r y \\
.05\end{array}$ & .02 & $\begin{array}{l}c \mu x \\
.02\end{array}$ & .05 & $\begin{array}{l}C M Z \\
.01\end{array}$ \\
\hline $\begin{array}{l}\text { MAX } \\
\text { MIM } \\
\text { FMS: }\end{array}$ & $\begin{array}{r}2 \cdot 18 \\
+15 \\
+28\end{array}$ & $\begin{array}{r}.07 \\
.07 \\
.02\end{array}$ & .02 & $\begin{array}{l}.05 \\
.02 \\
.01\end{array}$ & 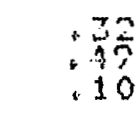 & $\begin{array}{l}.85 \\
.02 \\
.01\end{array}$ \\
\hline $\begin{array}{l}\text { QFACT } \\
\text { PFACT }\end{array}$ & $2 \cdot 23$ & $\begin{array}{l}2+43 \\
3+60\end{array}$ & $\frac{5}{5} \cdot 42$ & $\frac{2}{3} \cdot 0.5$ & 10.0 & $\begin{array}{l}3.03 \\
4.01\end{array}$ \\
\hline
\end{tabular}

IATA FOF FILE: H3500

\begin{tabular}{|c|c|c|c|c|c|c|}
\hline 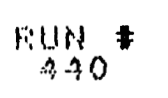 & 270.0 & 0.5 & $\mathrm{AZ}^{2}$ & $\begin{array}{r}\text { UEL } \\
+2 \cdot 2\end{array}$ & & \\
\hline COMF : & 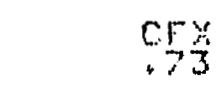 & $\begin{array}{r}\text { CFY } \\
-.0 S\end{array}$ & 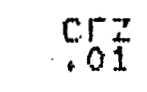 & $\begin{array}{l}\text { CHK } \\
.01\end{array}$ & cris & .04 \\
\hline $\begin{array}{l}\text { MAX } \\
\text { MIMU } \\
\text { FiMS }\end{array}$ & $\begin{array}{r}1.70 \\
+10 \\
+30\end{array}$ &.$\frac{07}{25}$ & .12 & .07 & $\begin{array}{r}54 \\
.50 \\
.14\end{array}$ & $\begin{array}{l}.0 A \\
.1 . z \\
.02\end{array}$ \\
\hline $\begin{array}{l}\text { GFACY } \\
\text { FFACT }\end{array}$ & $\frac{2}{3}: \frac{46}{5}$ & $\begin{array}{l}3.54 \\
4.13\end{array}$ & $\frac{7}{2}+43$ & 台:芜台 & $37+4 ?$ & 3.72 \\
\hline
\end{tabular}

\section{IATA FOR FILE: H3500}

\begin{tabular}{|c|c|c|c|c|c|c|}
\hline Fum & $\begin{array}{r}\text { HIH } \\
292.5\end{array}$ & 0.0 & 5.0 & $\begin{array}{r}U[1 \\
+2 \cdot 3\end{array}$ & & \\
\hline $\begin{array}{l}\text { COMF : } \\
\text { MEAH }\end{array}$ & $\begin{array}{cc}\cos \\
.93\end{array}$ & $\begin{array}{r}\text { CFY } \\
-.10\end{array}$ & $\begin{array}{c}\operatorname{cr} 2 \\
.00\end{array}$ & .02 & $\begin{array}{l}\text { Crsy } \\
.00\end{array}$ & $.0 \%$ \\
\hline $\begin{array}{l}\text { MAX } \\
\text { MIH } \\
\text { FMS }\end{array}$ & $\begin{array}{r}2.32 \\
.05 \\
.25\end{array}$ & $\begin{array}{r}.02 \\
.25 \\
.04\end{array}$ & .07 & $\begin{array}{l}.02 \\
.02 \\
.02\end{array}$ & .45 & $\begin{array}{l}.09 \\
.15 \\
.02\end{array}$ \\
\hline $\begin{array}{l}Q F A C T \\
F F A C T\end{array}$ & $\frac{2}{4} \cdot 49$ & 2.50 & $40.2 \frac{2}{3}$ & $\begin{array}{l}4.52 \\
2 \\
5\end{array}$ & $1 \frac{2}{5} \cdot 29$ & $2 \cdot 30$ \\
\hline
\end{tabular}




\begin{tabular}{|c|c|c|c|c|c|c|}
\hline $\log _{32}+2$ & $\begin{array}{l}15+1 ! \\
315.0\end{array}$ & ${ }_{0.0}^{E L}$ & $5 . \hat{A Z}$ & $\begin{array}{r}4 C L \\
42.5\end{array}$ & & \\
\hline $\begin{array}{l}\text { COHF : } \\
\text { HEAR : }\end{array}$ & $\begin{array}{c}C F \\
.43\end{array}$ & $\begin{array}{r}C 5 Y \\
-.03\end{array}$ & .02 & .02 & $\begin{array}{l}\text { CMY } \\
.00\end{array}$ & CH: \\
\hline $\begin{array}{l}\text { MAX } \\
\text { HIH } \\
\text { FHS }\end{array}$ & $\begin{array}{r}1+45 \\
+25 \\
+2 ?\end{array}$ & $\begin{array}{r}.06 \\
.04 \\
.02\end{array}$ & $\begin{array}{r}+92 \\
.02 \\
+02\end{array}$ & .04 & $\begin{array}{r}50 \\
.55 \\
.13 \\
.35\end{array}$ & .012 \\
\hline $\begin{array}{l}\text { QFACT } \\
\text { PFACT }\end{array}$ & 3.37 & $5+23$ & $3+24$ & $\overrightarrow{3}+5$ & 190.11 & $\frac{2}{3} \cdot 5$ \\
\hline IIATA F & JF FILE * & $H 3500$ & & & & \\
\hline $\operatorname{lill}_{\rightarrow \rightarrow 3}$ & $\begin{array}{l}4 I N 1 \\
357.5\end{array}$ & 0.5 & 5.9 & $\begin{array}{l}4 \div \frac{1}{9} \\
72 .\end{array}$ & & \\
\hline CoMP: & 65 & $\begin{array}{r}\text { CFY } \\
-.0 D\end{array}$ & $\operatorname{crz}_{02}$ & $\begin{array}{l}\text { CM. } \\
.00\end{array}$ & $\begin{array}{l}\text { CryY } \\
.01\end{array}$ & criz \\
\hline $\begin{array}{l}\text { MAY } \\
\text { MIM } \\
\text { FMS }\end{array}$ & $\begin{array}{r}1.45 \\
\cdot 47 \\
+25\end{array}$ & $\begin{array}{r}.02 \\
.18 \\
.05\end{array}$ & .07 & $\begin{array}{l}.05 \\
.05 \\
.05\end{array}$ & 点 & .02 \\
\hline $\begin{array}{l}\text { GFACT } \\
\text { FFACT }\end{array}$ & $\begin{array}{l}4 \cdot 97 \\
4.72\end{array}$ & 2.21 & $\frac{3}{3} \cdot \frac{45}{7}$ & $\begin{array}{r}2 \\
3.50\end{array}$ & $\begin{array}{r}97.43 \\
9.70\end{array}$ & $\begin{array}{l}2.00 \\
3.95\end{array}$ \\
\hline MATA F & DF FILE : & H350O & & & & \\
\hline $\operatorname{lin}_{44}$ & $\begin{array}{l}\text { HIHII } \\
0.0\end{array}$ & $\begin{array}{l}E L \\
0.0\end{array}$ & 5.0 & $\begin{array}{l}\text { V } \\
42 . \frac{1}{9}\end{array}$ & & \\
\hline $\begin{array}{l}\text { COMF } \\
\text { HEAN }\end{array}$ & $\begin{array}{c}C F \\
-.07\end{array}$ & $\begin{array}{l}\text { Cry } \\
+0 Z\end{array}$ & $\begin{array}{c}c . \\
.01\end{array}$ & $\begin{array}{l}\text { c.s. } \\
.02\end{array}$ & $\begin{array}{c}\text { CHy } \\
.01\end{array}$ & .00 \\
\hline $\begin{array}{l}\text { MAX: } \\
\text { MINS } \\
\text { FMS: }\end{array}$ & $\begin{array}{r}+32 \\
-12\end{array}$ & $\begin{array}{l}.02 \\
.11 \\
.02\end{array}$ & $\begin{array}{r}.0 .4 \\
.0 .4 \\
.01\end{array}$ & $\begin{array}{l}.05 \\
.02 \\
.01\end{array}$ & $\frac{2}{20}$ & $\begin{array}{l}.05 \\
.00 \\
.02\end{array}$ \\
\hline $\begin{array}{l}\text { QFACT } \\
\text { FFACT }\end{array}$ & $\begin{array}{r}39 \\
0.00\end{array}$ & $\begin{array}{l}3.35 \\
3000\end{array}$ & $\begin{array}{l}35 \\
2 \div 60\end{array}$ & 3.35 & 27.82 & $13 \cdot 35$ \\
\hline
\end{tabular}


Heliostat 3 , Configuration 3-J

\begin{tabular}{lllll}
\hline Case & Run & WD & EL & AZ \\
\hline Ver. Stow & 433 & 265 & 0 & 5 \\
& 451 & 247.5 & 0 & 5 \\
& 450 & 270 & 0 & 5 \\
& 449 & 292.5 & 0 & 5 \\
& 448 & 315 & 0 & 5 \\
& 447 & 337.5 & 0 & 5 \\
& 446 & 0 & 0 & 5 \\
\hline
\end{tabular}




\begin{tabular}{|c|c|c|c|c|c|c|}
\hline Rurst & $\begin{array}{l}\text { WIS!I } \\
20.0\end{array}$ & 0.0 & 5.2 & 4 & & \\
\hline $\begin{array}{l}\text { COMF : } \\
\text { HEAS : }\end{array}$ & cra & .05 & .01 & $\begin{array}{c}c .4 \% \\
.00\end{array}$ & $\begin{array}{l}\text { CHY } \\
.01\end{array}$ & $\begin{array}{c}c r z \\
.02\end{array}$ \\
\hline $\begin{array}{l}\text { MAX } \\
\text { MIYI } \\
\text { rise }\end{array}$ & $\begin{array}{r}.20 \\
.31 \\
.10\end{array}$ & $\begin{array}{r}.05 \\
+10 \\
+02\end{array}$ & $\begin{array}{l}10 \\
.02 \\
.02\end{array}$ & $\begin{array}{l}.04 \\
.05 \\
.01\end{array}$ & $\begin{array}{l}+30 \\
.05 \\
.05\end{array}$ & \\
\hline QFACT: & $A: \frac{2}{1}$ & $\frac{1}{3} \cdot \frac{17}{87}$ & 17.44 & 170.015 & 24.45 & 7.5 \\
\hline
\end{tabular}

\begin{tabular}{|c|c|c|c|c|c|c|}
\hline 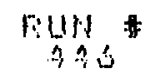 & WIHI & EL & 5.92 & $42 . \frac{1}{5}$ & & \\
\hline MOMF & $05 y$ & $\begin{array}{l}c 5 y \\
\cdots 01\end{array}$ & $\begin{array}{l}\text { cra } \\
.00\end{array}$ & $\begin{array}{l}\text { CHY } \\
.01\end{array}$ & $\begin{array}{l}\text { CHY } \\
.03\end{array}$ & $\begin{array}{l}C M z \\
.02\end{array}$ \\
\hline $\begin{array}{l}\text { MAY } \\
\text { HIYI } \\
\text { EHS }\end{array}$ & $\begin{array}{r}+40 \\
1.05 \\
+20\end{array}$ & $\begin{array}{r}.09 \\
.12 \\
.03\end{array}$ & $\begin{array}{l}.00 \\
.05 \\
.01\end{array}$ & $\begin{array}{l}.05 \\
.05 \\
.05\end{array}$ & $\begin{array}{l}+42 \\
+54 \\
+15 \\
+15\end{array}$ & $\begin{array}{r}.02 \\
.02 \\
.02\end{array}$ \\
\hline $\begin{array}{l}\text { CFACT } \\
\text { FEACT }\end{array}$ & $\begin{array}{l}7.55 \\
3.54\end{array}$ & $1 \frac{1}{3} \cdot \frac{25}{5}$ & 15.52 & $\begin{array}{l}5.05 \\
2.05\end{array}$ & $=0.52$ & \\
\hline
\end{tabular}

INATA FOF: FILE: H3501

\begin{tabular}{|c|c|c|c|c|c|c|}
\hline$\underset{37}{\operatorname{mun}}$ & $\lim _{3 \rightarrow 7}$ & EL & $A Z$ & $\begin{array}{l}\text { VEL } \\
32.7\end{array}$ & & \\
\hline CQHF : & CF & $\begin{array}{l}\text { CFY } \\
.0 \mathrm{~S}\end{array}$ & $\begin{array}{l}\mathrm{Cr} \\
.03\end{array}$ & $\begin{array}{l}\text { cris } \\
+00\end{array}$ & $\begin{array}{l}\text { CHYY } \\
.01\end{array}$ & $\begin{array}{c}\operatorname{cr} z \\
0.0\end{array}$ \\
\hline $\begin{array}{l}\text { MAX } \\
\text { MIHE } \\
\text { FHO }\end{array}$ & $\begin{array}{r}1+32 \\
1.00 \\
* 20\end{array}$ & $\begin{array}{l}10 \\
.10 \\
.03\end{array}$ & $\begin{array}{l}.03 \\
.05 \\
+01\end{array}$ & $\begin{array}{l}.96 \\
.05 \\
.05\end{array}$ & 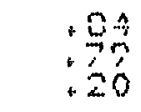 & $\begin{array}{l}.0 .1 \\
1.07 \\
.05\end{array}$ \\
\hline $\begin{array}{l}\text { QFACT } \\
\text { FFACT }\end{array}$ & 13.50 & $\frac{5}{5} \cdot 53$ & 3.67 & $1 \pm 0 \cdot \frac{9}{0}$ & 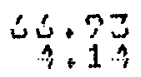 & $5 \cdot \frac{17}{37}$ \\
\hline
\end{tabular}

IATA FOF: FILE: H3501

\begin{tabular}{|c|c|c|c|c|c|c|}
\hline $\operatorname{linst}_{4 \rightarrow 3}$ & $\begin{array}{r}\text { WIW } \\
315.0\end{array}$ & 0.0 & $5: A Z$ & $\begin{array}{r}\text { VCL } \\
42.9\end{array}$ & & \\
\hline $\begin{array}{l}\text { GOMF: } \\
\text { MEAH }\end{array}$ & $\begin{array}{c}c 5 x \\
.39\end{array}$ & $\begin{array}{r}\operatorname{cry} \\
-+04\end{array}$ & $\begin{array}{l}c 5 z \\
.00\end{array}$ & crys & $\begin{array}{c}\text { CrgY } \\
.01\end{array}$ & $\operatorname{Crs}$ \\
\hline $\begin{array}{l}\text { MAE } \\
\text { HIEA } \\
\text { RME }\end{array}$ & 1. $\frac{61}{5}$ & $\begin{array}{r}.07 \\
.17 \\
+04\end{array}$ & $\begin{array}{l}.00 \\
.03 \\
.01\end{array}$ & $\begin{array}{l}.05 \\
.05 \\
.02\end{array}$ & 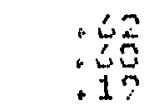 & $\begin{array}{r}07 \\
017 \\
07 \\
07\end{array}$ \\
\hline $\begin{array}{l}\text { QFACT } \\
\text { FFACT }\end{array}$ & $\begin{array}{l}4 \cdot 13 \\
7 \cdot 71\end{array}$ & $\begin{array}{l}3.94 \\
3.51\end{array}$ & $\begin{array}{r}14 \\
5.72\end{array}$ & $\frac{a}{2} \cdot 5$ & $92+0=$ & $z+1$ \\
\hline
\end{tabular}




\begin{tabular}{|c|c|c|c|c|c|c|}
\hline $\log n$ & WTMn & 50 & $5 . \frac{12}{0}$ & YCL & & \\
\hline $\operatorname{man}$ & 8 & 65 & $\mathrm{Cr}^{2}$ & $\begin{array}{l}0 r y \\
.01\end{array}$ & con & $6 \%$ \\
\hline $\begin{array}{l}\text { ny? } \\
\text { mon: }\end{array}$ & $\begin{array}{r}1.9 \\
13 \\
36\end{array}$ & $\begin{array}{l}05 \\
17 \\
.02\end{array}$ & $\begin{array}{l}.05 \\
.01\end{array}$ & .04 & 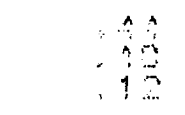 & $.0 \%$ \\
\hline orget & 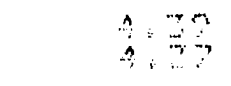 & $5: 00$ & $3.1=$ & $7 \times 29$ & 725,5 & $\hat{a}=5$ \\
\hline MATA Fo & PE FISE: & 113501 & & & & \\
\hline $\lim _{x=0}$ & $\begin{array}{r}\text { WIIT } \\
00.0\end{array}$ & 02 & 5.0 & $+2\left[\frac{1}{2}\right.$ & & \\
\hline mor & $\begin{array}{l}65 \\
20\end{array}$ & $6 r y$ & $0 \%$ & $\begin{array}{l}c r y \\
.01\end{array}$ & 60 & $\begin{array}{c}\operatorname{cm} z \\
.01\end{array}$ \\
\hline $\begin{array}{l}\text { my } \\
\text { mys }\end{array}$ & $\begin{array}{r}1.19 \\
28 \\
28\end{array}$ & .05 & $\begin{array}{l}0.4 \\
0.01 \\
0.13\end{array}$ & .04 & $\frac{\pi}{42}$ & .02 \\
\hline PFAC & 5.00 & $\begin{array}{l}5.60 \\
3.0 \%\end{array}$ & $93 \cdot 5$ & 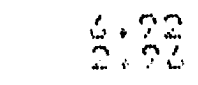 & 98.60 & 旨.9.8 \\
\hline$\because A T A \mathrm{FH}$ & IF FILE: & 43001 & & & & \\
\hline $\begin{array}{c}919 \\
94\end{array}$ & $24+2$ & $0: 0$ & $5 . \frac{A 2}{6}$ & 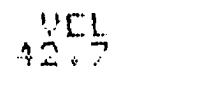 & & \\
\hline 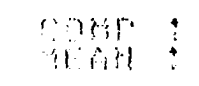 & 45 & $05 y$ & $c 5$ & .01 & 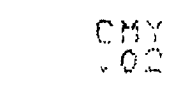 & $\cos 2$ \\
\hline 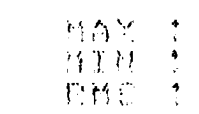 & $\begin{array}{c}1.96 \\
28\end{array}$ & $\begin{array}{r}.05 \\
.13 \\
.03\end{array}$ & $\begin{array}{l}.07 \\
.05 \\
01\end{array}$ & .0 .9 & $\begin{array}{l}10 \\
60 \\
110\end{array}$ & .09 \\
\hline gracr & $4 \cdot \begin{array}{c}1 \\
4 \\
4\end{array}$ & 4.35 & 3.98 & 5.51 & $\because x \cdot 0$ & $\begin{array}{r}2 ? .20 \\
2.22\end{array}$ \\
\hline
\end{tabular}


Heliostat 3 , Configuration $3-\mathrm{K}$

\begin{tabular}{lllll}
\hline Case & Run & WD & EL & AZ \\
\hline Ver. Stow & 434 & 265 & 0 & 5 \\
& 452 & 247.5 & 0 & 5 \\
& 453 & 270 & 0 & 5 \\
454 & 292.5 & 0 & 5 \\
455 & 315 & 0 & 5 \\
& 456 & 337.5 & 0 & 5 \\
458 & 0 & 0 & 5 \\
\hline
\end{tabular}


MATA FOA FILE: US5OA

\begin{tabular}{|c|c|c|c|c|c|c|c|}
\hline $\begin{array}{l}9 ! 119 \\
3 x-x\end{array}$ & & 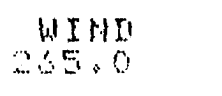 & 0.0 & 5.0 & 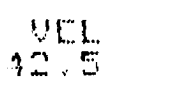 & & \\
\hline 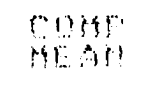 & $\vdots$ & $9 x$ & $\begin{array}{l}\mathrm{Cr} \\
.01\end{array}$ & $\begin{array}{l}0 . y \\
.01\end{array}$ & $\begin{array}{l}C+3 \\
0.92\end{array}$ & Cyy & $\begin{array}{r}\mathrm{EH} \\
01\end{array}$ \\
\hline $\begin{array}{l}40 \% \\
y B\end{array}$ & $\vdots$ & 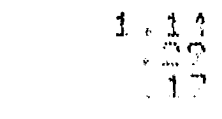 & .06 & $\begin{array}{l}0 \% \\
.05 \\
.02\end{array}$ & .07 & $\begin{array}{r}3 \\
-30 \\
-30\end{array}$ & $\begin{array}{r}.65 \\
.05 \\
.01\end{array}$ \\
\hline 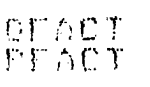 & 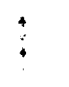 & $5 \cdot \frac{9}{3}$ & 14.95 & $\therefore \cdot \hat{A}$ & $\begin{array}{l}z \\
z \\
z\end{array}$ & 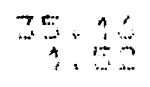 & 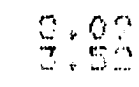 \\
\hline IATA & $\because 0$ & IF FILE: & 13502 & & & & \\
\hline 10114 & & $\begin{array}{l}4 I N I \\
0.5\end{array}$ & 0.5 & 5.9 & $\begin{array}{r}Y E 1 \\
\times 2: 3\end{array}$ & & \\
\hline $\begin{array}{l}\text { MHI } \\
\text { MII }\end{array}$ & $\vdots$ & $\begin{array}{c}r y \\
y \\
8\end{array}$ & $r y$ & $\begin{array}{l}c T^{-n} \\
01\end{array}$ & $\begin{array}{l}\text { CHy } \\
.00\end{array}$ & BMY & $\frac{0.1}{.01}$ \\
\hline 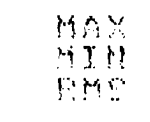 & $\vdots$ & 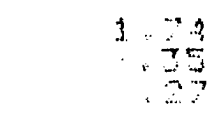 & $\begin{array}{l}.06 \\
.14 \\
.03\end{array}$ & $\begin{array}{l}00 \\
.95 \\
.01\end{array}$ & $\begin{array}{r}.05 \\
.95 \\
.01\end{array}$ & 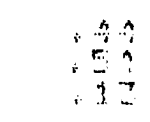 & $\begin{array}{l}.02 \\
.0= \\
.02\end{array}$ \\
\hline $5 r \cos$ & $\vdots$ & $\hat{y} \cdot 1$ & 出: & $\begin{array}{l}4.02 \\
7.11\end{array}$ & $2 \frac{9}{3} \cdot \frac{7}{4}$ & $13: 62$ & $1 \frac{1}{7} \cdot 57$ \\
\hline
\end{tabular}

MATA FOF FILE: 11302

\begin{tabular}{|c|c|c|c|c|c|c|c|}
\hline$\frac{519}{93}$ & & $\begin{aligned} & 4 \pi 3 \pi \\
= & 0\end{aligned}$ & $0: 0$ & $5: 0$ & $\begin{array}{r}1[1 \\
425\end{array}$ & & \\
\hline 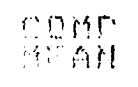 & $\vdots$ & $8 y$ & 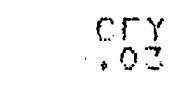 & 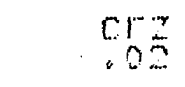 & $\begin{array}{l}\operatorname{cms} \\
.00\end{array}$ & $5 \%$ & $\begin{array}{l}\mathrm{CH} \\
. \mathrm{O}=\end{array}$ \\
\hline $\begin{array}{l}496 \\
419 \\
63 n\end{array}$ & $\vdots$ & 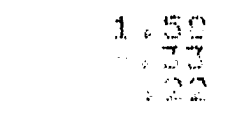 & $\begin{array}{r}.05 \\
.14 \\
.04\end{array}$ & $\begin{array}{r}0.4 \\
.0 \% \\
.01\end{array}$ & $\begin{array}{r}0.4 \\
+25 \\
+01\end{array}$ & $\begin{array}{l}.4 \\
30 \\
11 \\
.11\end{array}$ & 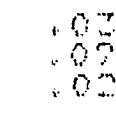 \\
\hline Art & 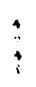 & $\begin{array}{l}A+B \\
\therefore\end{array}$ & 3.23 & $\overrightarrow{3}+4 \overrightarrow{3}$ & $\begin{array}{r}3.05 \\
7 \\
2\end{array}$ & $4.8 \div-\frac{7}{9}$ & $\begin{array}{l}5 \\
4+28\end{array}$ \\
\hline
\end{tabular}

MATA FOS FILF: 13502

\begin{tabular}{|c|c|c|c|c|c|c|c|}
\hline 9:19 & & $\begin{array}{l}419 ! \\
202\end{array}$ & EL & $\frac{\pi}{6}$ & 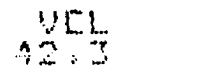 & & \\
\hline $\begin{array}{l}\text { naf } \\
\text { Mand }\end{array}$ & $\vdots$ & $6 y$ & $\begin{array}{r}65 y \\
0 \%\end{array}$ & $05 \frac{1}{1}$ & $\begin{array}{l}\mathrm{CHY} \\
.00\end{array}$ & $\begin{array}{c}c y \\
r y\end{array}$ & $\begin{array}{l}\text { chin } \\
0 \%\end{array}$ \\
\hline mas & $\vdots$ & 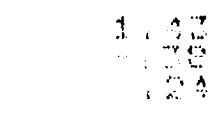 & .95 & $\begin{array}{r}96 \\
.05 \\
61\end{array}$ & $\begin{array}{r}05 \\
+65 \\
64 \\
04\end{array}$ & $\begin{aligned} \because c \\
\because n \\
\because 2 \\
12\end{aligned}$ & 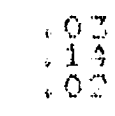 \\
\hline $\begin{array}{l}F A C Y \\
F A C T\end{array}$ & 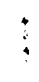 & $\begin{array}{ll}A & 7 \\
A & z\end{array}$ & $\frac{4}{7}+\frac{+y}{20}$ & $9+5$ & $2 \stackrel{4}{4}+3$ & $12 \cdot 78$ & $\overrightarrow{3} \cdot \frac{3}{3}$ \\
\hline
\end{tabular}




\begin{tabular}{|c|c|c|c|c|c|c|}
\hline 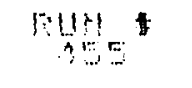 & $\begin{array}{ll}U 1 N T \\
\because 10\end{array}$ & 0.5 & 5.92 & บ는 & & \\
\hline mome: & 96 & $\begin{array}{c}c y \\
0.0\end{array}$ & $\operatorname{cr} 2$ & $\begin{array}{l}\text { cky } \\
.00\end{array}$ & Cry & 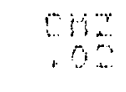 \\
\hline 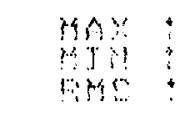 & $\begin{array}{r}32 \\
30 \\
20\end{array}$ & $\begin{array}{l}06 \\
111 \\
.02\end{array}$ & .02 & 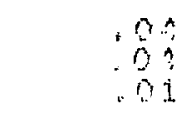 & 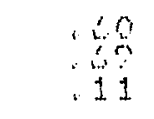 & .0 \\
\hline $\begin{array}{l}\text { EFACY } \\
\text { FAC? }\end{array}$ & $\because 98$ & 4.42 & 7. & $=\frac{3}{3}: \frac{7}{0}$ & $5+55$ & 5 \\
\hline MnTA $F$ & F: IILE & 43502 & & & & \\
\hline 9uts & $4 y_{5}$ & $05 \frac{1}{6}$ & $5: \hat{O}$ & $9=\frac{1}{2}$ & & \\
\hline orme & 05 & $\begin{array}{c}\operatorname{cry}^{-} \\
\cdots .01\end{array}$ & $\cos$ & 01 & cry & $c m_{1}^{\circ}$ \\
\hline $\begin{array}{l}\text { Wey } \\
\text { myen: }\end{array}$ & $\begin{array}{l}90 \\
92 \\
21\end{array}$ & $\begin{array}{l}.02 \\
.12 \\
.02\end{array}$ & $.0 \frac{7}{1}$ & .05 & 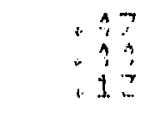 & \\
\hline FEAC & $? \geq \frac{3}{3}$ & 11.85 & 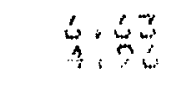 & 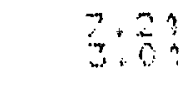 & $15 \cdot 19$ & $2 \cdot 8$ \\
\hline WATA & FILE: & 43502 & & & & \\
\hline ruts & $\ln \frac{I}{C_{1}}=\frac{I}{0}$ & 01 & 50 & $\begin{array}{l}y+3 \\
3=2\end{array}$ & & \\
\hline $\begin{array}{l}\text { gor } \\
\text { man! }\end{array}$ & 65 & $\begin{array}{c}65 \\
.20\end{array}$ & $\begin{array}{l}c 5 x \\
.00\end{array}$ & $\begin{array}{l}\cos \% \\
.00\end{array}$ & $\begin{array}{l}\operatorname{crs} \\
.01\end{array}$ & {$[x=$} \\
\hline 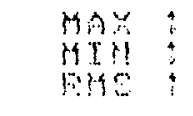 & $\begin{array}{l}150 \\
150 \\
258\end{array}$ & $\begin{array}{r}10 \\
\therefore 10 \\
: 00 \\
10\end{array}$ & $\begin{array}{l}.02 \\
.05 \\
.02\end{array}$ & $\begin{array}{l}.0 \hat{i} \\
.01 \\
.01\end{array}$ & 50 & \\
\hline Fract & 2.52 & $829 \%$ & 41.06 & $\because 9.20$ & 5.59 & 3.07 \\
\hline
\end{tabular}


Heliostat 3, Configuration 3-L

\begin{tabular}{lllll}
\hline Case & Run & WD & EL & AZ \\
\hline Ver. Stow & 486 & 247.5 & 0 & 5 \\
& 485 & 265 & 0 & 5 \\
& 484 & 270 & 0 & 5 \\
& 482 & 292.5 & 0 & 5 \\
& 481 & 315 & 0 & 5 \\
& 480 & 337.5 & 0 & 5 \\
& 479 & 0 & 0 & 5 \\
\hline
\end{tabular}




\begin{tabular}{|c|c|c|c|c|c|c|}
\hline rump & WIMII & ${ }_{0.0}^{5 L}$ & 5.0 & $\begin{array}{r}\text { VCL } \\
41.5\end{array}$ & & \\
\hline COMF: & $\begin{array}{l}\text { cro } \\
-24\end{array}$ & $\begin{array}{c}\operatorname{cry} \\
.02\end{array}$ & .01 & .01 & CMY & $\operatorname{crz}_{02}$ \\
\hline $\begin{array}{l}\text { MAX } \\
\text { HIH } \\
\text { FMS }\end{array}$ & $\begin{array}{r}1: 65 \\
: 20\end{array}$ & $\begin{array}{l}.07 \\
.10 \\
.02\end{array}$ & $\begin{array}{l}.04 \\
.04 \\
.01\end{array}$ & $\begin{array}{l}.06 \\
.04 \\
.01\end{array}$ & .91 & $\begin{array}{l}.02 \\
.02 \\
.02\end{array}$ \\
\hline $\begin{array}{l}\text { GEACT } \\
\text { FFACT }\end{array}$ & $4+54$ & 3.57 & ㄱ: & 5.57 & 总:呺 & $5 \cdot 12$ \\
\hline IIATA F & FI FILE : & H3503 & & & & \\
\hline Pun & 337.5 & ${ }_{0.0}^{E L}$ & $\begin{array}{r}A I \\
5+0\end{array}$ & $\begin{array}{r}Y C L \\
42+5\end{array}$ & & \\
\hline COMF : & cro & $\begin{array}{r}\text { CFY } \\
.02\end{array}$ & $\begin{array}{c}\operatorname{coz}^{2} \\
.01\end{array}$ & $\begin{array}{l}\text { Cro } \\
.01\end{array}$ & CHY & $\begin{array}{c}c 43 \\
.02\end{array}$ \\
\hline $\begin{array}{l}M A Y \\
M I H \\
\text { RHS }\end{array}$ & $\begin{array}{r}1 . \frac{1}{7} 9 \\
-25\end{array}$ & $\begin{array}{r}.05 \\
.09 \\
.02\end{array}$ & $\begin{array}{l}.93 \\
.07 \\
.01\end{array}$ & $.8 \frac{1}{8} .01$ & .54 & $\begin{array}{l}.05 \\
.15 \\
.05\end{array}$ \\
\hline $\begin{array}{l}\text { GFACT } \\
\text { FFACT }\end{array}$ & $16 \cdot 13$ & $\frac{5.02}{3} \cdot 2.2$ & $4 \cdot 54$ & $\begin{array}{r}10.57 \\
7.56\end{array}$ & $3 \frac{1}{3} \cdot 15$ & $4+51$ \\
\hline
\end{tabular}

IIATA FOR FILE: $H 3503$

\begin{tabular}{|c|c|c|c|c|c|c|}
\hline Rust & $315 \cdot 0$ & EL & 5.0 & VEL & & \\
\hline 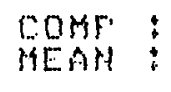 & 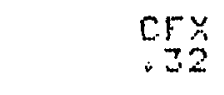 & $\begin{array}{r}\text { Cry } \\
-.01\end{array}$ & $\begin{array}{l}\mathrm{Crz} \\
.02\end{array}$ & $\begin{array}{l}\text { C.kY } \\
.00\end{array}$ & $\begin{array}{l}\text { CMY } \\
.0 S\end{array}$ & $\begin{array}{l}C M z \\
.03\end{array}$ \\
\hline $\begin{array}{l}\text { MAX } \\
\text { MIH } \\
\text { RMS }\end{array}$ & $\begin{array}{l}1.83 \\
\cdots \quad 35 \\
+30\end{array}$ & $\begin{array}{r}.07 \\
.11 \\
.05\end{array}$ & $\begin{array}{l}.02 \\
.09 \\
.01\end{array}$ & $\begin{array}{l}.05 \\
.05 \\
.02\end{array}$ & $\begin{array}{l}+45 \\
+53 \\
+10\end{array}$ & $\begin{array}{l}.05 \\
13 \\
05\end{array}$ \\
\hline $\begin{array}{l}\text { FACT } \\
\text { FACT }\end{array}$ & $\frac{5}{4} \div \frac{1}{3} \frac{1}{2}$ & 15.40 & $\begin{array}{l}3.73 \\
4+20\end{array}$ & $13 \cdot 2 己$ & 19.02 & $3 \cdot 02$ \\
\hline
\end{tabular}

IMATA FOF FILE: H35OJ

\begin{tabular}{|c|c|c|c|c|c|c|}
\hline Fun & $\begin{array}{r}\text { WIHn } \\
292.5\end{array}$ & 0.0 & $\begin{array}{r}A Z \\
5.0\end{array}$ & $\begin{array}{r}\text { VE- } \\
42.9\end{array}$ & & \\
\hline $\begin{array}{l}\text { COMF : } \\
\text { MEAN : }\end{array}$ & CF. & $\begin{array}{l}\operatorname{cry} \\
.00\end{array}$ & $\begin{array}{c}\mathrm{crz}_{2} \\
.00\end{array}$ & $\begin{array}{l}\text { C.Mx } \\
.00\end{array}$ & $\begin{array}{l}\text { CMY } \\
.02\end{array}$ & $\begin{array}{c}\operatorname{crs} 2 \\
.02\end{array}$ \\
\hline $\begin{array}{l}\text { MAX } \\
\text { MIHE } \\
\text { KMS }\end{array}$ & $\begin{array}{r}1 \cdot \frac{1}{3} \cdot \frac{1}{2} \\
: 20\end{array}$ & $\begin{array}{r}.07 \\
.03 \\
.02\end{array}$ & $\begin{array}{l}.04 \\
.05 \\
.01\end{array}$ & $\begin{array}{l}+05 \\
.04 \\
.01\end{array}$ & $\begin{array}{l}.42 \\
.70 \\
10\end{array}$ & $\begin{array}{l}09 \\
.10 \\
+02\end{array}$ \\
\hline $\begin{array}{l}\text { GFACT } \\
\text { FACT }\end{array}$ & $5 \cdot 39$ & $\begin{array}{r}47.01 \\
3.01\end{array}$ & $\begin{array}{r}14.43 \\
3.15\end{array}$ & $22 \cdot 72$ & $2 \frac{1}{3} \cdot \frac{65}{7}$ & $\begin{array}{l}3.50 \\
3.50 \\
1.56\end{array}$ \\
\hline
\end{tabular}




\begin{tabular}{|c|c|c|c|c|c|c|}
\hline rust & 270.0 & ${ }_{0.0}$ & 5.0 & UEL & & \\
\hline COMF & $\begin{array}{l}\text { CF. } \\
.30\end{array}$ & $\begin{array}{l}\text { C.rY } \\
.00\end{array}$ & $\operatorname{cr}_{-03}^{2}$ & .01 & CHy & $\begin{array}{c}C H z \\
.01\end{array}$ \\
\hline $\begin{array}{l}\text { MAO } \\
\text { KIHE } \\
\text { FHS }\end{array}$ & $\begin{array}{r}1.30 \\
.75 \\
.23\end{array}$ & $\begin{array}{r}.07 \\
.06 \\
.02\end{array}$ & $\begin{array}{r}.03 \\
+05 \\
.01\end{array}$ & $\begin{array}{l}.03 \\
.03 \\
.01\end{array}$ & $\begin{array}{r}52 \\
+55 \\
+10\end{array}$ & $\begin{array}{l}.05 \\
.05 \\
.02\end{array}$ \\
\hline QFACT & 4.55 & 35.45 & $\begin{array}{l}3+44 \\
4+61\end{array}$ & $3 \cdot \frac{22}{3}$ & 14.01 & 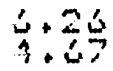 \\
\hline
\end{tabular}

IIATA FOF FILE:

\begin{tabular}{|c|c|c|c|c|c|c|}
\hline Fust & $\begin{array}{r}\text { WINII } \\
265.0\end{array}$ & 0.0 & 5.0 & $\begin{array}{r}\text { VEL } \\
42.7\end{array}$ & & \\
\hline $\begin{array}{l}\text { COHSF: } \\
\text { MEAM : }\end{array}$ & $\begin{array}{c}\text { CFX } \\
.31\end{array}$ & $\begin{array}{l}\text { C.FY } \\
.01\end{array}$ & crz & $\begin{array}{l}\text { Cr. } \\
.00\end{array}$ & $\begin{array}{l}\text { CHY } \\
.0 .4\end{array}$ & C.MI \\
\hline $\begin{array}{l}\text { MAX } \\
\text { HIME } \\
\text { FIHS }\end{array}$ & $\begin{array}{r}1.94 \\
-.39 \\
.25\end{array}$ & $\begin{array}{r}.08 \\
-.03 \\
.02\end{array}$ & .02 & $\begin{array}{r}.05 \\
.05 \\
+01\end{array}$ & .52 & $\begin{array}{l}.03 \\
.12 \\
02\end{array}$ \\
\hline $\begin{array}{l}\text { GFACT } \\
\text { FFACT }\end{array}$ & 3.178 & $12 \cdot 20$ & $\frac{2}{3}+50$ & $79+79$ & 13.00 & 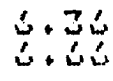 \\
\hline
\end{tabular}

IIATA FOR FILE: H3503

\begin{tabular}{|c|c|c|c|c|c|c|}
\hline FUN & 247.5 & $\begin{array}{c}E L \\
0.0\end{array}$ & 5.0 & $\begin{array}{l}\text { ULL } \\
+2.3\end{array}$ & & \\
\hline COMF: & $\begin{array}{c}C F x \\
.36\end{array}$ & $\begin{array}{l}\text { CFY } \\
.01\end{array}$ & .01 & $\begin{array}{l}\text { CMx } \\
.00\end{array}$ & $\begin{array}{l}\text { cisy } \\
.01\end{array}$ & $\begin{array}{l}\mathrm{CHZ} \\
.00\end{array}$ \\
\hline $\begin{array}{l}\text { MAX } \\
\text { MIN } \\
\text { RMS }\end{array}$ & $\begin{array}{r}1.45 \\
: 33 \\
+25\end{array}$ & $\begin{array}{r}.00 \\
.05 \\
.02\end{array}$ & $\begin{array}{l}.05 \\
.09 \\
.02\end{array}$ & $\begin{array}{l}.02 \\
.05 \\
.01\end{array}$ & $\begin{array}{r}+93 \\
.37 \\
.11\end{array}$ & $\begin{array}{l}.05 \\
.04 \\
.02\end{array}$ \\
\hline FFACT & 4.05 & $\frac{8.71}{3} \cdot 33$ & $\begin{array}{r}10+16 \\
1+20\end{array}$ & $42 \cdot 55$ & 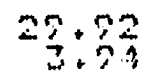 & 15.81 \\
\hline
\end{tabular}


Heliostat 3 , Configuration 3-M

\begin{tabular}{lllll}
\hline Case & Run & WD & EL & AZ \\
\hline Ver. Stow & 467 & 247.5 & 0 & 5 \\
& 469 & 265 & 0 & 5 \\
& 470 & 270 & 0 & 5 \\
471 & 292.5 & 0 & 5 \\
475 & 315 & 0 & 5 \\
477 & 337.5 & 0 & 5 \\
& 478 & 0 & 0 & 5 \\
\hline
\end{tabular}




\begin{tabular}{|c|c|c|c|c|c|c|}
\hline filly & $\begin{array}{r}\text { WIMII } \\
247.5\end{array}$ & 0.0 & $\begin{array}{c}A Z \\
5.0\end{array}$ & $\begin{array}{r}\text { VEL } \\
41: 5\end{array}$ & & \\
\hline $\begin{array}{l}\text { COMF } \\
\text { TEAN : }\end{array}$ & CES & $\begin{array}{l}\text { Cry } \\
.01\end{array}$ & $.00^{2}$ & .01 & CHS & $\begin{array}{l}\text { CMI } \\
+01\end{array}$ \\
\hline $\begin{array}{l}\text { MAX } \\
\text { MIHS } \\
\text { SHS }\end{array}$ & 1. & $\begin{array}{r}.12 \\
-.05 \\
.02\end{array}$ & $\begin{array}{l}.05 \\
.05 \\
.02\end{array}$ & $\begin{array}{l}.09 \\
.03 \\
.01\end{array}$ & $\begin{array}{l}45 \\
.34 \\
10\end{array}$ & $\begin{array}{l}0 \hat{1} \\
10 \\
02\end{array}$ \\
\hline GFACT & $\begin{array}{l}5.70 \\
5.32\end{array}$ & $\begin{array}{l}3.70 \\
4.38\end{array}$ & $1 \frac{1}{2} \cdot 72$ & 3.09 & $2 \pi \cdot \frac{1}{4}$ & $5 \cdot \frac{17}{3}$ \\
\hline IATA F & IF FILE : & $H 3504$ & & & & \\
\hline num & $\begin{array}{r}\text { WINI } \\
235+0\end{array}$ & 0.0 & $5 . A Z$ & $\begin{array}{l}\text { UEL } \\
42.2\end{array}$ & & \\
\hline $\begin{array}{l}\text { COHF } \\
\text { MEAN }\end{array}$ & $65 x$ & $\begin{array}{l}\text { cry } \\
.02\end{array}$ & $\begin{array}{l}c 5 z \\
.01\end{array}$ & $\begin{array}{l}\text { CHE } \\
.01\end{array}$ & $\begin{array}{l}\mathrm{CHY} \\
.04\end{array}$ & $\begin{array}{l}C H 2 \\
.00\end{array}$ \\
\hline $\begin{array}{l}\text { MAX } \\
\text { MINS } \\
\text { FMS }\end{array}$ & 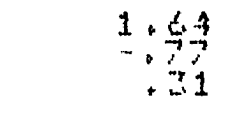 & $\begin{array}{r}.18 \\
-.10 \\
.03\end{array}$ & $\begin{array}{l}.05 \\
.05 \\
.02\end{array}$ & $\begin{array}{l}.05 \\
.05 \\
.02\end{array}$ & $: \begin{array}{l}71 \\
: 51 \\
0 \\
12\end{array}$ & $\begin{array}{l}.05 \\
.05 \\
.02\end{array}$ \\
\hline $\begin{array}{l}\text { BFACT } \\
\text { FFACT }\end{array}$ & 4.35 & $\begin{array}{l}7.29 \\
9.80\end{array}$ & $\frac{4.75}{2} .77$ & $3+3 \frac{1}{3}$ & $14+20$ & 10.50 \\
\hline
\end{tabular}

IATA FOFI FILE: H350A

\begin{tabular}{|c|c|c|c|c|c|c|}
\hline $\operatorname{mus}_{470}$ & 270.0 & EL & $A 2$ & 42.5 & & \\
\hline $\begin{array}{l}\text { CORF } \\
\text { MEAH }\end{array}$ & $\begin{array}{l}\text { CFY } \\
.35\end{array}$ & $\begin{array}{l}\text { cry } \\
.02\end{array}$ & $\frac{\operatorname{cra}}{.01}$ & CMY & $\begin{array}{l}\text { CMY } \\
.07\end{array}$ & .01 \\
\hline $\begin{array}{l}\text { MAX } \\
\text { MIN } \\
\text { FMS }\end{array}$ & $\begin{array}{r}1.98 \\
-.71 \\
.30\end{array}$ & $\begin{array}{l}17 \\
.05 \\
.03\end{array}$ & $\begin{array}{l}.05 \\
.05 \\
.02\end{array}$ & .05 & . & $\begin{array}{l}.00 \\
.05 \\
.02\end{array}$ \\
\hline FACT & 5.24 & 2.03 & $\stackrel{4}{2}: 43$ & $\frac{7}{3} \cdot 37$ & ?. & 4.00 \\
\hline
\end{tabular}

MATA FOR FILE : H3504

$\lim _{\rightarrow 1}$ WISI $292.5 \quad 0.0 \quad 5.0 \quad$ UEL

\begin{tabular}{|c|c|c|c|c|c|c|}
\hline $\begin{array}{l}\text { COMF } \\
\text { MEAH }\end{array}$ & $\begin{array}{l}C .5 \\
.14\end{array}$ & $\begin{array}{r}\text { CFY } \\
. .00\end{array}$ & $.0 \frac{1}{1}$ & $\begin{array}{c}\mathrm{CHX} \\
.02\end{array}$ & .01 & $\mathrm{CHz}_{\mathrm{O}}$ \\
\hline $\begin{array}{l}\text { MAX } \\
\text { MIMt } \\
\text { Fims }\end{array}$ & $\begin{array}{r}2.08 \\
=.85 \\
.28\end{array}$ & $\begin{array}{r}.11 \\
.09 \\
.03\end{array}$ & $\begin{array}{r}.05 \\
-.07 \\
.01\end{array}$ & .03 & $\begin{array}{r}5 \\
.55 \\
11\end{array}$ & $\begin{array}{r}.04 \\
.15 \\
.02\end{array}$ \\
\hline $\begin{array}{l}\text { QFACT } \\
\text { FFACT }\end{array}$ & $\begin{array}{r}14+47 \\
7.35\end{array}$ & 30.77 & $\frac{4}{2} \cdot 0 \frac{7}{6}$ & $4+\frac{7}{3}$ & $+\frac{1}{5}+\frac{1}{0}$ & $\begin{array}{c}5 \\
5 \\
5\end{array}$ \\
\hline
\end{tabular}




\begin{tabular}{|c|c|c|c|c|c|c|}
\hline FUn & 3IIHI & 0.0 & 5.0 & $\begin{array}{r}Y C L \\
42.3\end{array}$ & & \\
\hline $\begin{array}{l}\text { COHF : } \\
\text { HEAN }\end{array}$ & $\begin{array}{l}\text { CF. } \\
.29\end{array}$ & $\begin{array}{r}\text { CFY } \\
-.02\end{array}$ & $\begin{array}{l}\mathrm{crz} \\
.00\end{array}$ & Cry & CHY & $.0 \%$ \\
\hline $\begin{array}{l}\text { MAX } \\
\text { MINH } \\
\text { FMS }\end{array}$ & $\begin{array}{r}3.10 \\
1.30 \\
.95\end{array}$ & $\begin{array}{r}.09 \\
.13 \\
.03\end{array}$ & $\begin{array}{l}.01 \\
102 \\
102\end{array}$ & $\begin{array}{l}.05 \\
.05 \\
.02\end{array}$ & $\frac{1}{1}: \frac{1}{2} \frac{1}{2}$ & $\begin{array}{l}\frac{1}{2} \frac{1}{1} \\
: \frac{1}{0} \\
: \frac{1}{4}\end{array}$ \\
\hline $\begin{array}{l}\text { QFACT } \\
\text { FACT }\end{array}$ & 10.85 & $\begin{array}{l}7.99 \\
3.55\end{array}$ & $\begin{array}{r}34 \\
5.26 \\
4\end{array}$ & $13 \cdot 24$ & 15.72 & 5.73 \\
\hline IIATA F & OF FILE : & $H 350.4$ & & & & \\
\hline Fun & $3 \pm 7.5$ & 0.0 & $5 .{ }^{A Z}$ & $\begin{array}{r}\text { UEL } \\
+2 \cdot 2\end{array}$ & & \\
\hline $\begin{array}{l}\text { COHF } \\
\text { HEAH : }\end{array}$ & $\begin{array}{l}C F X \\
-4 i\end{array}$ & $\begin{array}{r}\text { CFY } \\
-.04\end{array}$ & .01 & $\begin{array}{l}\text { Crs } \\
.00\end{array}$ & .03 & $\begin{array}{l}\text { Cryz } \\
.05\end{array}$ \\
\hline $\begin{array}{l}\text { MAX } \\
\text { MIN } \\
\text { FiMS }\end{array}$ & $\begin{array}{r}1.80 \\
+30 \\
+26\end{array}$ & $\begin{array}{r}.01 \\
-.11 \\
.02\end{array}$ & $\begin{array}{l}.04 \\
.02 \\
.02\end{array}$ & $\begin{array}{l}.05 \\
.04 \\
.01\end{array}$ & $\begin{array}{r}.51 \\
+40 \\
+11\end{array}$ & $\begin{array}{l}.02 \\
.12 \\
.03\end{array}$ \\
\hline $\begin{array}{l}\text { QFACT } \\
\text { FFACT }\end{array}$ & $5: 99$ & $\frac{2}{3} .57$ & $3: 77$ & 35.72 & $15 \cdot 31$ & $\frac{2}{4}+15$ \\
\hline IIATA F & OF: FILE : & 43504 & & & & \\
\hline $\operatorname{lut}_{4 ? 3} *$ & $\begin{array}{r}\text { WI } \\
0.0 \mathrm{D}\end{array}$ & 0.0 & 5.0 & $\begin{array}{l}\text { VEL } \\
42.7\end{array}$ & & \\
\hline COHF : & $\begin{array}{r}C F \times \\
-40\end{array}$ & $\begin{array}{r}\text { CFY } \\
-.03\end{array}$ & $\begin{array}{l}\text { CFZ } \\
-.04\end{array}$ & C.s. & $.0 \mathrm{CHY}$ & $\begin{array}{l}\mathrm{cmz} \\
.05\end{array}$ \\
\hline $\begin{array}{l}\text { MAY : } \\
\text { MIME: }\end{array}$ & $\begin{array}{r}.88 \\
-1.50 \\
+25\end{array}$ & $\begin{array}{l}.02 \\
.14 \\
.02\end{array}$ & $\begin{array}{r}.00 \\
-.08 \\
.01\end{array}$ & $\begin{array}{r}.00 \\
.03 \\
.01\end{array}$ & $\begin{array}{l}.31 \\
.15 \\
12\end{array}$ & $\begin{array}{l}17 \\
.07 \\
.05\end{array}$ \\
\hline $\begin{array}{l}\text { QFACT: } \\
\text { FFACT }\end{array}$ & 3.70 & $\begin{array}{l}2 \cdot 23 \\
-7.51\end{array}$ & 2.35 & $2.5 \%$ & $13 \cdot 37$ & 2.70 \\
\hline
\end{tabular}

1 
Heliostat 3 , Configuration 3-N

\begin{tabular}{lcccc}
\hline Case & Run & WD & EL & AZ \\
\hline Ver. Stow & 502 & 247.5 & 0 & 5 \\
& 497 & 265 & 0 & 5 \\
503 & 270 & 0 & 5 \\
504 & 292.5 & 0 & 5 \\
505 & 315 & 0 & 5 \\
506 & 337.5 & 0 & 5 \\
& 507 & 0 & 0 & 5 \\
\hline
\end{tabular}




\begin{tabular}{|c|c|c|c|c|c|c|}
\hline $\operatorname{lum}_{49}$ & 4IM! & 0.5 & 5.92 & 42 들 & & \\
\hline $\begin{array}{l}\text { COMT: } \\
\text { HERH : }\end{array}$ & cru & $\begin{array}{r}C 5 Y \\
-.00\end{array}$ & $\begin{array}{l}.057 \\
.00\end{array}$ & $\begin{array}{l}\text { C.M. } \\
.01\end{array}$ & $\begin{array}{l}\text { Cry } \\
.02\end{array}$ & $\begin{array}{l}C+y= \\
+0=\end{array}$ \\
\hline $\begin{array}{l}\text { MAY : } \\
\text { MIHS } \\
\text { FUSE : }\end{array}$ & 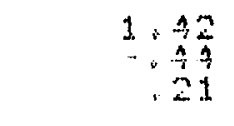 & $\begin{array}{l}.00 \\
.00 \\
.02\end{array}$ & $\begin{array}{r}.07 \\
.04 \\
.01\end{array}$ & $\begin{array}{l}.04 \\
.02 \\
.02\end{array}$ & $\begin{array}{l}.91 \\
.47 \\
.11\end{array}$ & $\begin{array}{l}.03 \\
.10 \\
.02\end{array}$ \\
\hline $\begin{array}{l}\text { GFACT } \\
\text { FACT }\end{array}$ & $5: 7^{4}$ & 79.99 & 100.27 & $\begin{array}{l}7+39 \\
3.90\end{array}$ & 24.470 & 3.5 .93 \\
\hline MATA F & OF FILE : & 43505 & & & & \\
\hline $\operatorname{lum}_{52}$ & $\begin{array}{r}41315 \\
247.5\end{array}$ & 0.0 & 5.0 & $\begin{array}{r}\text { VEL } \\
72.5\end{array}$ & & \\
\hline $\begin{array}{l}\text { Comf : } \\
\text { MEAt : }\end{array}$ & $\begin{array}{l}C 5 x \\
.25\end{array}$ & $\begin{array}{l}\text { CFY } \\
.00\end{array}$ & $.01 \frac{1}{1}$ & .02 & $\begin{array}{l}\text { Cry } \\
.01\end{array}$ & CHI \\
\hline $\begin{array}{l}\text { MAX } \\
\text { MIIS } \\
\text { FISE }\end{array}$ & $\begin{array}{r}1.37 \\
.25 \\
.25\end{array}$ & $\begin{array}{r}.07 \\
.07 \\
.02\end{array}$ & $\begin{array}{r}.07 \\
.04 \\
.02\end{array}$ & $\begin{array}{l}.03 \\
.05 \\
.01\end{array}$ & $\begin{array}{r}.97 \\
.50 \\
.10\end{array}$ & $\begin{array}{l}.02 \\
.05 \\
.01\end{array}$ \\
\hline $\begin{array}{l}\text { QFACT } \\
\text { FFACT }\end{array}$ & 4.92 & $5 \underline{3.00}$ & $3 \cdot 17$ & $\frac{A}{3}+\frac{1}{5} 7$ & 97.57 & 7.97 \\
\hline
\end{tabular}

IIATA FOF FILE: H35OS

\begin{tabular}{|c|c|c|c|c|c|c|}
\hline nut & $\begin{array}{r}4 I H I I \\
270.0\end{array}$ & $\begin{array}{c}E L \\
0.0\end{array}$ & & $\begin{array}{l}\text { VEL } \\
72.5\end{array}$ & & \\
\hline COMF & CFY & $\begin{array}{l}\text { CFY } \\
.00\end{array}$ & $\begin{array}{r}c .5 z \\
-.02\end{array}$ & $\begin{array}{l}\text { chy } \\
.01\end{array}$ & $\begin{array}{l}\text { cyy } \\
+02\end{array}$ & $\begin{array}{c}C H 2 \\
.01\end{array}$ \\
\hline $\begin{array}{l}\text { MAX } \\
\text { MHH } \\
\text { RHS }\end{array}$ & $\begin{array}{r}1.38 \\
\cdots \cdot 23 \\
.20\end{array}$ & $\begin{array}{r}.05 \\
-.05 \\
.05\end{array}$ & $\begin{array}{r}.05 \\
.02 \\
.02\end{array}$ & $\begin{array}{r}.02 \\
.05 \\
.01\end{array}$ & $\begin{array}{l}35 \\
.45 \\
.09\end{array}$ & .09 \\
\hline $\begin{array}{l}\text { GFACT } \\
\text { PFACT }\end{array}$ & $\frac{3}{5}+109$ & $7 \frac{2}{3} \cdot 1 \cdot \hat{3}$ & 3.85 & $4 \cdot \frac{17}{3}$ & $23.3 A$ & 3.27 \\
\hline
\end{tabular}

INATA FOF FILE : H350S

\begin{tabular}{|c|c|c|c|c|c|c|}
\hline Fun & $292: 5$ & 0.0 & $\frac{2}{0}$ & $\begin{array}{r}4 E_{1} \\
42.2\end{array}$ & & \\
\hline $\begin{array}{l}\text { COMF } \\
\text { MEAN }\end{array}$ & 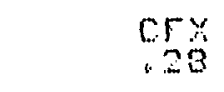 & $\begin{array}{l}\text { CFY } \\
\cdots .00\end{array}$ & .01 & .01 & $\begin{array}{l}\text { CMY } \\
.01\end{array}$ & $C_{0=}^{C H E}$ \\
\hline $\begin{array}{l}\text { MAE } \\
\text { RMES } \\
\text { FMS }\end{array}$ & $\begin{array}{r}1 \cdot 25 \\
\frac{2}{1} \\
10\end{array}$ & $\begin{array}{r}.07 \\
-.05 \\
.02\end{array}$ & $\begin{array}{l}.07 \\
.04 \\
.01\end{array}$ & $\begin{array}{l}.03 \\
.03 \\
.01\end{array}$ & $\begin{array}{r}.35 \\
.40 \\
.09\end{array}$ & $\begin{array}{r}.05 \\
.10 \\
.02\end{array}$ \\
\hline GFACT & $\frac{9}{5} \cdot \frac{51}{9}$ & $17 \cdot 33$ & 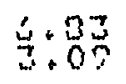 & 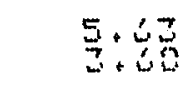 & $\begin{array}{r}20+40 \\
4.55\end{array}$ & $3.0 \%$ \\
\hline
\end{tabular}


MATA FOR FILE: 43505

\begin{tabular}{|c|c|c|c|c|c|c|}
\hline Rus: & $\begin{array}{l}\text { WINI } \\
315.0\end{array}$ & 0.0 & $\begin{array}{l}A Z \\
.0\end{array}$ & U2.7 & & \\
\hline Mng & $\frac{05 \%}{37}$ & $\begin{array}{r}c r y \\
-.01\end{array}$ & $\begin{array}{l}\operatorname{crz} \\
.02\end{array}$ & .01 & $\begin{array}{l}\text { CHy } \\
.00\end{array}$ & $\begin{array}{l}C M \\
.05\end{array}$ \\
\hline $\begin{array}{l}\text { MAX } \\
\text { MIYS }\end{array}$ & 1.97 & .05 & $\begin{array}{l}.05 \\
.06 \\
.01\end{array}$ & $\begin{array}{r}.05 \\
.05 \\
.01\end{array}$ & $\begin{array}{r}45 \\
.95 \\
.15\end{array}$ & .01 \\
\hline $\begin{array}{l}\text { GFACT } \\
F F A C T\end{array}$ & 4.03 & 7.03 & $\begin{array}{l}3 \\
2\end{array}$ & 4.75 & $152+\frac{1}{3}$ & $\frac{3}{4} \cdot \frac{7}{2}$ \\
\hline
\end{tabular}

IATA FOF: FILE : H3SOS

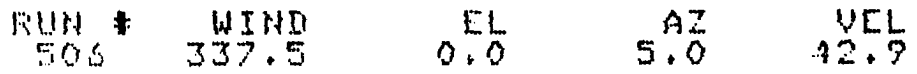

cogs: CFE

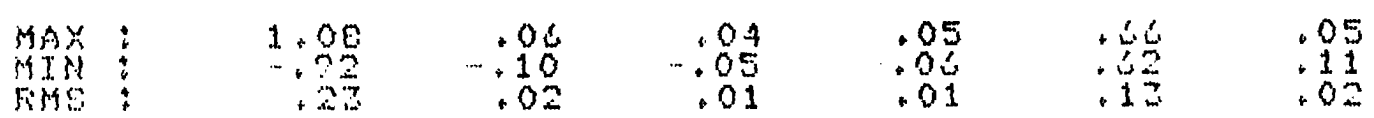

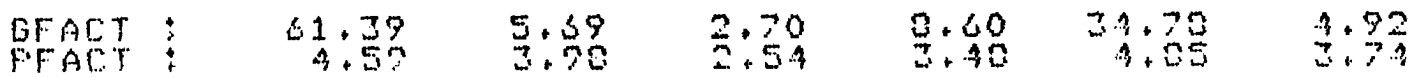

IATA FOF FILE: H350S

Furs

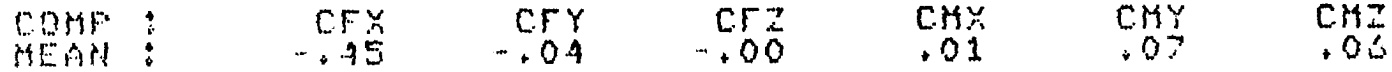

\begin{tabular}{|c|c|c|c|c|c|}
\hline $\begin{array}{l}\text { MAX : } \\
\text { HIH: } \\
\text { FUS: }\end{array}$ & $-1+\frac{1}{3} 9$ & $\begin{array}{l}.09 \\
: 11 \\
02\end{array}$ & $\begin{array}{l}.08 \\
.05 \\
.01\end{array}$ & $\begin{array}{l}.09 \\
.01 \\
.02\end{array}$ & $\begin{array}{l}44 \\
.45 \\
14\end{array}$ \\
\hline
\end{tabular}

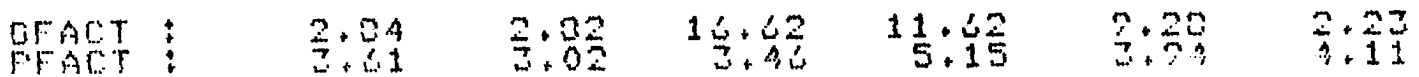


Heliostat 4, Configuration 4-A

\begin{tabular}{lllllll}
\hline Case & Run & WD & EL & AZ & Day & T'ime \\
\hline Smr AM & 465 & 265 & 40 & 350 & 172 & 8 AM \\
Smr Noon & 466 & 265 & 60 & 305 & 172 & 0 PN \\
Wntr AM & 461 & 265 & 25 & 330 & 355 & 8 AM \\
Wntr Noon & 464 & 265 & 40 & 305 & 355 & 0 PM \\
Witr PM & 463 & 265 & 25 & 270 & 172 & 4 MM \\
Ver. Stow & 459 & 265 & 0 & 330 & 355 & 8 AM \\
Hor. Stow & 460 & 265 & 90 & 330 & 355 & 8 AM \\
\hline
\end{tabular}


IITTA FOR FILE: 11100

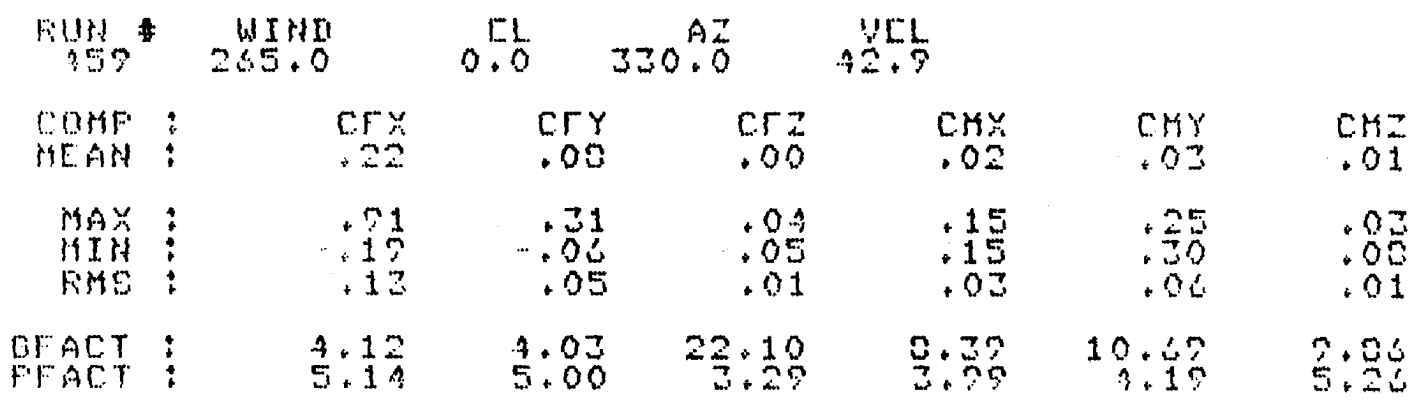

MATA FOF FILE: $H 4100$

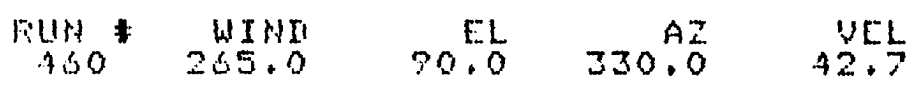

COHF : CFY CFY

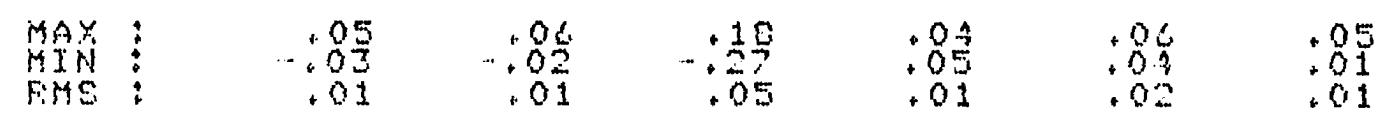

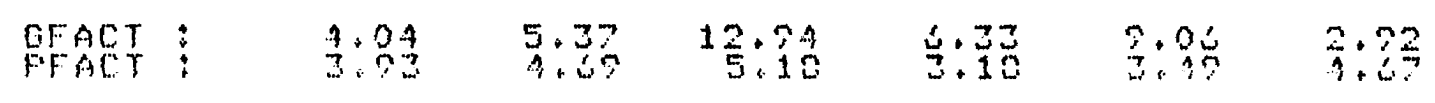

DATA FOF: FILE: H4100

FuH * WINT 255.0 25.

COMF: CFE CFY

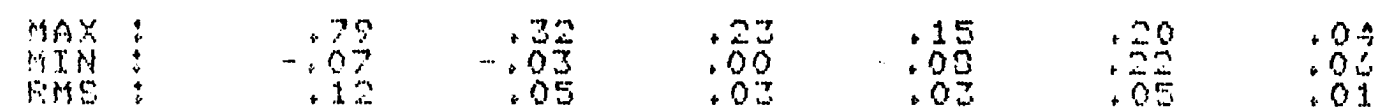

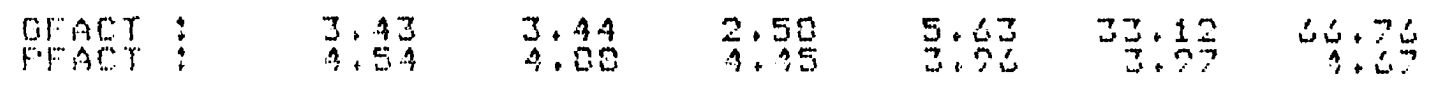

IATA FOF FILE: HA100

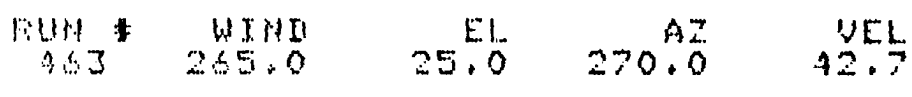

ronF: CFA $\quad$ CFY

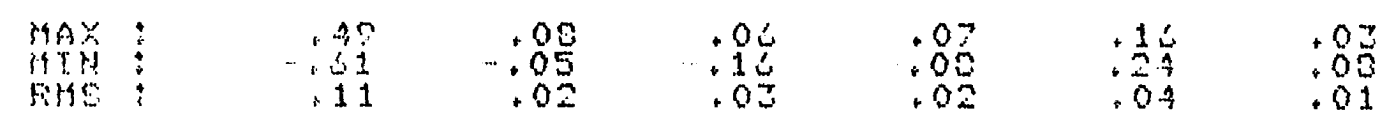

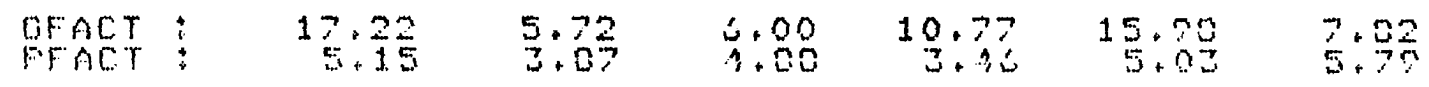




\begin{tabular}{|c|c|c|c|c|c|c|}
\hline nut: & $\begin{array}{r}\text { WIHI } \\
265: 0\end{array}$ & 90.5 & 305.0 & $\begin{array}{r}\text { VEl } \\
42.7\end{array}$ & & \\
\hline COMF : & .15 & $\begin{array}{l}\text { CFY } \\
.01\end{array}$ & c.r. & $\begin{array}{l}\cos 2 \\
.02\end{array}$ & $\begin{array}{c}C H Y \\
.02\end{array}$ & .01 \\
\hline $\begin{array}{l}\text { MAY } \\
\text { MIH! } \\
\text { Fist: }\end{array}$ & $\cdots: 24$ & $\begin{array}{r}1 \\
-1 \frac{1}{3} \\
+04\end{array}$ & $\begin{array}{r}.27 \\
.01 \\
.09\end{array}$ & . & $\begin{array}{l}21 \\
.50 \\
.00\end{array}$ & $\begin{array}{l}.05 \\
.05 \\
.01\end{array}$ \\
\hline $\begin{array}{l}\text { SFACT } \\
\text { FACT }\end{array}$ & $\begin{array}{l}5+01 \\
4+5 .\end{array}$ & 21.75 & $\frac{2.57}{3.75}$ & $\begin{array}{l}2.20 \\
3.58\end{array}$ & 11.05 & $2 \cdot 75$ \\
\hline IIATA F & JFI FILE : & $H 4100$ & & & & \\
\hline Futs & $\begin{array}{r}\text { 4I }+30 \\
255: 0\end{array}$ & 40.0 & $250 . A 2$ & $\begin{array}{r}\text { VEL } \\
42.5\end{array}$ & & \\
\hline $\begin{array}{l}\text { COMF : } \\
\text { MEAN }\end{array}$ & $\begin{array}{l}c .5 \\
.23\end{array}$ & $\begin{array}{l}\text { CFY } \\
.00\end{array}$ & CFz & $\begin{array}{l}\text { CMY } \\
.00\end{array}$ & $\begin{array}{l}\text { CMY } \\
.01\end{array}$ & .01 \\
\hline $\begin{array}{l}\text { MAY } \\
\text { FiHE } \\
\text { Fit }\end{array}$ & $\begin{array}{r}79 \\
\times 19 \\
.12\end{array}$ & $\begin{array}{r}.05 \\
. .05 \\
.01\end{array}$ & $\begin{array}{l}.31 \\
.00 \\
+05\end{array}$ & $\begin{array}{l}.05 \\
.05 \\
.01\end{array}$ & .23 & $\begin{array}{l}.0 .4 \\
.01 \\
.01\end{array}$ \\
\hline $\begin{array}{l}\text { PFACT } \\
\text { FFACT }\end{array}$ & $3+49$ & $\begin{array}{r}14 \cdot 24 \\
4.59\end{array}$ & $2+52$ & $206+\frac{1}{3} \frac{2}{2}$ & $+\frac{4}{5} \cdot \frac{45}{9}$ & 9.96 \\
\hline IATA F & OF: FILE : & H4100 & & & & \\
\hline Fumb & $\begin{array}{r}4 I M n \\
265.0\end{array}$ & 60.0 & 305.92 & $\begin{array}{r}\text { Yㄷㄴ } \\
42.9\end{array}$ & & \\
\hline COMG & $0 \%$ & $\begin{array}{l}\text { cry } \\
.03\end{array}$ & $\begin{array}{l}C F 2 \\
.23\end{array}$ & .02 & Cry & $\begin{array}{c}\cos 2 \\
.02\end{array}$ \\
\hline $\begin{array}{l}\text { MAX: } \\
\text { MIMS }\end{array}$ & $\begin{array}{r}.62 \\
.10 \\
.10\end{array}$ & $\begin{array}{r}+17 \\
.07 \\
.04\end{array}$ & $\begin{array}{l}.50 \\
.01 \\
.08\end{array}$ & $\begin{array}{l}10 \\
.14 \\
+04\end{array}$ & $\begin{array}{l}.20 \\
.12 \\
.05\end{array}$ & $\begin{array}{l}.04 \\
.01 \\
.01\end{array}$ \\
\hline 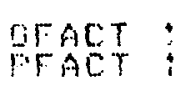 & 3.54 & 5.09 & $\frac{2}{4}+55$ & $\frac{7}{2} \cdot 3$ & $1 \frac{1}{3} \cdot \frac{53}{3}$ & $\frac{9}{5} \cdot 40$ \\
\hline
\end{tabular}

1 


\section{SELECTED DISTRIBUTION LIST}

Acurex Solar Corporation

485 Clyde Ave.

Mt. View, CA 94042

Mr. Don Duffy

Advanco Corporation 40701 Monterey Ave.

Palm Desert, CA 92260

Mr. Byron Washom

Arizona Public Service Company

P.O. Box 21666

Phoenix, AZ 85036

Mr. Eric Weber

Battelle Pacific NW Laboratory

P.O. Box 999

Richland, WA 99352

Dr. Ben Johnson

Mr. Tom A. Williams

Bechtel Corporation

P.O. Box 3965

San Francisco, CA 94119

Mr. Pascal DeLaquil

Black and Veatch Consulting Engineers

1500 Meadow Lake Parkway

Kansas City, MO 64114

Dr. Charles Grosskreutz

Brumleve, Mr. Tom

Consultant

1512 N. Gate Road

Walnut Creek, CA 94598

Dan-Ka Products, Inc.

3862 South Kalamath

Englewood, CO 80110

Mr. Daniel Sallis

Department of Civil Engineering

Colorado State University

Ft. Collins, CO 80523

Mr. John Peterka, Ph.D.

Professor, Civil Engineering
Department of Civil Engineering

University of Nebraska

60 th \& Dodge Street

Omaha, NE 68182-0178

Mr. Chris Tuan, Ph.D.

Department of Energy/ALO

P.O. Box 1500

Albuquerque, NM 87115

Mr. Dean Graves

Mr. Nyles Lackey

Department of Energy/HQ

Forrestal Building

1000 Independence Ave., SW

Washington, DC 20585

Dr. H. Coleman

Mr. S. Gronich

Mr. C. Mangold

Mr. M. Scheve

Mr. Frank Wilkins

Department of Energy/SAN

1333 Broadway

Oakland, CA 94536

Mr. Robert Hughey

Mr. William Lambert

Department of Energy/SAO

1617 Cole Blvd.

Golden, CO 80401

$\mathrm{Mr}$. Jerry Bellows

El Paso Electric

P.O. Box 982

El Paso, TX 79960

Mr. James E. Brown

Electric Power Research Institute

P.O. Box 10412

Palo Alto, CA 94303

Mr. Donald Augenstein

Entech, Incorporated

P.O. Box 612246

DFW Airport, TX 75261

Mr. Walter Hesse 
Georgia Institute of Technology

Atlanta, GA 30332

Mr. Tom Brown, Ph.D.

Jet Propulsion Laboratory

4800 Oak Grove Drive

Pasadena, CA 91109

Mr. William Owen

LaJet Energy Company

P.O. Box 3599

Abilene, TX 79604

Mr. Monte McGlaum

Luz Engineering Corp.

15720 Ventura Blvd.

Suite 504

Encino, CA 91436

Dr. David Kearney

Martin Marietta

P.O. Box 179

Denver, CO 80201

Mr. Tom Tracey

McDonnell Douglas Astronautics

Company

5301 Bolsa Ave.

Huntington Beach, CA 92647

Mr. Jim Rogan

Meridian Corporation

5113 Leesburg Pike

Suite 700

Falls Church, VA 22041

Mr. Dinesh Kumar

Mr. Jim Williamson

3M Corporation

3M Center Building, 207-1W-08

St. Paul, MN 55101

Mr. David Hill

Mr. Burton A. Benson

NASA Lewis Research Center

21000 Brookpark Road

Cleveland, $\mathrm{OH} 44135$

Dr. Dennis Flood
NASA-Johnson Space Center

NASA Road One - EPS

Houston, TX 77058

Mr. William Simon

Pacific Gas and Electric Company

3400 Crow Canyon Rd.

San Ramon, CA 94583

Mr. Gerry Braun

Rockwell International

Energy Systems Group

8900 DeSoto Ave.

Canoga Park, CA 91304

Mr. Tom H. Springer

Sandia National Laboratories

Solar Department 8453

Livermore, CA 94550

Mr. A Skinrood

Dr. R. A. Rinne

Sandia National Laboratories

Solar Energy Department 6220

P.O. Box 5800

Albuquerque, NM 87185

Mr. John Otts

Mr. James Leonard

Dr. Donald Schuler

Science Applications, Inc.

10401 Rosselle Street

San Diego, CA 92121

Dr. Barry Butler

Solar Energy Industries Association

1717 Massachusetts Ave. NW No. 503

Washington, DC 20036

Mr. Carlo La Porta

Mr. David Goren

Mr. Hal Seilstad

Solar Kinetics, Inc.

P.O. Box 47045

Dallas, TX 75247

Mr. Gus Hutchison 
Southern California Edison

2244 Walnut Grove Ave.

Rosemead, CA 91770

Mr. Joe Reeves

University of Arizona

Dept. of Mechanical and Aerospace

Engineering

Tucson, AZ 85721

Dr. Kumar Ramohalli

University of Arizona

Dept. of Electrical Engineering

Tucson, AZ 85721

Dr. Roger Jones

Solar Energy Research Institute

Mr. Bim Gupta

Dr. Dave Johnson

Dr. Larry Shannon

Mr. Al Lewandowski

Dr. L. M. Murphy 


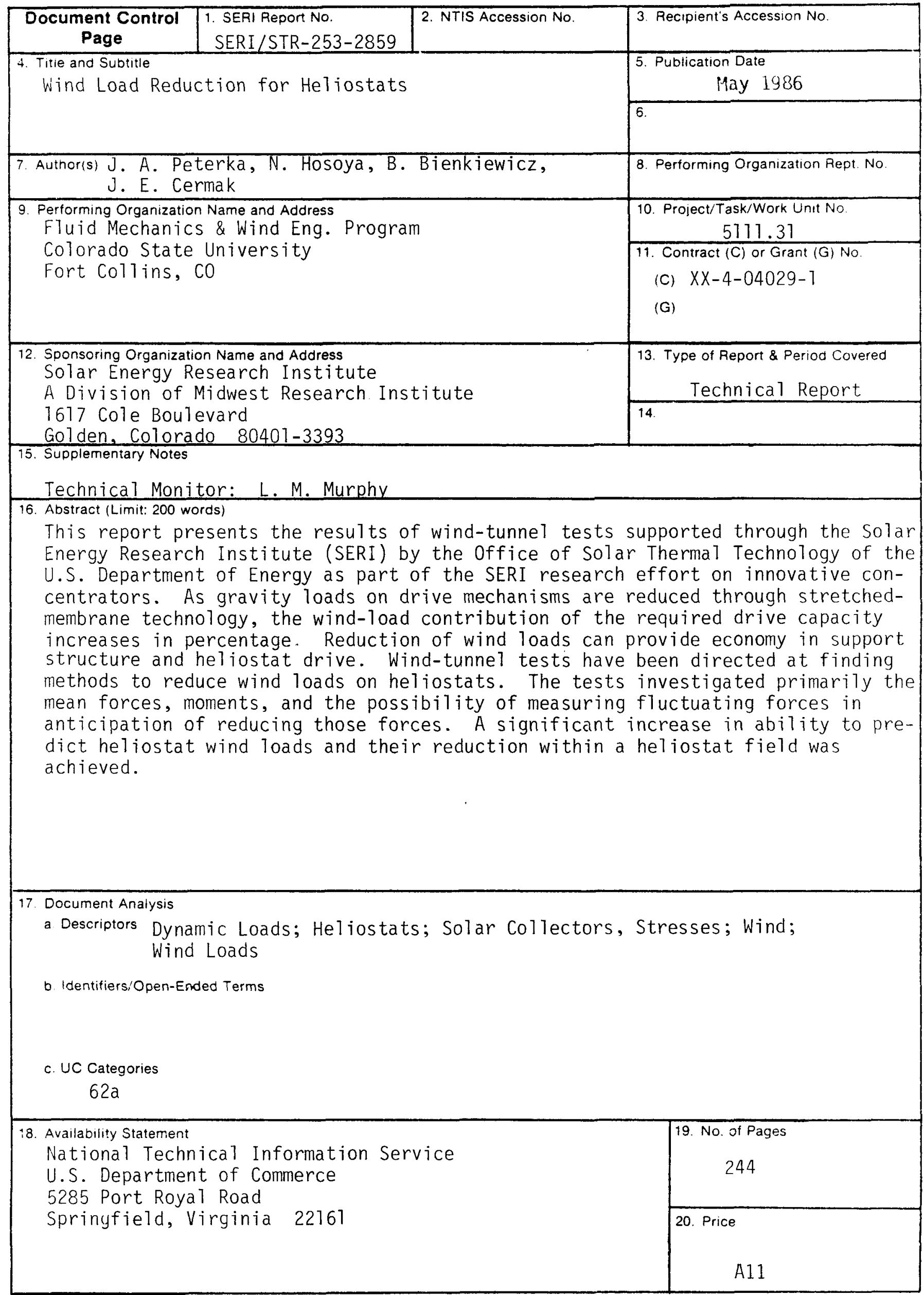

